*ak FMIS View/Frint Document Cover Sheet tow

This document was retrieved from the Documentation and Records Management (DRM) ISEARCH System. It is intended for Information only and may not be the most recent or updated version. Contact a Document Service Center (see Hanford Info for locations) if you need additional retrieval information.

Accession \#: D196111106

Document \#: WHC-SP-1181

Title/Desc:

MAINTENANCE \& OPERATIONS CONTRACTOR PLAN FOR TRANSITION TO THE PROJECT HANFORD MGMT CONTRACTOR [PHMC]

Pages: 267 


\title{
Maintenance and Operations Contractor Plan for Transition to the Project Hanford Management Contractor
}

\author{
M\&O Contractor Transition Team
}

Date Published

April 1996

Prepared for the U.S. Department of Energy Office of Emvironmental Restoration and Waste Management

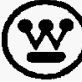

$$
\begin{array}{ll}
\text { Westinghouse } & \text { P.O Box } 1970 \\
\text { Hanford Company } & \text { Richland, Washington }
\end{array}
$$

Hanford Operations and Engineering Contractor for the U.S. Department of Energy under Contract DE-ACO6-87RL10930

Purpoes and Uee of Documemt This document was prepared for use within the U.S. Depertment of Ereigy and it: comectore. It is to be Uned only to perform, direct, of integrete work under U.S. Depertiment of Enemy oontrect:

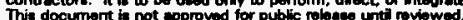

Pefent status This document copy, wince it is trenumitted in advance of patent clearence, in mede eveileble h conlidence eolely for use in performence of work under contrecte whis the U.S. Depertment of Eneray. This confidence, eolely for use in performance of work under contracte with the U.S. Depertmont of Eneroy. This document in not to be published nor its conternte otherwies disseminated or ueed for puppowes other then epecified

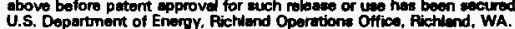


THIS PAGE INTENTIONALLY LEFT BLANK 
LEGAL DISCLAMMER

This report was propared as an account of work sponsored by en egency of the United Stetes Government. Neither the

United States Govarnment nor any egoney thereof, nor any of their employees, nor any of their contractors, subcontractors or their omployees, mekes any wartenty, express or implied, or assumes sny togal liability or responsibility for the accuracy, completeness, or any third party's use or the results of such use of any information, apparatus, product, or process disclosed, or represents that its use would not infringe privately owned rights. Roforence herein to any specific commercial product, process, or senvice by trede neme, trademark, manufecturer, of otherwise, does not necessarily constitute or imply its ondorsement, recommendation, or favoring by the United States Govermment or any agency thereof or its contractors or subcontractors. The viows and opinions of authors expressed herein do not nocesserily state or reflect those of the United States Government or any agency thereof.

This report has been reproduced from the best evailable capy. Available in paper copy and microfiche.

Aveitable to the U.S. Deparment of Enargy and its contractors from

Office of Scientific and Techrical Information

P.O. Box 62

Oak Ridge, TN 37831

(615) 576-8401

Primted in the Urixted States of America

DISCLM-3.CHP (1-91) 
THIS PAGE INTENTIONALLY

LEFT BLANK 


\section{RELEASE AUTHORIZATION}

Document Number: WHC-SP-1181, Rev. 0

Maintenance and Operations Contractor Plan for

Document Title: Transition to the Project Hanford Management Contractor

Release Date: $\quad 04 / 12 / 96$

This document was reviewed following the procedures described in WHC-CM-3-4 and is:

APPROVED FOR PUBLIC RELEASE

WHC Information Release Administration Specialist:

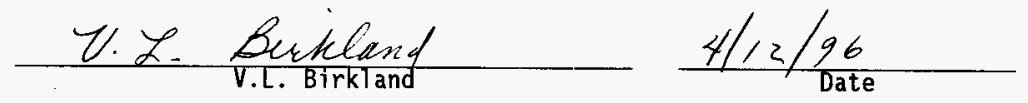





\section{TABLE OF CONTENTS}

1.0 INTRODUCTION $\ldots \ldots \ldots \ldots \ldots \ldots \ldots \ldots \ldots \ldots \ldots \ldots \ldots \ldots \ldots \ldots \ldots \ldots .1-1$

2.0 PLAN AND IMPLEMENTATION APPROACH $\ldots \ldots \ldots \ldots \ldots \ldots \ldots \ldots$

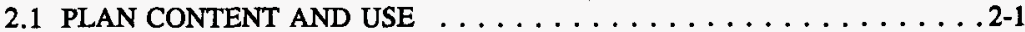

2.2 KEY ISSUES $\ldots \ldots \ldots \ldots \ldots \ldots \ldots \ldots \ldots \ldots \ldots \ldots \ldots \ldots \ldots \ldots \ldots \ldots .2-2$

2.3 ASSUMPTIONS $\ldots \ldots \ldots \ldots \ldots \ldots \ldots \ldots \ldots \ldots \ldots \ldots \ldots \ldots .2-3$

2.4 ACTION TRACKING AND RECORDKEEPING $\ldots \ldots \ldots \ldots \ldots \ldots 2-5$

2.5 COMPLETION VERIFICATION PROCESS $\ldots \ldots \ldots \ldots \ldots \ldots \ldots .2-6$

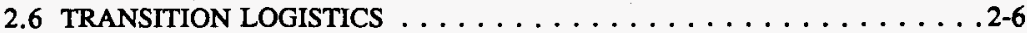

2.7 M\&O TRANSTTION MANPOWER AND COST ESTIMATE $\ldots \ldots \ldots \ldots 2-7$

2.8 TRANSITION SCHEDULE $\ldots \ldots \ldots \ldots \ldots \ldots \ldots \ldots \ldots \ldots . \ldots .7$

2.9 ACTION DESCRIPTIONS $\ldots \ldots \ldots \ldots \ldots \ldots \ldots \ldots \ldots \ldots . . \ldots \ldots$

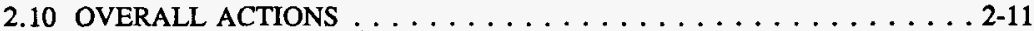

2.11 WHC CONTRACT CLOSEOUT ACTIVITIES $\ldots \ldots \ldots \ldots \ldots \ldots$ 2-12

3.0 MANAGEMENT AND INTEGRATION $\ldots \ldots \ldots \ldots \ldots \ldots \ldots \ldots \ldots$ 3-1

3.1 SITE PLANNING AND INTEGRATION $\ldots \ldots \ldots \ldots \ldots \ldots \ldots$.

3.1.1 Support to RL Strategic Planning . . . . . . . . . . . . 3-1

3.1.2 Project/Program Planning and Baseline Management . . . . . . . 3-1

3.1.3 Sitewide Systems Engineering . . . . . . . . . . . .

3.2 FINANCE AND ADMINISTRATION $\ldots \ldots \ldots \ldots \ldots \ldots \ldots \ldots . . \ldots \ldots$

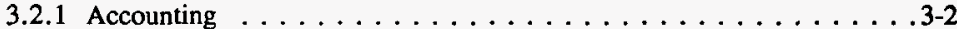

3.2.1.1 Accounts Receivables . . . . . . . . . . . . . . . 3-2

3.2.1.2 Department Integrated Standardized Core Accounting System (Discas) Interface for Plant and Equipment . . . . . 3-3

3.2.1.3 Transfer of General Ledger Accounts . . . . . . . . . . 3-3

3.2.1.4 Treasury . . . . . . . . . . . . . . . 3-3

3.2.1.5 Payroll Activities . . . . . . . . . . . . . 3-4

3.2.1.6 Tax Filings - City, County, Federal $\ldots \ldots \ldots \ldots$. . . . .

3.2.1.7 Fiscal Year-End - Closeout . . . . . . . . . . . . 3-4

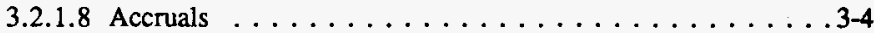

3.2.1.9 P-Cards . . . . . . . . . . . . . . . . 3-5

3.2.1.10 Travel . . . . . . . . . . . . . . 3-5

3.2.1.11 Work Authorization (Hanford Site) . . . . . . . . 3-6

3.2.1.12 Work For Others (Non-Hanford) $\ldots \ldots \ldots \ldots \ldots \ldots$. . . . . . .

3.2.1.13 Pensions . . . . . . . . . . . . . . . . 3-7

3.2.1.14 Savings Plans . . . . . . . . . . . . . . . . . . 3-7

3.2.1.15 Insurance Plans . . . . . . . . . . . . . . 3-7

3.2 .1 .16 Vacation . . . . . . . . . . . . . . . 3-8

3.2.1.17 Nuclear Materials Accounting $\ldots \ldots \ldots \ldots \ldots \ldots$ 3-8 


\section{TABLE OF CONTENTS (cont'd)}

3.2.2 Planning and Management Control Systems . . . . . . . . 3-8

3.2.2.1 Environmental Planning Data System . . . . . . . . . 3-8

3.2.2.2 Financial Data System . . . . . . . . . . . . . . . 3-9

3.2.2.3 Management Control System . . . . . . . . . . . 3-10

3.2.2.4 DOE Site and Performance Reporting . . . . . . . . 3-10

3.2.2.5 Project Analysis \& Reporting System . . . . . . . . . . 3-10

3.2.2.6 Miscellaneous Planning \& Reporting Databases . . . . . . 3-11

3.2.2.7 Overhead Rate Structure . . . . . . . . . . . 3-11

3.2.2.8 Financial Systems . . . . . . . . . . . . . . 3-12

3.3 HUMAN RESOURCES . . . . . . . . . . . . . . . . . . 3-12

3.3.1 Personnel Resources (WHC/BCSR) $\ldots \ldots \ldots \ldots \ldots \ldots \ldots$ 3-12

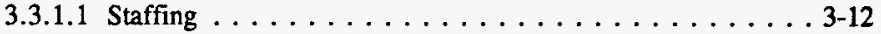

3.3.1.2 Site Access Support Services . . . . . . . . . . . 3-13

3.3.1.3 Outplacement . . . . . . . . . . . . . . . . . . 3-14

3.3.1.4 Personnel Records and Database Administration . . . . . . 3-14

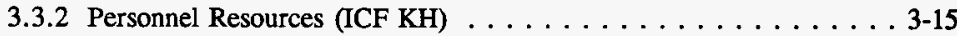

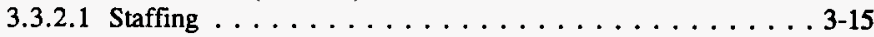

3.3.2.2 Site Access Support Services . . . . . . . . . 3-16

3.3.2.3 Outplacement . . . . . . . . . . . . . . . 3-16

3.3.2.4 Personnel Records and Database Administration . . . . . 3-17

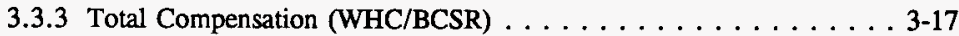

3.3.3.1 Salary Plan . . . . . . . . . . . . . . . . 3-17

3.3.3.2 Workers Compensation . . . . . . . . . . 3-18

3.3.3.3 Job Classifications and Salary Structure . . . . . . . 3-18

3.3.3.4 Performance Appraisals . . . . . . . . . . . . . . . . . 3-19

3.3.3.5 Benefits Administration . . . . . . . . . . . . 3-20

3.3.3.6 Return-to-Work . . . . . . . . . . . . . . . . 3-22

3.3.3.7 Pension and Savings . . . . . . . . . . . . . 3-23

3.3.3.8 Recognition Programs . . . . . . . . . . . . . . . . 3-24

3.3.3.9 Other . . . . . . . . . . . . . . . . 3-24

3.3.4 Total Compensation (ICF KH) $\ldots \ldots \ldots \ldots \ldots \ldots \ldots \ldots . . \ldots \ldots .24$

3.3.4.1 Salary Plan . . . . . . . . . . . . . . 3-24

3.3.4.2 Other . . . . . . . . . . . . . . . 3-25

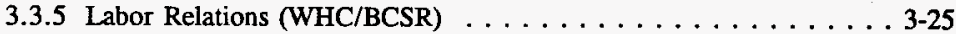

3.3.5.1 Collective Bargaining Agreements . . . . . . . . 3-25

3.3.5.2 Bargaining Unit Leadership/Demographics . . . . . . . 3-26

3.3.5.3 Notifications . . . . . . . . . . . . . . . . . 3-27

3.3.5.4 Grievance and Arbitration History/Caseload . . . . . . . . 3-27

3.3.6 Labor Relations (ICF KH) . . . . . . . . . . . . . . . 3-27

3.3.6.1 Collective Bargaining Agreements . . . . . . . . 3-28

3.3.6.2 Bargaining Unit Leadership/Demographics . . . . . . . . 3-29

3.3.6.3 Notifications . . . . . . . . . . . . . . . . . 3-29

3.3.6.4 Grievance and Arbitration History/Caseload . . . . . . . 3-29 


\section{TABLE OF CONTENTS (cont'd)}

3.3.7 Workforce Development and Diversity (WHC/BCSR) . . . . . 3-30

3.3.7.1 EEO/AAP . . . . . . . . . . . . . . 3-30

3.3.7.2 Diversity Recognition and Training . . . . . . . . . . 3-31

3.3.7.3 Educational Reimbursement Programs . . . . . . . . 3-31

3.3.7.4 Development Training . . . . . . . . . . . . 3-32

3.3.7.5 Education Enrichment Programs . . . . . . . . . 3-32

3.3.7.6 Graduate Rotational Program . . . . . . . . . . . 3-33

3.3.8 Workforce Development and Diversity (ICF KH) . . . . . 3-33

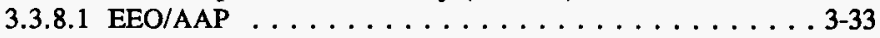

3.3.8.2 Diversity Recognition and Training . . . . . . . 3-34

3.3.8.3 Education Enrichment Programs . . . . . . . . . . 3-34

3.3.9 General Operations . . . . . . . . . . . . . . . . . 3-34

3.3.9.1 Transferring Vacation Hours for Family Medical

Emergencies . . . . . . . . . . . . . . . 3-34

3.3.9.2 Employee Work Schedules . . . . . . . . . . . . 3-35

3.3.9.3 Salary Continuance During Absences . . . . . . . . 3-35

3.3.9.4 Vacation and Holidays . . . . . . . . . . . . 3-36

3.3.9.5 Salaried Nonexempt Workforce Adjustment . . . . . . . 3-36

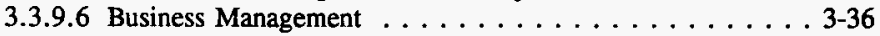

3.3.10 Re-engineering Support . . . . . . . . . . . . . . 3-37

3.4 EMPLOYEE CONCERNS $\ldots \ldots \ldots \ldots \ldots \ldots \ldots \ldots \ldots$. . . . . . . . . . . . .

3.5 ENVIRONMENTAL, SAFETY, QUALITY, AND HEALTH . . . . . . 3-38

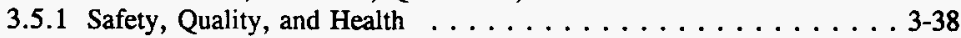

3.5.1.1 Program Management . . . . . . . . . . . . . . 3-38

3.5.1.2 Safety . . . . . . . . . . . . . . . . . 3-40

3.5.1.3 Radiological Control (RadCon) . . . . . . . . . . . . 3-43

3.5.1.4 Quality Assurance . . . . . . . . . . . . . 3-48

3.5.1.5 Compliance Assurance . . . . . . . . . . . . . . 3-51

3.5.2 Work Control . . . . . . . . . . . . . . . . . . . 3-51

3.5.3 Environmental Compliance . . . . . . . . . . . . . . . 3-52

3.5.3.1 Hanford Environmental Management Program . . . . . . 3-53

3.5.3.2 Reporting . . . . . . . . . . . . . . . . 3-63

3.5.3.3 Environmental Requirements Management . . . . . . . . 3-64

3.5.3.4 Non-Tri-Party Agreement Compliance Agreement . . . . . 3-64

3.5.3.5 Outsources . . . . . . . . . . . . . . 3-66

3.5.4 Tri-Party Agreement . . . . . . . . . . . . . . 3-66

3.5.5 Safety Analysis and Nuclear Engineering . . . . . . . . 3-68

3.5.6 Occupational Health Services . . . . . . . . . . . . . . 3-69

3.5.7 Centralized Occurrence Reporting . . . . . . . . . . . . 3-69

3.6 PROCUREMENT AND MATERIALS MANAGEMENT $\ldots \ldots \ldots \ldots$ 3-71

3.6.1 Procurement . . . . . . . . . . . . . . 3-71

3.6.1.1 Open Audit/Surveillance Items . . . . . . . 3-71 


\section{TABLE OF CONTENTS (cont'd)}

3.6.1.2 Identify Funding Reserves/Contingency Needs for Close Out Process . . . . . . . . . . . . . . . 3-71

3.6.1.3 Purchase Order Assignment/Transfer to One or More New Companies . . . . . . . . . . . . . . . . 3-72

3.6.1.4 Purchase Orders With Open Concerns . . . . . . . . 3-72

3.6.1.5 Purchase Orders in Termination Process . . . . . . . 3-72

3.6.1.6 Purchase Orders With Open Options . . . . . . . . . 3-73

3.6.1.7 Open Procurement Actions Beyond Transition . . . . . 3-73

3.6.1.8 Open Solicitations . . . . . . . . . . . . 3-74

3.6.1.9 Purchase Order Terms and Conditions . . . . . . . 3-74

3.6.1.10 Actions with DOE (RL \& HQ) . . . . . . . . . 3-75

3.6.1.11 Subcontractor Property Activities . . . . . . . . 3-75

3.6.1.12 Software License Agreements . . . . . . . . . . . 3-75

3.6.1.13 Procurement Reference Material . . . . . . . . . . 3-75

3.6.1.14 Automation System Information $\ldots \ldots \ldots \ldots \ldots$. . . . . . .

3.6.1.15 Outreach Activities . . . . . . . . . . . . . . 3-76

3.6 .2 Property Management . . . . . . . . . . . . . . 3-76

3.6.2.1 Capital and Sensitive Property . . . . . . . . . 3-76

3.6.2.2 Other Financially Controlled Inventories . . . . . . . . . 3-77

3.6.2.3 Export Control and High Risk Property . . . . . . . 3-77

3.6.2.4 Activities with DOE (RL \& HQ) . . . . . . . . . 3-77

3.6.2.5 Property Reference Material . . . . . . . . . . . 3-77

3.6.3 Economic Transfer . . . . . . . . . . . . . . . . 3-77

3.6.3.1 Intellectual Property . . . . . . . . . . . . . 3-77

3.6.3.2 Loan agreements, CRADAs and MOUs activities . . . . 3-78

3.6.3.3 Patent, Copyright, Royalty, and Invention activities . . . . 3-78

3.6.3.4 Asset Conversion Activities . . . . . . . . . . . . 3-78

3.6.3.5 Activities with DOE (RL \& HQ) . . . . . . . . . 3-78

3.6.4 Materials Management . . . . . . . . . . . . . . . . . 3-79

3.6.4.1 P-Card Assignment/Transfer to One or More New

Companies . . . . . . . . . . . . . . 3-79

3.6.4.2 Surplus Sales Assignment/Transfer to One or More New Companies . . . . . . . . . . . . . . . . . 3-79

3.6.4.3 Warehouse Consolidation Plan (gas dock elimination, surplus material move into 1163 and configuration of 1167 into excess store) . . . . . . . . . . . . . . 3-79

3.6.4.4 General Services Administration (GSA) Waiver Request . . 3-80

3.6.4.5 Activities with DOE (RL \& HQ) . . . . . . . . 3-80

3.6.4.6 Materials Management Reference Materials . . . . . . . 3-80

3.7 RE-ENGINEERING $\ldots \ldots \ldots \ldots \ldots \ldots \ldots \ldots \ldots \ldots \ldots \ldots$ 3-80

3.8 TECHNOLOGY MANAGEMENT $\ldots \ldots \ldots \ldots \ldots \ldots \ldots \ldots \ldots$ 3-81

3.8.1 National Technology Development Program (EM-50) . . . . . . 3-82 


\section{TABLE OF CONTENTS (cont'd)}

3.8.2 Technology Integration (Implementation), Site Technology

Coordination . . . . . . . . . . . . . . . . 3-83

3.9 GENERAL COUNSEL $\ldots \ldots \ldots \ldots \ldots \ldots \ldots \ldots \ldots \ldots \ldots$ 3-84

3.9.1 Litigation and Claims . . . . . . . . . . . . . . 3-84

3.9.2 Transition Agreement $\ldots \ldots \ldots \ldots \ldots \ldots \ldots \ldots \ldots \ldots$ 3-85

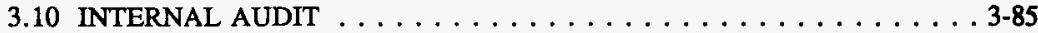

3.11 WORK FOR OTHERS $\ldots \ldots \ldots \ldots \ldots \ldots \ldots \ldots \ldots \ldots \ldots \ldots \ldots$

3.11.1 Support to EM-40 Headquarters . . . . . . . . . . . 3-86

3.11.2 Support to EM - Lease and Administration of Contractor Support

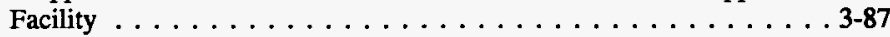

3.12 OTHER MANAGEMENT AND INTEGRATION ACTIVITIES $\ldots \ldots \ldots$ 3-88

3.12.1 Pollution Prevention/Waste Minimization . . . . . . . . . 3-88

3.12.2 U.S. Department of Energy Directives . . . . . . . . . . 3-89

3.12.3 Hanford PeopleCORE . . . . . . . . . . . . . . . 3-90

4.0 HANFORD PROJECTS $\ldots \ldots \ldots \ldots \ldots \ldots \ldots \ldots \ldots \ldots . \ldots \ldots$ 4-1

4.1 TANK WASTE REMEDIATION SYSTEM $\ldots \ldots \ldots \ldots \ldots \ldots \ldots$. $\ldots \ldots$

4.1.1 Management Systems . . . . . . . . . . . . . 4-2

4.1.2 Tank Farm Operations - Evaporator . . . . . . . . . 4-3

4.1.3 Tank Farm Operations - 200 East Tank Farms . . . . . . . . . 4-5

4.1.4 Tank Farm Operations - 200 West Tank Farms . . . . . . . . 4-8

4.1.5 Safety Issue Resolution/TWRS Engineering . . . . . . . . 4-11

4.1.6 Waste Characterization . . . . . . . . . . . . . 4-13

4.1.6.1 Waste Characterization - Technical Planning, Data

Evaluation, and Data Management Activities . . . . . . 4-13

4.1.6.2 Waste Characterization - Operations (Field) Activities . . 4-15

4.1.7 Waste Disposal Program . . . . . . . . . . . . . . 4-17

4.2 WASTE MANAGEMENT $\ldots \ldots \ldots \ldots \ldots \ldots \ldots \ldots \ldots$ 4-18

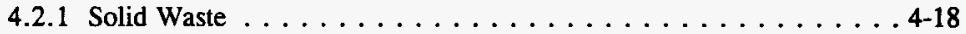

4.2.2 Liquid Effluents Program . . . . . . . . . . . . . 4-24

4.2.2.1200 Area Treated Effluent Disposal Facility . . . . . . . 4-25

4.2.2.2 Liquid Effluent Retention Facility . . . . . . . . . . . 4-27

4.2.2.3 200 Area Effluent Treatment Facility . . . . . . . . 4 4-29

4.2.2.4 300 Area Treated Effluent Disposal Facility . . . . . . . 4-32

4.2.2.5 340 Waste Handling Facility . . . . . . . . . . 4-34

4.3 SPENT NUCLEAR FUEL $\ldots \ldots \ldots \ldots \ldots \ldots \ldots \ldots \ldots \ldots .4 .36$

4.3.1 Spent Nuclear Fuel Project Integration $\ldots \ldots \ldots \ldots \ldots \ldots .4$ 4-37

4.3.2 Spent Nuclear Fuel Systems Integration . . . . . . . . . . . 4-38

4.3.3 K Basins Maintenance and Operations . . . . . . . . . . 4-39

4.3.4 K Basin Material Removal and Clean-Up Project . . . . . . . . . 4 4-39

4.3.5 Multi-Canister Overpack (MCO) Acquisition . . . . . . . . 4-40

4.3.6 Cask/Transportation System . . . . . . . . . . . . . 4-40

4.3.7 Canister Storage Building $\ldots \ldots \ldots \ldots \ldots \ldots \ldots .4 .41$ 


\section{TABLE OF CONTENTS (cont'd)}

4.3.8 Fuel Conditioning Processes . . . . . . . . . . . . . . 4-42

4.3.9 Other Hanford Fuel Retrieval Processing and Storage . . . . . . 4-42

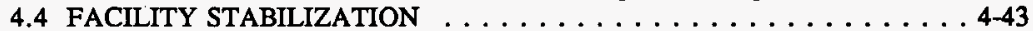

4.4 .1 PUREX $\ldots \ldots \ldots \ldots \ldots \ldots \ldots \ldots \ldots \ldots \ldots . \ldots \ldots 44$

4.4.2 Plutonium Finishing Plant $\ldots \ldots \ldots \ldots \ldots \ldots \ldots \ldots \ldots \ldots$

4.4.3 Fast Flux Test Facility . . . . . . . . . . . . . . 4-48

4.4.4 B Plant/Waste Encapsulation and Storage Facility . . . . . . . 4 4-50

4.4.5 300/400 Area Fuel Fabrication Facilities and 400 Area Fuels and

Materials Examination Facility . . . . . . . . . . . . 4-52

4.4.6 Transfer of 324 and 327 Building PNNL Facilities . . . . . . . 4-54

4.4.7 Materials and Future Transition Programs . . . . . . . . 4-57

4.5 ENVIRONMENTAL RESTORATION (ER) (INTEGRATION) $\ldots \ldots \ldots$. . .58

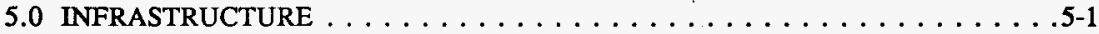

5.1 INFRASTRUCTURE OPERATIONS AND MAINTENANCE $\ldots \ldots \ldots 5-1$

5.1.1 Facility Acquisition, Operations, and Maintenance $\ldots \ldots \ldots \ldots$. . . .

5.1 .2 Utilities . . . . . . . . . . . . . . . . . .5-4

5.1.2.1 Steam Plant Operations . . . . . . . . . . . 5-4

5.1 .2 .2300 Area Water/Sewer Operations $\ldots \ldots \ldots \ldots \ldots .5$

5.1 .2 .3200 Area Water/Sewer Operations . . . . . . . 5-6

5.1.2.4 Site Electrical Systems . . . . . . . . . . . . . 5-7

5.1.3 Transportation Infrastructure and Services $\ldots \ldots \ldots \ldots \ldots .5$. . . . .

5.1.4 Fire and Emergency Response $\ldots \ldots \ldots \ldots \ldots \ldots \ldots$. . . . . . .

5.1.4.1 Fire Department Emergency Services . . . . . . . . . 5-11

5.1.4.2 Fire Systems Testing and Emergency Response . . . . 5 5-11

5.1.4.3 Services Provided to Others . . . . . . . . . . 5-11

5.1.4.4 Other - New RL Work Scope Planned for the HFD . . . . 5-12

5.1.4.5 Spccialized Training for Emergency Response Fire

Department Personnel . . . . . . . . . . . . . . 5-12

5.1 .4 .6 Fire Prevention . . . . . . . . . . . . . 5-12

5.1.4.7 Fire Systems Maintenance . . . . . . . . . . . 5-12

5.1.4.8 Respiratory Protection Equipment Services . . . . . . . 5-12

5.1.4.9 Contracts for Service and Purchase Requisitions that

Extend Past October 1, 1996 . . . . . . . . . . . 5-12

5.1.4.10 Emergency Services Facilities, Buildings, and Capital

Projects for Replacement . . . . . . . . . . . . . 5-13

5.1 .5 Fabrication Shops $\ldots \ldots \ldots \ldots \ldots \ldots \ldots \ldots \ldots$. . . . . . . . . .

5.1.6 Integrated Pest Management Services . . . . . . . . . . 5-17

5.1.7 Non-Radioactive/Non-Dangerous Waste Handling . . . . . . . . 5-18

5.1 .8 Site Crane \& Rigging Services $\ldots \ldots \ldots \ldots \ldots \ldots \ldots \ldots .5$ 5-20

5.1 .9 Landlord Program . . . . . . . . . . . . . . . . 5-22

5.1.10 Resource and Energy Management . . . . . . . . . . 5-23 


\section{TABLE OF CONTENTS (cont'd)}

5.2 REAL ESTATE AND MATERIAL MANAGEMENT $\ldots \ldots \ldots \ldots \ldots$ 5-24

5.2.1 Land-Use Planning and Management . . . . . . . . . . . 5-24

5.2.2 Calibration and Engineering Laboratories . . . . . . . . 5-26

5.2.2.1 Engineering Laboratories . . . . . . . . . . . . 5-26

5.2.2.2 Calibration Laboratory (AKA Standards Laboratory . . . . 5 5-27

5.3 INFORMATION AND COMMUNICATION $\ldots \ldots \ldots \ldots \ldots \ldots \ldots$ 5-28

5.3 .1 Operations and Support . . . . . . . . . . . . . 5-28

5.3.2 Information and Scientific Systems . . . . . . . . . . . . 5-29

5.3.3 President's Office/Technical Staff/Business Management . . . . 5-30

5.3.4 Documentation and Records Management . . . . . . . . . 5-30

5.3.5 Media Management Systems . . . . . . . . . . . . . 5-31

5.3.6 Telecommunications and Network Engineering $\ldots \ldots \ldots \ldots \ldots$. . . . . .

5.3.7 Baseline Summary Outline . . . . . . . . . . . . 5-33

5.3.8 Hanford Information System Inventory $\ldots \ldots \ldots \ldots \ldots \ldots .5 .34$

5.3.9 Data Management and Standards . . . . . . . . . . 5-35

6.0 OTHER CROSSCUTTING SERVICES $\ldots \ldots \ldots \ldots \ldots \ldots \ldots \ldots$. . . . . . .

6.1 SAFEGUARDS AND SECURITY $\ldots \ldots \ldots \ldots \ldots \ldots \ldots \ldots \ldots$ 6-1

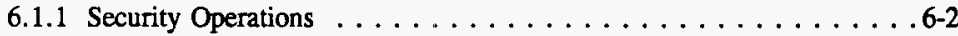

6.1 .2 Protective Forces . . . . . . . . . . . . . . . 6-3

6.1 .3 Information Security . . . . . . . . . . . . . . 6-3

6.1.4 Personnel Security and Central Badging . . . . . . . . . .

6.1.5 Protection Program Management . . . . . . . . . . . . 6-5

6.1.6 Material Control and Accountability . . . . . . . . 6-5

6.1 .7 Technical Security . . . . . . . . . . . . . . . 6-6

6.2 HANFORD ANALYTICAL SERVICES PROGRAM $\ldots \ldots \ldots \ldots \ldots 6-6$

6.2.1 Sample Management Office . . . . . . . . . . . . 6-7

6.2.2 High Level Analytical Services . . . . . . . . . . . . . 6-8

6.2.3 Low-Level Analytical Services . . . . . . . . . . . . . . 6-10

6.3 HANFORD SITE TRAFFIC MANAGER $\ldots \ldots \ldots \ldots \ldots \ldots \ldots$ 6-12

6.3.1 Hanford Site Transportation and Packaging Support . . . . . 6 6-12

6.3.2 DOE Headquarters EM Transportation Program Support . . . . . 6-14

6.3.3 Transportation and Packaging Work For Others Services . . . . 6-15

6.4 ARCHITECT ENGINEER/CONSTRUCTION MANAGEMENT . . . . . 6-16

6.4.1 Architect Engineer Services . . . . . . . . . . . . . . . . 6-17

6.4 .2 Construction Management Services . . . . . . . . . . . . . . 6-19

6.5 CONSTRUCTION PROJECT MANAGEMENT . . . . . . . . 6-21

6.6 ENVIRONMENTAL MONITORING AND SERVICES $\ldots \ldots \ldots \ldots .6-23$

6.6.1 Hanford Technical Services . . . . . . . . . . . . 6-2

6.6.1.1 RCRA Operational Monitoring Office . . . . . . . 6-

6.6.1.2 Geosciences . . . . . . . . . . . . . 6-23

6.6.1.3 Well Services . . . . . . . . . . . . . . 6-25

6.6.1.4 Near Field Monitoring . . . . . . . . . . 6-26 


\section{TABLE OF CONTENTS (cont'd)}

6.6.1.5 Geophysics Investigations . . . . . . . . . . . 6 6-27

6.6.1.6 Environmental Operations Sampling . . . . . . . 6-28

6.6.1.7 Special Projects . . . . . . . . . . . . . 6-29

6.6.2 CERCLA Monitoring . . . . . . . . . . . . 6-30

6.6.3 Groundwater Surveillance Monitoring Project $\ldots \ldots \ldots \ldots \ldots 6-31$

6.7 EXTERNAL/INTERNAL COMMUNICATIONS . . . . . . . 6-32

6.7.1 Interface with Site Communications/Public Affairs . . . . . . 6-32

6.7.2 Integration of WHC/ICF KH/BCSR Program Components Into the PHMC Program . . . . . . . . . . . . 6-32

6.7.3 Tri-Party Agreement Community Relations Plan . . . . . . . . 6-32

6.7.4 Introduce the PHMC Personnel to Hanford's External Publics . . . 6-33

6.7.5 Provide Employees With Necessary Information During Transition . 6-33

6.7.6 U.S. Department of Energy "Hanford Reach" and "DOE This Month" Communications . . . . . . . . . . . . . 6-34

6.7.7 Hanford Site Emergency Preparedness Communications . . . . . 6-35

6.7.8 WHC/ICF Kaiser/BCSR Farewell Activities . . . . . . . 6-35

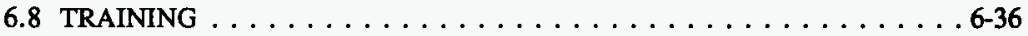

6.8.1 Training Services . . . . . . . . . . . . . 6-36

6.8.2 Hazardous Materials Management and Emergency Response . . . . 6-38

6.9 EMERGENCY PREPAREDNESS . . . . . . . . . . . . . . 6-39

6.9 .1 Emergency Management . . . . . . . . . . . . . 6-39

6.9.2 Radiological Assistance Program . . . . . . . . . . . 6-41

6.9.3 ESH Management Plan . . . . . . . . . . . . . 6-42

6.10 DIRECT SUPPORT TO RL $\ldots \ldots \ldots \ldots \ldots \ldots \ldots \ldots \ldots \ldots$ 
WHC-SP-1181

TABLE OF CONTENTS (cont'd)

FIGURE

2-1. Project Hanford Management Contractor Transition Schedule . . . . . . . . . 2-8

TABLE

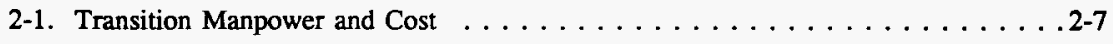




\section{ACRONYMS}

AAP

ADA

ADS

A-E

AOP

ASME

ASNT

ATP

BAT/AKART

$\mathrm{BCC}$

BCSR

BEMR

BHI

BOE

CAA

CAMB

CAMS

CAP

CDMP

CDR

CERCLA

CFR

CM

CMM

COE

$\mathrm{COO}$

CSB

CSG

CWA

CWC

D\&D

DNFSB

DOE

DOT

DQO

DST
Affirmative Action Plan

Americans With Disabilities

Activity Data Sheet

Architect-Engineer

Air Operating Permit

American Society of Mechanical Engineers

American Society for Nondestructive Testing

Acceptance Test Procedure

Best Available Technology/All Known And Reasonable

Treatment

Baseline Change Control

BCS Richland, Inc.

Baseline Environmental Management Report

Bechtel Hanford, Inc.

basis of estimate

Clean Air Act

Corrective Action Management Board

Corrective Action Management System

Cost Account Plan

Completed Decision Making Package

Conceptual Design Report

Comprehensive Environmental Response Compensation and

Liability Act

Code of Federal Regulations

construction management

Central Milestone Module

center of expertise

Conduct of Operations

Canister Storage Building

Central Support Group

Clean Water Act

Central Waste Complex

Decontamination and Decommissioning

Defense Nuclear Facilities Safety Board

U.S. Department of Energy

Department of Transportation

data quality objective

double-shell tank 


\section{ACRONYMS (cont'd)}

\begin{tabular}{|c|c|}
\hline EA & environmental assessment \\
\hline $\mathrm{ECN}$ & Engineering Change Notice \\
\hline Ecology & Washington State Department of Ecology \\
\hline ECP & Employee Concerns Program \\
\hline EEO & Equal Employment Opportunity \\
\hline EEOC & Equal Employment Opportunity Commission \\
\hline EIS & Environmental Impact Statement \\
\hline EL & Engineering Laboratories \\
\hline EM & Environmental Management \\
\hline EMP & Effluent Monitoring Plan \\
\hline EMT & Emergency Medical Technician \\
\hline EPA & Environmental Protection Agency \\
\hline EPCRA & Emergency Planning and Community Right-To-Know Act \\
\hline EPDS & Environmental Planning Data System \\
\hline ER & environmental restoration \\
\hline ERC & Environmental Restoration Contractor \\
\hline ERDF & Environmental Restoration Disposal Facility \\
\hline ESH & Environmental, Safety, and Health \\
\hline ETF & Effluent Treatment Facility \\
\hline ETS & Environmental Tracking System \\
\hline FDS & Financial Data System \\
\hline FEMP & Facility Effluent Monitoring Plan \\
\hline FFCA & Federal Facilities Compliance Agreement \\
\hline FFTF & Fast Flux Test Facility \\
\hline FMCS & Federal Mediation and Conciliation Service \\
\hline FMEF & Fuels and Materials Examination Facility \\
\hline FSM & Fire Systems Maintenance \\
\hline FTE & full-time equivalent \\
\hline FY & fiscal year \\
\hline GSA & General Services Administration \\
\hline GTR & Government Transportation Request \\
\hline GWM & Groundwater Management \\
\hline HAMMER & Hazardous Materials Management and Emergency Respon \\
\hline HAMTC & Hanford Atomic Metal Trades Council \\
\hline HASP & Hanford Analytical Services Program \\
\hline HATS & Hanford Action Tracking System \\
\hline HDPE & High-Density Polyethylene \\
\hline HEHF & Hanford Environmental Health Foundation \\
\hline HEIS & Hanford Environmental Information System \\
\hline HEMP & Hanford Environmental Management Program \\
\hline
\end{tabular}




\section{ACRONYMS (cont'd)}

\begin{tabular}{|c|c|}
\hline HFD & Hanford Fire Department \\
\hline HGIS & Hanford Geographic Information System \\
\hline HISI & Hanford Information System Inventory \\
\hline HLW & High-Level Waste \\
\hline HRC & Human Rights Commission \\
\hline HRP & Human Reliability Program \\
\hline HSRCM & Hanford Site Radiological Control Manual \\
\hline HSIS & Hanford Site Integrated Schedule \\
\hline HSSA & Hanford Site Stabilization Agreement \\
\hline HTS & Hanford Tracking System \\
\hline HQ & Headquarters \\
\hline IAEA & International Atomic Energy Agency \\
\hline ICF KH & ICF Kaiser Hanford \\
\hline IMUST & Inactive Miscellaneous Underground Tank \\
\hline INEL & Idaho National Engineering Laboratory \\
\hline IPC & Integration Project Coordinator \\
\hline IROF & Involuntary Reduction of Force \\
\hline ISS & Information and Scientific Systems \\
\hline JCS & Job Control System \\
\hline $\mathrm{JIC}$ & Joint Information Center \\
\hline LERF & Liquid Effluent Retention Facility \\
\hline LLBG & Low-Level Burial Ground \\
\hline LLMW & Low-Level Mixed Waste \\
\hline LLW & Low-Level Waste \\
\hline LP & Landlord Program \\
\hline $\mathrm{M} \& \mathrm{O}$ & Maintenance and Operations \\
\hline MC\&A & Material Control and Accountability \\
\hline MCO & Multi-Canister Overpack \\
\hline MCS & Management Control System \\
\hline MDD & Mission Direction Document \\
\hline MIP & Management Integration Plan \\
\hline MMS & Media Management Systems \\
\hline MOU & Memorandum of Understanding \\
\hline MRP & Management Requirements and Procedures \\
\hline MSDS & Material Safety Data Sheet \\
\hline MSA & Major System Acquisition \\
\hline MT & Magnetic Particle Testing \\
\hline MVT & Master Validation Table \\
\hline MYPP & Multi-Year Program Plan \\
\hline
\end{tabular}




\section{ACRONYMS (cont'd)}

NDA

NDE

NESHAP

NEx

NFPA

NIOSH

NIST

NLRB

NPDES

NRDWSF

NTS

OFCCP

OMP

ONC

OPC

OPEIU

ORPS

ORR

P\&E

PA

PAAA

PAPR

PAR

PFP

PHMC

PNNL

POC

PPA

PPM

PRTR

PSAP

PT

PTS

PUREX

QA

QC/NDT

R\&EM

RAP

RCRA
Nondestructive Assay

Nondestructive Examination

National Emission Standards for Hazardous Air Pollutants

Non-Exempt

National Fire Protection Association

National Institute of Safety and Health

National Institute of Standards and Technology

National Labor Relations Board

National Pollutant Discharge Elimination System

Nonradioactive Dangerous Waste Storage Facility

Noncompliance Tracking System

Office of Federal Contract Compliance Program

Operations and Maintenance Program

Occurrence Notification Center

Other Project Costs

Office of Professional Employees International Union

Occurrence Reporting Processing System

Official Readiness Review

Plant and Equipment

Performance Assessment:

Price Anderson Amendment Act

Powered Air Purifying Respirator

Project Analysis and Reporting System

Plutonium Finishing Plant

Project Hanford Management Contractor

Pacific Northwest National Laboratory

Point-of-Contact

Property Protected Area

Protection Program Management

Plutonium Recycle Test Reactor

Personnel Security Assurance Program

Penetrant Testing

Progress Tracking System

Plutonium-Uranium Extraction (Facility)

Quality Assurance

Quality Control and Nondestructive Technicians

Resource and Energy Management

Radiological Assistance Program

Resource Conservation and Recovery Act 


\section{ACRONYMS (cont'd)}

RDA

RDS

RFP

RIDS

RL

ROC

ROF

RMW

ROM

RT

SAIC

SALDS

SARP

SAS

SC\&RS

SCBA

SEP

SFS

SID

SL

SMO

SMP

SMS

SNF

SNM

SSPP

SST

STCG

SW

SWD

TBP

TEDF

TEC

TPA

TPAI

TPC

TRS

TRU

TRUM

TRUPACT

TRUSAF

TSD
Re-Deployment Assistance

Risk Data Sheet

Request For Proposal

Records Inventory and Disposition Schedules

U.S. Department of Energy-Richland Operations Office

Re-Employment Opportunity Center

Reduction of Force

Radioactive Mixed Waste

RCRA Operational Monitoring

Radiographic Testing

Science Applications International Corporation

State-Approved Land Disposal Site

Safety Analysis Report for Packaging

Safeguards and Security

Site Crane and Rigging Services

Self-Contained Breathing Apparatus

Safety Evaluation for Packaging

Site Fabrication Services

Standard Generalized Markup Language

Standards Laboratory

Sample Management Office

Software Management Plan

Site Management System

Spent Nuclear Fuel

Special Nuclear Material

Site Support Program Plan

Single-Shell Tank

Site Technical Coordination Group

Solid Waste

Solid Waste Disposal

Tri Butyl Phosphate

Treated Effluent Disposal Facility

Total Estimated Cost

Tri-Party Agreement

Tri-Party Agreement Integration

Total Project Cost

Training Services

Transuranic

Transuranic Mixed

Transuranic Package Transporter

Transuranic Assay and Storage Facility

Treatment, Storage, and Disposal 


\section{ACRONYMS (cont'd)}

TTP

TWRS

VA

VLDPE

VPP

WAC

WBS

WDOH

WEC

WESF

WHC

WIDS

WIPP

WRAP

WSCF
Technical Task Plan

Tank Waste Remediation System

Vulnerability Assessment

Very Low-Density Polyethylene

Voluntary Protection Plan

Washington Administrative Code, or

Waste Acceptance Criteria

Work Breakdown Structure

Washington State Department of Health

Westinghouse Electric Corporation

Waste Encapsulation and Storage Facility

Westinghouse Hanford Company

Waste Identification Data System

Waste Isolation Pilot Plant

Waste Receiving and Processing Module

Waste Sampling and Characterization Facility 
WHC-SP-1181

This page intentionally left blank. 


\subsection{INTRODUCTION}

This plan has been developed by Westinghouse Hanford Company (WHC), and its subcontractors ICF Kaiser Hanford (ICF KH) and BCS Richland, Inc. (BCSR), at the direction of the U.S. Department of Energy (DOE), Richland Operations Office (RL). WHC and its subcontractors are hereafter referred to as the Maintenance and Operations (M\&O) Contractor. The plan identifies actions involving the M\&O Contractor that are critical to (1) prepare for a smooth transition to the Project Hanford Management Contractor (PHMC), and (2) support and assist the PHMC and RL in achieving transition as planned, with no or minimal impact to ongoing baseline activities.

The plan is structured around two primary phases. The first is the pre-award phase, which started in mid-February 1996 and is currently scheduled to be completed on June 1,1996 , at which time the contract is currently planned to be awarded. The second is the follow-on four-month post-award phase from June 1, 1996, until October 1, 1996. Considering the magnitude and complexity of the scope of work being transitioned, completion in four months will require significant effort by all parties. To better ensure success, the M\&O Contractor has developed a pre-award phase that is intended to maximize readiness for transition. Priority is given to preparation for facility assessments and processing of personnel, as these areas are determined to be on the critical path for transition. In addition, the M\&O Contractor will put emphasis during the pre-award phase to close out open items prior to contract award, to include grievances, employee concerns, audit findings, compliance issues, etc.

Section 2.0 contains key issues and assumptions, an overall transition schedule, and generic definitions for some of the important repetitive actions. The remaining sections are basically organized around the statement of work contained within the PHMC Request for Proposal (RFP) and provide general descriptions of work, along with pre- and post-award actions to be completed. the date to be completed, and the responsible assignee(s). The preaward actions will initially be captured in an action tracking system and routinely statused until completion. The early post-award actions will subsequently then be tracked until this transition plan is integrated into a combined RL, PHMC, and M\&O Contractor transition plan, which is scheduled to be developed by July 1,1996 .

Pacific Northwest National Laboratory (PNNL), Bechtel Hanford, Inc. (BHI), and Hanford Environmental Health Foundation (HEHF) have been involved in the development of this plan. They have elected to include specific actions on their part to better ensure integration of their activities with those of the PHMC during transition. In addition, and in coordination with RL, selected RL actions have been included to facilitate development of the consolidated transition plan following contract award. 


\section{WHC-SP-1181}

This page intentionally left blank. 


\subsection{PLAN AND IMPLEMENTATION APPROACH}

This plan has been developed by a broad-based M\&O Contractor Transition Team representing all areas of WHC and its subcontractors, ICF KH and BCSR (hereafter referred to as the $M \& O$ Contractor). In addition, close coordination was maintained with and support was provided by the RL multi-contractor team and the RL extended transition team points of contact. The contents of the plan are based on the PHMC RFP (RL 1996a), and reflect the experience and knowledge of the participating team members to ensure an orderly and timely transition. In addition, transition plans from both prior Hanford transitions and recent transitions at other DOE sites were used in structuring this plan.

The M\&O Contractor transition effort is being managed and executed as a project. A Project Manager has been designated who is directly responsible to the President of WHC for successfully carrying out the M\&O Contractor transition activities necessary for a smooth transition to the PHMC. Each program and functional area has designated a transition team member, who reports directly to the Project Manager for activities governed by this plan.

\subsection{PLAN CONTENT AND USE}

This plan has been organized around the statement of work contained within the PHMC RFP and supports the RL Transition Plan for the PHMC (RL 1996b), dated February 1996. The plan identifies action items necessary to achieve a smooth transition in two phases. The first phase is the pre-award period (i.e., pre-transition) and the second phase is post-award (i.e., transition). In addition to M\&O Contractor actions, this plan includes actions from other Hanford contractors to ensure integration of their activities with those of the PHMC. Actions that are solely the responsibility of the PHMC per the RFP are not identified in this plan.

This plan will govern the execution of the pre-award phase. Therefore, all pre-award actions have been assigned a specific completion date and a responsible individual(s). The pre-award phase includes conduct of those actions that can be completed without knowledge or participation of the PHMC, with the goal to enhance the readiness to initiate transition.

The current RL transition plan shows the development of a consolidated RL, M\&O Contractor, and PHMC transition plan within one month following contract award. Therefore, this plan will be used to identify and track actions, along with the PHMC plan submitted with their proposal, during this first month of transition. Tentative dates for such actions have been identified in this plan, along with a responsible individual(s), recognizing these dates may change due to a variety of factors such as the PHMC's timeliness to mobilize and areas of first interest. 
Upon completion, the consolidated transition plan will be used to govern the remaining transition actions through contract takeover. To facilitate preparation of the consolidated plan, this plan includes actions that would involve the M\&O Contractor through the transition phase. Responsible individuals for the M\&O Contractor have been identified. Where practicable, dates or time periods for completing the actions have been estimated.

This plan does not include actions that are related to closeout of the M\&O contract. A separate M\&O Contractor team will be established to plan and execute this effort.

\subsection{KEY ISSUES}

The following identifies key issues which, if not resolved or clarified, could impact the ability for a smooth transition:

- This transition plan assumes a contract award date of June 1,1996 , followed by a four-month transition schedule, resulting in an October 1, 1996, contract takeover. The current RL Transition Plan basically distributes the four months into one month for mobilization and planning, two months to complete transition actions, and one month for readiness review and corrective action. Therefore, any action that reduces the schedule to less than four months in order to hold the October 1, 1996, takeover date could impact the capability for a smooth and complete transition.

- BHI and WHC are currently in the process of trying to resolve landlord responsibility issues that still remain from the previous Environmental Restoration Contractor (ERC) transition. RL has been party to these discussions. These issues need to be resolved prior to PHMC contract award. RL needs to clarify roles of all contractors, including PNNL, delineating who is responsible for what, so they do not effect the transition from the to the PHMC.

- Transfer of contractor responsibility as co-operator for Resource Conservation and Recovery Act of 1976 (RCRA) permitting documentation requires 90 days advance notification to the Washington State Department of Ecology (Ecology), and submittal of associated and recertified permitting documentation, unless this requirement is waived by Ecology. Assuming an October 1, 1996, takeover date, processing of all RCRA permitting documentation by July 1, 1996, is not feasible based on a June 1, 1996, contract award date. WHC cannot remain on permits after its contract expires. This is consistent with Section $\mathrm{E}$ of the "Agreement on Regulatory Interactions" between RL and WHC, dated August 14, 1995. 


\subsection{ASSUMPTIONS}

The following assumptions relate to the key issues described in Section 2.2:

- Transition will take four months from award to takeover:

- Contract award will be made on June 1, 1996. No contact will be made with the proposing teams prior to this date by the M\&O Transition Team concerning transition.

- Critical PHMC team members will have mobilized onsite within one week after contract award.

- The PHMC takeover will occur October 1, 1996. The current M\&O Contractor will maintain responsibility for all work up to that date. On October 1, 1996, all work will be transferred to the PHMC.

- The M\&O Contractor will continue beyond takeover on actions associated with contract closeout.

- RL will resolve outstanding landlord responsibility issues between WHC and BHI prior to contract award. In addition, RL will clarify any other remaining scope or responsibility issues between Hanford Contractors.

- RL shall submit notification to transfer co-operator responsibility for RCRA permitting documentation by July 1, 1996, or obtain relief from Ecology concerning the 90-day requirement for advance notification. If the July 1, 1996, date is not met, and relief from the 90 days is not obtained, WHC will be removed from the RCRA Permits as co-operator as of October 1, 1996. RL will remain as the sole owner/operator until such time as the PHMC is added as cooperator. This approach is consistent with Section $\mathrm{E}$ of the "Agreement on Regulatory Interactions" between RL and WHC, dated August 14, 1995. It is assumed an agreement with Ecology will be reached consistent with this approach.

The following additional assumptions are provided:

- Transition plans submitted with the PHMC proposals will not be made available to the M\&O Transition Team prior to contract award. Following award, the PHMC transition plan for the selected PHMC will be provided to the M\&O Transition Team. Note: The March 25, 1996, draft of this plan was placed in the DOE Reading Room for access by the proposing teams.

- Documented responses to bidder questions resulting from the pre-proposal conference still are valid unless countermanded in an amendment. 
- Few, if any, M\&O Contractor personnel will transfer to the PHMC team prior to the takeover date.

- Prior to contract award, RL will clarify PNNL and PHMC roles with respect to site planning and integration support to RL so that it can be properly addressed in transition.

- The PHMC will use many of the existing M\&O Contractor procedures and manuals following takeover until they have completed replacement of such procedures and manuals.

- The M\&O Contractor will continue as the lead for FY 1997 execution planning activities, but integrate closely with the PHMC Transition Team.

- Decisions concerning the transfer of program scope between existing contractors (BHI, WHC, and PNNL) will occur prior to contract award, or will be deferred until after contract takeover. Examples are the transfer of Environmental Management (EM)-50 technology tasks or environmental monitoring activities. RL will clarify any scope/responsibility issue, which may arise during transition, in an expedited manner.

- RL shall provide timely turnaround of their actions as defined in the transition plan (approvals, decisions, etc.).

- Tank Waste Remediation System (TWRS) Privatization will proceed as currently planned through the transition period.

- All formal transition actions, decisions, and agreements will be made with the PHMC integrating contractor, and not the major subcontractors. The PHMC integrating contractor will assign a transition manager as the primary point-ofcontact (POC). This is not intended to preclude day-to-day discussions, presentations, requests for information, facility inspections, etc., which will occur between all contractors involved.

- Unless notified otherwise by RL or the PHMC integrating contractor concerning recurring annual subcontracts, the M\&O Contractor will exercise such contracts for FY 1997 as necessary to ensure continuity of operations onsite.

- All actions completed per the transition plan will be adequately documented as closed by the assigned responsible individual(s).

- The M\&O Contractor will make space and services available to the PHMC Transition Team as requested. A sufficient amount of space will be reserved in advance, anticipating such a request. The costs of such space and services will be covered by the PHMC's transition budget. RL will obtain information on 
PHMC's needs in order to minimize any unnecessary expenditures or delays as soon as possible following contract award.

- For pre-existing conditions, WHC will not physically pre-assess areas where safety concerns preclude or discourage such preparatory actions.

- RL shall approve program change requests which are considered necessary to carry out the M\&O Contractor scope contained in this transition plan. For areas which do not submit change requests, the actions contained within this plan are considered to be included in the authorized scope of the direct or indirect program to which it is charged.

- All materials produced for formal briefings to the PHMC will be coordinated and reviewed with the appropriate RL POC. This does not apply to informal briefings (or discussion) and workshops, recognizing that the RL POCs may participate in such activities.

\subsection{ACTION TRACKING AND RECORDKEEPING}

An action tracking system has been established to track the actions contained in this action plan, and subsequently those contained in the consolidated action plan. The system includes for each action:

- Unique identification number cross-referenced to the appropriate transition plan section

- Program/functional area

- Action description

- Responsible individual(s)

- Planned completion date

- Actual completion date

- Status.

The system has multiple sorting capabilities, and will be the primary tool used in status meetings between the participating organizations.

Updates to actions (including addition of new actions, date changes, and actionee changes) will be made through the action tracking system. This will eliminate the need to continually update the plan itself. The plan would only be updated for a major change (i.e., 
extension of transition period) that would result in many actions having to be revised at one time.

WHC shall maintain a record of transition activities involving the M\&O Contractor. This will include documented completion of actions, transition agreements that are established, and any other pertinent data.

\subsection{COMPLETION VERIFICATION PROCESS}

Selected actions associated with programs and their facilities will require verification of completeness by the responsible RL program. Such actions will include, but are not limited to, property inventories, special nuclear material (SNM) inventories, and assessments of pre-existing conditions. This verification requirement will be integrated into the readiness review conducted during the latter stage of transition.

\subsection{TRANSITION LOGISTICS}

A key element towards a successful transition is ensuring that sufficient and adequate space and services are made available to transition participants in a timely manner. The M\&O Contractor is prepared to assist in this effort. This applies to central and satellite space within program/project areas, facilities, and functional areas. Specific action items have been identified in this plan for providing logistic support to include:

- Office space (both central and remote)

- Computers and network access

- Telephones

- Government vehicles

- Supplies

- Other services as necessary.

It is important to have as much information as possible in advance of the need date to be responsive and to eliminate any unnecessary costs. The M\&O Contractor recommends that RL query the selected PHMC immediately following contract award to obtain information on their logistical needs, and provide the range of needs in support of this planning effort.

Many actions have been included to identify and locate documentation for use by the PHMC. With exception of critical documentation, it is not intended to make copies to be provided to the PHMC, unless specifically requested. Existing documents may be located in a central area for ease of access. 


\subsection{M\&O TRANSITION MANPOWER AND COST ESTIMATE}

Table 2-1 identifies M\&O Contractor manpower and cost estimates for carrying out the actions contained in this plan. The estimates cover those activities that will be charged to the programs currently assigned to the M\&O Contractor. They do not include any costs incurred by the M\&O Contractor that, if incurred, would be charged to the PHMC transition account. Such activities would include cost for space and equipment provided to the PHMC Transition Team, or costs associated with modifications to current systems at the request of the PHMC for continued use after contract takeover.

M\&O Contractor programs will submit change packages as necessary to cover the costs in Table 2-1. Initially, change requests may be submitted to cover the pre-award activities only, which includes development of this plan. Once the contract award date is firmly established, additional change packages will be submitted to cover the transition period (or post-award phase). Some initial change requests may cover transition activities through contract takeover.

Table 2-1. Transition Manpower and Cost.

\begin{tabular}{|c|c|c|c|c|}
\hline \multirow{2}{*}{ Transition Area } & \multicolumn{2}{|c|}{ Pre-Award Phase } & \multicolumn{2}{|c|}{ Post-Award Fhase } \\
\hline & Man-Months & Cost $(\$ 000)$ & Man-Months: & $\operatorname{cost}(\$ 000)$ \\
\hline Project Management and Adminiatration & 10 & $\$ 100$ & 16 & $\$ 150$ \\
\hline Management and Integration & 38 & $\$ 280$ & 92 & $\$ 640$ \\
\hline Hanford Projects & 74 & $\$ 610$ & 134 & $\$ 1,000$ \\
\hline Infrastructure & 45 & $\$ 400$ & 60 & $\$ 500$ \\
\hline Other Crosscutting Services & 52 & $\$ 420$ & 70 & $\$ 610$ \\
\hline TOTAL & 219 & $\$ 1,810$ & 372 & $\$ 2,900$ \\
\hline
\end{tabular}

\subsection{TRANSITION SCIIEDURE}

Figure 2-1 provides the current transition schedule, summarizing the actions contained in this plan. This schedule is consistent with the schedule contained in the RL Transition Plan.

Based on prior experiences in transition, two transition areas are shown to be on the critical path. These are (1) completion of pre-existing condition assessments, and (2) the process of interviewing and extending offers to existing site personnel. One may also consider the area of property accountability as being close to the critical path. Based on this schedule, any effort to perform transition from contract award to contract takeover in less than four months would have a low probability of success unless one is willing to accept significant risk and potentially numerous actions to be completed after takeover. 
M O O Schedule for Transition to the PHMC

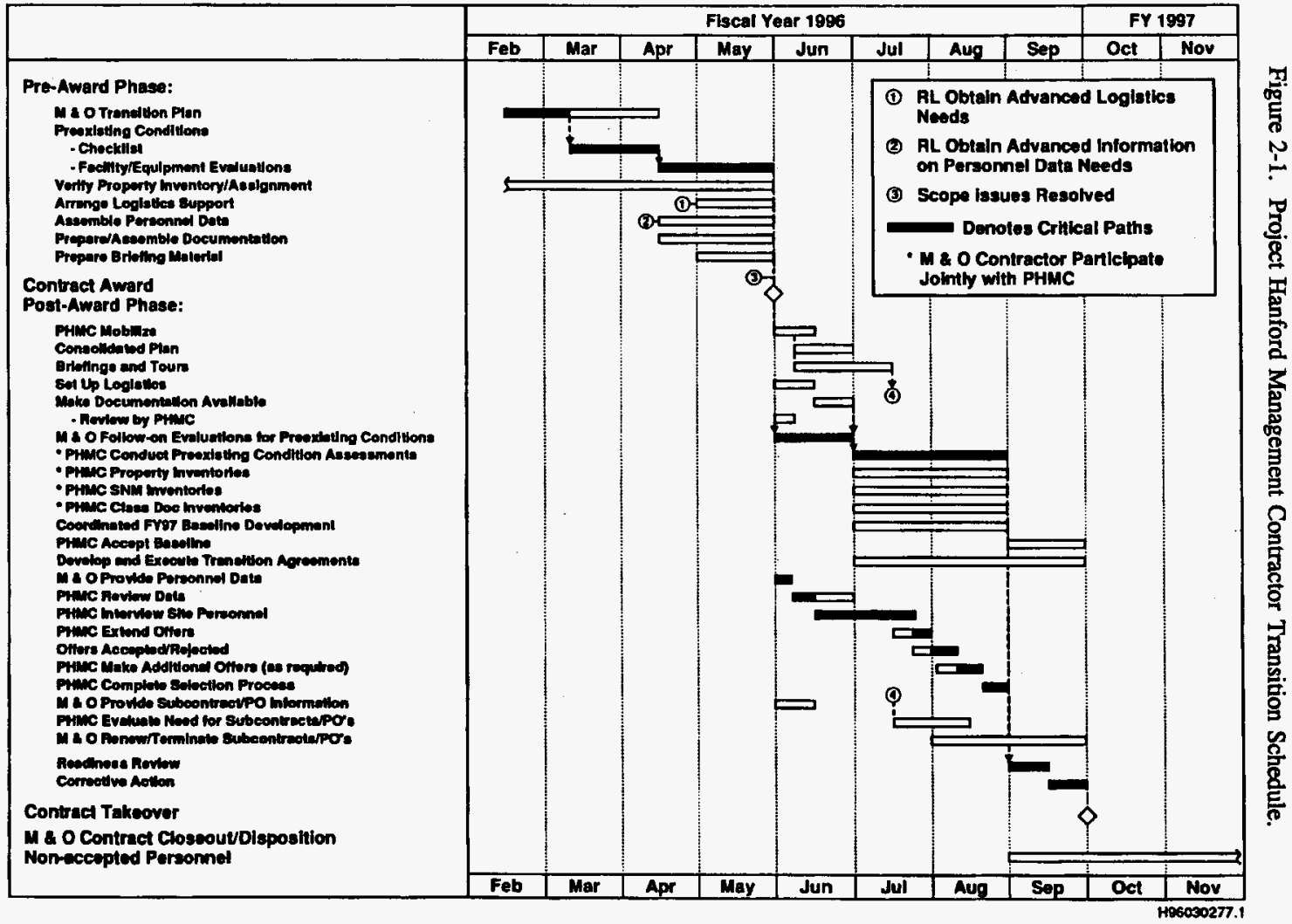




\subsection{ACTION DESCRIPTIONS}

The following sections in this plan identify many actions in support of transition, some of which are repeated for each program, project, or facility. The following provides further definition with respect to the scope and content of these repetitive actions:

- Key Program Documentation

- Key documentation includes, program/project "smart book," current multi-year program plans (MYPPs), work breakdown structures (WBS), Program/project Charter, activity data sheets (ADSs), risk data sheets (RDSs), cost account plans (CAPs), basis of estimate (BOE) library, change control log and file, cost and schedule status (i.e., Site Management System [SMS] report), progress tracking system (PTS) report, and current schedules. Includes other administrative plans and procedures. Also includes key technical reports, studies, and plans to include program level QA and safety documentation. For facilities not addressed separately, include applicable key facility documentation and pre-existing condition information.

- Program/Project Briefings

- Describe the mission and scope of the program or project. Provide the overall program status in terms of budget, schedule commitments, and compliance. Introduce key program personnel. Discuss pre-existing conditions not addressed at the facility or operational level. Provide listing of and introduce POCs, address any logistic issues, and discuss schedule and support for tours, pre-existing condition assessments, and conduct of inventories.

- Key Facility or Operations Documentation

- Documentation includes, facility or operations "smart book," primary facility drawings and in-process engineering change notices (ECNs); Operations and Maintenance records (e.g., shift operating $\operatorname{logs}$ ); safety and QA documentation; radiological control (RadCon) documentation; permits and regulatory submittals; environmental monitoring records; key construction project documentation; assessments and surveillance; training records; administrative; operating, and maintenance procedures; facility plans (e.g., building emergency plan, health and safety plan); subcontracts or agreements for outside support; and any other documentation essential to the continued safe operation of the facility. Include pre-existing condition documentation.

- Smart Books

- In conjunction with assembling key program/project or facility/operations documentation, "Smart Books" will be prepared. The purpose of the Smart Book is to summarize key data for use by the PHMC personnel, without making them wade through the large set of documents provided. Standard 
outlines for the smart books will be provided, and will include facility pictures, briefing material, documentation listings, POCs, subcontract listings and other relevant information.

\section{- Facility or Field Operation Briefings}

- Describe the facility (or operation) and its operating history. Introduce key facility POCs. Provide relevant budget and schedule information. Discuss all permits relative to the facility or operation. Discuss existing subcontracts and support agreements. Discuss QA, Safety, and RadCon programs. Discuss pre-existing conditions.

- Facility or Operations POC

- POCs should include the facility manager, managers of each organization at the facility, shift operations managers, system cognizant engineers and technical leads, persons in charge of ongoing activities, individuals responsible for contracts or agreements for outside support, and managers of supporting and/or client organizations in other programs.

For other operations or technical services, include key scientists/engineers and consultants.

- Pre-Existing Conditions

- A checklist for evaluating pre-existing conditions will be provided.

- In addition to other environmental, safety, and health (ESH) issues, preexisting conditions should address issues associated with environmental regulations, Occupational Safety and Health Administration (OSHA), Price Anderson Amendment Act (PAAA), and related DOE orders. Specifically include corrective actions from assessments and surveillances which have not yet been completed; occurrences and permit violations; ongoing regulatory compliance issues; limits on operations; unresolved environmental safety or health issues; conduct of operations ( $\mathrm{COO}$ ) issues and status of implementation; status of procedures and their relationship to the facility safety basis; and security risk evaluation issues. Include the planned resolution for each. Areas of uncertainty will be documented to ensure they are addressed jointly with the PHMC. Any physical (or walk-through) assessments done in advance of the PHMC will be done to facilitate readiness for transition. 


\subsection{OVERALL ACTIONS}

\begin{tabular}{|c|c|c|}
\hline Pre-Award Actions & Scheduled & setopes $(0)$ \\
\hline $\begin{array}{l}\text { 1. Establish Action Tracking System and enter pre-award actions for } \\
\text { tracking. }\end{array}$ & $4 / 19 / 96$ & JL Waite \\
\hline 2. Establish Transition Record file and initiate record keeping. & $5 / 01 / 96$ & JL Waite \\
\hline $\begin{array}{l}\text { 3. Assemble site listing of key POCs by program/project, facility, or } \\
\text { function. }\end{array}$ & $6 / 01 / 96$ & JL Waite \\
\hline $\begin{array}{l}\text { 4. Identify available central office space for use by the PHMC } \\
\text { Transition Team if needed. }\end{array}$ & $5 / 15 / 96$ & JL Waite \\
\hline 5. Develop pre-existing conditions checklist. & $4 / 30 / 96$ & $\begin{array}{l}\text { RA Dutton } \\
\text { RH Engelmann } \\
\text { AR Hawkins }\end{array}$ \\
\hline $\begin{array}{l}\text { 6. Develop standard outlines and content for program level and } \\
\text { project/facility/operations level "Smart Books." }\end{array}$ & $\begin{array}{c}4 / 15 / 96 \\
\text { (completed) }\end{array}$ & $\begin{array}{l}\text { JL Waite } \\
\text { CL Nansen }\end{array}$ \\
\hline Post-Award Actions & & \\
\hline 1. RL confirm space requirements for PHMC Transition Team. & $6 / 03 / 96$ & LA Marzette (RL) \\
\hline $\begin{array}{l}\text { 2. RL confirm any other logistic support to PHMC Transition Team } \\
\text { (i.e., government vehicles). }\end{array}$ & $6 / 03 / 96$ & LA Marzette (RL) \\
\hline $\begin{array}{l}\text { 3. PHMC Transition Team central office space available for use to } \\
\text { include fumiture, computers, telephones, and other office } \\
\text { equipment. }\end{array}$ & $6 / 15 / 96$ & JL Waite \\
\hline 4. All remaining logistical support provided to the PHMC Team. & $6 / 30 / 96$ & JL Waite \\
\hline 5. Provide site orientation briefing to the PHMC Team. & $6 / 15 / 96$ & $\begin{array}{l}\text { GW Jackson } \\
\text { J Erickson }\end{array}$ \\
\hline 6. Conduct site tour for the PHMC Team. & $6 / 15 / 96$ & $\begin{array}{l}\text { GW Jackson } \\
\text { J Erickson }\end{array}$ \\
\hline $\begin{array}{l}\text { 7. Develop PHMC briefing schedule in advance of consolidated } \\
\text { transition plan. }\end{array}$ & $6 / 15 / 96$ & $\begin{array}{l}\text { JL Waite } \\
\text { LA Marzette (RL) } \\
\text { PHMC }\end{array}$ \\
\hline $\begin{array}{l}\text { 8. Prepare a composite listing of intercontractor MOUs among the } \\
\text { M\&O Contractor and PNNL, BHI, and HEHF, and identify which } \\
\text { should be terminated verses those which should be modified for } \\
\text { use by the PHMC. }\end{array}$ & $6 / 15 / 96$ & $\begin{array}{l}\text { GW Jackson } \\
\text { MS Hanson (PNNL) } \\
\text { GF Jones (BHI) } \\
\text { JJ Maher III (HEHF) }\end{array}$ \\
\hline $\begin{array}{l}\text { 9. Prepare an update list of assumptions based on pre-award } \\
\text { activities. Include both general and area-specific sssumptions. }\end{array}$ & $6 / 15 / 96$ & JL Waite \\
\hline
\end{tabular}




\subsection{WHC CONTRACT CLOSEOUT ACTIVITIES}

A separate WHC team and associated plan will be established to address closeout of the WHC M\&O contract, to include its major subcontractors ICF KH and BCSR. The following transition action is necessary to support closeout:

\begin{tabular}{|c|c|c|}
\hline Post-Award Actions & $\begin{array}{l}\text { Scheduled } \\
\text { Date }\end{array}$ & Actones (s) \\
\hline $\begin{array}{l}\text { 1. PHMC/WHC/subcontractors Memorandum of Understanding } \\
\text { (MOU) negotiated to allow as needed access to former } \\
\text { employees, data, and facilities for closeout activities. (Will be } \\
\text { included as part of WHC/PHMC/DOE transfer agreement.) }\end{array}$ & 9/20/96 & $\begin{array}{l}\text { JM Knoll, Jr. } \\
\text { SR Morgan } \\
\text { CD Maxson } \\
\text { RL Benedetti } \\
\text { WJ Fuller (BCSR) } \\
\text { RL Contracting Officer }\end{array}$ \\
\hline
\end{tabular}




\subsection{MANAGEMENT AND INTEGRATION}

\subsection{SITE PLANNING AND INTEGRATION}

Site planning and integration includes RL strategic and mission planning, sitewide systems engineering and integration activities, and the development and maintenance of lifecycle program and project baselines.

\begin{tabular}{|c|c|c|}
\hline Pre-Award lactions & Schedaled & Actionee(s) \\
\hline $\begin{array}{l}\text { 1. Develop a list and assemble key site planning and } \\
\text { integration documentation and information for use by the } \\
\text { PHMC. Include information on databases. }\end{array}$ & $5 / 31 / 96$ & $\begin{array}{l}\text { ML Grygiel } \\
\text { JK Fish }\end{array}$ \\
\hline Post A vard letions & & \\
\hline $\begin{array}{l}\text { 1. Provide briefing to PHMC on the overall Hanford Site } \\
\text { Planning and Integration process }\end{array}$ & $7 / 01 / 96$ & $\begin{array}{l}\text { EW Higgins (RL) } \\
\text { MB Triplett (PNNL) } \\
\text { GW Jackson }\end{array}$ \\
\hline
\end{tabular}

\subsubsection{Support to RL Strategic Planning}

This activity is responsible to provide support to RL and PNNL in the development and integration of the strategic level planning documents (Hanford Strategic Thinking and the Mission Direction Document [MDD]), as well as to assist in the integration of program input to the MYPP planning process.

\begin{tabular}{|c|c|c|}
\hline Post-Award Actions & $\begin{array}{l}\text { Scheduled } \\
\text { Date }\end{array}$ & Retionee(s) \\
\hline $\begin{array}{l}\text { 1. Provide briefing or discussion with PHMC on M\&O } \\
\text { Contractor support to the RL strategic planning effort. }\end{array}$ & $\begin{array}{l}7 / 01 / 96- \\
7 / 15 / 96\end{array}$ & ST Smith \\
\hline
\end{tabular}

\subsubsection{Project/Program Planning and Baseline Management}

The current planning process centers around the development of MYPPs for the direct funded programs, and Site Support Program Plans (SSPPs) for indirect and direct support activities. Each program and support area will address their specific plans in other sections of this transition plan. This section addresses the overall planning processes currently in use, and current ongoing planning activities that the PHMC may need to get involved in.

Site baseline management involves the integration and maintenance of the overall technical, schedule, and cost baseline for the site. The technical baseline is further addressed in Section 3.1.3. 


\begin{tabular}{|c|c|c|}
\hline Pre Avrard Actions & $\begin{array}{l}\text { Scheduled } \\
\text { Date }\end{array}$ & lctionee(s) \\
\hline $\begin{array}{l}\text { 1. Assemble a set of the most recent MYPPs/SSPPs, and integrated } \\
\text { site baseline data (including HSIS) for use by the PHMC. }\end{array}$ & $5 / 31 / 96$ & JK Fish \\
\hline Post-Awerd Actions & & \\
\hline $\begin{array}{l}\text { 1. Provide briefing or discussion to the PHMC on the program } \\
\text { planning and integrated site baseline processes. }\end{array}$ & $\begin{array}{r}7 / 01 / 96- \\
7 / 15 / 96\end{array}$ & JK Fish \\
\hline $\begin{array}{l}\text { 2. Integrated with the PHMC for development of FY } 1997 \text { detailed } \\
\text { execution plans as part of the ongoing MYPP/SSPP revision. }\end{array}$ & $7 / 01 / 96$ & JK Fish \\
\hline
\end{tabular}

\subsubsection{Sitewide Systems Engineering}

This area is responsible for development and maintenance of the site level technical baseline, interface control documentation, operational scenarios to model the performance of the Hanford mission, publication of the site level specification and identification and tracking of technical integration issues. This activity also has responsibility to ensure integration of its products with the strategic level planning documentation being developed by PNNL and to ensure that technical considerations are included in the MYPP planning guidance provided to the program level. Plan.

This activity is governed by RLPD and RLID 430.1 and the Hanford Management

\begin{tabular}{|c|c|c|}
\hline Post-Award Actions & $\begin{array}{l}\text { Scheduled } \\
\text { Date }\end{array}$ & Actionee(s) \\
\hline $\begin{array}{l}\text { 1. Provide briefing or discussion with PHMC on sitewide } \\
\text { system engineering and integration activities. }\end{array}$ & $\begin{array}{l}7 / 01 / 96- \\
7 / 15 / 96\end{array}$ & PA Baynes \\
\hline
\end{tabular}

\subsection{FINANCE AND ADMINISTRATION}

\subsubsection{Accounting}

3.2.1.1 Accounts Receivables. The PHMC will be a RL non-integrated contract. Certain ledger accounts will be transferred and carried on RL's books.

\begin{tabular}{|c|c|c|}
\hline Post-Award Aetions & $\begin{array}{l}\text { Schethuled } \\
\text { Dote }\end{array}$ & Actionee (s) \\
\hline 1. Identify all receivables by account/company/employee. & $9 / 01 / 96$ & $\begin{array}{l}\text { J Armstrong (lead) } \\
\text { D Long } \\
\text { M Howard }\end{array}$ \\
\hline 2. Write-off and establish reserves where possible. & 9/01/96 & J Armstrong \\
\hline
\end{tabular}




\begin{tabular}{|l|c|l|l|}
\hline & Schediled & Date & \\
\hline 3. Identify accounts which are to be transferred to RL. & $9 / 01 / 96$ & P Felts \\
\hline $\begin{array}{l}\text { 4. Transfer General Ledger receivable accounts to } \\
\text { RL/PHMC/subcontractors. }\end{array}$ & $9 / 15 / 96$ & P Felts \\
& $\begin{array}{l}\text { RL } \\
\text { PHMC }\end{array}$ \\
\hline
\end{tabular}

\subsubsection{Department Integrated Standardized Core Accounting System (Discas) Interface} for Plant and Equipment. Establish a DISCAS interface with RL, which provides a monthly update of transactions for Plant and Equipment (P\&E). These affected accounts will no longer reside on the contractor's books.

\begin{tabular}{|c|c|c|}
\hline PreAvard Actions & Scheduled & hetrones $(s)$ \\
\hline $\begin{array}{l}\text { 1. Develop a comprehensive plan to identify P\&E accounts, } \\
\text { transactions, and automate the data transfer to RL. }\end{array}$ & $6 / 01 / 96$ & $\begin{array}{l}\text { I Shelt } \\
\text { A Lake }\end{array}$ \\
\hline Poct-A ward Actions & & \\
\hline $\begin{array}{l}\text { 1. Inplement the automated transfer prior to year-end } \\
\text { (if feasible). }\end{array}$ & $9 / 01 / 96$ & $\begin{array}{l}\text { J Shelt } \\
\text { BCSR } \\
\text { A Lake }\end{array}$ \\
\hline
\end{tabular}

3.2.1.3 Transfer of General Ledger Accounts. The PHMC will be a RL non-integrated contractor. As a result, certain general ledger accounts will no longer reside on the contractor's books. These accounts are to be transferred to RL.

\begin{tabular}{|c|c|c|}
\hline Presward Actions & Scheduled & Actiones(s) \\
\hline 1. Identify ledger accounts which are to be transferred to RL. & $6 / 01 / 96$ & $\begin{array}{l}\text { J Salancy } \\
\text { A Lake }\end{array}$ \\
\hline Post-A ward Actions & 8 & \\
\hline $\begin{array}{l}\text { 1. Identify all issues associated with an early transfer (prior to } \\
\text { FY-end closing) of accounts to RL. }\end{array}$ & $7 / 01 / 96$ & $\begin{array}{l}\text { J Salancy } \\
\text { A Lake }\end{array}$ \\
\hline 2. Transfer of account balances to $R L$. & TBD & $\begin{array}{l}\text { J Salancy } \\
\text { A Lake }\end{array}$ \\
\hline
\end{tabular}

3.2.1.4 Treasury. The current contractor government bank accounts and associated treasury activities will have to be dispositioned and transitioned. Also, a startup plan for the PHMC needs to be developed. The primary efforts will be to ensure treasury operations are current during transition. 


\begin{tabular}{|c|c|c|}
\hline Post-1 ward Actions & $\begin{array}{l}\text { Schedilled } \\
\text { Date }\end{array}$ & Hetionectis) \\
\hline $\begin{array}{l}\text { 1. Develop a detailed closeout/startup plan for all treasury } \\
\text { related activities. }\end{array}$ & $8 / 01 / 96$ & $\begin{array}{l}\text { M Byrd } \\
\text { PHMC }\end{array}$ \\
\hline
\end{tabular}

3.2.1.5 Payroll Activities. A range of payroll issues need to be addressed. These are general pay issues, system issues, tax issues, banking issues, etc.

\begin{tabular}{|c|c|c|}
\hline Post-Award Actiong & $\begin{array}{l}\text { Schetuled } \\
\text { Date }\end{array}$ & Actionee(s) \\
\hline $\begin{array}{l}\text { 1. Prepare a listing of all associated issues and a detailed plan of } \\
\text { implementation. }\end{array}$ & $8 / 01 / 96$ & M Byrd \\
\hline $\begin{array}{l}\text { 2. Agreements need to be made for the final M\&O Contractor } \\
\text { payroll and startup for the PHMC. }\end{array}$ & $8 / 01 / 96$ & $\begin{array}{l}\text { M Byrd } \\
\text { RL }\end{array}$ \\
\hline
\end{tabular}

3.2.1.6 Tax Filings - City, County, Federal. Tax reports will be required by prior and new site contractors. Develop a plan to assume a successful transition. Some of these may be identified in other parts of the plan.

\begin{tabular}{|l|c|l|}
\hline \multicolumn{1}{|c|}{ Post-Award Actions } & $\begin{array}{l}\text { Schetuled } \\
\text { Date }\end{array}$ & $7 / 01 / 96$ \\
\hline $\begin{array}{l}\text { 1. Identify all tax reports and filings which will need to be made } \\
\text { for the M\&O Contractors. }\end{array}$ & $\begin{array}{l}\text { J Salancy (lead) } \\
\text { D Staudacher } \\
\text { F Melton }\end{array}$ \\
\hline $\begin{array}{l}\text { 2. Develop a plan to file timely and accurate tax reports on } \\
\text { behalf of the M\&O Contractors. }\end{array}$ & $9 / 01 / 96$ & $\begin{array}{l}\text { J Salancy (lead) } \\
\text { D Staudacher } \\
\text { F Melton }\end{array}$ \\
\hline $\begin{array}{l}\text { 3. Prepare necessary documents to assure transition for proper } \\
\text { tax reporting by the PHMC. }\end{array}$ & $9 / 01 / 96$ & $\begin{array}{l}\text { J Salancy } \\
\text { PHMC }\end{array}$ \\
\hline
\end{tabular}

\subsubsection{Fiscal Year-End - Closeout. This section will be addressed as part of the} consolidated transition plan.

3.2.1.8 Accruals (Accounts payable and other fund liabilities). Forty to sixty million will be accrued for services performed but not paid as of September 30, 1996. They will have to be dispositioned.

\begin{tabular}{|l|c|l|}
\hline & $\begin{array}{c}\text { Schediled } \\
\text { Date }\end{array}$ & Post-Award Actions \\
\hline $\begin{array}{l}\text { 1. Develop a plan to effectively transfer the FY 1996 accruals to } \\
\text { the PHMC. }\end{array}$ & $8 / 01 / 96$ & $\begin{array}{l}\text { D Long (lead) } \\
\text { K Harris } \\
\text { C Stice } \\
\text { F Zvonar }\end{array}$ \\
\hline 2. Review Rocky Flats Process (or other applicable sites). & $7 / 01 / 96$ & K Harris \\
\hline
\end{tabular}




\begin{tabular}{|c|c|c|}
\hline Post-Antard Actions & $\begin{array}{l}\text { Scheduled } \\
\text { Date }\end{array}$ & Sctionee() \\
\hline $\begin{array}{l}\text { 3. Identify all areas for accruals (P-cards, services, Intra DOE } \\
\text { Work Orders, claims, etc). }\end{array}$ & $7 / 01 / 96$ & $\begin{array}{l}\text { D Long (lead) } \\
\text { K Harris } \\
\text { C Stice }\end{array}$ \\
\hline
\end{tabular}

\subsubsection{P-Cards}

\begin{tabular}{|l|c|l|}
\hline & $\begin{array}{c}\text { Schedaled } \\
\text { Date }\end{array}$ & vetionee(s) \\
\hline $\begin{array}{l}\text { 1. Develop a plan to close out P-cards under current WHC } \\
\text { contract. }\end{array}$ & $8 / 01 / 96$ & $\begin{array}{l}\text { R Dahlin (lead) } \\
\text { C Mayo }\end{array}$ \\
\hline 2. Develop a plan to implement P-cards for PHMC. & $8 / 01 / 96$ & $\begin{array}{l}\text { R Dahlin (lead) } \\
\text { C Mayo } \\
\text { PHMC }\end{array}$ \\
\hline
\end{tabular}

3.2.1.10 Travel. Develop plans for the closeout, transfer, and disposition of travel related activities. The plan should address the following issues/actions:

- Corporate Agreements in Use

- American Express Credit Cards

-- Cancel/Reissue employee cards under new corporate cards.

-- Cancel BTA/Reissue under new account (September charges won't hit until October/November so may want to discontinue use in August $=$ loss of revenue to Westinghouse Electric Corporation [WEC]).

- Rental Cars

-- Need to negotiate (or piggyback to existing) new agreements.

- WHC Agreements to be Reissued Under New Contractor(s) Name

- Government Transportation Request (GTR)

- Travel Agency Agreements

- Moving Company Agreements

- Employees in Other Locations

- Relocations

- Will have outstanding travel advances not costed.

-- Personal belongings in transit/storage.

- Relocation costs of Involuntary Reduction of Force (IROF) employees.

-- Relocation program in effect for 12 months after separation.

- Temporary Assignments

-- Will have outstanding travel advances not costed.

-- Will have continuing costs (including per diems, relocation costs). 
- Business Travel/Business Conference Expenses - Contract

- Contract allowable business travel/business conference uncosted.

-- Advances, prepayments, and airline tickets (trips in process or for future trips - after transition).

-- Other expenses for trips taken or in progress.

- Blanket Travel Authorizations (TAs)

-- Close out (collect advances)/reissue.

- Prepare/Reissue traveler government identification cards.

- Accounts receivables for employee travel expenses.

- Inventory tickets.

-- Disposition of existing tickets.

- Business Travel/Conference Expenses - Non-contract

- Non-contract business travel/conference expenses not submitted.

-- Process WEC reimbursed expenses.

-- Closeout/re-establish fee accounts.

- Miscellaneous

- Transfer all travel office equipment to the new contractor.

- Year-end accrual process?

\begin{tabular}{|c|c|c|}
\hline Post-Award Actions & Scheduled & Actionee(s) \\
\hline $\begin{array}{l}\text { 1. Develop a plan to resolve employee relocation, travel } \\
\text { advances, business conference expenses and other related } \\
\text { travel issues. }\end{array}$ & $7 / 15 / 96$ & M Howard \\
\hline $\begin{array}{l}\text { 2. Develop a plan to terminate current travel } \\
\text { agreements/contracts and implement new ones associated with } \\
\text { the PHMC. }\end{array}$ & $8 / 01 / 96$ & $\begin{array}{l}\text { M Howard } \\
\text { PHMC }\end{array}$ \\
\hline
\end{tabular}

3.2.1.11 Work Authorization (Hanford Site). A mechanism is needed for work authorization between the PHMC, subcontractors, and other Hanford contractors. Also, there are related tax impacts due to the contractual arrangements.

\begin{tabular}{|c|c|c|}
\hline , Post-A ward Actions & $\begin{array}{l}\text { Scheduled } \\
\text { Date }\end{array}$ & Actionee(s) \\
\hline $\begin{array}{l}\text { 1. Develop a listing of current sources of work to/from at the } \\
\text { Hanford Site. }\end{array}$ & $8 / 01 / 96$ & $\begin{array}{l}\text { K Harris } \\
\text { C Stice }\end{array}$ \\
\hline 2. Provide a summary of tax impacts. & $8 / 01 / 96$ & J Salancy \\
\hline 3. Develop work authorization mechanism. & $8 / 01 / 96$ & $\begin{array}{l}\text { RL } \\
\text { PHMC }\end{array}$ \\
\hline 4. Communicate and implement for FY 1997 start-up. & $9 / 30 / 96$ & PHMC \\
\hline
\end{tabular}


3.2.1.12 Work For Others (Non-Hanford). There are various mechanisms for the contracting of work performed by the M\&O Contractor. (MPOs, Intra DOE Work Orders, DOE RFSs, IWRs, IDWAs, etc.) See Section 3.11 for preparatory actions associated with work for others.

\begin{tabular}{|l|c|l|}
\hline 1.4 Scheduled & Actionee(s) \\
\hline 1. Identify all work for others. & $6 / 01 / 96$ & $\begin{array}{l}\text { J Knoll } \\
\text { P Felts }\end{array}$ \\
\hline 2. Closeout or reassign current contractual agreements. & $9 / 30 / 96$ & $\begin{array}{l}\text { J Knoll (lead) } \\
\text { P Felts } \\
\text { R Meyer } \\
\text { AN Lake } \\
\text { PHMC }\end{array}$ \\
\hline
\end{tabular}

3.2.1.13 Pensions. The PHMC will assume the current Pension Plan and related assets and liabilities. The Pension and Savings organization must maintain current operations and work towards assigning responsibility to new contractors(s). A draft detail plan is in place and will be modified and implemented as appropriate.

\begin{tabular}{|c|c|c|}
\hline Pre-Award Actions & $\begin{array}{l}\text { Scheduled } \\
\text { Date }\end{array}$ & Actionee(s) \\
\hline $\begin{array}{l}\text { 1. Prepare summary of all pension trust agreements and other } \\
\text { related contracts. }\end{array}$ & $6 / 01 / 96$ & M Byrd \\
\hline Post-Award Actions & & \\
\hline $\begin{array}{l}\text { 1. Trust agreements, investment management agreements, } \\
\text { service agreements, consultant agreements, third party } \\
\text { agreements, plan documents, summary plan descriptions, } \\
\text { actuarial reports, discrimination testing, annual reports, and } \\
\text { committee minutes should all be reviewed and reassigned. }\end{array}$ & $9 / 30 / 96$ & $\begin{array}{l}\text { M Byrd } \\
\text { PHMC }\end{array}$ \\
\hline
\end{tabular}

3.2.1.14 Savings Plans. The RFP requires bidders to propose a $401 \mathrm{~K}$ plan. A transition to the new plan(s) will have to be performed.

\begin{tabular}{|l|c|c|}
\hline & $\begin{array}{c}\text { Scheduled } \\
\text { Date }\end{array}$ & Actionee(s) \\
\hline 1. Identify all trust agreements and other related contracts. & $6 / 01 / 96$ & M Byrd \\
\hline $\begin{array}{l}\text { 1. Develop a transition plan to migrate to new 401K as } \\
\text { proposed by the PHMC. }\end{array}$ & $7 / 01 / 96$ & PHMC \\
\hline 2. Implement transition plan. & $10 / 01 / 96$ & PHMC \\
\hline
\end{tabular}

3.2.1.15 Insurance Plans. The RFP requires bidders to propose a health plan. The current plans will be transitioned to the new plans. 


\begin{tabular}{|c|c|c|}
\hline Prefiward Actions & $\begin{array}{l}\text { Schedaled } \\
\text { Dite }\end{array}$ & Metronee() \\
\hline $\begin{array}{l}\text { 1. Prepare summary of all insurance agreements and other } \\
\text { related contracts. }\end{array}$ & $6 / 01 / 96$ & M Byrd \\
\hline Post-Award Actions & & \\
\hline $\begin{array}{l}\text { 1. Develop a transition plan to migrate to the new health plan as } \\
\text { proposed by the PHMC. }\end{array}$ & $7 / 01 / 96$ & $\begin{array}{l}\text { M Byrd } \\
\text { PHMC }\end{array}$ \\
\hline 2. Implement transition plan. & $10 / 01 / 96$ & $\begin{array}{l}\text { M Byrd } \\
\text { PHMC }\end{array}$ \\
\hline
\end{tabular}

3.2.1.16 Vacation. The current accrued vacations liability for employees will need to be transferred to the PHMC or RL.

\begin{tabular}{|c|c|c|}
\hline Post-A ward Actions & $\begin{array}{l}\text { Scheduled } \\
\text { Date: }\end{array}$ & Lethonee(s) \\
\hline 1. DOE decision on Vacation Liability Methodology. & $8 / 01 / 96$ & A Lake (RL) \\
\hline $\begin{array}{l}\text { 2. Transfer employees accrued vacation hours and associated } \\
\text { liability to PHMC or RL (to be decided by RL). }\end{array}$ & $9 / 30 / 96$ & M Byrd \\
\hline
\end{tabular}

3.2.1.17 Nuclear Materials Accounting. This section will be addressed as part of the consolidated transition plan.

\subsubsection{Planning and Management Control Systems}

The Planning and Management Control Systems describe the processes used to manage the cost, schedule, and technical baselines of work performed by WHC for RL.

\begin{tabular}{|l|l|l|}
\hline \multicolumn{1}{|c|}{ Pre-Avard Actions } & $\begin{array}{c}\text { Scheduled } \\
\text { Date }\end{array}$ & Actionee(s) \\
\hline $\begin{array}{l}\text { 1. Develop a list and assemble key Planning and Management } \\
\text { Control System Documentation and information for use by } \\
\text { the PHMC. }\end{array}$ & $6 / 01 / 96$ & PJ Bence \\
\hline Post-Award Actions & $6 / 14 / 96$ & $\mathrm{KD}$ Cameron \\
\hline $\begin{array}{l}\text { 1. Provide a briefing to the PHMC on the overall Planning and } \\
\text { Management Control System philosophy for the site. }\end{array}$ & $7 / 12 / 96$ & PJ Bence \\
\hline $\begin{array}{l}\text { 2. Provide a workshop for the PHMC on assembled } \\
\text { documentation and processes used by the M\&O Contractor in } \\
\text { support of the Planning and Management Control Systems. }\end{array}$ & \\
\hline
\end{tabular}

\subsubsection{Environmental Planning Data System. The Environmental Planning Data System} (EPDS) includes the following modules: ADS, RDS/Priority List, Central Milestone Module (CMM), Baseline Change Control (BCC), and WBS Master Validation Table (MVT). These modules are used in support of the RL budget submittal process, progress tracking, and baseline control. 


\begin{tabular}{|l|l|l|}
\hline $\begin{array}{l}\text { E. Assemble a list of key documentation associated with the } \\
\text { EPDS and its associated modules. }\end{array}$ & $5 / 15 / 96$ & TE Tarcza \\
\hline 2. Verify that the ADS module is up-to-date. & $6 / 01 / 96$ & A Watts \\
\hline 3. Verify that the CMM module is up-to-date. & $6 / 01 / 96$ & BE Paul \\
\hline 4. Verify that the BCC module is up-to-date. & $6 / 01 / 96$ & WC Floberg \\
\hline 5. Verify that the RDS/Priority List module is up-to-date. & $6 / 01 / 96$ & KD Grindstaff \\
KN Lombardo
\end{tabular}

\subsubsection{Financial Data System (FDS).}

\begin{tabular}{|c|c|c|}
\hline Pre-Award Actions & Scheduled & Actionse(6) \\
\hline $\begin{array}{l}\text { 1. Develop a list and assemble key elements of the Financial } \\
\text { Data Systen's funds and Budget and Performance modules in } \\
\text { support of the WHC M\&O site portion. Other modules } \\
\text { covered in 3.2.2.8. }\end{array}$ & $6 / 01 / 96$ & $\begin{array}{l}\text { PJ Bence } \\
\text { DR Layfield }\end{array}$ \\
\hline Post-Award Actions & & \\
\hline $\begin{array}{l}\text { 1. Provide a workshop for the PHMC on assembled } \\
\text { documentation and processes used by the M\&O Contractor. }\end{array}$ & $6 / 28 / 96$ & $\begin{array}{l}\text { PJ Bence } \\
\text { DR Layfield }\end{array}$ \\
\hline
\end{tabular}

\subsection{Funds Module.}

\begin{tabular}{|l|l|l|}
\hline Pre-Award Actions & Scheduled \\
Date & & \\
\hline $\begin{array}{l}\text { 1. Develop FUNDS reconciliation for funds table to the M\&O } \\
\text { Contractor organizational breakdown structure. }\end{array}$ & $6 / 01 / 96$ & $\begin{array}{l}\text { CT Hewitt } \\
\text { MK Qualheim }\end{array}$ \\
\hline $\begin{array}{l}\text { 1. Transfer database in support of the WHC curtent year } \\
\text { funding/cost profile }\end{array}$ & & \\
- Expense & $9 / 06 / 96$ & CT Hewitt \\
- Capital & $9 / 06 / 96$ & MK Qualheim \\
- Special & $9 / 06 / 96$ & ML Vodney \\
- RL/HQ. & $9 / 06 / 96$ & ML Vodney \\
\hline
\end{tabular}




\subsection{Budget Module.}

\begin{tabular}{|c|c|c|}
\hline Posts A ward Actions & Scheduled & $x(100,0)$ \\
\hline 1. Provide Budget data files of the approved Program Baseline. & $9 / 27 / 96$ & DR Layfield \\
\hline
\end{tabular}

\subsection{Performance Module.}

\begin{tabular}{|c|c|c|}
\hline Poct-Anged Setions & Soleduled & Actornes $(s)$ \\
\hline $\begin{array}{l}\text { 1. Provide information and documentation on the processes used } \\
\text { by the M\&O Contractor in support of performance. }\end{array}$ & $8 / 01 / 96$ & $\begin{array}{l}\text { PJ Bence } \\
\text { DR Layfield }\end{array}$ \\
\hline
\end{tabular}

\subsubsection{Management Control System.}

\begin{tabular}{|l|c|c|c|}
\hline Scheduled & Date & & \\
\hline $\begin{array}{l}\text { 1. Provide a briefing or discussion of the M\&O Contractor's } \\
\text { Management Control System Policy and Procedures. Provide } \\
\text { copies of the pertinent procedures and documents. }\end{array}$ & $7 / 14 / 96$ & $\begin{array}{l}\text { PJ Bence } \\
\text { RS Wade }\end{array}$ \\
\hline
\end{tabular}

\subsubsection{DOE Site and Performance Reporting.}

\begin{tabular}{|c|c|c|}
\hline Pre-Award Actions & $\begin{array}{l}\text { Scheduled } \\
\text { Date }\end{array}$ & Actionee(s) \\
\hline $\begin{array}{l}\text { 1. Develop a list of the DOE site and performance reporting } \\
\text { systems being utilized by the M\&O Contractor and their } \\
\text { processes. }\end{array}$ & $6 / 01 / 96$ & C Edwards \\
\hline Post-A ward Letions & म. & \\
\hline $\begin{array}{l}\text { 1. Provide a transition plan that will ensure system integrity for } \\
\text { the following reporting systems: } \\
\text { - Program Management and Control System (PMCS) } \\
\text { - Progress Tracking System (PTS) } \\
\text { - Site Management System (SMS). }\end{array}$ & $7 / 19 / 96$ & C Edwards \\
\hline
\end{tabular}

3.2.2.5 Project Analysis \& Reporting System. Project Analysis \& Reporting System (PARS) is a cost and performance analytical tool designed for ease of use by Cost Account Managers and management. All Hanford Site employees have access. PARS extracts data from the FDS. 


\begin{tabular}{|c|c|c|}
\hline Pred dorty ptous & Sopented & setopos $(6)$ \\
\hline $\begin{array}{l}\text { 1. Assemble documentation associated with PARS including: } \\
\text { - Sample reports } \\
\text { - System description } \\
\text { - Manuals } \\
\text { - Procedures. }\end{array}$ & $5 / 15 / 96$ & $\begin{array}{l}\text { RF Blake } \\
\text { RA Marahall }\end{array}$ \\
\hline 2. Verify that the PARS module is up-to-date. & $6 / 01 / 96$ & $\begin{array}{l}\text { R Blake } \\
\text { R Marshall }\end{array}$ \\
\hline 2004 ward & & \\
\hline $\begin{array}{l}\text { 1. Make PARS documentation available for use by the PHMC. } \\
\text { Provide copies upon request. }\end{array}$ & $6 / 10 / 96$ & $\begin{array}{l}\text { R Blake } \\
\text { R Marshall }\end{array}$ \\
\hline
\end{tabular}

\subsubsection{Miscellaneous Planning \& Reporting Databases.}

\begin{tabular}{|l|c|c|c|}
\hline & Scheduled & Date & \\
\hline $\begin{array}{l}\text { 1. Develop a list and assemble key Planning and Reporting } \\
\text { Databases utilized by the M\&O Contractor in support of the } \\
\text { site. }\end{array}$ & $6 / 01 / 96$ & PJ Bence \\
\hline \multicolumn{1}{|c|}{ Post-Award Actions } & \\
\hline $\begin{array}{l}\text { 1. Provide a workshop for the PHMC on the miscellaneous } \\
\text { processes in support of the control functions for the M\&O } \\
\text { Contractor. }\end{array}$ & $7 / 19 / 96$ & PJ Bence \\
\hline
\end{tabular}

3.2.2.7 Overhead Rate Structure. It is assumed the overhead rate structure will change under the PHMC concept. The necessary actions and changes will need to occur to implement the new overtiead methodology.

\begin{tabular}{|l|l|l|l|}
\hline & Scheduled & \\
\hline
\end{tabular}




\subsubsection{Financial Systems.}

\begin{tabular}{|c|c|c|}
\hline Pre-Awrid Actions & $\begin{array}{l}\text { Schediled } \\
\text { Date }\end{array}$ & Actioner (o) \\
\hline $\begin{array}{l}\text { 1. Prepare an overview of the Financial Systems currently in } \\
\text { use. }\end{array}$ & $6 / 01 / 96$ & $\begin{array}{l}\text { P Felts } \\
\text { BCSR }\end{array}$ \\
\hline Post-Award Actions & & \\
\hline $\begin{array}{l}\text { 1. Identify any system changes necessary to support the PHMC } \\
\text { concept. }\end{array}$ & $7 / 01 / 96$ & $\begin{array}{l}\text { PHMC } \\
\text { D Pouley } \\
\text { BCSR }\end{array}$ \\
\hline
\end{tabular}

\subsection{HUMAN RESOURCES}

Human Resources will ensure continuity in all of its functional areas and fulfill ongoing commitments during contract transition. A comprehensive listing, by function, of key issues, actions, and activities is set forth below. Through this process all essential data will be readily available to carry out a smooth and informed transition of the workforce to the incoming PHMC contractor.

\subsubsection{Personnel Resources (WHC/BCSR)}

Develop actions to prepare, describe, and deliver essential information and records regarding staffing, site access support, outplacement, personnel records and database management.

\subsubsection{Staffing.}

\begin{tabular}{|c|c|c|}
\hline Pre-Awerd Adions & $\begin{array}{l}\text { Scheduled } \\
\text { Date }\end{array}$ & Actionee(s) \\
\hline $\begin{array}{l}\text { 1. Establish selection procem and eppropriately formatted pre- } \\
\text { interview information oc the beckground and experience of } \\
\text { incumbent employees. }\end{array}$ & $5 / 15 / 96$ & $\begin{array}{l}\text { RW Holleran } \\
\text { DE Hansen }\end{array}$ \\
\hline $\begin{array}{l}\text { 2. Develop a hiring process flow chart for all categories of } \\
\text { employees. }\end{array}$ & $6 / 01 / 96$ & $\begin{array}{l}\text { RW Holleran } \\
\text { DE Hansen }\end{array}$ \\
\hline $\begin{array}{l}\text { 3. Develop procedure for placement options for all incumbent } \\
\text { employees, e.g. selected; not selected; offer refused; WARN, } \\
\text { etc. }\end{array}$ & $6 / 01 / 96$ & $\begin{array}{l}\text { RW Holleran } \\
\text { DE Hansen }\end{array}$ \\
\hline $\begin{array}{l}\text { 4. Develop appropriate reports depicting status of all } \\
\text { staffing/placement activities. }\end{array}$ & $6 / 01 / 96$ & $\begin{array}{l}\text { RW Holleran } \\
\text { DE Hansen }\end{array}$ \\
\hline $\begin{array}{l}\text { 5. Update the contractual site-wide bargaining unit recall lists } \\
\text { and non-exempt/non-bargaining recall list. }\end{array}$ & $6 / 01 / 96$ & $\begin{array}{l}\text { RW Holleran } \\
\text { DE Hansen }\end{array}$ \\
\hline $\begin{array}{l}\text { 6. Update the Re-Deployment Assistance (RDA) list of non- } \\
\text { bargaining employees requiring job re-assignments. }\end{array}$ & $6 / 01 / 96$ & $\begin{array}{l}\text { RW Holleran } \\
\text { DE Hansen }\end{array}$ \\
\hline
\end{tabular}




\begin{tabular}{|c|c|c|}
\hline Pre-1ward Actions & Scheduled & $1 \mathrm{CH}_{0+\mathrm{e}}(\mathrm{s})$ \\
\hline 7. Prepare staffing plans, status, and rational for the PHMC. & 6/01/96 & $\begin{array}{l}\text { RW Holleran } \\
\text { DE Hansen }\end{array}$ \\
\hline \multicolumn{3}{|l|}{ Poct Awird Actions } \\
\hline $\begin{array}{l}\text { 1. Recommend to PHMC use of a centralized clearinghouse to } \\
\text { manage placements during transition. Coordinate employment } \\
\text { interview schedules, determine offer letter requirements and } \\
\text { maintain tracking system for interviews, offers, accepts and } \\
\text { declines. }\end{array}$ & $6 / 03 / 96$ & $\begin{array}{l}\text { RW Holleran } \\
\text { DE Hancen }\end{array}$ \\
\hline $\begin{array}{l}\text { 2. Provide PHMC with WHC flow chart depicting } \\
\text { ataffing/placement processes. }\end{array}$ & $6 / 03 / 96$ & $\begin{array}{l}\text { RW Holleren } \\
\text { DE Hansen }\end{array}$ \\
\hline $\begin{array}{l}\text { 3. Provide PHMC with procedure identified by WHC for } \\
\text { disposition of all non-transitioning incumbent employees. }\end{array}$ & $7 / 01 / 96$ & $\begin{array}{l}\text { RW Holleran } \\
\text { DE Hansen }\end{array}$ \\
\hline $\begin{array}{l}\text { 4. Provide PHMC with reports, as appropriate, depicting pre- } \\
\text { turnover status of all staffing/placement activities. }\end{array}$ & $7 / 01 / 96$ & $\begin{array}{l}\text { RW Holleran } \\
\text { DE Hansen }\end{array}$ \\
\hline $\begin{array}{l}\text { 5. Provide PHMC with sitewide bargaining unit recall lists and } \\
\text { galaried non-exempt downgrade and recall lists. }\end{array}$ & $10 / 01 / 96$ & $\begin{array}{l}\text { RW Holleran } \\
\text { DE Hansen }\end{array}$ \\
\hline 6. Provide appropriate information on excess employees. & $10 / 01 / 96$ & $\begin{array}{l}\text { RW Holleran } \\
\text { DE Hansen }\end{array}$ \\
\hline 7. Provide staffing plans, status, and rational to the PHMC. & $10 / 01 / 96$ & $\begin{array}{l}\text { RW Holleran } \\
\text { DE Hansen }\end{array}$ \\
\hline
\end{tabular}

\subsubsection{Site Access Support Services.}

\begin{tabular}{|c|c|c|}
\hline Pre-Award Actions & $\begin{array}{l}\text { Scheduled } \\
\text { Date }\end{array}$ & Letionee(s) \\
\hline $\begin{array}{l}\text { 1. Develop description of Pre-Employment Investigations } \\
\text { process flow. }\end{array}$ & $6 / 01 / 96$ & ER Jackson \\
\hline $\begin{array}{l}\text { 2. Develop description of Human Reliability Program (HRP) } \\
\text { processes including Personnel Security Assurance Program } \\
\text { (PSAP), U.S. Department of Transportation (DOT), and all } \\
\text { other substance abuse testing programs. }\end{array}$ & $6 / 01 / 96$ & $\begin{array}{l}\text { ER Jackson } \\
\text { BD Corder }\end{array}$ \\
\hline $\begin{array}{l}\text { 3. Develop description of Foreign National Visitors assignments } \\
\text { process, including usage statistics. }\end{array}$ & $6 / 01 / 96$ & ER Jackson \\
\hline Post-Award Actions & & \\
\hline 1. Provide process flow of Pre-Employment Investigations. & 9/01/96 & ER Jackson \\
\hline $\begin{array}{l}\text { 2. Provide description of Human Reliability Program (HRP) } \\
\text { processes including PSAP, DOT, and all other substances } \\
\text { abuse testing programs. }\end{array}$ & 9/01/96 & $\begin{array}{l}\text { ER Jackson } \\
\text { BD Corder }\end{array}$ \\
\hline $\begin{array}{l}\text { 3. Provide Foreign National Visitors assignments process, } \\
\text { including usage statistics. }\end{array}$ & 9/01/96 & ER Jackson \\
\hline
\end{tabular}




\subsubsection{Outplacement.}

\begin{tabular}{|c|c|c|}
\hline 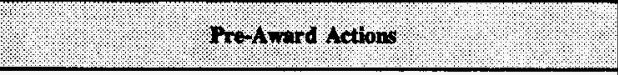 & $\begin{array}{l}\text { Scheduled } \\
\text { Date }\end{array}$ & Actionee (s) \\
\hline $\begin{array}{l}\text { 1. Contact General Counsel for determination of ownership of } \\
\text { "former employee" Re-Employment Opportunity Center } \\
\text { (ROC) files and usage database. }\end{array}$ & $5 / 15 / 96$ & CH Smith \\
\hline $\begin{array}{l}\text { 2. Collect copies of Hanford Workforce Restructuring Plan and } \\
\text { the ROC brochure. }\end{array}$ & $6 / 01 / 96$ & CH Smith \\
\hline $\begin{array}{l}\text { 3. Prepare a list of all former employees with } 3161 \text { Plan hiring } \\
\text { preference. }\end{array}$ & 6/01/96 & CH Smith \\
\hline $\begin{array}{l}\text { 4. Prepare guidance worksheets and coordinate applicable } \\
\text { resources for preparation of resumes, or the like, prior to } \\
\text { employees interviewing with the PHMC contractor. }\end{array}$ & $6 / 01 / 96$ & $\begin{array}{l}\text { CH Smith } \\
\text { CA Locke }\end{array}$ \\
\hline $\begin{array}{l}\text { 5. Develop a recommendation for the PHMC contractor to } \\
\text { support continuation of outplacement services already in } \\
\text { progress. }\end{array}$ & $6 / 01 / 96$ & CH Smith \\
\hline Post-Anard Actions & & \\
\hline $\begin{array}{l}\text { 1. Coordinate with PHMC contractor to ensure job search, } \\
\text { interviewing and transition resources for employees not } \\
\text { receiving or declining employment offers. }\end{array}$ & $6 / 03 / 96$ & CH Smith \\
\hline $\begin{array}{l}\text { 2. Provide to the PHMC contractor copies of Hanford } \\
\text { Workforce Restructuring Plan and the ROC brochure. }\end{array}$ & $6 / 03 / 96$ & CH Smith \\
\hline $\begin{array}{l}\text { 3. Provide a list of all former employees with } 3161 \text { Plan hiring } \\
\text { preference. }\end{array}$ & $6 / 03 / 96$ & CH Smith \\
\hline $\begin{array}{l}\text { 4. Provide guidance worksheets and coordinate applicable } \\
\text { resources for preparation of resumes, or the like, prior to } \\
\text { employees interviewing with the PHMC contractor. }\end{array}$ & $6 / 03 / 96$ & $\begin{array}{l}\text { CH Smith } \\
\text { CA Locke }\end{array}$ \\
\hline $\begin{array}{l}\text { 5. Provide recommendation for the PHMC contractor to support } \\
\text { continuation of outplacement services already in progress. }\end{array}$ & $7 / 01 / 96$ & CH Smith \\
\hline
\end{tabular}

\subsubsection{Personnel Records and Database Administration.}

\begin{tabular}{|c|c|c|}
\hline Pre-Award Actions & $\begin{array}{l}\text { Scheduled } \\
\text { Date }\end{array}$ & Actionee(s) \\
\hline $\begin{array}{l}\text { 1. Determine, in conjunction with General Counsel, extent of } \\
\text { employee records and data to be transferred to PHMC. }\end{array}$ & $6 / 01 / 96$ & $\begin{array}{l}\text { CA Locke } \\
\text { CK MacLeod }\end{array}$ \\
\hline $\begin{array}{l}\text { 2. Review readiness to provide PHMC appropriate employee } \\
\text { demographic data, by organization, such as name, position, } \\
\text { classification, employment status and service date. }\end{array}$ & $6 / 01 / 96$ & CA Locke \\
\hline 3. Plan a demonstration of PeopleSOFT capabilities for PHMC. & $6 / 01 / 96$ & CA Locke \\
\hline $\begin{array}{l}\text { 4. Prepare update for system modification priorities and } \\
\text { troubleshooting activities to the PeopleSoft database. }\end{array}$ & $6 / 01 / 96$ & CA Locke \\
\hline 5. Develop detailed organization charts/lists. & 6/01/96 & CA Locke \\
\hline
\end{tabular}




\begin{tabular}{|l|c|c|}
\hline $\begin{array}{l}\text { Resources department personnel. } \\
\text { Develop listing of computer hardware/software for Human }\end{array}$ & $6 / 01 / 96$ & CA Locke \\
\hline $\begin{array}{l}\text { 6rovide personnel data and final lists transferring incumbent } \\
\text { employees to PHMC. }\end{array}$ & $10 / 01 / 96$ & CA Locke \\
\hline $\begin{array}{l}\text { 2. Coordinate with PHMC to determine method for transferring } \\
\text { required employee personnel information. }\end{array}$ & $9 / 01 / 96$ & CA Locke \\
\hline $\begin{array}{l}\text { 3. Obtain appropriate personnel information releases from } \\
\text { employees. }\end{array}$ & $10 / 01 / 96$ & CA Locke \\
\hline 4. Demonstrate PeopleSOFT to PHMC contractor. & $10 / 01 / 96$ & CA Locke \\
\hline 5. Provide appropriate organization charts/lists to PHMC. & $6 / 03 / 96$ & CA Locke \\
\hline $\begin{array}{l}\text { 6. Provide a listing of computer hardware/software for Human } \\
\text { Resources department personnel. }\end{array}$ & $10 / 01 / 96$ & CA Locke \\
\hline 7. Initiate status change forms for employees. & $10 / 01 / 96$ & CA Loke \\
\hline
\end{tabular}

\subsubsection{Personnel Resources (ICF KH)}

Develop actions, where activity is unique or not provided by WHC, to prepare, describe, and deliver essential information and records regarding staffing, site access support, outplacement, personnel records, and database management. ICF KH will coordinate with WHC to avoid duplication in briefings or submission of multiple formats.

\subsubsection{Staffing.}

\begin{tabular}{|l|l|l|}
\hline $\begin{array}{l}\text { Schetuled } \\
\text { Date }\end{array}$ & \\
\hline $\begin{array}{l}\text { 1. Establish selection process and appropriately formatted pre- } \\
\text { incumbent employees. Coordinate with WHC, as } \\
\text { appropriate, to avoid multiple formats. }\end{array}$ & $5 / 15 / 96$ & $\begin{array}{l}\text { VC Hodgin } \\
\text { CG Larsen }\end{array}$ \\
\hline $\begin{array}{l}\text { 2. Develop a hiring process flow chart for all categories of } \\
\text { employees. }\end{array}$ & $6 / 01 / 96$ & $\begin{array}{l}\text { VC Hodgin } \\
\text { CG Larsen }\end{array}$ \\
\hline $\begin{array}{l}\text { 3. Develop procedure for placement options for all incumbent } \\
\text { employees, e.g., selected; not selected; offer refused; } \\
\text { WARN, etc. }\end{array}$ & $6 / 01 / 96$ & $\begin{array}{l}\text { VC Hodgin } \\
\text { CG Larsen }\end{array}$ \\
\hline $\begin{array}{l}\text { 4. Develop appropriate reports depicting status of all } \\
\text { staffing/placement activities. }\end{array}$ & $6 / 01 / 96$ & $\begin{array}{l}\text { VC Hodgin } \\
\text { CG Larsen }\end{array}$ \\
\hline $\begin{array}{l}\text { 5. Update the contractual sitewide bargaining unit recall lists } \\
\text { and non-exempt/non-bargaining recall list. }\end{array}$ & $6 / 01 / 96$ & $\begin{array}{l}\text { VC Hodgin } \\
\text { CG Larsen }\end{array}$ \\
\hline $\begin{array}{l}\text { 6. Update the Re-Deployment Assistance (RDA) list of non- } \\
\text { bargaining employees requiring job re-assignments. }\end{array}$ & $6 / 01 / 96$ & $\begin{array}{l}\text { VC Hodgin } \\
\text { JJ Chunn }\end{array}$ \\
\hline
\end{tabular}




\begin{tabular}{|c|c|c|}
\hline Prestoro fetions & ocheduled & Yetronec(s) \\
\hline 7. Prepare staffing plans, status, and rational for the PHMC. & $6 / 01 / 96$ & $\begin{array}{l}\text { VC Hodgin } \\
\text { CG Larsen }\end{array}$ \\
\hline Post_Award Actions & & \\
\hline $\begin{array}{l}\text { 1. Recommend to PHMC use of a centralized clearing house to } \\
\text { manage placements during transition. Coordinate } \\
\text { employment interview schedules, determine offer letter } \\
\text { requirements and maintain tracking system for interviews, } \\
\text { offers, accepts and declines. }\end{array}$ & $6 / 03 / 96$ & $\begin{array}{l}\text { VC Hodgin } \\
\text { CG Laraen }\end{array}$ \\
\hline $\begin{array}{l}\text { 2. Provide PHMC with flow chart depicting staffing/placement } \\
\text { processes. }\end{array}$ & $6 / 03 / 96$ & $\begin{array}{l}\text { VC Hodgin } \\
\text { CG Larsen }\end{array}$ \\
\hline $\begin{array}{l}\text { 3. Provide PHMC with procedure identified for disposition of } \\
\text { all incumbent employees. }\end{array}$ & $7 / 01 / 96$ & $\begin{array}{l}\text { VC Hodgin } \\
\text { CG Larsen }\end{array}$ \\
\hline $\begin{array}{l}\text { 4. Provide PHMC with reports, as appropriate, depicting status } \\
\text { of all staffing/placement activities. }\end{array}$ & $7 / 01 / 96$ & $\begin{array}{l}\text { VC Hodgin } \\
\text { CG Larsen } \\
\text { JJ Chunn }\end{array}$ \\
\hline 5. Provide appropriate information on excess employees. & $10 / 01 / 96$ & $\begin{array}{l}\text { VC Hodgin } \\
\text { JJ Chunn }\end{array}$ \\
\hline 6. Provide staffing plans, status, and rational to the PHMC. & $10 / 01 / 96$ & $\begin{array}{l}\text { VC Hodgin } \\
\text { CG Larsen }\end{array}$ \\
\hline
\end{tabular}

\subsubsection{Site Access Support Services}

\begin{tabular}{|c|c|c|}
\hline Pre-1ward Actions & Scheduled & sethoned (s) \\
\hline $\begin{array}{l}\text { 1. Develop description of Pre-Employment Investigations } \\
\text { process flow. }\end{array}$ & $6 / 01 / 96$ & CG Larsen \\
\hline Post-A ward Actions & & \\
\hline 1. Provide process flow of Pre-Employment Investigations. & $9 / 01 / 96$ & CG Larsen \\
\hline
\end{tabular}

\subsubsection{Outplacement.}

\begin{tabular}{|l|l|l|l|}
\hline & Scheduled & Date & \\
\hline $\begin{array}{l}\text { 1. Prepare a list of all former employees with 3161 Plan hiring } \\
\text { preference. }\end{array}$ & $6 / 01 / 96$ & CG Larsen \\
\hline
\end{tabular}




\subsubsection{Personnel Records and Database Administration.}

\begin{tabular}{|c|c|c|}
\hline Hre-Avard hetions & Schemiled & Atetionee(f) \\
\hline $\begin{array}{l}\text { 1. Determine, in conjunction with General Counsel, extent of } \\
\text { employee records and data to be transferred to PHMC. }\end{array}$ & $6 / 01 / 96$ & $\begin{array}{l}\text { vC Hodgin } \\
\text { Olsen }\end{array}$ \\
\hline $\begin{array}{l}\text { 2. Review readiness to provide PHMC appropriate employee } \\
\text { demographic data, by organization, such as name, position, } \\
\text { classification, employment status and service date. }\end{array}$ & 6/01/96 & $\begin{array}{l}\text { VC Hodgin } \\
\text { CG Larsen }\end{array}$ \\
\hline 3. Develop detailed organization charts/lists. & $6 / 01 / 96$ & $\begin{array}{l}\text { VC Hodgin } \\
\text { CG Larsen }\end{array}$ \\
\hline $\begin{array}{l}\text { 4. Develop listing of computer hardware/software for Human } \\
\text { Resources department personnel. }\end{array}$ & $6 / 01 / 96$ & CG Larsen \\
\hline Post-Award Actions & & \\
\hline $\begin{array}{l}\text { 1. Provide personnel data and final lists transferring incumbent } \\
\text { employees to PHMC. }\end{array}$ & $10 / 01 / 96$ & $\begin{array}{l}\text { VC Hodgin } \\
\text { CG Larsen }\end{array}$ \\
\hline 2. Initiate status change forms for employees. & $10 / 01 / 96$ & CG Larsen \\
\hline $\begin{array}{l}\text { 3. Obtain appropriate personnel information releases from } \\
\text { employees. }\end{array}$ & $10 / 01 / 96$ & $\begin{array}{l}\text { VC Hodgin } \\
\text { CG Larsen }\end{array}$ \\
\hline 4. Provide appropriate organization charts/lists to PHMC. & $10 / 01 / 96$ & $\begin{array}{l}\text { VC Hodgin } \\
\text { CG Larsen }\end{array}$ \\
\hline $\begin{array}{l}\text { 5. Provide a listing of computer hardware/software for Human } \\
\text { Resources department personnel. }\end{array}$ & $10 / 01 / 96$ & CG Larsen \\
\hline
\end{tabular}

\subsubsection{Total Compensation (WHC/BCSR)}

Determine, develop, and prepare appropriate data regarding the salary plan, worker's compensation, job classifications, salary structures, and other compensation programs. Perform the same requirements as they relate to benefit plan documentation, plan coverages and status, return-to-work, pension, savings, recognition programs and the like.

\subsubsection{Salary Plan.}

\begin{tabular}{|c|c|c|}
\hline Pre-Award Actions & $\begin{array}{l}\text { Schetuled } \\
\text { Date }\end{array}$ & Actioneets) \\
\hline $\begin{array}{l}\text { 1. Prepare a list of calendar year salary plan actions scheduled } \\
\text { for October, November, and December } 1996 \text {. }\end{array}$ & $6 / 01 / 96$ & $\begin{array}{l}\text { FA Powers } \\
\text { H Lacher }\end{array}$ \\
\hline $\begin{array}{l}\text { 2. Determine ownership of prior year salary increase plans and } \\
\text { disposition accordingly. }\end{array}$ & $6 / 01 / 96$ & $\begin{array}{l}\text { FA Powers } \\
\text { H Lacher }\end{array}$ \\
\hline $\begin{array}{l}\text { 3. Determine distribution of remaining salary increase funds } \\
\text { which are usually distributed as adjustments in the } \\
\text { November/December time frame. Estimate available funds } \\
\text { and recommend a distribution method. }\end{array}$ & $6 / 01 / 96$ & $\begin{array}{l}\text { FA Powers } \\
\text { H Lacher }\end{array}$ \\
\hline
\end{tabular}




\begin{tabular}{|c|c|c|}
\hline Post-A ward Actions & $\begin{array}{l}\text { Scheduled } \\
\text { Date }\end{array}$ & Actionee(s) \\
\hline $\begin{array}{l}\text { 1. Status PHMC contractor regarding salary actions for } \\
\text { October, November, and December } 1996 \text { for those } \\
\text { employees accepting offers. }\end{array}$ & $10 / 01 / 96$ & $\begin{array}{l}\text { FA Powers } \\
\text { H Lacher }\end{array}$ \\
\hline $\begin{array}{l}\text { 2. Review salary planning process and provide PHMC } \\
\text { contractor a list of prior year salary increase plans as } \\
\text { appropriate. }\end{array}$ & $10 / 01 / 96$ & $\begin{array}{l}\text { FA Powers } \\
\text { H Lacher }\end{array}$ \\
\hline $\begin{array}{l}\text { 3. Coordinate with PHMC to determine distribution of } \\
\text { remaining salary increase funds which are usually distributed } \\
\text { as adjustments in the November/December time frame. } \\
\text { Estimate available funds and recommend a distribution } \\
\text { method. }\end{array}$ & $10 / 01 / 96$ & $\begin{array}{l}\text { FA Powers } \\
\text { H Lacher }\end{array}$ \\
\hline
\end{tabular}

\subsubsection{Workers Compensation.}

\begin{tabular}{|c|c|c|}
\hline Pre-Award Actions & $\begin{array}{l}\text { Scheduled } \\
\text { Date }\end{array}$ & Actioned(b) \\
\hline $\begin{array}{l}\text { 1. Develop a computerized report to compile a preliminary } \\
\text { listing of Workers Compensation liabilities including names, } \\
\text { provisions under which they left, status including liabilities } \\
\text { associated with all potential outcomes (ICF KH, BCSR, and } \\
\text { WHC). }\end{array}$ & $6 / 01 / 96$ & $\begin{array}{l}\text { FA Powers } \\
\text { H Lacher }\end{array}$ \\
\hline Post-Award Actions & \% & 4 \\
\hline $\begin{array}{l}\text { 1. Provide the PHMC contractor a complete preliminary list of } \\
\text { employees on Workers Compensation by company (CCF KH, } \\
\text { BCSR, and WHC). }\end{array}$ & $6 / 03 / 96$ & $\begin{array}{l}\text { FA Powers } \\
\text { H Lacher }\end{array}$ \\
\hline $\begin{array}{l}\text { 2. Generate a final report for turnover of Workers } \\
\text { Compensation liabilities including names, provisions under } \\
\text { which they left, and the liabilities associated with all potential } \\
\text { outcomes. }\end{array}$ & $10 / 01 / 96$ & $\begin{array}{l}\text { FA Powers } \\
\text { H Lacher }\end{array}$ \\
\hline
\end{tabular}

\subsubsection{Job Classifications and Salary Structure.}

\begin{tabular}{|c|c|c|}
\hline Pre-Award Actions & $\begin{array}{l}\text { Schediled } \\
\text { Date }\end{array}$ & Actionee(s) \\
\hline $\begin{array}{l}\text { 1. Prepare a complete list of employees by name, organization, } \\
\text { title, grade and actual salary. }\end{array}$ & 6/01/96 & $\begin{array}{l}\text { FA Powers } \\
\text { H Lacher }\end{array}$ \\
\hline $\begin{array}{l}\text { 2. Prepare an analysis of the broadbanding salary structure pilot } \\
\text { and recommendation regarding future implementation. }\end{array}$ & 6/01/96 & $\begin{array}{l}\text { FA Powers } \\
\text { H Lacher }\end{array}$ \\
\hline 3. Prepare a complete listing of all jobs by title and grade. & 6/01/96 & $\begin{array}{l}\text { FA Powers } \\
\text { H Lacher }\end{array}$ \\
\hline $\begin{array}{l}\text { 4. Generate a report which compiles a preliminary listing of all } \\
\text { employees who qualify for certification pay or stipends and } \\
\text { the criteria under which these are paid. }\end{array}$ & 6/01/96 & $\begin{array}{l}\text { FA Powers } \\
\text { H Lacher }\end{array}$ \\
\hline
\end{tabular}




\begin{tabular}{|c|c|c|}
\hline Pred Awrid setions & sonediled & $(0,490+2)$ \\
\hline $\begin{array}{l}\text { 5. Develop a list of bargaining unit, salaried non-exempt and } \\
\text { exempt salary structures. }\end{array}$ & $6 / 01 / 96$ & $\begin{array}{l}\text { FA Powers } \\
\text { H Lacher }\end{array}$ \\
\hline $\begin{array}{l}\text { 6. Prepare copies of all compensation policies including } \\
\text { temporary upgrades, appointments of fellow/advisory } \\
\text { engineers, and signature authority as well as a detailed } \\
\text { description of any other practices. }\end{array}$ & $6 / 01 / 96$ & $\begin{array}{l}\text { FA Powers } \\
\text { H Lacher }\end{array}$ \\
\hline \multicolumn{3}{|l|}{ Post $/$ ward Actions } \\
\hline 1. Provide a complete listing of all jobs by title and grade. & $6 / 03 / 96$ & $\begin{array}{l}\text { FA Powers } \\
\text { H Lacher }\end{array}$ \\
\hline $\begin{array}{l}\text { 2. Provide an analysis of the broadbanding pilot and } \\
\text { recommendation regarding future implementation. }\end{array}$ & $9 / 01 / 96$ & $\begin{array}{l}\text { FA Powers } \\
\text { H Lacher }\end{array}$ \\
\hline $\begin{array}{l}\text { 3. Generate and transmit a final turnover report listing all } \\
\text { PHMC contractor accepted employees by name, organization, } \\
\text { title, grade and actual salary. }\end{array}$ & $10 / 01 / 96$ & $\begin{array}{l}\text { FA Powers } \\
\text { H Lacher }\end{array}$ \\
\hline $\begin{array}{l}\text { 4. Generate a final turnover report listing all employees who } \\
\text { qualify for certification pay or stipends. }\end{array}$ & $10 / 01 / 96$ & $\begin{array}{l}\text { FA Powers } \\
\text { H Lacher }\end{array}$ \\
\hline $\begin{array}{l}\text { 5. Provide a list of bargaining unit, salaried non-exempt and } \\
\text { exempt salary structures. }\end{array}$ & $10 / 01 / 96$ & $\begin{array}{l}\text { FA Powers } \\
\text { H Lacher }\end{array}$ \\
\hline $\begin{array}{l}\text { 6. Provide copies of all compensation policies including } \\
\text { temporary upgrades, appointments of fellow/advisory } \\
\text { engineers, and signature authority as well as a detailed } \\
\text { description of any other practices. }\end{array}$ & $10 / 01 / 96$ & $\begin{array}{l}\text { FA Powers } \\
\text { H Lacher }\end{array}$ \\
\hline
\end{tabular}

\subsubsection{Performance Appraisals.}

\begin{tabular}{|l|c|l|}
\hline & $\begin{array}{l}\text { Scheduled } \\
\text { Date }\end{array}$ & Pre-Award Actions \\
\hline $\begin{array}{l}\text { 1. Complete an analysis of the pilot of The Employee Appraiser } \\
\text { software and prepare a recommendation regarding future } \\
\text { implementation. }\end{array}$ & $6 / 01 / 96$ & $\begin{array}{l}\text { FA Powers } \\
\text { H Lacher }\end{array}$ \\
\hline $\begin{array}{l}\text { 1. Deliver a complete analysis of the pilot of The Employee } \\
\text { Appraiser software and make a recommendation regarding } \\
\text { future implementation. }\end{array}$ & $9 / 01 / 96$ & $\begin{array}{l}\text { FA Powers } \\
\text { H Lacher }\end{array}$ \\
\hline
\end{tabular}




\subsubsection{Benefits Administration.}

\section{Plan Documentation}

\begin{tabular}{|c|c|c|}
\hline Pre-Award Actions & $\begin{array}{c}\text { Scheduled } \\
\text { Date }\end{array}$ & Actionee(s) \\
\hline $\begin{array}{l}\text { 1. Identify and gather copies of all formal plan documents, } \\
\text { summary plan descriptions, and administrative contracts with } \\
\text { third parties. Review these for language pertaining to } \\
\text { sponsor change or plan termination. }\end{array}$ & $6 / 01 / 96$ & $\begin{array}{l}\text { FA Powers } \\
\text { JR Sorensen }\end{array}$ \\
\hline $\begin{array}{l}\text { 2. Document practices and procedures not otherwise } \\
\text { documented (e.g., not in MRPs, etc.) and all known } \\
\text { commitments made by previous contractors. }\end{array}$ & $6 / 01 / 96$ & $\begin{array}{l}\text { FA Powers } \\
\text { JR Sorensen }\end{array}$ \\
\hline $\begin{array}{l}\text { 3. To the extent they are available, collect copies of all } \\
\text { predecessor plan documents and summary plan descriptions } \\
\text { and a listing of the people to whom they apply (also covered } \\
\text { separately under the specific heading). }\end{array}$ & $6 / 01 / 96$ & $\begin{array}{l}\text { FA Powers } \\
\text { JR Sorensen }\end{array}$ \\
\hline Post-Award Actions & & \\
\hline $\begin{array}{l}\text { 1. Provide, as appropriate, to PHMC practices and procedures } \\
\text { not otherwise documented (e.g., not in MRPs, etc.) and all } \\
\text { known commitments made by previous contractors. }\end{array}$ & $8 / 01 / 96$ & $\begin{array}{l}\text { FA Powers } \\
\text { JR Sorensen }\end{array}$ \\
\hline $\begin{array}{l}\text { 2. Provide appropriate copies, to the extent they are available, } \\
\text { of all predecessor plan documents and summary plan } \\
\text { descriptions and a listing of the people to whom they apply } \\
\text { (also covered separately under the specific heading). }\end{array}$ & $8 / 01 / 96$ & $\begin{array}{l}\text { FA Powers } \\
\text { JR Sorensen }\end{array}$ \\
\hline $\begin{array}{l}\text { 3. Notify third-party vendors/administrators of all benefit plans } \\
\text { of the upcoming contract changeover and arrange with them } \\
\text { and the PHMC contractor, for transfer, modification or } \\
\text { termination of agreements between them and PHMC. }\end{array}$ & $8 / 01 / 96$ & $\begin{array}{l}\text { FA Powers } \\
\text { JR Sorensen }\end{array}$ \\
\hline
\end{tabular}

\section{Plan Coverage and Status}

\begin{tabular}{|c|c|c|}
\hline Pre-Award Actions & $\begin{array}{l}\text { Scheduled } \\
\text { Datre }\end{array}$ & fetiones (s) \\
\hline $\begin{array}{l}\text { 1. Develop a report which compiles a preliminary listing of } \\
\text { long-term disability participants including identification of } \\
\text { specific provisions and the funding sources which apply to } \\
\text { each individual or group of individuals. Will include } \\
\text { commitments for medical, life or continued pension } \\
\text { accruals. Must ensure a link between these and any future } \\
\text { plans. }\end{array}$ & $6 / 01 / 96$ & $\begin{array}{l}\text { FA Powers } \\
\text { JR Sorensen }\end{array}$ \\
\hline $\begin{array}{l}\text { 2. Develop a report which compiles a preliminary listing of all } \\
\text { plan liabilities and commitments, verify assumption of same } \\
\text { by the PHMC contractor, as appropriate. }\end{array}$ & $6 / 01 / 96$ & $\begin{array}{l}\text { FA Powers } \\
\text { JR Sorensen }\end{array}$ \\
\hline
\end{tabular}




\begin{tabular}{|c|c|c|}
\hline Are-Award Actions & $\begin{array}{l}\text { Scheduled } \\
\text { Date }\end{array}$ & fotopec() \\
\hline $\begin{array}{l}\text { 3. Develop a report which compiles a listing of short-term } \\
\text { disability participants including salary continuance status, } \\
\text { payment amount being received, the obligation to return } \\
\text { them to their same position, and the potential liability if } \\
\text { they go to long-term disability after } 6 \text { months. }\end{array}$ & $6 / 01 / 96$ & $\begin{array}{l}\text { FA Powers } \\
\text { JR Sorensen }\end{array}$ \\
\hline $\begin{array}{l}\text { 4. Identify any other obligations (e.g., sick leave accrual from } \\
\text { BCSR) carried over from predecessor contractors. }\end{array}$ & $6 / 01 / 96$ & $\begin{array}{l}\text { FA Powers } \\
\text { JR Sorensen }\end{array}$ \\
\hline $\begin{array}{l}\text { 5. Develop a health care plan report which identifies } \\
\text { participants by plan (including vision and dental), current } \\
\text { eligibility and enrollment status, and contribution amounts } \\
\text { by company. Break down into activities (several groups } \\
\text { within this), retired (several groups within this), } \\
\text { terminations, COBRA, leaves of absence, etc. }\end{array}$ & 6/01/96 & $\begin{array}{l}\text { FA Powers } \\
\text { JR Sorensen }\end{array}$ \\
\hline 6. Complete an eligibility review for all plan participants. & 6/01/96 & $\begin{array}{l}\text { FA Powers } \\
\text { JR Sorensen }\end{array}$ \\
\hline $\begin{array}{l}\text { 7. Develop a computerized report which compiles a } \\
\text { preliminary list of plan participants receiving termination } \\
\text { benefits, their current status, and dates when coverage level } \\
\text { or contribution rates will change. }\end{array}$ & $6 / 01 / 96$ & $\begin{array}{l}\text { FA Powers } \\
\text { JR Sorensen }\end{array}$ \\
\hline $\begin{array}{l}\text { 8. Develop a report which compiles a preliminary listing of } \\
\text { employees who are enrolled in Personal Accident insurance } \\
\text { for employee and spouse, including contribution amounts. }\end{array}$ & $6 / 01 / 96$ & $\begin{array}{l}\text { FA Powers } \\
\text { JR Sorensen }\end{array}$ \\
\hline $\begin{array}{l}\text { 9. Develop a report which compiles a preliminary listing of all } \\
\text { employees who are on family. educational, military or } \\
\text { personal leave. Include any benefits coverages they have, } \\
\text { expected return dates and ohligations to place them in a } \\
\text { position upon return. }\end{array}$ & $6 / 01 / 96$ & $\begin{array}{l}\text { FA Powers } \\
\text { JR Sorensen }\end{array}$ \\
\hline 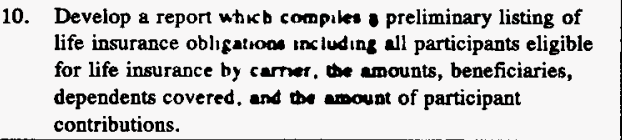 & 6/01/96 & $\begin{array}{l}\text { FA Powers } \\
\text { JR Sorensen }\end{array}$ \\
\hline $\begin{array}{l}\text { 11. Develop report of Income Protectoon Plan participants; } \\
\text { active employees, w well as those receiving benefits from } \\
\text { the plan. }\end{array}$ & $6 / 01 / 96$ & $\begin{array}{l}\text { FA Powers } \\
\text { JR Sorensen }\end{array}$ \\
\hline \multicolumn{3}{|l|}{ Post-Award Actions } \\
\hline $\begin{array}{l}\text { 1. Provide the PHMC a report listing short-term disability } \\
\text { participants including salary continuance status, payment } \\
\text { amount being received, the obligation to return them to } \\
\text { their same position, and the potential liability if they go to } \\
\text { long-term disability after } 6 \text { months. }\end{array}$ & 6/03/96 & $\begin{array}{l}\text { FA Powers } \\
\text { JR Sorensen }\end{array}$ \\
\hline $\begin{array}{l}\text { 2. Arrange for assumption or transition of Flexible Spending } \\
\text { Accounts for the remainder of the calendar year. }\end{array}$ & 8/01/96 & $\begin{array}{l}\text { FA Powers } \\
\text { JR Sorensen }\end{array}$ \\
\hline $\begin{array}{l}\text { 3. Determine whether WHC will continue to provide payroll } \\
\text { deduction for Group Universal Life insurance coverage. }\end{array}$ & 8/01/96 & $\begin{array}{l}\text { FA Powers } \\
\text { JR Sorensen }\end{array}$ \\
\hline
\end{tabular}




\begin{tabular}{|c|c|c|}
\hline Poot-Arard letions & Scheduled & Actipoper) \\
\hline $\begin{array}{l}\text { 4. Generate and provide a final turnover report for the PHMC } \\
\text { listing all long-term disability participants including } \\
\text { identification of specific plan provisions and the funding } \\
\text { sources which apply to each. Include commitments for } \\
\text { medical, life, or continued pension accruals. }\end{array}$ & $10 / 01 / 96$ & $\begin{array}{l}\text { FA Powers } \\
\text { JR Sorensen }\end{array}$ \\
\hline $\begin{array}{l}\text { 5. Generate a final PHMC turnover report listing all plan } \\
\text { liabilities and commitments. }\end{array}$ & $10 / 01 / 96$ & $\begin{array}{l}\text { FA Powers } \\
\text { JR Sorensen }\end{array}$ \\
\hline $\begin{array}{l}\text { 6. Generate a final turnover report listing short-term disability } \\
\text { participants including salary continuance status, payment } \\
\text { amount being received, the obligation to return them to } \\
\text { their same position, and the potential liability if they go to } \\
\text { long-term disability. }\end{array}$ & $10 / 01 / 96$ & $\begin{array}{l}\text { FA Powers } \\
\text { JR Sorensen }\end{array}$ \\
\hline $\begin{array}{l}\text { 7. Generate a final turnover health care plan report which } \\
\text { identifies participants by plan (including vision and dental), } \\
\text { current eligibility and enrollment status, and contribution } \\
\text { amounts by company. Break down into actives (several } \\
\text { groups within this), retired (several groups within this), } \\
\text { terminations, COBRA, leaves of absence, etc. }\end{array}$ & $10 / 01 / 96$ & $\begin{array}{l}\text { FA Powers } \\
\text { JR Sorensen }\end{array}$ \\
\hline $\begin{array}{l}\text { 8. Generate a final turnover list of plan participants receiving } \\
\text { termination benefits, their current status, and dates when } \\
\text { coverage level or contribution rates will change. }\end{array}$ & $10 / 01 / 96$ & $\begin{array}{l}\text { FA Powers } \\
\text { JR Sorensen }\end{array}$ \\
\hline $\begin{array}{l}\text { 9. Generate a final turnover list of employees who are } \\
\text { enrolled in Personal Accident insurance for employee and } \\
\text { spouse, including contribution amounts. }\end{array}$ & $10 / 01 / 96$ & $\begin{array}{l}\text { FA Powers } \\
\text { JR Sorensen }\end{array}$ \\
\hline $\begin{array}{l}\text { 10. Generate a final turnover ina of all employees who are on } \\
\text { family, educational, miliury or personal leave. Include } \\
\text { any benefit coverages they have, expected return dates and } \\
\text { obligations, if any, to plece them in a position upon return. }\end{array}$ & $10 / 01 / 96$ & $\begin{array}{l}\text { FA Powers } \\
\text { R Sorensen }\end{array}$ \\
\hline $\begin{array}{l}\text { 11. Generate a final turoover repor lirting life insurance } \\
\text { obligations includins all partcipants eligible for life } \\
\text { insurance by carriet. the amounts, dependents covered, and } \\
\text { the amount of partucipant cootributions. }\end{array}$ & $10 / 01 / 96$ & $\begin{array}{l}\text { FA Powers } \\
\text { JR Sorensen }\end{array}$ \\
\hline $\begin{array}{l}\text { 12. Generate a final turbover lim of employees who are } \\
\text { enrolled in the Income Procecuos Plan. Include a list of } \\
\text { former employees who are receiving benefits from the } \\
\text { plan. }\end{array}$ & $10 / 01 / 96$ & $\begin{array}{l}\text { FA Powers } \\
\text { JR Sorensen }\end{array}$ \\
\hline $\begin{array}{l}\text { 13. Provide notification to those individuals who have benefits } \\
\text { (i.e., COBRA, leave of absence, and retiree status) of any } \\
\text { plan changes affecting coverage. }\end{array}$ & $10 / 01 / 96$ & $\begin{array}{l}\text { FA Powers } \\
\text { JR Sorensen }\end{array}$ \\
\hline
\end{tabular}

\subsubsection{Return-to-Work.}

\begin{tabular}{|l|c|l|l|}
\hline 1 Actionee(s) \\
\hline $\begin{array}{l}\text { 1. Document the return-to-work process including all applicable } \\
\text { MRPs and other appropriate policies/ practices. }\end{array}$ & $6 / 01 / 96$ & $\begin{array}{l}\text { FA Powers } \\
\text { JR Sorensen }\end{array}$ \\
\hline
\end{tabular}




\begin{tabular}{|l|l|l|}
\hline & $\begin{array}{c}\text { Schednled } \\
\text { Date }\end{array}$ & Pre-tward Actions \\
\hline $\begin{array}{l}\text { 2. Prepare a list of any employees being tracked under the } \\
\text { Workability program expected return date and obligations, if } \\
\text { any, for a position upon return. }\end{array}$ & $6 / 01 / 96$ & $\begin{array}{l}\text { FA Powers } \\
\text { JR Sorensen }\end{array}$ \\
\hline $\begin{array}{l}\text { 1. Provide the PHMC documented relationships between the } \\
\text { return-to-work coordinator, HEHF, and Workability. }\end{array}$ & $8 / 01 / 96$ & $\begin{array}{l}\text { FA Powers } \\
\text { JR Sorensen }\end{array}$ \\
\hline $\begin{array}{l}\text { 2. Prepare and transmit a final turnover report listing any } \\
\text { employees under the managed diaability program expected } \\
\text { return date and obligation, if any, for a position upon return. }\end{array}$ & $10 / 01 / 96$ & $\begin{array}{l}\text { FA Powers } \\
\text { JR Sorensen }\end{array}$ \\
\hline
\end{tabular}

\subsubsection{Pension and Savings.}

\begin{tabular}{|c|c|c|}
\hline Pre-Award Actions & $\begin{array}{l}\text { Scheduled } \\
\text { Date }\end{array}$ & Actionee(s) \\
\hline $\begin{array}{l}\text { 1. Develop a report which compiles a preliminary listing of all } \\
\text { vested terminated employees and the accrued pension } \\
\text { liability. }\end{array}$ & $6 / 01 / 96$ & $\begin{array}{l}\text { MJ Byrd } \\
\text { FA Powers } \\
\text { JR Sorensen }\end{array}$ \\
\hline $\begin{array}{l}\text { 2. Develop a report which identifies all active employees and } \\
\text { their accrued pension benefit. }\end{array}$ & $6 / 01 / 96$ & $\begin{array}{l}\text { MJ Byrd } \\
\text { FA Powers } \\
\text { JR Sorensen }\end{array}$ \\
\hline $\begin{array}{l}\text { 3. Prepare a list of current participants in the Savings Plan } \\
\text { including deductions, year-to-date contributions, outstanding } \\
\text { loans and the company match commitment. }\end{array}$ & $6 / 01 / 96$ & $\begin{array}{l}\text { FA Powers } \\
\text { JR Sorensen }\end{array}$ \\
\hline (1) Post-Award Actions & \% & औ \\
\hline $\begin{array}{l}\text { 1. Provide documentation on the membership and role of the } \\
\text { Pension and Savings Committee and arrange for transfer of } \\
\text { responsibilities to the PHMC contractor. Include a complete } \\
\text { accounting of all plan funds. }\end{array}$ & 9/01/96 & $\begin{array}{l}\text { MJ Byrd } \\
\text { FA Powers } \\
\text { JR Sorensen }\end{array}$ \\
\hline $\begin{array}{l}\text { 2. Determine with PHMC, continuation of liaison to the } \\
\text { Retirees Association. }\end{array}$ & 9/01/96 & $\begin{array}{l}\text { FA Powers } \\
\text { JR Sorensen }\end{array}$ \\
\hline $\begin{array}{l}\text { 3. Generate a final turnover list of all active employees and their } \\
\text { accrued pension benefit as of } 9 / 30 / 96 \text {. }\end{array}$ & 9/01/96 & $\begin{array}{l}\text { MJ Byrd } \\
\text { FA Powers } \\
\text { JR Sorensen } \\
\end{array}$ \\
\hline $\begin{array}{l}\text { 4. Generate a final turnover list of all current retirees (and } \\
\text { dependents if applicable), the plan and provisions under } \\
\text { which they retired, the medical and life insurance they have, } \\
\text { and the contribution amounts being paid. }\end{array}$ & $10 / 01 / 96$ & $\begin{array}{l}\text { FA Powers } \\
\text { JR Sorensen }\end{array}$ \\
\hline $\begin{array}{l}\text { 5. Generate a final turnover list of all vested terminated } \\
\text { employees and the accrued pension liability. }\end{array}$ & $10 / 01 / 96$ & $\begin{array}{l}\text { FA Powers } \\
\text { JR Sorensen }\end{array}$ \\
\hline $\begin{array}{l}\text { 6. If the PHMC offers a savings plan, provide list of current } \\
\text { participants including deductions, year-to-date contributions, } \\
\text { outstanding loans and the company match commitment. }\end{array}$ & $10 / 01 / 96$ & $\begin{array}{l}\text { FA Powers } \\
\text { JR Sorensen }\end{array}$ \\
\hline
\end{tabular}




\subsubsection{Recognition Programs.}

\begin{tabular}{|c|c|c|}
\hline Pre $A$ urard fetions & $\begin{array}{l}\text { Scheduled } \\
\text { Date }\end{array}$ & 4totonents) \\
\hline $\begin{array}{l}\text { 1. Prepare a description of current employee recognition and } \\
\text { service award programs and budget. Coordinate with PHMC } \\
\text { to determine compatibility with their programs and adapt } \\
1997 \text { eligibility list for transitioned employees. }\end{array}$ & $6 / 01 / 96$ & $\begin{array}{l}\text { FA Powers } \\
\text { JR Sorensen }\end{array}$ \\
\hline Post-Amard letions & & \\
\hline $\begin{array}{l}\text { 1. Provide description of current employee recognition and } \\
\text { service award programs including budget. Generate a list of } \\
\text { employees who will be eligible for service awards in } \\
\text { FY } 1997 \text { by company and level of award. }\end{array}$ & $9 / 01 / 96$ & $\begin{array}{l}\text { FA Powers } \\
\text { JR Sorensen }\end{array}$ \\
\hline
\end{tabular}

\subsubsection{Other.}

\begin{tabular}{|c|c|c|}
\hline Post-Award Actions & Scheduled & Actonee(s) \\
\hline $\begin{array}{l}\text { 1. Identify the status and cost of the Hanford Family Care } \\
\text { Services contract with Working Solutions, Inc. Arrange for } \\
\text { termination or continuation of the contract. }\end{array}$ & $7 / 01 / 96$ & $\begin{array}{l}\text { FA Powers } \\
\text { JR Sorensen }\end{array}$ \\
\hline $\begin{array}{l}\text { 2. Provide details of current Employee Assistance Program } \\
\text { services. }\end{array}$ & $7 / 01 / 96$ & $\begin{array}{l}\text { FA Powers } \\
\text { JR Sorensen }\end{array}$ \\
\hline $\begin{array}{l}\text { 3. Determine whether the PHMC contractor will allow BHI to } \\
\text { continue purchasing benefit plans. Provide funding } \\
\text { arrangement information. }\end{array}$ & $7 / 01 / 96$ & $\begin{array}{l}\text { FA Powers } \\
\text { JR Sorensen }\end{array}$ \\
\hline
\end{tabular}

\subsubsection{Total Compensation (ICF KH)}

\subsubsection{Salary Plan.}

\begin{tabular}{|l|c|c|}
\hline Scheduled & Date & Pre-Award Lctions \\
\hline $\begin{array}{l}\text { 1. Prepare a list of calendar year salary plan actions scheduled } \\
\text { for October, November, and December } 1996 .\end{array}$ & $6 / 01 / 96$ & BJ Smetana \\
\hline $\begin{array}{l}\text { 2. Determine ownership of prior year salary increase plans and } \\
\text { disposition accordingly. }\end{array}$ & $6 / 01 / 96$ & BJ Smetana \\
\hline $\begin{array}{l}\text { 3. Determine distribution of remaining salary increase funds } \\
\text { which are usually distributed as adjustments in the } \\
\text { November/December time frame. Estimate available funds } \\
\text { and recommend a distribution method. }\end{array}$ & $6 / 01 / 96$ & BJ Smetana \\
\hline
\end{tabular}




\begin{tabular}{|c|c|c|}
\hline Poot-A mard Actions & $\begin{array}{l}\text { Scheduled } \\
\text { Date }\end{array}$ & lettones $(6)$ \\
\hline $\begin{array}{l}\text { 1. Status PHMC contractor regarding salary actions for } \\
\text { October, November, and December } 1996 \text { for those } \\
\text { employees accepting offers. }\end{array}$ & $10 / 01 / 96$ & BJ Smetana \\
\hline $\begin{array}{l}\text { 2. Review salary planning process and provide PHMC } \\
\text { contractor a list of prior year salary increase plans as } \\
\text { appropriate. }\end{array}$ & $10 / 01 / 96$ & BJ Smetana \\
\hline $\begin{array}{l}\text { 3. Coordinate with PHMC contractor to determine distribution } \\
\text { of remaining salary increase funds which are usually } \\
\text { distributed as adjustments in the November/December time } \\
\text { frame. Estimate available and recommend a distribution } \\
\text { method. }\end{array}$ & $10 / 01 / 96$ & BJ Smetana \\
\hline
\end{tabular}

\subsubsection{Other.}

\begin{tabular}{|c|c|c|}
\hline Post-Award Actions & $\begin{array}{l}\text { Sclieduled } \\
\text { Date }\end{array}$ & Actionee(s) \\
\hline $\begin{array}{l}\text { 1. Identify the status and cost of the Hanford Family Care } \\
\text { Services contract with Working Solutions, Inc. Arrange for } \\
\text { termination or continuation of the contract. }\end{array}$ & $7 / 01 / 96$ & JJ Chunn \\
\hline $\begin{array}{l}\text { 2. Provide details of current Employee Assistance Program } \\
\text { services. }\end{array}$ & 7/01/96 & JJ Chunn \\
\hline
\end{tabular}

\subsubsection{Labor Relations (WHC/BCSR)}

Determine, develop, and prepare appropriate data and information regarding the bargaining unit workforce, bargaining agents, collective bargaining agreements, union officials, grievance and arbitration history and caseloads. Further, address the labor relations climate and Federal agency notification requirements.

\subsubsection{Collective Bargaining Agreements.}

\begin{tabular}{|c|c|c|}
\hline Pre-Award fetions & $\begin{array}{l}\text { Scheduled } \\
\text { Date }\end{array}$ & Hetionoe(s) \\
\hline $\begin{array}{l}\text { 1. Prepare summary of the collective bargaining agreements } \\
\text { with the Hanford Atomic Metal Trades Council (HAMTC) } \\
\text { and Hanford Guards Union (HGU) describing future } \\
\text { contractual obligations for actions such as negotiations, } \\
\text { general wage increases, etc. }\end{array}$ & $5 / 01 / 96$ & $\begin{array}{l}\text { BD Corder } \\
\text { GF Saskowsky }\end{array}$ \\
\hline $\begin{array}{l}\text { 2. HAMTC Appendix "A" bargaining issues: Prepare a } \\
\text { summary and status of any ongoing negotiations with } \\
\text { affiliated local unions. }\end{array}$ & $5 / 01 / 96$ & $\begin{array}{l}\text { BD Corder } \\
\text { GF Saskowsky }\end{array}$ \\
\hline $\begin{array}{l}\text { 3. Prepare copies of current labor agreements and other letters } \\
\text { and memorandums of understanding. }\end{array}$ & $5 / 01 / 96$ & $\begin{array}{l}\text { BD Corder } \\
\text { GF Saskowsky }\end{array}$ \\
\hline
\end{tabular}




\begin{tabular}{|c|c|c|}
\hline Pre-Award Actions & $\begin{array}{l}\text { Scheduled } \\
\text { Date. }\end{array}$ & Actionee(6) \\
\hline $\begin{array}{l}\text { 4. Assemble necessary information to support ongoing } \\
\text { transitional consultation with the PHMC on collective } \\
\text { bargaining issues, historical past practices, and negotiations } \\
\text { strategy. }\end{array}$ & $5 / 01 / 96$ & $\begin{array}{l}\text { BD Corder } \\
\text { GF Saskowsky }\end{array}$ \\
\hline Post-A ward Actions & & ४ \\
\hline $\begin{array}{l}\text { 1. Brief PHMC on collective bargaining agreements with } \\
\text { HAMTC and HGU describing future contractual obligations } \\
\text { for actions such as negotiations, general wage increases, etc. }\end{array}$ & $6 / 03 / 96$ & $\begin{array}{l}\text { BD Corder } \\
\text { GF Saskowsky }\end{array}$ \\
\hline $\begin{array}{l}\text { 2. Provide PHMC contractor with details and status of HAMTC } \\
\text { Appendix " } A \text { " bargaining issues, including a summary and } \\
\text { status of any ongoing negotiations with affiliated local unions. }\end{array}$ & 6/03/96 & $\begin{array}{l}\text { BD Corder } \\
\text { GF Saskowsky }\end{array}$ \\
\hline $\begin{array}{l}\text { 3. Provide copies of current labor agreements and other letters } \\
\text { and memorandums of understanding. }\end{array}$ & $6 / 03 / 96$ & $\begin{array}{l}\text { BD Corder } \\
\text { GF Saskowsky }\end{array}$ \\
\hline $\begin{array}{l}\text { 4. Provide ongoing transitional consultation with the PHMC on } \\
\text { collective bargaining issues, historical past practices, and } \\
\text { negotiations strategy. }\end{array}$ & $6 / 03 / 96$ & $\begin{array}{l}\text { BD Corder } \\
\text { GF Saskowsky }\end{array}$ \\
\hline
\end{tabular}

\subsubsection{Bargaining Unit Leadership/Demographics.}

\begin{tabular}{|l|l|l|}
\hline Prefward Actions & $\begin{array}{l}\text { Schetuled } \\
\text { Date }\end{array}$ & Actionee(s) \\
\hline $\begin{array}{l}\text { 1. Develop appropriate charts demonstrating union leadership } \\
\text { structure for HAMTC and HGU including business agents, } \\
\text { officers, affiliated locals, project chief stewards and the } \\
\text { steward structure in general. }\end{array}$ & $5 / 01 / 96$ & $\begin{array}{l}\text { BD Corder } \\
\text { GF Saskowsky }\end{array}$ \\
\hline $\begin{array}{l}\text { 2. Summarize the HAMTC and HGU workforce population by } \\
\text { years of service, classification and location (areas). }\end{array}$ & $6 / 01 / 96$ & $\begin{array}{l}\text { BD Corder } \\
\text { GF Saskowsky }\end{array}$ \\
\hline $\begin{array}{l}\text { 1. Provide PHMC contractor copies of charts demonstrating } \\
\text { union leadership structure for HAMTC and HGU including } \\
\text { business agents, officers, affiliated locals, project chief } \\
\text { stewards and the steward structure in general. }\end{array}$ & $6 / 03 / 96$ & $\begin{array}{l}\text { BD Corder } \\
\text { GF Saskowsky }\end{array}$ \\
\hline $\begin{array}{l}\text { 2. Brief PHMC on workforce demographics and provide } \\
\text { summary listings covering the HAMTC and HGU worikforce } \\
\text { population by years of service, classification and location } \\
\text { (areas). }\end{array}$ & $6 / 03 / 96$ & $\begin{array}{l}\text { BD Corder } \\
\text { GF Saskowsky }\end{array}$ \\
\hline $\begin{array}{l}\text { 3. Provide PHMC a listing of numbers and names of bargaining } \\
\text { unit employees on termination status with recall rights by } \\
\text { classification. }\end{array}$ & $9 / 01 / 96$ & $\begin{array}{l}\text { BD Corder } \\
\text { GF Saskowsky }\end{array}$ \\
\hline
\end{tabular}




\subsubsection{Notifications.}

\begin{tabular}{|l|l|l|l|}
\hline & Scheduled & Date & \\
\hline $\begin{array}{l}\text { 1. Prepare notification to Federal Mediation and Conciliation } \\
\text { Service (FMCS) and the unions that the collective bargaining } \\
\text { agreements with HAMTC and HGU are terminating effective } \\
10 / 01 / 96 .\end{array}$ & $6 / 01 / 96$ & BD Corder \\
\hline $\begin{array}{l}\text { 1. Notify the FMCS that the collective bargaining agreements } \\
\text { with HAMTC and HGU are terminating effective 10/01/96. }\end{array}$ & $8 / 01 / 96$ & BD Corder \\
\hline $\begin{array}{l}\text { 2. Notify the unions that the collective bargaining agreements } \\
\text { between WHC and HAMTC/HGU will terminate effective } \\
10 / 01 / 96 .\end{array}$ & $8 / 01 / 96$ & BD Corder \\
\hline
\end{tabular}

\subsubsection{Grievance and Arbitration History/Caseload.}

\begin{tabular}{|c|c|c|}
\hline Pre-Award Aetions & Scheduled & Actiones (s) \\
\hline $\begin{array}{l}\text { 1. Prepare summaries and analysis of arbitration cases and } \\
\text { grievances filed by calendar year from January } 1,1993 \\
\text { through present time. Analysis to include summary by issue } \\
\text { and outcome of arbitration. }\end{array}$ & $5 / 31 / 96$ & $\begin{array}{l}\text { BD Corder } \\
\text { GF Saskowsky }\end{array}$ \\
\hline $\begin{array}{l}\text { 2. Prepare summaries of any open NLRB charges and past cases } \\
\text { of major significance to the Hanford Site. }\end{array}$ & $6 / 01 / 96$ & $\begin{array}{l}\text { BD Corder } \\
\text { GF Saskowsky }\end{array}$ \\
\hline $\begin{array}{l}\text { 3. Determine, with General Counsel review, ownership of in } \\
\text { process grievances and arbitrations at turnover. }\end{array}$ & $6 / 01 / 96$ & $\begin{array}{l}\text { BD Corder } \\
\text { GF Saskowsky }\end{array}$ \\
\hline Post-Award Actions & & \\
\hline $\begin{array}{l}\text { 1. Provide a summary analysis of arbitration cases and } \\
\text { grievances filed by calendar year from January } 1,1993 \text {, } \\
\text { through present time. Analysis to include summary by issue } \\
\text { and outcome of arbitration. }\end{array}$ & $9 / 01 / 96$ & $\begin{array}{l}\text { BD Corder } \\
\text { GF Saskowsky }\end{array}$ \\
\hline $\begin{array}{l}\text { 2. Provide summaries of any open National Labor Relations } \\
\text { Board (NLRB) charges and past cases of major significance } \\
\text { to the Hanford Site. }\end{array}$ & $10 / 01 / 96$ & $\begin{array}{l}\text { BD Corder } \\
\text { GF Saskowsky }\end{array}$ \\
\hline $\begin{array}{l}\text { 3. Provide listing of General Counsel reviewed grievances in } \\
\text { process or pending/scheduled for arbitration including } \\
\text { subject, local union and date of arbitration if available. Also, } \\
\text { arbitration cases pending award. }\end{array}$ & $10 / 01 / 96$ & $\begin{array}{l}\text { BD Corder } \\
\text { GF Saskowsky }\end{array}$ \\
\hline
\end{tabular}

\subsubsection{Labor Relations (ICF KH)}

ICF KH Labor Relations will assist WHC Labor Relations in transferring HAMTC information. 
The following collective bargaining agreements are unique to ICF KH and information listed below, as applicable, will be provided for each as indicated:

- Hanford Site Stabilization Agreement (HSSA)

- Office and Professional Employees International Union (OPEIU)

- Quality Control and Nondestructive Technicians (QC/NDT).

\subsubsection{Collective Bargaining Agreements.}

\begin{tabular}{|c|c|c|}
\hline Pre-Award lactions & $\begin{array}{l}\text { Scheduled } \\
\text { Date }\end{array}$ & Actionee $(s)$ \\
\hline $\begin{array}{l}\text { 1. Prepare summary of the collective bargaining agreements } \\
\text { describing further contractual obligations for actions such as } \\
\text { negotiations, general wage increases, etc. }\end{array}$ & $5 / 01 / 96$ & JA Carter \\
\hline 2. Prepare a summary and status of any ongoing negotiations. & $5 / 01 / 96$ & JA Carter \\
\hline $\begin{array}{l}\text { 3. Prepare copies of current labor agreements and other letters } \\
\text { and MOUs. }\end{array}$ & $5 / 01 / 96$ & JA Carter \\
\hline $\begin{array}{l}\text { 4. Assemble necessary information to support ongoing } \\
\text { transitional consultation with the PHMC on collective } \\
\text { bargaining issues, historical past practices, and negotiations } \\
\text { strategy. }\end{array}$ & $5 / 01 / 96$ & JA Carter \\
\hline $\begin{array}{l}\text { 5. Prepare information necessary for continuing consultation to } \\
\text { the PHMC with regard to the application and administration } \\
\text { of the HSSA and the transitioning of the existing construction } \\
\text { workforce to the PHMC. }\end{array}$ & $6 / 01 / 96$ & JA Carter \\
\hline Post-Award Actions & & \\
\hline $\begin{array}{l}\text { 1. Brief PHMC on collective bargaining agreements describing } \\
\text { further contractual obligations for actions such as } \\
\text { negotiations, general wage increases, etc. }\end{array}$ & 6/03/96 & JA Carter \\
\hline $\begin{array}{l}\text { 2. Provide new contractor with details and status of bargaining } \\
\text { issues, including a summary and status of any ongoing } \\
\text { negotiations with unions. }\end{array}$ & 6/03/96 & JA Carter \\
\hline $\begin{array}{l}\text { 3. Provide copies of current labor agreements and other letters } \\
\text { and MOUs. }\end{array}$ & $6 / 03 / 96$ & JA Carter \\
\hline $\begin{array}{l}\text { 4. Provide ongoing transitional consultation with the PHMC on } \\
\text { collective bargaining issues, historical past practices, and } \\
\text { negotiations strategy. }\end{array}$ & $6 / 03 / 96$ & JA Carter \\
\hline $\begin{array}{l}\text { 5. Provide continuing consultation to the PHMC with regard to } \\
\text { the application and administration of the HSSA and the } \\
\text { transitioning of the existing construction workforce to the } \\
\text { PHMC. }\end{array}$ & 9/30/96 & JA Carter \\
\hline
\end{tabular}




\subsubsection{Bargaining Unit Leadership/Demographics.}

\begin{tabular}{|l|c|l|l|}
\hline & Seliodiled & \\
\hline $\begin{array}{l}\text { 1. Develop appropriate charts demonstrating union leadership } \\
\text { structure including business agents, officers, affiliated locals, } \\
\text { project chief stewards, and the steward structure in general. }\end{array}$ & $6 / 01 / 96$ & JA Carter \\
\hline $\begin{array}{l}\text { 2. Summarize the bargaining workforce population by years of } \\
\text { eervice, classification, and location (areas). }\end{array}$ & $6 / 01 / 96$ & JA Carter \\
\hline $\begin{array}{l}\text { 1. Provide new contractor copies of charts demonstrating union } \\
\text { leadership structure for bargaining units, including business } \\
\text { agents, officers, affiliated locals, project chief stewards, and } \\
\text { the steward structure in general. }\end{array}$ & $6 / 03 / 96$ & JA Carter \\
\hline $\begin{array}{l}\text { 2. Brief PHMC on workforce demographics and provide } \\
\text { summary listings covering the bargaining unit workforce } \\
\text { populations by years of service, classification, and location } \\
\text { (areas). }\end{array}$ & $6 / 03 / 96$ & JA Carter \\
\hline $\begin{array}{l}\text { 3. Provide PHMC a listing of numbers of bargaining unit } \\
\text { employees terminated with recall rights by classification. }\end{array}$ & $9 / 01 / 96$ & JA Carter \\
\hline
\end{tabular}

\subsubsection{Notifications.}

\begin{tabular}{|c|c|c|}
\hline Pre-Award Actions & $\begin{array}{l}\text { Schedived } \\
\text { Date }\end{array}$ & (4) laionse(s) \\
\hline $\begin{array}{l}\text { 1. Prepare notification to FMCS and the unions that the } \\
\text { collective bargaining agreements with HAMTC and HGU are } \\
\text { terminating effective } 10 / 01 / 96 \text {. }\end{array}$ & $6 / 01 / 96$ & JA Carter \\
\hline Post-A wrard Actions & & \\
\hline $\begin{array}{l}\text { 1. Prepare notification to FMCS and the union that the } \\
\text { collective bargaining agreements are terminating effective } \\
10 / 01 / 96 \text {. }\end{array}$ & $8 / 01 / 96$ & JA Carter \\
\hline $\begin{array}{l}\text { 2. Notify the FMCS that the collective bargaining agreements } \\
\text { are terminating effective } 10 / 01 / 96 \text {. }\end{array}$ & $8 / 01 / 96$ & JA Carter \\
\hline $\begin{array}{l}\text { 3. Notify the unions that the collective bargaining agreements } \\
\text { between them and ICF KH will terminate effective 10/01/96. }\end{array}$ & $8 / 01 / 96$ & JA Carter \\
\hline
\end{tabular}

\subsubsection{Grievance and Arbitration History/Caseload.}

\begin{tabular}{|c|c|c|}
\hline Pre-Award Actions & $\begin{array}{l}\text { Scheduled } \\
\text { Date }\end{array}$ & Yctionee(s) \\
\hline $\begin{array}{l}\text { 1. Prepare a summary and analysis of arbitration cases and } \\
\text { grievances filed by calendar year from January 1, 1993, } \\
\text { through present time. Analysis to include summary by issue } \\
\text { and outcome of arbitration. }\end{array}$ & $5 / 31 / 96$ & JA Carter \\
\hline
\end{tabular}




\begin{tabular}{|c|c|c|}
\hline Pre-Avard Actions & $\begin{array}{l}\text { Scheduled } \\
\text { Date }\end{array}$ & Actionee(s) \\
\hline $\begin{array}{l}\text { 2. Prepare a summary of any open NLRB charges and past } \\
\text { cases of major significance to the Hanford Site. }\end{array}$ & 6/01/96 & JA Carter \\
\hline $\begin{array}{l}\text { 3. Determine, with General Counsel review, ownership of in- } \\
\text { process grievances and arbitrations at turnover. }\end{array}$ & $6 / 01 / 96$ & JA Carter \\
\hline Pod-A ward letions & & (1) \\
\hline $\begin{array}{l}\text { 1. Provide a summary analysis of arbitration cases and } \\
\text { grievances filed by calendar year from January } 1,1993 \text {, } \\
\text { through present time. Analysis to include summary by issue } \\
\text { and outcome of arbitration. }\end{array}$ & 9/01/96 & JA Carter \\
\hline $\begin{array}{l}\text { 2. Provide a summary of any open NLRB charges and past } \\
\text { cases of major significance to the Hanford Site. }\end{array}$ & $10 / 01 / 96$ & JA Carter \\
\hline $\begin{array}{l}\text { 3. Provide a list of General Counsel-reviewed grievances in } \\
\text { process or pending/scheduled for arbitration including } \\
\text { subject, local union, and date of arbitration if available. Also } \\
\text { arbitration cases pending award. }\end{array}$ & $10 / 01 / 96$ & JA Carter \\
\hline
\end{tabular}

\subsubsection{Workforce Development and Diversity (WHC/BCSR)}

Determine, develop, and prepare appropriate data regarding Equal Employment Opportunity (EEO), Affirmative Action Plan (AAP) activities and Americans with Disabilities Act (ADA) compliance, personal/professional development, education reimbursement, and community outreach/educational enrichment programs.

\subsubsection{EEO/AAP.}

\begin{tabular}{|c|c|c|}
\hline Pre-Award Actions & Scheduled & Actionee(s) \\
\hline $\begin{array}{l}\text { 1. Coordinate with Personnel Resources to compile all pertinent data } \\
\text { for preparation of a } 1997 \text { AAP with special emphasis on applicant } \\
\text { tracking records, promotions and placement statistics, and labor } \\
\text { market data. }\end{array}$ & $6 / 01 / 96$ & $\begin{array}{l}\text { CA Marrero } \\
\text { RW Holleran }\end{array}$ \\
\hline $\begin{array}{l}\text { 2. Prepare a briefing and provide copies of the current AAP } \\
\text { including a statistical profile reflecting fernale and minority } \\
\text { representation by category/classification in the workforce. }\end{array}$ & $6 / 01 / 96$ & $\begin{array}{l}\text { G Mitchell } \\
\text { CK Macleod }\end{array}$ \\
\hline $\begin{array}{l}\text { 3. Conduct a comprehensive review of internal and external } \\
\text { complaints and cases by agency, and status for General Counsel } \\
\text { determination of ownership and protocol for PHMC contractor } \\
\text { briefing. }\end{array}$ & $6 / 01 / 96$ & $\begin{array}{l}\text { CA Marrero } \\
\text { G Mitchell }\end{array}$ \\
\hline $\begin{array}{l}\text { 4. Compile a list of external oversight agency contacts (Human } \\
\text { Rights Commission [HRC], Equal Employment Opportunity } \\
\text { Commission [EEOC], Office of Federal Contract Compliance } \\
\text { Program [OFCCP], etc.) in Region X. }\end{array}$ & $6 / 01 / 96$ & $\begin{array}{l}\text { CA Marrero } \\
\text { G Mitchell }\end{array}$ \\
\hline
\end{tabular}




\begin{tabular}{|c|c|c|c|}
\hline & Post-Award Actions & Schoduled & Actonet $(s)$ \\
\hline 1. & $\begin{array}{l}\text { Provide recommendations and input to the PHMC related to } \\
\text { specific data, goal setting and preparation strategy to develop } \\
\text { and implement a } 1997 \text { AAP. }\end{array}$ & $9 / 01 / 96$ & $\begin{array}{l}\text { CA Marrero } \\
\text { G Mitchell }\end{array}$ \\
\hline 2. & $\begin{array}{l}\text { Provide PHMC a briefing and provide copies of the current } \\
\text { AAP including a statistical profile reflecting female and } \\
\text { minority representation by category/classification in the } \\
\text { workforce. }\end{array}$ & $9 / 01 / 96$ & $\begin{array}{l}\text { G Mitchell } \\
\text { CK Macleod }\end{array}$ \\
\hline 3. & $\begin{array}{l}\text { Brief PHMC contractor on internal and external complaints and } \\
\text { cases by agency and status based on General Counsel } \\
\text { determination of ownership and protocol. }\end{array}$ & $9 / 01 / 96$ & $\begin{array}{l}\text { CA Marrero } \\
\text { G Mitchell }\end{array}$ \\
\hline 4. & $\begin{array}{l}\text { Review agency contacts listings with PHMC contractor } \\
\text { including a briefing on relationships and protocol. Provide } \\
\text { listing of external oversight agency contacts (HRC, EEOC, } \\
\text { OFCCP, etc.) in Region } \underline{X} \text {. }\end{array}$ & $9 / 01 / 96$ & $\begin{array}{l}\text { CA Marrero } \\
\text { G Mitchell }\end{array}$ \\
\hline
\end{tabular}

\subsubsection{Diversity Recognition and Training.}

\begin{tabular}{|c|c|c|}
\hline Pre-Award Actions & $\begin{array}{l}\text { Schediled } \\
\text { Date }\end{array}$ & Actoplos $(s)$ \\
\hline $\begin{array}{l}\text { 1. Develop a briefing document including current activities in } \\
\text { support of nationally recognized diversity events (Black History } \\
\text { Month, Women's History Month, etc.) and a timeline/calendar of } \\
\text { observances. }\end{array}$ & $6 / 01 / 96$ & $\begin{array}{l}\text { CA Marrero } \\
\text { G Mitchell }\end{array}$ \\
\hline $\begin{array}{l}\text { 2. Prepare a listing and brief description of training programs and } \\
\text { requirements in areas related to Title VII, i.e., "EEO } 2000^{n} \text { and } \\
\text { "Sexual Harassment in the Workplace." }\end{array}$ & $6 / 01 / 96$ & $\begin{array}{l}\text { CA Marrero } \\
\text { G Mitchell }\end{array}$ \\
\hline \multicolumn{3}{|l|}{ Post-Award Actions } \\
\hline $\begin{array}{l}\text { 1. Brief PHMC on diversity recognition events and activities } \\
\text { including a timeline/calendar of observances. Determine } \\
\text { continuation of approach or an alternative. }\end{array}$ & $9 / 01 / 96$ & $\begin{array}{l}\text { CA Marrero } \\
\text { G Mitchell }\end{array}$ \\
\hline $\begin{array}{l}\text { 2. Provide PHMC contractor a listing and brief description of } \\
\text { training programs and requirements in areas related to 'Title VII, } \\
\text { i.e., "EEO } 2000^{*} \text { and "Sexual Harassment in the Workplace." }\end{array}$ & $9 / 01 / 96$ & $\begin{array}{l}\text { CA Marrero } \\
\text { G Mitchell }\end{array}$ \\
\hline
\end{tabular}

\subsubsection{Educational Reimbursement Programs.}

\begin{tabular}{|c|c|c|}
\hline Pre-Awaid Actions & $\begin{array}{l}\text { Scheduled } \\
\text { Dote }\end{array}$ & Actionese(s) \\
\hline $\begin{array}{l}\text { 1. Develop a briefing packet including copies of existing policy, } \\
\text { participation statistics by contractor and costs related to the } \\
\text { Educational Reimbursement Program for active employees. }\end{array}$ & $6 / 01 / 96$ & $\begin{array}{l}\text { CA Marrero } \\
\text { KR Banta }\end{array}$ \\
\hline $\begin{array}{l}\text { 2. Prepare a briefing including statistical and cost information for } \\
\text { the Educational Reimbursement Program coverage of terminated } \\
\text { employees eligible for benefits described in the Hanford } \\
\text { Workforce Restructuring Plan ( } 3161 \text { Plan). }\end{array}$ & $6 / 01 / 96$ & $\begin{array}{l}\text { CA Marrero } \\
\text { KR Banta }\end{array}$ \\
\hline
\end{tabular}




\begin{tabular}{|c|c|c|}
\hline Posh Aprarditons & $\begin{array}{l}\text { Schediuled } \\
\text { Date }\end{array}$ & 10 ctionee $(6)$ \\
\hline $\begin{array}{l}\text { 1. Brief PHMC on existing policy, participation statistics by } \\
\text { contractor and costs related to the Educational Reimbursement } \\
\text { Program for sctive employees and provide copies of policy, } \\
\text { forms and other guidelines. Determine if the same or alternate } \\
\text { program will be used prior to Fall college enrollments. }\end{array}$ & $7 / 01 / 96$ & $\begin{array}{l}\text { CA Marrero } \\
\text { KR Banta }\end{array}$ \\
\hline $\begin{array}{l}\text { 2. Brief PHMC on statistical and cost information for the } \\
\text { Educational Reimbursement Program coverage of terminated } \\
\text { employees eligible for benefits described in the Hanford } \\
\text { Workforce Restructuring Plan ( } 3161 \text { Plans). }\end{array}$ & $7 / 01 / 96$ & $\begin{array}{l}\text { CA Marrero } \\
\text { KR Banta }\end{array}$ \\
\hline
\end{tabular}

\subsubsection{Development Training}

\begin{tabular}{|c|c|c|}
\hline Pre-Award Actions & $\begin{array}{l}\text { Scheduled } \\
\text { Date }\end{array}$ & Actionee $(s)$ \\
\hline $\begin{array}{l}\text { 1. Develop a briefing packet that includes information on } \\
\text { development training courses, delivery methods and } \\
\text { processes. }\end{array}$ & $5 / 31 / 96$ & $\begin{array}{l}\text { CA Marrero } \\
\text { KR Banta }\end{array}$ \\
\hline Post-Award Actions & & \\
\hline $\begin{array}{l}\text { 1. Provide PHMC briefing on training courses, delivery } \\
\text { methods and processes. }\end{array}$ & $7 / 01 / 96$ & $\begin{array}{l}\text { CA Marrero } \\
\text { KR Banta }\end{array}$ \\
\hline
\end{tabular}

\subsubsection{Education Enrichment Programs.}

\begin{tabular}{|c|c|c|}
\hline Pre-Award Actions & $\begin{array}{l}\text { Scheduled } \\
\text { Date }\end{array}$ & tetiones (s) \\
\hline $\begin{array}{l}\text { 1. Prepare a comprehensive briefing and supporting } \\
\text { documentation covering strategic education partnerships, } \\
\text { university/college and } \mathrm{K}-12 \text { education involvement. }\end{array}$ & $6 / 01 / 96$ & $\begin{array}{l}\text { CA Marrero } \\
\text { DG Carter } \\
\text { JE Bongers (BCSR) }\end{array}$ \\
\hline $\begin{array}{l}\text { 2. Prepare information covering student development and } \\
\text { internship activities at pre-college (school-to-work initiative), } \\
\text { college (Partnership for Environmental Technology } \\
\text { Education) and university (Associated Western Universities) } \\
\text { levels. }\end{array}$ & $6 / 01 / 96$ & $\begin{array}{l}\text { CA Marrero } \\
\text { DG Carter }\end{array}$ \\
\hline Post-Award Actions & & \\
\hline $\begin{array}{l}\text { 1. Provide PHMC with a comprehensive briefing and supporting } \\
\text { documentation covering strategic education partnerships, } \\
\text { university/college and K-12 eduction involvement. } \\
\text { Determine path forward with PHMC contractor. }\end{array}$ & $7 / 01 / 96$ & $\begin{array}{l}\text { CA Marrero } \\
\text { DG Carter } \\
\text { JE Bongers }\end{array}$ \\
\hline $\begin{array}{l}\text { 2. Brief new contractor and provide information covering } \\
\text { student development and internship activities at pre-college } \\
\text { (school-to-work initiative), college (Partnership for } \\
\text { Environmental Technology Education) and university } \\
\text { (Associated Western Universities) levels. }\end{array}$ & $7 / 01 / 96$ & $\begin{array}{l}\text { CA Marrero } \\
\text { DG Carter }\end{array}$ \\
\hline
\end{tabular}




\subsubsection{Graduate Rotational Program.}

\begin{tabular}{|l|l|l|}
\hline $\begin{array}{l}\text { 1. Prepare a briefing on the Graduate Rotational Program for } \\
\text { strategic recruitment of technical undergraduates for the } \\
\text { purpose of hiring them in rotational work experiences at } \\
\text { Hanford. }\end{array}$ & $6 / 01 / 96$ & $\begin{array}{l}\text { G Mitchell } \\
\text { DE Hansen }\end{array}$ \\
\hline
\end{tabular}

\subsubsection{Workforce Development and Diversity (ICF KH)}

\subsubsection{EEO/AAP.}

\begin{tabular}{|c|c|c|}
\hline Pre-Award Actions & $\begin{array}{l}\text { Scheduled } \\
\text { Date }\end{array}$ & Actionee(s) \\
\hline $\begin{array}{l}\text { 1. Prepare a briefing and provide copies of the current AAP } \\
\text { including a statistical profile reflecting female and minority } \\
\text { representation by category/classification in the workforce. }\end{array}$ & $6 / 01 / 96$ & $\begin{array}{l}\text { VC Hodgin } \\
\text { Lewis }\end{array}$ \\
\hline $\begin{array}{l}\text { 2. Conduct a comprehensive review of internal and external } \\
\text { complaints and cases by agency and status for General Counsel } \\
\text { determination of ownership and protocol for PHMC contractor } \\
\text { briefing. }\end{array}$ & $6 / 01 / 96$ & $\begin{array}{l}\text { VC Hodgin } \\
\text { Lewis }\end{array}$ \\
\hline $\begin{array}{l}\text { 3. Compile a list of external oversight agency contacts (HRC, } \\
\text { EEOC, OFCCP, etc.) in Region } \underline{X} \text {. }\end{array}$ & $6 / 01 / 96$ & $\begin{array}{l}\text { VC Hodgin } \\
\text { Lewis } \\
\end{array}$ \\
\hline \multicolumn{3}{|l|}{ Post-Award Actions } \\
\hline $\begin{array}{l}\text { 1. Provide PHMC a briefing and provide copies of the current } \\
\text { AAP including a statistical profile reflecting female and minority } \\
\text { representation by category/classification in the workforce. }\end{array}$ & 9/01/96 & $\begin{array}{l}\text { VC Hodgin } \\
\text { Lewis }\end{array}$ \\
\hline $\begin{array}{l}\text { 2. Brief PHMC contractor on internal and external complaints and } \\
\text { cases by agency and status based on General Counsel } \\
\text { determination of ownership and protocol. }\end{array}$ & 9/01/96 & $\begin{array}{l}\text { VC Hodgin } \\
\text { Lewis }\end{array}$ \\
\hline $\begin{array}{l}\text { 3. Review agency contacts listings with PHMC contractor } \\
\text { including a briefing on relationships and protocol. Provide } \\
\text { listing of external oversight agency contacts (HRC, EEOC, } \\
\text { OFCCP, etc.) in Region X. }\end{array}$ & 9/01/96 & $\begin{array}{l}\text { VC Hodgin } \\
\text { Lewis }\end{array}$ \\
\hline
\end{tabular}




\subsubsection{Diversity Recognition and Training.}

\begin{tabular}{|c|c|c|}
\hline Prothard 1 ctons & scheduled & Actiones (s) \\
\hline $\begin{array}{l}\text { 1. Develop a briefing document including current activities in } \\
\text { support of nationally recognized diversity events (Black } \\
\text { History Month, Women's History Month, etc.) and a } \\
\text { timeline/calendar of observances. }\end{array}$ & $6 / 01 / 96$ & $\begin{array}{l}\text { VC Hodgin } \\
\text { Lewis }\end{array}$ \\
\hline Post-A ward Actions & & \\
\hline $\begin{array}{l}\text { 1. Brief PHMC on diversity recognition events and activities } \\
\text { including a timeline/calendar of observances. Determine } \\
\text { continuation of approach or an alternative. }\end{array}$ & $9 / 01 / 96$ & $\begin{array}{l}\text { VC Hodgin } \\
\text { Lewis }\end{array}$ \\
\hline
\end{tabular}

\subsubsection{Education Enrichment Programs.}

\begin{tabular}{|l|l|l|}
\hline & Scheduled & Date \\
\hline $\begin{array}{l}\text { 1. Prepare information covering student development and } \\
\text { internship activities at pre-college (school-to-work initiative), } \\
\text { college (Partnership for Environmental Technology } \\
\text { Education) and university (Associated Western Universities) } \\
\text { levels. }\end{array}$ & $6 / 01 / 96$ & $\mathrm{JJ}$ Chunn \\
\hline $\begin{array}{l}\text { 1. Brief new contractor and provide information covering } \\
\text { student development and internship activities at pre-college } \\
\text { (school-to-work initiative), college (Partnership for }\end{array}$ & $\mathbf{7 / 0 1 / 9 6}$ & $\mathrm{JJ}$ Chunn \\
$\begin{array}{l}\text { Environmental Technology Education) and university } \\
\text { (Associated Western Universities) levels. }\end{array}$ & \\
\hline
\end{tabular}

\subsubsection{General Operations}

Determine, develop, and prepare appropriate data regarding employee relations, special programs, general policies, business management and the like.

\subsubsection{Transferring Vacation Hours for Family Medical Emergencies.}

\begin{tabular}{|l|l|l|l|}
\hline & Scheduled & Date & Actionse(s) \\
\hline $\begin{array}{l}\text { 1. Prepare a briefing on the voluntary transfer of vacation from one } \\
\text { employee to another due to family medical emergencies including } \\
\text { historical usage statistics. }\end{array}$ & $6 / 01 / 96$ & JT Eckert \\
\hline $\begin{array}{l}\text { 2. Develop a current participation status report reflecting donors, } \\
\text { recipients and committed vacation hours. }\end{array}$ & $6 / 01 / 96$ & JT Eckert \\
\hline
\end{tabular}




\begin{tabular}{|c|c|c|}
\hline Post-Award Actions & $\begin{array}{l}\text { Scheduled } \\
\text { Date. }\end{array}$ & Actioner(s) \\
\hline $\begin{array}{l}\text { 1. Brief PHMC on the voluntary transfer of vacation from one } \\
\text { employee to another due to family medical emergencies including } \\
\text { historical usage statistics. }\end{array}$ & $8 / 01 / 96$ & JT Eckert \\
\hline $\begin{array}{l}\text { 2. Provide current participation status report reflecting donor, } \\
\text { recipients and committed vacation hours. Coordinate with PHMC } \\
\text { to determine continuation of the program or an approach to } \\
\text { process participants likely to carry forward past contract turnover. }\end{array}$ & $8 / 01 / 96$ & JT Eckert \\
\hline
\end{tabular}

\subsubsection{Employee Work Schedules.}

\begin{tabular}{|c|c|c|}
\hline Pre-Award Actions & $\begin{array}{l}\text { Scheduled } \\
\text { Date. }\end{array}$ & Sctionee(s) \\
\hline $\begin{array}{l}\text { 1. Develop a briefing on non-bargaining unit standard work schedules, } \\
8 \mathrm{X} 9 \text { '6, 12-hour shifts and assignment to nonstandard work } \\
\text { schedules. }\end{array}$ & $6 / 01 / 96$ & M Hawkins \\
\hline $\begin{array}{l}\text { 2. Prepare copies of work schedules, policy statements and statistical } \\
\text { data identifying employees assigned to } 8 \text { X 9's and 12-hour shifts. }\end{array}$ & $6 / 01 / 96$ & JM Hawkins \\
\hline Post-Award Actions & & \\
\hline $\begin{array}{l}\text { 1. Brief PHMC on non-bargaining unit standard work schedules, } 8 \mathrm{X} \\
\text { 9's, 12-hour shifts and assignment to nonstandard work schedules. }\end{array}$ & 9/01/96 & JM Hawkins \\
\hline $\begin{array}{l}\text { 2. Provide copies of work schedules, policy statements and statistical } \\
\text { data identifying employees assigned to } 8 \mathrm{X} \text { 9's and 12-hour shifts. }\end{array}$ & 9/01/96 & JM Hawkins \\
\hline
\end{tabular}

\subsubsection{Salary Continuance During Absences.}

\begin{tabular}{|l|c|l|}
\hline & $\begin{array}{c}\text { Scheduled } \\
\text { Date }\end{array}$ & Actionee(s) \\
\hline $\begin{array}{l}\text { 1. Pevelop briefing on the requirements and guidelines for the } \\
\text { continuation of salary payments during periods of employee } \\
\text { absence. Cover inclusion, by reference, in collective bargaining } \\
\text { agreements. }\end{array}$ & $6 / 01 / 96$ & MK Strankman \\
\hline $\begin{array}{l}\text { 1. Brief PHMC on the requirements and guidelines for the continuation } \\
\text { of salary payments during periods of employee absence. Cover } \\
\text { inclusion, by reference, in collective bargaining agreements. }\end{array}$ & $9 / 01 / 96$ & MK Strankman \\
\hline
\end{tabular}




\subsubsection{Vacation and Holidays.}

\begin{tabular}{|c|c|c|}
\hline Pre Award Actions & Schedilled & lettonee () \\
\hline $\begin{array}{l}\text { 1. Prepare a briefing on the accrual method of vacation accumulation } \\
\text { and the holiday schedule including qualification criteria. Cover } \\
\text { inclusion in collective bargaining agreements. }\end{array}$ & $6 / 01 / 96$ & FA Blowe \\
\hline $\begin{array}{l}\text { 2. Develop a preliminary accrued vacation liability report and identify } \\
\text { scheduled holidays through the balance of the calendar year. }\end{array}$ & $6 / 01 / 96$ & FA Blowe \\
\hline Post-A ward Actions & & \\
\hline $\begin{array}{l}\text { 1. Brief PHMC on the accrual method of vacation accumulation and } \\
\text { the holiday schedule including qualification criteria. Cover } \\
\text { inclusion in collective bargaining agreements. }\end{array}$ & $9 / 01 / 96$ & FA Blowe \\
\hline $\begin{array}{l}\text { 2. Review preliminary accrued vacation liability report with PHMC } \\
\text { and determine vacation policy to be used after turnover. Establish } \\
\text { amount of accrued vacation transferable to provide a basis for pre- } \\
\text { urnover vacation payout. }\end{array}$ & $9 / 01 / 96$ & FA Blowe \\
\hline
\end{tabular}

\subsubsection{Salaried Nonexempt Workforce Adjustment.}

\begin{tabular}{|l|c|c|}
\hline Scheduled & Date & Actionee(s) \\
\hline $\begin{array}{l}\text { 1. Prepare a briefing on responsibility and requirements for } \\
\text { adjustments in the salaried nonexempt workforce, including transfer, } \\
\text { promotion, and reduction of force (ROF). }\end{array}$ & $6 / 01 / 96$ & KA Hill \\
\hline $\begin{array}{l}\text { 1. Brief the PHMC on responsibility and requirements for adjustments } \\
\text { in the salaried nonexempt workforce, including transfer, promotion, } \\
\text { and ROF. }\end{array}$ & $9 / 01 / 96$ & KA Hill \\
\hline
\end{tabular}

\subsubsection{Business Management.}

\begin{tabular}{|c|c|c|}
\hline Pre-Award Actions & $\begin{array}{l}\text { Scheduled } \\
\text { Date }\end{array}$ & Actionge(s) \\
\hline $\begin{array}{l}\text { 1. Develop the FY } 1997 \text { SSPP including supporting budgets, } \\
\text { workscope definition and milestone commitments. }\end{array}$ & $5 / 01 / 96$ & $\begin{array}{l}\text { HA Sieber } \\
\text { RC Boldt }\end{array}$ \\
\hline Post A ward Actions & & \\
\hline $\begin{array}{l}\text { 1. Coordinate with PHMC to obtain input to the FY } 1997 \text { SSPP } \\
\text { including supporting budgets, workscope definition and milestone } \\
\text { commitments. }\end{array}$ & $7 / 01 / 96$ & $\begin{array}{l}\text { HA Sieber } \\
\text { RC Boldt }\end{array}$ \\
\hline
\end{tabular}




\subsubsection{Re-engineering Support}

Determine, develop, and prepare appropriate data and information regarding pilot programs and processes dealing with the human resources aspects of re-engineering initiatives.

\begin{tabular}{|c|c|c|}
\hline Pro-Award Actions & $\begin{array}{l}\text { Scheduled } \\
\text { Datte }\end{array}$ & Actionee(s) \\
\hline $\begin{array}{l}\text { 1. Prepare a briefing that will identify divisions, departments or } \\
\text { facilities where re-engineered processes have been implemented } \\
\text { utilizing piloted human resources programs and procedures for such } \\
\text { activities as staffing, compensation, performance assessment and } \\
\text { training. Include information on results or expected outcomes of } \\
\text { pilots. }\end{array}$ & $5 / 01 / 96$ & JF Oswald \\
\hline $\begin{array}{l}\text { 2. Assemble information and data specific to in-place, self-directed } \\
\text { work teams including names, classifications, roles and work } \\
\text { locations to minimize disruption to in-place or planned re- } \\
\text { engineering initiatives. }\end{array}$ & $5 / 01 / 96$ & JF Oswald \\
\hline Post-Award Actions & & \\
\hline $\begin{array}{l}\text { 1. Provide PHMC briefing regarding divisions, departments or } \\
\text { facilities where re-engineered processes have been implemented } \\
\text { utilizing piloted human resources programs and procedures for such } \\
\text { activities as staffing, compensation, performance assessment and } \\
\text { training. Coordinate with PHMC to continue and/or modify } \\
\text { approach, based on results or expected outcomes of pilots. }\end{array}$ & $6 / 15 / 96$ & JF Oswald \\
\hline $\begin{array}{l}\text { 2. Provide PHMC information and data specific to in-place self- } \\
\text { directed work teams including names, classifications, roles and } \\
\text { work locations to minimize disruption to in-place or planned re- } \\
\text { engineering initiatives. Recommend an approach and discuss } \\
\text { feasibility of intact transition to PHMC. }\end{array}$ & $6 / 15 / 96$ & JF Oswald \\
\hline
\end{tabular}

\subsection{EMPLOYEE CONCERNS}

The Employee Concerns Program (ECP) responds to and tracks to closure concerns raised by WHC/BCSR employees.

\begin{tabular}{|c|c|c|}
\hline Pre-Award Actions & $\begin{array}{l}\text { Scheduled } \\
\text { Date }\end{array}$ & Actionee $(\mathrm{s})$ \\
\hline $\begin{array}{l}\text { 1. Determine how to disposition and/or resolve employee concerns } \\
\text { that remain open at end of WHC contract }(9 / 30 / 96) \text {. Will need } \\
\text { input/guidance from General Counsel's Office. }\end{array}$ & $5 / 31 / 96$ & MG Dickinson \\
\hline $\begin{array}{l}\text { 2. Determine disposition of closed employee-concerns files; } \\
\text { computerized database; and logbooks. Will need input/guidance } \\
\text { from General Counsel's Office. }\end{array}$ & $5 / 31 / 96$ & MG Dickinson \\
\hline
\end{tabular}




\begin{tabular}{|l|c|c|}
\hline Sost-Awerd Actions & Gediled & \\
\hline $\begin{array}{l}\text { 1. Transfer, if appropriate, information necessary to } \\
\text { resolve/complete those concerns that remain open at the time } \\
\text { start of the PHMC contract. }\end{array}$ & $9 / 30 / 96$ & MG Dickinson \\
\hline $\begin{array}{l}\text { 2. Transfer, as determined appropriate, employee concerns files } \\
\text { and information. }\end{array}$ & $9 / 30 / 96$ & MG Dickinson \\
\hline
\end{tabular}

\subsection{ENVIRONMENTAL, SAFETY, QUALITY, AND HEALTH}

\subsubsection{Safety, Quality, and Health}

\subsubsection{Program Management.}

\subsection{Budget.}

\begin{tabular}{|c|c|c|}
\hline Pre-Award Actions & $\begin{array}{l}\text { Scheduled } \\
\text { Date }\end{array}$ & Actionee(s) \\
\hline 1. Assemble current budget documentation (SSPP). & $5 / 31 / 96$ & JK Pettey \\
\hline Post-Award Actions & & \\
\hline 1. Provide status of overall organization budget. & $8 / 01 / 96$ & JK Pettey \\
\hline $\begin{array}{l}\text { 2. Provide briefing on S\&H management planning process and status } \\
\text { (EH planning process, including RDS and ADS). }\end{array}$ & $8 / 01 / 96$ & JK Pettey \\
\hline 3. Provide budget documentation. & $7 / 01 / 96$ & JK Pettey \\
\hline
\end{tabular}

\subsection{Administration.}

\begin{tabular}{|c|c|c|}
\hline Pre-Award Actions & Scheduled & Actiones $(\mathrm{s})$ \\
\hline $\begin{array}{l}\text { 1. Document status of customer commitments against current } \\
\text { program plan. }\end{array}$ & $5 / 31 / 96$ & SJ Richardson \\
\hline Post-Award Actions & & \\
\hline 1. Provide organization charts, staff directory. & $7 / 01 / 96$ & VB Moore \\
\hline 2. Provide training status. & $9 / 02 / 96$ & VB Moore \\
\hline
\end{tabular}




\subsection{Corrective Action Management System (CAMS).}

\begin{tabular}{|c|c|c|}
\hline Pre-Award Actions & $\begin{array}{l}\text { Scheduled } \\
\text { Date }\end{array}$ & Actionee(s) \\
\hline $\begin{array}{l}\text { 1. Assemble documentation on CAMS (WHC-CM-1-4) and } \\
\text { Corrective Action Management Board (CAMB). }\end{array}$ & $5 / 31 / 96$ & RB Balthazor \\
\hline $\begin{array}{l}\text { 2. Document current policy status and status of outstanding } \\
\text { CAMB actions. }\end{array}$ & $5 / 31 / 96$ & RB Balthazor \\
\hline 4.14 .4 Post-Award Actions & 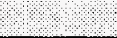 & \\
\hline 1. Provide briefing on CAMS. & $8 / 01 / 96$ & RB Balthazor \\
\hline 2. Provide CAMS documentation. & 7/01/96 & RB Balthazor \\
\hline
\end{tabular}

3.5.1.1.4 Hanford Action Tracking System. The Hanford Action Tracking System (HATS) provides a mechanism to monitor, status, and trend corrective actions at Hanford. The re-engineering process designated HATS as the preferred official corrective action system, and can also handle non-corrective action items. Actions are tracked from initiation through closure. HATS is designed to cover a multi-contractor environment, providing a global perspective of corrective action activities. HATS is being considered for use in documenting pre-existing conditions.

\begin{tabular}{|c|c|c|}
\hline Pre-A ward Actions & $\begin{array}{c}\text { Scbeduled } \\
\text { Date }\end{array}$ & Actionee(s) \\
\hline $\begin{array}{l}\text { 1. Publish a HATS/CAM Procen Guide to define business rules } \\
\text { and user responsibilities. }\end{array}$ & $5 / 01 / 96$ & $\begin{array}{l}\text { MK Britton } \\
\text { JM Knoll, Jr. }\end{array}$ \\
\hline Pout-Awerd Actions & & \\
\hline $\begin{array}{l}\text { 1. Provide the HATS Busunem Procen User's Guide and a list of } \\
\text { open items that require chnoure. }\end{array}$ & $7 / 01 / 96$ & $\begin{array}{l}\text { MK Britton } \\
\text { JM Knoll, Jr. } \\
\end{array}$ \\
\hline $\begin{array}{l}\text { 2. Conduct a presentation and o demoastration of the HATS } \\
\text { functionality. (Include relempanchip to CICS) }\end{array}$ & $7 / 15 / 96$ & \begin{tabular}{|l} 
MK Britton \\
JM Knoll, Jr.
\end{tabular} \\
\hline $\begin{array}{l}\text { 3. Provide training and documedentroe to ensure a smooth } \\
\text { transition of HATS adminustrative responsibilities and } \\
\text { requirements. }\end{array}$ & $9 / 30 / 96$ & $\begin{array}{l}\text { MK Britton } \\
\text { JM Knoll, Jr. }\end{array}$ \\
\hline
\end{tabular}

\subsection{Points of Contact.}

\begin{tabular}{|l|c|c|}
\hline 4 & $\begin{array}{c}\text { Scheduled } \\
\text { Date }\end{array}$ & Actionee(s)
\end{tabular}




\subsubsection{Safety.}

\subsection{Administration.}

\begin{tabular}{|c|c|c|}
\hline Pre-A ward Actions & $\begin{array}{l}\text { Scheduled } \\
\text { Date }\end{array}$ & Sotonects) \\
\hline 1. Assemble current safety plans, policies and procedures. & $5 / 31 / 96$ & GR Franz \\
\hline $\begin{array}{l}\text { 2. Assemble documentation for Industrial Safety (IS) and Fire } \\
\text { Protection (FP) only: This relates to the assembly of all } \\
\text { WHC programmatic (levels } 1 \text { and 2) policies, standards, and } \\
\text { procedures relating to IS and FP, as well as all company } \\
\text { level safety program plans, gosls, objectives, and milestones. }\end{array}$ & $5 / 31 / 96$ & JR Bell \\
\hline 3. Verify contents of safety resource library. & $5 / 31 / 96$ & JR Bell \\
\hline Past-A ward Actions & & \\
\hline $\begin{array}{l}\text { 1. Brief PHMC on medical services provided by HEHF } \\
\text { (program and budget status). }\end{array}$ & $8 / 01 / 96$ & JJ Maher III \\
\hline $\begin{array}{l}\text { 2. Brief PHMC on medical services provided by HEHF (budget } \\
\text { status). }\end{array}$ & $8 / 01 / 96$ & $\begin{array}{l}\text { AW Spillman } \\
\text { RO Volk }\end{array}$ \\
\hline 3. Transfer medical surveillance enrollment records. & $9 / 29 / 96$ & $\begin{array}{l}\text { MK Britton } \\
\text { JJ Maher III }\end{array}$ \\
\hline 4. Transfer safety resource library. & $9 / 29 / 96$ & JR Bell \\
\hline 5. Brief PHMC on safety awareness program. & $8 / 01 / 96$ & GR Franz \\
\hline
\end{tabular}

\subsection{Accident Investigation and OSHA Injury Records.}

\begin{tabular}{|l|l|l|}
\hline \multicolumn{1}{|c|}{ Pre-Awer Actions } & Scheduled & Date
\end{tabular}




\subsection{Regulatory Implementation}

\begin{tabular}{|l|c|c|}
\hline $\begin{array}{l}\text { 1. One page summary (DSI, Internal Memo, etc.) providing a } \\
\text { brief overview of the status on issuing and/or developing } \\
\text { Implementation Plans (IPs)/Exemption Requests (ERs) for the } \\
\text { balance of the Phase I Rules. }\end{array}$ & $6 / 01 / 96$ & DM Busche \\
\hline $\begin{array}{l}\text { 1. Summary report detailing the status of issuing Rules and/or } \\
\text { preparing IP/ERs for each WHC managed Nuclear Facility } \\
\text { (e.g., positioning, negotiated w/regulator, compliance } \\
\text { baselines, validation status, etc.) }\end{array}$ & $9 / 02 / 96$ & DM Busche \\
\hline 2. Project Files (for each rule and each facility). & & \\
\hline 3. Document repository for IPs/ERs prepared. & $9 / 02 / 96$ & DM Busche \\
\hline
\end{tabular}

\subsection{Subcontractor/Procurement Oversight.}

\begin{tabular}{|c|c|c|}
\hline $\begin{array}{l}\text { + } \\
+ \\
+\end{array}$ & $\begin{array}{l}\text { Schediled } \\
\text { Date }\end{array}$ & Actoones $(S)$ \\
\hline $\begin{array}{l}\text { 1. Document status of ongoing subcontractor/procurement } \\
\text { oversight. }\end{array}$ & $6 / 01 / 96$ & $\begin{array}{l}\text { DL Bloom } \\
\text { BG Baker } \\
\text { RD Melbihess }\end{array}$ \\
\hline Post-Award Actions & & \\
\hline $\begin{array}{l}\text { 1. Transfer subcontractor/procurement oversight status } \\
\text { information. }\end{array}$ & $9 / 02 / 96$ & $\begin{array}{l}\text { DL Bloom } \\
\text { BG Baker } \\
\text { RD Melbihess }\end{array}$ \\
\hline
\end{tabular}

\subsection{Industrial Hygiene and Safety (including IH Equipment Lab)}

\begin{tabular}{|l|l|l|l|}
\hline 1. & $\begin{array}{l}\text { Verify equipment list (2); verify calibration status (3) of IH } \\
\text { Equipment Laboratory (IHEL). Note: These actions relate } \\
\text { only to exposure monitoring equipment directly controlled } \\
\text { by IHEL. IHEL maintains exposure monitoring equipment } \\
\text { for WHC and ICF KH. }\end{array}$ & $6 / 01 / 96$ & GR Franz \\
\hline 2. & $\begin{array}{l}\text { Verify equipment list: via IHEL Microsoft Access } \\
\text { database. Verification includes database review and contact } \\
\text { with equipment assignees. }\end{array}$ & $6 / 01 / 96$ & DS Shoop \\
\hline 3. & Verify calibration status: via IHEL equipment files. & $6 / 01 / 96$ & DS Shoop \\
ML Zabel
\end{tabular}




\begin{tabular}{|c|c|c|}
\hline Post Awrard Actions & $\begin{array}{l}\text { Scheduled } \\
\text { Date }\end{array}$ & Actionee(s) \\
\hline $\begin{array}{l}\text { 1. Document status of IHEL: operating procedures available } \\
\text { on HLAN and will be reviewed with PHMC, plus day-to- } \\
\text { day IHEL operation. }\end{array}$ & $9 / 02 / 96$ & $\begin{array}{l}\text { DS Shoop } \\
\text { ML Zabel }\end{array}$ \\
\hline $\begin{array}{l}\text { 2. Transfer equipment and calibration status for IHEL: via } \\
\text { formal transfer of IHEL database. Physical verification of } \\
\text { equipment list is assumed. }\end{array}$ & 9/02/96 & $\begin{array}{l}\text { DS Shoop } \\
\text { ML Zabel }\end{array}$ \\
\hline $\begin{array}{l}\text { 3. Provide status of ongoing industrial hygiene program } \\
\text { development efforts: Written Industrial Hygiene Programs } \\
\text { and IH Technical Procedures are maintained on HLAN in } \\
\text { WHC-CM-1-11/WHC-CM } 4-40 \text { and WHC-IP-0030 and will } \\
\text { be reviewed with PHMC. }\end{array}$ & 9/02/96 & $\begin{array}{l}\text { DS Shoop } \\
\text { SL Alexander }\end{array}$ \\
\hline $\begin{array}{l}\text { 4. Provide status of ongoing fire protection standards: Time } \\
\text { depend on number of standards in process. }\end{array}$ & $9 / 02 / 96$ & JR Bell \\
\hline $\begin{array}{l}\text { 5. Provide status of Enhanced Work Planning Demonstration } \\
\text { Project: via review with PHMC of project background, } \\
\text { Phase I report, budget, Phase II status, and project } \\
\text { milestones. }\end{array}$ & $9 / 02 / 96$ & $\begin{array}{l}\text { DS Shoop } \\
\text { LM Calderon }\end{array}$ \\
\hline $\begin{array}{l}\text { Provide status of program to track chemical, physical, } \\
\text { biological exposure and transfer any available records: The } \\
\text { WHC Industrial Hygiene Programs organization maintains } \\
\text { exposure records for WHC and ICF KH in hard copy and } \\
\text { on Flow Gemini database. It is being replaced with } \\
\text { Sentry } 2000 \text {. } \\
\text { Overview implementation status, data conversion, technical } \\
\text { procedures contained in WHC-IP-0030, and operating } \\
\text { parameters associated with exposure database; formally } \\
\text { transfer Sentry } 2000 \text { software and file server to PHMC; } \\
\text { describe process for development, maintenance and storage } \\
\text { of employee exposure records; review and update exposure } \\
\text { records, RIDs; formally transfer records. }\end{array}$ & $9 / 02 / 96$ & $\begin{array}{l}\text { DS Shoop } \\
\text { KD Moorman }\end{array}$ \\
\hline $\begin{array}{l}\text { 7. Brief PHMC on ergonomic software: ErgoSmart software } \\
\text { is available to all Hanford employees via HLAN, with } \\
\text { supporting technical procedures contained in WHC-0030. }\end{array}$ & 9/02/96 & $\begin{array}{l}\text { DS Shoop } \\
\text { KD Moorman }\end{array}$ \\
\hline 8. Formally transfer ergonomic software. & $9 / 02 / 96$ & $\begin{array}{l}\text { DS Shoop } \\
\text { KD Moorman }\end{array}$ \\
\hline $\begin{array}{l}\text { 9. Brief new contractor on Voluntary Protection Program } \\
\text { (VPP) status. }\end{array}$ & $9 / 02 / 96$ & JR Bell \\
\hline $\begin{array}{l}\text { 10. Transfer VPP application materials: This action requires } \\
\text { no advanced preparation. }\end{array}$ & $9 / 02 / 96$ & JR Bell \\
\hline
\end{tabular}

\subsection{Fire Safety}

\begin{tabular}{|l|c|c|c|}
\hline \multirow{4}{*}{ Pre-Award Actions } & $\begin{array}{c}\text { Scheduled } \\
\text { Date }\end{array}$ & Netionee(s) \\
\hline 1. Document status of ongoing fire safety oversight. & $6 / 01 / 96$ & JR Bell \\
\hline
\end{tabular}




\begin{tabular}{|l|l|l|}
\hline Post-Award hrtions & $\begin{array}{c}\text { Scheduled } \\
\text { Date }\end{array}$ & Netionee( $)$ \\
\hline $\begin{array}{l}\text { 1. Transfer fire and property damage records: we keep no such } \\
\text { official records on behalf of the company, other than the } \\
\text { annual summary reports which are in office files. Action: } \\
\text { turn over all office files. }\end{array}$ & $9 / 02 / 96$ & JR Bell \\
\hline 2. Transfer fire safety oversight status information. & $9 / 02 / 96$ & JR Bell \\
\hline
\end{tabular}

\subsubsection{Radiological Control (RadCon).}

\subsection{Administration.}

\begin{tabular}{|c|c|c|}
\hline Pre-Award Actions & $\begin{array}{l}\text { Scheduled } \\
\text { Date }\end{array}$ & Aetlonee(s) \\
\hline $\begin{array}{l}\text { 1. Prepare summary package outlining RadCon Central Support } \\
\text { Group (CSG), Center of Expertise (COE) and Site Dosimetry } \\
\text { Services } \\
\text { - Include program and budget formulation (both direct and } \\
\text { indirect activities. }\end{array}$ & $6 / 01 / 96$ & DJ Newland \\
\hline Post-A ward Actions & & \\
\hline $\begin{array}{l}\text { 1. Brief PHMC on current status of CSG/COE activities and } \\
\text { Site Dosimetry Services } \\
\text { - Provide program and budget status detail (SSPP, ADS, } \\
\text { RDS). }\end{array}$ & $9 / 01 / 96$ & DJ Newland \\
\hline $\begin{array}{l}\text { 2. Discuss with PHMC transition actions associated to } \\
\text { decentraled RadCon Organization } \\
\text { - HPT transfer to the line } \\
\text { - Funding transitions } \\
\text { - Bargaining Unit support }\end{array}$ & $9 / 02 / 96$ & DJ Newland \\
\hline $\begin{array}{l}\text { 3. Provide PHMC status of outstanding customer commitments } \\
\text { (RadCon Path Forward) } \\
\text { - Sitewide RadCon Action Plans } \\
\text { - Facility Specific Action Plans }\end{array}$ & $9 / 02 / 96$ & DJ Newland \\
\hline
\end{tabular}

\subsection{Centralized RadCon CSG and COE.}

\begin{tabular}{|l|l|l|}
\hline \multicolumn{1}{|c|}{ Pro-Award Actions } & $\begin{array}{l}\text { Scheduled } \\
\text { Date }\end{array}$ & Actionee(s) \\
\hline $\begin{array}{l}\text { 1. Prepare to brief PHMC on centralized RadCon support } \\
\text { activities and sitewide implementation roles } \\
\text { Technical Radiological Engineering support. }\end{array}$ & $6 / 01 / 96$ & PB Chadly \\
\hline Post-Award Actions & $9 / 02 / 96$ & PB Chadly \\
\hline $\begin{array}{l}\text { 1. Assemble CSG and COE completed decision making } \\
\text { packages (CDMP) for transfer. }\end{array}$ & \\
\hline
\end{tabular}


2. Provide PHMC with CSG and COE sitewide infrastructure and requirements implementation process

- Address sitewide requirements consistency issues.

3. Provide PHMC overview/status of Hanford Site Radiological Control Manual (HRSCM) support services.

4. Brief PHMC on external contractor procured services/support status.

\begin{tabular}{|c|l|}
\hline $\begin{array}{c}\text { Scheduled } \\
\text { Dete }\end{array}$ & Actionee(f) \\
\hline $9 / 02 / 96$ & PB Chadly \\
\hline $9 / 02 / 96$ & PB Chadly \\
\hline $9 / 02 / 96$ & PB Chadly \\
\hline
\end{tabular}

3.5.1.3.3 Site Personnel Dosimetry Services. Note: If the PHMC decides not to utilize the sitewide radiation protection services, the other Hanford contractors and DOE-RL will need to develop new strategies to obtain/maintain these services. Therefore, a separate transition plan dealing with all Hanford contractors and RL will need to be developed.

\begin{tabular}{|c|c|c|}
\hline Pre-Award Actions & $\begin{array}{l}\text { Scheduled } \\
\text { Date }\end{array}$ & Actionee(s) \\
\hline $\begin{array}{l}\text { 1. Prepare to brief PHMC on all sitewide radiation protection } \\
\text { services currently provided by PNNL } \\
\text { - In-vivo counting (whole-body, lung, organ, skeletal) } \\
\text { - In-vitro bioassay program } \\
\text { - External dosimeter processing/reporting } \\
\text { - External expert contracting services/oversight } \\
\text { - Historical and current radiation exposure and RadCon } \\
\text { program records project } \\
\text { - RadCon instrumentation calibration, maintenance and } \\
\text { - testing programs } \\
\text { - Sitewide radiation protection committee structure, charters } \\
\text { and membership }\end{array}$ & 6/01/96 & $\begin{array}{l}\text { LR McKay } \\
\text { ME Hevland (PNNL) }\end{array}$ \\
\hline \multicolumn{3}{|l|}{ Post-A ward Actions } \\
\hline $\begin{array}{l}\text { 1. Assemble auditable personnel dosimetry record } \\
\text { documentation. }\end{array}$ & 9/02/96 & $\begin{array}{l}\text { LR McKay } \\
\text { ME Hevland (PNNL) }\end{array}$ \\
\hline $\begin{array}{l}\text { 2. Brief PHMC on internal dosimetry program } \\
\text { - Prepare/transfer routine internal dosimetry records } \\
\text { - Provide emergency response evaluation results } \\
\text { - Transfer employee "home location" logistics database. }\end{array}$ & 9/02/96 & $\begin{array}{l}\text { LR McKay } \\
\text { ME Hevland (PNNL) }\end{array}$ \\
\hline $\begin{array}{l}\text { 3. Brief PHMC on external dosimetry program } \\
\text { - Prepare/transfer routine external dosimetry records } \\
\text { - Provide dose reconstruction and exposure evaluation data } \\
\text { - Transfer worker restrictions data. }\end{array}$ & 9/02/96 & $\begin{array}{l}\text { LR McKay } \\
\text { ME Hevland (PNNL) }\end{array}$ \\
\hline $\begin{array}{l}\text { 4. Brief PHMC on subcontractor dosimetry program } \\
\text { - Provide dosimetry interface matrix for plants/projects } \\
\text { - Prepare/transfer subcontractor dosimetry records. }\end{array}$ & 9/02/96 & $\begin{array}{l}\text { LR McKay } \\
\text { ME Hevland (PNNL) }\end{array}$ \\
\hline 5. Transfer personnel dosimetry records database and software. & 9/02/96 & $\begin{array}{l}\text { LR McKay } \\
\text { ME Hevland (PNNL) }\end{array}$ \\
\hline $\begin{array}{l}\text { 6. Transfer Dosimetry and Medical Services Manual } \\
\text { (WHC-CM-4-16). }\end{array}$ & 9/02/96 & $\begin{array}{l}\text { LR McKay } \\
\text { ME Hevland (PNNL) }\end{array}$ \\
\hline
\end{tabular}




\subsection{RadCon Technical Standards.}

\begin{tabular}{|c|c|c|}
\hline Pre-Award Actions & $\begin{array}{l}\text { Schedilled } \\
\text { Date }\end{array}$ & Yet'onee(s) \\
\hline $\begin{array}{l}\text { 1. Prepare to brief PHMC on established minimum RadCon } \\
\text { technical standards and documents } \\
\text { - Applied Radiological Controls (WHC-CM-4-14) } \\
\text { - Dosimetry and Medical Services Manual } \\
\text { (WHC-CM-4-16) } \\
\text { - Health Physics Procedures (WHC-IP-0718) } \\
\text { - Access Control Entry System User Manual } \\
\text { (WHC-IP-0993) } \\
\text { - WHC Occupational As Low As Reasonably Achievable } \\
\text { (ALARA) Program } \\
\text { (WHC-IP-1043). }\end{array}$ & $6 / 01 / 96$ & PB Chadly \\
\hline Post-Award Actions & & \\
\hline $\begin{array}{l}\text { 1. Assemble/transfer current RadCon Path Forward plan, } \\
\text { standards, policies and implementing procedures } \\
\text { - Validate current RadCon standards } \\
\text { - Assemble/status outstanding field change requests } \\
\text { - Provide central and facility specific copies to new } \\
\text { contractor. }\end{array}$ & $9 / 02 / 96$ & PB Chadly \\
\hline
\end{tabular}

\subsection{Fixed/Portable Radiological Monitoring Equipment.}

\begin{tabular}{|c|c|c|}
\hline Post-Award Actions & $\begin{array}{l}\text { Scheduled } \\
\text { Date }\end{array}$ & Actiones (s) \\
\hline $\begin{array}{l}\text { 1. Document current status of RadCon monitoring } \\
\text { equipment/Lab } \\
\text { - Verify current RadCon instrumentation and equipment list } \\
\text { - Verify calibration standards and records } \\
\text { - Provide current technical standards, procurement data, } \\
\text { technical basis and supporting data for instruments } \\
\text { - Transfer equipment, calibration standards, and records. }\end{array}$ & $9 / 02 / 96$ & $\begin{array}{l}\text { LR McKay } \\
\text { ME Hevland (PNNL) }\end{array}$ \\
\hline $\begin{array}{l}\text { 2. Provide PHMC status of ongoing RadCon equipment } \\
\text { decentralization and prepare for transfer } \\
\text { - Pooled instrument calibration and maintenance } \\
\text { - Interface with Hanford Instrument Evaluation Committee. }\end{array}$ & $9 / 02 / 96$ & $\begin{array}{l}\text { LR McKay } \\
\text { ME Hevland (PNNL) }\end{array}$ \\
\hline
\end{tabular}




\subsection{RadCon Technical Compliance/Documentation.}

\begin{tabular}{|c|c|c|}
\hline Post-Award fetions & $\begin{array}{l}\text { Schedured } \\
\text { d dete }\end{array}$ & Atitoned $(\mathrm{s})$ \\
\hline $\begin{array}{l}\text { 1. Assemble supporting data for compliance actions } \\
\text { (10 Code of Federal Regulations [CFR] 835, RCM, etc.) } \\
\text { - Document status of compliance actions in progress } \\
\text { - Transfer RadCon RPP and S/RID compliance data } \\
\text { - Transfer RadCon implementation activity database } \\
\text { - Provide COE CDMP closure packages, compliance plans } \\
\text { and schedules on implementation actions } \\
\text { - Provide Interpretive Authority Technical Equivalency } \\
\text { Determination (TED) data demonstrating compliance. }\end{array}$ & $9 / 02 / 96$ & PB Chadly \\
\hline
\end{tabular}

\subsection{Radiological Access Control Entry System (ACES).}

\begin{tabular}{|c|c|c|}
\hline Pre-Award Actions & Scheduled & tetionee( 8$)$ \\
\hline 1. Verify accuracy of ACES database. & $6 / 01 / 96$ & LR McKay \\
\hline Post Award Actions & & \\
\hline 1. Brief/train PHMC on employee/visitor ACES operation. & $9 / 02 / 96$ & LR McKay \\
\hline $\begin{array}{l}\text { 2. Transfer ACES database system including records, software, } \\
\text { hardware licensing agreements } \\
\text { - ACES training standards/records } \\
\text { - ACES password database. }\end{array}$ & $9 / 02 / 96$ & LR McKay \\
\hline $\begin{array}{l}\text { 3. Transfer Access Control Entry System User Manual } \\
\text { (WHC-IP-0993). }\end{array}$ & $9 / 02 / 96$ & LR McKay \\
\hline
\end{tabular}

\subsection{Occupational ALARA Program.}

\begin{tabular}{|c|c|c|}
\hline Pre-Award Actions & $\begin{array}{l}\text { Scheduled } \\
\text { Date }\end{array}$ & Actionests) \\
\hline $\begin{array}{l}\text { 1. Prepare to brief PHMC on existing occupational ALARA } \\
\text { program } \\
\text { - Facility and site infrastructure } \\
\text { - Centralized Radiological Engineering support } \\
\text { - Centralized Contamination Control Improvement Program } \\
\text { - Radiological Emergency Assistance Center/Training Site } \\
\text { (REAC/TS). }\end{array}$ & $6 / 01 / 96$ & PB Chadly \\
\hline \multicolumn{3}{|l|}{ Post-Award Actions } \\
\hline 1. Assemble ALARA Program documentation and current plans. & $9 / 02 / 96$ & PB Chadly \\
\hline $\begin{array}{l}\text { 2. Transfer current program documentation, record reports, and } \\
\text { supplemental training plans } \\
\text { - WHC Occupational ALARA Program (WHC-IP-1043) } \\
\text { - Optimization methodology and training materials. }\end{array}$ & $9 / 02 / 96$ & PB Chadly \\
\hline
\end{tabular}




\subsection{RadCon COE Internal Assessment Program.}

\begin{tabular}{|l|c|c|}
\hline $\begin{array}{l}\text { 1. Prepare to brief PHMC on current COE assessment program and } \\
\text { support provided to the Facility Evaluation Board (FEB). }\end{array}$ & $6 / 01 / 96$ & PB Chadly \\
\hline $\begin{array}{l}\text { 1. Document status of ongoing sitewide RadCon assessments. } \\
\text { Transfer assessment status tracking and trending program and }\end{array}$ & $9 / 02 / 96$ & PB Chadly \\
\hline associated records. & $9 / 96$ & PB Chadly \\
\hline $\begin{array}{l}\text { 3. Transfer all formal interpretations (both facility and site level) } \\
\text { documentation on RadCon requirements. }\end{array}$ & $9 / 02 / 96$ & PB Chadly \\
\hline 4. Provide company level RadCon S/RID review and approval data. & $9 / 02 / 96$ & PB Chadly \\
\hline
\end{tabular}

\subsection{RadCon Site Training.}

\begin{tabular}{|l|c|c|}
\hline & Schediled & Date \\
\hline 1. Prepare to brief PHMC onsite RadCon training program. & $6 / 01 / 96$ & PB Chadly \\
\hline $\begin{array}{l}\text { 1. Provide PHMC with training lesson plans, review/approval data, } \\
\text { and training effectiveness evaluations. }\end{array}$ & $9 / 02 / 96$ & PB Chadly \\
\hline $\begin{array}{l}\text { 2. Provide PHMC current status on RadCon Technician and } \\
\text { recertification. }\end{array}$ & $9 / 02 / 96$ & PB Chadly \\
\hline 3. Transfer associated radiological training records. & $9 / 02 / 96$ & PB Chadly \\
\hline
\end{tabular}

\subsection{RadCon Sitewide Project/Sub-Contractor Support.}

\begin{tabular}{|c|c|c|}
\hline Pre-Award Actions & $\begin{array}{l}\text { Scheduled } \\
\text { Date }\end{array}$ & Actionee(s) \\
\hline $\begin{array}{l}\text { 1. Prepare to brief PHMC on existing RadCon construction } \\
\text { projects, landlord, vehicle maintenance, laundry facility, RCRA, } \\
\text { shipping/receiving, HPT supplemental crew and diversified } \\
\text { subcontractor support activities } \\
\text { - ICF Kaiser } \\
\text { - INS } \\
\text { - PNNL } \\
\text { - IRM } \\
\text { - BCSR, as appropriate. }\end{array}$ & $6 / 01 / 96$ & RL Watts \\
\hline Pest-Award Actions & & \\
\hline 1. Transfer existing contracts to PHMC. & $9 / 02 / 96$ & RL Watts \\
\hline
\end{tabular}




\subsubsection{Quality Assurance (QA).}

\subsection{General Organization.}

\begin{tabular}{|c|c|c|}
\hline Post-Award Actions & $\begin{array}{l}\text { Scheduled } \\
\text { Date }\end{array}$ & actionse(t) \\
\hline 1. Provide organization charts and charters. & $7 / 01 / 96$ & H Spanheimer \\
\hline 2. Provide organization points of contact list and staff directory. & $7 / 01 / 96$ & H Spanheimer \\
\hline
\end{tabular}

\subsection{QA Program.}

\begin{tabular}{|l|l|l|}
\hline 1. Provide Quality Assurance policies and procedures of: & Scheduled & Dete \\
- Quality Assurance Manual (WHC-CM-4-2) & $7 / 01 / 96$ & H Spanheimer \\
- Quality Assurance Instructions (WHC-CM-4-5) & \\
- "Approval Designators" (WHC-CM-3-5, Section 12.7) & \\
- WHC Company-level QA S/RID & \\
- WHC QA Policy (WHC-CM-1). & \\
\hline
\end{tabular}

\subsection{10 Code of Federal Regulations (CFR) 830.120 Regulatory}

\section{Compliance Status.}

\begin{tabular}{|c|c|c|}
\hline Post-Award Letions & $\begin{array}{l}\text { Scheduled } \\
\text { Date } \\
\end{array}$ & Actionee $(\mathrm{s})$ \\
\hline $\begin{array}{l}\text { 1. Provide Regulatory Compliance status of Noncompliance } \\
\text { Notification Reports reported to-date/history. }\end{array}$ & $9 / 02 / 96$ & RN Sherman \\
\hline $\begin{array}{l}\text { 2. Summary of pending investigations/issues. } \\
\text { - Summary Report with background; specific rule } \\
\text { noncompliance; and status of investigation, including root } \\
\text { cause and corrective action(s). } \\
\text { NOTE: Assume one investigation/issue is in progress and } \\
\text { adjust time needed to complete summary report based on } \\
\text { the number of open events. }\end{array}$ & $9 / 02 / 96$ & RN Sherman \\
\hline $\begin{array}{l}\text { 3. Summary of outstanding corrective action commitments } \\
\text { - Summary report of open commitments and corrective } \\
\text { actions with identification of source of commitment. }\end{array}$ & $9 / 02 / 96$ & RN Sherman \\
\hline
\end{tabular}

\subsection{WHC Management Assessment Program.}

\begin{tabular}{|l|l|l|l|}
\hline Post-Award Actions & Scheduled & Date & \\
\hline 1. Provide annual 1995 Management Assessment Report. & $9 / 02 / 96$ & GM Stevens \\
\hline $\begin{array}{l}\text { 2. Provide copy of APIP and summarize current implementation } \\
\text { status. }\end{array}$ & $9 / 02 / 96$ & GM Stevens \\
\hline
\end{tabular}




\subsection{Suspect/Counterfeit Materials Program.}

\begin{tabular}{|c|c|c|}
\hline Presmard actions & Schediled & betioneds) \\
\hline 1. Update investigations in process. & $6 / 01 / 96$ & JN Nansen \\
\hline Pot-Award Actions & & \\
\hline 1. Brief PHMC on program history and current status. & $9 / 02 / 96$ & JN Nansen \\
\hline 2. Brief PHMC on training program. & $9 / 02 / 96$ & RD Moerman \\
\hline 3. Provide copy of calendar year (CY) 1995 annual report. & $9 / 02 / 96$ & CR Hoover \\
\hline
\end{tabular}

\subsection{Quality Assurance Oversight/Assessment Activities.}

\begin{tabular}{|c|c|c|}
\hline Rost-Award Actions & $\begin{array}{l}\text { Scheduled } \\
\text { Date, }\end{array}$ & Actionee $(s)$ \\
\hline $\begin{array}{l}\text { 1. Provide copy and status of CY } 1996 \text { oversight/assessment } \\
\text { schedule. }\end{array}$ & $9 / 02 / 96$ & JA Peltier \\
\hline 2. Provide FY 1996 Annual QA Management Assessment Plan. & $9 / 02 / 96$ & H Spanheimer \\
\hline
\end{tabular}

\subsection{Natural Phenomena Hazards Mitigation.}

\begin{tabular}{|c|c|c|}
\hline $\begin{array}{l}\text { मै. Pre-Award Actions } \\
\end{array}$ & $\begin{array}{l}\text { Scheduled } \\
\text { Date }\end{array}$ & Actonee(s) \\
\hline $\begin{array}{l}\text { 1. Provide a status repon of the ongoing program to implement } \\
\text { DOE Order } 5480.28 \text {, NPH Mitigation. }\end{array}$ & $6 / 01 / 96$ & TJ Conrads \\
\hline $\begin{array}{l}\text { 2. Provide copies of SP-1174, "WHC Structural Analysis and } \\
\text { Design Criteria." }\end{array}$ & $6 / 01 / 96$ & TJ Conrads \\
\hline Pon-Awert Actions & $\therefore$ & \\
\hline 1. Provide copy of implemeotmose documents issued to-date. & $9 / 02 / 96$ & TJ Conrads \\
\hline 2. Provide summary atus repon of order implementation. & $9 / 02 / 96$ & TJ Conrads \\
\hline
\end{tabular}

\subsection{Codes and Standards Compliance Activities.}

\begin{tabular}{|c|c|c|}
\hline Pre-Award Actions & $\begin{array}{l}\text { Scheduled } \\
\text { Date }\end{array}$ & Actionee(s) \\
\hline $\begin{array}{l}\text { 1. Update list of WHC S/RIDs and outstanding commitments: } \\
\text { copies of company S/RIDs should be provided by the } \\
\text { responsible interpretative authorities. }\end{array}$ & $6 / 01 / 96$ & $\begin{array}{l}\text { MD Jackson } \\
\text { DS Shoop } \\
\text { JR Bell }\end{array}$ \\
\hline 10, Post-Award Actions & & \\
\hline $\begin{array}{l}\text { 1. Provide historical background and summary of DNFSB } \\
\text { recommendations related to S/RID program. }\end{array}$ & $9 / 02 / 96$ & MD Jackson \\
\hline 2. Provide copy of WHC S/RID program procedures & $9 / 02 / 96$ & MD Jackson \\
\hline
\end{tabular}




\subsection{Nuclear Safety Regulatory Compliance.}

\begin{tabular}{|l|c|l|}
\hline 1. Copy of Nuclear Safety Rule Compliance (WHC-IP-1112). & $9 / 02 / 96$ & TA Demitruk \\
\hline 2. Copy of milestone transmittal letter and basis for facility list. & $9 / 02 / 96$ & TA Demitruk \\
\hline $\begin{array}{l}\text { 3. Provide status of RL Docket file: } \\
\text { - Listing of document types submitted }\end{array}$ & $9 / 02 / 96$ & TA Demitruk \\
- Summary overview report & & \\
- Computer operator desk instructions/users manual & & \\
\hline
\end{tabular}

\subsection{Price Anderson Amendment Act.}

\begin{tabular}{|c|c|c|}
\hline Post-Award Actions & $\begin{array}{l}\text { Scheduled } \\
\text { Date }\end{array}$ & Actionee(s) \\
\hline $\begin{array}{l}\text { 1. Brief PHMC on status of Nuclear Safety Rules and } \\
\text { Implementation Plans for Hanford nuclear facilities } \\
\text { - Presentation/briefing to include details of reports and, } \\
\text { status, and areas needing specific attention (i.e., regulator } \\
\text { negotiated positions, legally enforceable due dates, etc.). }\end{array}$ & $9 / 02 / 96$ & DM Lucoff \\
\hline $\begin{array}{l}\text { 2. Brief PHMC on status of pending Nuclear Safety Rules and } \\
\text { future implementation requirements. } \\
\text { - Presentation/briefing topics: details of reports and files, } \\
\text { status, areas needing specific attention (i.e., regulator } \\
\text { negotiated positions, legally enforceable due dates, etc.). }\end{array}$ & $9 / 02 / 96$ & DM Lucoff \\
\hline $\begin{array}{l}\text { 3. Brief PHMC on training requirements for personnel involved } \\
\text { with PAAA rule implemenution and non-compliance } \\
\text { reporting. Provide training a necessary. } \\
\text { - Presentation/briefing upks: reports and files, status, areas } \\
\text { needing specific attentson (1.e., regulator negotiated } \\
\text { positions, legally enforceable due dates, etc.). }\end{array}$ & $9 / 02 / 96$ & $\begin{array}{l}\text { DM Busche } \\
\text { RN Sherman }\end{array}$ \\
\hline
\end{tabular}

\subsection{Regulatory Implementation.}

\begin{tabular}{|c|c|c|}
\hline $\begin{array}{l}1+2 \\
+ \\
\end{array}$ & Scheduled & Actionet (s) \\
\hline $\begin{array}{l}\text { 1. Summarize pending self-reporting activities. } \\
\text { (Assume one investigation/issue is in progress and adjust time } \\
\text { needed to complete summary report based on the number of } \\
\text { open events.) }\end{array}$ & $6 / 01 / 96$ & RN Sherman \\
\hline $\begin{array}{l}\text { 2. Summarize pending/open corrective actions associated with } \\
\text { NNRs. } \\
\text { (Assume one investigation/issue is in progress and adjust time } \\
\text { needed to complete summary report based on the number of } \\
\text { open events.) }\end{array}$ & $6 / 01 / 96$ & RN Sherman \\
\hline Post-Award Actions & & \\
\hline $\begin{array}{l}\text { 1. Provide current status of minor noncompliances identified in } \\
\text { the WHC internal reporting database. }\end{array}$ & $9 / 02 / 96$ & RN Sherman \\
\hline
\end{tabular}




\begin{tabular}{|c|c|c|}
\hline Post-A ward / Actions & $\begin{array}{l}\text { Seheduled } \\
\text { Date }\end{array}$ & Netiones $(6)$ \\
\hline $\begin{array}{l}\text { 2. Provide copies of summary reports provided to DOE on a } \\
\text { quarterly basis describing minor noncompliances added to the } \\
\text { local database Reference existing WBS } \\
\text { Designator } 6.7 .2 .5 .2 .3 .06 \text {. }\end{array}$ & $9 / 02 / 96$ & RN Sherman \\
\hline
\end{tabular}

\subsection{DNFSB Coordination/Site General Status.}

\begin{tabular}{|l|c|c|}
\hline $\begin{array}{l}\text { 1. Brief PHMC on status of DNFSB activities affecting } \\
\text { WHC/Hanford. }\end{array}$ & $6 / 01 / 96$ & JW Hales \\
\hline $\begin{array}{l}\text { Date } \\
\text { 1. Provide copies of all active DNFSB recommendations. }\end{array}$ & $9 / 02 / 96$ & $\begin{array}{l}\text { JW Hales } \\
\text { SA Bauer }\end{array}$ \\
\hline $\begin{array}{l}\text { 2. Provide copies of monthly status reports on WHC/Hanford } \\
\text { DNFSB commitments for past six months. }\end{array}$ & $9 / 02 / 96$ & $\begin{array}{l}\text { JW Hales } \\
\text { SA Bauer }\end{array}$ \\
\hline $\begin{array}{l}\text { 3. Brief PHMC on status of DNFSB activities and near-term } \\
\text { commitments/deliverables for WHC/Hanford facilities. }\end{array}$ & $9 / 02 / 96$ & JW Hales \\
\hline
\end{tabular}

3.5.1.5 Compliance Assurance. All records of the Compliance Assurance organization will be dispersed to records management and to the Facility Evaluation Board prior to contract transition.

\subsubsection{Work Control}

Operations and Maintenance Programs (OMP) coordinates, at the company level, programs that support work control within the company. OMP oversees the COO and maintenance, and provide "services" such as performance monitoring, procedure development, statistical analysis and MIP development. We also oversee the Job Control System which is used by all facilities for documenting work control. Numerous management "self-assessments" are coordinated by OMP and provide current status of our facilities in these areas. All of these functions will transition in some form to the PHMC contractor. OMP will coordinate this transition.

\begin{tabular}{|c|c|c|}
\hline Pre-Award Actions & $\begin{array}{l}\text { Scheduled } \\
\text { Date }\end{array}$ & Actionee $(s)$ \\
\hline 1. Identify WHC Directives which govern ConOps. & $4 / 15 / 96$ & CP Ames \\
\hline $\begin{array}{l}\text { 2. Outline Assessment Status for All Facilities and prepare } \\
\text { briefing. }\end{array}$ & $5 / 01 / 96$ & CP Ames \\
\hline 3. Prepare Brief on CMMS Issues. & $5 / 01 / 96$ & ER Hamm \\
\hline 4. Prepare Brief on Budget Issues. & $5 / 01 / 96$ & JC Bickford \\
\hline 5. Prepare Brief on Configuration Management Initiative. & $5 / 01 / 96$ & ER Hamm \\
\hline
\end{tabular}




\begin{tabular}{|l|c|l|}
\hline & $\begin{array}{c}\text { Scheduled } \\
\text { Date }\end{array}$ & Pre-Award Actions \\
6. Prepare Brief on Lesson Learned Program. & $5 / 01 / 96$ & JC Bickford \\
\hline 7. Prepare Brief on current Performance Indicators. & $5 / 01 / 96$ & SS Prevette \\
\hline Post-Award Actions & & \\
\hline 1. Provide copies of WHC directives in use. & $6 / 10 / 96$ & RD Raaz \\
\hline 2. Present briefings as requested by PHMC. & TBD & RD Raax \\
\hline
\end{tabular}

\subsubsection{Environmental Compliance}

The Environmental Services organization is involved (directly or indirectly) with the preparation of documents, reporting, and negotiations concerning environmental compliance within the M\&O Contractor. Programs and facilities have identified actions involving program and facility specific environmental information, including assemblage of key existing documentation, preparation of briefings, documenting a listing of key knowledgeable individuals as POCs, and identifying specific permits. The following actions are established to address sitewide environmental compliance and permitting actions:

\begin{tabular}{|l|l|l|}
\hline \multicolumn{1}{|c|}{ Pre-Award Actions } & $\begin{array}{l}\text { Scheduled } \\
\text { Date }\end{array}$ & Ictionee(s) \\
\hline $\begin{array}{l}\text { 1. Develop a list of key sitewide environmental compliance and } \\
\text { permitting documentation. (Provide to RL POC for review). } \\
\text { Prepare and distribute guidance to actionees on material to be } \\
\text { included in "Smart Books" and briefings. }\end{array}$ & $5 / 15 / 96$ & RH Engelmann \\
\hline $\begin{array}{l}\text { 2. Assemble key documentation, or identify its location, for use } \\
\text { by the PHMC. Includes development of a "Smart Book" to } \\
\text { include information developed below. }\end{array}$ & $6 / 01 / 96$ & $\begin{array}{l}\text { RH Engelmann } \\
\text { SM Price } \\
\text { J Luke } \\
\text { LP Diediker }\end{array}$ \\
$\begin{array}{l}\text { GC Cummins } \\
\text { RC Brune } \\
\text { JO Skolrud } \\
\text { EM Greager } \\
\text { DJ Carrell }\end{array}$ \\
\hline $\begin{array}{l}\text { 3. Identify briefings and prepare briefing material to address } \\
\text { sitewide environmental activities for presentation to the } \\
\text { PHMC. Topics to be covered should include sitewide } \\
\text { environmental reports, sitewide RCRA permitting, central } \\
\text { regulatory analyses and requirements activities, etc. (coordinate } \\
\text { number and content of briefings with RL POC). }\end{array}$ & $6 / 01 / 96$ & $\begin{array}{l}\text { WT Dixon } \\
\text { RH Engelmann } \\
\text { SM Price }\end{array}$ \\
& $\begin{array}{l}\text { JJ Luke } \\
\text { LP Diediker } \\
\text { GC Cummins } \\
\text { RC Brune } \\
\text { JO Skolrud } \\
\text { EM Greager } \\
\text { DJ Carrell }\end{array}$ \\
\hline $\begin{array}{l}\text { 5. Develop a list of key M\&O Contractor environmental POCs. } \\
\text { subcontracts. Include any Task Orders still planned to be } \\
\text { executed prior to contract takeover. }\end{array}$ & $6 / 01 / 96$ & RH Engelmann \\
\hline
\end{tabular}




\begin{tabular}{|c|c|c|}
\hline Pre-Avard Letions & $\begin{array}{l}\text { Scheduled } \\
\text { Date. }\end{array}$ & Altiones) \\
\hline $\begin{array}{l}\text { 6. Verify the accuracy of property assignments withis the } \\
\text { Environmental Services organization. }\end{array}$ & 6/01/96 & $\begin{array}{l}\text { WT Dixon } \\
\text { SM Cooley }\end{array}$ \\
\hline $\begin{array}{l}\text { 7. Identify space and equipment within the Environmental } \\
\text { Services area for use by the PHMC, if needed. }\end{array}$ & $6 / 01 / 96$ & $\begin{array}{l}\text { WT Dixon } \\
\text { SM Cooley }\end{array}$ \\
\hline 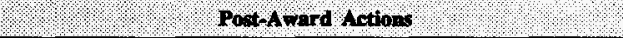 & & \\
\hline $\begin{array}{l}\text { 1. Provide the PHMC access to the key documentation. Provide } \\
\text { copies upon request. }\end{array}$ & $6 / 15 / 96$ & $\begin{array}{l}\text { WT Dixon } \\
\text { MW Cline }\end{array}$ \\
\hline $\begin{array}{l}\text { 2. PHMC identify any space and equipment requirements within } \\
\text { the environmental services area. }\end{array}$ & $6 / 10 / 96$ & PHMC \\
\hline 3. Space and equipment available to the PHMC. & $6 / 15 / 96$ & $\begin{array}{l}\text { WT Dixon } \\
\text { SM Cooley }\end{array}$ \\
\hline 4. Provide environmental briefings to the PHMC. & $\begin{array}{l}7 / 01 / 96- \\
8 / 15 / 96\end{array}$ & $\begin{array}{l}\text { WT Dixon } \\
\text { RH Engelmann }\end{array}$ \\
\hline 5. Perform a joint property inventory with the PHMC. & by $8 / 30 / 96$ & $\begin{array}{l}\text { WT Dixon } \\
\text { SM Cooley }\end{array}$ \\
\hline
\end{tabular}

3.5.3.1 Hanford Environmental Management Program. The Hanford Environmental Management Program (HEMP) was established in 1986 to create and implement a structured approach toward achieving sitewide environmental compliance. The purpose of the HEMP is to provide guidance and support across WHC mission areas to ensure facilities/programs achieve compliance with environmental requirements and regulatory agreements. This program also provides RL with a mechanism to coordinate specific environmental activities between multiple contractors. For sitewide reports and permits, WHC coordinates the activities of all Hanford Site contractors as directed by RL. WHC also provides a focal point for regulator access and technical data requests, and sitewide compliance resolution.

\begin{tabular}{|c|c|c|}
\hline Pre-Award Actions & $\begin{array}{l}\text { Schethiled } \\
\text { Date. }\end{array}$ & Actionee(s) \\
\hline $\begin{array}{l}\text { 1. Compile key program documents (e.g., FY } 1996 \text { MYPP, } \\
\text { ADS, RDS), and prepare briefing. }\end{array}$ & 6/01/96 & $\begin{array}{l}\text { RH Engeimann } \\
\text { DA Moore }\end{array}$ \\
\hline \multicolumn{3}{|l|}{ Pest-A ward Actions } \\
\hline 1. Conduct briefing meeting with PHMC. & $\begin{array}{l}\text { 7/01/96- } \\
8 / 01 / 96 \\
\end{array}$ & \begin{tabular}{|l|} 
RH Engelmann \\
DA Moore \\
\end{tabular} \\
\hline 2. Provide HEMP management status to PHMC. & 9/24/96 & $\begin{array}{l}\text { RH Engelmann } \\
\text { DA Moore }\end{array}$ \\
\hline 3. Turnover records to PHMC. & $10 / 01 / 96$ & $\begin{array}{l}\text { RH Engelmann } \\
\text { DA Moore }\end{array}$ \\
\hline
\end{tabular}

3.5.3.1.1 Regulatory Reporting. WHC coordinates the preparation of 11 sitewide regulatory reports for RL that will require continued input from the contractor team. These reports are the Emergency Planning and Community Right-To-Know Act (EPCRA) Section 311 Material Safety Data Sheet List Upgrades (four quarterly reports), EPCRA Section 312 Tier Two Emergency and Hazardous Chemical Inventory, EPCRA 313 Toxic Chemical 
Release Inventory, Annual Polychlorinated Biphenyl (PCB) Document Log, Annual PCB Report, Annual Dangerous Waste Report, Land Disposal Restrictions Status Report (Tri-Party Agreement Milestone M-26-01), and RCRA 3016 Inventory of Federal Agency Hazardous Waste Facilities (on even numbered calendar years).

\begin{tabular}{|c|c|c|}
\hline Poot-Award Actions & $\begin{array}{l}\text { Schetuled } \\
\text { Date }\end{array}$ & (1etionee ( $)$ \\
\hline $\begin{array}{l}\text { 1. Establish PHMC POCs for each of the specified sitewide } \\
\text { regulatory reports. }\end{array}$ & $7 / 01 / 96-8 / 15 / 96$ & JO Skolrud \\
\hline $\begin{array}{l}\text { 2. Identify PHMC certification authority for specified sitewide } \\
\text { regulatory reports, as required. }\end{array}$ & $7 / 01 / 96-8 / 15 / 96$ & Jo Skolrud \\
\hline $\begin{array}{l}\text { 3. Establish and maintain a network of reporting representatives } \\
\text { from each facility, or program, as needed to assure timely } \\
\text { completion of specified reports. }\end{array}$ & $7 / 01 / 96-8 / 15 / 96$ & JO Skolrud \\
\hline $\begin{array}{l}\text { 4. Provide specialized training and database system access } \\
\text { authorization for PHMC database administrators and } \\
\text { reporting representatives to allow access to needed data files } \\
\text { and data transmittal capabilities. }\end{array}$ & $7 / 01 / 96-8 / 15 / 96$ & JO Skolrud \\
\hline 5. Provide status on workscope to PHMC. & 9/24/96 & JO Skolrud \\
\hline 6. Turnover working files to PHMC. & $10 / 01 / 96$ & JO Skolrud \\
\hline $\begin{array}{l}\text { 7. Conduct briefing with PHMC on management and conduct of } \\
\text { Regulatory Reporting. }\end{array}$ & $8 / 15 / 96$ & JO Skolrud \\
\hline
\end{tabular}

3.5.3.1.2 Sitewide RCRA Permitting. The Hanford Facility is a single RCRA facility consisting of 66 treatment, storage, and/or disposal (TSD) units. Nine of these units have been officially clean closed in accordance with the Washington Administrative Code (WAC). WHC has management responsibility for 33 of the remaining 57 units, with other Hanford Site contractors having management responsibilities for the remaining units. The units managed by WHC must be transitioned to the PHMC. The RCRA permitting documentation involved in this transition includes the: (1) Hanford Facility RCRA Permit, (2) Hanford Facility Part A Permit Application (which includes one Form 1 and a Form 3 for each TSD unit), (3) Hanford Facility Dangerous Waste Part B Permit Application, and (4) Closure Plans.

Transfer of WHC responsibility as co-operator for RCRA permitting documentation requires 90 days advance notification to Ecology, and submittal of the associated, recertified permitting documentation, unless this requirement is waived by Ecology. Assuming an October 1, 1996, takeover date, processing of all RCRA permitting documentation by July 1, 1996, is not feasible based on a June 1, 1996, contract award date. If circumstances preclude the PHMC from assuming co-operator responsibilities for RCRA permitting documentation by October 1, 1996, WHC will exercise Section E of the Agreement on Regulatory Interactions between RL and WHC dated August 14, 1995. This memorandum of agreement states that "...in the event of a change in contract scope or termination or expiration of the Contract, RL will require the successor contractor to accept transfer of all permits for which WHC is a permittee or signatory, or in the alternative, RL will accept such responsibility, subject to the provisions of Contract, and WHC shall be relieved of liability and responsibility for permit violations where the operative facts occur after the 
effective date of the change in scope, termination, or expiration of this contract." The WHC will pursue a documented agreement with Ecology that Ecology will: (1) waive the 90-day requirement for submittal of recertified permitting documentation, and (2) agree that WHC will be removed from the RCRA permitting documentation as co-operator as of October 1 , 1996, leaving RL as the owner/operator if the PHMC is not yet ready to assume co-operator responsibilities.

\begin{tabular}{|c|c|c|}
\hline Pre-1werd Aetions & $\begin{array}{l}\text { Scheatiled } \\
\text { Date }\end{array}$ & Actionse() \\
\hline $\begin{array}{l}\text { 1. Complete written agreement among RL, WHC, and Ecology } \\
\text { waiving the } 90 \text {-day RCRA permitting documentation } \\
\text { submittal requirements and clarifying assumption of co- } \\
\text { operator responsibilities. }\end{array}$ & $5 / 03 / 96$ & SM Price \\
\hline Post-Award Action & $\sqrt{8}$ & (4) \\
\hline 1. Provide RCRA permitting briefing(s) to the PHMC. & $\begin{array}{l}6 / 15 / 96- \\
8 / 30 / 96 \\
\end{array}$ & SM Price \\
\hline 2. Turnover RCRA permitting files to PHMC. & $10 / 01 / 96$ & SM Price \\
\hline
\end{tabular}

Further details on the plan for accomplishing transition of RCRA permitting documentation is provided below:

3.5.3.1.3 Hanford Facility RCRA Permit. The Hanford Facility RCRA Permit (RCRA Permit) is currently in two portions. The Dangerous Waste Portion (DW Portion), was issued by Ecology to RL (Owner/Operator), WHC (Co-operator), BHI (Co-operator), and PNNL (Co-operator), effective September 28, 1994, through September 27, 2004. The Hazardous and Solid Waste Amendments Portion, was issued by the U.S. Environmental Protection Agency (EPA) to RL (Owner/Operator) only effective September 28, 1994, through September 27, 2004. Due to the number of Hanford Site TSD units, a stepwise permitting process is being used. Any TSD units not yet included in the RCRA Permit (DW Portion) will be incorporated in a future, annual permit modification. Until incorporated, TSD units will continue to operate under interim status.

Permit Condition I.A.2. (DW Portion) states that WHC is identified as a Permittee for activities subject to the Conditions of this Permit where its agents, employees, or subcontractors have operational and/or management responsibilities and control. The Permittee for the DW Portion will need to be transferred from WHC to the PHMC.

Permit Condition I.E.14. (DW Portion) states that the Permit may be transferred to a new co-operator in accordance with the provisions of WAC 173-303-830(2). WAC 173-303-830(2)(b) allows that changes in the ounership or operational control of a facility may be made as a Class 1 modification (requires notification) with prior written approval of Ecology. The new owner or operator must submit a revised permit application no later than 90 days prior to the scheduled change.

Because Permit Condition II.H.3. (DW Portion) states that the permittees are exempt from the requirements of WAC 173-303-620 (Financial Requirements) no action to transfer or demonstrate compliance with financial requirements is required. 


\begin{tabular}{|c|c|c|}
\hline Pod-Anerd Actions & $\begin{array}{l}\text { Gelheduled } \\
\text { Dete }\end{array}$ & Tetionee( \\
\hline $\begin{array}{l}\text { 1. Submit Class } 1 \text { modification notifying Ecology of a change in } \\
\text { the permittee from WHC to the PHMC. (A determination } \\
\text { must be made with RL, and agreed to by Ecology, as to the } \\
\text { permittee[s] [Co-operator\{s\}] to transfer RCRA Permit } \\
\text { responsibilities to the PHMC by October } 1,1996 \text {. If } \\
\text { Ecology does not approve the submitted Class } 1 \text { modification, } \\
\text { RL would accept sole responsibility per MOA.) }\end{array}$ & $7 / 01 / 96$ & SM Price \\
\hline $\begin{array}{l}\text { 2. Support Ecology's written approval of the Class } 1 \\
\text { modification regarding a change in the permittee from WHC } \\
\text { to the PHMC. (The only formal notification by Ecology to } \\
\text { release WHC from RCRA Permit responsibilities is the } \\
\text { approval of the Class } 1 \text { modification requesting transfer.) }\end{array}$ & $\begin{array}{l}6 / 01 / 06- \\
10 / 01 / 96\end{array}$ & $\begin{array}{l}\text { SM Price } \\
\text { PHMC }\end{array}$ \\
\hline $\begin{array}{l}\text { 3. Support the PHMC's certification and resubmittal to Ecology } \\
\text { of the } 616 \text { Nonradioactive Dangerous Waste Storage Facility } \\
\text { (NRDWSF) permit application. (The } 616 \text { NRDWSF is the } \\
\text { only final status operating TSD unit in the RCRA Permit } \\
\text { with permit applications certified by WHC. Both Part A and } \\
\text { Part B permit applications must be resubmitted [certified] by } \\
\text { the PHMC by July 1, 1996, unless the agreement identified } \\
\text { in Section 3.5.3.1.2 is obtained.) }\end{array}$ & $\begin{array}{l}7 / 01 / 96- \\
10 / 01 / 96\end{array}$ & $\begin{array}{l}\text { SM Price } \\
\text { PHMC }\end{array}$ \\
\hline $\begin{array}{l}\text { 4. Support the PHMC's certification and resubmittal to Ecology } \\
\text { of the Part A, Form } 3 \text { for the 105-DR Large Sodium Fire } \\
\text { Facility. (The Part A, Form 3, for the 105-DR Large } \\
\text { Sodium Fire Facility (Closure Plan), the only remaining TSD } \\
\text { unit undergoing closure in the RCRA Permit, must be } \\
\text { resubmitted (certified) by the PHMC by July 1, 1996, unless } \\
\text { the agreement identified in Section 3.5.3.1.2 is obtained.) }\end{array}$ & $\begin{array}{c}7 / 01 / 96- \\
10 / 01 / 96\end{array}$ & $\begin{array}{l}\text { SM Price } \\
\text { PHMC }\end{array}$ \\
\hline
\end{tabular}

An Ecology-initiated Class 3 modification of the RCRA Permit also is scheduled for 1996. Currently the schedule calls for: (1) all information from WHC to be certified and formally delivered to Ecology by July 1, 1996, (2) the 45-day Public Comment Period to commence mid-July 1996, (3) a permit decision to be issued in late September 1996, and, (4) the permit to be in effect in late-October 1996. Three WHC-managed TSD units and a training plan for 616 NRDWSF are expected to be added to the RCRA Permit by the modification.

\begin{tabular}{|c|c|c|}
\hline Poet-Award Actions & $\begin{array}{c}\text { Scheduled } \\
\text { Date. }\end{array}$ & Actionee(s) \\
\hline $\begin{array}{l}\text { 1. Support the PHMC's certification and resubmittal to Ecology } \\
\text { of the PUREX Storage Tunnels Part A, Form } 3 \text { and Part B } \\
\text { permit applications; the Part A, Form } 3 \text { permit applications } \\
\text { for the 3718-F Alkali Metal Treatment unit and the } 4843 \\
\text { Alkali Metal Storage unit (units undergoing closure); and the } \\
\text { training plan for the } 616 \text { NRDWSF. (All permitting } \\
\text { documentation must be resubmitted [certified] by the PHMC } \\
\text { by July 1, 1996, unless the agreement identified in Section } \\
\text { 3.5.3.1.2 is obtained.) }\end{array}$ & $\begin{array}{l}7 / 01 / 96 \\
10 / 01 / 96\end{array}$ & $\begin{array}{l}\text { SM Price } \\
\text { PHMC }\end{array}$ \\
\hline
\end{tabular}


The PHMC will likely want to submit formal comments to Ecology during the Public Comment Period, and evaluate any need for appeal during the 30 days following issuance of the modification decision.

\begin{tabular}{|l|l|l|}
\hline & Scheduled & Date \\
\hline $\begin{array}{l}\text { 1. Support review by the PHMC of a RCRA Permit required } \\
\text { report describing the methods and procedures to be used by } \\
\text { the permittees to achieve compliance with the sitewide } \\
\text { underground dangerous waste piping mapping and marking } \\
\text { permit conditions (Condition II.U., DW Portion). (This } \\
\text { report is due to Ecology by September 27, 1996. The report } \\
\text { describes the products Ecology can expect each } \\
\begin{array}{l}\text { September 27, through 2004. The method includes a central } \\
\text { mapping/information database for processing and presenting } \\
\text { these products.) }\end{array}\end{array}$ & $\begin{array}{l}7 / 01 / 96- \\
\text { SM Price } \\
\text { PHMC }\end{array}$ \\
\hline $\begin{array}{l}\text { Transfer responsibility for taking the lead in coordinating } \\
\text { collective submittals and information for the four permittees } \\
\text { in accordance with the sitewide conditions of the RCRA } \\
\text { permit. }\end{array}$ & $10 / 01 / 96$ & SM Price \\
\hline
\end{tabular}

A Research, Development, and Demonstration (RD\&D) Permit for the Waste Water Pilot Plant (WWPP) was issued by the EPA, separate from the Hanford Facility RCRA Permit. This permit was issued to RL (owner/operator) and WHC (co-operator) and also will need to be transferred to the PHMC.

\begin{tabular}{|c|c|c|}
\hline Post-A ward Actions & $\begin{array}{l}\text { Scheduled } \\
\text { Date }\end{array}$ & Actionets) \\
\hline $\begin{array}{l}\text { 1. Support the PHMC's certification and resubmittal to Ecology } \\
\text { of the RDD\&D permit application for the WWPP. (This } \\
\text { RD\&D permit application must be resubmitted [certified] by } \\
\text { the PHMC by July } 1,1996 \text {, unless the agreement identified } \\
\text { in Section } 3.5 .3 .1 .2 \text { is obtained. If use of this RD\&D permit } \\
\text { is opted for by the PHMC, EPA may need to be a participant } \\
\text { in the agreement.) }\end{array}$ & $\begin{array}{l}7 / 01 / 96 \\
10 / 01 / 96\end{array}$ & $\begin{array}{l}\text { SM Price } \\
\text { PHMC }\end{array}$ \\
\hline
\end{tabular}

\subsection{Hanford Facility Part A Permit Application. There is one Form 1 and} 33 Hanford Facility Dangerous Waste Part A Permit Application Form 3s that must be addressed. Washington Administrative Code 173-303-805(7)(a)(iv) requires these Part A forms to be resubmitted (certified) by the PHMC to Ecology no later than 90 days prior to the scheduled change in management control. Assuming the change becomes effective on October 1, 1996, the Part A forms must be resubmitted no later than July 1, 1996, unless the agreement identified in Section 3.5.3.1.2 is obtained.

\begin{tabular}{|c|c|c|}
\hline Pre-Award Actions & $\begin{array}{l}\text { Scheduled } \\
\text { Date }\end{array}$ & Altionee $(s)$ \\
\hline $\begin{array}{l}\text { 1. Perform a WHC review of the Part A, Form } 1 \text { and the } \\
\text { Part A, Form } 3 \text { permit applications (managed by WHC) } \\
\text { against their respective TSD unit to determine trueness, } \\
\text { accuracy, and completeness. }\end{array}$ & $6 / 01 / 96$ & SM Price \\
\hline
\end{tabular}




\begin{tabular}{|l|l|l|l|}
\hline & selheduled & \\
\hline 1. Support the PHMC's certification and resubmittal to Ecology & $7 / 01 / 96-$ & SM Price \\
of a Part A, Form 1 and 33 Part A, Form 3 permit & $10 / 01 / 96$ & PHMC \\
applications. The Part A, Form 1 and Form 3s must be & \\
resubmitted [certified] by the PHMC by July 1, 1996, unless \\
the agreement identified in Section 3.5.1.2 is obtained.)
\end{tabular}

3.5.3.1.5 Hanford Facility Dangerous Waste Part B Permit Application. Of the 33 units for which WHC retains responsibility, 1 unit has been granted final status, 10 units are slated to receive final status, and the remaining units will be closed as discussed above.

WAC 173-303-830(2)(b) requires that the PHMC submit revised Part B permit applications (certified) for the 10 units seeking final status to Ecology no later than 90 days prior to the scheduled change in management control. Assuming the change becomes effective on October 1, 1996, the Part B permit applications must be resubmitted no later than July 1, 1996. In light of the Hanford Facility permitting strategy identified in Section 3.5.3.1.2 and the enormous effort resubmittal of the Part B permit applications represents, a written agreement must be achieved between all parties concerned (e.g., WHC, RL, the PHMC, and Ecology). This agreement must address the following:

- Relieve the PHMC of the need to submit revised Part B permit applications no later than 90 days prior to the scheduled date for assuming management responsibility.

- Identify permit application submittal dates supportive of the permit modification schedule contained in the Hanford Facility RCRA Permit.

- Remove WHC from the RCRA Part B permit applications as co-operator as of October 1, 1996.

- Relieve the PHMC's liability associated with the trueness, accuracy, or completeness of the permit applications until such time as the permit applications are certified and resubmitted.

\begin{tabular}{|c|c|c|}
\hline Post-Award lctions & Sobeduled & Actionses $(s)$ \\
\hline $\begin{array}{l}\text { 1. Complete a written agreement on Part B permit application } \\
\text { processing among RL, WHC, the PHMC, and Ecology. }\end{array}$ & $7 / 01 / 96$ & $\begin{array}{l}\text { SM Price } \\
\text { PHMC }\end{array}$ \\
\hline
\end{tabular}

Operational control of the sole final status TSD unit (616 NRDWSF) that WHC has management responsibility for, shall be transferred in accordance with Condition I.E.14. of the Hanford Facility RCRA Permit (see Section 3.5.3.1.3.).

3.5.3.1.6 Closure Plans. Currently, there are 14 interim status TSD units that are not operating and will be closed under RCRA. Eight of these TSD units for which WHC has management responsibility have already been certified clean closed and revised Part A, Form 3 permit applications submitted to Ecology. Closure plans have been or will be developed 
for the remaining 6 units and submitted to Ecology for approval and for eventual inclusion in the Hanford Facility RCRA Permit. The Part A, Form 3 permit applications for the remaining TSD units undergoing closure assigned to the PHMC will have to be recertified (see Section 3.5.3.1.4).

Additionally, integration between Hanford Site contractors with TSD management responsibilities will be necessary to maintain a consistent closure strategy among all the Hanford Site TSD unit closures (e.g., issues such as RCRA/CERCLA integration, as well as the use of health-based standards, sampling integration, and remediation integration).

\begin{tabular}{|l|l|l|}
\hline & Sclieduled & Date \\
\hline 1. Transfer the management responsibilities for TSD unit & $7 / 01 / 96-$ & SM Price \\
closures to the PHMC. (TSD units include the 105-DR & $10 / 01 / 96$ & \\
Large Sodium Fire Facility, 303-K Radioactive Mixed Waste & \\
Storage Facility, 300 Area Waste Acid Treatment System, \\
3718-F Alkali Metal Treatment and Storage Facility, 4843 \\
Alkali Metal Storage Facility, and Single-Shell Tanks. Three \\
of these TSD units, the 105-DR Large Sodium Fire Facility, \\
the 3718-F Alkali Metal Treatment and Storage Facility, and \\
the 4843 Alkali Metal Storage Facility, should be certified \\
clean closed by the end of CY 1996.)
\end{tabular}

3.5.3.1.7 Sitewide Air and Water Permitting. There are two categories of Clean Air Act (CAA) and Clean Water Act of 1977 (CWA) related permits onsite; facility specific and sitewide. All CAA and CWA permits are owned by RL. That is, neither WHC nor any other contractor is a co-signer to a permit application and the permits have been issued to RL. Therefore, there is no requirement to sign over any CAA or CWA permit, or permit application, to the PHMC. PHMC will need to verify that CWA and CAA permit conditions are being met.

\begin{tabular}{|l|c|l|}
\hline \multicolumn{1}{|c|}{ Post-Award Actions } & $\begin{array}{c}\text { Scheduled } \\
\text { Date }\end{array}$ & Actionee (s) \\
\hline $\begin{array}{l}\text { 1. Brief PHMC on scope of Air and Water Permitting } \\
\text { workscope. }\end{array}$ & $\begin{array}{c}7 / 01 / 96- \\
8 / 15 / 96\end{array}$ & EM Greager \\
\hline 2. Provide status of workscope to PHMC. & $9 / 24 / 96$ & EM Greager \\
\hline 3. Turnover files to PHMC. & $10 / 01 / 96$ & EM Greager \\
\hline
\end{tabular}

\section{CAA Permits}

Facility specific CAA permits are issued by the Washington State Departments of Health (WDOH), Ecology, and/or the EPA to RL under a regulatory order of approval with activity-specific conditions and/or operating limitations. The conditions/limitations are incorporated into the "Hanford Site Air Operating Permit (AOP) Application," (DOE/RL-95-07) initially submitted from RL to Ecology on May 26, 1995. Each facility owner is responsible for meeting the conditions of its permit(s). 
The current sitewide CAA license, FF-01, is with the DOH. Once the Hanford Site AOP Application (DOE/RL-95-07) is finalized as a site AOP, expected in November 1997, the FF-01 license will be canceled. Until issuance of the permit, frequent/periodic updates to the application ensures its currentness and allows for the continued operation onsite of all points that emit regulated constituents to atmosphere.

\begin{tabular}{|c|c|c|}
\hline Post-Award Actions & $\begin{array}{c}\text { Scheduled } \\
\text { Date } \\
\end{array}$ & Actionee(s) \\
\hline $\begin{array}{l}\text { 1. Brief PHMC on scope of AOP activities, transmit copy of } \\
\text { application and description of WHC workscope. }\end{array}$ & $\begin{array}{l}7 / 01 / 96 \\
8 / 15 / 96\end{array}$ & LP Diediker \\
\hline $\begin{array}{l}\text { 2. Brief/status PHMC on FF-01 permit, transmit copy of } \\
\text { permit. }\end{array}$ & $\begin{array}{l}7 / 01 / 96- \\
8 / 15 / 96 \\
\end{array}$ & LP Diediker \\
\hline 3. AOP files turnover to PHMC. & $10 / 01 / 96$ & LP Diediker \\
\hline
\end{tabular}

\section{CWA Permits}

All CWA permits are owned by RL. There are two types of CWA related permits: National Pollutant Discharge Elimination System (NPDES) and WAC 173-216. NPDES permits allow for discharges to the Columbia River. WAC 173-216 permits allow for discharges to the soil column.

NPDES Permit WA-000374-3 was issued to RL on December 7, 1981, and is a sitewide permit. The permit was originally issued to cover the discharge from eight outfalls on the Hanford Site. Because of shutdown and deactivation activities there are now only four active outfalls covered by the permit. Those outfalls are 003 and 004 in the $100 \mathrm{~K}$ Area, N-Springs in the $100 \mathrm{~N}$ Area, and 013 in the 300 Area. Outfall 013 services a PNNL facility. BHI monitors $\mathrm{N}$-Springs. The 003 and 004 Outfalls service facilities that will transfer to the PHMC.

NPDES Permit WA-002591-7 was issued to RL on October 31, 1994, for discharges from the 300 Area Treated Effluent Disposal Facility.

NPDES Permit WA-R-00-000F was issued to RL on August 27, 1992. It permits the discharge of stormwater runoff from industrial points on the Hanford Site to the Columbia River.

\begin{tabular}{|c|c|c|}
\hline Post-Award Actions & $\begin{array}{l}\text { Scheduled } \\
\text { Date }\end{array}$ & Actionce(s) \\
\hline $\begin{array}{l}\text { 1. Brief PHMC on the NPDES permits, transmit copies of } \\
\text { permits and other key information. }\end{array}$ & $\begin{array}{l}7 / 01 / 96 \\
8 / 15 / 96\end{array}$ & LP Diediker \\
\hline 2. Turnover of files to PHMC. & $10 / 01 / 96$ & LP Diediker \\
\hline
\end{tabular}

WAC 173-216 permits have been issued for the 200 Area Effluent Treatment Facility (ETF), the 200 Area Treated Effluent Disposal Facility (TEDF), and those discharges identified in Consent Order DE 91NM-177 (216 Consent Order). The construction of septic systems are permitted with the Department of Health. Management of potable water is conducted in accordance with Department of Health regulations. 


\begin{tabular}{|l|l|l|}
\hline & Soloduled & \\
\hline $\begin{array}{l}\text { 1. Brief PHMC on the WAC 173-216 permits, transmit copies } \\
\text { of permits and other key information. }\end{array}$ & $\begin{array}{c}7 / 01 / 96- \\
8 / 15 / 96\end{array}$ & LP Diediker \\
\hline 2. Turnover of files to PHMC. & $10 / 01 / 96$ & LP Diediker \\
\hline
\end{tabular}

\subsection{Regulator Inspection Support.}

Regulatory Inspection POC--WHC currently acts as the initial point of contact for all environmental regulatory inspections of the Hanford Site. It is assumed that the PHMC will be performing this role as well. Pertinent information associated with these inspections will be maintained by the PHMC in a central inspection file.

\begin{tabular}{|c|c|c|}
\hline Pre-Awprd Actions & $\begin{array}{l}\text { Scheduled } \\
\text { Date }\end{array}$ & Actionse(s) \\
\hline $\begin{array}{l}\text { 1. Complete the new standardized access requirements system } \\
\text { for regulatory agency inspectors. }\end{array}$ & $6 / 01 / 96$ & SA Szendre \\
\hline $\begin{array}{l}\text { 2. Complete MOU with PNNL for regulatory agency inspection } \\
\text { point of contact responsibilities. }\end{array}$ & $6 / 01 / 96$ & SA Szendre \\
\hline $\begin{array}{l}\text { 3. Complete MOU with the ERC Team for regulatory agency } \\
\text { inspection point of contact responsibilities. }\end{array}$ & $6 / 01 / 96$ & SA Szendre \\
\hline \multicolumn{3}{|l|}{ Post-Award Actions } \\
\hline $\begin{array}{l}\text { 1. Brief PHMC on duties, responsibilities, and processes } \\
\text { involved with providing POC services for Hanford Site } \\
\text { regulatory agency inspections. }\end{array}$ & $\begin{array}{l}7 / 01 / 96- \\
8 / 15 / 96\end{array}$ & $\begin{array}{l}\text { SM Price } \\
\text { SA Szendre }\end{array}$ \\
\hline $\begin{array}{l}\text { 2. Brief PHMC on duties, responsibilities, and processes } \\
\text { involved with maintaining central inspection files. }\end{array}$ & $\begin{array}{l}7 / 01 / 96- \\
8 / 15 / 96\end{array}$ & SA Szendre \\
\hline 3. Status PHMC on issues. & $9 / 24 / 96$ & SA Szendre \\
\hline $\begin{array}{l}\text { 4. Turnover all inspection records and related files to the } \\
\text { PHMC. }\end{array}$ & $10 / 01 / 96$ & SA Szendre \\
\hline
\end{tabular}

RCRA Facility Permit Inspection--Condition II.O of the Hanford Site RCRA Permit includes the requirement for annual inspections of the 100, 200 East, 200 West, 300, 400, and 1100 Areas, along with inspections of the banks of the Columbia River twice per year.

\begin{tabular}{|l|c|c|}
\hline & Post-Award Actions & Scheduled \\
Date & & \\
\hline 1. Conduct 1996 inspections per established schedule. & $9 / 30 / 96$ & DB Beagles \\
\hline $\begin{array}{l}\text { 2. Brief PHMC on the duties, responsibilities, and } \\
\text { processes involved with the RCRA permit general } \\
\text { inspections. }\end{array}$ & $7 / 01 / 96-8 / 15 / 96$ & DB Beagles \\
\hline 3. Turnover records to PHMC. & $10 / 01 / 96$ & DB Beagles \\
\hline
\end{tabular}


Notices of Violation (NOV) Database and Reporting-A database of NOV and enforcement actions is maintained to meet RL reporting requirements and certain permitting requirements associated with permit expansions under interim status. This database includes sitewide information and will be the responsibility of the PHMC.

\begin{tabular}{|l|c|l|}
\hline \multicolumn{1}{|c|}{ Poot Arrard Actions } & Selieduled & Date \\
\hline $\begin{array}{l}\text { 1. Complete as many NOV corrective actions as possible and } \\
\text { close as many of the open items on the NOV database as } \\
\text { possible. }\end{array}$ & $9 / 30 / 96$ & $\begin{array}{l}\text { LT St. Georges } \\
\text { affected ECOB }\end{array}$ \\
\hline $\begin{array}{l}\text { 2. Brief the PHMC on the duties, responsibilities, and processes } \\
\text { associated with maintaining and reporting the NOV database. }\end{array}$ & $\begin{array}{c}7 / 01 / 96- \\
8 / 15 / 96\end{array}$ & LT St. Georges \\
\hline 3. Turnover records to PHMC. & $10 / 01 / 96$ & LT St. Georges \\
\hline
\end{tabular}

3.5.3.1.9 National Envinonmental Policy Act of 1969 (NEPA) Compliance. WHC screens proposed activities for NEPA compliance requirements, and prepares documents (environmental assessments [EA], categorical exclusions, Environmental Impact Statement [EIS] technical support documents) used by RL in the NEPA process.

Copies of all Hanford EISs and EAs and related records are maintained centrally. Copies of categorical exclusions for M\&O Contractor managed activities are also held centrally.

HEMP also funds Hanford Site NEPA training support, and input to sitewide planning efforts and procedures.

\begin{tabular}{|l|l|l|}
\hline & $\begin{array}{c}\text { Scheduled } \\
\text { Date }\end{array}$ & Actionee(s) \\
\hline 1. Brief PHMC on NEPA management and workscope. & $\begin{array}{c}7 / 01 / 96- \\
8 / 15 / 96\end{array}$ & RH Engelmann \\
\hline 2. Provide status of in-preparation NEPA documents to PHMC. & $9 / 26 / 96$ & RH Engelmann \\
\hline 3. Turnover files to PHMC. & $10 / 01 / 96$ & RH Engelmann \\
\hline
\end{tabular}

3.5.3.1.10 Crosscutting Noncompliances. In July 1995, RL and its contractors identified all known potential non-permitted treatment, storage, and disposal activities at Hanford, and all potential environmental noncompliant conditions. There were identified 160 individual potential noncompliant conditions on the Hanford Site. Of these, 66 are facility-specific and the remaining 94 involve, or crosscut, more than one RL Program or facility. The pre-existing conditions survey will provide additional details.

\begin{tabular}{|c|c|c|}
\hline Post-A pard Aetions & $\begin{array}{l}\text { Schediled } \\
\text { Date }\end{array}$ & petionee(s) \\
\hline $\begin{array}{l}\text { 1. Efforts will be made to formally resolve all known } \\
\text { noncompliances prior to contract takeover. }\end{array}$ & $9 / 30 / 96$ & $\begin{array}{l}\text { RH Engelmann } \\
\text { WR Brown }\end{array}$ \\
\hline
\end{tabular}




\subsubsection{Reporting.}

3.5.3.2.1 Effluent Reporting. The PHMC will be required by Federal, Washington State and RL requirements to monitor and report on Hanford Site effluents. Reports required:

- Monthly NPDES reporting to Ecology, Washington State Department of Health, and EPA

- Discharge Information System (EIS/ODIS) report to INEL

- Environmental releases report to RL

- Radionuclide Air Emissions Report to EPA and Washington State Department of Health

- Biannual/PTRAEU Report to Washington State Department of Health

- Monthly Excess Emission Report on Baghouse Bypass notifications to Ecology

- Annual Air Emissions Inventory Update to Ecology (to include the report pursuant to WAC 173-400-105, "Annual Inventory of Non-Radiological Airborne Emissions")

- Quarterly Reports on Status of the Federal Facilities Compliance Agreement (FFCA) to Washington State Department of Health and EPA

- WHC Operational Environmental Monitoring Annual Report to Washington State Department of Health

\begin{tabular}{|c|c|c|}
\hline Post A wrard Actions & $\begin{array}{l}\text { scheduled } \\
\text { Date }\end{array}$ & Achones(s) \\
\hline 1. Brief PHMC on activities scope, transmit key documents. & $\begin{array}{l}7 / 01 / 96- \\
8 / 15 / 96\end{array}$ & LP Diediker \\
\hline 2. Provide workscope status to PHMC. & $9 / 24 / 96$ & LP Diediker \\
\hline 3. Turnover of records, etc. & $10 / 01 / 96$ & LP Diediker \\
\hline
\end{tabular}

\subsection{Effluent Monitoring Plan and Facility Effluent Monitoring Plan} Reporting. The PHMC will be required to provide updating on existing EMP and Facility Effluent Monitoring Plans (FEMPs) as required by DOE Orders (will be per 10 CFR 834). These are on a three-year cycle with the next due date in November 1997.

\begin{tabular}{|c|c|c|}
\hline Post-Award Actions & $\begin{array}{l}\text { Scheduled } \\
\text { Date }\end{array}$ & Actionee(s) \\
\hline $\begin{array}{l}\text { 1. Brief PHMC on activities scope, and provide access to key } \\
\text { documents. }\end{array}$ & $8 / 15 / 96$ & LP Diediker \\
\hline 2. Turnover of records, etc. & $10 / 01 / 96$ & LP Diediker \\
\hline
\end{tabular}


3.5.3.3 Environmental Requirements Management. WHC provides regulatory analysis/interpretation/consultation to $R L$ and projects, as requested, to resolve environmental issues. This support covers the applicability of several environmental areas (e.g., RCRA, CAA, CWA) to help RL and projects adequately plan for future missions as they become identified. Additionally, a proactive approach is pursued by WHC in providing comments to positively influence proposed Federal, state, and local rulemakings in the interest of RL and the Hanford Site.

\begin{tabular}{|c|c|c|}
\hline Poot-A werd Actions & $\begin{array}{l}\text { Sclueduled } \\
\text { Dete }\end{array}$ & Letioned \\
\hline $\begin{array}{l}\text { 1. Brief PHMC on workscope, and provide access to key } \\
\text { documents. }\end{array}$ & $\begin{array}{l}7 / 01 / 96- \\
8 / 15 / 96\end{array}$ & $\begin{array}{l}\text { RH Engelmann } \\
\text { WE Toebe } \\
\text { DJ Carrell } \\
\text { LP Diediker } \\
\text { NE Darling } \\
\end{array}$ \\
\hline 2. Turnover of files and documents. & 10/01/96 & $\begin{array}{l}\text { RH Engelmann } \\
\text { WE Toebe } \\
\text { DJ Carrell } \\
\text { LP Diediker } \\
\text { NE Darling }\end{array}$ \\
\hline
\end{tabular}

\subsubsection{Non-Tri-Party Agreement Compliance Agreement.}

3.5.3.4.1 NESHAPS Federal Facility Compliance Agreement. A Federal Facility Compliance Agreement (FFCA) was signed on February 7, 1994, by RL and EPA, Region 10. Milestones in the FFCA bring all stack sampling systems on the Hanford Site into compliance with requirements in 40 CFR 61 Subpart $H$. WHC is bringing its stacks into compliance. WHC has met or exceeded all of it's milestones. Because the FFCA agreement is between RL and EPA, the new contractor/s will be subject to the FFCA and will be required to meet remaining milestones.

\begin{tabular}{|c|c|c|}
\hline Post-Award Actions & $\begin{array}{l}\text { Scheduled } \\
\text { Date }\end{array}$ & Actionee(s) \\
\hline 1. Brief PHMC on NESHAPS FFCA, transmit key documents. & 8/01/96 & $\begin{array}{l}\text { LP Diediker } \\
\text { WE Davis }\end{array}$ \\
\hline $\begin{array}{l}\text { 2. Conduct a comprehensive tour of all facilities baving a major } \\
\text { NESHAP stack. }\end{array}$ & 9/01/96 & $\begin{array}{l}\text { LP Diediker } \\
\text { WE Davis }\end{array}$ \\
\hline 3. Provide current status of compliance activities to PHMC. & $9 / 24 / 96$ & $\begin{array}{l}\text { LP Diediker } \\
\text { WE Davis }\end{array}$ \\
\hline 4. Turnover of records. & $10 / 01 / 96$ & $\begin{array}{l}\text { LP Diediker } \\
\text { WE Davis }\end{array}$ \\
\hline
\end{tabular}

3.5.3.4.2 216 Consent Order. Ecology and DOE negotiated the 216 Consent Order, formally known as Consent Order No. DE 91NM-177 in 1991. The purpose of the consent order is to control the discharge of liquid effiuents on the Hanford Reservation in accordance with applicable state water quality criteria and establish a schedule for permits to be secured for effluent streams discharged at the Hanford Site. Milestones for three of the following types of liquid effluent streams were established; Phase I streams, Phase II streams 
and miscellaneous streams. Most of the milestones for the Phase I and Phase II liquid effluent streams are complete. Milestones for the miscellaneous streams included submitting an inventory of disposal sites, submitting a plan to identify and evaluate all miscellaneous streams and submitting a plan and schedule for disposition and regulatory compliance for all remaining miscellaneous streams. The plans and schedule were submitted as required and is currently being implemented onsite. Specific transition actions pertaining to this Order are contained in Section 4.2.2. To be further addressed in actions contained in Section 4.2.1, Solid Waste.

3.5.3.4.3 Heart of America Settlement. As per agreement in the Heart of America Northwest. et al.. vs Westinghouse, et al, WHC agreed to notify Heart of America in instances where "For releases where immediate written notification is provided to regulatory agencies, Defendants, or one Defendant, as appropriate, shall provide a copy of any written notification provided under the statutes and regulation cited above to Plaintiffs by facsimile within one business day following such notification." Procedures for notifying Heart of America, in such instances as noted above, have been incorporated into the WHC Environmental Compliance Manual (ECM) 7-5. Heart of America and the offsite environmental regulatory agency are notified by WHC External Communications of a release within twenty-four hours of the event. See WHC-CM-7-5, Environmental Compliance, Page 5 of 53. To be further addressed in actions contained in Section 4.1, TWRS.

3.5.3.4.4 RL/EPA Region 10 Compliance Agreement for Trench 94 (PCBs). Region 10 of EPA and RL, are parties to this agreement which was entered into under the authority of Executive Order (E.O.) 12088. This agreement was being entered into to bring burial ground 218-E-12B, Trench 94, into compliance with the Toxic Substance Control Act (TSCA). Trench 94 is that area set aside for disposal of submarine reactor compartments in the 200 East Area 218-E-12B Burial Ground.

Full compliance with Section 15 of TSCA would be attained by EPA's granting of approval pursuant to TSCA, to the RL to operate a chemical waste landfill at Trench 94, for the sole purpose of landfilling submarine reactor compartments containing PCB-contaminated components (with low level of radioactivity). To be further addressed in actions contained in Section 4.2.1, Solid Waste.

\subsection{DOE/NNPP/EPA Federal Facility Compliance Agreement on Storage of} PCBs. Agreement was tentatively reached between DOE, NNPP (U.S. Navy Naval Nuclear Propulsion Program), and EPA on storage of PCB articles and containers of waste beyond the one year TSCA limit. This agreement has been signed by DOE and NNPP and is currently in EPA possession for signature.

The agreement requires Annual Status Report detailing specific information including covered PCB wastes, justification for continued storage beyond one year, and a summary of the compliance status and steps taken to mitigate storage deficiencies.

The agreement specifies $\mathrm{M} \& \mathrm{O}$ contractors as "co-operators" of DOE sites. Transfer to PHMC is necessary. To be further addressed in actions contained in Section 4.2.1, Solid Waste. 


\subsubsection{Outsources.}

3.5.3.6.1 SAIC/CH2M Hill Subcontract. Significant subcontract' support for environmental compliance issues is provided to WHC and ICF KH through two multi-million dollar, multi-year, task order Purchase Orders managed by Environmental Services:

- MTB-SVV-315924 (now MTB-SVV-452401) issued to Science Applications International Corporation (SAIC) with Hart-Crowser and Foster Wheeler as subcontractors.

- MRS-SVV-296951 (now TRS-SVV-416662) issued to CH2M Hill with IT and Environmental Issues as subcontractors.

\begin{tabular}{|c|c|c|}
\hline Post-Award Actions & $\begin{array}{l}\text { Scheduled } \\
\text { Date }\end{array}$ & Sutionge $(0)$ \\
\hline $\begin{array}{l}\text { 1. Brief PHMC. Summarize all active task orders. Identify } \\
\text { those that will not be complete at time of transition. }\end{array}$ & $\begin{array}{l}7 / 01 / 96- \\
8 / 15 / 96\end{array}$ & RG Upchurch \\
\hline $\begin{array}{l}\text { 2. Provide status to PHMC of the Environmental Services' } \\
\text { subcontracts as to dollars and DPLH remaining. }\end{array}$ & 9/24/96 & RG Upchurch \\
\hline 3. Turn over task order management files. & $10 / 01 / 96$ & $\begin{array}{l}\text { RH Engelmann } \\
\text { RG Upchurch }\end{array}$ \\
\hline
\end{tabular}

3.5.3.6.2 Work for Others. Currently, WHC is conducting limited permitting and closure plan work for BHI and PNNL.

\begin{tabular}{|l|c|l|}
\hline 1 Post-Award Actions & $\begin{array}{c}\text { Scheduled } \\
\text { Date }\end{array}$ & \\
\hline 1. Brief PHMC on scope of work for others. & $\begin{array}{c}7 / 01 / 96- \\
8 / 15 / 96\end{array}$ & SM Price \\
\hline $\begin{array}{l}\text { 2. Negotiate transfer of work from WHC with BHI, PNNL and } \\
\text { PHMC. }\end{array}$ & $9 / 01 / 96$ & SM Price \\
\hline
\end{tabular}

\subsubsection{Tri-Party Agreement}

The Tri-Party Agreement is a legal binding document signed by DOE, EPA, and Ecology. The Tri-Party Agreement establishes a Hanford Sitewide RCRA/CERCLA compliance agreement comprised of programmatic milestones and completion dates for waste management and environmental restoration (ER) activities spanning the time frame of 1989 through 2028. WHC Tri-Party Agreement Integration (TPAI) organization has the lead responsibility for providing integration management for the successful completion of all TriParty Agreement programmatic milestones for all site contractors.

${ }^{1}$ WHC Procurement will work with the new contractor to transition existing contracts. 
Some of the activities performed by TPAI in supporting RL include: maintaining TriParty Agreement change control systems, implement amendments to the Tri-Party Agreement document, conduct monthly IAMIT meetings for RL, EPA, and Ecology, conduct monthly Tri-Party Agreement Training Course, provide status of Tri-Party Agreement milestones, and maintain the Tri-Party Agreement Handbook.

\begin{tabular}{|c|c|c|}
\hline Pre-Award Actions & $\begin{array}{l}\text { Schedulied } \\
\text { Date }\end{array}$ & Actionee(s) \\
\hline $\begin{array}{l}\text { 1. Assemble copies of the Environmental Tracking System } \\
\text { (ETS) reports available to provide status of Tri-Party } \\
\text { Agreement milestones and identify responsible personnel. }\end{array}$ & 6/01/96 & R Morrison \\
\hline $\begin{array}{l}\text { 2. Assemble copies of the current Tri-Party Agreement } \\
\text { document available for PHMC review. }\end{array}$ & $6 / 01 / 96$ & R Morrison \\
\hline $\begin{array}{l}\text { 3. Develop list of unresolved Tri-Party Agreement Disputes, } \\
\text { open change requests, on going and anticipated regulator } \\
\text { negotiations and unresolved Tri-Party Agreement document } \\
\text { issues. }\end{array}$ & $5 / 17 / 96$ & R Morrison \\
\hline $\begin{array}{l}\text { 4. Assemble copies of latest IAMTT meeting minutes to identify } \\
\text { current issues of discussion between RL, EPA and Ecology. }\end{array}$ & $5 / 17 / 96$ & F Calapristi \\
\hline $\begin{array}{l}\text { 5. Assembly status of current and future Tri-Party Agreernent } \\
\text { negotiations. }\end{array}$ & $5 / 17 / 96$ & R Morrison \\
\hline $\begin{array}{l}\text { 6. Develop list of missed Tri-Party Agreement milestones for } \\
\text { consideration of potentual luabilities. }\end{array}$ & $5 / 17 / 96$ & L Arnold \\
\hline 4 : Post-Amard Actions & 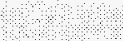 & ? \\
\hline $\begin{array}{l}\text { 1. Provide personnel and amengment list for the Tri-Party } \\
\text { Agreement Integration orgasuretion. }\end{array}$ & 6/07/96 & L Arnold \\
\hline $\begin{array}{l}\text { 2. Make copies of the Tn.Party Agroement document, with } \\
\text { latest changes, available for PHMC review. }\end{array}$ & 6/07/96 & R Morrison \\
\hline $\begin{array}{l}\text { 3. Provide list of unresolvad In-Party Agreement Disputes, } \\
\text { open change requests. oagong antucipated regulator } \\
\text { negotiations and uarecolved Tn-Party Agreement document } \\
\text { issues. }\end{array}$ & $6 / 10 / 96$ & R Morrison \\
\hline $\begin{array}{l}\text { 4. Make copy of Tri-Pury Aeroement Handbook available for } \\
\text { PHMC review. }\end{array}$ & $6 / 10 / 96$ & F Calapristi \\
\hline $\begin{array}{l}\text { 5. Make copies of the ETS reports available to provide } \\
\text { June 1, } 1996 \text { status of Tri-Party Agreement milestones and } \\
\text { identify responsible personnel. }\end{array}$ & $6 / 14 / 96$ & R Morrison \\
\hline $\begin{array}{l}\text { 6. Arrange introductory meeting between RL, EPA, Ecology } \\
\text { and PHMC. }\end{array}$ & $6 / 14 / 96$ & L Arnold \\
\hline $\begin{array}{l}\text { 7. Provide status of current and future Tri-Party Agreement } \\
\text { negotiations. }\end{array}$ & $6 / 17 / 96$ & R Morrison \\
\hline 8. Schedule Tri-Party Agreement Training course for PHMC. & $6 / 21 / 96$ & F Calapristi \\
\hline $\begin{array}{l}\text { 9. Provide copies of May LAMIT approved meeting minutes to } \\
\text { identify current issues of discussion between RL, EPA and } \\
\text { Ecology. }\end{array}$ & 6/28/96 & F Calapristi \\
\hline
\end{tabular}




\begin{tabular}{|c|c|c|c|}
\hline & Posth ward fohons & schedripes & Herionee() \\
\hline 10. & $\begin{array}{l}\text { Deliver all Tri-Party Agreement training course materials } \\
\text { (files, records, overheads, handouts) to PHMC management } \\
\text { so they may continue to provide training as needed. }\end{array}$ & $9 / 20 / 96$ & R Moerman \\
\hline
\end{tabular}

\subsubsection{Safety Analysis and Nuclear Engineering}

The Safety Analysis and Nuclear Engineering (SA\&NE) organization provides the nuclear and radiological expertise required to achieve the safe management, operations, handling and disposition of nuclear facilities and radioactive materials on the Hanford Site. This organization's expertise provides the safety authorization basis, environmental performance assessments, nuclear fuel characterization, and criticality and shielding analyses in support of nuclear design and operational safety.

SA\&NE functions as an integral part of major projects, thereby providing cost competitive expertise based on decades of site-specific knowledge utilizing a balanced mix of experience and skills.

SA\&NE is currently providing or has recently completed deliverables for site projects and programs including TWRS, SNF, Transition Facilities, Solid Waste, and Special Initiatives.

\begin{tabular}{|c|c|c|}
\hline 4: Pre-Award Actions & $\begin{array}{l}\text { Scheduled } \\
\text { Date }\end{array}$ & Actionee(s) \\
\hline $\begin{array}{l}\text { 1. Assemble key program/propect documentation, and identify } \\
\text { its location, for use by the PHMC. }\end{array}$ & $6 / 01 / 96$ & A Ramble \\
\hline $\begin{array}{l}\text { 2. Identify number of briefings. and prepare organizational } \\
\text { briefing(s) for presentution in the PHMC. }\end{array}$ & $5 / 15 / 96$ & A Ramble \\
\hline $\begin{array}{l}\text { 3. Develop a listing of key prozram/project individuals as } \\
\text { POCs. }\end{array}$ & 6/01/96 & A Ramble \\
\hline $\begin{array}{l}\text { 4. Develop listing of program project aubcontracts. Include any } \\
\text { subcontracts still planned wo be executed prior to contract } \\
\text { takeover. }\end{array}$ & $6 / 01 / 96$ & A Ramble \\
\hline 40 Post-Award Actions & 4 & \\
\hline $\begin{array}{l}\text { 1. Provide any documentation or information requested by the } \\
\text { PHMC, if available, that was not identified during the pre- } \\
\text { award phase. }\end{array}$ & $7 / 01 / 96$ & A Ramble \\
\hline 2. Provide organizational briefing to the PHMC. & $7 / 15 / 96$ & A Ramble \\
\hline 3. Provide program/projects briefing to the PHMC. & $8 / 01 / 96$ & A Ramble \\
\hline
\end{tabular}




\subsubsection{Occupational Health Services}

Occupational Health Services are provided to the Hanford Site by the HEHF. The Contractor shall obtain for itself and require of all subcontractors performing work on the Hanford Site the following services from HEHF: occupational medical evaluations including return to work evaluations and work restriction reviews, medical surveillance evaluations, occupational primary care, health care centers/first aid, work conditioning, case management, work site health programs including blood-borne pathogens and immunizations, behavioral health services including employee assistance programs, Material Safety Data Sheet (MSDS) services, and health information services such as medical records and medical scheduling.

\section{Key Assumption}

- PeopleCore System will be carried forward and maintained by the PHMC.

Note: PeopleCore is a sitewide central database which consolidates information about people from diverse automated and non-automated systems. PeopleCore provides single point of entry for data collection, maintenance, and dissemination of information for all Hanford Site contractors. It is the basis for all of HEHF's medical system personnel rosters, and automatically updates these rosters on a daily basis.

\begin{tabular}{|c|c|c|}
\hline Post-Award Actions & $\begin{array}{c}\text { Scheduled } \\
\text { Date }\end{array}$ & Actionee(s) \\
\hline $\begin{array}{l}\text { 1. The PHMC shall coordinate with HEHF and reach agreement } \\
\text { regarding service requirements and delivery, and coordination } \\
\text { and integration between the Contractor's safety and health } \\
\text { organization(s) and HEHF including data gathering and } \\
\text { sharing. The agreement should emphasize a comprehensive } \\
\text { public health approach as being integral to a well run and } \\
\text { safety program, and address cost and resource effectiveress. } \\
\text { (This agreement shall be subject to approval and validation } \\
\text { by RL.) }\end{array}$ & $9 / 01 / 96$ & $\begin{array}{l}\text { JJ Maher III } \\
\text { R Volk }\end{array}$ \\
\hline $\begin{array}{l}\text { 2. HEHF has a significant number of support services which are } \\
\text { provided by the current M\&O Contractor that are critical for } \\
\text { the general operations of Occupational Health Services. } \\
\text { These services are procured under ongoing work orders } \\
\text { issued by HEHF. Contact HEHF to obtain listing of work } \\
\text { orders. }\end{array}$ & 9/01/96 & $\begin{array}{l}\text { JJ Maher III } \\
\text { S Fritts }\end{array}$ \\
\hline
\end{tabular}

\subsubsection{Centralized Occurrence Reporting}

The Occurrence Notification Center (ONC) is currently managed and staffed by WHC. The group provides centralized infrastructure support to the Hanford Site Occurrence Reporting Program. This support includes software and hardware maintenance, training, tracking, occurrence report distribution to include input into the Occurrence Report Processing System (ORPS). The ONC also provides ancillary services to Emergency 
Preparedness by way of operation and maintenance of communications and notification systems for declared emergencies.

\begin{tabular}{|c|c|c|}
\hline Pre-Award Actions & $\begin{array}{l}\text { Scheduled } \\
\text { Date }\end{array}$ & Actionee( ) \\
\hline $\begin{array}{l}\text { 1. Assemble key program (or project) documentation, or identify its } \\
\text { location, for use by the PHMC. Includes development of a } \\
\text { program/project "Smart Book," including listings and descriptions } \\
\text { developed above. }\end{array}$ & $6 / 01 / 96$ & DJ Connell \\
\hline 2. Develop an ONC POC List. & $6 / 01 / 96$ & DJ Connell \\
\hline $\begin{array}{l}\text { 3. Prepare a presentation which covers the work scope, staffing, and } \\
\text { budget of the ONC. }\end{array}$ & $6 / 01 / 96$ & DJ Connell \\
\hline $\begin{array}{l}\text { 4. Develop listing and description of program (or project) databases, } \\
\text { including facility (or operations) databases. }\end{array}$ & $6 / 01 / 96$ & DJ Connell \\
\hline $\begin{array}{l}\text { 5. Develop a listing of special agreement services provided to RL, } \\
\text { other DOE sites, and other entities. }\end{array}$ & $6 / 01 / 96$ & DJ Connell \\
\hline $\begin{array}{l}\text { 6. Develop a list of current work order services or other form of } \\
\text { agreement to provide work, or obtain work, for other } \\
\text { organizations, on and offsite. }\end{array}$ & $6 / 01 / 96$ & DJ Connell \\
\hline $\begin{array}{l}\text { 7. Verify the accuracy of property assignments at the } \\
\text { program/project organizational level and correct any deficiencies. }\end{array}$ & $6 / 01 / 96$ & DJ Connell \\
\hline 8. Determine pre-existing conditions. & $6 / 01 / 96$ & DJ Connell \\
\hline Post-A ward Actions & ॰ & 8 \\
\hline $\begin{array}{l}\text { 1. Provide the PHMC access to the key program (or project) } \\
\text { documentation, any program level generated pre-existing } \\
\text { conditions information, listing of POCs, and listing of } \\
\text { subcontracts. Provide copies upon request. }\end{array}$ & $\begin{array}{l}\text { prior to } \\
6 / 15 / 96\end{array}$ & DJ Connell \\
\hline $\begin{array}{l}\text { 2. Determine occurrence reporting relationships and responsibilities } \\
\text { of the PHMC and subcontractors. }\end{array}$ & 8/01/96 & DJ Connell \\
\hline $\begin{array}{l}\text { 3. Identify needed changes to authority files, ORPS designators, } \\
\text { procedures, and training. }\end{array}$ & 9/01/96 & DJ Connell \\
\hline $\begin{array}{l}\text { 4. Complete changes to authority files, ORPS designators, and } \\
\text { procedures. }\end{array}$ & 9/15/96 & DJ Connell \\
\hline 5. Implement changes to occurrence report process. & 9/30/96 & DJ Connell \\
\hline $\begin{array}{l}\text { 6. Perform a joint program/project level property and equipment } \\
\text { inventory with PHMC personnel. }\end{array}$ & by $8 / 30 / 96$ & DJ Connell \\
\hline $\begin{array}{l}\text { 7. Revise authority files, ORPS designators, and train new } \\
\text { personnel. }\end{array}$ & 9/30/96 & DJ Connell \\
\hline $\begin{array}{l}\text { 8. Identify data crossover for retrofit ORPS data with new ORPS } \\
\text { designators. }\end{array}$ & 9/30/96 & DJ Connell \\
\hline
\end{tabular}




\subsection{PROCUREMENT AND MATERIALS MANAGEMENT}

\subsubsection{Procurement}

\begin{tabular}{|c|c|c|}
\hline rresward fetions & Scheduled & fetiones) \\
\hline 1. Assemble current organizational data (charts and charters). & $5 / 31 / 96$ & JJ Jones \\
\hline 2. List of property assigned to PMM. & $5 / 31 / 96$ & R Dahlin \\
\hline 3. Status of re-engineering initiatives and actions. & $5 / 31 / 96$ & R Meyer \\
\hline $\begin{array}{l}\text { 4. Assemble copy of all current reports provided to external } \\
\text { customers. }\end{array}$ & $5 / 31 / 96$ & E Beavers \\
\hline 5. Provide overview of financial situation and work plans. & $5 / 31 / 96$ & J Stewart \\
\hline Post Award Actions & $\$$ & \\
\hline 1. Resolve any open issues on property by turn over. & $9 / 30 / 96$ & R Dahlin \\
\hline $\begin{array}{l}\text { 2. Provide update on organization data, re-engineering, financial } \\
\text { status, and work plans. }\end{array}$ & $9 / 30 / 96$ & RJ Meyer \\
\hline
\end{tabular}

\subsubsection{Open Audit/Surveillance Items.}

\begin{tabular}{|l|c|c|}
\hline Scheduled & Date & Pre-Award Actions \\
\hline 1. Identify and status all open items. & $5 / 31 / 96$ & $\begin{array}{l}\text { E Higginbothom } \\
\text { B Hubbard }\end{array}$ \\
\hline $\begin{array}{l}\text { 1. Complete as many audit/surveillance items as possible by nurn } \\
\text { over. }\end{array}$ & $9 / 30 / 96$ & $\begin{array}{l}\text { E Higginbothom } \\
\text { B Hubbard }\end{array}$ \\
\hline $\begin{array}{l}\text { 2. Transfer action to appropriate contractor(s) or WHC close out } \\
\text { team. }\end{array}$ & $9 / 30 / 96$ & $\begin{array}{l}\text { E Higginbothom } \\
\text { B Hubbard }\end{array}$ \\
\hline
\end{tabular}

\subsubsection{Identify Funding Reserves/Contingency Needs for Close Out Process.}

\begin{tabular}{|c|c|c|}
\hline Pre-Award Actions & $\begin{array}{l}\text { Scheduled } \\
\text { pate }\end{array}$ & Letionet (s) \\
\hline $\begin{array}{l}\text { 1. Compile listing of orders and funding necessary of purchase } \\
\text { orders in the close out process. }\end{array}$ & $5 / 31 / 96$ & $\begin{array}{l}\text { E Higginbothom } \\
\text { B Hubbard }\end{array}$ \\
\hline Post-A ward Actions & & \\
\hline 1. Update funding reserves/contingency needs for close outs. & $9 / 30 / 96$ & $\begin{array}{l}\text { E Higginbothom } \\
\text { B Hubbard }\end{array}$ \\
\hline $\begin{array}{l}\text { 2. Transfer action to appropriate contractor(s) or WHC close out } \\
\text { team. }\end{array}$ & $9 / 30 / 96$ & $\begin{array}{l}\text { E Higginbothom } \\
\text { B Hubbard }\end{array}$ \\
\hline
\end{tabular}




\subsubsection{Purchase Order Assignment/Transfer to One or More New Companies.}

\begin{tabular}{|l|c|l|l|}
\hline 1. Develop purchase order assignment letter. & Scheduled & Date & \\
& $5 / 31 / 96$ & $\begin{array}{l}\text { M Taylor } \\
\text { S Ace }\end{array}$ \\
\hline 1. Develop listing of orders to new company(s). & $6 / 30 / 96$ & $\begin{array}{l}\text { M Taylor } \\
\text { S Ace }\end{array}$ \\
\hline 2. Determine approach for all unassigned orders. & $6 / 30 / 96$ & $\begin{array}{l}\text { M Taylor } \\
\text { S Ace }\end{array}$ \\
\hline 3. Issue assignment letters. & $9 / 30 / 96$ & $\begin{array}{l}\text { R Hadley } \\
\text { J Smith } \\
\text { B Hubbard }\end{array}$ \\
\hline
\end{tabular}

\subsubsection{Purchase Orders With Open Concerns.}

\begin{tabular}{|l|c|l|l|}
\hline Scheduled & Date & Pre-Award Actions \\
\hline $\begin{array}{l}\text { 1. Identify all purchase orders with protests, claims or any other } \\
\text { open legal issue. }\end{array}$ & $5 / 31 / 96$ & $\begin{array}{l}\text { R Hadley } \\
\text { J Smith } \\
\text { S Ace } \\
\text { R Dutton }\end{array}$ \\
\hline $\begin{array}{l}\text { 1. Update list of purchase orders with protests, claims or any } \\
\text { other open legal issue. }\end{array}$ & $9 / 30 / 96$ & $\begin{array}{l}\text { R Hadley } \\
\text { J Smith } \\
\text { S Ace } \\
\text { R Dutton }\end{array}$ \\
\hline $\begin{array}{l}\text { 2. Transfer action to appropriate contractor(s) or WHC close out } \\
\text { team. }\end{array}$ & $9 / 30 / 96$ & $\begin{array}{l}\text { R Hadley } \\
\text { J Smith } \\
\text { S Ace }\end{array}$ \\
\hline
\end{tabular}

\subsubsection{Purchase Orders in Termination Process.}

\begin{tabular}{|l|c|c|c|}
\hline $\begin{array}{l}\text { Pre-Award Actions } \\
\text { 1. Identify all purchase orders in termination (convenience or } \\
\text { default) process. }\end{array}$ & $\mathbf{5 / 3 1 / 9 6}$ & $\begin{array}{l}\text { R Hadley } \\
\text { J Smith } \\
\text { S Ace } \\
\text { T Gasperino }\end{array}$ \\
\hline $\begin{array}{l}\text { 1. Update list of purchase orders in termination (convenience or } \\
\text { default) process. }\end{array}$ & $9 / 30 / 96$ & $\begin{array}{l}\text { R Hadley } \\
\text { J Smith } \\
\text { S Ace } \\
\text { T Gasperino }\end{array}$ \\
\hline
\end{tabular}




\begin{tabular}{|c|c|c|}
\hline Post-Award / Actions & scuenouled & detion-s \\
\hline $\begin{array}{l}\text { 2. Transfer action to appropriate contractor(s) or WHC close out } \\
\text { team. }\end{array}$ & $9 / 30 / 96$ & $\begin{array}{l}\text { R Hadley } \\
\text { J Smith } \\
\text { S Ace } \\
\text { T Gasperino } \\
\text { R Dutton }\end{array}$ \\
\hline
\end{tabular}

\subsubsection{Purchase Orders With Open Options.}

\begin{tabular}{|c|c|c|}
\hline (ै) Pre-Award Actions & Scheduled & rotonses) \\
\hline $\begin{array}{l}\text { 1. Identify all purchase orders with options that could be } \\
\text { exercised for services after September } 30,1996 \text {. }\end{array}$ & $5 / 31 / 96$ & $\begin{array}{l}\text { R Hadley } \\
\text { J Smith } \\
\text { S Ace } \\
\text { T Gasperino }\end{array}$ \\
\hline Post-Award Actions & & \\
\hline $\begin{array}{l}\text { 1. Update list of purchase orders with options that could be } \\
\text { exercised for services after September } 30,1996 \text {. }\end{array}$ & $9 / 30 / 96$ & $\begin{array}{l}\text { R Hadley } \\
\text { J Smith } \\
\text { S Ace } \\
\text { T Gasperino }\end{array}$ \\
\hline
\end{tabular}

\subsubsection{Open Procurement Actions Beyond Transition.}

\begin{tabular}{|c|c|c|}
\hline Pre-Award Actions & Scheduled & Actiones $($ ) \\
\hline $\begin{array}{l}\text { 1. List all purchase orders that will be open beyond } \\
\text { September } 30,1996 \text {. }\end{array}$ & $5 / 31 / 96$ & $\begin{array}{l}\text { R Hadley } \\
\text { I Smith } \\
\text { S Ace } \\
\text { T Gasperino }\end{array}$ \\
\hline $\begin{array}{l}\text { 2. List all Memorandum orders with other DOE sites that will be } \\
\text { open beyond September } 30,1996 \text {. }\end{array}$ & $5 / 31 / 96$ & $\begin{array}{l}\text { R Hadley } \\
\text { J Smith } \\
\text { T Gasperino }\end{array}$ \\
\hline $\begin{array}{l}\text { 3. List all undefinitized purchase orders in work as of contract } \\
\text { award. }\end{array}$ & $5 / 31 / 96$ & $\begin{array}{l}\text { R Hadley } \\
\text { J Smith } \\
\text { T Gasperino }\end{array}$ \\
\hline $\begin{array}{l}\text { 4. List all Interwork Agreements with WEC and Boeing } \\
\text { subsidiaries that will be open beyond September } 30,1996 \text {. }\end{array}$ & $5 / 31 / 96$ & $\begin{array}{l}\text { R Hadley } \\
\text { J Smith }\end{array}$ \\
\hline $\begin{array}{l}\text { 5. List all Interworks agreements with ICF KH subsidiaries that } \\
\text { will be open beyond September } 30,1996 \text {. }\end{array}$ & $5 / 31 / 96$ & C Maxson \\
\hline $\begin{array}{l}\text { 6. List all special agreements (travel agency, air lines/car rentals, } \\
\text { head hunters, etc.) that will be open beyond } \\
\text { September } 30,1996 \text {. }\end{array}$ & $5 / 31 / 96$ & $\begin{array}{l}\text { R Hadley } \\
\text { J Smith } \\
\text { C Maxson }\end{array}$ \\
\hline
\end{tabular}




\section{WHC-SP-1181}

\begin{tabular}{|l|l|l|}
\hline 1. Update all action lists in section 3.6.8 (A) above. & $\begin{array}{l}\text { Scheduled } \\
\text { Date }\end{array}$ & $\begin{array}{l}\text { Actionee( }) \\
9 / 30 / 96\end{array}$ \\
\hline 2. Transfer open orders to appropriate contractor(s). & $\begin{array}{l}\text { R Hadley } \\
\text { J Smith } \\
\text { S Ace } \\
\text { T Gasperino }\end{array}$ \\
\hline 3. Initiate termination for unassigned open orders. & $9 / 30 / 96$ & $\begin{array}{l}\text { R Hadley } \\
\text { J Smith } \\
\text { T Gasperino }\end{array}$ \\
\hline
\end{tabular}

\subsubsection{Open Solicitations.}

\begin{tabular}{|c|c|c|}
\hline Pre-Award Actions & $\begin{array}{l}\text { Scheduled } \\
\text { Date }\end{array}$ & Actionee(s) \\
\hline 1. List of all solicitations and awards in process at award time. & $5 / 31 / 96$ & $\begin{array}{l}\text { R Hadley } \\
\text { J Smith } \\
\text { T Gasperino }\end{array}$ \\
\hline Post-Award Actions & & \\
\hline $\begin{array}{l}\text { 1. Update list of all solicitations that will be open beyond } \\
\text { September } 30,1996 \text {. }\end{array}$ & 9/30/96 & $\begin{array}{l}\text { R Hadley } \\
\text { J Smith } \\
\text { T Gasperino }\end{array}$ \\
\hline $\begin{array}{l}\text { 2. Transfer open solicitations to appropriate contractor(s) or } \\
\text { cancel solicitations. }\end{array}$ & $9 / 30 / 96$ & $\begin{array}{l}\text { R Hadley } \\
\text { J Smith } \\
\text { T Gasperino }\end{array}$ \\
\hline
\end{tabular}

\subsubsection{Purchase Order Terms and Conditions.}

\begin{tabular}{|c|c|c|}
\hline Pre-Award Actions & $\begin{array}{l}\text { Scheduled } \\
\text { Date }\end{array}$ & Actionee(s) \\
\hline $\begin{array}{l}\text { 1. Current set of terms and conditions incorporated into purchase } \\
\text { orders. }\end{array}$ & $5 / 31 / 96$ & $\begin{array}{l}\text { R Hadley } \\
\text { J Smith } \\
\text { T Gasperino }\end{array}$ \\
\hline Post-Awrard Actions & 4 & 48 \\
\hline $\begin{array}{l}\text { 1. Selected contractor terms and conditions incorporated into } \\
\text { purchase orders. }\end{array}$ & 9/30/96 & $\begin{array}{l}\text { R Hadley } \\
\text { J Smith } \\
\text { T Gasperino }\end{array}$ \\
\hline
\end{tabular}




\subsubsection{Actions with DOE (RL \& HQ).}

\begin{tabular}{|l|l|l|l|}
\hline $\begin{array}{l}\text { Sclieduled } \\
\text { Date }\end{array}$ & $\begin{array}{l}\text { Pre-Award Actions } \\
\text { 1. Provide a listing of procurement actions/initiatives with DOE } \\
\text { (RL \& HQ). }\end{array}$ & $5 / 31 / 96$ & $\begin{array}{l}\text { R Hadley } \\
\text { J Smith } \\
\text { C Maxson }\end{array}$ \\
\hline $\begin{array}{l}\text { 1. Update listing of procurement actions/initiatives with DOE } \\
\text { (RL \& HQ). }\end{array}$ & $9 / 30 / 96$ & $\begin{array}{l}\text { R Hadley } \\
\text { J Smith } \\
\text { C Maxson }\end{array}$ \\
\hline
\end{tabular}

\subsubsection{Subcontractor Property Activities.}

\begin{tabular}{|c|c|c|}
\hline Post-Award Actions & $\begin{array}{l}\text { Scheduled } \\
\text { Date }\end{array}$ & Actionee (s) \\
\hline 1. Complete subcontractor property reviews. & $9 / 30 / 96$ & $\begin{array}{l}\text { R Hadley } \\
\text { J Smith } \\
\text { C Maxson }\end{array}$ \\
\hline
\end{tabular}

\subsubsection{Software License Agreements.}

\begin{tabular}{|c|c|c|}
\hline ( & $\begin{array}{l}\text { Scheduled } \\
\text { Date }\end{array}$ & Actionee(s) \\
\hline 1. List all software licenses. & $5 / 31 / 96$ & $\begin{array}{l}\text { R Hadley } \\
\text { B Hubbard }\end{array}$ \\
\hline 2. Develop software license transfer agreement. & $5 / 31 / 96$ & $\begin{array}{l}\text { R Hadley } \\
\text { B Hubbard } \\
\text { R Dutton }\end{array}$ \\
\hline ( & 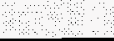 & \\
\hline 1. Transfer of software licenses to one or more new companies. & $9 / 30 / 96$ & $\begin{array}{l}\text { R Hadley } \\
\text { B Hubbard }\end{array}$ \\
\hline $\begin{array}{l}\text { 2. Terminate all software license agreements not transferred to } \\
\text { new contractor(s). }\end{array}$ & $9 / 30 / 96$ & $\begin{array}{l}\text { R Hadley } \\
\text { B Hubbard }\end{array}$ \\
\hline $\begin{array}{l}\text { 3. Negotiate any necessary modifications to sitewide license } \\
\text { agreements. }\end{array}$ & $9 / 30 / 96$ & $\begin{array}{l}\text { R Hadley } \\
\text { B Hubbard } \\
\text { R Dutton }\end{array}$ \\
\hline
\end{tabular}

\subsubsection{Procurement Reference Material.}

\begin{tabular}{|l|c|c|l|}
\hline $\begin{array}{l}\text { Pre-Award Actions } \\
\text { 1. Assemble extra set of all current terms and conditions, } \\
\text { manuals, procedures, forms and work place instructions for } \\
\text { procurement functions. }\end{array}$ & $5 / 31 / 96$ & $\begin{array}{l}\text { M Taylor } \\
\text { B Hubbard } \\
\text { T Gasperino }\end{array}$ \\
\hline
\end{tabular}




\begin{tabular}{|l|c|c|c|}
\hline $\begin{array}{l}\text { Selinediled } \\
\text { 2. Develop a list of forms that include references or logos that } \\
\text { may need to be changed. }\end{array}$ & $5 / 31 / 96$ & $\begin{array}{l}\text { M Taylor } \\
\text { B Hubbard }\end{array}$ \\
\hline
\end{tabular}

\subsubsection{Automation System Information.}

\begin{tabular}{|c|c|c|}
\hline rrotwridsctions & Scheduled & Setionee(t) \\
\hline 1. List of all automated systems with interface details. & $5 / 31 / 96$ & $\begin{array}{l}\text { M Taylor } \\
\text { S Ace }\end{array}$ \\
\hline $\begin{array}{l}\text { 2. Status of all major system modifications or requirements } \\
\text { changes in process. }\end{array}$ & $5 / 31 / 96$ & $\begin{array}{l}\text { M Taylor } \\
\text { S Ace }\end{array}$ \\
\hline Post-Award Actions & & \\
\hline $\begin{array}{l}\text { 1. Update status of all major system modifications or } \\
\text { requirements in process. }\end{array}$ & $9 / 30 / 96$ & $\begin{array}{l}\text { M Taylor } \\
\text { S Ace }\end{array}$ \\
\hline
\end{tabular}

\subsubsection{Outreach Activities.}

\begin{tabular}{|l|c|c|c|}
\hline Pre-Award Actions & Scheduled & Date & \\
\hline $\begin{array}{l}\text { 1. Develop summary and assemble listing of all Outreach } \\
\text { commitments of WHC. }\end{array}$ & $5 / 31 / 96$ & C Crain \\
\hline
\end{tabular}

\subsubsection{Property Management}

\subsubsection{Capital and Sensitive Property.}

\begin{tabular}{|c|c|c|}
\hline Pre-Award Actions & Ccheduled & Achonets \\
\hline $\begin{array}{l}\text { 1. Provide a current inventory of all capital and sensitive property } \\
\text { for WHC/BCSR and ICF Kaiser operations. }\end{array}$ & $5 / 31 / 96$ & R Dahlin \\
\hline Post-Award Actions & & \\
\hline $\begin{array}{l}\text { 1. Statistical Sample Verification of } 3.7 .1 \text { above, } 95 \% \text { confidence } \\
\text { level. }\end{array}$ & $9 / 30 / 96$ & R Dahlin \\
\hline 2. Transfer ownership officially to new contractor(s). & $9 / 30 / 96$ & R Dahlin \\
\hline
\end{tabular}




\subsubsection{Other Financially Controlled Inventories.}

\begin{tabular}{|l|c|l|l|}
\hline & $\begin{array}{c}\text { Scheduled } \\
\text { Date }\end{array}$ & Actionee(s) \\
\hline $\begin{array}{l}\text { 1. Provide a current inventory of all capital and sensitive property } \\
\text { for WHC/BCSR and ICF KH operations. }\end{array}$ & $5 / 31 / 96$ & R Dahlin \\
\hline $\begin{array}{l}\text { 1. Complete all other inventories of financially controlled property } \\
\text { and reconcile. }\end{array}$ & $9 / 30 / 96$ & R Dahlin \\
\hline 2. Transfer ownership officially to new contractor(s). & $9 / 30 / 96$ & R Dahlin \\
\hline
\end{tabular}

\subsubsection{Export Control and High Risk Property.}

\begin{tabular}{|c|c|c|}
\hline Post-Award Actions & $\begin{array}{l}\text { Scheduled } \\
\text { Date }\end{array}$ & Actionee(s) \\
\hline $\begin{array}{l}\text { 1. Provide listing of current inventory of all export control and } \\
\text { high risk surplus/excess property in disposal cycle. }\end{array}$ & $8 / 01 / 96$ & R Dahlin \\
\hline
\end{tabular}

\subsubsection{Activities with DOE (RL \& HQ).}

\begin{tabular}{|c|c|c|}
\hline Pre-Award Actions & $\begin{array}{l}\text { Scheduled } \\
\text { Date }\end{array}$ & Actionee(s) \\
\hline 1. List of actions/initiatives with DOE. & $5 / 31 / 96$ & R Dahlin \\
\hline Post-Amerd Actions & & \\
\hline 1. Update list of actions'inutuetivet mth DOE. & 9/30/96 & R Dahlin \\
\hline
\end{tabular}

\subsubsection{Property Reference M1aterial.}

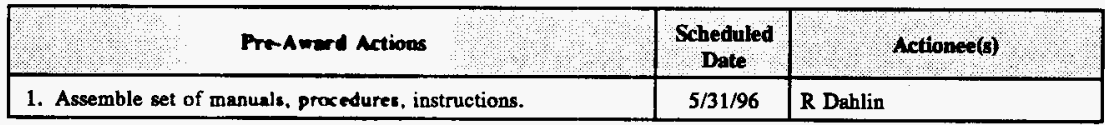

\subsubsection{Economic Transfer}

\subsubsection{Intellectual Property.}

\begin{tabular}{|l|l|l|l|}
\hline \multicolumn{1}{|c|}{ Pre-Award Actions } & Scheduled & Date
\end{tabular}




\begin{tabular}{|c|c|c|}
\hline Postrurd petings & Sohed ved & tethones $(6)$ \\
\hline 1. Transfer/preserve WEC rights as is appropriate. & $9 / 30 / 96$ & $\begin{array}{l}\text { B Silva } \\
\text { R Dutton }\end{array}$ \\
\hline
\end{tabular}

\subsubsection{Loan agreements, CRADAs and MOUs activities.}

\begin{tabular}{|c|c|c|}
\hline 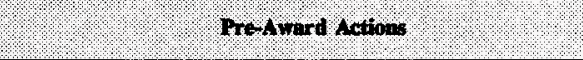 & Solnednted & potopos $(8)$ \\
\hline $\begin{array}{l}\text { 1. Complete reviews and provide listing of loan agreements, } \\
\text { CRADAs and MOUs, etc. that will be open beyond } \\
\text { September } 30,1996 \text {. }\end{array}$ & $5 / 31 / 96$ & $\begin{array}{l}\text { B Silva } \\
\text { R Dutton }\end{array}$ \\
\hline Post-Award Actions & & \\
\hline $\begin{array}{l}\text { 1. Transfer existing agreements such as licenses, CRADAs, } \\
\text { Patents, Copy Rights, Trademarks, Invention Disclosures. }\end{array}$ & $9 / 30 / 96$ & $\begin{array}{l}\text { B Silva } \\
\text { R Dutton }\end{array}$ \\
\hline
\end{tabular}

\subsubsection{Patent, Copyright, Royalty, and Invention activities.}

\begin{tabular}{|c|c|c|}
\hline Pre-Award Actions & $\begin{array}{l}\text { Scheduled } \\
\text { Datte }\end{array}$ & Actiones $(s)$ \\
\hline $\begin{array}{l}\text { 1. Complete review and provide listing of all patent, copyright, } \\
\text { royalty and invention activity that will be open as of } \\
\text { September } 30,1996 \text {. }\end{array}$ & $5 / 31 / 96$ & $\begin{array}{l}\text { B Silva } \\
\text { R Dutton }\end{array}$ \\
\hline 1.: Post-Award Actions & & \\
\hline 1. Transfer activities as is approptute to new contractor(s). & $9 / 30 / 96$ & $\begin{array}{l}\text { B Silva } \\
\text { R Dutton }\end{array}$ \\
\hline
\end{tabular}

\subsubsection{Asset Conversion Activities.}

\begin{tabular}{|c|c|c|}
\hline ४ & $\begin{array}{l}\text { Scheduled } \\
\text { Date }\end{array}$ & Actionests) \\
\hline 1. Provide status of oped aseet converaion projects. & $5 / 31 / 96$ & B Silva \\
\hline Post-Award Actions & & \\
\hline 1. Update status of open asset conversion projects. & $9 / 30 / 96$ & B Silva \\
\hline 2. Transfer activities as is appropriate to new contractor(s). & $9 / 30 / 96$ & B Silva \\
\hline
\end{tabular}

\subsubsection{Activities with DOE (RL \& HQ).}

\begin{tabular}{|c|c|c|}
\hline Pretiward Actions & $\begin{array}{l}\text { Scheduled } \\
\text { Date }\end{array}$ & Actionee ( $)$ \\
\hline 1. Compile list of actions/initiatives with DOE. & $5 / 31 / 96$ & B Silva \\
\hline
\end{tabular}




\section{Post-Award Actions}

\begin{tabular}{|c|c|}
\hline $\begin{array}{l}\text { Schediled } \\
\text { Dute. }\end{array}$ & Hethoned $(\mathrm{s})$ \\
\hline $9 / 30 / 96$ & B Silva \\
\hline
\end{tabular}

\subsubsection{Materials Management}

\subsubsection{P-Card Assignment/Transfer to One or More New Companies.}

\begin{tabular}{|c|c|c|}
\hline Pre-A ward Actions & $\begin{array}{l}\text { Scheduled } \\
\text { Date. }\end{array}$ & thripoes \\
\hline 1. Develop assignment clause or letter. & $5 / 31 / 96$ & R Dahlin \\
\hline Post-Award Actions & 4,4 & \\
\hline $\begin{array}{l}\text { 1. Determine P-card split and financial liability splits between new } \\
\text { companies. }\end{array}$ & $8 / 31 / 96$ & $\begin{array}{l}\text { R Dahlin } \\
\text { C Mayo }\end{array}$ \\
\hline 2. Implement transfer. & 9/30/96 & $\begin{array}{l}\text { R Dahlin } \\
\text { C Mayo }\end{array}$ \\
\hline
\end{tabular}

\subsubsection{Surplus Sales Assignment/Transfer to One or More New Companies.}

\begin{tabular}{|l|c|l|}
\hline Pre-Award Actions & $\begin{array}{c}\text { Scheduled } \\
\text { Date }\end{array}$ & Actionee(s) \\
\hline 1. Develop assignment clause or letter. & $5 / 31 / 96$ & R Dahlin \\
\hline $\begin{array}{l}\text { 1. Determine split and financial liability splits between new } \\
\text { companies. }\end{array}$ & $8 / 31 / 96$ & R Dahlin \\
\hline 2. Implement transfer. & $9 / 30 / 96$ & R Dahlin \\
\hline
\end{tabular}

\subsubsection{Warehouse Consolidation Plan (gas dock elimination, surplus material move} into 1163 and configuration of 1167 into excess store).

\begin{tabular}{|l|c|c|}
\hline 1.40 Pre-Award Actions & $\begin{array}{c}\text { Scheduled } \\
\text { Date }\end{array}$ & \\
\hline 1. Complete strategy plan. & $5 / 31 / 96$ & R Dahlin \\
\hline Post-Award Actions & & \\
\hline 1. Implement strategy. & $9 / 30 / 96$ & R Dahlin \\
\hline
\end{tabular}




\subsubsection{General Services Administration (GSA) Waiver Request.}

\begin{tabular}{|c|c|c|}
\hline Prestward Actions & $\begin{array}{l}\text { Scheduled } \\
\text { Date }\end{array}$ & tetones (s) \\
\hline $\begin{array}{l}\text { 1. Implement disposal General Services Administration (GSA) } \\
\text { waiver based on DOE-HQ approval by April } 15,1996 \text {. If } \\
\text { April } 15 \text { date missed, need to reschedule effort action date } \\
\text { based on work prioritization. }\end{array}$ & $5 / 31 / 96$ & R Dahlin \\
\hline
\end{tabular}

\subsubsection{Activities with DOE (RL \& HQ).}

\begin{tabular}{|c|c|c|}
\hline Pro-Award Actions & $\begin{array}{l}\text { Scheduled } \\
\text { pate }\end{array}$ & Actionee(s) \\
\hline 1. List of actions/initiatives with DOE. & $5 / 31 / 96$ & R Dahlin \\
\hline Post-Award Actions & & \\
\hline 1. Update list of issues/initiatives with DOE. & $9 / 30 / 96$ & R Dahlin \\
\hline
\end{tabular}

\subsubsection{Materials Management Reference Materials.}

\begin{tabular}{|c|c|c|c|}
\hline & Pre-Award Actions & Scheduled & \\
Date & &
\end{tabular}

\subsection{RE-ENGINEERING}

Assumptions:

- Re-engineering of the Hanford Site will continue through the award and transition periods.

- Re-engineered facilities will be "contractor proof" in that they will maintain their re-engineered structure and redesigned work processes by the PHMC.

Re-engineering is a fundamental rethinking and redesign of the business processes to achieve dramatic improvements in safety, performance, speed, service and cost. At Hanford, WHC, ICF KH, and BCSR, re-engineering has been or is being undertaken in three major areas:

- Support and administration areas that provide services to virtually all components of the company.

Examples: Finance, Communications, Infrastructure, Procurement, Contracts, General Counsel, Information Technology, etc. 
- Plants/Facilities: PUREX, Fast Flux Test Facility (FFTF), PFP, Solid Waste, T-Plant, B-Plant, SNF, TWRS (West Tanks, East Tanks, Evaporator, Characterization).

- Requirements Management: Safety, Quality, Environmental, S/Rids, RadCon, Emergency Preparedness, etc. To date, re-engineering has been implemented at one plant, PUREX, and is in progress at two other facilities, B-Plant and West Tank Farms.

A draft schedule shows that during calendar year 1996, a total of seven facilities/plants will be re-engineered. Plant re-engineering includes information technology redesign (evaluate JCS work control, project scheduling, etc.), evaluating and defining the culture and the values, redesign of the Management Systems including the performance metrics, defining a new and flatter organization (designing cross functional work teams), developing streamlined work processes, and defining new job descriptions including skills and responsibilities.

In the Support and Administration area, re-engineering has played a major role in setting up a CIO function, streamlining the supply chain function, reducing the size and number of computing resources, simplifying and standardizing department overhead costing, restructuring of the finance organization into a teaming arrangement, aligning infrastructure services to commercial practices, providing communications planning and support for reengineering, alignment of the Human Resources with the re-engineered functions and improving the planning, integration and control process.

In the Requirements Management area, COEs are being set up in four major areas (RadCon, Environmental, Safety, Quality). These centers are staffed on a part-time basis with Plant people to ensure Plants play a major upfront ownership role in the requirements arena with a corresponding major downsizing of centralized organizations.

\begin{tabular}{|l|c|l|}
\hline \multicolumn{1}{|c|}{ Post-Award Actions } & $\begin{array}{c}\text { Seheduled } \\
\text { Date }\end{array}$ & Actionee(s) \\
\hline $\begin{array}{l}\text { 1. Provide detailed re-engineering presentation including path } \\
\text { forward. }\end{array}$ & $7 / 22 / 96$ & RJ Bliss \\
\hline $\begin{array}{l}\text { 2. Provide lists and location of all re-engineering documents and } \\
\text { files. }\end{array}$ & $7 / 22 / 96$ & RG Slocum \\
\hline
\end{tabular}

\subsection{TECHNOLOGY MANAGEMENT}

The M\&O Contractor participation in the National Program is twofold; (1) participates in performing technology development tasks that are near or at the implementation phase and (2) performs an integration role between providers of technology and the potential end users (projects) by functioning as part of and in support of the Site Technology Coordination Group (STCG). 


\subsubsection{National Technology Development Program (EM-50)}

The EM-50 Program is an applied technology program with a strong focus on implementing technology. The WHC role is primarily implementation accomplished by direct involvement in the final phases of technology development (Engineering Development and Implementation). This involvement is limited to those technologies that the National Program views WHC to be the most appropriate contractor to complete the final phases of an assigned technology. As many as 22 other contractors are also involved in similar efforts at their sites requiring WHC and other Site contractors to interface broadly with the technology community to ensure technology is appropriately considered. Section 3.8.2, "Technology Integration," describes this effort.

The EM-50 Program at Hanford is managed by a joint contractor team with representation from BHI, PNNL, and WHC. The team interfaces routinely with the RL Technology Program Officer of the Science and Technology Programs Division.

In addition to WHC participation on the joint contractor management team, WHC manages tasks defined by Technical Task Plans (TTPs) with a combined budget of $\$ 7.6 \mathrm{M}$.

The tasks are managed by a WHC Program Office with a budget allocated from each TTP. Participation on the joint contractor team is direct funded by RL providing funding for administrative and management support to the joint team. These functions are defined in a RL EM-50 Program Management Plan.

\begin{tabular}{|c|c|c|}
\hline Pre-Award Actions & Scheduled & Actionee(s) \\
\hline $\begin{array}{l}\text { 1. Using the PHMC RFP as a basis, review each currently } \\
\text { assigned technology project with RL and determine if it fits the } \\
\text { role of the PHMC as described in Section C. }\end{array}$ & $4 / 19 / 96$ & JD Berger \\
\hline $\begin{array}{l}\text { 2. Determine the appropriate course of action, assisted by the RL } \\
\text { Technology Program Officer, for disposition of technology } \\
\text { projects that do not fit the defined PHMC role. }\end{array}$ & $5 / 03 / 96$ & JD Berger \\
\hline $\begin{array}{l}\text { 3. Assemble key program (or project) documentation, or identify } \\
\text { its location, for use by the PHMC. Includes development of a } \\
\text { program/project "Smart Book," including listings and } \\
\text { descriptions developed below. }\end{array}$ & $6 / 01 / 96$ & JD Berger \\
\hline $\begin{array}{l}\text { 4. Identify number of briefings, and prepare program (or project) } \\
\text { briefing(s) for presentation to PHMC (coordinate number and } \\
\text { content with RL POC). }\end{array}$ & $6 / 01 / 96$ & J̣D Berger \\
\hline $\begin{array}{l}\text { 5. Develop a listing of key program (or project) individuals as } \\
\text { POCs, including facility and operations personnel. }\end{array}$ & $6 / 01 / 96$ & JD Berger \\
\hline $\begin{array}{l}\text { 6. Develop listing and description of program (or project) } \\
\text { subcontracts, including facility (or operations) subcontracts. } \\
\text { Include any subcontracts still planned to be executed prior to } \\
\text { contract takeover. }\end{array}$ & $6 / 01 / 96$ & JD Berger \\
\hline $\begin{array}{l}\text { 7. Develop listing and description of program (or project) } \\
\text { databases, including facility (or operations) databases. }\end{array}$ & $6 / 01 / 96$ & JD Berger \\
\hline
\end{tabular}




\begin{tabular}{|c|c|c|}
\hline Prosward Actoms & oblediled & focthols $(6)$ \\
\hline $\begin{array}{l}\text { 8. Identify space and equipment within program (or project) area } \\
\text { and facilities for use by the PHMC. }\end{array}$ & $6 / 01 / 96$ & JD Berger \\
\hline $\begin{array}{l}\text { 9. Establish a schedule for the actions defined above in } \\
\text { dispositioning technology projects that do not fit the role of the } \\
\text { PHMC. }\end{array}$ & $6 / 01 / 96$ & JD Berger \\
\hline Post-A ward setions & & \\
\hline $\begin{array}{l}\text { 1. Provide the PHMC access to the key program (or project) } \\
\text { documentation, any program level generated pre-existing } \\
\text { conditions information, listing of POCs, and listing of } \\
\text { cubcontracts. Provide copies upon request. }\end{array}$ & $6 / 15 / 96$ & JD Berger \\
\hline $\begin{array}{l}\text { 2. Program/project and facility space and services available for } \\
\text { use by the PHMC. }\end{array}$ & $6 / 15 / 96$ & JD Berger \\
\hline $\begin{array}{l}\text { 3. Provide a review briefing to the PHMC, or the designated } \\
\text { subcontractor, covering pertinent aspects of the technology } \\
\text { projects being transferred. }\end{array}$ & $7 / 01 / 96$ & JD Berger \\
\hline $\begin{array}{l}\text { 4. Host a tour of each technology project to familiarize the } \\
\text { PHMC with each project including current cost, scope and } \\
\text { schedule. }\end{array}$ & $7 / 01 / 96$ & JD Berger \\
\hline $\begin{array}{l}\text { 5. Discuss the concept of the joint contractor technology } \\
\text { management team including the role of the PHMC. Introduce } \\
\text { PHMC personnel to the individuals making up the team and to } \\
\text { the Program Management Plan that describes the activities the } \\
\text { team is responsible for. }\end{array}$ & $9 / 15 / 96$ & JD Berger \\
\hline
\end{tabular}

\subsubsection{Technology Integration (Implementation), Site Technology Coordination Group Support (STCG)}

The Technology Integration role functions as part of the joint RL contractor team and provides a major benefit to the contractor making decisions on end use of technology. The personnel filling this role facilitate a technology user organization connection with the providers of technology (EM-50 and others) and the projects that can potentially benefit. It is a "brokering" or facilitation role where candidate technologies are identified and "providers" are connected to "users." This effort functions as part of the STCG and is further facilitated by a direct tie to the Site Technical Integration effort and the Systems Engineering approach.

\begin{tabular}{|c|c|c|}
\hline Pre-Award Actions & $\begin{array}{l}\text { Schedulea } \\
\text { Date }\end{array}$ & Actone (s) \\
\hline $\begin{array}{l}\text { 1. Assemble key program (or project) documentation, or identify } \\
\text { its location, for use by the PHMC. Includes development of a } \\
\text { program/project "Smart Book," including listings and } \\
\text { descriptions developed below. }\end{array}$ & $6 / 01 / 96$ & JD Berger \\
\hline $\begin{array}{l}\text { 2. Identify number of briefings, and prepare program (or project) } \\
\text { briefing(s) for presentation to PHMC. }\end{array}$ & $6 / 01 / 96$ & JD Berger \\
\hline
\end{tabular}




\begin{tabular}{|c|c|c|}
\hline Pre-Awra Actions & $\begin{array}{l}\text { Schedulod } \\
\text { Date }\end{array}$ & Actiopee(s) \\
\hline $\begin{array}{l}\text { 3. Develop a listing of key program (or project) individuals as } \\
\text { POCs, including facility and operations personnel. }\end{array}$ & 6/01/96 & JD Berger \\
\hline $\begin{array}{l}\text { 4. Identify space and equipment within program (or project) area } \\
\text { and facilities for use by the PHMC. }\end{array}$ & 6/01/96 & JD Berger \\
\hline Post-Anard Aetions & . & \\
\hline $\begin{array}{l}\text { 1. Provide the PHMC access to the key program (or project) } \\
\text { documentation, any program level generated pre-existing } \\
\text { conditions information, listing of POCs, and listing of } \\
\text { subcontracts. Provide copies upon request. }\end{array}$ & $6 / 15 / 96$ & JD Berger \\
\hline $\begin{array}{l}\text { 2. PHMC identify space and equipment needs within } \\
\text { program/project area and facilities. }\end{array}$ & $6 / 08 / 96$ & JD Berger \\
\hline $\begin{array}{l}\text { 3. Program/project and facility space and services available for } \\
\text { use by the PHMC. }\end{array}$ & $6 / 15 / 96$ & JD Berger \\
\hline 4. Provide program/project briefing(s) to the PHMC. & $\begin{array}{c}6 / 15- \\
7 / 01 / 96\end{array}$ & JD Berger \\
\hline 5. Provide program/project level tour to the PHMC. & $\begin{array}{c}6 / 15- \\
7 / 01 / 96\end{array}$ & ID Berger \\
\hline
\end{tabular}

\subsection{GENERAL COUNSEL}

The General Counsel area includes Contract Administration and Legal matter for WHC. This section will outline the transitional requirements for both sections of the Office of General Counsel. The completion of a comprehensive Transition Agreement which will cover the transfer of WHC's obligations and liabilities to the PHMC or RL, and which rights to access records, information, and former employees in the defense of any retained litigation or in the event of post-transition litigation and/or claims, is considered the critical item that must be accomplished prior the completion of the transition process.

\subsubsection{Litigation and Claims}

\begin{tabular}{|l|l|l|}
\hline $\begin{array}{l}\text { 1. Prepare summary of status of litigation matters, administration } \\
\text { hearings, arbitrations and subcontractor claims and a } \\
\text { recommendation for transfer of some or all of these matter to } \\
\text { PHMC. Provide summary and recommendation to RL Office } \\
\text { of Chief Counsel. }\end{array}$ & $8 / 15 / 96$ & RA Dutton \\
\hline $\begin{array}{l}\text { 2. Negotiate transfer of some or all of the litigation, } \\
\text { administrative hearing, arbitrations and subcontractor claims to } \\
\text { PHMC including transfer of files and associated documents and } \\
\text { evidence. }\end{array}$ & 9/15/96 & RA Dutton \\
\hline
\end{tabular}




\subsubsection{Transition Agreement}

\begin{tabular}{|c|c|c|}
\hline postsward yetions & Wowedinted & Kretionee(s) \\
\hline $\begin{array}{l}\text { 1. Conduct discussion on scope and terms of Transition } \\
\text { Agreement with PHMC contractor counsel and RL Office of } \\
\text { Chief Counsel and others as may be necessary. }\end{array}$ & 9/01/96 & RA Dutton \\
\hline $\begin{array}{l}\text { 2. Prepare a draft of Transition Agreement and circulate for } \\
\text { comment. }\end{array}$ & $9 / 15 / 96$ & RA Dutton \\
\hline $\begin{array}{l}\text { 3. Assemble all documents to be incorporated into Transition } \\
\text { Agreement. }\end{array}$ & $9 / 15 / 96$ & $\begin{array}{l}\text { RA Dutton } \\
\text { IK Knoll }\end{array}$ \\
\hline 4. Negotiate, Finalize and Execute Agreement. & $9 / 30 / 96$ & $\begin{array}{l}\text { RA Dutton } \\
\text { PHMC } \\
\text { DOE }\end{array}$ \\
\hline
\end{tabular}

\subsection{INTERNAL AUDIT}

\begin{tabular}{|c|c|c|}
\hline Pro-Award Actions: & $\begin{array}{l}\text { Sehedule } \\
\text { Date }\end{array}$ & 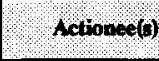 \\
\hline 1. Assemble current organizational data (charts and charters). & $5 / 31 / 96$ & LH Greenberg \\
\hline 2. Prepare FY 1997 Audit Plan and obtain RL approval. & $5 / 31 / 96$ & LH Greenberg \\
\hline 3. Identify and status all open audit findings. & $5 / 31 / 96$ & LH Greenbers \\
\hline Post-Award Actions & & \\
\hline $\begin{array}{l}\text { 1. Brief PHMC on overview of Internal Audit functions } \\
\text { - Audit organizational status } \\
\text { - M\&O Contractor \& OIG Cooperative Audit Strategy } \\
\text { - Open audit findings } \\
\text { - FY } 1997 \text { Audit Plan. }\end{array}$ & $7 / 01-8 / 01 / 96$ & LH Greenberg \\
\hline 2. Complete FY 1996 Audit Plan. & $9 / 30 / 96$ & LH Greenberg \\
\hline 3. Transfer actions to PHMC. & $9 / 30 / 96$ & LH Greenberg \\
\hline
\end{tabular}

\subsection{WORK FOR OTHERS}

Work For Others is established and meets the requirements as called for in Clauses H-17 and H-18 of the Model Contract. There are over 161 active orders with DOE, other government entities (local, state, and Federal), U.S. commercial and foreign businesses. These active orders have a total value of more than $\$ 99.0 \mathrm{M}$ and provide cost savings by helping lower overhead costs.

\begin{tabular}{|c|c|c|}
\hline Pre-Award Actions & $\begin{array}{c}\text { Scheoriled } \\
\text { Detes }\end{array}$ & Sctionet $(s)$ \\
\hline 1. Complete/finalize desk procedures to include transition actions. & $5 / 03 / 96$ & $\begin{array}{l}\text { AT Broady } \\
\text { J.M.Knoll }\end{array}$ \\
\hline
\end{tabular}




\begin{tabular}{|c|c|c|}
\hline Pre- , ward Actions & Senedited & Hethes $(s)$ \\
\hline 2. Update and complete active listing of WFO orders. & $5 / 03 / 96$ & $\begin{array}{l}\text { AT Broady } \\
\text { MM Knoll }\end{array}$ \\
\hline $\begin{array}{l}\text { 3. Develop eponsor address listing and draft assignment } \\
\text { notification to sponsors. }\end{array}$ & $5 / 17 / 96$ & $\begin{array}{l}\text { AT Broady } \\
\text { JM Knoll }\end{array}$ \\
\hline $\begin{array}{l}\text { 4. Review how transitions could impact assigned WFO personnel } \\
\text { to determine potential adverse affects. }\end{array}$ & $5 / 03 / 96$ & $\begin{array}{l}\text { AT Broady } \\
\text { JM Knoll }\end{array}$ \\
\hline 5. Prepare Briefing and orientation for newly assigned personnel. & $5 / 24 / 96$ & $\begin{array}{l}\text { AT Broady } \\
\text { MM Knoll }\end{array}$ \\
\hline Pod d ward Actons & & \\
\hline 1. Transmit assignment notifications to sponsors. & $6 / 30 / 96$ & $\begin{array}{l}\text { AT Broady } \\
\text { JM Knoll }\end{array}$ \\
\hline 2. Prepare order listing for newly assigned personnel. & $6 / 30 / 96$ & $\begin{array}{l}\text { AT Broady } \\
\text { M Knoll }\end{array}$ \\
\hline 3. Review and revise procedures to include new contractor. & $6 / 30 / 96$ & $\begin{array}{l}\text { AT Broady } \\
\text { JM Knoll }\end{array}$ \\
\hline $\begin{array}{l}\text { 4. Transfer all WFO files and listings to newly assigned } \\
\text { personnel. }\end{array}$ & $6 / 30 / 96$ & $\begin{array}{l}\text { AT Broady } \\
\text { MM Knoll }\end{array}$ \\
\hline
\end{tabular}

\subsubsection{Support to EM-40 Headquarters}

Technical support is provided to the DOE HQ EM-40 Program in the areas of management systems development, regulatory analysis, programmatic objective implementation, and environmental compliance review. The support provided by the M\&O Contractor is supplemented with additional technical expertise, when required, utilizing existing contracts. Funding for this effort comes directly from HQ via an HQ ADS. Reporting is directly to HQ with information copies to RL EM-40 Program Manager.

\begin{tabular}{|c|c|c|}
\hline Pre-Award Actions & Scheduled & Actionee $(s)$ \\
\hline $\begin{array}{l}\text { 1. Discuss description of the out year (FY } 1997 \text { plus) expectation } \\
\text { for this effort with HQ. }\end{array}$ & $6 / 01 / 96$ & J Berger \\
\hline $\begin{array}{l}\text { 2. Assemble key program (or project) documentation, or identify } \\
\text { its location, for use by the PHMC. Includes development of a } \\
\text { program/project "Smart Book," including listings and } \\
\text { descriptions developed below. }\end{array}$ & $6 / 01 / 96$ & JD Berger \\
\hline $\begin{array}{l}\text { 3. Identify number of briefings, and prepare program (or project) } \\
\text { briefing(s) for presentation to PHMC. }\end{array}$ & $6 / 01 / 96$ & JD Berger \\
\hline $\begin{array}{l}\text { 4. Develop a listing of key program (or project) individuals as } \\
\text { POCs, including facility and operations personnel. }\end{array}$ & $6 / 01 / 96$ & JD Berger \\
\hline
\end{tabular}




\begin{tabular}{|c|c|c|}
\hline Post-Award Ictions & Selhediled & Hefloyse (6) \\
\hline $\begin{array}{l}\text { 1. Provide the PHMC access to the key program (or project) } \\
\text { documentation, any program level generated pre-existing } \\
\text { conditions information, listing of POCs, and listing of } \\
\text { subcontracts. Provide copies upon request. }\end{array}$ & $6 / 15 / 96$ & JD Berger \\
\hline $\begin{array}{l}\text { 2. Provide year-to-date reports to the PHMC documenting work } \\
\text { performed. Brief the PHMC on all aspect of this work. }\end{array}$ & 7/01/96 & JD Berger \\
\hline $\begin{array}{l}\text { 3. Meet with key individuals, both contractor and DOE, to } \\
\text { receive briefings on work status and future expectations. }\end{array}$ & $8 / 01 / 96$ & JD Berger \\
\hline
\end{tabular}

\subsubsection{Support to EM - Lease and Administration of Contractor Support Facility}

The Trevion I Building 4th floor lease of 8,000 square feet of office space is used by 40 to 50 DOE Contractors assigned to provide specialized technical support to DOE Environmental Management (EM) Headquarters Divisions. WHC leases the 4th floor facility with the concurrence of RL and via direction and funding provided by the Office of Acquisition Management, EM-16. The contractor support facility is managed via subcontract with a local Germantown, Maryland firm. This building administration arrangement minimizes the support necessary from WHC and minimizes travel.

The administration subcontract provides space allocation, office supplies, computer, printer, copier, fax machine maintenance and suite security. The subcontractor also ensures compliance with building policy and coordinates resolution of issues with the owner as well as providing WHC monthly reports of building status.

All furnishings, including kitchen equipment, office furniture, conference room items, and computer desks were provided by WHC from direct funding provided by the DOE. The facility is equipped to handle 50 full time occupants or a combination of personnel assigned on short term projects and $\mathbf{3 0}$ to $\mathbf{4 0}$ full time occupants. Three large conference areas can be modified with modular partitions to accommodate a variety of needs.

\begin{tabular}{|l|l|l|}
\hline \multicolumn{1}{|c|}{ Pre-Award Actions } & $\begin{array}{c}\text { Scheduled } \\
\text { Date }\end{array}$ & Actionee(s) \\
\hline $\begin{array}{l}\text { 1. Determine future plans for this activity. How long will this } \\
\text { service be needed? Is the current facility adequate? }\end{array}$ & $5 / 15 / 96$ & JD Berger \\
\hline $\begin{array}{l}\text { 2. Determine when contracts for facility lease and administration } \\
\text { need to be extended/rebid. }\end{array}$ & $5 / 15 / 96$ & JD Berger \\
\hline $\begin{array}{l}\text { 3. Assemble key program (or project) documentation, or identify } \\
\text { its location, for use by the PHMC. Includes development of a } \\
\text { program/project "Smart Book," including listings and } \\
\text { descriptions developed below. }\end{array}$ & $6 / 01 / 96$ & ID Berger \\
\hline $\begin{array}{l}\text { 4. Identify number of briefings, and prepare program (or project) } \\
\text { briefing(s) for presentation to PHMC (coordinate number and } \\
\text { content with RL POC). }\end{array}$ & $6 / 01 / 96$ & JD Berger \\
\hline
\end{tabular}




\begin{tabular}{|c|c|c|}
\hline 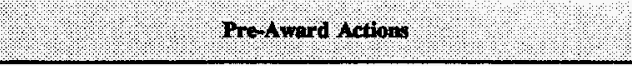 & $\begin{array}{l}\text { Scheduled } \\
\text { Date }\end{array}$ & Ictionee( \\
\hline $\begin{array}{l}\text { 5. Deveiop a listing of key program (or project) individuals as } \\
\text { POC, including facility and operations personnel. }\end{array}$ & $6 / 01 / 96$ & JD Berger \\
\hline $\begin{array}{l}\text { 6. Develop listing and description of program (or project) } \\
\text { subcontracts, including facility (or operations) subcontracts. } \\
\text { Include any subcontracts still planned to be executed prior to } \\
\text { contract takeover. }\end{array}$ & $6 / 01 / 96$ & JD Berger \\
\hline Post-Award Actions & & \\
\hline $\begin{array}{l}\text { 1. Provide the PHMC access to the key program (or project) } \\
\text { documentation, any program level generated pre-existing } \\
\text { conditions information, listing of POCs, and listing of } \\
\text { subcontracts. Provide copies upon request. }\end{array}$ & $6 / 15 / 96$ & JD Berger \\
\hline $\begin{array}{l}\text { 2. Brief the PHMC on the actions necessary to successfully } \\
\text { manage the Trevion I building. Identify the interfaces. }\end{array}$ & $7 / 01 / 96$ & JD Berger \\
\hline $\begin{array}{l}\text { 3. Visit the facility, meet with subcontractors (building owner and } \\
\text { administrator), inspect equipment, furniture, etc. to facilitate } \\
\text { turnover of all WHC controlled equipment, furniture, supplies } \\
\text { to the PHMC. Meet the interface contacts and discuss future } \\
\text { needs and funding arrangements. }\end{array}$ & $8 / 30 / 96$ & ID Berger \\
\hline $\begin{array}{l}\text { 4. Extend or rebid current contracts for facility lease and } \\
\text { administration. }\end{array}$ & $9 / 30 / 96$ & JD Berger \\
\hline
\end{tabular}

\subsection{OTHER MANAGEMENT AND INTEGRATION ACTIVITIES}

\subsubsection{Pollution Prevention/Waste Minimization}

The Pollution Prevention/Waste Minimization (P2/WMin) Program is implemented to meet Federal, State, and DOE regulations, policies, and reporting requirements. WHC provides planning, management, reporting, and oversight support for all P2/WMin on the Hanford Site. The activities include P2/WMin sitewide coordination, planning, reporting, training, awareness, assessments, incentives, maintenance of tracking systems, toxics reduction, recycling and affirmative procurement programs. A Center of Excellence has been formed within WHC, at RL direction, to provide a streamlined, low-cost enabler of P2/WMin activities across the site. Its purpose is to enable contractor/project generators to actively seek and implement initiatives to eliminate waste generation.

The P2/WMin Program established at Hanford is seen as a leader in the DOE complex and received 4 of the 10 national awards in 1995, as best in class. P2/WMin is DOE's preferred approach to EM because of its many cost and environmental benefits. In FY 1995, the Program documented a total of $\$ 26.4 \mathrm{M}$ in pollution prevention savings, and avoided generation of over 4.3 million kilograms of sanitary waste, 206,000 kilograms of hazardous waste, 32,000 cubic meters of radioactive waste, as well expenditure of 296,000 kilowatt hours of energy. 
The P2/WMin FY 1996 MYPP provides baseline requirements, milestones, and regulatory drivers. P2/WMin Program meets the requirements of the RFP DE-RP06-06RL13200, Sections B.1.b (Page C-15) and F (Page C-33).

\begin{tabular}{|c|c|c|}
\hline fre-Award Actions & $\begin{array}{l}\text { Sechoduled } \\
\text { Date }\end{array}$ & Actionee(s) \\
\hline $\begin{array}{l}\text { 1. Develop site guide to WHC, ICF KH, BCSR, PNNL and } \\
\text { ERC P2/WMin documents/records and reports. }\end{array}$ & $5 / 15 / 96$ & $\begin{array}{l}\text { JM Knoll, Jr. } \\
\text { JR Kirkendall }\end{array}$ \\
\hline $\begin{array}{l}\text { 2. Prepare a briefing on overall requirements/drivers/funding on } \\
\text { P2/WMin. }\end{array}$ & $5 / 24 / 96$ & $\begin{array}{l}\text { JM Knoll, Jr. } \\
\text { JR Kirkendall }\end{array}$ \\
\hline Post-Award Actions & ra & 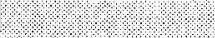 \\
\hline $\begin{array}{l}\text { 1. Brief PHMC on status, plans, and progress on P2/WMin } \\
\text { overall requirements/drivers/funding/ROI project successes } \\
\text { for RL and HQ ADSs. }\end{array}$ & $6 / 17 / 96$ & $\begin{array}{l}\text { JM Knoll, Jr. } \\
\text { JR Kirkendall }\end{array}$ \\
\hline $\begin{array}{l}\text { 2. Provide a list of P2/WMin POCs for major program areas, } \\
\text { Hanford Site Prime Contractors (ERC, PNNL, HEHF), } \\
\text { and RL. }\end{array}$ & $6 / 17 / 96$ & $\begin{array}{l}\text { JM Knoll, Jr. } \\
\text { JR Kirkendall }\end{array}$ \\
\hline $\begin{array}{l}\text { 3. Provide contractor team point of contacts for P2/WMin } \\
\text { sitewide regulatory/DOE reports and certification } \\
\text { requirements and transmittal of annual data reporting forms } \\
\text { to assure timely completion of reports. }\end{array}$ & $6 / 17 / 96$ & $\begin{array}{l}\text { JM Knoll, Jr. } \\
\text { J Kirkendall }\end{array}$ \\
\hline $\begin{array}{l}\text { 4. Brief PHMC on Pollution Prevention Opportunity Assessment } \\
\text { (P2OA) process. Provide materials and tools WHC used to } \\
\text { conduct P20As. }\end{array}$ & $6 / 17 / 96$ & $\begin{array}{l}\text { JM Knoll, Jr. } \\
\text { JR Kirkendall }\end{array}$ \\
\hline $\begin{array}{l}\text { 5. Provide P2/WMin site guide, documentation, and records } \\
\text { associated with P2/WMin. Establish management and staff } \\
\text { needs. }\end{array}$ & $6 / 21 / 96$ & $\begin{array}{l}\text { JM Knoll, Jr. } \\
\text { JR Kirkendall }\end{array}$ \\
\hline $\begin{array}{l}\text { 6. Turnover files on P2/WMin generator set-aside fee pilot } \\
\text { implementation to the PHMC to support RFP requirement. }\end{array}$ & $7 / 30 / 96$ & $\begin{array}{l}\text { JM Knoll, Jr. } \\
\text { JR Kirkendall }\end{array}$ \\
\hline
\end{tabular}

\subsubsection{U.S. Department of Energy Directives}

The current RFP clause H.14 covers the model contract requirements for Laws, Regulations, and DOE Directives. In turn, this clause describes how the new contractor will be expected to comply/integrate the DOE Directives. The listing of DOE Directives for compliance is contained in Section J, Appendix C of the RFP. There currently exists a process/system that receives the directives from the Contracting Officer, identifies the organization responsible for implementation, maintains the company DOE assigned database and works with organizations in preparing and submitting waivers requests to DOE.

\begin{tabular}{|c|c|c|}
\hline Pre-Award Actions & $\begin{array}{c}\text { Schediled } \\
\text { Date }\end{array}$ & Actionee (s) \\
\hline $\begin{array}{l}\text { 1. Compare current listing of Directives that are for WHC } \\
\text { compliance with the listing in the RFP and identify a listing of } \\
\text { differences. }\end{array}$ & $5 / 31 / 96$ & $\begin{array}{l}\text { AT Broady } \\
\text { JM Knoll, Jr. }\end{array}$ \\
\hline
\end{tabular}




\begin{tabular}{|c|c|c|}
\hline Prophrof Actions & $\begin{array}{l}\text { Scheduled } \\
\text { pete }\end{array}$ & tetiones (s) \\
\hline $\begin{array}{l}\text { 2. Complete listing of active waivers (deviations/exemptions), and } \\
\text { pending waivers. }\end{array}$ & $5 / 17 / 96$ & $\begin{array}{l}\text { AT Broady } \\
\text { JM Knoll, Jr. }\end{array}$ \\
\hline $\begin{array}{l}\text { 3. Uaing listing developed under item } 1 \text { above and the information } \\
\text { regarding organizations in the RFP identify proposed } \\
\text { organizations that will be responsible for unassigned directives. }\end{array}$ & $5 / 31 / 96$ & $\begin{array}{l}\text { AT Broady } \\
\text { JM Knoll, Jr. }\end{array}$ \\
\hline 4. Revise, as required, the Directives Desk Procedures. & $5 / 24 / 96$ & $\begin{array}{l}\text { AT Broady } \\
\text { M Knoll, Jr. }\end{array}$ \\
\hline \multicolumn{3}{|l|}{ Post A ward /ctions } \\
\hline $\begin{array}{l}\text { 1. Conduct briefing to newly assigned personnel on files and } \\
\text { listings pertaining to the administration of the Directives } \\
\text { including delta developed under No. } 1 \text { of Pre-Award actions. }\end{array}$ & $9 / 30 / 96$ & $\begin{array}{l}\text { AT Broady } \\
\text { JM Knoll, Jr. }\end{array}$ \\
\hline $\begin{array}{l}\text { 2. Transfer all completed files and listings pertaining to } \\
\text { Directives. }\end{array}$ & $9 / 30 / 96$ & $\begin{array}{l}\text { AT Broady } \\
\text { JM Knoll, Jr. }\end{array}$ \\
\hline $\begin{array}{l}\text { 3. Conduct training on the Directives Desk Procedures and } \\
\text { operation and maintenance of database. }\end{array}$ & $9 / 30 / 96$ & $\begin{array}{l}\text { AT Broady } \\
\text { JM Knoll, Jr. }\end{array}$ \\
\hline
\end{tabular}

\subsubsection{Hanford PeopleCORE}

Hanford PeopleCORE is a sitewide central database that collects information about Hanford RL and contractor people. It is the official source for distributing the information to over 100 key applications (e.g., site phone directory, medical records). Hanford PeopleCORE provides a single point for data collection, maintenance, and dissemination of information for all Hanford contractors and services.

\begin{tabular}{|l|c|l|}
\hline Scheduled & Date & Pre-Award Actions \\
\hline 1. Publish a Hanford PeopleCORE Contractor's User's Guide. & $5 / 29 / 96$ & $\begin{array}{l}\text { MK Britton } \\
\text { JM Knoll, Jr. }\end{array}$ \\
\hline $\begin{array}{l}\text { 1. Brief all new contractors and major subcontractors on Hanford } \\
\text { Site's PeopleCORE system and the site's utilization, including } \\
\text { feeder requirements to Hanford systems. }\end{array}$ & $7 / 15 / 96$ & MK Britton \\
\hline $\begin{array}{l}\text { 2. Identify issues, level of support, resources necessary to develop data } \\
\text { interfaces and processes for new contractor people information. }\end{array}$ & $\mathbf{8 / 0 1 / 9 6}$ & MK Britton \\
\hline $\begin{array}{l}\text { 3. Provide training and documentation to ensure a smooth transition of } \\
\text { Hanford PeopleCORE administrative responsibilities and } \\
\text { requirements. }\end{array}$ & $9 / 30 / 96$ & MK Britton \\
\hline
\end{tabular}




\subsection{HANFORD PROJECTS}

\subsection{TANK WASTE REMEDIATION SYSTEM}

TWRS manages the waste tank farms and the present and future tank waste disposal facilities. TWRS manages the operation of facilities associated with the receipt, containment, storage, and handling of liquid high-level wastes (HLWs) at Hanford, excluding some of those facilities generating the waste. In addition, TWRS develops and executes a program for the characterization, retrieval, pretreatment, and treatment for disposal of single-shell tank (SST) and double-shell tank (DST) wastes.

\begin{tabular}{|c|c|c|}
\hline 14 Pre-Award Actions & $\begin{array}{l}\text { Scheduled } \\
\text { Date }\end{array}$ & Actionee(b) \\
\hline $\begin{array}{l}\text { 1. In outline form for all facilities/operations to follow, } \\
\text { define the appropriate contents and level of detail to be } \\
\text { included in facility/operations briefings to the PHMC. } \\
\text { (Review with RL POC.) }\end{array}$ & $5 / 01 / 96$ & DL Lenseigne \\
\hline $\begin{array}{l}\text { 2. Assemble key program (or project) documentation, or } \\
\text { identify its location, for use by the PHMC. Includes } \\
\text { development of a program "Smart Book," including } \\
\text { listings and descriptions provided from Sections 4.1.1 } \\
\text { through 4.1.7. }\end{array}$ & $6 / 01 / 96$ & DL Lenseigne \\
\hline $\begin{array}{l}\text { 3. Identify number of briefings, and prepare program (or } \\
\text { project) briefing(s) for presentation to PHMC. } \\
\text { (Coordinate number and content with RL POC.) }\end{array}$ & $6 / 01 / 96$ & DL Lenseigne \\
\hline $\begin{array}{l}\text { 4. Identify space and equipment within program (or project) } \\
\text { area and facilities for use by the PHMC. }\end{array}$ & $6 / 01 / 96$ & DL Lenseigne \\
\hline Post-Award Actions & & s. \\
\hline $\begin{array}{l}\text { 1. PHMC identify space and equipment needs within } \\
\text { program/project area and facilities. }\end{array}$ & by $6 / 08 / 96$ & PHMC \\
\hline $\begin{array}{l}\text { 2. Provide the PHMC access to the key program (or project) } \\
\text { documentation, any program level generated pre-existing } \\
\text { conditions information, listing of POCs, and listing of } \\
\text { subcontracts. Provide copies upon request. }\end{array}$ & prior to $6 / 15 / 96$ & DL Lenseigne \\
\hline $\begin{array}{l}\text { 3. Provide program/project and facility space and services } \\
\text { for use by the PHMC. }\end{array}$ & $6 / 15 / 96$ & DL Lenseigne \\
\hline 4. Provide program/project briefing(s) to the PHMC. & $6 / 15 / 96-7 / 01 / 96$ & RD Wojtasek \\
\hline 5. Provide program/project level tour to the PHMC. & $6 / 15 / 96-7 / 01 / 96$ & RD Wojtasek \\
\hline
\end{tabular}




\subsubsection{Management Systems}

Manage the overall TWRS Program within funding levels provided by DOE to ensure that WHC business goals and objectives are achieved. Provide program management and other administrative functions required to identify and develop future missions, submit programmatic and financial reports to RL, and control budgets and other assigned resources.

\begin{tabular}{|c|c|c|}
\hline Pre-Award Actions & $\begin{array}{l}\text { Scheduled } \\
\text { Date }\end{array}$ & Retionee(s) \\
\hline $\begin{array}{l}\text { 1. Identify key documents. Provide listing to } \\
\text { program/project level for consolidation and RL review. }\end{array}$ & $5 / 01 / 96$ & WJ Schlauder \\
\hline $\begin{array}{l}\text { 2. Develop a listing of key program/project individuals as } \\
\text { POCs. Input to consolidated program listing. }\end{array}$ & $5 / 15 / 96$ & WJ Schlauder \\
\hline $\begin{array}{l}\text { 3. Develop a listing and description of subcontracts } \\
\text { supporting the program/project and input to } \\
\text { consolidated program/project listing. Include any } \\
\text { subcontracts planned to be executed prior to contract } \\
\text { takeover. }\end{array}$ & $5 / 15 / 96$ & DL Lenseigne \\
\hline $\begin{array}{l}\text { 4. Develop a listing and description of databases. Provide } \\
\text { list to program/project level for consolidation. }\end{array}$ & $5 / 15 / 96$ & $\begin{array}{l}\text { CE Wilson III } \\
\text { JD Thomson }\end{array}$ \\
\hline $\begin{array}{l}\text { 5. Identify number of briefings, and prepare briefing(s) } \\
\text { for the PHMC. (Coordinate number and content with } \\
\text { RL POC.) }\end{array}$ & $6 / 01 / 96$ & WJ Schlauder \\
\hline $\begin{array}{l}\text { 6. Assemble key documentation, or identify its location, } \\
\text { for use by the PHMC. Includes preparation of a } \\
\text { "Smart Book," to include information developed in the } \\
\text { following actions. }\end{array}$ & $6 / 01 / 96$ & WJ Schlauder \\
\hline $\begin{array}{l}\text { 7. Develop a list of open action items and commitments, } \\
\text { including status (Environmental, Tri-Party Agreement, } \\
\text { DNFSB as examples). Provide to RL POC for review. }\end{array}$ & $6 / 01 / 96$ & WJ Schlauder \\
\hline $\begin{array}{l}\text { 8. Develop a list of current work order services or other } \\
\text { form of agreement to provide work for other } \\
\text { organizations, on and offsite. }\end{array}$ & $6 / 01 / 96$ & WJ Schlauder \\
\hline $\begin{array}{l}\text { 9. Develop a list of current work order services or other } \\
\text { form of agreement to obtain work from other } \\
\text { organizations, on and offsite. }\end{array}$ & $6 / 01 / 96$ & WJ Schlauder \\
\hline $\begin{array}{l}\text { 10. Verify the accuracy of property assignments for the } \\
\text { facility (or operations) and correct any deficiencies. }\end{array}$ & $6 / 01 / 96$ & WJ Schlauder \\
\hline $\begin{array}{l}\text { 11. Identify space and equipment within program/project } \\
\text { area and facilities for use by the PHMC. }\end{array}$ & $6 / 01 / 96$ & WJ Schlauder \\
\hline Post-Award Actions & & \\
\hline $\begin{array}{l}\text { 1. Provide the PHMC access to key documentation, pre- } \\
\text { existing condition information, commitment data, } \\
\text { inventory data, etc. (as established in the pre-award } \\
\text { phase). Provide copies upon request. }\end{array}$ & by $6 / 15 / 96$ & WJ Schlauder \\
\hline
\end{tabular}




\begin{tabular}{|l|c|l|}
\hline \multicolumn{1}{|c|}{ Post-Award Actions } & Scheduled & Nationee(s) \\
\hline $\begin{array}{l}\text { 2. Provide any additional documentation or information } \\
\text { requested by the PHMC, if available, that were not } \\
\text { identified during the pre-aWard phase. }\end{array}$ & by $7 / 01 / 96$ & WJ Schlauder \\
\hline 3. Provide briefing(s) to the PHMC. & $7 / 01 / 96-7 / 15 / 96$ & WJ Schlauder \\
\hline 4. Provide a tour to the PHMC. & $7 / 01 / 96-7 / 15 / 96$ & WJ Schlauder \\
\hline $\begin{array}{l}\text { 5. Provide any recurring reports that the PHMC would } \\
\text { like to receive during transition. }\end{array}$ & by $7 / 15 / 96$ & WJ Schlauder \\
\hline $\begin{array}{l}\text { 6. Provide an updated status of ongoing work prior to } \\
\text { contract turnover. }\end{array}$ & by $9 / 30 / 96$ & WJ Schlauder \\
\hline
\end{tabular}

\subsubsection{Tank Farm Operations - Evaporator}

Ensure environmentally sound operations of the 242-A Evaporator Facility, including all auxiliary equipment associated with handling tank waste for evaporative waste volume reduction. Perform functions required for evaporation of water from dilute waste and support to facilities and processes. These functions include preventative and corrective maintenance, facility concentration operations including waste transfer to feed tanks, surveillance monitoring, health physics activities, industrial hygiene and safety functions, and engineering and analysis. Responsible for managing and controlling upgrades to facilities and infrastructure and enhancing the safety of facility operations.

Responsible for the following facilities:

1. 242-A Evaporator Facility

2. 241-AP Tank Farm

3. 241-AW Tank Farm

4. Liquid Effuent Retention Facility (operation only).

\begin{tabular}{|c|c|c|c|}
\hline & Pre-Award Actions & $\begin{array}{c}\text { Scheduled } \\
\text { Date }\end{array}$ & Actionee(s) \\
\hline 1. & $\begin{array}{l}\text { Develop and validate } \bullet \text { comprebensive list of facilities } \\
\text { owned \& operated by the Evaporator Project. }\end{array}$ & $3 / 29 / 96$ & AK Gardner \\
\hline 2. & $\begin{array}{l}\text { Identify key documents for the facility or operations. } \\
\text { Provide listing to program/project level for } \\
\text { consolidation and RL review. }\end{array}$ & $5 / 01 / 96$ & $\begin{array}{l}\text { MD Stratton (Evaporator) } \\
\text { LA Garner (Environmental) } \\
\text { LE Simmons (Training) } \\
\end{array}$ \\
\hline & $\begin{array}{l}\text { Develop a comprehensive list of open-ended } \\
\text { compliance issues/(Hanford Action Tracking System } \\
\text { [HATS] as an example).Provide to RL POC for } \\
\text { review. }\end{array}$ & $5 / 01 / 96$ & $\begin{array}{l}\text { CL Day-Phalen (HATS) } \\
\text { LA Garner (Environmental) } \\
\text { LM Calderon (Safety) } \\
\text { CL Caldwell (RadCon) } \\
\text { I Weber (QA) } \\
\text { MD Stratton (CONOPs) } \\
\text { EJ Austin (S/RIDs Phase 1) }\end{array}$ \\
\hline
\end{tabular}









\begin{tabular}{|c|c|c|c|}
\hline & Pred A ward Actions & $\begin{array}{l}\text { Schedoled } \\
\text { Dute }\end{array}$ & $x+10 n-1)$ \\
\hline 17. & $\begin{array}{l}\text { Develop a list of permits governing the facility or } \\
\text { operation, and provide a description of each as to } \\
\text { where and how it applies. }\end{array}$ & $6 / 01 / 96$ & LA Garner \\
\hline 18. & $\begin{array}{l}\text { Verify the accuracy of property assignments for the } \\
\text { facility or operations and correct any deficiencies. }\end{array}$ & $6 / 01 / 96$ & $\begin{array}{l}\text { CL Day-Phalen (Portable } \\
\text { capital equipment \& software } \\
\text { accountability) } \\
\text { AK Gardner (other) }\end{array}$ \\
\hline 19. & $\begin{array}{l}\text { Identify space and equipment within program/project } \\
\text { area and facilities for use by the PHMC. }\end{array}$ & $6 / 01 / 96$ & RD Gustaveon \\
\hline & Post-A ward Actions & & \\
\hline 1. & $\begin{array}{l}\text { Provide the PHMC access to key documentation, pre- } \\
\text { existing condition information, commitment data, } \\
\text { inventory data, etc. (as established in the pre-award } \\
\text { phase). Provide copies upon request. }\end{array}$ & by $6 / 15 / 96$ & $\begin{array}{l}\text { RD Gustavson } \\
\text { MD Stratton }\end{array}$ \\
\hline 2. & $\begin{array}{l}\text { Provide any additional documentation or information } \\
\text { requested by the PHMC, if available, that were not } \\
\text { identified during the pre-award phase. }\end{array}$ & by $7 / 01 / 96$ & $\begin{array}{l}\text { RD Gustavson } \\
\text { MD Stratton }\end{array}$ \\
\hline 3. & Provide facility or operations briefing(s) to the PHMC. & $7 / 01 / 96-7 / 15 / 96$ & $\begin{array}{l}\text { RD Gustavson } \\
\text { MD Stratton }\end{array}$ \\
\hline 4. & Provide a facility or operations tour to the PHMC. & $7 / 01 / 96-7 / 15 / 96$ & $\begin{array}{l}\text { RD Gustavson } \\
\text { MD Stratton }\end{array}$ \\
\hline & $\begin{array}{l}\text { Provide recurring reports that the PHMC would like to } \\
\text { receive during transition. }\end{array}$ & by $7 / 15 / 96$ & $\begin{array}{l}\text { RD Gustavson } \\
\text { MD Stratton }\end{array}$ \\
\hline 6. & Provide necessary training to PHMC personnel. & $7 / 01 / 96-8 / 01 / 96$ & $\begin{array}{l}\text { RD Gustavson } \\
\text { MD Stratton }\end{array}$ \\
\hline & $\begin{array}{l}\text { Assist the PHMC in the conduct of a Pre-Existing } \\
\text { Condition Assessment }\end{array}$ & $7 / 15 / 96-8 / 30 / 96$ & $\begin{array}{l}\text { RD Gustavson } \\
\text { MD Stratton }\end{array}$ \\
\hline & $\begin{array}{l}\text { Perform a joint property and equipment inventory with } \\
\text { the PHMC. }\end{array}$ & by $8 / 30 / 96$ & $\begin{array}{l}\text { RD Gustavson } \\
\text { MD Stratton }\end{array}$ \\
\hline & $\begin{array}{l}\text { Provide an updated status of ongoing work prior to } \\
\text { contract turnover. }\end{array}$ & by $9 / 30 / 96$ & $\begin{array}{l}\text { RD Gustavson } \\
\text { MD Stratton }\end{array}$ \\
\hline 10. & Provide a categorized inventory of wastes. & by $9 / 30 / 96$ & $\begin{array}{l}\text { RD Gustavson } \\
\text { MD Stratton }\end{array}$ \\
\hline
\end{tabular}

\subsubsection{Tank Farm Operations - 200 East Tank Farms}

Ensure environmentally sound operations of the 200 East tank farms, including all facilities and auxiliary equipment associated with handling and storing tank waste. Manage the 200 East Tank Farm Transition Project to establish the SST farms (200 East) into a controlled, clean, and stable configuration in a timely and cost-effective manner. Conduct all activities pertaining to the operation of a permitted TSD facility within the boundary of the current Interim Safety Basis and environmental permits in a manner that ensures compliance 
with all applicable Federal, state, and local environmental regulations. Perform all support functions required for routine surveillance, operation, and maintenance of the 200 East Area Transition Project facilities to safely monitor status and control of the underground storage of waste. These functions include preventative and corrective maintenance, safe facility operations, waste transfer, onsite acceptance of wastes for tank storage, surveillance monitoring, health physics activities, industrial hygiene and safety functions, engineering and analysis, managing and controlling upgrades to facilities and infrastructure, and preparation for facility turnover to the Waste Disposal Program.

List of major facilities:

- 241-A, -C, -B, -BX, -BY, -AN, -AX, -AZ, and -AY Tank Farms

- 204-AR Unloading Facility

- 209-E Critical Mass Laboratory

- 209-EA 90-Day Storage

- 2400E Dry Material Handling Facility

- 244-AR, Sludge Vault Storage and Processing Facility

- 272-AW TFO Support Facility

- 216-B-62, 216-B-63, 216-A-08, 216-B-55, 216-A-30, 216-A-37-2, 216-A-45 Cribs

- 216-B-3 Expansion Ponds

- 244-BX Vault

- 244-CR Vault

- 244-A Lift Station

- 216-E-16-101 PSW Grout Vault

- 243-G Grout Processing Facility

- Cross-Site Transfer Line (East of 241-EW Vent Station)

- 274-AW Office Building

- 278-AW Document Storage Area

- Miscellaneous transfer piping and units ancillary to Tank Farm facilities

- 14 Inactive Miscellaneous Storage Tanks (IMUSTs).

\begin{tabular}{|c|c|c|}
\hline Pre-Award Actions & $\begin{array}{l}\text { Scheduled } \\
\text { Date }\end{array}$ & Actionee(s) \\
\hline 1. Produce and verify facility ownership listing. & $3 / 29 / 96$ & P Hinojosa \\
\hline $\begin{array}{l}\text { 2. Identify key documents for the facility or } \\
\text { operations. Provide listing to program/project } \\
\text { level for consolidation and RL review. }\end{array}$ & $5 / 01 / 96$ & $\begin{array}{l}\text { P Honojosa (East) } \\
\text { LA Garner (Environmental) } \\
\text { LE Simmons (Training) } \\
\end{array}$ \\
\hline $\begin{array}{l}\text { 3. Develop a comprehensive list of open-ended } \\
\text { compliance issues (HATS as an example). Provide } \\
\text { to RL POC for review. }\end{array}$ & $5 / 01 / 96$ & $\begin{array}{l}\text { CL Day-Phalen (HATS) } \\
\text { LA Garner (Environmental) } \\
\text { LM Calderon (Safety) } \\
\text { CL Caldwell (RadCon) } \\
\text { J Weber (QA) } \\
\text { P Hinojosa (CONOPs) } \\
\text { EF Austin (S/RIDs Phase 1) }\end{array}$ \\
\hline $\begin{array}{l}\text { 4. Identify POCs for the facility or operations and } \\
\text { input to consolidated program/project listing. }\end{array}$ & $5 / 15 / 96$ & P Hinojosa \\
\hline
\end{tabular}




\begin{tabular}{|c|c|c|}
\hline Prestrard Actions & ochediled & 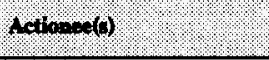 \\
\hline $\begin{array}{l}\text { 5. Develop a listing and description of subcontracts } \\
\text { supporting the facility or operation and input to } \\
\text { coneolidated program/project listing. Include any } \\
\text { subcontracts planned to be executed prior to } \\
\text { contract takeover. }\end{array}$ & $5 / 15 / 96$ & RA Finke \\
\hline $\begin{array}{l}\text { 6. Develop a listing and description of facility or } \\
\text { operations databases. Provide list to } \\
\text { program/project level for consolidation. }\end{array}$ & $5 / 15 / 96$ & RB Bass \\
\hline $\begin{array}{l}\text { 7. Identify number of briefings, and prepare facility } \\
\text { or operations briefing(s) for the PHMC. } \\
\text { (Coordinate number and content with RL POC.) }\end{array}$ & $6 / 01 / 96$ & P Hinojosa \\
\hline $\begin{array}{l}\text { 8. Assemble key facility or operations documentation, } \\
\text { or identify its location, for use by the PHMC. } \\
\text { Includes preparation of a facility or operations } \\
\text { "Smart Book," to include information developed in } \\
\text { the following actions. }\end{array}$ & $6 / 01 / 96$ & P Hinojosa \\
\hline $\begin{array}{l}\text { 9. Using the pre-existing checklist, compile pre- } \\
\text { existing condition information on the facility and/or } \\
\text { major equipment. Conduct, or schedule as a } \\
\text { separate post-award action(s), any assessments } \\
\text { required to address all relevant items on the } \\
\text { checklist. Provide completed checklist to RL POC } \\
\text { for review. }\end{array}$ & $6 / 01 / 96$ & $\begin{array}{l}\text { P Hinojosa } \\
\text { RD Gustavson } \\
\text { JF Brown (Environmental) } \\
\text { LM Calderon (Safety) } \\
\text { CL Caldwell (RadCon) } \\
\text { J Weber (QA) } \\
\text { EI Austin (S/RIDS Phase I) }\end{array}$ \\
\hline $\begin{array}{l}\text { 10. Develop a list of open action items and } \\
\text { commitments, including status (Environmental, Tri- } \\
\text { Party Agreement, DNFSB, Secretarial Safety } \\
\text { Initiatives, etc.). Provide to RL POC for review. }\end{array}$ & $6 / 01 / 96$ & $\begin{array}{l}\text { RL Nelson } \\
\text { LA Garner (Environmental) }\end{array}$ \\
\hline $\begin{array}{l}\text { 11. Identify current inventories of feed for the facility, } \\
\text { and materials to support continued operation (i.e., } \\
\text { essential materials, tools and equipment). }\end{array}$ & $6 / 01 / 96$ & $\begin{array}{l}\text { P Hinojosa (MatEquip) } \\
\text { WB Barton (Waste) }\end{array}$ \\
\hline $\begin{array}{l}\text { 12. Develop a list of facility or operation-specific } \\
\text { mandatory training and qualification requirements } \\
\text { for employees. }\end{array}$ & $6 / 01 / 96$ & LE Simmons \\
\hline $\begin{array}{l}\text { 13. Develop a listing of information which constintes } \\
\text { the "authorization basis" for the facility. }\end{array}$ & $6 / 01 / 96$ & $\begin{array}{l}\text { ML Cowen } \\
\text { JJ Klos }\end{array}$ \\
\hline $\begin{array}{l}\text { 14. Develop a description of ongoing and planned } \\
\text { construction projects. }\end{array}$ & $6 / 01 / 96$ & RD Gustaveon \\
\hline $\begin{array}{l}\text { 15. Develop a listing of special agreement services } \\
\text { provided to RL, other DOE sites, and other } \\
\text { entities. }\end{array}$ & $6 / 01 / 96$ & $\begin{array}{l}\text { P Hinojosa (Vadose zone, } \\
\text { Purgewater storage, LERF } \\
\text { Operation, etc.) }\end{array}$ \\
\hline $\begin{array}{l}\text { 16. Develop a list of current work order services or } \\
\text { other form of agreement to provide work for other } \\
\text { organizations, on and offsite. }\end{array}$ & $6 / 01 / 96$ & MI Yeager \\
\hline $\begin{array}{l}\text { 17. Develop a list of current work order services or } \\
\text { other form of agreement to obtain work from other } \\
\text { organizations, on and offsite. }\end{array}$ & $6 / 01 / 96$ & MI Yeager \\
\hline
\end{tabular}




\begin{tabular}{|c|c|c|}
\hline Pre-Award lactions & $\begin{array}{l}\text { Scheduled } \\
\text { Date. }\end{array}$ & Actionee(s) \\
\hline $\begin{array}{l}\text { 18. Develop a list of permits governing the facility or } \\
\text { operation, and provide a description of each as to } \\
\text { where and how it applies. }\end{array}$ & $6 / 01 / 96$ & LA Garner \\
\hline $\begin{array}{l}\text { 19. Verify the accuracy of property assignments for the } \\
\text { facility or operations and correct any deficiencies. }\end{array}$ & $6 / 01 / 96$ & $\begin{array}{l}\text { CL Day-Phalen (Portable capital } \\
\text { equipment \& software } \\
\text { accountability) } \\
\text { P Hinojosa (other) } \\
\end{array}$ \\
\hline $\begin{array}{l}\text { 20. Identify space and equipment within } \\
\text { program/project area and facilities for use by the } \\
\text { PHMC. }\end{array}$ & $6 / 01 / 96$ & RD Gustavson \\
\hline 14. Post-Award Actions & & \\
\hline $\begin{array}{l}\text { 1. Provide the PHMC access to key documentation, } \\
\text { pre-existing condition information, commitment } \\
\text { data, inventory data, etc. (as established in the pre- } \\
\text { award phase). Provide copies upon request. }\end{array}$ & by $6 / 15 / 96$ & $\begin{array}{l}\text { RD Gustavson } \\
\text { P Hinojosa }\end{array}$ \\
\hline $\begin{array}{l}\text { 2. Provide any additional documentation or } \\
\text { information requested by the PHMC, if available, } \\
\text { that were not identified during the pre-award } \\
\text { phase. }\end{array}$ & by $7 / 01 / 96$ & $\begin{array}{l}\text { RD Gustavson } \\
\text { P Hinojosa }\end{array}$ \\
\hline $\begin{array}{l}\text { 3. Provide facility or operations briefing(s) to the } \\
\text { PHMC. }\end{array}$ & $7 / 01 / 96-7 / 15 / 96$ & $\begin{array}{l}\text { RD Gustavson } \\
\text { P Hinojosa }\end{array}$ \\
\hline 4. Provide a facility or operations tour to the PHMC. & $7 / 01 / 96-7 / 15 / 96$ & $\begin{array}{l}\text { RD Gustavson } \\
\text { P Hinojosa } \\
\end{array}$ \\
\hline $\begin{array}{l}\text { 5. Provide any recurring reports that the PHMC } \\
\text { would like to receive during transition. }\end{array}$ & by $7 / 15 / 96$ & $\begin{array}{l}\text { RD Gustavson } \\
\text { P Hinojosa } \\
\end{array}$ \\
\hline 6. Provide necessary training to PHMC personnel. & $7 / 01 / 96-8 / 01 / 96$ & $\begin{array}{l}\text { RD Gustavson } \\
\text { P Hinojosa }\end{array}$ \\
\hline $\begin{array}{l}\text { 7. Assist the PHMC in the conduct of a Pre-Existing } \\
\text { Condition Assessment }\end{array}$ & $7 / 15 / 96-8 / 30 / 96$ & \begin{tabular}{|l} 
RD Gustavson \\
P Hinojosa
\end{tabular} \\
\hline $\begin{array}{l}\text { 8. Provide an updated status of ongoing work prior to } \\
\text { contract turnover. }\end{array}$ & by $9 / 30 / 96$ & $\begin{array}{l}\text { RD Gustavson } \\
\text { P Hinojosa } \\
\end{array}$ \\
\hline $\begin{array}{l}\text { 9. Perform a joint property and equipment inventory } \\
\text { with the PHMC. }\end{array}$ & by $8 / 30 / 96$ & $\begin{array}{l}\text { RD Gustavson } \\
\text { P Hinojosa } \\
\end{array}$ \\
\hline 10. Provide a categorized inventory of wastes. & by $9 / 30 / 96$ & $\begin{array}{l}\text { RD Gustavson } \\
\text { P Hinojosa }\end{array}$ \\
\hline
\end{tabular}

\subsubsection{Tank Farm Operations - 200 West Tank Farms}

Ensure environmentally sound operations of the 200 West tank farms, including all facilities and auxiliary equipment associated with handling and storing tank waste. Manage the 200 West Tank Farm Transition Project to establish the SST farms (200 West) into a controlled, clean, and stable configuration in a timely and cost-effective manner. Conduct all activities pertaining to the operation of a permitted TSD facility within the boundary of the 
current Interim Safety Basis and environmental permits in a manner that ensures compliance with all applicable Federal, state, and local environmental regulations. Perform all support functions required for routine surveillance, operation, and maintenance of the 200 West Area Transition Project facilities to safely monitor status and control of the underground storage of waste. These functions include preventative and corrective maintenance, facility operations including waste transfer, onsite acceptance of wastes for tank storage, surveillance monitoring, health physics activities, industrial hygiene and safety functions, and engineering and analysis. Responsible for managing and controlling upgrades to facilities and infrastructure, enhancing the safety of facility operations, and preparing for the eventual facility turnover to the Waste Disposal Program.

List of major facilities:

- 207-T Retention Basins

- 241-S, -SX, -SY, -T, -TX, -TY, -U Tank Farms

- 216-S-25, 216-S-26, 216-U-16, 216-U-17, 216-W-LC, 216-Z-20 Cribs

- 216-T-1, 216-T-4-2, 216-U-14 Ditches

- 216-Z-21 Pond

- 242-S and 242-T Evaporator Facilities

- 244-S, -U, -TX DCRT Vaults

- 241-TX-302C Catch Tank

- 241-S-302 Catch Tank

- 2727-W, and -WA Sodium Storage Facility

- 272-WA TFO Support Facility

- 278-WA Document Control Center

- 241-EW Vent Station

- Cross-Site Transfer Lines (West of and including the 241-EW Vent Station)

- 22 IMUSTs

- Miscellaneous transfer piping and units ancillary to tank farm facilities.

\begin{tabular}{|c|c|c|}
\hline Pre-Award Actions & $\begin{array}{l}\text { Scheduled } \\
\text { Date }\end{array}$ & Actionee(s) \\
\hline $\begin{array}{l}\text { 1. Develop and validate a comprehensive list of facilities } \\
\text { owned \& operated by West Tank Farm Transition } \\
\text { Project. }\end{array}$ & $3 / 29 / 96$ & LC Mercado \\
\hline $\begin{array}{l}\text { 2. Identify key documents for the facility or operations. } \\
\text { Provide listing to program/project level for } \\
\text { consolidation and RL review. }\end{array}$ & $5 / 01 / 96$ & $\begin{array}{l}\text { LC Mercado (West) } \\
\text { LA Garner (Environmental) } \\
\text { LE Simmons (Training) }\end{array}$ \\
\hline $\begin{array}{l}\text { 3. Develop a comprehensive list of open-ended } \\
\text { compliance issues (HATS as an example). Provide to } \\
\text { RL POC for review. }\end{array}$ & $5 / 01 / 96$ & $\begin{array}{l}\text { CL Day-Phalen (HATS) } \\
\text { LA Garner (Environmenta) } \\
\text { LM Calderon (Safety) } \\
\text { SL Bump (RadCon) } \\
\text { J Weber (QA) } \\
\text { LC Mercado (CONOPs) } \\
\text { EJ Austin (S/RIDs Phase 1) }\end{array}$ \\
\hline $\begin{array}{l}\text { 4. Identify POCs for the facility or operations and input to } \\
\text { consolidated program/project listing. }\end{array}$ & $5 / 15 / 96$ & LC Mercado \\
\hline
\end{tabular}




\begin{tabular}{|c|c|c|c|}
\hline & Pre-Award Actions & $\begin{array}{l}\text { Schedilud } \\
\text { Dote }\end{array}$ & Hotopec() \\
\hline 5. & $\begin{array}{l}\text { Develop a listing and description of subcontracts } \\
\text { supporting the facility or operation and input to } \\
\text { consolidated program/project listing. Include any } \\
\text { subcontracts planned to be executed prior to contract } \\
\text { takeover. }\end{array}$ & $5 / 15 / 96$ & RA Finke \\
\hline 6. & $\begin{array}{l}\text { Develop a listing and description of facility or } \\
\text { operations databases. Provide list to program/project } \\
\text { level for consolidation. }\end{array}$ & $5 / 15 / 96$ & RB Bass \\
\hline 7. & $\begin{array}{l}\text { Identify number of briefings, and prepare facility (or } \\
\text { operations) briefing(s) for the PHMC. (Coordinate } \\
\text { number and content with RL POC.) }\end{array}$ & $6 / 01 / 96$ & LC Mercado \\
\hline 8. & $\begin{array}{l}\text { Assemble key facility or operations documentation, or } \\
\text { identify its location, for use by the PHMC. Includes } \\
\text { preparation of a facility or operations "Smart Book," to } \\
\text { include information develop in the following actions. }\end{array}$ & $6 / 01 / 96$ & $\begin{array}{l}\text { LC Mercado } \\
\text { RD Gustavson }\end{array}$ \\
\hline 9. & $\begin{array}{l}\text { Using the pre-existing checklist, compile pre-existing } \\
\text { condition information on the facility and/or major } \\
\text { equipment. Conduct, or schedule as a separate post- } \\
\text { award action(s), any assessments required to address all } \\
\text { relevant items on the checklist. Provide completed } \\
\text { checklist to RL POC for review. }\end{array}$ & 6/01/96 & $\begin{array}{l}\text { LC Mercado } \\
\text { RD Gustavson } \\
\text { JM Barnett (Environmental) } \\
\text { LA Calderon (Safety) } \\
\text { SL Bump (RadCon) } \\
\text { J Weber (QA) } \\
\text { EJ Austin (S/RID Phase I) } \\
\end{array}$ \\
\hline 10. & $\begin{array}{l}\text { Develop a list of open action items and commitments, } \\
\text { including status (Environmental, Tri-Party Agreement, } \\
\text { DNFSB, Secretarial Safety Initiatives, etc.). Provide } \\
\text { to RL POC for review. }\end{array}$ & $6 / 01 / 96$ & $\begin{array}{l}\text { RL Nelson } \\
\text { L.A Garner (Environmental) }\end{array}$ \\
\hline 11. & $\begin{array}{l}\text { Identify current inventories of feed for the facility, and } \\
\text { materials to support continued operation (i.e., essential } \\
\text { materials, tools and equipment). }\end{array}$ & $6 / 01 / 96$ & $\begin{array}{l}\text { WB Barton (Waste) } \\
\text { LC Mercado (Mat/Equip) }\end{array}$ \\
\hline 12. & $\begin{array}{l}\text { Develop a list of facility or operation-specific } \\
\text { mandatory training and qualification requirements for } \\
\text { employees. }\end{array}$ & 6/01/96 & LE Simmons \\
\hline & $\begin{array}{l}\text { Develop a listing of information which constitutes the } \\
\text { "authorization basis" for the facility. }\end{array}$ & $6 / 01 / 96$ & $\begin{array}{l}\text { ML Cowen } \\
\text { JJ Klos } \\
\end{array}$ \\
\hline & $\begin{array}{l}\text { Develop a description of ongoing and planned } \\
\text { construction projects. }\end{array}$ & $6 / 01 / 96$ & RD Gustavson \\
\hline & $\begin{array}{l}\text { Develop a list of current work order services or other } \\
\text { form of agreement to provide work for other } \\
\text { organizations, on and offsite. }\end{array}$ & $6 / 01 / 96$ & JS Thomas \\
\hline & $\begin{array}{l}\text { Develop a list of current work order services or other } \\
\text { form of agreement to obtain work from other } \\
\text { organizations, on and offsite. }\end{array}$ & 6/01/96 & JS Thomas \\
\hline & $\begin{array}{l}\text { Develop a list of permits governing the facility or } \\
\text { operation, and provide a description of each as to } \\
\text { where and how it applies. }\end{array}$ & $6 / 01 / 96$ & LA Garner \\
\hline
\end{tabular}




\begin{tabular}{|c|c|c|c|}
\hline & Pre-A ward hetions & $\begin{array}{l}\text { Schedaled } \\
\text { Date }\end{array}$ & (retionec(o) \\
\hline & $\begin{array}{l}\text { Verify the accuracy of property assignments for the } \\
\text { facility or operations and correct any deficiencies. }\end{array}$ & 6/01/96 & $\begin{array}{l}\text { CL Day-Phalen (portabie capital } \\
\text { equipment \& eoftware } \\
\text { accountability) } \\
\text { LC Mercado (other) }\end{array}$ \\
\hline 19. & $\begin{array}{l}\text { Identify space and equipment within program/project } \\
\text { area and facilities for use by the PHMC. }\end{array}$ & $6 / 01 / 96$ & RD Gustavson \\
\hline & Post-Avard Actions & & \%: \\
\hline 1. & $\begin{array}{l}\text { Provide the PHMC access to key documentation, pre- } \\
\text { oxisting condition information, commitment data, } \\
\text { inventory data, etc. (as established in the pre-award } \\
\text { phase). Provide copies upon request. }\end{array}$ & by $6 / 15 / 96$ & $\begin{array}{l}\text { RD Guetavion } \\
\text { LC Mercado }\end{array}$ \\
\hline 2. & $\begin{array}{l}\text { Provide any additional documentation or information } \\
\text { requested by the PHMC, if available, that were not } \\
\text { identified during the pre-award phase. }\end{array}$ & by $7 / 01 / 96$ & $\begin{array}{l}\text { RD Gustavson } \\
\text { LC Mercado }\end{array}$ \\
\hline 3. & Provide facility or operations briefing(s) to the PHMC. & $\begin{array}{l}7 / 01 / 96- \\
7 / 15 / 96\end{array}$ & $\begin{array}{l}\text { RD Gustavson } \\
\text { LC Mercado }\end{array}$ \\
\hline & Provide a facility or operations tour to the PHMC. & $\begin{array}{l}7 / 01 / 96- \\
7 / 15 / 96 \\
\end{array}$ & $\begin{array}{l}\text { RD Gustavson } \\
\text { LC Mercado }\end{array}$ \\
\hline 5. & $\begin{array}{l}\text { Provide any recurring reports that the PHMC would } \\
\text { like to receive during transition. }\end{array}$ & by $7 / 15 / 96$ & $\begin{array}{l}\text { RD Gustavson } \\
\text { LC Mercado } \\
\end{array}$ \\
\hline 6. & Provide necessary training to PHMC personnel. & $\begin{array}{l}\text { 7/01/96- } \\
8 / 01 / 96\end{array}$ & $\begin{array}{l}\text { RD Gustavson } \\
\text { LC Mercado }\end{array}$ \\
\hline & $\begin{array}{l}\text { Assist the PHMC in the conduct of a Pre-Existing } \\
\text { Condition Assessment. }\end{array}$ & $\begin{array}{l}7 / 15 / 96- \\
8 / 01 / 96 \\
\end{array}$ & $\begin{array}{l}\text { RD Gustavson } \\
\text { LC Mercado } \\
\end{array}$ \\
\hline 8. & $\begin{array}{l}\text { Perform a joint property and equipment inventory with } \\
\text { the PHMC. }\end{array}$ & by $8 / 30 / 96$ & $\begin{array}{l}\text { RD Gustavson } \\
\text { LC Mercado } \\
\end{array}$ \\
\hline 9. & $\begin{array}{l}\text { Provide an updated starus of ongoing work prior to } \\
\text { contract turnover. }\end{array}$ & by $9 / 30 / 96$ & $\begin{array}{l}\text { RD Gustavson } \\
\text { LC Mercado } \\
\end{array}$ \\
\hline & Provide a categorized inventory of wastes. & by $9 / 30 / 96$ & $\begin{array}{l}\text { RD Gustaveon } \\
\text { LC Mercado }\end{array}$ \\
\hline
\end{tabular}

\subsubsection{Safety Issue Resolution/TWRS Engineering}

The TWRS Chief Engineer is the TWRS Design Authority and has overall responsibility for establishing, implementing, and maintaining the TWRS technical baseline, the TWRS Authorization Basis and SMS and provides safety analysis, licensing, process engineering, and related technical services to TWRS facility and project organizations, using systems engineering processes, tools, and methodologies. 


\begin{tabular}{|c|c|c|}
\hline Pre Award Sctions & Woherised & Setionec $(3)$ \\
\hline $\begin{array}{l}\text { 1. Identify key documents. Provide listing to } \\
\text { program/project level for consolidation and } R L \\
\text { review. }\end{array}$ & $5 / 01 / 96$ & DJ Berryman \\
\hline $\begin{array}{l}\text { 2. Identify POCs and input to the consolidated } \\
\text { program/project listing. }\end{array}$ & $5 / 15 / 96$ & DJ Berryman \\
\hline $\begin{array}{l}\text { 3. Develop a listing and description of subcontracts } \\
\text { cupporting the facility (or operation) and input to } \\
\text { consolidated program/project listing. Include any } \\
\text { subcontracts planned to be executed prior to contract } \\
\text { takeover. }\end{array}$ & $5 / 15 / 96$ & DJ Berryman \\
\hline $\begin{array}{l}\text { 4. Develop a listing and description of databases. } \\
\text { Provide list to program/project level for consolidation. }\end{array}$ & $5 / 15 / 96$ & $\begin{array}{l}\text { RJ Cash } \\
\text { SR Long }\end{array}$ \\
\hline $\begin{array}{l}\text { 5. Identify number of briefings, and prepare briefing(s) } \\
\text { for the PHMC. (Coordinate number and content with } \\
\text { RL POC.) }\end{array}$ & $6 / 01 / 96$ & AM Umek \\
\hline $\begin{array}{l}\text { 6. Assemble key documentation, or identify its location, } \\
\text { for use by the PHMC. Includes preparation of a } \\
\text { "Smart Book," which will include information } \\
\text { developed in the following actions. }\end{array}$ & $6 / 01 / 96$ & DJ Berryman \\
\hline $\begin{array}{l}\text { 7. Deveiop a list of open action items and commitments, } \\
\text { including status (Environmental, Tri-Party Agreement, } \\
\text { DNFSB as examples). Provide to RL POC for } \\
\text { review. }\end{array}$ & $6 / 01 / 96$ & SR Long \\
\hline $\begin{array}{l}\text { 8. Develop a listing of informatuon which constitutes the } \\
\text { "authorization basis" for the fecility. }\end{array}$ & $6 / 01 / 96$ & $\begin{array}{l}\text { M Cowen } \\
\text { JJ Klos }\end{array}$ \\
\hline $\begin{array}{l}\text { 9. Develop a list of curre nt wort order services or other } \\
\text { form of agreement to provide work for other } \\
\text { organizations, on and offute. }\end{array}$ & $6 / 01 / 96$ & $\begin{array}{l}\text { TL Denton } \\
\text { JP Sloughter }\end{array}$ \\
\hline $\begin{array}{l}\text { 10. Develop a list of current wort ofder services or other } \\
\text { form of agreement wohlus work from other } \\
\text { organizations, on and oflute. }\end{array}$ & $6 / 01 / 96$ & $\begin{array}{l}\text { DJ Berryman } \\
\text { DA Raap } \\
\text { TL Denton }\end{array}$ \\
\hline $\begin{array}{l}\text { 11. Verify the accuracy of property assignments for the } \\
\text { facility (or operations) and correct any deficiencies. }\end{array}$ & $6 / 01 / 96$ & LA Robinson \\
\hline $\begin{array}{l}\text { 12. Identify space and equipment within program/project } \\
\text { area and facilities for use by the PHMC. }\end{array}$ & $6 / 01 / 96$ & DJ Berryman \\
\hline Post-Award Actions & & \\
\hline $\begin{array}{l}\text { 1. Provide the PHMC access to key documentation, pre- } \\
\text { existing condition information, commitment data, } \\
\text { inventory data, etc. (as established in the pre-award } \\
\text { phase). Provide copies upon request. }\end{array}$ & by $6 / 15 / 96$ & DJ Berryman \\
\hline $\begin{array}{l}\text { 2. Provide program/project and facility space and services } \\
\text { for use by PHMC. }\end{array}$ & $6 / 15 / 96$ & DJ Berryman \\
\hline $\begin{array}{l}\text { 3. Provide any additional documentation or information } \\
\text { requested by the PHMC, if available, that were not } \\
\text { identified during the pre-award phase. }\end{array}$ & by $7 / 01 / 96$ & DJ Berryman \\
\hline
\end{tabular}




\begin{tabular}{|c|c|c|c|}
\hline & Post-Award Actions & $\begin{array}{l}\text { Scheduled } \\
\text { Date. }\end{array}$ & Actionee(s) \\
\hline 4. & Provide briefing(s) to the PHMC. & $7 / 01 / 96-7 / 15 / 96$ & AM Umek \\
\hline 5. & $\begin{array}{l}\text { Provide any recurring reports that the PHMC would } \\
\text { like to receive during transition. }\end{array}$ & by $7 / 15 / 96$ & DJ Berryman \\
\hline 6. & $\begin{array}{l}\text { Perform a joint property and equipment inventory } \\
\text { with the PHMC. }\end{array}$ & by $8 / 30 / 96$ & LA Robinson \\
\hline 7. & $\begin{array}{l}\text { Provide an updated status of ongoing work prior to } \\
\text { contract turnover. }\end{array}$ & by $9 / 30 / 96$ & TC Varljen \\
\hline
\end{tabular}

\subsubsection{Waste Characterization}

Manage the tank waste characterization project to ensure that data necessary for the continued safe operation of the tank farms and the eventual disposal of the waste are attained in a timely and cost-effective manner. This includes identifying data needs, identifying technical bases for data acquisition and use, deploying equipment, maintaining equipment, acquiring samples, analyzing samples, evaluating data, maintaining Characterization databases and publishing Tank Characterization Reports. Characterization Project also ensures timely completion of the Tri-Party Agreement characterization milestones for tank characterization and execution of the DNFSB 93-5 Implementation Plan.

\subsubsection{Waste Characterization - Technical Planning, Data Evaluation, and Data Management Activities.}

\begin{tabular}{|c|c|c|}
\hline 8 Pre-Award Actions & $\begin{array}{l}\text { Scheduled } \\
\text { Date }\end{array}$ & Actionee(s) \\
\hline $\begin{array}{l}\text { 1. Identify key documents. Provde listing to } \\
\text { program/project level fox consolidation and RL } \\
\text { review. }\end{array}$ & $5 / 01 / 96$ & DJ McCain \\
\hline $\begin{array}{l}\text { 2. Develop a comprehenave lia of open-ended } \\
\text { compliance issues (HATS is an erample). Provide to } \\
\text { RL POC for review. }\end{array}$ & $5 / 01 / 96$ & LD Pennington \\
\hline $\begin{array}{l}\text { 3. Identify POCs and input wo coneolidated } \\
\text { program/project listing. }\end{array}$ & $5 / 15 / 96$ & DJ McCain \\
\hline $\begin{array}{l}\text { 4. Develop a listing and deccription of subcontracts } \\
\text { supporting the program/project or facility (or } \\
\text { operation) and input to consolidated program/project } \\
\text { listing. Include any subcontracts planned to be } \\
\text { executed prior to contract takeover. }\end{array}$ & $5 / 15 / 96$ & GA Hanson \\
\hline $\begin{array}{l}\text { 5. Develop a listing and description of databases. } \\
\text { Provide list to program/project level for consolidation. }\end{array}$ & $5 / 15 / 96$ & JB Schaffer \\
\hline $\begin{array}{l}\text { 6. Identify number of briefings, and prepare briefing(s) } \\
\text { for the PHMC. (Coordinate number and content with } \\
\text { RL POC.) }\end{array}$ & $6 / 01 / 96$ & SJ Eberlein \\
\hline
\end{tabular}




\begin{tabular}{|c|c|c|c|}
\hline 7. & $\begin{array}{l}\text { Assemble key facility documentation, or identify its } \\
\text { location, for use by the PHMC. Includes preparation } \\
\text { of a "Smart Book," to include information develop in } \\
\text { the following actions. }\end{array}$ & $6 / 01 / 96$ & AE Young \\
\hline 8. & $\begin{array}{l}\text { Develop a list of open action items and commitments, } \\
\text { including status (Environmental, Tri-Party Agreement, } \\
\text { DNFSB as examples). Provide to RL POC for } \\
\text { review. }\end{array}$ & $6 / 01 / 96$ & LD Pennington \\
\hline 9. & $\begin{array}{l}\text { Develop a list of facility (or operation)-specific } \\
\text { mandatory training and qualification requirements for } \\
\text { employees. }\end{array}$ & $6 / 01 / 96$ & DJ McCain \\
\hline 10. & $\begin{array}{l}\text { Develop a listing of special agreement services } \\
\text { provided to RL, other DOE sites, and other entities. }\end{array}$ & $6 / 01 / 96$ & GA Hanson \\
\hline 11. & $\begin{array}{l}\text { Develop a list of current work order services or other } \\
\text { form of agreement to provide work for other } \\
\text { organizations, on and offsite. }\end{array}$ & $6 / 01 / 96$ & GA Hanson \\
\hline 12. & $\begin{array}{l}\text { Develop a list of current work order services or other } \\
\text { form of agreement to obtain work from other } \\
\text { organizations, on and offsite. }\end{array}$ & $6 / 01 / 96$ & GA Hanson \\
\hline 13. & $\begin{array}{l}\text { Verify the accuracy of property assignments for the } \\
\text { facility (or operations) and correct any deficiencies. }\end{array}$ & 6/01/96 & YR Cantu \\
\hline 14. & $\begin{array}{l}\text { Identify space and equipment within program/project } \\
\text { area and facilities for use by the PHMC. }\end{array}$ & $6 / 01 / 96$ & DJ MeCain \\
\hline 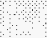 & Post-A ward Actions & & \\
\hline 1. & $\begin{array}{l}\text { Provide the PHMC access to key documentation, pre- } \\
\text { existing condition information, commitment data, } \\
\text { inventory data, etc. (as established in the pre-award } \\
\text { phase). Provide copies upon request. }\end{array}$ & by $6 / 15 / 96$ & AL Young \\
\hline 2. & $\begin{array}{l}\text { Provide program/project and facility space and } \\
\text { services for use by PHMC. }\end{array}$ & $6 / 15 / 96$ & DJ McCain \\
\hline 3. & $\begin{array}{l}\text { Provide any additional documentation or information } \\
\text { requested by the PHMC, if available, that were not } \\
\text { identified during the pre-award phase. }\end{array}$ & by $7 / 01 / 96$ & AL Young \\
\hline 4. & Provide briefing(s) to the PHMC. & $7 / 01 / 96-7 / 15 / 96$ & $\begin{array}{l}\text { DJ McCain } \\
\text { SJ Eberlein }\end{array}$ \\
\hline 5. & Provide a tour to the PHMC. & $7 / 01 / 96-7 / 15 / 96$ & $\begin{array}{l}\text { DJ MoCain } \\
\text { SJ Eberlein }\end{array}$ \\
\hline 6. & $\begin{array}{l}\text { Provide any recurring reports that the PHMC would } \\
\text { like to receive during transition. }\end{array}$ & by $7 / 15 / 96$ & DJ McCain \\
\hline 7. & Provide necessary training to PHMC personnel. & $7 / 01 / 96-8 / 01 / 96$ & SJ Eberiein \\
\hline 8. & $\begin{array}{l}\text { Perform a joint property and equipment inventory } \\
\text { with the PHMC. }\end{array}$ & by $8 / 30 / 96$ & YR Cantu \\
\hline 9. & $\begin{array}{l}\text { Provide an updated status of ongoing work prior to } \\
\text { contract turnover. }\end{array}$ & by $9 / 30 / 96$ & SJ Eberlein \\
\hline
\end{tabular}




\begin{tabular}{|c|c|c|c|}
\hline & Post-Award Actons & Solediled & $1+0 p+6)$ \\
\hline & Provide a categorized inventory of wastes. & by $9 / 30 / 96$ & $\begin{array}{l}\text { DJ McCain } \\
\text { I Husa }\end{array}$ \\
\hline
\end{tabular}

4.1.6.2 Waste Characterization - Operations (Field) Activities. Responsible for the following: Push-mode Core Sampling System (Truck \#1), Rotary-mode Core Sampling System \#1 (Truck \#2), Rotary-mode Core Sampling System \#2 (Truck \#3), and Rotary-mode Core Sampling System \#3 (Truck \#4).

\begin{tabular}{|c|c|c|}
\hline Pre-Award Actions & $\begin{array}{l}\text { Scheduled } \\
\text { Date. }\end{array}$ & Actiones (s) \\
\hline $\begin{array}{l}\text { 1. Identify key documents. Provide listing to } \\
\text { program/project level for consolidation and RL review. }\end{array}$ & $5 / 01 / 96$ & TJ Kelley \\
\hline $\begin{array}{l}\text { 2. Develop a comprehensive list of open-ended } \\
\text { compliance issues (HATS as an example). Provide to } \\
\text { RL POC for review. }\end{array}$ & $5 / 01 / 96$ & $\begin{array}{l}\text { TJ Kelley } \\
\text { LD Pennington }\end{array}$ \\
\hline $\begin{array}{l}\text { 3. Identify POCs and input to consolidated } \\
\text { program/project listing. }\end{array}$ & $5 / 15 / 96$ & JG Burton \\
\hline $\begin{array}{l}\text { 4. Develop a listing and description of subcontracts } \\
\text { supporting the program/project or facility (or } \\
\text { operation) and input to consolidated program/project } \\
\text { listing. Include any subcontracts planned to be } \\
\text { executed prior to contract takeover. }\end{array}$ & $5 / 15 / 96$ & $\begin{array}{l}\text { TJ Kelley } \\
\text { GA Hanson }\end{array}$ \\
\hline $\begin{array}{l}\text { 5. Develop a listing and description of databases. Provide } \\
\text { list to program/project level for consolidation. }\end{array}$ & $5 / 15 / 96$ & RE Raymond \\
\hline $\begin{array}{l}\text { 6. Identify number of briefings, and prepare briefing(s) } \\
\text { for the PHMC. (Coordinate number and content with } \\
\text { RL POC.) }\end{array}$ & $6 / 01 / 96$ & LF Ermold \\
\hline $\begin{array}{l}\text { 7. Assemble key documentation, or identify its location, } \\
\text { for use by the PHMC. Includes preparation of a } \\
\text { "Smart Book," to include information develop in the } \\
\text { following actions. }\end{array}$ & $6 / 01 / 96$ & TJ Kelley \\
\hline $\begin{array}{l}\text { 8. Using the pre-existing checklist, compile pre-existing } \\
\text { condition information on the facility and/or major } \\
\text { equipment. Conduct, or schedule as a separate post- } \\
\text { award action(s), any assessments required to adequately } \\
\text { address all relevant items on the checklist. Provide } \\
\text { completed checklist to RL POC for review. }\end{array}$ & $6 / 01 / 96$ & JG Burton \\
\hline $\begin{array}{l}\text { 9. Develop a list of open action items and commitments, } \\
\text { including status (Environmental, Tri-Party Agreement, } \\
\text { DNFSB as examples). Provide to RL POC for review. }\end{array}$ & $6 / 01 / 96$ & $\begin{array}{l}\text { TJ Kelley } \\
\text { LD Pennington }\end{array}$ \\
\hline $\begin{array}{l}\text { 10. Identify current inventories of feed for the facility, and } \\
\text { materials to support continued operation (i.e., essential } \\
\text { materials, tools and equipment). }\end{array}$ & $6 / 01 / 96$ & JG Burton \\
\hline $\begin{array}{l}\text { 11. Develop a list of facility (or operation)-specific } \\
\text { mandatory training and qualification requirements for } \\
\text { employees. }\end{array}$ & 6/01/96 & GA Hanson \\
\hline
\end{tabular}




\begin{tabular}{|c|c|c|}
\hline Pre-Award Actions & $\begin{array}{l}\text { Sohediled } \\
\text { Date }\end{array}$ & Actionee(s) \\
\hline $\begin{array}{l}\text { 12. Develop a listing of information which constitutes the } \\
\text { "authorization basis" for the facility. }\end{array}$ & 6/01/96 & $\begin{array}{l}\text { ML Cowen } \\
\text { JJ Klos }\end{array}$ \\
\hline $\begin{array}{l}\text { 13. Develop a description of ongoing and planned } \\
\text { construction projects. }\end{array}$ & 6/01/96 & TJ Kelley \\
\hline $\begin{array}{l}\text { 14. Develop a listing of special agreement services } \\
\text { provided to RL, other DOE sites, and other entities. }\end{array}$ & $6 / 01 / 96$ & $\begin{array}{l}\text { TJ Kelley } \\
\text { GA Hanson }\end{array}$ \\
\hline $\begin{array}{l}\text { 15. Develop a list of current work order services or other } \\
\text { form of agreement to provide work for other } \\
\text { organizations, on and offite. }\end{array}$ & $6 / 01 / 96$ & $\begin{array}{l}\text { TJ Kelley } \\
\text { GA Hanson }\end{array}$ \\
\hline $\begin{array}{l}\text { 16. Develop a list of current work order services or other } \\
\text { form of agreement to obtain work from other } \\
\text { organizations, on and offsite. }\end{array}$ & 6/01/96 & $\begin{array}{l}\text { TJ Kelley } \\
\text { GA Hanson }\end{array}$ \\
\hline $\begin{array}{l}\text { 17. Develop a list of permits governing the facility (or } \\
\text { operation), and provide a description of each as to } \\
\text { where and how it applies. }\end{array}$ & $6 / 01 / 96$ & RD Gustavson \\
\hline $\begin{array}{l}\text { 18. Verify the accuracy of property assignments for the } \\
\text { facility (or operations) and correct any deficiencies. }\end{array}$ & 6/01/96 & $\begin{array}{l}\text { JG Burton } \\
\text { TJ Kelley } \\
\text { RE Raymond }\end{array}$ \\
\hline $\begin{array}{l}\text { 19. Identify space and equipment within program/project } \\
\text { area and facilities for use by the PHMC. }\end{array}$ & 6/01/96 & PD Culver \\
\hline Post-Award Actions & & \\
\hline $\begin{array}{l}\text { 1. Provide the PHMC access to key documentation, pre- } \\
\text { existing condition information, commitment data, } \\
\text { inventory data, etc. (as established in the pre-award } \\
\text { phase). Provide copies upon request. }\end{array}$ & by $6 / 15 / 96$ & TJ Kelley \\
\hline $\begin{array}{l}\text { 2. Provide program/project and facility space and services } \\
\text { for use by PHMC. }\end{array}$ & $6 / 15 / 96$ & PD Culver \\
\hline $\begin{array}{l}\text { 3. Provide any additional documentation or information } \\
\text { requested by the PHMC, if available, that were not } \\
\text { identified during the pre-award phase. }\end{array}$ & by $7 / 01 / 96$ & TJ Kelley \\
\hline 4. Provide briefing(s) to the PHMC. & $7 / 01 / 96-7 / 15 / 96$ & $\begin{array}{l}\text { LF Ermold } \\
\text { JG Burton }\end{array}$ \\
\hline 5. Provide a tour to the PHMC. & $7 / 01 / 96-7 / 15 / 96$ & JG Burton \\
\hline 6. Provide necessary training to PHMC personnel. & 7/01/96-8/01/96 & JG Burton \\
\hline $\begin{array}{l}\text { 7. Assist the PHMC in the conduct of a Pre-Existing } \\
\text { Condition Assessment. }\end{array}$ & $7 / 15 / 96-8 / 30 / 96$ & JG Burton \\
\hline $\begin{array}{l}\text { 8. Provide an updated status of ongoing work prior to } \\
\text { contract turnover. }\end{array}$ & by $9 / 30 / 96$ & TJ Kelley \\
\hline 9. Provide a categorized inventory of wastes. & by $9 / 30 / 96$ & $\begin{array}{l}\text { JG Burton } \\
\text { R. E. Raymond }\end{array}$ \\
\hline
\end{tabular}

\footnotetext{
organization.

${ }^{1}$ Waste generated during field sampling activities. Tank inventories covered by Technical Basis
} 


\subsubsection{Waste Disposal Program}

The charter of the TWRS Disposal Program includes four projects: (1) the Waste Retrieval Project, (2) the Low-Level Waste (LLW) Project, (2) the HLW Project, and (4) the Storage and Disposal Project. The Waste Retrieval Project includes SST and DST retrieval and tank farm closure. The LLW Project covers pretreatment and immobilization of lowactivity waste for disposition onsite. The HLW Project is responsible for the pretreatment and immobilization of HLW for final disposition offsite. The Storage and Disposal Project includes LLW disposal onsite, the interim storage of HLW until final disposition offsite, and the treatment and interim storage of cesium and strontium capsules. The TWRS Disposal Program charter also includes all M\&O Contractor support to the Privatization effort for the LLW and the HLW Vitrification programs.

Facilities within the Disposal Program include Near-Surface Vaults 218-E-16-102, $-103,-104$, and -105 . These four vaults are all empty and clean.

All key equipment currently owned or expected to be owned by the end of FY 1996 by the Disposal Program falls within the Waste Retrieval Project. Current equipment includes the Tank C-106 Retrieval Sluicing System for Project W-320 and Tank AZ-101 Retrieval System (decant pump, two mixer pumps, and instrumentation) for Project W-151. By the end of FY 1996, we expect to procure a Sonic Probe to supplement mixer pumps during DST retrieval.

\begin{tabular}{|c|c|c|}
\hline Pre-Award Actions & $\begin{array}{l}\text { Scheduled } \\
\text { Date }\end{array}$ & Actionee(s) \\
\hline $\begin{array}{l}\text { 1. Identify key documents. Provide listing to program/project level } \\
\text { for consolidation and RL review. }\end{array}$ & $5 / 01 / 96$ & $\begin{array}{c}\text { TW Crawford } \\
\end{array}$ \\
\hline 2. Identify POCs and input to consolidated program/project listing. & $5 / 15 / 96$ & TW Crawford \\
\hline $\begin{array}{l}\text { 3. Develop a listing and description of subcontracts supporting the } \\
\text { program/project or facility (or operation) and input to consolidated } \\
\text { program/project listing. Include any subcontracts planned to be } \\
\text { executed prior to contract takeover. }\end{array}$ & $5 / 15 / 96$ & TW Crawford \\
\hline $\begin{array}{l}\text { 4. Develop a listing and description of databases. Provide list to } \\
\text { program/project level for consolidation. }\end{array}$ & $5 / 15 / 96$ & TW Crawford \\
\hline $\begin{array}{l}\text { 5. Identify number of briefings, and prepare briefing(s) for the } \\
\text { PHMC. (Coordinate number and content with RL POC.) }\end{array}$ & 6/01/96 & TW Crawford \\
\hline $\begin{array}{l}\text { 6. Assemble key documentation, or identify its location, for use by } \\
\text { the PHMC. Includes preparation of a facility (or operations) } \\
\text { "Smart Book," to include information develop in the following } \\
\text { actions. }\end{array}$ & $6 / 01 / 96$ & TW Crawford \\
\hline $\begin{array}{l}\text { 7. Using the pre-existing checklist, compile pre-existing condition } \\
\text { information on the facility and/or major equipment. Conduct, or } \\
\text { schedule as a separate post-award action(s), any assessments } \\
\text { required to adequately address all relevant items on the checklist. } \\
\text { Provide completed checklist to RL POC for review. }\end{array}$ & $6 / 01 / 96$ & TW Crawford \\
\hline $\begin{array}{l}\text { 8. Develop a list of open action items and commitments, including } \\
\text { status (Environmental, Tri-Party Agreement, DNFSB as examples). } \\
\text { Provide to RL POC for review. }\end{array}$ & $6 / 01 / 96$ & TW Crawford \\
\hline
\end{tabular}




\begin{tabular}{|c|c|c|}
\hline Pro-Aprard/ctions & $\begin{array}{l}\text { Schethled } \\
\text { Date }\end{array}$ & Ittonee $(6)$ \\
\hline $\begin{array}{l}\text { 9. Develop a listing of information which constitutes the } \\
\text { "authorization basis" for the facility. }\end{array}$ & 6/01/96 & TW Crawford \\
\hline $\begin{array}{l}\text { 10. Develop a description of ongoing and planned construction } \\
\text { projects. }\end{array}$ & $6 / 01 / 96$ & TW Crawford \\
\hline $\begin{array}{l}\text { 11. Develop a list of current work order services or other form of } \\
\text { agreement to provide work for other organizations, on and offsite. }\end{array}$ & 6/01/96 & TW Crawford \\
\hline $\begin{array}{l}\text { 12. Develop a list of current work order services or other form of } \\
\text { agreement to obtain work from other organizations, on and offsite. }\end{array}$ & 6/01/96 & TW Crawford \\
\hline $\begin{array}{l}\text { 13. Identify apace and equipment within program/project area and } \\
\text { facilities for use by the PHMC. }\end{array}$ & $6 / 01 / 96$ & TW Crawford \\
\hline $\begin{array}{l}\text { 14. Develop a list of permits governing the facility (or operation), and } \\
\text { provide a description of each as to where and how it applies. }\end{array}$ & 6/01/96 & TW Crawford \\
\hline $\begin{array}{l}\text { 15. Verify the accuracy of property assignments for the facility (or } \\
\text { operations) and correct any deficiencies. }\end{array}$ & $6 / 01 / 96$ & TW Crawford \\
\hline 4. 4 Post-Award Actions & & \\
\hline $\begin{array}{l}\text { 1. Provide the PHMC access to key documentation, pre-existing } \\
\text { condition information, commitment data, inventory data, etc. (as } \\
\text { established in the pre-award phase). Provide copies upon request. }\end{array}$ & by $6 / 15 / 96$ & TW Crawford \\
\hline $\begin{array}{l}\text { 2. Provide program/project and facility space and services for use by } \\
\text { PHMC. }\end{array}$ & $6 / 15 / 96$ & TW Crawford \\
\hline $\begin{array}{l}\text { 3. Provide any additional documentation or information requested by } \\
\text { the PHMC, if available, that were not identified during the pre- } \\
\text { award phase. }\end{array}$ & by $7 / 01 / 96$ & TW Crawford \\
\hline 4. Provide briefing(s) to the PHMC. & 7/01/96-7/15/96 & TW Crawford \\
\hline 5. Provide a tour to the PHMC. & 7/01/96-7/15/96 & TW Crawford \\
\hline $\begin{array}{l}\text { 6. Provide any recurring reports that the PHMC would like to receive } \\
\text { during transition. }\end{array}$ & by $7 / 15 / 96$ & TW Crawford \\
\hline $\begin{array}{l}\text { 7. Assist the PHMC in the conduct of a Pre-Existing Condition } \\
\text { Assessment. }\end{array}$ & $7 / 15 / 96-8 / 30 / 96$ & TW Crawford \\
\hline 8. Perform a joint property and equipment inventory with the PHMC. & by $8 / 30 / 96$ & TW Crawford \\
\hline $\begin{array}{l}\text { 9. Provide an updated status of ongoing work prior to contract } \\
\text { turnover. }\end{array}$ & by $9 / 30 / 96$ & TW Crawford \\
\hline
\end{tabular}

\subsection{WASTE MANAGEMENT}

\subsubsection{Solid Waste} for RL.

The Solid Waste (SW) Program at the Hanford Site is currently directed by WHC 
The SW Program is responsible for the development (and RL approval) of the Hanford Site Waste Acceptance Criteria (WAC), and the effective and efficient treatment, storage, and disposal of the hazardous, radioactive, and mixed solid wastes generated on the site, as well as selected offsite generators. The program's goal is to receive, store, treat, and dispose of solid radioactive and nonradioactive dangerous wastes in a safe, environmentally compliant, and cost effective manner.

The SW Program provides a comprehensive approach for the storage, treatment, and disposal of current and future solid waste received at the Hanford Site (from onsite and offsite generators) in a manner compliant with the current and evolving regulations, orders, permits, and safety analyses (Federal, state, DOE, and WHC).

The SW Program addresses activities required for the exhumation and disposal of selected wastes currently in retrievable storage; provides a central focus for control of cost, scope, and schedule of Hanford Site non sanitary, non-HLW solid waste activities; and, provides a vehicle for ready communication of the scope of those activities to onsite and offsite organizations.

During remediation activities, large volumes of radioactive, nonradioactive hazardous, and mixed solid waste are expected to be produced; thus, creating the need for subsequent decontamination, treatment, storage, and/or waste disposal. The SW Program mission is to manage current and future contaminated solid waste streams in a safe, responsible, cost effective, and legally compliant manner.

Complex regulatory and technical requirements drive the scope of the SW Program services to receive, store, treat, and dispose of the radioactive and nonradioactive dangerous waste in support of the cleanup and remediation efforts onsite. In addition, the SW Program provides management and oversight to projects and programs which support the overall program mission.

The Solid Waste Disposal (SWD) facilities listed below have primary functions of treatment, storage, and disposal:

- Treatment

- 2336-W Waste Receiving and Processing Module (WRAP) 1

- Radioactive Mixed Waste (RMW) Treatment (via offsite contracts)

- 221-T Canyon (High Level Decon. Processing)

- 2706-T (LLW Processing)

- Thermal Treatment (via offsite contract)

- Tri Butyl Phosphate (TBP) Treatment (via offsite contract)

- Storage

- 616 Facility

- Transuranic Assay and Storage Facility (TRUSAF)

- Central Waste Complex (CWC)

- W-112 Enhanced Radioactive Mixed Waste Storage 
- Disposal

- Low Level Burial Grounds

- RMW Disposal (Trenches)

- Dangerous (Nonradioactive) Waste Disposal (offsite contract).

\section{Facility Descriptions}

2336-W Waste Receiving and Processing Module 1: WRAP 1 will receive retrieved and newly generated solid contact-handled radioactive wastes in 55-gal drums (some overpacked in salvage drums). Treatment activities will include repackaging and certification of design through-put waste streams. Drums will be inspected, assayed, and opened if necessary. Waste contents can be sorted and segregated, empty drums and wastes compacted as appropriate, repackaged, certified and shipped to final disposal (Waste Isolation Pilot Plant [WIPP]). Construction of the WRAP 1 facility scheduled to be complete in June 1996, with operation starting in March 1997. WRAP 1 will have a design operating life of 30 years on a one-shift per day basis. 6,825 drums of contact-handled waste can be received annually. This includes 2,100 drums of newly generated TRU/TRUMW, 2,625 drums of retrieved suspect transuranic (TRU) (assumed to be comprised of 50\% TRU and 50\% LLW), and 2,100 drums of newly generated LLW/low-level mixed waste (LLMW). The facility can also receive, certify, and ship 70 standard waste boxes of contact-handled TRU annually. WRAP 1 will be capable of accommodating the minimum required annual waste throughput in no more 175 operating days per year, with no more than five operating days per week. This is equivalent to $70 \%$, or 35 weeks, of the 50 weeks in a normal operating year. TRU/TRUM waste processed will meet TRUPACT II shipping requirements and the waste acceptance criteria for disposal at WIPP, which will receive waste from WRAP 1 until 2023. WRAP 1 contains two gloveboxes in which limited small-scale treatment can be performed for more difficult waste in support of Federal Facilities Compliance Agreement (FFCA) or other small-scale treatment options. Currently funding guidance only provides for LLW processing and nondestructive examination (NDE)/nondestructive assay (NDA) activities, with start-up of TRU processing beyond FY 2002.

RMW Treatment Offsite Contracts: Since 1985, the Hanford Site has been storing LLMW for future treatment and disposal. It has been decided that the most efficient way to treat this waste is through utilization of offsite commercial contractors. Solid Waste has been investigating several different methods of treatment and has contracts either in place or in the process of being placed for these treatments. These include:

- TBP Treatment

- RMW Stabilization

- Macroencapsulation

- Volume Reduction with ATG (DOE contract SWD manages)

- Thermal Treatment.

221-T: The T Plant facility consists of a number of structures in the 200 West Area of the Hanford Site. The two primary treatment and storage facilities are the 221-T canyon and the 2706-T Building. T Plant was constructed in 1943 through 1944 to extract plutonium from production reactor fuel. The plant performed this function until it was deactivated in 1956. Most of the original process equipment was subsequently removed. In 1957, 
T Plant was placed in service as a beta-gamma decontamination facility and a support complex for experiments or other operations requiring containment or isolation. Today, 221-T is used for decontamination of high-level radioactive and hazardous contaminated items and process equipment. Support structures also have limited treatment of mixed and dangerous waste. The 221-T Facility has a waste container load/unload bay in the railcar tunnel that is used to place waste containers into the canyon for storage or treatment. This bay is also used for the cleanout and annual certification and maintenance of the liquid waste railcars.

2706-T: The 2706-T Building is a pre-engineered building erected during 1959 and 1960 to perform low level radioactive decontamination of railroad equipment. The facility has an open top tank to catch liquids during decontamination and piping to transfer the waste liquids to holding tanks. The building is fed by a railroad spur and the facility can accept a full size locomotive for treatment. 2706-T underwent extensive refurbishment in 1992 through 1994 and recently the building was expanded to include an additional 3000 square feet of floor area. This will allow an area to perform microencapsulation of waste under cover and in a separate containment if required. The additional space will also be utilized for staging of heavy equipment before decontamination and after decontamination for final release surveys. A second containment has been set up to allow limited alpha treatment in the facility making the facility a "dual survey" facility. The tank and piping systems are being upgraded with capital funds to bring the tank and liquid piping systems into full compliance by September 1999. The safety basis is being updated to change the facility from a fissile exempt facility to a fissile isolated facility. This will allow decontamination of alpha equipment and allow receipt of measurable amounts of fissile material into the facility. The 2706-T Facility is also used for LLW verification, sampling and repackaging for the Hanford Site and other waste generators. In the area surrounding the facility, there are numerous storage pads to stage waste, store empty containers and perform treatment. A $60-\mathrm{ft}$ by $90-\mathrm{ft}, 4,000 \mathrm{psf}$ pad to macroencapsulate tank farms long length equipment is being poured to allow that work to be performed in the area. A $70-\mathrm{ft}$ by 28 -ft change/office trailer was recently added to the complex and provides space for engineering and supervision offices and a step off pad for controlled movement in and out of the facility. A second, older change facility exists and could be used for a second step off pad when work load requires. The entire area is bounded by a fence which is used to control access and designate the permitted area.

Nonradioactive Dangerous Waste Storage Facility (616 Building): The Nonradioactive Dangerous Waste Storage Facility (NRDWSF) is classified as container storage and provides a centralized storage unit to receive, store, and prepare shipments of nonradioactive dangerous waste. This facility has the capacity to store $\mathbf{4 0 0}$ containers of dangerous waste. This facility is used on an exception basis only - preferred procedure is for generators to ship directly to offsite treatment, storage, or disposal facilities. The costs of offsite shipment are borne by the generators.

\section{4-T Transuranic Assay and Storage Facility (TRUSAF): The 224-T Facility} primarily stores TRU waste that meets the Hanford Site Solid WAC. This TRU waste will require processing through WRAP 1 to meet the WIPP WAC when finalized. In addition, other properly characterized and packaged LLMW could be accepted for storage. TRUSAF has the capacity to store 2,000, 55-gal (208 liter) drums. Waste is received primarily in 
DOT approved or equivalent $17 \mathrm{C}$ or $17 \mathrm{H}$ UN1A2 55 -gal containers or other DOT approved packages and overpacks.

Central Waste Complex (CWC): The CWC consists of a number of buildings, storage modules, and projects. Included among these facilities are twelve small mixed waste storage buildings (2402-W, 2402-WB, 2402-WC, 2402-WD, 2402-WE, 2402-WF, 2402-WG, 2402-WH, 2402-WI, 2402-WJ, 2402-WK, and 2402-WL), low-flash-point mixed waste storage modules, one plutonium/polychlorinated biphenyl mixed waste storage building (2401-W), large mixed waste storage (2403-WA, 2403-WB, 2403-WC, and 2403-WD) and a mixed waste storage pad. These facilities provide interim storage of mixed waste awaiting appropriate treatment and disposal methods being developed for waste disposition. The 2401-W, 2402-W series, 2403-W series, low-flashpoint mixed storage modules, alkali metal waste storage modules, and south alkali metal storage modules are currently operational. The 2401-W storage building has the capacity to store 1,072, 208-liter (55-gallon) drum equivalents of waste. Each of the $2402-W$ series buildings has the capacity to store 1,072 , 208-liter (55-gal) drum equivalents of waste. The 2403-W series buildings have a combined capacity of 52,300, 208-liter (55-gal) drum equivalents. Buildings 2403-WA, -WB, and -WC will accommodate 11,600, 208-liter (55-gal) drum equivalents each; and 2403-WD will accommodate 17,500, 208-liter (55-gal) drum equivalents. There are 19 low-flashpoint mixed storage modules providing a total capacity to store 400 , 208-liter (55-gal) drum equivalents of waste. There are 4 alkali metal waste storage modules providing a total capacity to store 84,208 -liter (55-gal) drum equivalents of waste. There are 8 south alkali metal waste storage modules providing a total capacity to store 168 , 208-liter (55-gal) drum equivalents of waste. Waste in all CWC facilities is segregated according to hazardous characteristics. The characteristic types of LLMW/TRUM stored will include acids, caustics, oxidizers, and Toxicity Characteristic Leaching Procedure toxic waste.

W-112 Enhanced Radieactive and Mixed Waste Storage Phase V Project: The Phase $\mathrm{V}$ storage facility will store wastes and distribute waste to various radioactive solid waste treatment facilities. This project is an addition to the existing $\mathrm{CWC}$ and includes the following storage buildings: 2404-WA, 2404-WB, 2404-WC. The Phase V project will provide interim and long term storage for waste. Project W-112 will also provide general office (2740-W) and maintenance space (2620-W) for the WRAP 1. Phase V will store contact-handled solid wastes received from offsite and onsite generators. Phase V will be used for interim storage of LLW and LLMW transferred from storage to treatment or disposal facilities. Storage will be provided for 13,300 drum equivalent (55-gallon drums) of contact-handled waste. Phase V will be able to accommodate low-level, mixed, and TRU wastes that do not meet the performance assessment. Classified and remote handled wastes will not be stored in the Phase V Facility.

Low-Level Burial Grounds: The Low-Level Burial Grounds (LLBG) provide disposal capability for Category 1 and Category $3 \mathrm{LLW}$. The SW Program currently disposes of LLW in burial grounds in the 200 Areas. The LLBG is capable of accommodating all forecasted Category 1 and Category 3 LLW. 
Radioactive Mixed Waste Disposal: The RMW Disposal facility (RMW Trenches \#31 and \#34, and Burial Ground \#218-W-5) will provide permanent disposal capability for LLMW. Two disposal trenches have been constructed for RMW. Each disposal system will have a design life of 50 years minimum, 20 years are required for the operational phase, and 30 years for post closure monitoring. The capacity of each of the two RMW trench is a minimum of $7,600 \mathrm{~m}^{3}\left(270,000 \mathrm{ft}^{3}\right)$ of waste for a total of $15,200 \mathrm{~m}^{3}\left(540,000 \mathrm{ft}^{3}\right)$ of waste. Additional disposal capacity will be built in the future as demand requires. The site selected for disposal of mixed waste packages is the 218-W-5 Burial Ground. The RMW disposal system will meet the minimum functional standards for a dangerous waste landfill as required by Washington State regulations (Ecology 1991).

\begin{tabular}{|c|c|c|}
\hline Pre-A ward Actions & $\begin{array}{l}\text { Schedaled } \\
\text { Date }\end{array}$ & lationee(o) \\
\hline $\begin{array}{l}\text { 1. Assemble key program documentation, or identify its } \\
\text { location, for use by the PHMC. Includes development } \\
\text { of a program "Snart Book." }\end{array}$ & $6 / 01 / 96$ & ME Kurts \\
\hline $\begin{array}{l}\text { 2. Develop a listing of key program individuals as POCs, } \\
\text { including facility and operations personnel. }\end{array}$ & $6 / 01 / 96$ & ME Kurts \\
\hline $\begin{array}{l}\text { 3. Develop listing and description of program databases, } \\
\text { including facility (or operations) databases. }\end{array}$ & $6 / 01 / 96$ & ME Kurts \\
\hline $\begin{array}{l}\text { 4. Verify the accuracy of property assignments at the } \\
\text { program organizational level and correct any } \\
\text { deficiencies. }\end{array}$ & $6 / 01 / 96$ & NP Daniel \\
\hline $\begin{array}{l}\text { 5. Identify applicable milestones and commitments, } \\
\text { determine their status, and supply a copy of the } \\
\text { milestone description sheet. }\end{array}$ & $6 / 01 / 96$ & EF Mares \\
\hline $\begin{array}{l}\text { 6. Develop a list of open action items and commitments } \\
\text { and open-ended compliance issues. }\end{array}$ & $6 / 01 / 96$ & MI Heinemeyer \\
\hline $\begin{array}{l}\text { 7. Develop a list of facility-specific mandatory training and } \\
\text { qualification requirements for employees. }\end{array}$ & $6 / 01 / 96$ & ML Heinemeyer \\
\hline $\begin{array}{l}\text { 8. Develop a listing of information which constitutes the } \\
\text { "authorization basis" for all the SWM facilities. }\end{array}$ & $6 / 01 / 96$ & PL Hapke \\
\hline $\begin{array}{l}\text { 9. Develop a listing of information which constitutes the } \\
\text { "authorization basis" for the } \mathrm{T} \text { Plant facilities. }\end{array}$ & $6 / 01 / 96$ & MS Wright \\
\hline $\begin{array}{l}\text { 10. Develop a listing and description of ongoing and } \\
\text { planned construction projects for SWD. }\end{array}$ & $6 / 01 / 96$ & DE McKenney \\
\hline $\begin{array}{l}\text { 11. Develop a listing of "work for others" agreements, to } \\
\text { include onsite work orders and offsite special requests } \\
\text { and HQ ADSs. }\end{array}$ & $6 / 01 / 96$ & ME Kurts \\
\hline $\begin{array}{l}\text { 12. Using the pre-existing condition checklist, compile pre- } \\
\text { existing condition information for all SWD facilities. } \\
\text { Conduct, or schedule as a separate post-closure } \\
\text { action(s), any assessments required to adequately } \\
\text { address all relevant items on the checklist. }\end{array}$ & $6 / 01 / 96$ & $\begin{array}{l}\text { RD Pierce } \\
\text { BM Barnes }\end{array}$ \\
\hline
\end{tabular}




\begin{tabular}{|c|c|c|}
\hline Poet-A ward Actions & $\begin{array}{l}\text { Seheduled } \\
\text { Date }\end{array}$ & $1+t_{10}=0(0)$ \\
\hline $\begin{array}{l}\text { 1. Provide the PHMC access to the key program } \\
\text { documentation, any program level generated pre-existing } \\
\text { conditions information, listing of POCs, etc. Provide } \\
\text { copies upon request. }\end{array}$ & $6 / 15 / 96$ & DE MC Kenney \\
\hline $\begin{array}{l}\text { 2. Program and facility space and services available for use } \\
\text { by the PHMC. }\end{array}$ & $6 / 15 / 96$ & ML Heinemeyer \\
\hline $\begin{array}{l}\text { 3. Provide Solid Waste Program business overview } \\
\text { briefing to the PHMC. }\end{array}$ & $6 / 10 / 96-6 / 20 / 96$ & ML Heinemeyer \\
\hline 4. Provide T Plant business review briefing to the PHMC. & $6 / 10 / 96-6 / 20 / 96$ & MS Wright \\
\hline $\begin{array}{l}\text { 5. Provide a WRAP } 1 \text { business review briefing to the } \\
\text { PHMC. }\end{array}$ & $6 / 10 / 96-6 / 20 / 96$ & PI Hapke \\
\hline 6. Provide SWM facilities tour to the PHMC. & $6 / 10 / 96-6 / 28 / 96$ & PL Hapke \\
\hline 7. Provide T Plant facilities tour to the PHMC. & $6 / 10 / 96-6 / 28 / 96$ & MS Wright \\
\hline 8. Provide WRAP 1 facilities tour to the PHMC. & $6 / 10 / 96-6 / 28 / 96$ & PL Hapke \\
\hline $\begin{array}{l}\text { 9. Discuss list of non-compliant issues and status with } \\
\text { PHMC and RL POC. }\end{array}$ & $6 / 28 / 96$ & $\begin{array}{l}\text { RD Pierce } \\
\text { CK Girres } \\
\text { KM McDonald }\end{array}$ \\
\hline $\begin{array}{l}\text { 10. Provide PHMC any recurring reports that they would } \\
\text { like to receive during transition. }\end{array}$ & $7 / 15 / 96-9 / 30 / 96$ & ML Heinemeyer \\
\hline 11. Provide necessary training to PHMC personnel. & $7 / 01 / 96-9 / 30 / 96$ & ML Heinemeyer \\
\hline $\begin{array}{l}\text { 12. Assist the PHMC in the conduct of Pre-existing } \\
\text { Condition Assessments. }\end{array}$ & $7 / 01 / 96-8 / 30 / 96$ & $\begin{array}{l}\text { RD Pierce } \\
\text { CK Girres } \\
\text { KM McDonald } \\
\end{array}$ \\
\hline $\begin{array}{l}\text { 13. Provide an updated status on ongoing work prior to } \\
\text { contract turnover. }\end{array}$ & 9/30/96 & WH Hamilton, Jr. \\
\hline
\end{tabular}

\subsubsection{Liquid Effluents Program}

The Liquid Effluents Program provides integrated liquid effluent management to support cleanup of the Hanford Site. The program mission is to manage current and future liquid effluent streams in a safe, responsible, cost-effective, and legally-compliant manner. The approach to implementing treatment and disposal of liquid effluents is based on the following strategy: stream characterization; identification of best available technology/all known and reasonable treatment (BAT/AKART); regulator acceptance of BAT/AKART; and obtaining appropriate state and/or Federal permits.

\begin{tabular}{|l|c|c|}
\hline \multicolumn{1}{|c|}{ Pre-Award Actions } & $\begin{array}{c}\text { Scheduled } \\
\text { Date }\end{array}$ & \\
\hline $\begin{array}{l}\text { 1. Develop a list of key program (or project) } \\
\text { documentation. Provide to RL POC for review. }\end{array}$ & $5 / 15 / 96$ & FT Green \\
\hline
\end{tabular}




\begin{tabular}{|c|c|c|}
\hline Pre-Award Actions & $\begin{array}{l}\text { Scheduled } \\
\text { Date }\end{array}$ & Actiobere(s) \\
\hline $\begin{array}{l}\text { 2. Assemble key program (or project) documentation, or } \\
\text { identify its location, for use by the PHMC. Includes } \\
\text { development of a program/project "Smart Book," } \\
\text { including listings and descriptions developed below. }\end{array}$ & $6 / 01 / 96$ & FT Green \\
\hline $\begin{array}{l}\text { 3. Identify number of briefings, and prepare program (or } \\
\text { project) briefing(s) for presentation to PHMC. } \\
\text { (Coordinate number and content with RL POC.) }\end{array}$ & $6 / 01 / 96$ & FT Green \\
\hline $\begin{array}{l}\text { 4. Develop a listing of Key program (or project) } \\
\text { individuals as POCs, including facility and operations } \\
\text { personnel. }\end{array}$ & $6 / 01 / 96$ & FT Green \\
\hline $\begin{array}{l}\text { 5. Develop listing and description of program (or project) } \\
\text { databases, including facility (or operations) databases. }\end{array}$ & $6 / 01 / 96$ & FT Green \\
\hline $\begin{array}{l}\text { 6. Verify the accuracy of property assignments at the } \\
\text { program/project organizational level and correct any } \\
\text { deficiencies. }\end{array}$ & $6 / 01 / 96$ & FT Green \\
\hline $\begin{array}{l}\text { 7. Identify space and equipment within program (or } \\
\text { project) area and facilities for use by the PHMC. }\end{array}$ & $6 / 01 / 96$ & FT Green \\
\hline $\begin{array}{l}\text { 8. In outline form for all facilities/operations to follow, } \\
\text { define the appropriate contents and level of detail to be } \\
\text { included in facility/operations briefings to the PHMC. } \\
\text { (Review with RL POC.) }\end{array}$ & $5 / 01 / 96$ & FT Green \\
\hline 4ै Post-Award Actions & 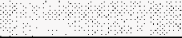 & 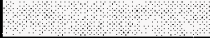 \\
\hline $\begin{array}{l}\text { 1. Provide the PHMC access to the key program (or } \\
\text { project) documentation, any program level generated } \\
\text { pre-existing conditions information, listing of POCs, } \\
\text { and listing of subcontracts. Provide copies upon } \\
\text { request. }\end{array}$ & prior to $6 / 15 / 96$ & FT Green \\
\hline $\begin{array}{l}\text { 2. Program/project and facility space and services available } \\
\text { for use by the PHMC. }\end{array}$ & $6 / 15 / 96$ & FT Green \\
\hline 3. Provide program/project briefing(s) to the PHMC. & $6 / 15 / 96-7 / 01 / 96$ & FT Green \\
\hline 4. Provide program/project level tour to the PHMC. & 6/15/96-7/01/96 & FT Green \\
\hline $\begin{array}{l}\text { 5. Perform a joint program/project level property and } \\
\text { equipment inventory with PHMC personnel. }\end{array}$ & by $8 / 30 / 96$ & FT Green \\
\hline
\end{tabular}

4.2.2.1 200 Area Treated Effluent Disposal Facility. The 200 Area TEDF is a collection and disposal system for non-RCRA permitted waste streams which already meet discharge requirements. Implementation of BAT/AKART is the responsibility of the generating facilities. Facilities which discharge to the 200 Area TEDF include the PFP, 222-S Laboratory, T Plant, 284-W Power Plant, PUREX Plant, B Plant, and 242-A-81 Water Services Building. Each facility must comply with discharge limits in the WAC 173-216 State Waste Discharge Permit without further treatment. 
The 200 Area TEDF began operation in April 1995 and has a 30-year life. Design capacity of the 200 Area TEDF is $2300 \mathrm{gal} / \mathrm{min}$, although the Discharge Permit presently limits the average monthly flow to $640 \mathrm{gal} / \mathrm{min}$. About $130 \mathrm{Mgal}$ of treated effluent was discharged in 1995. The 200 Area TEDF includes more that 12 miles of polyvinyl chloride (PVC) pipe up to $14 \mathrm{in}$. in diameter. The effluent is discharged to two, 5-acre disposal ponds located east of the 200 East Area.

\begin{tabular}{|c|c|c|}
\hline Pre-Award Actions & $\begin{array}{l}\text { Scheduled } \\
\text { Date }\end{array}$ & Actionee(s) \\
\hline $\begin{array}{l}\text { 1. Identify number of briefings, and prepare facility (or } \\
\text { operations) briefing(s) for the PHMC. (Coordinate } \\
\text { number and content with RL POC.) }\end{array}$ & $6 / 01 / 96$ & DW Lindsey \\
\hline $\begin{array}{l}\text { 2. Identify key documents for the facility (or operations). } \\
\text { Provide listing to program/project level for } \\
\text { consolidation and RL review. }\end{array}$ & $5 / 01 / 96$ & DW Lindsey \\
\hline $\begin{array}{l}\text { 3. Assemble key facility (or operations) documentation, } \\
\text { or identify its location, for use by the PHMC. } \\
\text { Includes preparation of a facility (or operations) } \\
\text { "Smart Book," to include information develop in the } \\
\text { following actions. }\end{array}$ & $6 / 01 / 96$ & DW Lindsey \\
\hline $\begin{array}{l}\text { 4. Identify POCs for the facility (or operations) and input } \\
\text { to consolidated program/project listing. }\end{array}$ & $5 / 15 / 96$ & DW Lindsey \\
\hline $\begin{array}{l}\text { 5. Develop a listing and description of subcontracts } \\
\text { supporting the facility (or operation) and input to } \\
\text { consolidated program/project listing. Include any } \\
\text { subcontracts planned to be executed prior to contract } \\
\text { takeover. }\end{array}$ & $5 / 15 / 96$ & DW Lindsey \\
\hline $\begin{array}{l}\text { Using the pre-existing checklist, compile pre-existing } \\
\text { condition information on the facility and/or major } \\
\text { equipment. Conduct, or schedule as a separate post- } \\
\text { award action(s), any assessments required to } \\
\text { adequately address all relevant items on the checklist. } \\
\text { Provide completed checklist to RL POC for review. }\end{array}$ & $6 / 01 / 96$ & DW Lindsey \\
\hline $\begin{array}{l}\text { 7. Develop a list of open action items and commitments, } \\
\text { including status (Environmental, Tri-Party Agreement, } \\
\text { DNFSB as examples). Provide to RL POC for } \\
\text { review. }\end{array}$ & $6 / 01 / 96$ & DW Lindsey \\
\hline $\begin{array}{l}\text { 8. Identify current inventories of feed for the facility, and } \\
\text { materials to support continued operation (i.e., essential } \\
\text { materials, tools and equipment). }\end{array}$ & $6 / 01 / 96$ & DW Lindsey \\
\hline $\begin{array}{l}\text { 9. Develop a list of facility (or operation)-specific } \\
\text { mandatory training and qualification requirements for } \\
\text { employees. }\end{array}$ & 6/01/96 & DW Lindsey \\
\hline $\begin{array}{l}\text { 10. Develop a listing of information which constitutes the } \\
\text { "authorization basis" for the facility. }\end{array}$ & $6 / 01 / 96$ & DW Lindsey \\
\hline $\begin{array}{l}\text { 11. Develop a description of ongoing and planned } \\
\text { construction projects. }\end{array}$ & $6 / 01 / 96$ & DW Lindsey \\
\hline
\end{tabular}




\begin{tabular}{|c|c|c|}
\hline Pre-Award Aetions & $\begin{array}{l}\text { Scheduled } \\
\text { Date }\end{array}$ & Actionee(s) \\
\hline $\begin{array}{l}\text { 12. Develop a listing and description of facility (or } \\
\text { operations) databases. Provide list to program/project } \\
\text { level for consolidation. }\end{array}$ & $5 / 15 / 96$ & DW Lindsey \\
\hline $\begin{array}{l}\text { 13. Develop a list of current work order services or other } \\
\text { form of agreement to provide work for other } \\
\text { organizations, on and offsite. }\end{array}$ & $6 / 01 / 96$ & DW Lindsey \\
\hline $\begin{array}{l}\text { 14. Develop a list of current work order services or other } \\
\text { form of agreement to obtain work from other } \\
\text { organizations, on and offsite. }\end{array}$ & $6 / 01 / 96$ & DW Lindsey \\
\hline $\begin{array}{l}\text { 15. Develop a list of permits governing the facility (or } \\
\text { operation), and provide a description of each as to } \\
\text { where and how it applies. }\end{array}$ & $6 / 01 / 96$ & DW Lindsey \\
\hline $\begin{array}{l}\text { 16. Verify the accuracy of property assignments for the } \\
\text { facility (or operations) and correct any deficiencies. }\end{array}$ & $6 / 01 / 96$ & DW Lindsey \\
\hline 4 Post-Award Actions & & $4: 4.4$ \\
\hline $\begin{array}{l}\text { 1. Provide facility (or operations) briefing(s) to the } \\
\text { PHMC. }\end{array}$ & $7 / 01 / 96-7 / 15 / 96$ & DW Lindsey \\
\hline 2. Provide a facility (or operations) tour to the PHMC. & $7 / 01 / 96-7 / 15 / 96$ & DW Lindsey \\
\hline $\begin{array}{l}\text { 3. Provide the PHMC access to key documentation, pre- } \\
\text { existing condition information, commitment data, } \\
\text { inventory data, etc. (as established in the pre-award } \\
\text { phase) Provide copies upon request. }\end{array}$ & by $6 / 15 / 96$ & DW Lindsey \\
\hline $\begin{array}{l}\text { 4. Provide any additional documentation or information } \\
\text { requested by the PHMC, if available, that was not } \\
\text { identified during the pre-award phase. }\end{array}$ & by $7 / 01 / 96$ & DW Lindsey \\
\hline $\begin{array}{l}\text { 5. Provide PHMC any recurring reports that they would } \\
\text { like to receive during transition. }\end{array}$ & by $7 / 15 / 96$ & DW Lindsey \\
\hline 6. Provide necessary training to PHMC personnel. & $7 / 01 / 96-8 / 01 / 96$ & DW Lindsey \\
\hline $\begin{array}{l}\text { 7. Provide an updated status of ongoing work prior to } \\
\text { contract turnover. }\end{array}$ & by $9 / 30 / 96$ & DW Lindsey \\
\hline $\begin{array}{l}\text { 8. Perform a joint property and equipment inventory with } \\
\text { the PHMC. }\end{array}$ & by $8 / 30 / 96$ & DW Lindsey \\
\hline 9. Provide a categorized inventory of wastes. & by $9 / 30 / 96$ & DW Lindsey \\
\hline $\begin{array}{l}\text { 10. Assist the PHMC in the conduct of Pre-existing } \\
\text { Condition Assessments. }\end{array}$ & by $8 / 30 / 96$ & DW Lindsey \\
\hline
\end{tabular}

\subsubsection{Liquid Effluent Retention Facility. The LERF consists of three RCRA-compliant} surface impoundments for storing process condensate from the 242-A Evaporator and other feeds. The LERF provides equalization of the flow and $\mathrm{pH}$ of the feed to the ETF. Each LERF basin has a capacity of $6.5 \mathrm{Mgal}$. Two basins are used for normal operation and the third is used as contingency in the event a leak should develop in an operational basin. The basins are constructed of two high-density polyethylene (HDPE) flexible membrane liners. A system is provided to detect, collect, and remove leachate from between the primary and 
secondary liners. Beneath the secondary HDPE liner is a soil/bentonite barrier should the primary and secondary liners fail. Each basin has a mechanically-tensioned floating membrane cover constructed of very low-density polyethylene (VLDPE) to keep-out unwanted material and to minimize evaporation of the basin contents. The LERF began operation in April 1994 and is designed for a 20-year life. A total of $8.7 \mathrm{Mgal}$ of process condensate were stored in the LERF at the end of 1995.

\begin{tabular}{|c|c|c|}
\hline Pre-Award Actions & $\begin{array}{l}\text { Scheduled } \\
\text { Date }\end{array}$ & Actionof(h) \\
\hline $\begin{array}{l}\text { 1. Identify number of briefings, and prepare facility (or } \\
\text { operations) briefing(s) for the PHMC. (Coordinate } \\
\text { number and content with RL POC.) }\end{array}$ & $6 / 01 / 96$ & DW Lindsey \\
\hline $\begin{array}{l}\text { 2. Identify key documents for the facility (or operations). } \\
\text { Provide listing to program/project level for } \\
\text { consolidation and RL review. }\end{array}$ & $5 / 01 / 96$ & DW Lindsey \\
\hline $\begin{array}{l}\text { 3. Assemble key facility (or operations) documentation, } \\
\text { or identify its location, for use by the PHMC. } \\
\text { Includes preparation of a facility (or operations) } \\
\text { "Smart Book," to include information develop in the } \\
\text { following actions. }\end{array}$ & $6 / 01 / 96$ & DW Lindsey \\
\hline $\begin{array}{l}\text { 4. Identify POCs for the facility (or operations) and input } \\
\text { to consolidated program/project listing. }\end{array}$ & $5 / 15 / 96$ & DW Lindsey \\
\hline $\begin{array}{l}\text { 5. Develop a listing and description of subcontracts } \\
\text { supporting the facility (or operation) and input to } \\
\text { consolidated program/project listing. Include any } \\
\text { subcontracts planned to be executed prior to contract } \\
\text { takeover. }\end{array}$ & $5 / 15 / 96$ & DW Lindsey \\
\hline $\begin{array}{l}\text { 6. Using the pre-existing checklist, compile pre-existing } \\
\text { condition information on the facility and/or major } \\
\text { equipment. Conduct, or schedule as a separate post- } \\
\text { award action(s), any assessments required to } \\
\text { adequately address all relevant items on the checklist. } \\
\text { Provide completed checklist to RL POC for review. }\end{array}$ & $6 / 01 / 96$ & DW Lindsey \\
\hline $\begin{array}{l}\text { 7. Develop a list of open action items and commitments, } \\
\text { including status (Environmental, Tri-Party Agreement, } \\
\text { DNFSB as examples). Provide to RL POC for } \\
\text { review. }\end{array}$ & $6 / 01 / 96$ & DW Lindsey \\
\hline $\begin{array}{l}\text { 8. Identify current inventories of feed for the facility, and } \\
\text { materials to support continued operation (i.e., essential } \\
\text { materials, tools and equipment). }\end{array}$ & $6 / 01 / 96$ & DW Lindsey \\
\hline $\begin{array}{l}\text { 9. Develop a list of facility (or operation)-specific } \\
\text { mandatory training and qualification requirements for } \\
\text { employees. }\end{array}$ & $6 / 01 / 96$ & DW Lindsey \\
\hline $\begin{array}{l}\text { 10. Develop a listing of information which constitutes the } \\
\text { "authorization basis" for the facility. }\end{array}$ & $6 / 01 / 96$ & DW Lindsey \\
\hline $\begin{array}{l}\text { 11. Develop a description of ongoing and planned } \\
\text { construction projects. }\end{array}$ & $6 / 01 / 96$ & DW Lindsey \\
\hline
\end{tabular}




\begin{tabular}{|c|c|c|}
\hline Pre-Award Actions & Scheduled & $1+\operatorname{ton}=0(s)$ \\
\hline $\begin{array}{l}\text { 12. Develop a listing and description of facility (or } \\
\text { operations) databases. Provide list to program/project } \\
\text { level for consolidation. }\end{array}$ & $5 / 15 / 96$ & DW Lindsey \\
\hline $\begin{array}{l}\text { 13. Develop a list of current work order services or other } \\
\text { form of agreement to provide work for other } \\
\text { organizations, on and offsite. }\end{array}$ & $6 / 01 / 96$ & DW Lindeey \\
\hline $\begin{array}{l}\text { 14. Develop a list of current work order services or other } \\
\text { form of agreement to obtain work from other } \\
\text { organizations, on and offsite. }\end{array}$ & $6 / 01 / 96$ & DW Lindsey \\
\hline $\begin{array}{l}\text { 15. Develop a list of permits governing the facility (or } \\
\text { operation), and provide a description of each as to } \\
\text { where and how it applies. }\end{array}$ & $6 / 01 / 96$ & DW Lindsey \\
\hline $\begin{array}{l}\text { 16. Verify the accuracy of property assignments for the } \\
\text { facility (or operations) and correct any deficiencies. }\end{array}$ & $6 / 01 / 96$ & DW Lindsey \\
\hline \multicolumn{3}{|l|}{ Post-Award Actions } \\
\hline $\begin{array}{l}\text { 1. Provide facility (or operations) briefing(s) to the } \\
\text { PHMC. }\end{array}$ & $7 / 01 / 96-7 / 15 / 96$ & DW Lindsey \\
\hline 2. Provide a facility (or operations) tour to the PHMC. & $7 / 01 / 96-7 / 15 / 96$ & DW Lindsey \\
\hline $\begin{array}{l}\text { 3. Provide the PHMC access to key documentation, pre- } \\
\text { existing condition information, commitment data, } \\
\text { inventory data, etc. (as established in the pre-award } \\
\text { phase), Provide copies upon request. }\end{array}$ & by $6 / 15 / 96$ & DW Lindsey \\
\hline $\begin{array}{l}\text { 4. Provide any additional documentation or information } \\
\text { requested by the PHMC, if available, that was not } \\
\text { identified during the pre-award phase. }\end{array}$ & by $7 / 01 / 96$ & DW Lindsey \\
\hline $\begin{array}{l}\text { 5. Provide PHMC any recurring reports that they would } \\
\text { like to receive during transition. }\end{array}$ & by $7 / 15 / 96$ & DW Lindsey \\
\hline 6. Provide necessary training to PHMC personnel. & $7 / 01 / 96-8 / 01 / 96$ & DW Lindsey \\
\hline $\begin{array}{l}\text { 7. Provide an updated status of ongoing work prior to } \\
\text { contract turnover. }\end{array}$ & by $9 / 30 / 96$ & DW Lindsey \\
\hline $\begin{array}{l}\text { 8. Perform a joint property and equipment inventory with } \\
\text { the PHMC. }\end{array}$ & by $8 / 30 / 96$ & DW Lindsey \\
\hline 9. Provide a categorized inventory of wastes. & by $9 / 30 / 96$ & DW Lindsey \\
\hline $\begin{array}{l}\text { 10. Assist the PHMC in the conduct of Pre-existing } \\
\text { Condition Assessments. }\end{array}$ & by $8 / 30 / 96$ & DW Lindsey \\
\hline
\end{tabular}

\subsubsection{200 Area Effluent Treatment Facility. The ETF provides for (1) collection of} liquid effluents; (2) treatment to reduce the concentration of radioactive and hazardous waste constituents in the effluent streams to acceptable levels; (3) tanks to verify the treated effluent meets discharge requirements; and (4) disposal of the treated effluent. The treatment process constitutes BAT and includes ultraviolet light/peroxide (UV/Ox) destruction of organic wmpounds, reverse osmosis to remove dissolved solids, and ion exchange to remove the last 
traces of contaminants. Treatment capacity of the ETF is $150 \mathrm{gal} / \mathrm{min}$. The ETF began operation in December 1995 and has a 30-year design life.

The treated effluent from the ETF is discharged via a dedicated pipeline to a stateapproved land disposal site (SALDS). The SALDS consists of an underground drain field. A delisting petition was approved by the EPA and exempts the treated process condensate from the requirements of hazardous waste regulations under RCRA, and imposes certain effluent quality restrictions. High concentrations of ammonia in the evaporator process condensate also make this stream a dangerous waste subject to WAC 173-303 Dangerous Waste Regulations. The SALDS was permitted under the WAC 173-216 State Waste Discharge Permit Program.

Secondary waste from treating the process condensate (or other feed streams) is a LLMW that will be concentrated, dried, and packaged in 55-gal drums. The ETF is a RCRA permitted storage facility and this secondary waste material is temporarily stored until it is transferred to the CWC for subsequent treatment (if needed to meet Land Disposal Restriction treatment standards) and disposal in the Mixed Waste Trench.

\begin{tabular}{|c|c|c|}
\hline Pre-Award Actions & $\begin{array}{l}\text { Scheduled } \\
\text { Date }\end{array}$ & Actionee(s) \\
\hline $\begin{array}{l}\text { 1. Identify number of briefings, and prepare facility (or } \\
\text { operations) briefing(s) for the PHMC. (Coordinate } \\
\text { number and content with RL POC.) }\end{array}$ & $6 / 01 / 96$ & DW Lindsey \\
\hline $\begin{array}{l}\text { 2. Identify key documents for the facility (or operations). } \\
\text { Provide listing to program/project level for } \\
\text { consolidation and RL review. }\end{array}$ & $5 / 01 / 96$ & DW Lindsey \\
\hline $\begin{array}{l}\text { 3. Assemble key facility (or operations) documentation, } \\
\text { or identify its location, for use by the PHMC. } \\
\text { Includes preparation of a facility (or operations) } \\
\text { "Smart Book," to include information develop in the } \\
\text { following actions. }\end{array}$ & $6 / 01 / 96$ & DW Lindsey \\
\hline $\begin{array}{l}\text { 4. Identify POCs for the facility (or operations) and input } \\
\text { to consolidated program/project listing. }\end{array}$ & $5 / 15 / 96$ & DW Lindsey \\
\hline $\begin{array}{l}\text { 5. Develop a listing and description of subcontracts } \\
\text { supporting the facility (or operation) and input to } \\
\text { consolidated program/project listing. Include any } \\
\text { subcontracts planned to be executed prior to contract } \\
\text { takeover. }\end{array}$ & $5 / 15 / 96$ & DW Lindsey \\
\hline $\begin{array}{l}\text { Using the pre-existing checklist, compile pre-existing } \\
\text { condition information on the facility and/or major } \\
\text { equipment. Conduct, or schedule as a separate post- } \\
\text { award action(s), any assessments required to } \\
\text { adequately address all relevant items on the checklist. } \\
\text { Provide completed checklist to RL POC for review. }\end{array}$ & $6 / 01 / 96$ & DW Lindsey \\
\hline $\begin{array}{l}\text { 7. Develop a list of open action items and commitments, } \\
\text { including status (Environmental, Tri-Party Agreement, } \\
\text { DNFSB as examples). Provide to RL POC for } \\
\text { review. }\end{array}$ & $6 / 01 / 96$ & DW Lindsey \\
\hline
\end{tabular}




\begin{tabular}{|c|c|c|}
\hline Pre-Award Letons & Shediled & Actopen $(\beta)$ \\
\hline $\begin{array}{l}\text { 8. Identify current inventories of feed for the facility, and } \\
\text { materials to support continued operation (i.e., essential } \\
\text { materials, tools and equipment). }\end{array}$ & $6 / 01 / 96$ & DW Lindsey \\
\hline $\begin{array}{l}\text { 9. Develop a list of facility (or operation)-specific } \\
\text { mandatory training and qualification requirements for } \\
\text { employees. }\end{array}$ & $6 / 01 / 96$ & DW Lindsey \\
\hline $\begin{array}{l}\text { 10. Develop a listing of information which constitutes the } \\
\text { "authorization basis" for the facility. }\end{array}$ & $6 / 01 / 96$ & DW Lindsey \\
\hline $\begin{array}{l}\text { 11. Develop a description of ongoing and planned } \\
\text { construction projects. }\end{array}$ & $6 / 01 / 96$ & DW Lindsey \\
\hline $\begin{array}{l}\text { 12. Develop a listing and description of facility (or } \\
\text { operations) databases. Provide list to program/project } \\
\text { level for consolidation. }\end{array}$ & $5 / 15 / 96$ & DW Lindsey \\
\hline $\begin{array}{l}\text { 13. Develop a list of current work order services or other } \\
\text { form of agreement to provide work for other } \\
\text { organizations, on and offsite. }\end{array}$ & $6 / 01 / 96$ & DW Lindsey \\
\hline $\begin{array}{l}\text { 14. Develop a list of current work order services or other } \\
\text { form of agreement to obtain work from other } \\
\text { organizations, on and offsite. }\end{array}$ & $6 / 01 / 96$ & DW Lindsey \\
\hline $\begin{array}{l}\text { 15. Develop a list of permits governing the facility (or } \\
\text { operation), and provide a deacription of each as to } \\
\text { where and how it applies. }\end{array}$ & $6 / 01 / 96$ & DW Lindsey \\
\hline $\begin{array}{l}\text { 16. Verify the accuracy of property asignments for the } \\
\text { facility (or operations) and correct any deficiencies. }\end{array}$ & $6 / 01 / 96$ & DW Lindsey \\
\hline 19 Post-Awnd Action & & \\
\hline $\begin{array}{l}\text { 1. Provide facility (or operations) brefing(s) to the } \\
\text { PHMC. }\end{array}$ & $7 / 01 / 96-7 / 15 / 96$ & DW Lindsey \\
\hline 2. Provide a facility (or operatoon) lour to the PHMC. & $7 / 01 / 96-7 / 15 / 96$ & DW Lindsey \\
\hline 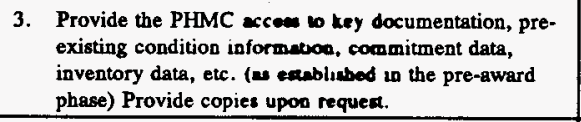 & by $6 / 15 / 96$ & DW Lindsey \\
\hline $\begin{array}{l}\text { 4. Provide any additional documentation or information } \\
\text { requested by the PHMC, if available, that was not } \\
\text { identified during the pre-award phase. }\end{array}$ & by $7 / 01 / 96$ & DW Lindsey \\
\hline $\begin{array}{l}\text { 5. Provide PHMC any recurring reports that they would } \\
\text { like to receive during transition. }\end{array}$ & by $7 / 15 / 96$ & DW Lindsey \\
\hline 6. Provide necessary training to PHMC personnel. & $7 / 01 / 96-8 / 01 / 96$ & DW Lindsey \\
\hline $\begin{array}{l}\text { 7. Provide an updated status of ongoing work prior to } \\
\text { contract tumover. }\end{array}$ & by $9 / 30 / 96$ & DW Lindsey \\
\hline $\begin{array}{l}\text { 8. Perform a joint property and equipment inventory with } \\
\text { the PHMC. }\end{array}$ & by $8 / 30 / 96$ & DW Lindsey \\
\hline 9. Provide a categorized inventory of wastes. & by $9 / 30 / 96$ & DW Lindsey \\
\hline
\end{tabular}




\begin{tabular}{|c|c|c|c|}
\hline & Post-Award Actions & Schetuled & Petsonects) \\
\hline & $\begin{array}{l}\text { Assist the PHMC in the conduct of Pre-existing } \\
\text { Condition Assessments. }\end{array}$ & by $8 / 30 / 96$ & DW Lindsey \\
\hline
\end{tabular}

4.2.2.4 300 Area Treated Effluent Disposal Facility. Wastewater from laboratories, research facilities, office buildings, and fuel fabrication facilities in the 300 Area is treated in the 300 Area TEDF. The wastewater consists of once-through cooling water, steam condensate, and other liquid wastes generated in non-contact radioactive processes. The 300 Area TEDF is designed for continuous receipt of waste waters with storage capacity of up to 5 days at the design flow rate of $300 \mathrm{gal} / \mathrm{min}$. The facility treats the wastewater using BAT. The treatment process includes iron co-precipitation to remove heavy metals, thiol functional resin ion exchange to remove mercury, and ultraviolet light/hydrogen peroxide oxidation to destroy organics and cyanide. The 300 Area TEDF began operation in December 1994 and has a 30-year design life. About $83 \mathrm{Mgal}$ of wastewater were treated in 1995.

The treated liquid effluent is monitored and discharged through an outfall to the Columbia River under a NPDES permit. Sludge from the iron co-precipitation process is dewatered and used for backfill in the LLW trench.

\begin{tabular}{|c|c|c|}
\hline Pre-Award Actions & $\begin{array}{l}\text { Scheduled } \\
\text { Date }\end{array}$ & Actionee(s) \\
\hline $\begin{array}{l}\text { 1. Identify number of briefings, and prepare facility (or } \\
\text { operations) briefing(s) for the PHMC. (Coordinate } \\
\text { number and content with RL POC.) }\end{array}$ & $6 / 01 / 96$ & LW Roberts \\
\hline $\begin{array}{l}\text { 2. Identify key documents for the facility (or operations). } \\
\text { Provide listing to program/project level for } \\
\text { consolidation and RL review. }\end{array}$ & $5 / 01 / 96$ & LW Roberts \\
\hline $\begin{array}{l}\text { 3. Assemble key facility (or operations) documentation, } \\
\text { or identify its location, for use by the PHMC. } \\
\text { Includes preparation of a facility (or operations) } \\
\text { "Smart Book," to include information develop in the } \\
\text { following actions. }\end{array}$ & $6 / 01 / 96$ & LW Roberts \\
\hline $\begin{array}{l}\text { 4. Identify POCs for the facility (or operations) and input } \\
\text { to consolidated program/project listing. }\end{array}$ & $5 / 15 / 96$ & LW Roberts \\
\hline $\begin{array}{l}\text { 5. Deveiop a listing and description of subcontracts } \\
\text { supporting the facility (or operation) and input to } \\
\text { consolidated program/project listing. Include any } \\
\text { subcontracts planned to be executed prior to contract } \\
\text { takeover. }\end{array}$ & $5 / 15 / 96$ & LW Roberts \\
\hline $\begin{array}{l}\text { 6. Using the pre-existing checklist, compile pre-existing } \\
\text { condition information on the facility and/or major } \\
\text { equipment. Conduct, or schedule as a separate post- } \\
\text { award action(s), any assessments required to } \\
\text { adequately address all relevant items on the checklist. } \\
\text { Provide completed checklist to RL POC for review. }\end{array}$ & $6 / 01 / 96$ & LW Roberts \\
\hline
\end{tabular}




\begin{tabular}{|c|c|c|}
\hline Pre-Award Actions & ocheouled & tentonee(s) \\
\hline $\begin{array}{l}\text { 7. Develop a list of open action items and commitments, } \\
\text { including status (Environmental, Tri-Party Agreement, } \\
\text { DNFSB as examples). Provide to RL POC for } \\
\text { review. }\end{array}$ & $6 / 01 / 96$ & LW Roberts \\
\hline $\begin{array}{l}\text { 8. Identify current inventories of feed for the facility, and } \\
\text { materials to support continued operation (i.e., essential } \\
\text { materials, tools and equipment). }\end{array}$ & $6 / 01 / 96$ & LW Roberts \\
\hline $\begin{array}{l}\text { 9. Develop a list of facility (or operation)-epecific } \\
\text { mandatory training and qualification requirements for } \\
\text { employees. }\end{array}$ & $6 / 01 / 96$ & LW Roberts \\
\hline $\begin{array}{l}\text { 10. Develop a listing of information which constitutes the } \\
\text { "authorization basis" for the facility. }\end{array}$ & $6 / 01 / 96$ & LW Roberts \\
\hline $\begin{array}{l}\text { 11. Develop a description of ongoing and planned } \\
\text { construction projects. }\end{array}$ & $6 / 01 / 96$ & LW Roberts \\
\hline $\begin{array}{l}\text { 12. Develop a listing and description of facility (or } \\
\text { operations) databases. Provide list to program/project } \\
\text { level for consolidation. }\end{array}$ & $5 / 15 / 96$ & LW Roberts \\
\hline $\begin{array}{l}\text { 13. Develop a list of current work order services or other } \\
\text { form of agreement to provide work for other } \\
\text { organizations, on and offsite. }\end{array}$ & $6 / 01 / 96$ & LW Roberts \\
\hline $\begin{array}{l}\text { 14. Develop a list of current work order services or other } \\
\text { form of agreement to obtain work from other } \\
\text { organizations, on and offsite. }\end{array}$ & $6 / 01 / 96$ & LW Roberts \\
\hline $\begin{array}{l}\text { 15. Develop a list of permits governing the facility (or } \\
\text { operation), and provide a description of each as to } \\
\text { where and how it applies. }\end{array}$ & $6 / 01 / 96$ & LW Roberts \\
\hline $\begin{array}{l}\text { 16. Verify the accuracy of property assignments for the } \\
\text { facility (or operations) and correct any deficiencies. }\end{array}$ & $6 / 01 / 96$ & LW Roberts \\
\hline Post-Award Actions & & \\
\hline $\begin{array}{l}\text { 1. Provide facility (or operations) briefing(s) to the } \\
\text { PHMC. }\end{array}$ & $7 / 01 / 96-7 / 15 / 96$ & LW Roberts \\
\hline 2. Provide a facility (or operations) tour to the PHMC. & $7 / 01 / 96-7 / 15 / 96$ & LW Roberts \\
\hline $\begin{array}{l}\text { 3. Provide the PHMC access to key documentation, pre- } \\
\text { existing condition information, commitment data, } \\
\text { inventory data, etc. (as established in the pre-award } \\
\text { phase) Provide copies upon request. }\end{array}$ & by $6 / 15 / 96$ & LW Roberts \\
\hline $\begin{array}{l}\text { 4. Provide any additional documentation or information } \\
\text { requested by the PHMC, if available, that was not } \\
\text { identified during the pre-award phase. }\end{array}$ & by $7 / 01 / 96$ & LW Roberts \\
\hline $\begin{array}{l}\text { 5. Provide PHMC any recurring reports that they would } \\
\text { like to receive during transition. }\end{array}$ & by $7 / 15 / 96$ & LW Roberts \\
\hline 6. Provide necessary training to PHMC personnel. & $7 / 01 / 96-8 / 01 / 96$ & LW Roberts \\
\hline $\begin{array}{l}\text { 7. Provide an updated status of ongoing work prior to } \\
\text { contract turnover. }\end{array}$ & by $9 / 30 / 96$ & LW Roberts \\
\hline
\end{tabular}




\begin{tabular}{|c|c|c|}
\hline Poot-Award Actions & Scheinler & fetfones (s) \\
\hline $\begin{array}{l}\text { 8. Perform a joint property and equipment inventory with } \\
\text { the PHMC. }\end{array}$ & by $8 / 30 / 96$ & LW Roberts \\
\hline 9. Provide a categorized inventory of wastes. & by $9 / 30 / 96$ & LW Roberts \\
\hline $\begin{array}{l}\text { 10. Assist the PHMC in the conduct of Pre-existing } \\
\text { Condition Assessments. }\end{array}$ & by $8 / 30 / 96$ & LW Roberts \\
\hline
\end{tabular}

4.2.2.5 340 Waste Handling Facility. The 340 Complex provides receipt, storage, and loadout capability for low-level liquid waste generated during laboratory operations in the 300 Area. The waste is accumulated and stored in two, 15,000-gal tanks located in a covered, below-grade vault in the 340 Building. Six additional 8,000-gal tanks in the adjacent 340-A Building provide backup storage capability. The waste is pumped into rail cars and transported to the 204-AR Unloading Facility, for neutralization and transfer to DSTs in the 200 Area for storage. The 340 Facility does not have a RCRA permit and wastes cannot be stored for more than 90 days.

The 340 Facility will cease operation on or before the year 2000 following implementation of a replacement system in an existing facility such as the 325 Building. A new waste handling facility with storage and loadout capability will be provided for the 325 Building. This replacement facility will also serve any other generators which are still operating. The 340 Facility will be cleaned out and custody will be transferred to the Facility Stabilization Program for deactivation.

\begin{tabular}{|c|c|c|}
\hline Pre-Award Actions & $\begin{array}{l}\text { Scheduled } \\
\text { Date }\end{array}$ & Actionee( $($ ) \\
\hline $\begin{array}{l}\text { 1. Identify number of briefings, and prepare facility (or } \\
\text { operations) briefing(s) for the PHMC. (Coordinate } \\
\text { number and content with RL POC.) }\end{array}$ & $6 / 01 / 96$ & LW Roberts \\
\hline $\begin{array}{l}\text { 2. Identify key documents for the facility (or operations). } \\
\text { Provide listing to program/project level for } \\
\text { consolidation and RL review. }\end{array}$ & $5 / 01 / 96$ & LW Roberts \\
\hline $\begin{array}{l}\text { 3. Assemble key facility (or operations) documentation, } \\
\text { or identify its location, for use by the PHMC. } \\
\text { Includes preparation of a facility (or operations) } \\
\text { "Smart Book," to include information develop in the } \\
\text { following actions. }\end{array}$ & $6 / 01 / 96$ & LW Roberts \\
\hline $\begin{array}{l}\text { 4. Identify POCs for the facility (or operations) and input } \\
\text { to consolidated program/project listing. }\end{array}$ & $5 / 15 / 96$ & LW Roberts \\
\hline $\begin{array}{l}\text { 5. Develop a listing and description of subcontracts } \\
\text { supporting the facility (or operation) and input to } \\
\text { consolidated program/project listing. Include any } \\
\text { subcontracts planned to be executed prior to contract } \\
\text { takeover. }\end{array}$ & $5 / 15 / 96$ & LW Roberts \\
\hline
\end{tabular}




\begin{tabular}{|c|c|c|}
\hline Pre-Avard Actions & $\begin{array}{l}\text { Schetuled } \\
\text { Date }\end{array}$ & Actionee(6) \\
\hline $\begin{array}{l}\text { 6. Using the pre-existing checklist, compile pre-existing } \\
\text { condition information on the facility and/or major } \\
\text { equipment. Conduct, or schedule as a separate post- } \\
\text { award action(s), any assessments required to } \\
\text { adequately address all relevant items on the checklist. } \\
\text { Provide completed checklist to RL POC for review. }\end{array}$ & 6/01/96 & LW Roberts \\
\hline $\begin{array}{l}\text { 7. Develop a list of open action items and commitments, } \\
\text { including status (Environmental, Tri-Party Agreement, } \\
\text { DNFSB as examples). Provide to RL POC for } \\
\text { review. }\end{array}$ & 6/01/96 & LW Roberts \\
\hline $\begin{array}{l}\text { 8. Identify current inventories of feed for the facility, and } \\
\text { materials to support continued operation (i.e., essential } \\
\text { materials, tools and equipment). }\end{array}$ & $6 / 01 / 96$ & LW Roberts \\
\hline $\begin{array}{l}\text { 9. Develop a list of facility (or operation)-specific } \\
\text { mandatory training and qualification requirements for } \\
\text { employees. }\end{array}$ & $6 / 01 / 96$ & LW Roberts \\
\hline $\begin{array}{l}\text { 10. Develop a listing of information which constitutes the } \\
\text { "authorization basis" for the facility. }\end{array}$ & $6 / 01 / 96$ & LW Roberts \\
\hline $\begin{array}{l}\text { 11. Develop a description of ongoing and planned } \\
\text { construction projects. }\end{array}$ & 6/01/96 & LW Roberts \\
\hline $\begin{array}{l}\text { 12. Develop a listing and description of facility (or } \\
\text { operations) databases. Provide list to program/project } \\
\text { level for consolidation. }\end{array}$ & $5 / 15 / 96$ & LW Roberts \\
\hline $\begin{array}{l}\text { 13. Develop a list of current work order services or other } \\
\text { form of agreement to provide work for other } \\
\text { organizations, on and offsite. }\end{array}$ & $6 / 01 / 96$ & LW Roberts \\
\hline $\begin{array}{l}\text { 14. Develop a list of current work order services or other } \\
\text { form of agreement to obtain work from other } \\
\text { organizations, on and offsite. }\end{array}$ & $6 / 01 / 96$ & LW Roberts \\
\hline $\begin{array}{l}\text { 15. Develop a list of permits governing the facility (or } \\
\text { operation), and provide a description of each as to } \\
\text { where and how it applies. }\end{array}$ & $6 / 01 / 96$ & LW Roberts \\
\hline $\begin{array}{l}\text { 16. Verify the accuracy of property assignments for the } \\
\text { facility (or operations) and correct any deficiencies. }\end{array}$ & $6 / 01 / 96$ & LW Roberts \\
\hline 44 Post-Award Actions & $8 \%$ & 184 \\
\hline $\begin{array}{l}\text { 1. Provide facility (or operations) briefing(s) to the } \\
\text { PHMC. }\end{array}$ & $7 / 01 / 96-7 / 15 / 96$ & LW Roberts \\
\hline 2. Provide a facility (or operations) tour to the PHMC. & $7 / 01 / 96-7 / 15 / 96$ & LW Roberts \\
\hline $\begin{array}{l}\text { 3. Provide the PHMC access to key documentation, pre- } \\
\text { existing condition information, commitment data, } \\
\text { inventory data, etc. (as established in the pre-award } \\
\text { phase). Provide copies upon request. }\end{array}$ & by $6 / 15 / 96$ & LW Roberts \\
\hline $\begin{array}{l}\text { 4. Provide any additional documentation or information } \\
\text { requested by the PHMC, if available, that was not } \\
\text { identified during the pre-award phase. }\end{array}$ & by $7 / 01 / 96$ & LW Roberts \\
\hline
\end{tabular}




\begin{tabular}{|c|c|c|}
\hline Post-Award Actions & $\begin{array}{l}\text { Schedinled } \\
\text { Date }\end{array}$ & Petionee (6) \\
\hline $\begin{array}{l}\text { 5. Provide PHMC any recurring reports that they would } \\
\text { like to receive during transition. }\end{array}$ & by $7 / 15 / 96$ & LW Roberts \\
\hline 6. Provide necessary training to PHMC personnel. & 7/01/96-8/01/96 & LW Roberts \\
\hline $\begin{array}{l}\text { 7. Provide an updated status of ongoing work prior to } \\
\text { contract turnover. }\end{array}$ & by $9 / 30 / 96$ & LW Roberts \\
\hline $\begin{array}{l}\text { 8. Perform a joint property and equipment inventory with } \\
\text { the PHMC. }\end{array}$ & by $8 / 30 / 96$ & LW Roberts \\
\hline 9. Provide a categorized inventory of wastes. & by $9 / 30 / 96$ & LW Roberts \\
\hline $\begin{array}{l}\text { 10. Assist the PHMC in the conduct of Pre-existing } \\
\text { Condition Assessments. }\end{array}$ & by $8 / 30 / 96$ & LW Roberts \\
\hline
\end{tabular}

\subsection{SPENT NUCLEAR FUEL}

The scope of the SNF Project covers the maintenance and preparation of the $\mathbf{K}$ Basins for safe storage and removal of all SNF, debris, and sludge; constructing, as necessary, new systems and facilities to condition and store the fuel prior to final disposition; relocating K Basins spent nuclear fuel to the interim storage facility; and accepting stewardship of spent nuclear fuel from other Hanford locations. The Project Management Plan sets forth the management basis for the SNF Project.

\begin{tabular}{|c|c|c|}
\hline Pre-Award Actions & $\begin{array}{l}\text { Scheduled } \\
\text { Date }\end{array}$ & Actionee(s) \\
\hline $\begin{array}{l}\text { 1. Provide documents that led to overall SNF Project } \\
\text { Strategy }\end{array}$ & $5 / 31 / 96$ & EW Gerber \\
\hline $\begin{array}{l}\text { 2. Provide key SNF Project Management documents } \\
\text { (Project Plan, Project Management Plan, SNF Interim } \\
\text { Storage Plan, MOUs, etc.). }\end{array}$ & $5 / 31 / 96$ & $\begin{array}{l}\text { CC Hages } \\
\text { SL Magnani }\end{array}$ \\
\hline $\begin{array}{l}\text { 3. Develop a list of key project documentation. Provide } \\
\text { to RL POC for review. }\end{array}$ & $5 / 15 / 96$ & CC Hages \\
\hline $\begin{array}{l}\text { 4. Identify key project documentation location for use by } \\
\text { the PHMC. }\end{array}$ & $6 / 01 / 96$ & CC Hages \\
\hline $\begin{array}{l}\text { 5. Identify number of briefings for presentation to } \\
\text { PHMC. (Coordinate number and content with RL } \\
\text { POC.) }\end{array}$ & $6 / 01 / 96$ & CC Hages \\
\hline $\begin{array}{l}\text { 6. Develop a listing of Key project individuals as POCs, } \\
\text { including facility and operations personnel. }\end{array}$ & $6 / 01 / 96$ & CC Hages \\
\hline $\begin{array}{l}\text { 7. Develop listing and description of project subcontracts, } \\
\text { including operations subcontracts. Include any } \\
\text { subcontracts still planned to be executed prior to } \\
\text { contract takeover. }\end{array}$ & $5 / 15 / 96$ & CC Hages \\
\hline $\begin{array}{l}\text { 8. Provide copies of all SNF subcontracts the PHMC will } \\
\text { assume. }\end{array}$ & $6 / 01 / 96$ & CC Hages \\
\hline
\end{tabular}




\begin{tabular}{|c|c|c|}
\hline Pre-Award Actions & $\begin{array}{l}\text { Scheduled } \\
\text { Date }\end{array}$ & Mationed $(6)$ \\
\hline $\begin{array}{l}\text { 9. Develop listing and description of project databases, } \\
\text { including operations databases. }\end{array}$ & $6 / 01 / 96$ & CC Hages \\
\hline $\begin{array}{l}\text { 10. Verify the accuracy of property assignments at the } \\
\text { project organizational level and correct any } \\
\text { deficiencies. }\end{array}$ & 6/01/96 & CC Hages \\
\hline $\begin{array}{l}\text { 11. Identify space and equipment within project area and } \\
\text { facilities for use by the PHMC. }\end{array}$ & 6/01/96 & CC Hages \\
\hline $\begin{array}{l}\text { 12. In outline form for all operations to follow, define the } \\
\text { appropriate contents and level of detail to be included } \\
\text { in operations briefings to the PHMC. (Review with } \\
\text { RL POC.) }\end{array}$ & $5 / 01 / 96$ & CC Hages \\
\hline 144 Post-Award Actions & & \\
\hline $\begin{array}{l}\text { 1. Provide the PHMC access to the key project } \\
\text { documentation, listing of POCs, and listing of } \\
\text { subcontracts. Provide copies upon request. }\end{array}$ & $6 / 10 / 96$ & CC Hages \\
\hline $\begin{array}{l}\text { 2. Project and facility space and services available for use } \\
\text { by the PHMC }\end{array}$ & $6 / 14 / 96$ & CC Hages \\
\hline 3. Conduct briefing on SNF Project Strategy & $6 / 14 / 96$ & EW Gerber \\
\hline $\begin{array}{l}\text { 4. Conduct high-level briefing on Project charter and } \\
\text { mission }\end{array}$ & $6 / 14 / 96$ & JC Fulton \\
\hline $\begin{array}{l}\text { 5. Perform a joint project level property and equipment } \\
\text { inventory with PHMC personnel. }\end{array}$ & by $8 / 30 / 96$ & CC Hages \\
\hline
\end{tabular}

\subsubsection{Spent Nuclear Fuel Project Integration}

Project Integration encompasses the development of required project management planning documentation, establishment of the project technical, schedule, and cost baselines, and definition of work priorities and funding requirements. Project Evaluation defines project reporting, review, and commitment tracking requirements. Project Execution covers the implementation of the project control system through work authorization, baseline status accounting, and BCC. Workscope includes integration of environmental, safety, quality assurance, regulatory involvement, and external interfaces.

\begin{tabular}{|c|c|c|}
\hline Pre-Award Actions & $\begin{array}{c}\text { Scheduled } \\
\text { Date }\end{array}$ & Actionedo \\
\hline $\begin{array}{l}\text { 1. Provide key documents on cost, schedule, reporting, } \\
\text { administration, safety, and stakeholders involvement to } \\
\text { include Level } 0,1 \text {, and } 2 \text { resource loaded schedules, } \\
\text { MYPP, ADS, RDS, SMP, RRP, Construction Project } \\
\text { Data Sheet, configuration control, etc. }\end{array}$ & $5 / 31 / 96$ & CC Hages \\
\hline
\end{tabular}




\begin{tabular}{|l|c|l|}
\hline & Schediled & Date \\
1. Conduct budget and schedule briefings. & $6 / 14 / 96$ & $\begin{array}{l}\text { JL Denning } \\
\text { SL Magnani }\end{array}$ \\
\hline 2. Conduct safety and regulatory briefings. & $6 / 14 / 96$ & GC Mooers \\
\hline 3. Conduct briefing on tribal and stakeholder participation. & $6 / 14 / 96$ & GC Mooers \\
\hline 4. Conduct briefing on administration procedures. & $6 / 14 / 96$ & CC Hages \\
\hline $\begin{array}{l}\text { 5. Turnover soft copies and programs for the key project } \\
\text { documents, baselines, and reporting mechanism. }\end{array}$ & $6 / 10 / 96$ & CC Hages \\
\hline
\end{tabular}

\subsubsection{Spent Nuclear Fuel Systems Integration}

The Systems Integration element encompasses the overall technical direction for the SNF Project. The workscope includes identification and evaluation of technical approaches for safe dry interim storage of $\mathrm{K}$ Basins SNF, implementation of the overall technical strategy for the Project, and resolution of technical issues as necessary to implement the strategy. System Integration consists of six major sub-elements:

- Systems Engineering

- Process Engineering

- Technology Acquisition

- Characterization

- Technical Program Integration

- EIS.

\begin{tabular}{|c|c|c|}
\hline Pre-Award Actions & $\begin{array}{l}\text { Seheduled } \\
\text { Date }\end{array}$ & Actionee(s) \\
\hline 1. Provide key documents on SNF Systems Integration. & $5 / 31 / 96$ & EW Gerber \\
\hline $\begin{array}{l}\text { 2. Provide background information on the EIS and Record } \\
\text { of Decision. }\end{array}$ & $5 / 31 / 96$ & GC Mooers \\
\hline (ैost-Award Actions: & & \\
\hline $\begin{array}{l}\text { 1. Conduct the briefing for the Integrated Process Strategy } \\
\text { and Process Flow. }\end{array}$ & $6 / 14 / 96$ & EW Gerber \\
\hline $\begin{array}{l}\text { 2. Conduct briefing on Technology Acquisition, and } \\
\text { Characterization. }\end{array}$ & $7 / 01 / 96$ & EW Gerber \\
\hline 3. Conduct briefing on EIS and ROD. & $7 / 08 / 96$ & GC Mooers \\
\hline
\end{tabular}




\subsubsection{K Basins Maintenance and Operations}

$\mathrm{K}$ Basins M\&O manages and directs all activities at the $\mathrm{K}$ Basins in a safe, economic, and environmentally sound manner, including facility operations and maintenance; handling and storage of spent nuclear fuel; and operational support of the SNF Project. All activities are conducted in compliance with DOE contractual orders and applicable Federal, state, and local laws.

\begin{tabular}{|c|c|c|}
\hline Pre-Award Actions & $\begin{array}{l}\text { Scheduled } \\
\text { Date }\end{array}$ & Actionoe(s) \\
\hline $\begin{array}{l}\text { 1. Using the pre-existing condition checklist being } \\
\text { developed for the site ( } 4 / 15 \text { availability) compile pre- } \\
\text { existing condition information on the K Basins and } \\
\text { major equipment at the basins. Conduct, or schedule as } \\
\text { a separate post-closure action(s), any assessments } \\
\text { required to adequately address all relevant items on the } \\
\text { checklist (latest completion: } 7 / 15 \text { ). }\end{array}$ & $6 / 01 / 96$ & DW Siddoway \\
\hline $\begin{array}{l}\text { 2. Provide } K \text { Basin fuel inventory and turnover } \\
\text { documentation and certification. }\end{array}$ & $6 / 01 / 96$ & DW Siddoway \\
\hline 44 Post-Award Actions & & \\
\hline $\begin{array}{l}\text { 1. Provide the PHMC K Basin baseline documentation, } \\
\text { pre-existing reports, and Dose and Resource Assessment } \\
\text { information. }\end{array}$ & $6 / 14 / 96$ & DW Siddoway \\
\hline 2. Conduct briefing on $\mathrm{K}$ Basin operations. & $6 / 21 / 96$ & DW Siddoway \\
\hline 3. Conduct facility tour(s). & 7/01-7/03/96 & DW Siddoway \\
\hline $\begin{array}{l}\text { 4. Conduct briefing on fuel accountability and turnover } \\
\text { mechanism. }\end{array}$ & $7 / 01 / 96$ & DW Siddoway \\
\hline $\begin{array}{l}\text { 5. Conduct briefing on facility turnover and acceptance of } \\
\text { pre-existing conditions. }\end{array}$ & $7 / 03 / 96$ & DW Siddoway \\
\hline $\begin{array}{l}\text { 6. Assist the PHMC in the conduct of Pre-existing } \\
\text { Condition Assessments. }\end{array}$ & by $8 / 30 / 96$ & DW Siddoway \\
\hline
\end{tabular}

\subsubsection{K Basin Material Removal and Clean-Up Project}

The K Basin Material Removal and Clean-Up Project manages and executes projects and subprojects within K Area required to support the Path Forward, and projects required to support $\mathrm{K}$ Basins day-to-day maintenance and operations function consistent with SNF Project requirements. Projects/subprojects scope will be defined by specific acquisition strategies, but typically will be from design through turnover to operations. The Fuel Removal System Design and Acquisition Project is being managed separate from water, sludge, and debris. 


\begin{tabular}{|c|c|c|}
\hline Pre-Award Actions & $\begin{array}{l}\text { Schedulod } \\
\text { Date }\end{array}$ & Actopes $(s)$ \\
\hline 1. Provide facility projects documentation. & $5 / 31 / 96$ & MJ Wiemers \\
\hline 2. Provide Fuel Removal Systems documentation. & $5 / 31 / 96$ & BS Carlisle \\
\hline $\begin{array}{l}\text { 3. Provide Fuel Removal Water Purification } \\
\text { documentation. }\end{array}$ & $5 / 31 / 96$ & DS Takasumi \\
\hline Post-Award Actions & & \\
\hline 1. Conduct facility projects overview briefing. & $6 / 14 / 96$ & MJ Wiemers \\
\hline $\begin{array}{l}\text { 2. Conduct dose reduction, water, sludge, and debris } \\
\text { project status briefing. }\end{array}$ & $6 / 21 / 96$ & MI Wiemers \\
\hline 3. Conduct Fuel Removal Systems briefing. & $6 / 14 / 96$ & BS Carlisle \\
\hline
\end{tabular}

\subsubsection{Multi-Canister Overpack (MCO) Acquisition}

The MCO represents activities that provide a container or containment boundary with known integrity for SNF. The MCO is common to systems associated with fuel retrieval, transport, drying, staging, conditioning, and storage. Therefore, the MCO is designed to the most restrictive constraints imposed by requirements for Fuel Retrieval, K Basins SNF Vacuum Drying, Cask and Transportation, Canister Storage Building (CSB), and Hot Vacuum Conditioning systems. The current MCO concept is a cylindrical vessel with removable fuel baskets that are capable of being stacked within the cylinder.

\begin{tabular}{|c|c|c|}
\hline 1. Pre-Award Actions & $\begin{array}{l}\text { Scheduled } \\
\text { Date }\end{array}$ & hetionee(s) \\
\hline $\begin{array}{l}\text { 1. Provide MCO design fanncation and testing } \\
\text { documentation and sufery pepon. }\end{array}$ & $5 / 31 / 96$ & WD Gallo \\
\hline Post-Award Artions & & \\
\hline 1. Conduct briefing on MICO design and safety basis. & $6 / 14 / 96$ & WD Gallo \\
\hline 2. Conduct briefing on MiCO Fahrication and Testing. & $6 / 21 / 96$ & WD Gallo \\
\hline
\end{tabular}

\subsubsection{Cask/Transportation System}

The cask and transportation activities involve physically removing the MCO/Cask from the basin loadout pit, moving the cask to the K Basin SNF Vacuum Drying, and transporting the loaded cask from the $\mathrm{K}$ Basin to the CSB (or hot vacuum conditioning as an alternative) located in the 200 Area. Empty MCOs are also received at the CSB, transported, and placed in the $\mathrm{K}$ Basin loadout. 


\begin{tabular}{|c|c|c|}
\hline Pre-Award Actions & $\begin{array}{l}\text { Scheduled } \\
\text { Date }\end{array}$ & Actionee(s) \\
\hline $\begin{array}{l}\text { 1. Provide Cask/Transportation Systems Acquisition, } \\
\text { Testing and Qualification documentation and Regulatory } \\
\text { Compliance information. }\end{array}$ & $5 / 31 / 96$ & WD Gallo \\
\hline 2. Provide Basin facility upgrade plans for transportation. & $5 / 31 / 96$ & WD Gallo \\
\hline 3. Provide SARP. & $5 / 31 / 96$ & WD Gallo \\
\hline (4. 4 Post-Award Actions & ? & ই \\
\hline $\begin{array}{l}\text { 1. Conduct briefings on Cask/Transportation design and } \\
\text { safety basis. }\end{array}$ & $6 / 14 / 96$ & WD Gallo \\
\hline $\begin{array}{l}\text { 2. Conduct briefings on Basin Upgrades for } \\
\text { Transportation. }\end{array}$ & $6 / 21 / 96$ & WD Gallo \\
\hline
\end{tabular}

\subsubsection{Canister Storage Building}

The CSB will be used for dry staging and storage of the $\mathrm{K}$ Basins fuel. The CSB will utilize the partially constructed building in the 200 East Area that was originally intended for a vitrification plant.

\begin{tabular}{|c|c|c|}
\hline Pre-Award Actions & $\begin{array}{l}\text { Scheduled } \\
\text { Date }\end{array}$ & Actionee(s) \\
\hline 1. Provide CSB Project Manapement Plan. & $5 / 31 / 96$ & MK Mahaffey \\
\hline $\begin{array}{l}\text { 2. Provide Fluor Title II wns III whement of work and } \\
\text { performance specification S.043. }\end{array}$ & $5 / 31 / 96$ & J Bazinet \\
\hline 3. Provide CSB Startup Plan and Operations Staffing Plan. & $5 / 31 / 96$ & C Thompson \\
\hline $\begin{array}{l}\text { 4. Provide Phased SARs and SRID. (depending on } \\
\text { funding). }\end{array}$ & 6/01/96 & J Garvin \\
\hline 5. Provide Construction Mtanazement Plan. & $6 / 01 / 96$ & J Mortimer \\
\hline Post-Aunard Actimas & 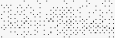 & \\
\hline $\begin{array}{l}\text { 1. Conduct briefings on Project Masagement, Design, and } \\
\text { Construction Status. }\end{array}$ & $6 / 14 / 96$ & MK Mahaffey \\
\hline $\begin{array}{l}\text { 2. Conduct briefing on CSB Regulatory Strategy and } \\
\text { Permitting. }\end{array}$ & $6 / 21 / 96$ & GC Mooers \\
\hline 3. Conduct tour of CSB construction. & $6 / 21 / 96$ & J Mortimer \\
\hline $\begin{array}{l}\text { 4. Conduct briefing on CSB Authorization Basis Strategy } \\
\text { and documentation. }\end{array}$ & $6 / 21 / 96$ & GC Mooers \\
\hline 5. Presentation on CSB Operations and ORR & $6 / 28 / 96$ & C Thompson \\
\hline
\end{tabular}




\section{WHC-SP-1181}

\subsubsection{Fuel Conditioning Processes}

Cold Vacuum Drying. The Cold Vacuum Drying Facility will remove the water from the MCO before shipping it to the CSB by a combination of draining and cold vacuum drying. Vacuum drying will require only 24 to 48 hours to remove all of the free water. Removal of the free water greatly reduces the hydrogen generation rate and allows the MCO to be shipped to the CSB with the process valves closed, greatly enhancing safety during shipping.

The Hot Vacuum Conditioning is expected to be performed within the CSB. This process involves heating the fuel and any residual sludge to $300^{\circ} \mathrm{C}$, where it is held under vacuum to remove water that is chemically bound to fuel and corrosion products, and to decompose uranium hydride that may be present. After the drying and uranium hydride decomposition step is completed, the fuel is cooled and exposed to a mixture of inert gas and controlled concentrations of oxygen. This step exposes the fuel to increasing levels of oxygen in a controlled manner to oxidize high surface area uranium metal. Thus, high surface area unoxidized uranium metal formed by the decomposition of hydrides, or during fuel handling, will be eliminated. When this partial oxidation step is completed, the MCOs will be cooled, backfilled with inert gas and sealed for interim storage in the CSB.

\begin{tabular}{|c|c|c|}
\hline Pre-Award Actions & $\begin{array}{l}\text { Scheduled } \\
\text { Date }\end{array}$ & Actioned(s) \\
\hline $\begin{array}{l}\text { 1. Provide Cold Vacuum Drying Facility Design, } \\
\text { Acquisition, Safety, and Regulatory documentation. }\end{array}$ & $5 / 31 / 96$ & FW Bradshaw \\
\hline $\begin{array}{l}\text { 2. Provide Hot Conditioning System Design, Acquisition, } \\
\text { Safety, and Regulatory documentation. }\end{array}$ & $5 / 31 / 96$ & WD Gallo \\
\hline Post-Award Actions & & 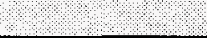 \\
\hline 1. Conduct briefings on Cold Vacuum Drying. & $6 / 14 / 96$ & FW Bradshaw \\
\hline 2. Conduct briefings on Hot Conditioning. & $7 / 01 / 96$ & WD Gallo \\
\hline
\end{tabular}

\subsubsection{Other Hanford Fuel Retrieval Processing and Storage}

The Other Fuel Management sub-project encompasses SNF Project activities necessary to attain safe interim storage of the SNF at the Hanford Site that is not currently stored at the K Basins and transfer of these materials to INEL in accordance with the SNF and INEL EIS Record of Decision. Some of the SNF stored at the 200 Area ISA may be transferred to INEL for long-term interim storage. 


\begin{tabular}{|c|c|c|}
\hline Pre-Award Actions & $\begin{array}{l}\text { Schothled } \\
\text { Date }\end{array}$ & Setioneto \\
\hline \multirow{2}{*}{$\begin{array}{l}\text { 1. Provide Other Hanford SNF documentation and } \\
\text { certification. } \\
\text { NOTE: Plans and schedules for Other Hanford SNF is } \\
\text { contained in DOE-owned SNF Interim Storage Plan } \\
\text { and Program Plan (identified under 4.3) }\end{array}$} & $5 / 15 / 96$ & EW Gerber \\
\hline & & \\
\hline Post-Awrard Actions & & \\
\hline 1. Conduct briefings on Other Hanford Fuels. & $6 / 14 / 96$ & EW Gerber \\
\hline $\begin{array}{l}\text { 2. Conduct briefings on Other Hanford SNF accountability } \\
\text { and turnover mechanism }\end{array}$ & $6 / 14 / 96$ & EW Gerber \\
\hline
\end{tabular}

\subsection{FACILITY STABILIZATION}

\begin{tabular}{|c|c|c|}
\hline Pre-Award Actions & $\begin{array}{l}\text { Scheduled } \\
\text { Date }\end{array}$ & Actionee(s) \\
\hline $\begin{array}{l}\text { 1. Develop a list of key Transition Projects documentation. } \\
\text { Provide to RL POC for review. }\end{array}$ & $5 / 15 / 96$ & CL Nansen \\
\hline $\begin{array}{l}\text { 2. Assemble key Transition Projects documentation, or } \\
\text { identify its location, for use by the PHMC. Includes } \\
\text { development of a Transition Projects "Smart Book," } \\
\text { including listings and descriptions developed below. }\end{array}$ & $6 / 01 / 96$ & CL Nansen \\
\hline $\begin{array}{l}\text { 3. Identify number of briefings, and prepare Transition } \\
\text { Projects briefing(s) for presentation to PHMC. } \\
\text { (Coordinate number and content with RL. POC.) }\end{array}$ & $6 / 01 / 96$ & CL Nansen \\
\hline $\begin{array}{l}\text { 4. Develop a listing of key Transition Projects individuals } \\
\text { as POCs, including facility and operations personnel. }\end{array}$ & $6 / 01 / 96$ & CL Nansen \\
\hline $\begin{array}{l}\text { 5. Develop listing and description of Transition Projects } \\
\text { subcontracts. Include any subeontracts still planned to } \\
\text { be executed prior to contract takeover. }\end{array}$ & $6 / 01 / 96$ & CL Nansen \\
\hline $\begin{array}{l}\text { 6. Develop listing and description of Transition Projects } \\
\text { databases. }\end{array}$ & $6 / 01 / 96$ & CL Nansen \\
\hline $\begin{array}{l}\text { 7. Identify space and equipment within } 2753 \mathrm{E} \text { and facilities } \\
\text { for use by the PHMC. }\end{array}$ & 6/01/96 & CL Nansen \\
\hline $\begin{array}{l}\text { 8. In outline form for all facilities/operations to follow, } \\
\text { define the appropriate contents and level of detail to be } \\
\text { included in facility/operations briefings to the PHMC. } \\
\text { (Review with RL POC.) }\end{array}$ & $5 / 01 / 96$ & CL Nansen \\
\hline Post-Award Actions & & ४ \\
\hline $\begin{array}{l}\text { 1. Provide the PHMC access to the key Transition Projects } \\
\text { documentation, any program level generated pre- } \\
\text { existing conditions information, listing of POCs, and } \\
\text { listing of subcontracts. Provide copies upon request. }\end{array}$ & prior to $6 / 15 / 96$ & CL Nansen \\
\hline
\end{tabular}




\begin{tabular}{|l|c|l|}
\hline \multicolumn{1}{|c|}{ Post-Award Actions } & $\begin{array}{c}\text { Scheduled } \\
\text { Date }\end{array}$ & by $6 / 8 / 96$ \\
\hline $\begin{array}{l}\text { 2. PHMC identify space and equipment needs within } \\
\text { Transition Projects area and facilities. }\end{array}$ & PHMC \\
\hline $\begin{array}{l}\text { 3. Transition Projects and facility space and services } \\
\text { available for use by the PHMC. }\end{array}$ & $6 / 15 / 96$ & CL Nansen \\
\hline 4. Provide Transition Projects briefing(s) to the PHMC. & $6 / 15 / 96-7 / 01 / 96$ & CL Nansen \\
\hline 5. Provide Transition Projects tours to the PHMC. & $6 / 15 / 96-7 / 01 / 96$ & CL Nansen \\
\hline
\end{tabular}

\subsubsection{PUREX}

This complex was a processing facility to extract plutonium in spent fuels. In the process, uranium was separated, sent to the Uranium Trioxide $\left(\mathrm{UO}_{3}\right)$ Plant to become Uranium fluoride and then returned to Fernald to be enriched and reprocessed into fuel billets for new fuel elements for $\mathrm{N}$ Reactor, thus closing the fuel cycle.

In December, 1992, DOE authorized termination of the facilities and directed that actions be initiated to proceed with shutdown planning and subsequent transition to a passively safe and environmentally secure state pending final disposition. The $\mathrm{UO}_{3}$ transition was completed in FY 1995; the PUREX transition is targeted to be completed in FY 1997.

\begin{tabular}{|c|c|c|}
\hline Pre-Award Actions & $\begin{array}{c}\text { Scheduled } \\
\text { Date }\end{array}$ & Actionee(s) \\
\hline $\begin{array}{l}\text { 1. Identify number of briefings, and prepare PUREX } \\
\text { briefing(s) for the PHMC. (Coordinate number and } \\
\text { content with RL POC.) }\end{array}$ & 6/01/96 & DE Kammenzind \\
\hline $\begin{array}{l}\text { 2. Identify key documents for PUREX. Provide listing to } \\
\text { CL Nansen for consolidation and RL review. }\end{array}$ & $5 / 01 / 96$ & DE Kammenzind \\
\hline $\begin{array}{l}\text { 3. Assemble key PUREX documentation, or identify its } \\
\text { location, for use by the PHMC. Includes preparation } \\
\text { of a PUREX "Smart Book," to include information } \\
\text { developed in the following actions. }\end{array}$ & 6/01/96 & DE Kammenzind \\
\hline 4. Identify POCs for PUREX and input to CL Nansen. & $5 / 15 / 96$ & DE Kammenzind \\
\hline $\begin{array}{l}\text { 5. Develop a listing and description of subcontracts } \\
\text { supporting PUREX and input to CL Nansen. Include } \\
\text { any subcontracts planned to be executed prior to } \\
\text { contract takeover. }\end{array}$ & $5 / 15 / 96$ & DE Kammenzind \\
\hline $\begin{array}{l}\text { Using the pre-existing checklist, compile pre-existing } \\
\text { condition information on PUREX facilities and/or } \\
\text { major equipment. Conduct, or schedule as a separate } \\
\text { post-award action(s), any assessments required to } \\
\text { adequately address all relevant items on the checklist. } \\
\text { Provide completed checklist to RL POC for review. }\end{array}$ & $6 / 01 / 96$ & DE Kammenzind \\
\hline
\end{tabular}




\begin{tabular}{|c|c|c|}
\hline (1.1. & $\begin{array}{l}\text { Scheduled } \\
\text { Dete. }\end{array}$ & Actioneds) \\
\hline $\begin{array}{l}\text { 7. Identify applicable milestones and commitments (e.g., } \\
\text { Tri-Party Agreement, DNFSB and consent order } \\
\text { milestones), and determine their stans. Describe the } \\
\text { form of the deliverable and what constitutes } \\
\text { completion. Provide to RL POC for review. }\end{array}$ & $6 / 01 / 96$ & DE Kammenzind \\
\hline $\begin{array}{l}\text { 8. Identify current inventories of feed for PUREX, and } \\
\text { materials to support continued operation (i.e., essential } \\
\text { materials, tools and equipment). }\end{array}$ & 6/01/96 & DE Kammenzind \\
\hline $\begin{array}{l}\text { 9. Develop a list of PUREX-specific mandatory training } \\
\text { and qualification requirements for employees. }\end{array}$ & $6 / 01 / 96$ & DE Kammenzind \\
\hline $\begin{array}{l}\text { 10. Develop a listing of information which constitutes the } \\
\text { "guthorization basis" for the PUREX facility. }\end{array}$ & 6/01/96 & DE Kammenzind \\
\hline $\begin{array}{l}\text { 1. Develop a description of ongoing and planned } \\
\text { construction projects. }\end{array}$ & 6/01/96 & DE Kammenzind \\
\hline $\begin{array}{l}\text { 12. Develop a listing of special agreement services } \\
\text { provided to RL, other DOE sites, and other entities. }\end{array}$ & 6/01/96 & DE Kammenzind \\
\hline $\begin{array}{l}\text { 13. Develop a listing and description of PUREX } \\
\text { databases. Provide list to CL Nansen for } \\
\text { consolidation. }\end{array}$ & $5 / 15 / 96$ & DE Kammenzind \\
\hline $\begin{array}{l}\text { 14. Develop a list of current work order services or other } \\
\text { form of agreement to provide work for other } \\
\text { organizations, on and offsite. }\end{array}$ & $6 / 01 / 96$ & DE Kammenzind \\
\hline $\begin{array}{l}\text { 15. Develop a list of current work order services or other } \\
\text { form of agreement to obtain work from other } \\
\text { organizations, on and offsite. }\end{array}$ & $6 / 01 / 96$ & DE Kammenzind \\
\hline $\begin{array}{l}\text { 16. Develop a list of permits governing PUREX and } \\
\text { provide a description of each as to where and how it } \\
\text { applies. }\end{array}$ & $6 / 01 / 96$ & DE Kammenzind \\
\hline $\begin{array}{l}\text { 17. Verify the accuracy of property assignments for } \\
\text { PUREX and correct any deficiencies. }\end{array}$ & $6 / 01 / 96$ & DE Kammenzind \\
\hline $\begin{array}{l}\text { 18. Identify and make available key PUREX-specific } \\
\text { training and qualification records and documents. }\end{array}$ & $6 / 01 / 96$ & DE Kammenzind \\
\hline Post-Award Actions & & \\
\hline 1. Provide PUREX briefing(s) to the PHMC. & $7 / 01 / 96-7 / 15 / 96$ & DE Kammenzind \\
\hline 2. Provide a PUREX facilities tour to the PHMC. & $7 / 01 / 96-7 / 15 / 96$ & DE Kammenzind \\
\hline $\begin{array}{l}\text { 3. Provide PHMC access to key documentation, pre- } \\
\text { existing condition information, commitment data, } \\
\text { inventory data, etc. (as established in the pre-award } \\
\text { phase). Provide copies upon request. }\end{array}$ & by $6 / 15 / 96$ & DE Kammenzind \\
\hline $\begin{array}{l}\text { 4. Provide any additional documentation or information } \\
\text { requested by the PHMC, if available, that was not } \\
\text { identified during the pre-award phase. }\end{array}$ & by $7 / 01 / 96$ & DE Kammenzind \\
\hline
\end{tabular}




\begin{tabular}{|c|c|c|}
\hline Post-Award Actions & $\begin{array}{l}\text { Schedivled } \\
\text { Date }\end{array}$ & fotionect \\
\hline $\begin{array}{l}\text { 5. PHMC identify any recurring reports that they would } \\
\text { like to receive during transition. }\end{array}$ & by $7 / 15 / 96$ & PHMC \\
\hline $\begin{array}{l}\text { 6. Provide necessary PUREX-specific training to PHMC } \\
\text { personnel. }\end{array}$ & $7 / 01 / 96-8 / 01 / 96$ & DE Kammenzind \\
\hline $\begin{array}{l}\text { 7. Provide an updated status of ongoing work prior to } \\
\text { contract turnover. }\end{array}$ & by $9 / 30 / 96$ & DE Kammenzind \\
\hline 8. Perform a joint inventory of SNM with the PHMC. & by $8 / 30 / 96$ & DE Kammenzind \\
\hline $\begin{array}{l}\text { 9. Perform a joint property and equipment inventory with } \\
\text { the PHMC. }\end{array}$ & by $8 / 30 / 96$ & DE Kammenzind \\
\hline 10. Provide a categorized inventory of wastes. & by $9 / 30 / 96$ & DE Kammenzind \\
\hline $\begin{array}{l}\text { 11. Provide an updated status of end point criteria to } \\
\text { PHMC. }\end{array}$ & by $9 / 30 / 96$ & DE Kammenzind \\
\hline $\begin{array}{l}\text { 12. Assist the PHMC in the conduct of a Pre-existing } \\
\text { Condition Assessment }\end{array}$ & $7 / 15 / 96-8 / 30 / 96$ & DE Kammenzind \\
\hline
\end{tabular}

\subsubsection{Plutonium Finishing Plant}

This complex contains vaults for storage of special nuclear material and processing systems with associated support systems for stabilizing plutonium materials.

\begin{tabular}{|c|c|c|}
\hline Pre-Award Actions & $\begin{array}{l}\text { Scheduled } \\
\text { Date }\end{array}$ & Actionee(o) \\
\hline $\begin{array}{l}\text { 1. Identify number of briefings, and prepare PFP } \\
\text { briefing(s) for the PHMC. (Coordinate number and } \\
\text { content with RL POC.) }\end{array}$ & $6 / 01 / 96$ & CM Kronvall \\
\hline $\begin{array}{l}\text { 2. Identify key documents for PFP. Provide listing to } \\
\text { CL Nansen for consolidation and RL review. }\end{array}$ & $5 / 01 / 96$ & CM Kronvall \\
\hline $\begin{array}{l}\text { 3. Assemble key PFP documentation, or identify its } \\
\text { location, for use by the PHMC. Includes preparation } \\
\text { of a PFP "Smart Book," to include information } \\
\text { developed in the following actions. }\end{array}$ & $6 / 01 / 96$ & CM Kronvall \\
\hline 4. Identify POCs for PFP and input to CL Nansen. & $5 / 15 / 96$ & CM Kronvall \\
\hline $\begin{array}{l}\text { 5. Develop a listing and description of subcontracts } \\
\text { supporting PFP and input to CL Nansen. Include any } \\
\text { subcontracts planned to be executed prior to contract } \\
\text { takeover. }\end{array}$ & $5 / 15 / 96$ & CM Kronvall \\
\hline $\begin{array}{l}\text { Using the pre-existing checklist, compile pre-existing } \\
\text { condition information on the PFP facilities and/or major } \\
\text { equipment. Conduct, or schedule as a separate post- } \\
\text { award action(s), any assessments required to adequately } \\
\text { address all relevant items on the checklist. Provide } \\
\text { completed checklist to RL POC for review. }\end{array}$ & 6/01/96 & CM Kronvall \\
\hline
\end{tabular}


7. Identify applicable milestones and commitments (e.g. Tri-Party Agreement, DNFSB and consent order milestones), and determine their status. Describe the form of the deliverable and what constitutes completion. Provide to RL POC for review.

8. Identify current inventories of feed for PFP, and materials to support continued operation (i.e., essential materials, tools and equipment).

9. Develop a list of PFP-specific mandatory training and qualification requirements for employees.

10. Develop a listing of information which constitutes the "authorization basis" for PFP.

11. Develop a description of ongoing and planned construction projects.

12. Develop a listing of special agreement services provided to RL, other DOE sites, and other entities.

13. Develop a listing and description of PFP databases. Provide list to CL Nansen for consolidation.

14. Develop a list of current work order services or other form of agreement to provide work for other organizations, on and offsite.

15. Develop a list of current work order services or other form of agreement to obtain work from other organizations, on and offsite.

16. Develop a list of permits governing PFP, and provide a description of each as to where and how it applies.

17. Verify the accuracy of property assignments for PFP and correct any deficiencies.

18. Identify and make available PFP-specific training and qualification records and documents.

\section{$\sqrt{3+5}$}

$6 / 01 / 96$ Dote:

CM Kronvall

100000

\begin{tabular}{l|l} 
CM Kronvall \\
\end{tabular}




\begin{tabular}{|c|c|c|}
\hline Post-A ward Actions & $\begin{array}{l}\text { Schedoled } \\
\text { Date }\end{array}$ & Hofionoef(B) \\
\hline $\begin{array}{l}\text { 6. Provide necessary PFP-specific training to PHMC } \\
\text { personnel. }\end{array}$ & 7/01/96-8/01/96 & CM Kronvall \\
\hline $\begin{array}{l}\text { 7. Provide an updated status of ongoing work prior to } \\
\text { contract turnover. }\end{array}$ & by $9 / 30 / 96$ & $\begin{array}{l}\text { CM Kronvall } \\
\text { EC Vogt }\end{array}$ \\
\hline 8. Perform a joint inventory of SNM with the PHMC. & by $8 / 30 / 96$ & $\begin{array}{l}\text { CM Kronvall } \\
\text { BR Fitzpatrick }\end{array}$ \\
\hline $\begin{array}{l}\text { 9. Perform a joint property and equipment inventory with } \\
\text { the PHMC. }\end{array}$ & by $8 / 30 / 96$ & CM Kronvall \\
\hline 10. Provide a categorized inventory of wastes. & by $9 / 30 / 96$ & CM Kronvall \\
\hline 11. Provide an updated status of end point criteria to PHMC. & by $9 / 30 / 96$ & CM Kronvall \\
\hline $\begin{array}{l}\text { 12. Assist the PHMC in the conduct of a Pre-existing } \\
\text { Condition Assessment. }\end{array}$ & $7 / 15 / 96-8 / 30 / 96$ & CM Kronvall \\
\hline
\end{tabular}

\subsubsection{Fast Flux Test Facility}

The liquid metal reactor and support facilities served as the technology and materials development center for the liquid metal and breeder reactor programs. The FFTF is shut down and being prepared for tumover to D\&D. Options for altemative use are currently under consideration.

\begin{tabular}{|c|c|c|}
\hline Pre-Award Actions & Scheduled & Actones $(s)$ \\
\hline $\begin{array}{l}\text { 1. Identify number of briefings, and prepare FFTF briefing(s) for the } \\
\text { PHMC. (Coordinate number and content with RL POC.) }\end{array}$ & $6 / 01 / 96$ & DB Klos \\
\hline $\begin{array}{l}\text { 2. Identify key documents for FFTF. Provide listing to CL Nansen } \\
\text { for consolidation and RL review. }\end{array}$ & $5 / 01 / 96$ & DB Klos \\
\hline $\begin{array}{l}\text { 3. Assemble FFTF documentation, or identify its location, for use by } \\
\text { the PHMC. Includes preparation of an FFTF "Smart Book," to } \\
\text { include information developed in the following actions. }\end{array}$ & $6 / 01 / 96$ & DB Klos \\
\hline 4. Identify POCs for FFTF and input to CL Nansen. & $5 / 15 / 96$ & DB Klos \\
\hline $\begin{array}{l}\text { 5. Develop a listing and description of stbcontracts supporting FFTF } \\
\text { and input to CL Nansen. Include any subcontracts planned to be } \\
\text { executed prior to contract takeover. }\end{array}$ & $5 / 15 / 96$ & DB Klos \\
\hline $\begin{array}{l}\text { 6. Using the pre-existing checklist, compile pre-existing condition } \\
\text { information on FFTF facilities and/or major equipment. Conduct, } \\
\text { or schedule as a separate post-award action(s), any assessments } \\
\text { required to adequately address all relevant items on the checklist. } \\
\text { Provide completed checklist to RL POC for review. }\end{array}$ & $6 / 01 / 96$ & DB Klos \\
\hline $\begin{array}{l}\text { 7. Identify applicable milestones and commitments (e.g., Tri-Party } \\
\text { Agreement, DNFSB and consent order milestones), and determine } \\
\text { their status. Describe the form of the deliverable and what } \\
\text { constitutes completion. Provide to RL POC for review. }\end{array}$ & $6 / 01 / 96$ & DB Klos \\
\hline
\end{tabular}




\begin{tabular}{|c|c|c|}
\hline Pre-1ward Actions & $\begin{array}{l}\text { Scheduled } \\
\text { Date }\end{array}$ & Lefiones) \\
\hline $\begin{array}{l}\text { 8. Identify current inventories of feed for FFTF, and materials to } \\
\text { support continued operation (i.e., essential materials, tools and } \\
\text { equipment). }\end{array}$ & $6 / 01 / 96$ & DB Klos \\
\hline $\begin{array}{l}\text { 9. Develop a list of FFTF-specific mandatory training and } \\
\text { qualification requirements for employees. }\end{array}$ & $6 / 01 / 96$ & DB Klos \\
\hline $\begin{array}{l}\text { 10. Develop a listing of information which constitutes the } \\
\text { "authorization basis" for FFTF. }\end{array}$ & 6/01/96 & DB Klos \\
\hline $\begin{array}{l}\text { 11. Develop a description of ongoing and planned construction } \\
\text { projects. }\end{array}$ & $6 / 01 / 96$ & DB Klos \\
\hline $\begin{array}{l}\text { 12. Develop a listing of special agreement services provided to RL, } \\
\text { other DOE sites, and other entities. }\end{array}$ & $6 / 01 / 96$ & DB Klos \\
\hline $\begin{array}{l}\text { 13. Develop a listing and description of FFTF databases. Provide list } \\
\text { to CL Nansen for consolidation. }\end{array}$ & $5 / 15 / 96$ & DB Klos \\
\hline $\begin{array}{l}\text { 14. Develop a list of current work order services or other form of } \\
\text { agreement to provide work for other organizations, on and offsite. }\end{array}$ & 6/01/96 & DB Klos \\
\hline $\begin{array}{l}\text { 15. Develop a list of current work order services or other form of } \\
\text { agreement to obtain work from other organizations, on and offsite. }\end{array}$ & $6 / 01 / 96$ & DB Klos \\
\hline $\begin{array}{l}\text { 16. Develop a list of permits governing FFTF, and provide a } \\
\text { description of each as to where and how it applies. }\end{array}$ & $6 / 01 / 96$ & DB Klos \\
\hline $\begin{array}{l}\text { 17. Verify the accuracy of property assignments for FFTF and correct } \\
\text { any deficiencies. }\end{array}$ & 6/01/96 & DB Klos \\
\hline $\begin{array}{l}\text { 18. Identify and make available FFTF-specific training and } \\
\text { qualification records and documents. }\end{array}$ & $6 / 01 / 96$ & DB Klos \\
\hline Post-A ward Actions & & \\
\hline 1. Provide FFTF briefing(s) to the PHMC. & $7 / 01 / 96-7 / 15 / 96$ & DB Klos \\
\hline 2. Provide FFTF facilities tour to the PHMC. & $7 / 01 / 96-7 / 15 / 96$ & DB Klos \\
\hline $\begin{array}{l}\text { 3. Provide PHMC access to key documentation, pre-existing } \\
\text { condition information, commitment data, inventory data, etc. (as } \\
\text { established in the pre-award phase). Provide copies upon request. }\end{array}$ & by $6 / 15 / 96$ & DB Klos \\
\hline $\begin{array}{l}\text { 4. Provide any additional documentation or information requested by } \\
\text { the PHMC, if available, that was not identified during the pre- } \\
\text { award phase. }\end{array}$ & by $7 / 01 / 96$ & DB Klos \\
\hline $\begin{array}{l}\text { 5. PHMC identify any recurring reports that they would like to } \\
\text { teceive during transition. }\end{array}$ & by $7 / 15 / 96$ & DB Klos \\
\hline 6. Provide necessary FFTF-specific training to PHMC personnel. & $7 / 01 / 96-8 / 01 / 96$ & DB Klos \\
\hline $\begin{array}{l}\text { 7. Provide an updated status of ongoing work prior to contract } \\
\text { turnover. }\end{array}$ & by $9 / 30 / 96$ & DB Klos \\
\hline 8. Perform a joint inventory of SNM with the PHMC. & by $8 / 30 / 96$ & DB Klos \\
\hline 9. Perform a joint property and equipment inventory with the PHMC. & by $8 / 30 / 96$ & DB Klos \\
\hline 10. Provide a categorized inventory of wastes. & by $9 / 30 / 96$ & DB Klos \\
\hline 11. Provide an updated status of end point criteria to PHMC. & by $9 / 30 / 96$ & DB Klos \\
\hline
\end{tabular}


12. Assist the PHMC in the conduct of a Pre-existing Condition Assessment

\subsubsection{B Plant/Waste Encapsulation and Storage Facility}

The B Plant facility was originally constructed as a chemical separations canyon facility to recover nuclear materials through a Bismuth Phosphate Process and was later operated as a "waste fractionization plant" to remove cesium and strontium from tank farm liquid waste. The B Plant deactivation order was received in September 1995. The Waste Encapsulation and Storage Facility (WESF) was constructed to encapsulate and store cesium and strontium obtained from the B Plant processing. The B Plant facility systems are required to maintain the safe environment for WESF, which currently stores the cesium and strontium capsules. An accelerated hazards reduction plan has been proposed that will complete plant deactivation by October 1998.

\begin{tabular}{|c|c|c|}
\hline Pre-Award Actions & $\begin{array}{l}\text { Scheduled } \\
\text { Date }\end{array}$ & Actionee() \\
\hline $\begin{array}{l}\text { 1. Identify number of briefings, and prepare } \\
\text { B Plant/WESF briefing(s) for the PHMC. (Coordinate } \\
\text { number and content with RL POC.) }\end{array}$ & $6 / 01 / 96$ & JL Pappan \\
\hline $\begin{array}{l}\text { 2. Identify key documents for B Plant/WESF. Provide } \\
\text { listing to CL Nansen fot conmolidation and RL review. }\end{array}$ & $5 / 01 / 96$ & $\begin{array}{l}\text { JL Pappan } \\
\text { TL Main }\end{array}$ \\
\hline $\begin{array}{l}\text { 3. Assemble key B PlanuWESF documentation, or } \\
\text { identify its location. for use by the PHMC. Includes } \\
\text { preparation of a B Plant WESF "Smart Book," to } \\
\text { include information develimed in the following actions. }\end{array}$ & $6 / 01 / 96$ & JL Pappan \\
\hline $\begin{array}{l}\text { 4. Identify POCs for B Plant WESF and input to } \\
\text { CL Nansen. }\end{array}$ & $5 / 15 / 96$ & IL Pappan \\
\hline $\begin{array}{l}\text { 5. Develop a listing and dewiption of subcontracts } \\
\text { supporting B Plant/u'ESF and input to CL Nansen. } \\
\text { Include any subcontracts planned to be executed prior } \\
\text { to contract takeover. }\end{array}$ & $5 / 15 / 96$ & JL Pappan \\
\hline $\begin{array}{l}\text { 6. Using the pre-existing checklist, compile pre-existing } \\
\text { condition information on B Plant/WESF facilities } \\
\text { and/or major equipment. Conduct, or schedule as a } \\
\text { separate post-award action(s), any assessments } \\
\text { required to adequately address all relevant items on the } \\
\text { checklist. Provide completed checklist to RL POC for } \\
\text { review. }\end{array}$ & $6 / 01 / 96$ & JL Pappan \\
\hline $\begin{array}{l}\text { 7. Identify applicable milestones and commitments (e.g., } \\
\text { Tri-Party Agreement, DNFSB and consent order } \\
\text { milestones), and determine their status. Describe the } \\
\text { form of the deliverable and what constitutes } \\
\text { completion. Provide to RL POC for review. }\end{array}$ & $6 / 01 / 96$ & JL Pappan \\
\hline
\end{tabular}




\begin{tabular}{|c|c|c|}
\hline 1. Pre-Award Actions & $\begin{array}{l}\text { Scheduled } \\
\text { Date }\end{array}$ & Setioneros) \\
\hline $\begin{array}{l}\text { 8. Identify current inventories of feed for B Plant/WESF, } \\
\text { and materials to support continued operation (i.e., } \\
\text { essential materials, tools and equipment). }\end{array}$ & $6 / 01 / 96$ & IL Pappan \\
\hline $\begin{array}{l}\text { 9. Develop a list of B Plant/WESF-specific mandatory } \\
\text { training and qualification requirements for employees. }\end{array}$ & 6/01/96 & JL Pappan \\
\hline $\begin{array}{l}\text { 10. Develop a listing of information which constitutes the } \\
\text { "authorization basis" for B Plant/WESF. }\end{array}$ & $6 / 01 / 96$ & JL Pappan \\
\hline $\begin{array}{l}\text { 11. Develop a description of ongoing and planned } \\
\text { construction projects. }\end{array}$ & $6 / 01 / 96$ & JL Pappan \\
\hline $\begin{array}{l}\text { 12. Develop a listing of special agreement services } \\
\text { provided to RL, other DOE sites, and other entities. }\end{array}$ & $6 / 01 / 96$ & JL Pappan \\
\hline $\begin{array}{l}\text { 13. Develop a listing and description of B Plant/WESF } \\
\text { databases. Provide list to CL Nansen for } \\
\text { consolidation. }\end{array}$ & $5 / 15 / 96$ & JL Pappan \\
\hline $\begin{array}{l}\text { 14. Develop a list of current work order services or other } \\
\text { form of agreement to provide work for other } \\
\text { organizations, on and offsite. }\end{array}$ & $6 / 01 / 96$ & JL Pappan \\
\hline $\begin{array}{l}\text { 15. Develop a list of current work order services or other } \\
\text { form of agreement to obtain work from other } \\
\text { organizations, on and offsite. }\end{array}$ & $6 / 01 / 96$ & JL Pappan \\
\hline $\begin{array}{l}\text { 16. Develop a list of permits governing B Plant/WESF, } \\
\text { and provide a description of each as to where and how } \\
\text { it applies. }\end{array}$ & $6 / 01 / 96$ & JL Pappan \\
\hline $\begin{array}{l}\text { 17. Verify the accuracy of property assignments for } \\
\text { B Plant/WESF and correct any deficiencies. }\end{array}$ & 6/01/96 & IL Pappan \\
\hline $\begin{array}{l}\text { 18. Identify and make available B Plant/WESF -specific } \\
\text { training and qualification records and documents. }\end{array}$ & $6 / 01 / 96$ & JL Pappan \\
\hline Post-Award Actions & & \% \\
\hline 1. Provide B Plant/WESF briefing(s) to the PHMC. & $7 / 01 / 96-7 / 15 / 96$ & JL Pappan \\
\hline 2. Provide B Plant/WESF facilities tour to the PHMC. & $7 / 01 / 96-7 / 15 / 96$ & JL Pappan \\
\hline $\begin{array}{l}\text { 3. Provide PHMC access to key documentation, pre- } \\
\text { existing condition information, commitment data, } \\
\text { inventory data, etc. (as established in the pre-award } \\
\text { phase). Provide copies upon request. }\end{array}$ & by $6 / 15 / 96$ & JL Pappan \\
\hline $\begin{array}{l}\text { 4. Provide any additional documentation or information } \\
\text { requested by the PHMC, if available, that was not } \\
\text { identified during the pre-award phase. }\end{array}$ & by $7 / 01 / 96$ & JL Pappan \\
\hline $\begin{array}{l}\text { 5. PHMC identify any recurring reports that they would } \\
\text { like to receive during transition. }\end{array}$ & by $7 / 15 / 96$ & JL Pappan \\
\hline $\begin{array}{l}\text { 6. Provide necessary B Plant/WESF-specific training to } \\
\text { PHMC personnel. }\end{array}$ & $7 / 01 / 96-8 / 01 / 96$ & JL Pappan \\
\hline $\begin{array}{l}\text { 7. Provide an updated status of ongoing work prior to } \\
\text { contract turnover. }\end{array}$ & by $9 / 30 / 96$ & JL Pappan \\
\hline
\end{tabular}




\begin{tabular}{|l|c|c|}
\hline 8. Perform a joint inventory of SNM with the PHMC. & by $8 / 30 / 96$ & IL Pappan \\
\hline $\begin{array}{l}\text { 9. Perform a joint property and equipment inventory with } \\
\text { the PHMC. }\end{array}$ & by $8 / 30 / 96$ & JL Pappan \\
\hline 10. Provide a categorized inventory of wastes. & by $9 / 30 / 96$ & JL Pappan \\
\hline 11. Provide an updated status of end point criteria to & by $9 / 30 / 96$ & JL Pappan \\
\hline PHMC. & $7 / 15 / 96-8 / 30 / 96$ & JL Pappan \\
\hline 12. Assist the PHMC in the conduct of a Pre-existing & & \\
\hline
\end{tabular}

\subsubsection{0/400 Area Fuel Fabrication Facilities and 400 Area Fuels and Materials Examination Facility}

Facilities include the 15 fuel fabrication facilities that were used for fabricating Hanford's production reactor fuel. The facilities are being deactivated but continue to store large quantities of uranium metal and have active RCRA TSDs. Also included are the fuels development Laboratory (308 Building) which was most recently used to fabricate FFTF plutonium fuels and the Plutonium Recycle Test Reactor (PRTR) (309 Building). Both are being deactivated, the 308 Building is scheduled for turnover to the ERC by October 1, 1996. Also included in this grouping is the Fuels and Materials Examination Facility (FMEF) in the 400 Area. It was never utilized for its original mission and is therefore not radiologically contaminated. It supports various Hanford missions by renting valuable space and capabilities. It continues to be studied for FFTF support activities and national weapons plutonium disposition alternatives.

\begin{tabular}{|c|c|c|}
\hline Pre-Award Actions & $\begin{array}{l}\text { Scheduled } \\
\text { Date }\end{array}$ & Actionee(8) \\
\hline $\begin{array}{l}\text { 1. Identify number of briefings, and prepare } 300 / 400 \\
\text { Area Fuel Fabrications briefing(s) for the PHMC } \\
\text { (Coordinate number and content with RL POC.) }\end{array}$ & $6 / 01 / 96$ & JM Steffen \\
\hline $\begin{array}{l}\text { 2. Identify key documents for the } 300 / 400 \text { Area Fuel } \\
\text { Fabrications. Provide listing to CL Nansen for } \\
\text { consolidation and RL review. }\end{array}$ & $5 / 01 / 96$ & JM Steffen \\
\hline $\begin{array}{l}\text { 3. Assemble } 300 / 400 \text { Area Fuel Fabrication Facilities } \\
\text { documentation, or identify its location, for use by the } \\
\text { PHMC. Includes preparation of a } 300 / 400 \text { Area Fuel } \\
\text { Fabrications "Smart Book," to include information } \\
\text { developed in the foliowing actions. }\end{array}$ & $6 / 01 / 96$ & JM Steffen \\
\hline $\begin{array}{l}\text { 4. Identify POCs for the } 300 / 400 \text { Area Fuel Fabrication } \\
\text { Facilities and input to CL Nansen. }\end{array}$ & $5 / 15 / 96$ & M Steffen \\
\hline
\end{tabular}




\begin{tabular}{|c|c|c|}
\hline Pre-Award Actions & $\begin{array}{l}\text { Scheduled } \\
\text { Date. }\end{array}$ & Actionee $(8)$ \\
\hline $\begin{array}{l}\text { 5. Develop a listing and description of subcontracts } \\
\text { supporting the } 300 / 400 \text { Area Fuel Fabrication } \\
\text { Facilities and input to CL Nansen. Include any } \\
\text { subcontracts planned to be executed prior to contract } \\
\text { takeover. }\end{array}$ & $5 / 15 / 96$ & IM Steffen \\
\hline $\begin{array}{l}\text { 6. Using the pre-existing checklist, compile pre-existing } \\
\text { condition information on the } 300 / 400 \text { Area Fuel } \\
\text { Fabrications Facilities and/or major equipment. } \\
\text { Conduct, or schedule as a separate post-award } \\
\text { action(s), any assessments required to adequately } \\
\text { address all relevant items on the checklist. Provide } \\
\text { completed checklist to RL POC for review. }\end{array}$ & $6 / 01 / 96$ & M Steffen \\
\hline $\begin{array}{l}\text { 7. Identify applicable milestones and commitments (e.g., } \\
\text { Tri-Party Agreement, DNFSB and consent order } \\
\text { milestones), and determine their status. Describe the } \\
\text { form of the deliverable and what constitutes } \\
\text { completion. Provide to RL POC for review. }\end{array}$ & $6 / 01 / 96$ & M Steffen \\
\hline $\begin{array}{l}\text { 8. Identify current inventories of feed for the } 300 / 400 \\
\text { Area Fuel Fabrications, and materials to support } \\
\text { continued operation (i.e., essential materials, tools and } \\
\text { equipment). }\end{array}$ & $6 / 01 / 96$ & JM Steffen \\
\hline $\begin{array}{l}\text { 9. Develop a list of } 300 / 400 \text { Area Fuel Fabrications - } \\
\text { specific mandatory training and qualification } \\
\text { requirements for employees. }\end{array}$ & $6 / 01 / 96$ & JM Steffen \\
\hline $\begin{array}{l}\text { 10. Develop a listing of information which constitutes the } \\
\text { "authorization basis" for the } 300 / 400 \text { Area Fuel } \\
\text { Fabrications. }\end{array}$ & $6 / 01 / 96$ & JM Steffen \\
\hline $\begin{array}{l}\text { 11. Develop a description of ongoing and planned } \\
\text { construction projects. }\end{array}$ & $6 / 01 / 96$ & JM Steffen \\
\hline $\begin{array}{l}\text { 12. Develop a listing of special agreement services } \\
\text { provided to RL, other DOE sites, and other entities. }\end{array}$ & $6 / 01 / 96$ & JM Steffen \\
\hline $\begin{array}{l}\text { 13. Develop a listing and description of } 300 / 400 \text { Area Fuel } \\
\text { Fabrications databases. Provide list to } \mathrm{CL} \text { Nansen for } \\
\text { consolidation. }\end{array}$ & $5 / 15 / 96$ & JM Steffen \\
\hline $\begin{array}{l}\text { 14. Develop a list of current work order services or other } \\
\text { form of agreement to provide work for other } \\
\text { organizations, on and offsite. }\end{array}$ & $6 / 01 / 96$ & JM Steffen \\
\hline $\begin{array}{l}\text { 15. Develop a list of current work order services or other } \\
\text { form of agreement to obtain work from other } \\
\text { organizations, on and offsite. }\end{array}$ & $6 / 01 / 96$ & JM Steffen \\
\hline $\begin{array}{l}\text { 16. Develop a list of permits governing the } 300 / 400 \text { Area } \\
\text { Fuel Fabrications, and provide a description of each as } \\
\text { to where and how it applies. }\end{array}$ & $6 / 01 / 96$ & JM Steffen \\
\hline $\begin{array}{l}\text { 17. Verify the accuracy of property assignments for the } \\
300 / 400 \text { Area Fuel Fabrications and correct any } \\
\text { deficiencies. }\end{array}$ & 6/01/96 & JM Steffen \\
\hline
\end{tabular}




\begin{tabular}{|c|c|c|}
\hline Pre-Award Actions & Schetuled & Actioneth) \\
\hline $\begin{array}{l}\text { 18. Identify and make available } 300 / 400 \text { Area Fuel } \\
\text { Fabrications -specific training and qualification records } \\
\text { and documents. }\end{array}$ & 6/01/96 & M Steffen \\
\hline 404 Post-Award Actions & 48 & 1.8 \\
\hline $\begin{array}{l}\text { 1. Provide } 300 / 400 \text { Area Fuel Facility briefing(s) to the } \\
\text { PHMC. }\end{array}$ & $7 / 01 / 96-7 / 15 / 96$ & M Steffen \\
\hline $\begin{array}{l}\text { 2. Provide } 300 / 400 \text { Area Fuel Facility tour to the } \\
\text { PHMC. }\end{array}$ & $7 / 01 / 96-7 / 15 / 96$ & JM Steffen \\
\hline $\begin{array}{l}\text { 3. Provide PHMC access to key documentation, pre- } \\
\text { existing condition information, commitment data, } \\
\text { inventory data, etc. (as established in the pre-award } \\
\text { phase). Provide copies upon request. }\end{array}$ & by $6 / 15 / 96$ & JM Steffen \\
\hline $\begin{array}{l}\text { 4. Provide any additional documentation or information } \\
\text { requested by the PHMC, if available, that was not } \\
\text { identified during the pre-award phase. }\end{array}$ & by $7 / 01 / 96$ & JM Steffen \\
\hline $\begin{array}{l}\text { 5. PHMC identify any recurring reports that they would } \\
\text { like to receive during transition. }\end{array}$ & by $7 / 15 / 96$ & PHMC \\
\hline $\begin{array}{l}\text { 6. Provide necessary } 300 / 400 \text { Area Fuel Facility-specific } \\
\text { training to PHMC personnel. }\end{array}$ & $7 / 01 / 96-8 / 01 / 96$ & M Steffen \\
\hline $\begin{array}{l}\text { 7. Provide an updated status of ongoing work prior to } \\
\text { contract turnover. }\end{array}$ & by $9 / 30 / 96$ & JM Steffen \\
\hline 8. Perform a joint inventory of SNM with the PHMC. & by $8 / 30 / 96$ & M Steffen \\
\hline $\begin{array}{l}\text { 9. Perform a joint property and equipment inventory with } \\
\text { the PHMC. }\end{array}$ & by $8 / 30 / 96$ & JM Steffen \\
\hline 10. Provide a categorized inventory of wastes. & by $9 / 30 / 96$ & MM Steffen \\
\hline $\begin{array}{l}\text { 11. Provide an updated stanus of end point criteria to } \\
\text { PHMC. }\end{array}$ & by $9 / 30 / 96$ & JM Steffen \\
\hline $\begin{array}{l}\text { 12. Assist the PHMC in the conduct of a Pre-existing } \\
\text { Condition Assessment. }\end{array}$ & $7 / 15 / 96-8 / 30 / 96$ & M Steffen \\
\hline
\end{tabular}

\subsubsection{Transfer of 324 and 327 Building PNNL Facilities}

The 324 Building provided for the development of liquid metal systems and the study of chemical and metallurgical processes in a single integrated facility for the orderly progress of nonradioactive or radioactive developmental studies from laboratory or bench-scale to full engineering scale pilot plant demonstrations. It houses a nuclear materials storage vault, plutonium glovebox laboratory, enriched uranium hood, and assay equipment for fuel and waste material.

The 327 Building provided specially shielded, ventilated and equipped laboratories for physical and metallurgical examination of irradiated nuclear materials including measurements, metallography, transient testing at room and elevated temperatures, density 
and in-cell machining. Radiation effects can be determined as and aid in developing stable materials.

\begin{tabular}{|c|c|c|}
\hline Pre-Award Actions & $\begin{array}{l}\text { Scheduled } \\
\text { Date }\end{array}$ & Petionee(s) \\
\hline $\begin{array}{l}\text { 1. Identify number of briefings, and prepare Buildings } \\
324 \text { and } 327 \text { briefing(s) for the PHMC. (Coordinate } \\
\text { number and content with RL POC.) }\end{array}$ & $6 / 01 / 96$ & $\begin{array}{l}\text { TW Halverson } \\
\text { D Knowlton (PNNL) }\end{array}$ \\
\hline $\begin{array}{l}\text { 2. Identify key documents for Buildings } 324 \text { and } 327 \text {. } \\
\text { Provide listing to CL Nansen for consolidation and RL } \\
\text { review. }\end{array}$ & $5 / 15 / 96$ & $\begin{array}{l}\text { TW Halverson } \\
\text { D Knowlton (PNNL) }\end{array}$ \\
\hline $\begin{array}{l}\text { 3. Assemble key Buildings } 324 \text { and } 327 \text { documentation, } \\
\text { or identify its location, for use by the PHMC. } \\
\text { Includes preparation of a Buildings } 324 \text { and } 327 \\
\text { "Smart Book," to include information developed in the } \\
\text { following actions. }\end{array}$ & $6 / 01 / 96$ & $\begin{array}{l}\text { TW Halverson } \\
\text { D Knowlton (PNNL) }\end{array}$ \\
\hline $\begin{array}{l}\text { 4. Identify POCs for Buildings } 324 \text { and } 327 \text { and input to } \\
\text { CL Nansen. }\end{array}$ & $5 / 15 / 96$ & $\begin{array}{l}\text { TW Halverson } \\
\text { D Knowlton (PNNL) }\end{array}$ \\
\hline $\begin{array}{l}\text { 5. Develop a listing and description of subcontracts } \\
\text { supporting Buildings } 324 \text { and } 327 \text { and input to } \\
\text { CL Nansen. Include any subcontracts planned to be } \\
\text { executed prior to contract takeover. }\end{array}$ & $5 / 15 / 96$ & $\begin{array}{l}\text { TW Halverson } \\
\text { D Knowiton (PNNL) }\end{array}$ \\
\hline $\begin{array}{l}\text { 6. Using the pre-existing checklist, compile pre-existing } \\
\text { condition information on Buildings } 324 \text { and } 327 \text { and/or } \\
\text { major equipment. Conduct, or schedule as a separate } \\
\text { post-award action(s), any assessments required to } \\
\text { adequately address all relevant items on the checklist. } \\
\text { Provide completed checklist to RL POC for review. }\end{array}$ & $6 / 01 / 96$ & $\begin{array}{l}\text { TW Halverson } \\
\text { D Knowlton (PNNL) }\end{array}$ \\
\hline $\begin{array}{l}\text { 7. Identify applicable milestones and commitments (e.g., } \\
\text { Tri-Party Agreement, DNFSB and consent order } \\
\text { milestones), and determine their status. Describe the } \\
\text { form of the deliverable and what constitutes } \\
\text { completion. Provide to RL POC for review. }\end{array}$ & $6 / 01 / 96$ & $\begin{array}{l}\text { TW Halverson } \\
\text { D Knowlton (PNNL) }\end{array}$ \\
\hline $\begin{array}{l}\text { 8. Identify current inventories of feed for Buildings } 324 \\
\text { and } 327 \text {, and materials to support continued operation } \\
\text { (i.e., essential materials, tools and equipment). }\end{array}$ & $6 / 01 / 96$ & $\begin{array}{l}\text { TW Halverson } \\
\text { D Knowlton (PNNL) }\end{array}$ \\
\hline $\begin{array}{l}\text { 9. Develop a list of Buildings } 324 \text { and } 327 \text {-specific } \\
\text { mandatory training and qualification requirements for } \\
\text { employees. }\end{array}$ & $6 / 01 / 96$ & $\begin{array}{l}\text { TW Halverson } \\
\text { D Knowlton (PNNL) }\end{array}$ \\
\hline $\begin{array}{l}\text { 10. Develop a listing of information which constitutes the } \\
\text { "authorization basis" for Buildings } 324 \text { and } 327 \text {. }\end{array}$ & 6/01/96 & $\begin{array}{l}\text { TW Halverson } \\
\text { D Knowlton (PNNL) }\end{array}$ \\
\hline $\begin{array}{l}\text { 11. Develop a description of ongoing and planned } \\
\text { construction projects. }\end{array}$ & $6 / 01 / 96$ & $\begin{array}{l}\text { TW Halverson } \\
\text { D Knowlton (PNNL) }\end{array}$ \\
\hline $\begin{array}{l}\text { 12. Develop a listing of special agreement services } \\
\text { provided to RL, other DOE sites, and other entities. }\end{array}$ & $6 / 01 / 96$ & $\begin{array}{l}\text { TW Halverson } \\
\text { D Knowlton (PNNL) }\end{array}$ \\
\hline $\begin{array}{l}\text { 13. Develop a listing and description of Buildings } 324 \text { and } \\
327 \text { databases. Provide list to for consolidation. }\end{array}$ & $5 / 15 / 96$ & $\begin{array}{l}\text { TW Halverson } \\
\text { D Knowlton (PNNL) }\end{array}$ \\
\hline
\end{tabular}




\begin{tabular}{|c|c|c|c|}
\hline & Pre-Award Actions & $\begin{array}{l}\text { Schedivied } \\
\text { Date }\end{array}$ & Petiones $(0)$ \\
\hline 14. & $\begin{array}{l}\text { Develop a list of current work order services or other } \\
\text { form of agreement to provide work for other } \\
\text { organizations, on and offsite. }\end{array}$ & $6 / 01 / 96$ & $\begin{array}{l}\text { TW Halverson } \\
\text { D Knowlton (PNNL) }\end{array}$ \\
\hline 15. & $\begin{array}{l}\text { Develop a list of current work order services or other } \\
\text { form of agreement to obtain work from other } \\
\text { organizations, on and offsite. }\end{array}$ & $6 / 01 / 96$ & $\begin{array}{l}\text { TW Halverson } \\
\text { D Knowlton (PNNL) }\end{array}$ \\
\hline 16. & $\begin{array}{l}\text { Develop a list of permits governing Buildings } 324 \text { and } \\
327 \text {, and provide a description of each as to where and } \\
\text { bow it applies. }\end{array}$ & $6 / 01 / 96$ & $\begin{array}{l}\text { TW Halverson } \\
\text { D Knowlton (PNNL) }\end{array}$ \\
\hline 17. & $\begin{array}{l}\text { Verify the accuracy of property assignments for } \\
\text { Buildings } 324 \text { and } 327 \text { and correct any deficiencies. }\end{array}$ & $6 / 01 / 96$ & $\begin{array}{l}\text { TW Halverson } \\
\text { D Knowiton (PNNL) }\end{array}$ \\
\hline \multirow[t]{2}{*}{18.} & $\begin{array}{l}\text { Identify and make available Buildings } 324 \text { and } 327 \text { - } \\
\text { specific training and qualification records and } \\
\text { documents. }\end{array}$ & $6 / 01 / 96$ & $\begin{array}{l}\text { TW Halverson } \\
\text { D Knowlton (PNNL) }\end{array}$ \\
\hline & Post-Award Actions & & \\
\hline 1. & $\begin{array}{l}\text { Provide } 324 \text { and } 327 \text { Facilities briefing(s) to the } \\
\text { PHMC. }\end{array}$ & $7 / 01 / 96-7 / 15 / 96$ & $\begin{array}{l}\text { TW Halverson } \\
\text { D Knowlton (PNNL) }\end{array}$ \\
\hline 2. & Provide 324 and 327 Facilities tour to the PHMC. & $7 / 01 / 96-7 / 15 / 96$ & $\begin{array}{l}\text { TW Halverson } \\
\text { D Knowlton (PNNL) }\end{array}$ \\
\hline 3. & $\begin{array}{l}\text { Provide PHMC access to key documentation, pre- } \\
\text { existing condition information, commitment data, } \\
\text { inventory data, etc. (as established in the pre-award } \\
\text { phase). Provide copies upon request. }\end{array}$ & by $6 / 15 / 96$ & $\begin{array}{l}\text { TW Halverson } \\
\text { D Knowlton (PNNL) }\end{array}$ \\
\hline 4. & $\begin{array}{l}\text { Provide any additional documentation or information } \\
\text { requested by the PHMC, if available, that was not } \\
\text { identified during the pre-award phase. }\end{array}$ & by $7 / 01 / 96$ & $\begin{array}{l}\text { TW Halverson } \\
\text { D Knowlton (PNNL) }\end{array}$ \\
\hline 5 & $\begin{array}{l}\text { PHMC identify any recurring reports that they would } \\
\text { like to receive during transition. }\end{array}$ & by $7 / 15 / 96$ & $\begin{array}{l}\text { TW Halverson } \\
\text { D Knowlton (PNNL) }\end{array}$ \\
\hline & $\begin{array}{l}\text { Provide necessary } 324 \text { and } 327 \text { Facilities -specific } \\
\text { training to PHMC personnel. }\end{array}$ & $7 / 01 / 96-8 / 01 / 96$ & $\begin{array}{l}\text { TW Halverson } \\
\text { D Knowlton (PNNL) }\end{array}$ \\
\hline & $\begin{array}{l}\text { Provide an updated status of ongoing work prior to } \\
\text { contract turnover. }\end{array}$ & by $9 / 30 / 96$ & $\begin{array}{l}\text { TW Halverson } \\
\text { D Knowlton (PNNL) }\end{array}$ \\
\hline & Perform a joint inventory of SNM with the PHMC. & by $8 / 30 / 96$ & $\begin{array}{l}\text { TW Halverson } \\
\text { D Knowlton (PNNL) }\end{array}$ \\
\hline 9. & $\begin{array}{l}\text { Perform a joint property and equipment inventory with } \\
\text { the PHMC. }\end{array}$ & by $8 / 30 / 96$ & $\begin{array}{l}\text { TW Halverson } \\
\text { D Knowlton (PNNL) }\end{array}$ \\
\hline 10. & Provide a categorized inventory of wastes. & by $9 / 30 / 96$ & $\begin{array}{l}\text { TW Halverson } \\
\text { D Knowlton (PNNL) }\end{array}$ \\
\hline 11. & $\begin{array}{l}\text { Provide an updated status of end point criteria to } \\
\text { PHMC. }\end{array}$ & by $9 / 30 / 96$ & $\begin{array}{l}\text { TW Halverson } \\
\text { D Knowlton (PNNL) }\end{array}$ \\
\hline & $\begin{array}{l}\text { Assist the PHMC in the conduct of a Pre-existing } \\
\text { Condition Assessment }\end{array}$ & $7 / 15 / 96-8 / 30 / 96$ & $\begin{array}{l}\text { TW Halverson } \\
\text { D Knowlton (PNNL) }\end{array}$ \\
\hline
\end{tabular}




\subsubsection{Materials and Future Transition Programs}

Long-term disposition of nuclear material and special nuclear materials. Identification and development of facilities and facility group transitions above and beyond the current scopes of work.

Note: Materials and Future Transition Programs section covers facility stabilization areas that may not be covered by individual plants. It may therefore be possible that response to some Section 4.4.7 actions may become "Not applicable" when a specific item is completely covered by individual plants.

\begin{tabular}{|c|c|c|}
\hline Pre-Award Actions & $\begin{array}{l}\text { Scheduled } \\
\text { Date }\end{array}$ & Actionee(s) \\
\hline $\begin{array}{l}\text { 1. Prepare Materials and Future Transition Programs } \\
\text { briefing for the PHMC. (Coordinate with RL POC.) }\end{array}$ & $6 / 01 / 96$ & RR Borisch \\
\hline $\begin{array}{l}\text { 2. Identify key documents for the Materials and Future } \\
\text { Transition Programs. Provide listing to CL Nansen } \\
\text { for consolidation and RL review. }\end{array}$ & $5 / 01 / 96$ & RR Borisch \\
\hline $\begin{array}{l}\text { 3. Assemble Materials and Future Transition Programs } \\
\text { documentation, or identify its location, for use by the } \\
\text { PHMC. Includes preparation of a Materials and } \\
\text { Future Transition Programs "Smart Book," to include } \\
\text { information developed in the following actions. }\end{array}$ & $6 / 01 / 96$ & RR Borisch \\
\hline $\begin{array}{l}\text { 4. Identify POCs for the Materials and Future Transition } \\
\text { Programs and input to CL Nansen. }\end{array}$ & $5 / 15 / 96$ & RR Borisch \\
\hline $\begin{array}{l}\text { 5. Develop a listing and description of subcontracts } \\
\text { supporting the Materials and Future Transition } \\
\text { Programs and input to consolidated program/project } \\
\text { listing. Include any subcontracts planned to be } \\
\text { executed prior to contract takeover. }\end{array}$ & $5 / 15 / 96$ & RR Borisch \\
\hline $\begin{array}{l}\text { 6. Identify applicable milestones and commitments (e.g., } \\
\text { Tri-Party Agreement, DNFSB and consent order } \\
\text { milestones), and determine their status. Describe the } \\
\text { form of the deliverable and what constitutes } \\
\text { completion. Provide to RL POC for review. }\end{array}$ & $6 / 01 / 96$ & RR Borisch \\
\hline $\begin{array}{l}\text { 7. Develop a listing of special agreement services } \\
\text { provided to RL, other DOE sites, and other entities. }\end{array}$ & $6 / 01 / 96$ & RR Borisch \\
\hline $\begin{array}{l}\text { 8. Develop a listing of Materials and Future Transition } \\
\text { Programs facility POCs to be available for the } \\
\text { transition phase. Provide list to CL Nansen for } \\
\text { consolidation. }\end{array}$ & $5 / 15 / 96$ & RR Borisch \\
\hline $\begin{array}{l}\text { 9. Develop a listing and description of Materials and } \\
\text { Future Transition Programs databases. Provide list to } \\
\text { CL Nansen for consolidation. }\end{array}$ & $5 / 15 / 96$ & RR Borisch \\
\hline $\begin{array}{l}\text { 10. Develop a list of current work order services or other } \\
\text { form of agreement to provide work for other } \\
\text { organizations, on and offsite. }\end{array}$ & $6 / 01 / 96$ & RR Borisch \\
\hline
\end{tabular}




\begin{tabular}{|c|c|c|}
\hline Pre-Award Ietions & Schetuled & Actionee(s) \\
\hline $\begin{array}{l}\text { 11. Develop a list of current work order services or other } \\
\text { form of agreement to obtain work from other } \\
\text { organizations, on and offsite. }\end{array}$ & $6 / 01 / 96$ & RR Borisch \\
\hline $\begin{array}{l}\text { 12. Verify the accuracy of property assignments for } \\
\text { Materials and Future Transition Programs and correct } \\
\text { any deficiencies. }\end{array}$ & $6 / 01 / 96$ & RR Borisch \\
\hline $\begin{array}{l}\text { 13. Identify and make available Materials and Future } \\
\text { Transition Programs -specific training and qualification } \\
\text { records and documents. }\end{array}$ & $6 / 01 / 96$ & RR Borisch \\
\hline Fost-A ward Actions & & \\
\hline $\begin{array}{l}\text { 1. Provide Materials and Future Transition briefing(s) to } \\
\text { the PHMC. }\end{array}$ & $7 / 01 / 96-7 / 15 / 96$ & RR Borisch \\
\hline $\begin{array}{l}\text { 2. Provide PHMC access to key documentation, } \\
\text { commitment data, inventory data, etc. (as established } \\
\text { in the pre-award phase). Provide copies upon request. }\end{array}$ & by $6 / 15 / 96$ & RR Borisch \\
\hline $\begin{array}{l}\text { 3. Provide any additional documentation or information } \\
\text { requested by the PHMC, if available, that was not } \\
\text { identified during the pre-award phase. }\end{array}$ & by $7 / 01 / 96$ & RR Borisch \\
\hline $\begin{array}{l}\text { 4. PHMC identify any recurring reports that they would } \\
\text { like to receive during transition. }\end{array}$ & by $7 / 15 / 96$ & PHMC \\
\hline $\begin{array}{l}\text { 5. Provide an updated status of ongoing work prior to } \\
\text { contract nurnover. }\end{array}$ & by $9 / 30 / 96$ & RR Borisch \\
\hline $\begin{array}{l}\text { 6. Perform a joint property and equipment inventory with } \\
\text { the PHMC. }\end{array}$ & by $8 / 30 / 96$ & RR Borisch \\
\hline
\end{tabular}

\subsection{ENVIRONMENTAL RESTORATION (ER) (INTEGRATION)}

BHI is under separate prime contract to RL to perform ER work for the Hanford Site. The contract runs through FY 1999, with an option for three additional years.

ER work includes removal of contamination in soils and groundwater, D\&D of Site facilities, exhuming of buried waste, installation of environmental barriers (where appropriate), and the management and support activities required to accomplish this work. Operation of the Environmental Restoration Disposal Facility (ERDF) for disposal of ER exhumed and generated wastes is included within the BHI workscope. BHI has responsibility for management and coordination of the sitewide groundwater monitoring activities (refer to Section 6.6.2), sitewide asbestos removal/abatement program, and (non-radioactive) underground storage tank removal. BHI also is responsible for management of three sitewide environmental databases: the Hanford Geological Information System (GIS), Hanford Environmental Information System (HEIS), and Waste Information Data System (WIDS). 
The PHMC integrates data generated and provided by BHI for the Richland ER Project into the overall Hanford Site data systems, including the Sitewide Baseline. Examples of such data include cost, schedule, milestone, and any other data requested by DOE. The PHMC is responsible for integrating data and planning information from BHI into the sitewide planning and reporting systems, and is not responsible for management of or accomplishing any of the workscope within the BHI contract.

Refer to Section 6.2.2 of this plan for a description of the BHI ER Project workscope for CERCLA monitoring and related pre- and post-award transition actions.

\begin{tabular}{|c|c|c|}
\hline Pre-Award Actions & $\begin{array}{l}\text { Scheduled } \\
\text { Dale }\end{array}$ & Actionee(s) \\
\hline $\begin{array}{l}\text { 1. Identify all applicable documents serving to define the } \\
\text { business relationships and workscope interactions } \\
\text { between the PHMC and BHI organizations (contractual } \\
\text { requirements, Sitewide agreements, procedures, MOUs, } \\
\text { end point criteria, etc.). }\end{array}$ & $5 / 15 / 96$ & $\begin{array}{l}\text { DD von der Linden } \\
\text { JH Dunkirk }\end{array}$ \\
\hline $\begin{array}{l}\text { 2. Prepare a briefing for PHMC management } \\
\text { representatives, describing relations and interactions } \\
\text { between } B H I \text { and the PHMC in all applicable } \\
\text { workscope areas and functions. }\end{array}$ & $5 / 30 / 96$ & GF Jones \\
\hline $\begin{array}{l}\text { 3. Identify appropriate BHI points-of -contact for the } \\
\text { PHMC. }\end{array}$ & $5 / 15 / 96$ & GF Jones \\
\hline $\begin{array}{l}\text { 4. Document the status of ongninf und near-term planned } \\
\text { sub-projects and activities reyuiring involvement by or } \\
\text { support from the PHMC. }\end{array}$ & $5 / 01 / 96$ & $\begin{array}{l}\text { MC Hughes } \\
\text { SC Foelber }\end{array}$ \\
\hline 5. List all active work orders und their expiration dates. & $4 / 22 / 96$ & DD von der Linden \\
\hline $\begin{array}{l}\text { 6. Determine which support mervices work orders will be } \\
\text { continued and/or renewed. } \\
\text { discontinuation. Document ramonale. }\end{array}$ & $5 / 15 / 96$ & (Numerous) \\
\hline $\begin{array}{l}\text { 7. Determine and document murse of Site facilities being } \\
\text { transitioned from W'HIC Io ER. Include status of pre- } \\
\text { existing conditions records. an apropriate. }\end{array}$ & $5 / 24 / 96$ & $\begin{array}{l}\text { MC Hughes } \\
\text { JH Dunkirk }\end{array}$ \\
\hline $\begin{array}{l}\text { 8. Complete resolution ot open contract liability issues } \\
\text { involving WHC, BHI, und DOE. }\end{array}$ & $8 / 01 / 96$ & $\begin{array}{l}\text { DD von der Linden } \\
\text { JH Dunkirk }\end{array}$ \\
\hline 4 4 Post-Award Actions & & \\
\hline $\begin{array}{l}\text { 1. Provide briefing for the PHMC relevant to interactions } \\
\text { between } B H I \text { and PHMC in all applicable workscope } \\
\text { areas and functions. }\end{array}$ & $6 / 15 / 96$ & $\begin{array}{l}\text { MC Hughes } \\
\text { SC Foelber } \\
\text { (and others) }\end{array}$ \\
\hline $\begin{array}{l}\text { 2. Identify to PHMC those work orders for PHMC } \\
\text { services that BHI will continue and/or renew, and those } \\
\text { work orders BHI is discontinuing (including effective } \\
\text { date of discontinuation). }\end{array}$ & $6 / 15 / 96$ & (Numerous) \\
\hline $\begin{array}{l}\text { 3. Establish routine points of contact and agreed method } \\
\text { and frequency of interface between PHMC and BHI } \\
\text { during Transition Period. }\end{array}$ & $6 / 15 / 96$ & $\begin{array}{l}\text { MC Hughes } \\
\text { SC Foelber }\end{array}$ \\
\hline
\end{tabular}


WHC-SP-1181

\begin{tabular}{|l|c|c|}
\hline $\begin{array}{l}\text { 4. Reach concurrence and document agreement on } \\
\text { continuation of all work in-progress involving support } \\
\text { from PHMC. }\end{array}$ & $6 / 15 / 96$ & TBD \\
\hline $\begin{array}{l}\text { 5. If requested, provide the PHMC representatives a tour } \\
\text { of ER Project sites to clarify areas of BHI responsibility } \\
\text { (vs. PHMC or other contractor), including discussion of } \\
\text { relevant pre-existing site conditions. }\end{array}$ & TBD & TBD \\
\hline 6. Update/renew active work orders, as appropriate. & $9 / 13 / 96$ & (Numerous) \\
\hline
\end{tabular}




\subsection{INFRASTRUCTURE}

\subsection{INFRASTRUCTURE OPERATIONS AND MAINTENANCE}

\subsubsection{Facility Acquisition, Operations, and Maintenance}

The Maintenance Services organization provides expertise and funding for acquisition, management, disposal, and reporting of 25 commercial and intercontractor lease space. Provide oversite of major modifications to leased facilities. Serve as RL contact point for General Services Administration (GSA) leased facilities and site outleasing activities. Property/space and maintenance management to 378 government facilities and 25 commercial lease facilities are also provided. Facilities and Maintenance management manages the Occupancy Pool budget and maintenance activities in general purpose facilities in addition to programmatic support. Maintenance Services organization also provides landlord related maintenance (corrective, preventive, and predictive) for approximately 378 general purpose facilities (GPF), such as office buildings, mobile offices, fabrication shops, and warehouses. As of January 1996, this included 1.4 million square feet of useable floor space. Additionally, maintenance provides support to programmatic facilities, including PFP, PUREX, T and B Plants, Tank Farms, Solid Waste Disposal, Spent Nuclear Fuels, Transportation, Analytical Labs, FMEF, PNNL, Central Stores, Hanford Fire Department (HFD), Steam \& Water Utilities (Powerhouse), HAMMER Training Facility, and TEDF. Maintenance Services provides custodial services and management direction for approximately 222 general purpose facilities in the $100,200,300,400$, and 600 Areas. Included is 145 Programmatic facilities.

Assumptions

- Continued use of inexpensive commercial lease space

- Vacating of high cost government space to commercial lease

- Increase role in the RL outlease activities

- Facilities (GPF/Programmatic) will continue to be vacated onsite

- RL will expect input on vacate activity frequently

- Increased shutdown activities

- Increased reporting to RL-SID

- Occupancy and custodial candidates for outsourcing 
- Funding for upgrades and maintenance to the infrastructure system will be maintained to ensure services, economic transition, and diversification will succeed.

- A graded approach will be utilized in the development and implementation of commercial standards to ensure that the magnitude of resources and materials are commensurate with the safe and reliable operation of the maintenance program.

- Condition Assessment Surveys will be developed by Facility Management to recognize electrical, mechanical, and structural deficiencies in the infrastructure buildings. This process will be the means by which infrastructure assets are maintained.

- Computerized Maintenance Management System will continue to be the methodology to identify, plan/schedule and document performance measures for maintenance work requests.

- Continued vacating of facilities will reduce custodial staff.

- Possible HAMTC issues related to outsourcing.

\begin{tabular}{|c|c|c|}
\hline Pre-Award Actions & $\begin{array}{l}\text { Scheduled } \\
\text { Date }\end{array}$ & Xetionee (s) \\
\hline $\begin{array}{l}\text { 1. Identify number of briefings, and prepare briefing(s) } \\
\text { for the PHMC on facility acquisition, occupancy, } \\
\text { maintenance, and custodial. (Provide for RL POC } \\
\text { review) }\end{array}$ & $5 / 15 / 96$ & JG Holyoak \\
\hline $\begin{array}{l}\text { 2. Identify key documents for facility acquisition, } \\
\text { occupancy, maintenance, and custodial. (Provide for } \\
\text { RL POC review) }\end{array}$ & $5 / 01 / 96$ & JG Holyoak \\
\hline $\begin{array}{l}\text { 3. Assemble key documentation, or identify its location, } \\
\text { for use by the PHMC. Includes preparation of a } \\
\text { "Smart Book," to include information develop in the } \\
\text { following actions. }\end{array}$ & 6/01/96 & JG Holyoak \\
\hline $\begin{array}{l}\text { 4. Develop a listing and description of subcontracts. } \\
\text { Include any subcontracts planned to be executed prior } \\
\text { to contract takeover. }\end{array}$ & $5 / 15 / 96$ & JG Holyoak \\
\hline $\begin{array}{l}\text { 5. Using the pre-existing checklist, compile pre-existing } \\
\text { condition information on facilities and/or major } \\
\text { equipment. Conduct, or schedule as a separate post- } \\
\text { award action(s), any assessments required to } \\
\text { adequately address all relevant items on the checklist. } \\
\text { Provide completed checklist for RL POC review. }\end{array}$ & 6/01/96 & JG Holyoak \\
\hline $\begin{array}{l}\text { 6. Develop a list of open action items and commitments, } \\
\text { including status. (Environmental, Tri-Party } \\
\text { Agreement, DNFSB as examples) }\end{array}$ & $6 / 01 / 96$ & JG Holyoak \\
\hline
\end{tabular}




\begin{tabular}{|c|c|c|}
\hline ҮBOK[OH OY & $96 / 0 \varepsilon / 8-96 / \mathcal{S I} / L$ & 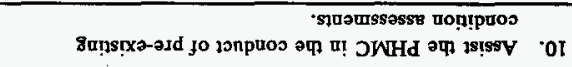 \\
\hline JeORTOH Or & $96 / 0 \varepsilon / 6 \mathrm{Kq}$ & 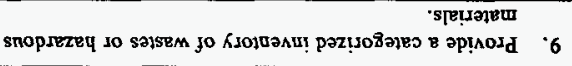 \\
\hline YeOK[OH OF & $96 / 0 \varepsilon / 8 \mathrm{Kq}$ & 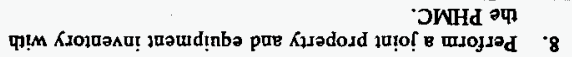 \\
\hline Ү्रвOKIOH Dr & $96 / 0 \varepsilon / 6 K q$ & 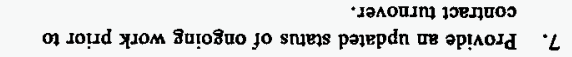 \\
\hline 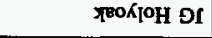 & 96/I0/8-96/IO/L & 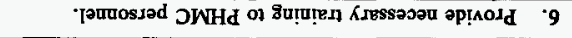 \\
\hline xeokioH Or & $96 / 51 / L \times 9$ & 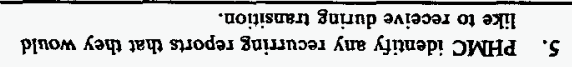 \\
\hline XEOKIOH OS & $96 / 10 / L K q$ & 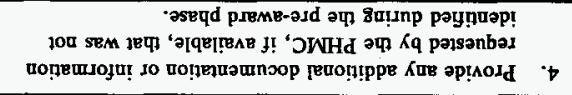 \\
\hline YеOKјOH Or & $96 / S I / 9 K q$ & 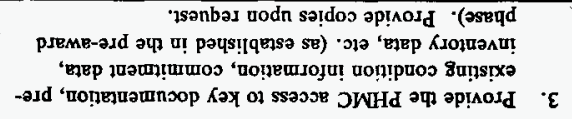 \\
\hline शвоКlOH Dr & $96 / S I / L-96 / T 0 / L$ & 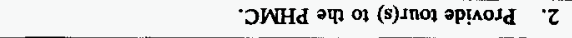 \\
\hline YEOKIOH Or & $96 / S I / L-96 / I 0 / L$ & 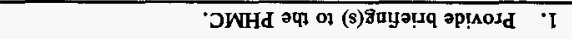 \\
\hline & & SUOIDV PJEAV-15Od \\
\hline YEOKiOH Or & $96 / 10 / 9$ & 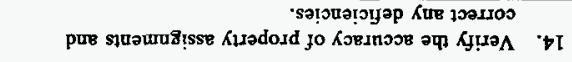 \\
\hline त्र००К & $96 / L 0 / 9$ & 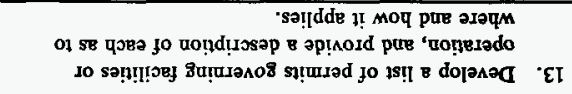 \\
\hline TeOS1OH OS & $96 / 10 / 9$ & 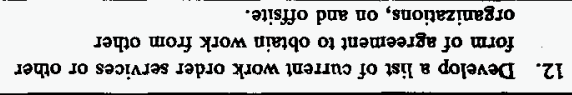 \\
\hline ro0रlOH Or & 96/10/9 & 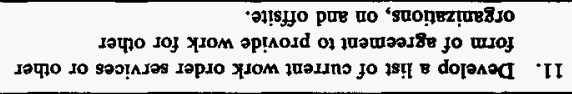 \\
\hline ग曰0K10H DI & 96/SI/S & 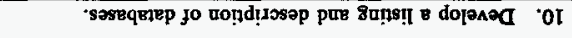 \\
\hline ग\%०К애 OS & $96 / S t / S$ & 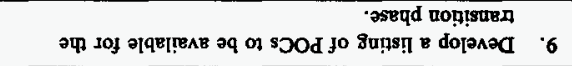 \\
\hline TEOK[OH DI & $96 / 10 / 9$ & 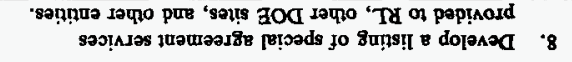 \\
\hline ТөО人[OH OR & $96 / 10 / 9$ & 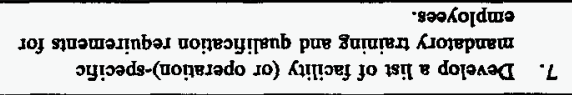 \\
\hline $60 m 0919$ & parparps & suoppY P PRAV PAd \\
\hline
\end{tabular}




\subsubsection{Utilities}

5.1.2.1 Steam Plant Operations. Utilities operates three steam generating plants; 300 Powerhouse, 200 East Powerhouse and the 200 West Package Boiler, and the steam distribution systems. Presently, there is an ESPC proposal to find and institute alternate energy sources, which allows for the decommissioning of the steam plants. The central steam generating plants are scheduled to be shutdown prior to November 1, 1996, if the ESPC contract award and implementation are successful.

\begin{tabular}{|c|c|c|}
\hline 4 Pre-Award Actions & $\begin{array}{l}\text { Scheduled } \\
\text { Date }\end{array}$ & Actionee(t) \\
\hline 1. Identify steam plant transition team. & $5 / 01 / 96$ & $\begin{array}{l}\text { HL Debban } \\
\text { WA Retterer }\end{array}$ \\
\hline $\begin{array}{l}\text { 2. Identify number of briefings, and prepare briefing(s) } \\
\text { for the PHMC. }\end{array}$ & $6 / 01 / 96$ & $\begin{array}{l}\text { HIL Debban } \\
\text { WA Retterer }\end{array}$ \\
\hline $\begin{array}{l}\text { 3. Identify key documents for the facility/operations. } \\
\text { Assemble or identify their location for the PHMC. }\end{array}$ & 6/01/96 & $\begin{array}{l}\text { HL Debban } \\
\text { POC }\end{array}$ \\
\hline $\begin{array}{l}\text { 4. Develop a listing and description of subcontracts and } \\
\text { other supporting activity organizations. Include any } \\
\text { subcontracts planned to be executed prior to contract } \\
\text { takeover. }\end{array}$ & $6 / 01 / 96$ & $\begin{array}{l}\text { HL Debban } \\
\text { POC }\end{array}$ \\
\hline $\begin{array}{l}\text { 5. Using the pre-existing checklist, compile pre-existing } \\
\text { condition information on facilities and/or major } \\
\text { equipment. Conduct, or schedule as a separate post- } \\
\text { award action(s), any assessments required to } \\
\text { adequately address all relevant items on the checklist. } \\
\text { Provide completed checklist for RL POC for review. } \\
\text { Include a list of open-ended compliance issues: } \\
\text { HATS, SATS, shutdown plans, ESPC, etc. }\end{array}$ & $6 / 01 / 96$ & $\begin{array}{l}\text { HL Debban } \\
\text { POC }\end{array}$ \\
\hline 6. Develop a listing of POCs for operations/ facilities. & $5 / 01 / 96$ & $\begin{array}{l}\text { HL Debban } \\
\text { WA Retterer }\end{array}$ \\
\hline \multicolumn{3}{|l|}{ Post-Award Actions } \\
\hline 1. Provide facility briefing(s) to the PHMC. & $7 / 01 / 96$ & POC \\
\hline 2. Provide facility tour(s) to the PHMC. & $8 / 01 / 96$ & POC \\
\hline $\begin{array}{l}\text { 3. Provide the PHMC access to key documentation, pre- } \\
\text { existing condition information, commitment data, } \\
\text { inventory data, etc. (as established in the pre-award } \\
\text { phase). Provide copies upon request. }\end{array}$ & by $6 / 15 / 96$ & POC \\
\hline $\begin{array}{l}\text { 4. Provide additional documentation as requested to } \\
\text { PHMC. }\end{array}$ & ongoing & POC \\
\hline $\begin{array}{l}\text { 5. Provide PHMC with events as they occur, in a timely } \\
\text { manner. }\end{array}$ & ongoing & POC \\
\hline 6. Provide necessary training to PHMC personnel. & $10 / 01 / 96$ & POC \\
\hline
\end{tabular}




\begin{tabular}{|c|c|c|c|}
\hline & Post-Award Actions & Scheduled & totionses) \\
\hline 7. & $\begin{array}{l}\text { Provide an updated status of ongoing work prior to } \\
\text { contract turnover. }\end{array}$ & $9 / 15 / 96$ & POC \\
\hline 8. & $\begin{array}{l}\text { Perform a joint property and equipment inventory } \\
\text { with the PHMC. }\end{array}$ & $9 / 01 / 96$ & DR Herman \\
\hline 9. & $\begin{array}{l}\text { Provide a categorized inventory of wastes or } \\
\text { hazardous materials. }\end{array}$ & $8 / 15 / 96$ & POC \\
\hline & $\begin{array}{l}\text { Assist the PHMC in the conduct of pre-existing } \\
\text { condition assessments. }\end{array}$ & $7 / 15 / 96-8 / 30 / 96$ & POC \\
\hline
\end{tabular}

5.1.2.2 300 Area Water/Sewer Operations. The 300 Area Utilities organization produces potable water for distribution to facilities in the 300 Area, including maintenance and operation of the sewer collection system. At the present time there are two bids for this function to DOE for consideration; one from ICF KH and the other from the City of Richland. If the responsibility for the production and distribution of potable water is turned over to the City of Richland, the PHMC may have nothing to do with this function. If the responsibility, or any portion, remains with $\mathrm{ICF} \mathrm{KH}$, then that responsibility must be transitioned to the PHMC.

\begin{tabular}{|c|c|c|}
\hline Pre-Award Actions & $\begin{array}{l}\text { Scheduled } \\
\text { Date }\end{array}$ & Actionee(s) \\
\hline 1. Identify 300 area water/sewer transition team. & $5 / 01 / 96$ & $\begin{array}{l}\text { HIL Debban } \\
\text { WA Retterer }\end{array}$ \\
\hline $\begin{array}{l}\text { 2. Identify number of briefings, and prepare briefing(s) } \\
\text { for the PHMC. }\end{array}$ & 6/01/96 & $\begin{array}{l}\text { HL Debban } \\
\text { WA Retterer }\end{array}$ \\
\hline $\begin{array}{l}\text { 3. Identify key documents for the facility/operations. } \\
\text { Assemble or identify their location for the PHMC. }\end{array}$ & 6/01/96 & $\begin{array}{l}\text { HL. Debban } \\
\text { POC }\end{array}$ \\
\hline $\begin{array}{l}\text { 4. Develop a listing and description of subcontracts and } \\
\text { other supporting activity organizations. Include any } \\
\text { subcontracts planned to be executed prior to contract } \\
\text { takeover. }\end{array}$ & $6 / 01 / 96$ & $\begin{array}{l}\text { HL. Debban } \\
\text { POC }\end{array}$ \\
\hline $\begin{array}{l}\text { 5. Using the pre-existing checklist, compile pre-existing } \\
\text { condition information on facilities and/or major } \\
\text { equipment. Conduct, or schedule as a separate post- } \\
\text { award action(s), any assessments required to } \\
\text { adequately address all relevant items on the checklist. } \\
\text { Provide completed checklist for RL POC for review. } \\
\text { Include a list of open-ended compliance issues: HATS, } \\
\text { SATS, shutdown plans, ESPC, etc. }\end{array}$ & 6/01/96 & $\begin{array}{l}\text { HIL. Debban } \\
\text { POC }\end{array}$ \\
\hline 6. Develop a listing of POCs for operations/ facilities. & $5 / 01 / 96$ & $\begin{array}{l}\text { HL Debban } \\
\text { WA Retterer }\end{array}$ \\
\hline Post-Award Actions & $\%$ & 18 \\
\hline 1. Provide facility briefing(s) to the PHMC. & $7 / 01 / 96$ & POC \\
\hline
\end{tabular}




\begin{tabular}{|c|c|c|}
\hline Post-A ward Actions & $\begin{array}{l}\text { Scheduled } \\
\text { Date }\end{array}$ & Letionee $(\mathbf{s})$ \\
\hline 2. Provide facility tour(s) to the PHMC. & $8 / 01 / 96$ & POC \\
\hline $\begin{array}{l}\text { 3. Provide the PHMC access to key documentation, pre- } \\
\text { existing condition information, commitment data, } \\
\text { inventory data, etc. (as established in the pre-award } \\
\text { phase). Provide copies upon request. }\end{array}$ & by $6 / 15 / 96$ & POC \\
\hline $\begin{array}{l}\text { 4. Provide additional documentation as requested to } \\
\text { PHMC. }\end{array}$ & ongoing & POC \\
\hline $\begin{array}{l}\text { 5. Provide PHMC with events as they occur, in a timely } \\
\text { manner. }\end{array}$ & ongoing & POC \\
\hline 6. Provide necessary training to PHMC personnel. & $10 / 01 / 96$ & POC \\
\hline $\begin{array}{l}\text { 7. Provide an updated status of ongoing work prior to } \\
\text { contract turnover. }\end{array}$ & $9 / 15 / 96$ & POC \\
\hline $\begin{array}{l}\text { 8. Perform a joint property and equipment inventory with } \\
\text { the PHMC. }\end{array}$ & 9/01/96 & DR Herman \\
\hline $\begin{array}{l}\text { 9. Provide a categorized inventory of wastes or hazardous } \\
\text { materials. }\end{array}$ & $8 / 15 / 96$ & POC \\
\hline $\begin{array}{l}\text { 10. Assist the PHMC in the conduct of pre-existing } \\
\text { condition assessments. }\end{array}$ & $7 / 15 / 96-8 / 30 / 96$ & POC \\
\hline
\end{tabular}

\subsubsection{200 Area Water/Sewer Operations. The 200 Area Utilities organization maintains} and operates the export water system, the 200 East water treatment plant, the 200 West water treatment plant and associated distribution systems. This organization also performs septic system pumping, maintenance and reporting.

\begin{tabular}{|c|c|c|}
\hline Pre-Award Actions & $\begin{array}{l}\text { Scheduled } \\
\text { Date }\end{array}$ & Actionet(s) \\
\hline 1. Identify 200 area water/sewer transition team. & $5 / 01 / 96$ & $\begin{array}{l}\text { HL Debban } \\
\text { WA Retterer }\end{array}$ \\
\hline $\begin{array}{l}\text { 2. Identify number of briefings, and prepare briefing(s) } \\
\text { for the PHMC. }\end{array}$ & $6 / 01 / 96$ & $\begin{array}{l}\text { HL Debban } \\
\text { WA Retterer }\end{array}$ \\
\hline $\begin{array}{l}\text { 3. Identify key documents for the facility/operations. } \\
\text { Assemble or identify their location for the PHMC. }\end{array}$ & 6/01/96 & $\begin{array}{l}\text { HL. Debban } \\
\text { POC }\end{array}$ \\
\hline $\begin{array}{l}\text { 4. Develop a listing and description of subcontracts and } \\
\text { other supporting activity organizations. Include any } \\
\text { subcontracts planned to be executed prior to contract } \\
\text { takeover. }\end{array}$ & $6 / 01 / 96$ & $\begin{array}{l}\text { HL Debban } \\
\text { POC }\end{array}$ \\
\hline $\begin{array}{l}\text { Using the pre-existing checklist, compile pre-existing } \\
\text { condition information on facilities and/or major } \\
\text { equipment. Conduct, or schedule as a separate post- } \\
\text { award action(s), any assessments required to } \\
\text { adequately address all relevant items on the checklist. } \\
\text { Provide completed checklist for RL POC for review. } \\
\text { Include a list of open-ended compliance issues: HATS, } \\
\text { SATS, shutdown plans, ESPC, etc. }\end{array}$ & 6/01/96 & $\begin{array}{l}\text { HL. Debban } \\
\text { POC }\end{array}$ \\
\hline
\end{tabular}




\begin{tabular}{|c|c|c|}
\hline Pre-Award Actions & Scheduled & fetioned $(s)$ \\
\hline 6. Develop a listing of POCs for operations/ facilities. & $5 / 01 / 96$ & $\begin{array}{l}\text { HL Debban } \\
\text { WA Retterer }\end{array}$ \\
\hline Post-Award Actions & & \\
\hline 1. Provide facility briefing(s) to the PHMC. & $7 / 01 / 96$ & POC \\
\hline 2. Provide facility tour(s) to the PHMC. & $8 / 01 / 96$ & POC \\
\hline $\begin{array}{l}\text { 3. Provide the PHMC access to key documentation, pre- } \\
\text { existing condition information, commitment data, } \\
\text { inventory data, etc. (as established in the pre-award } \\
\text { phase) Provide copies upon request. }\end{array}$ & by $6 / 15 / 96$ & POC \\
\hline $\begin{array}{l}\text { 4. Provide additional documentation as requested to } \\
\text { PHMC. }\end{array}$ & ongoing & POC \\
\hline $\begin{array}{l}\text { 5. Provide PHMC with events as they occur, in a timely } \\
\text { manner. }\end{array}$ & ongoing & POC \\
\hline 6. Provide necessary training to PHMC personnel. & $10 / 01 / 96$ & POC \\
\hline $\begin{array}{l}\text { 7. Provide an updated status of ongoing work prior to } \\
\text { contract turnover. }\end{array}$ & $9 / 15 / 96$ & POC \\
\hline $\begin{array}{l}\text { 8. Perform a joint property and equipment inventory with } \\
\text { the PHMC. }\end{array}$ & $9 / 01 / 96$ & DR Herman \\
\hline $\begin{array}{l}\text { 9. Provide a categorized inventory of wastes or hazardous } \\
\text { materials. }\end{array}$ & $8 / 15 / 96$ & $\mathrm{POC}$ \\
\hline $\begin{array}{l}\text { 10. Assist the PHMC in the conduct of pre-existing } \\
\text { condition assessments. }\end{array}$ & $7 / 15 / 96-8 / 30 / 96$ & POC \\
\hline
\end{tabular}

\subsubsection{Site Electrical Systems.}

\section{Assumption:}

- DOE has put out an RFP for the operation and maintenance of the high voltage electrical systems. This transition schedule is based on Utilities keeping the contract.

\begin{tabular}{|c|c|c|}
\hline Pre-Award Actions & $\begin{array}{l}\text { Scheruled: } \\
\text { Date }\end{array}$ & sttonsos $(s)$ \\
\hline 1. Identify electrical transition team. & $5 / 01 / 96$ & $\begin{array}{l}\text { HL Debban } \\
\text { WA Retterer }\end{array}$ \\
\hline $\begin{array}{l}\text { 2. Identify number of briefings, and prepare briefing(s) } \\
\text { for the PHMC. }\end{array}$ & $6 / 01 / 96$ & $\begin{array}{l}\text { HL Debban } \\
\text { WA Retterer }\end{array}$ \\
\hline $\begin{array}{l}\text { 3. Identify key documents for the facility/operations. } \\
\text { Assemble or identify their location for the PHMC. }\end{array}$ & $6 / 01 / 96$ & $\begin{array}{l}\text { HL Debban } \\
\text { POC }\end{array}$ \\
\hline
\end{tabular}




\begin{tabular}{|c|c|c|}
\hline Pre-Award Actions & Scheduled & Setionee $(6)$ \\
\hline $\begin{array}{l}\text { 4. Develop a listing and description of subcontracts and } \\
\text { other supporting activity organizations. Include any } \\
\text { subcontracts planned to be executed prior to contract } \\
\text { takeover. }\end{array}$ & $6 / 01 / 96$ & $\begin{array}{l}\text { HL Debban } \\
\text { POC }\end{array}$ \\
\hline $\begin{array}{l}\text { 5. Using the pre-existing checklist, compile pre-existing } \\
\text { condition information on facilities and/or major } \\
\text { equipment. Conduct, or schedule as a separate post- } \\
\text { award action(s), any assessments required to adequately } \\
\text { address all relevant items on the checklist. Provide } \\
\text { completed checklist for RL POC for review. Include a } \\
\text { list of open-ended compliance issues: HATS, SATS, } \\
\text { shutdown plans, ESPC, etc. }\end{array}$ & $6 / 01 / 96$ & $\begin{array}{l}\text { HL Debban } \\
\text { POC }\end{array}$ \\
\hline 6. Develop a listing of POCs for operations/facilities. & $5 / 01 / 96$ & $\begin{array}{l}\text { HL Debban } \\
\text { WA Retterer }\end{array}$ \\
\hline Post-Award Actions & & \\
\hline 1. Provide facility briefing(s) to the PHMC. & $7 / 01 / 96$ & POC \\
\hline 2. Provide facility tour(s) to the PHMC. & $8 / 01 / 96$ & POC \\
\hline $\begin{array}{l}\text { 3. Provide the PHMC access to key documentation, pre- } \\
\text { existing condition information, commitment data, } \\
\text { inventory data, etc. (as established in the pre-award } \\
\text { phase). Provide copies upon request. }\end{array}$ & by $6 / 15 / 96$ & POC \\
\hline $\begin{array}{l}\text { 4. Provide additional documentation as requested to } \\
\text { PHMC. }\end{array}$ & ongoing & POC \\
\hline $\begin{array}{l}\text { 5. Provide PHMC with events as they occur, in a timely } \\
\text { manner. }\end{array}$ & ongoing & POC \\
\hline 6. Provide necessary training to PHMC personnel. & $10 / 01 / 96$ & POC \\
\hline $\begin{array}{l}\text { 7. Provide an updated status of ongoing work prior to } \\
\text { contract turnover. }\end{array}$ & $9 / 15 / 96$ & POC \\
\hline $\begin{array}{l}\text { 8. Perform a joint property and equipment inventory with } \\
\text { the PHMC. }\end{array}$ & $9 / 01 / 96$ & DR Herman \\
\hline $\begin{array}{l}\text { 9. Provide a categorized inventory of wastes or hazardous } \\
\text { materials. }\end{array}$ & $8 / 15 / 96$ & POC \\
\hline $\begin{array}{l}\text { 10. Assist the PHMC in the conduct of pre-existing } \\
\text { condition assessments. }\end{array}$ & $7 / 15 / 96-8 / 30 / 96$ & POC \\
\hline
\end{tabular}

\subsubsection{Transportation Infrastructure and Services}

Manage Hanford Sitewide fleet, including inventory, procurement, and costing for Hanford Site contractors. Interface with GSA, RL, and contractors on GSA-provided fleet issues. Provide preventive maintenance and repair services for Hanford Site vehicle and construction equipment. Services include fueling, lubrication, and field and shop maintenance. Provide personnel and material transportation services for the Hanford Site. 
Modes used include rail, truck, bus, and light vehicle. Transportation support functions include road and rail system maintenance, snow removal, and fire support.

Assumptions:

- MOU between RL and GSA will remain in effect.

- Current organization will remain intact.

- GSA will use Fleet Maintenance for preventive maintenance (PM) and repair.

- BHI will continue to use Fleet Maintenance for PM and repair work via work order.

- Fleet Maintenance personnel will not be consolidated into one facility prior to October 1996.

- HAMTC and Building Trades will not be merged.

- Currently planned consolidation of transportation functions will occur.

\begin{tabular}{|c|c|c|}
\hline Pre-Award Actions & $\begin{array}{l}\text { Scheduled } \\
\text { Date }\end{array}$ & Actionee(s) \\
\hline $\begin{array}{l}\text { 1. Identify number of briefings, and prepare briefing(s) } \\
\text { on transportation management, maintenance, and } \\
\text { operations for the PHMC. (Provide for RL POC } \\
\text { review.) }\end{array}$ & $5 / 15 / 96$ & $\begin{array}{l}\text { AC Metz (Management) } \\
\text { JF Woods (Maintenance) } \\
\text { RG Ciccone (Operations) }\end{array}$ \\
\hline $\begin{array}{l}\text { 2. Identify key documents for the transportation } \\
\text { management, maintenance, and operations. (Provide } \\
\text { for RL POC review.) }\end{array}$ & $5 / 01 / 96$ & $\begin{array}{l}\text { AC Metz } \\
\text { JF Woods } \\
\text { RG Ciccone }\end{array}$ \\
\hline $\begin{array}{l}\text { 3. Assemble key documentation, or identify its location, } \\
\text { for use by the PHMC. Includes preparation of a } \\
\text { "Smart Book," to include information develop in the } \\
\text { following actions. }\end{array}$ & $6 / 01 / 96$ & $\begin{array}{l}\text { AC Metz } \\
\text { JF Woods } \\
\text { RG Ciccone }\end{array}$ \\
\hline $\begin{array}{l}\text { 4. Develop a listing and description of subcontracts. } \\
\text { Include any subcontracts planned to be executed prior } \\
\text { to contract takeover. }\end{array}$ & $5 / 15 / 96$ & BA Holmberg \\
\hline $\begin{array}{l}\text { 5. Using the pre-existing checklist, compile pre-existing } \\
\text { condition information on facilities and/or major } \\
\text { equipment. Conduct, or schedule as a separate post- } \\
\text { award action(s), any assessments required to adequately } \\
\text { address all relevant items on the checklist. (Provide } \\
\text { completed checklist for RL POC review.) }\end{array}$ & $6 / 01 / 96$ & $\begin{array}{l}\text { BA Holmberg } \\
\text { AC Metz } \\
\text { JF Woods } \\
\text { RG Ciccone }\end{array}$ \\
\hline $\begin{array}{l}\text { 6. Develop a list of open action items and commitments, } \\
\text { including status (Environmental, Tri-Party Agreement, } \\
\text { DNFSB as examples). }\end{array}$ & $6 / 01 / 96$ & BA Holmberg \\
\hline
\end{tabular}




\begin{tabular}{|c|c|c|}
\hline Pre-Avard Actions & $\begin{array}{l}\text { Scheduled } \\
\text { Date }\end{array}$ & Actionor(s) \\
\hline $\begin{array}{l}\text { 7. Develop a list of facility (or operation)-specific } \\
\text { mandatory training and qualification requirements for } \\
\text { employees. }\end{array}$ & $6 / 01 / 96$ & BA Holmberg \\
\hline $\begin{array}{l}\text { 8. Develop a listing of special agreement services } \\
\text { provided to RL, other DOE sites, and other entities. }\end{array}$ & $6 / 01 / 96$ & BA Holmberg \\
\hline $\begin{array}{l}\text { 9. Develop a listing of POCs to be available for the } \\
\text { transition phase. }\end{array}$ & $5 / 15 / 96$ & BA Holmberg \\
\hline 10. Develop a listing and description of databases. & $5 / 15 / 96$ & BA Holmberg \\
\hline $\begin{array}{l}\text { 11. Develop a list of current work order services or other } \\
\text { form of agreement to provide work for other } \\
\text { organizations, on and offsite. }\end{array}$ & $6 / 01 / 96$ & BA Holmberg \\
\hline $\begin{array}{l}\text { 12. Develop a list of current work order services or other } \\
\text { form of agreement to obtain work from other } \\
\text { organizations, on and offsite. }\end{array}$ & $6 / 01 / 96$ & BA Holmberg \\
\hline $\begin{array}{l}\text { 13. Develop a list of permits governing facilities or } \\
\text { operation, and provide a description of each as to } \\
\text { where and how it applies. }\end{array}$ & $6 / 01 / 96$ & BA Holmberg \\
\hline $\begin{array}{l}\text { 14. Verify the accuracy of property assignments and } \\
\text { correct any deficiencies. }\end{array}$ & 6/01/96 & BA Holmberg \\
\hline Post-Award Actions & & \\
\hline 1. Provide briefing(s) to the PHMC. & $7 / 01 / 96-7 / 15 / 96$ & $\begin{array}{l}\text { AC Metz (Management) } \\
\text { JF Woods (Maintenance) } \\
\text { RG Ciccone (Operations) }\end{array}$ \\
\hline 2. Provide tour(s) to the PHMC. & $7 / 01 / 96-7 / 15 / 96$ & \begin{tabular}{|l|} 
AC Metz \\
JF Woods \\
RG Ciccone \\
\end{tabular} \\
\hline $\begin{array}{l}\text { 3. Provide the PHMC access to key documentation, pre- } \\
\text { existing condition information, commitment data, } \\
\text { inventory data, etc. (as established in the pre-award } \\
\text { phase). Provide copies upon request. }\end{array}$ & by $6 / 15 / 96$ & $\begin{array}{l}\text { AC Metz } \\
\text { JF Woods } \\
\text { RG Ciccone }\end{array}$ \\
\hline $\begin{array}{l}\text { 4. Provide any additional documentation or information } \\
\text { requested by the PHMC, if available, that was not } \\
\text { identified during the pre-award phase. }\end{array}$ & by $7 / 01 / 96$ & BA Holmberg \\
\hline $\begin{array}{l}\text { 5. PHMC identify any recurring reports that they would } \\
\text { like to receive during transition. }\end{array}$ & by $7 / 15 / 96$ & BA Holmberg \\
\hline 6. Provide necessary training to PHMC personnel. & $7 / 01 / 96-8 / 01 / 96$ & BA Holmberg \\
\hline $\begin{array}{l}\text { 7. Provide an updated status of ongoing work prior to } \\
\text { contract tumover. }\end{array}$ & by $9 / 30 / 96$ & BA Holmberg \\
\hline $\begin{array}{l}\text { 8. Perform a joint property and equipment inventory with } \\
\text { the PHMC. }\end{array}$ & by $8 / 30 / 96$ & BA Holmberg \\
\hline $\begin{array}{l}\text { 9. Provide a categorized inventory of wastes or hazardous } \\
\text { materials. }\end{array}$ & by $9 / 30 / 96$ & BA Holmberg \\
\hline
\end{tabular}




\begin{tabular}{|c|c|c|c|}
\hline & Post- A ward Actions & Schenuled & Actionee(s) \\
\hline & $\begin{array}{l}\text { Assist the PHMC in the conduct of pre-existing } \\
\text { condition assessments. }\end{array}$ & $7 / 15 / 96-8 / 30 / 96$ & BA Holmberg \\
\hline
\end{tabular}

\subsubsection{Fire and Emergency Response}

The HFD is a multi-disciplined, multi-functional organization which provides fire suppression, rescue, emergency medical and ambulance services, and hazardous material responses that are capable of containing, controlling, and terminating emergency situations which could threaten the operations, employees, or interest of the DOE operated Hanford Site or the various companies and agencies operating on the Hanford Site. This includes emergency support to Bonneville Power Administration facilities, mutual aid response to surrounding fire districts under a Mutual Aid Agreement, and contractual firefighting and ambulance response to Washington Public Power Supply System (Supply System). The fire department's sitewide safety and fire prevention program includes fire alarm system functional testing and inspection, preventive and corrective maintenance, respiratory protection, building tours and inspections, prefire planning, and employee fire safety training.

5.1.4.1 Fire Department Fmergency Services. The emergency services group operates out of four fire stations, strategically located on the Hanford Site to provide emergency support services and staffed with trained fire fighters and fire service officers. Personnel are typically cross-trained in several areas; a fire fighter may hold certifications as an Emergency Medical Technician (EMT), a paramedic, a hazardous material technician, a driver/pumper operator, an emergency dispatcher, a high angle/low angle and confined space rescue responder, etc. Similarly, fire service officers are trained in incident command and in various emergency response specialties. This cross training optimizes personnel utilization by assuring certified responders are available to cover a wide range of emergency situations with the lowest possible number of response staff. All emergency services are provided on a 24-hour-a-day, 7-day-a neek basis.

5.1.4.2 Fire Systems Testing and Emergency Response. The fire systems testing and emergency response group provide operational assurance testing of fire alarm, fire suppression, and fire water distribution systems in and around site building and facilities. Testing is performed to the frequencies required by NFPA codes and standards as implemented by DOE Orders and as modified by approved waivers and exemption requests.

5.1.4.3 Services Provided to Others. HFD services are being utilized to varying degrees by private companies doing business on the site and by other government agencies. Current recipients of these services include the Supply System complex, Kaiser Aluminum, Bonneville Power Administration Ashe Sub-Station, the Port of Benton 400 Area Incubator Project Facilities, and the Laser Interferometer Gravitational-Wave Observatory. 
5.1.4.4 Other - New RL Work Scope Planned for the HFD. WHC has the lead (as designated by RL) for a Hanford Site Emergency Response Organization re-engineering project, which is a performanced-based RL milestone. This effort will provide a streamlined emergency response organization, while maintaining a strong response capability for the site. The HFD is providing key input and support to the re-engineering project, which when implemented will expand HFD responsibilities sitewide for incident management during emergencies.

\subsubsection{Specialized Training for Emergency Response Fire Department Personnel.} Except for the special classification of paramedic, all HFD personnel are qualified as Firefighter I before being allowed to respond to an emergency. Job specific training includes, but is not limited to, pumper operators, EMT, officer, hazardous materials, special operations, and fire cause \& origin. Both DOE and the Washington State mandates certain levels of competencies based on National Codes and Standards.

5.1.4.6 Fire Prevention. The Fire Marshal has the responsibility for fire prevention programs, public education, fire investigation, pre-design and pre-construction briefings, plans review, and special considerations for decommissioning and decontamination projects.

Annual programmatic activities include, but are not limited to, fire prevention, handson fire extinguisher training for site employees, Fire Marshal Permits (as listed in the NFPA Fire Prevention Code), final authority for pre-fire plans, developing and directing the inspection programs for flammable \& reactive waste sites, HFD building tours \& inspections, hydrant flow testing, and storage, use, and disposal of combustible, flammable, explosive or reactive material.

5.1.4.7 Fire Systems Maintenance. Fire Systems Maintenance (FSM) provides preventive, and repair maintenance of fire alarm, fire suppression, and fire water distribution systems in and around site buildings and facilities. Maintenance is performed to the frequencies required by National Fire Procection Association codes and standards as implemented by DOE orders, and as modified by approved waivers and current exemption requests.

5.1.4.8 Respiratory Prodection Equipment Services. Self-contained breathing apparatus (SCBA), escape packs. Powered Air Purifying Respirator (PAPR) systems, regulator and cylinder maintenance, inspection, certified testing and servicing are provided for Hanford contractors who operate/use respiratory protection equipment. Services include the performance of inspections and tests on SCBA valves and low and high pressure breathing air cylinders, PAPR, and escape packs, and the refilling of approximately 4,400 SCBA cylinders per year.

\subsubsection{Contracts for Service and Purchase Requisitions that Extend Past}

October 1, 1996. The HFD has numerous contracts in place that either extend over more than one year or have renewal clauses. This is necessary to in order to avoid any delay in the receipt of equipment or services required to support emergency operations. 


\subsubsection{Emergency Services Facilities, Buildings, and Capital Projects for} Replacement. Many of the HFD facilities and buildings were built decades ago and are nearing the end of their useful life without major restoration and/or replacement. These facilities exist throughout the Hanford Site.

The following actions will be completed to ensure a complete and orderly turnover of HFD operations to the PHMC.

\begin{tabular}{|c|c|c|}
\hline 48 Pre-Award Actions & $\begin{array}{l}\text { Seheduled } \\
\text { Date }\end{array}$ & Ictioneets) \\
\hline $\begin{array}{l}\text { 1. Develop a list of key HFD documentation. Provide to } \\
\text { RL POC for review. }\end{array}$ & $5 / 15 / 96$ & DE Good \\
\hline $\begin{array}{l}\text { 2. Assemble key HFD documentation, or identify its } \\
\text { location, for use by the PHMC. Includes development } \\
\text { of a HFD "Smart Book," including listings and } \\
\text { descriptions developed below. }\end{array}$ & $6 / 01 / 96$ & WR Hayes \\
\hline $\begin{array}{l}\text { 3. Identify number of briefings, and prepare HFD briefing } \\
\text { for presentation to PHMC. (Coordinate number and } \\
\text { content with RL POC.) }\end{array}$ & $6 / 01 / 96$ & DE Good \\
\hline $\begin{array}{l}\text { 4. Develop a listing of key HFD individuals as POCs, } \\
\text { including facility and operations personnel. }\end{array}$ & $6 / 01 / 96$ & DE Good \\
\hline $\begin{array}{l}\text { 5. Develop listing and description of HFD subcontracts, } \\
\text { including facility (or operations) subcontracts. Include } \\
\text { any subcontracts still planned to be executed prior to } \\
\text { contract takeover. }\end{array}$ & $6 / 01 / 96$ & WR Hayes \\
\hline $\begin{array}{l}\text { 6. Develop listing and description of HFD databases, } \\
\text { including facility (or operations) databases. }\end{array}$ & $6 / 01 / 96$ & WR Hayes \\
\hline $\begin{array}{l}\text { 7. Verify the accuracy of property assignments at the HFD } \\
\text { organizational level and correct any deficiencies. }\end{array}$ & $6 / 01 / 96$ & WR Hayes \\
\hline $\begin{array}{l}\text { 8. Identify space and equipment within HFD facilities for } \\
\text { use by the PHMC. }\end{array}$ & $6 / 01 / 96$ & WR Hayes \\
\hline $\begin{array}{l}\text { 9. Using the pre-existing condition checklist, compile pre- } \\
\text { existing condition information on any facilities or major } \\
\text { equipment not addressed separately. Conduct, or } \\
\text { schedule as a separate post-closure action(s), any } \\
\text { assessments required to adequately address all relevant } \\
\text { items on the checklist. }\end{array}$ & $6 / 01 / 96$ & DE Good \\
\hline $\begin{array}{l}\text { 10. Conduct an assessment (e.g., OSHA assessment) of } \\
\text { HFD facilities. (If there are any significant assessments } \\
\text { required for facilities or major equipment then include } \\
\text { them as separate actions). }\end{array}$ & $5 / 15 / 96$ & DE Good \\
\hline $\begin{array}{l}\text { 11. In outline form for all facilities/operations to follow, } \\
\text { define the appropriate contents and level of detail to be } \\
\text { included in facility/operations briefings to the PHMC. } \\
\text { (Review with RL POC.) }\end{array}$ & $5 / 01 / 96$ & DE Good \\
\hline
\end{tabular}




\begin{tabular}{|c|c|c|}
\hline Pre-Award Actions & Scheduled & Setonge(s) \\
\hline $\begin{array}{l}\text { 12. Develop a list of open action items and commitments, } \\
\text { including status (Environmental, Tri-Party Agreement, } \\
\text { DNFSB as examples). Provide to RL POC for review. }\end{array}$ & $6 / 01 / 96$ & WR Hayes \\
\hline $\begin{array}{l}\text { 13. Identify current inventories of materials to support } \\
\text { continued operation (i.e., essential materials, tools and } \\
\text { equipment). }\end{array}$ & $6 / 01 / 96$ & WR Hayes. \\
\hline $\begin{array}{l}\text { 14. Develop a list of facility (or operation)-specific } \\
\text { mandatory training and qualification requirements for } \\
\text { employees. }\end{array}$ & $6 / 01 / 96$ & WR Hayes \\
\hline $\begin{array}{l}\text { 15. Develop a listing of information which constitutes the } \\
\text { "authorization basis" for each facility. }\end{array}$ & $6 / 01 / 96$ & WR Hayes \\
\hline $\begin{array}{l}\text { 16. Develop a description of ongoing and planned } \\
\text { construction projects. }\end{array}$ & $6 / 01 / 96$ & WR Hayes \\
\hline $\begin{array}{l}\text { 17. Develop a listing of special agreement services provided } \\
\text { to RL, other DOE sites, and other entities. }\end{array}$ & $6 / 01 / 96$ & WR Hayes \\
\hline $\begin{array}{l}\text { 18. Develop a listing of facility POCs to be available for the } \\
\text { transition phase. Provide list to program/project level } \\
\text { for consolidation. }\end{array}$ & $5 / 15 / 96$ & DE Good \\
\hline $\begin{array}{l}\text { 19. Develop a list of current work order services or other } \\
\text { form of agreement to provide work for other } \\
\text { organizations, on and offsite. }\end{array}$ & $6 / 01 / 96$ & WR Hayes \\
\hline $\begin{array}{l}\text { 20. Develop a list of current work order services or other } \\
\text { form of agreement to obtain work from other } \\
\text { organizations, on and offsite. }\end{array}$ & $6 / 01 / 96$ & WR Hayes \\
\hline $\begin{array}{l}\text { 21. Develop a list of permits governing the facility (or } \\
\text { operation), and provide a description of each as to where } \\
\text { and how it applies. }\end{array}$ & $6 / 01 / 96$ & WR Hayes \\
\hline \multicolumn{3}{|l|}{ Post-Award Actions } \\
\hline $\begin{array}{l}\text { 1. Provide the PHMC access to the key HFD } \\
\text { documentation, any program level generated pre-existing } \\
\text { conditions information, listing of POCs, and listing of } \\
\text { subcontracts. Provide copies upon request. }\end{array}$ & prior to $6 / 15 / 96$ & WR Hayes \\
\hline $\begin{array}{l}\text { 2. PHMC identify space and equipment needs within HFD } \\
\text { area and facilities. }\end{array}$ & by $6 / 08 / 96$ & WR Hayes \\
\hline $\begin{array}{l}\text { 3. HFD facility space and services available for use by the } \\
\text { PHMC. }\end{array}$ & $6 / 15 / 96$ & WR Hayes \\
\hline 4. Provide HFD briefing to the PHMC. & $6 / 15 / 96-7 / 01 / 96$ & DE Good \\
\hline 5. Provide HFD tours to the PHMC. & $6 / 15 / 96-7 / 01 / 96$ & DE Good \\
\hline $\begin{array}{l}\text { 6. Perform a joint HFD property and equipment inventory } \\
\text { with PHMC personnel. }\end{array}$ & by $8 / 30 / 96$ & DE Good \\
\hline $\begin{array}{l}\text { 7. Provide any additional documentation or information } \\
\text { requested by the PHMC, if available, that was not } \\
\text { identified during the pre-award phase. }\end{array}$ & by $7 / 01 / 96$ & WR Hayes \\
\hline
\end{tabular}




\begin{tabular}{|c|c|c|}
\hline Post A ward Actions & soheduled & lotopen \\
\hline $\begin{array}{l}\text { 8. PHMC identify any recurring reports that they would } \\
\text { like to receive during transition. }\end{array}$ & by $7 / 15 / 96$ & PHMC \\
\hline 9. Provide necessary training to PHMC personnel. & $7 / 01-8 / 01 / 96$ & WR Hayes \\
\hline $\begin{array}{l}\text { 10. Provide an updated status of ongoing work prior to } \\
\text { contract turnover. }\end{array}$ & by $9 / 30 / 96$ & DE Good \\
\hline 11. Provide a categorized inventory of wastes. & by $9 / 30 / 96$ & WR Hayes \\
\hline $\begin{array}{l}\text { 12. Provide an updated status of end point criteria to } \\
\text { PHMC. }\end{array}$ & by $9 / 30 / 96$ & DE Good \\
\hline
\end{tabular}

\subsubsection{Fabrication Shops}

The Site Fabrication Services (SFS) mission is to maintain a center of excellence with capability for the fabrication of prototype assemblies, repair of parts, components, and specified equipment repairs. Specific emphasis will be placed on prototypical and emergency work, which requires extensive engineering and manufacturing coordination. SFS will encourage a one-stop shopping center by maintaining a work brokering activity that will - te and manage fabrication and manufacturing work to others onsite or offsite as required pport clean-up activities and schedules.

Assumptions:

- Welding Procedures and Welder Qualifications are currently ICF KH and cannot be transferred to the new contractor. The new contractor must use their own procedures and re-qualify the welders to their program for code work. If the contractor does not have the necessary procedures to perform the work currently in progress, they will need to qualify those procedures prior to the continuation of that work.

- The ICF KH QA Program cannot be transferred to the new contractor. The new contractor must submit for approval their own program. This will require the recertification of all ICF $\mathrm{KH}$ Inspection Personnel.

- The ICF KH American Society of Mechanical Engineers (ASME) Code Stamps cannot be transferred to the new contractor. If code work is required, the new contractor will be required to be surveyed and qualified by the code community.

- Magnetic Particle Testing, Liquid Penetrant Testing, Radiographic Testing, and Ultrasonic Testing is currently performed by WHC as a service to SFS. When this function is terminated, the new contractor will have to supply their own NDE Service, or hire these services in accordance with their QA Program if they are to comply with American Society for Nondestructive Testing requirements. 


\begin{tabular}{|c|c|c|}
\hline Pre Award Actions & $\begin{array}{l}\text { Scheduled } \\
\text { Date }\end{array}$ & Actioness) \\
\hline $\begin{array}{l}\text { 1. Identify number of briefings, and prepare briefing(s) } \\
\text { for the PHMC. (Provide for RL POC review.) }\end{array}$ & $5 / 15 / 96$ & MA Butterworth \\
\hline $\begin{array}{l}\text { 2. Identify key documents for the facility (or operations). } \\
\text { (Provide for RL POC review.) }\end{array}$ & $5 / 01 / 96$ & MA Butterworth \\
\hline $\begin{array}{l}\text { 3. Assemble key documentation, or identify its location, } \\
\text { for use by the PHMC. Includes preparation of a } \\
\text { "Smart Book," to include information develop in the } \\
\text { following actions. }\end{array}$ & 6/01/96 & MA Butterworth \\
\hline $\begin{array}{l}\text { 4. Develop a listing and description of subcontracts. } \\
\text { Include any subcontracts planned to be executed prior } \\
\text { to contract takeover. }\end{array}$ & $5 / 15 / 96$ & MA Butterworth \\
\hline $\begin{array}{l}\text { 5. Using the pre-existing checklist, compile pre-existing } \\
\text { condition information on facilities and/or major } \\
\text { equipment. Conduct, or schedule as a separate post- } \\
\text { award action(s), any assessments required to adequately } \\
\text { address all relevant items on the checklist. Provide } \\
\text { completed checklist for RL POC review. }\end{array}$ & $6 / 01 / 96$ & MA Butterworth \\
\hline $\begin{array}{l}\text { 6. Develop a list of open action items and commitments, } \\
\text { including status (Environmental, Tri-Party Agreement, } \\
\text { DNFSB as examples). }\end{array}$ & $6 / 01 / 96$ & MA Butterworth \\
\hline $\begin{array}{l}\text { 7. Develop a list of facility (or operation)-specific } \\
\text { mandatory training and qualification requirements for } \\
\text { employees. }\end{array}$ & 6/01/96 & MA Butterworth \\
\hline $\begin{array}{l}\text { 8. Develop a listing of special agreement services } \\
\text { provided to RL, other DOE sites, and other entities. }\end{array}$ & 6/01/96 & MA Butterworth \\
\hline $\begin{array}{l}\text { 9. Develop a listing of POCs to be available for the } \\
\text { transition phase. }\end{array}$ & $5 / 15 / 96$ & MA Butterworth \\
\hline 10. Develop a listing and description of databases. & $5 / 15 / 96$ & MA Butterworth \\
\hline $\begin{array}{l}\text { 11. Develop a list of current work order services or other } \\
\text { form of agreement to provide work for other } \\
\text { organizations, on and offsite. }\end{array}$ & $6 / 01 / 96$ & MA Butterworth \\
\hline $\begin{array}{l}\text { 12. Develop a list of current work order services or other } \\
\text { form of agreement to obtain work from other } \\
\text { organizations, on and offsite. }\end{array}$ & $6 / 01 / 96$ & MA Butterworth \\
\hline $\begin{array}{l}\text { 13. Develop a list of permits governing facilities or } \\
\text { operation, and provide a description of each as to } \\
\text { where and how it applies. }\end{array}$ & $6 / 01 / 96$ & MA Butterworth \\
\hline $\begin{array}{l}\text { 14. Verify the accuracy of property assignments and } \\
\text { correct any deficiencies. }\end{array}$ & $6 / 01 / 96$ & MA Butterworth \\
\hline Post-Award Actions & & \\
\hline 1. Provide briefing(s) to the PHMC. & $7 / 01 / 96-7 / 15 / 96$ & MA Butterworth \\
\hline 2. Provide tour(s) to the PHMC. & $7 / 01 / 96-7 / 15 / 96$ & MA Butterworth \\
\hline
\end{tabular}




\begin{tabular}{|c|c|c|}
\hline Post Award Actions & Schedoled & $+(0,0)=2(s)$ \\
\hline $\begin{array}{l}\text { 3. Provide the PHMC access to key documentation, pre- } \\
\text { existing condition information, commitment data, } \\
\text { inventory data, etc. (as established in the pre-award } \\
\text { phase). Provide copies upon request. }\end{array}$ & by $6 / 15 / 96$ & MA Butterworth \\
\hline $\begin{array}{l}\text { 4. Provide any additional documentation or information } \\
\text { requested by the PHMC; if available, that was not } \\
\text { identified during the pre-award phase. }\end{array}$ & by $7 / 01 / 96$ & MA Butterworth \\
\hline $\begin{array}{l}\text { 5. PHMC identify any recurring reports that they would } \\
\text { like to receive during transition. }\end{array}$ & by $7 / 15 / 96$ & MA Butterworth \\
\hline 6. Provide necessary training to PHMC personnel. & $7 / 01 / 96-8 / 01 / 96$ & MA Butterworth \\
\hline $\begin{array}{l}\text { 7. Provide an updated status of ongoing work prior to } \\
\text { contract turnover. }\end{array}$ & by $9 / 30 / 96$ & MA Butterworth \\
\hline $\begin{array}{l}\text { 8. Perform a joint property and equipment inventory with } \\
\text { the PHMC. }\end{array}$ & by $8 / 30 / 96$ & MA Butterworth \\
\hline $\begin{array}{l}\text { 9. Provide a categorized inventory of wastes or hazardous } \\
\text { materials. }\end{array}$ & by $9 / 30 / 96$ & MA Butterworth \\
\hline $\begin{array}{l}\text { 10. Assist the PHMC in the conduct of pre-existing } \\
\text { condition assessments. }\end{array}$ & $7 / 15 / 96-8 / 30 / 96$ & MA Butterworth \\
\hline
\end{tabular}

\subsubsection{Integrated Pest Management Services}

Integrated Pest Management manages and controls plant and animal pest species at Hanford using a combination of safe and cost effective control alternatives such as administrative controls, engineering controls, and chemical applications.

\begin{tabular}{|c|c|c|}
\hline Pre-Award Actions & $\begin{array}{l}\text { Scheduled } \\
\text { Date }\end{array}$ & Actionee(s) \\
\hline $\begin{array}{l}\text { 1. Prepare for a comprehensive briefing for the PHMC describing } \\
\text { all operational aspects of the organization. }\end{array}$ & $5 / 01 / 96$ & CL Looney \\
\hline $\begin{array}{l}\text { 2. Identify all key documents relevant to the organizations } \\
\text { operations, including a listing of key facility and field } \\
\text { documents. (Provide a listing to RL for review.) }\end{array}$ & $5 / 01 / 96$ & CL Looney \\
\hline $\begin{array}{l}\text { 3. Obtain copies of key facility documents and assemble, or identify } \\
\text { their location for access. Deveiop an operations "Smart Book." }\end{array}$ & $5 / 15 / 96$ & CL Looney \\
\hline 4. Identify points-of-contact for the organization. & $5 / 15 / 96$ & CL Looney \\
\hline $\begin{array}{l}\text { 5. Using the pre-existing conditions checklist, evaluate pre-existing } \\
\text { conditions. Conduct assessments as necessary to adequately } \\
\text { address checklist. }\end{array}$ & $5 / 15 / 96$ & CL Looney \\
\hline $\begin{array}{l}\text { 6. Identify applicable milestones and commitments (e.g., Tri-Party } \\
\text { Agreement and Consent Order milestones), and determine their } \\
\text { status. Describe the form of the deliverable and what constitutes } \\
\text { completion. }\end{array}$ & $5 / 15 / 96$ & CL Looney \\
\hline
\end{tabular}




\section{WHC-SP-1181}

\begin{tabular}{|c|c|c|}
\hline (14. 40 Pre-Award Actions & $\begin{array}{l}\text { Schedulad? } \\
\text { Date. }\end{array}$ & Actiones (6) \\
\hline $\begin{array}{l}\text { 7. Verify the accuracy of property assignments for the facility and } \\
\text { correct any deficiencies. }\end{array}$ & $5 / 15 / 96$ & CL Looney \\
\hline 8. Verify the completeness of all RIDS and correct any deficiencies. & $5 / 15 / 96$ & CL Looney \\
\hline Post-Award Actions & & \\
\hline $\begin{array}{l}\text { 1. Provide a comprehensive briefing for the PHMC complete with } \\
\text { all facility, personnel, and organizational documentation. } \\
\text { (Provide copies upon request.) }\end{array}$ & $6 / 15 / 96$ & CL Looney \\
\hline $\begin{array}{l}\text { 2. Conduct a comprehensive tour of all facilities and field } \\
\text { operations relevant to the organization. }\end{array}$ & $6 / 15 / 96$ & CL Looney \\
\hline $\begin{array}{l}\text { 3. Assist the PHMC in a survey of pre-existing conditions at the } \\
\text { facility. }\end{array}$ & $8 / 01 / 96$ & CL Looney \\
\hline $\begin{array}{l}\text { 4. Provide an update on work completed and work in progress } \\
\text { during the transition to the PHMC. }\end{array}$ & 9/15/96 & CL Looney \\
\hline $\begin{array}{l}\text { 5. Complete a joint property and equipment inventory with the } \\
\text { PHMC. }\end{array}$ & 9/15/96 & CL Looney \\
\hline 6. Provide any additional documentation requested by the PHMC. & ongoing & CL Looney \\
\hline 7. Provide necessary training to PHMC personnel. & $8 / 01 / 96$ & CL Looney \\
\hline 8. Provide a categorized inventory of wastes. & 9/30/96 & CL Looney \\
\hline
\end{tabular}

\subsubsection{Non-Radioactive/Non-Dangerous Waste Handling}

Provide disposal services for municipal, asbestos, drummed, and medical wastes. Manage contracts and interface with the City of Richland, Waste Management of Kennewick, and Basin Disposal, Inc. Pick up waste containers throughout the Hanford Site and deliver to the City of Richland landfill. Transport hazardous materials.

\section{Assumptions:}

- Contracts with the City of Richland, Waste Management of Kennewick, and Basin Disposal, Inc., will remain in effect.

- Municipal waste will be delivered to the City of Richland landfill by HAMTC workers.

- Hazardous material transportation groups will be consolidated.

\begin{tabular}{|c|c|c|}
\hline Pre-Award Actions & $\begin{array}{l}\text { Scheduled } \\
\text { Date }\end{array}$ & Actioned (s) \\
\hline $\begin{array}{l}\text { 1. Identify number of briefings, and prepare briefing(s) } \\
\text { for the PHMC. (Provide for RL POC review.) }\end{array}$ & $5 / 15 / 96$ & KD Johnson \\
\hline
\end{tabular}




\begin{tabular}{|c|c|c|}
\hline Pre-Award Aetions & Scheduled & Hetopes) \\
\hline $\begin{array}{l}\text { 2. Identify key documents for the facility (or operations). } \\
\text { (Provide for RL POC review.) }\end{array}$ & $5 / 01 / 96$ & KD Johnson \\
\hline $\begin{array}{l}\text { 3. Assemble key documentation, or identify its location, } \\
\text { for use by the PHMC. Includes preparation of a } \\
\text { "Smart Book," to include information develop in the } \\
\text { following actions. }\end{array}$ & $6 / 01 / 96$ & KD Johnson \\
\hline $\begin{array}{l}\text { 4. Develop a listing and description of subcontracts. } \\
\text { Include any subcontracts planned to be executed prior } \\
\text { to contract takeover. }\end{array}$ & $5 / 15 / 96$ & KD Johnson \\
\hline $\begin{array}{l}\text { 5. Using the pre-existing checklist, compile pre-existing } \\
\text { condition information on facilities and/or major } \\
\text { equipment. Conduct, or schedule as a separate post- } \\
\text { award action(s), any assessments required to adequately } \\
\text { address all relevant items on the checklist. Provide } \\
\text { completed checklist for RL POC review. }\end{array}$ & $6 / 01 / 96$ & KD Johnson \\
\hline $\begin{array}{l}\text { 6. Develop a list of open action items and commitments, } \\
\text { including status (Environmental, Tri-Party Agreement, } \\
\text { DNFSB as examples). }\end{array}$ & $6 / 01 / 96$ & KD Johnson \\
\hline $\begin{array}{l}\text { 7. Develop a list of facility (or operation)-specific } \\
\text { mandatory training and qualification requirements for } \\
\text { employees. }\end{array}$ & $6 / 01 / 96$ & KD Johnson \\
\hline $\begin{array}{l}\text { 8. Develop a listing of special agreement services } \\
\text { provided to } R L \text {, other DOE sites, and other entities. }\end{array}$ & $6 / 01 / 96$ & KD Johnson \\
\hline $\begin{array}{l}\text { 9. Develop a listing of POCs to be available for the } \\
\text { transition phase. }\end{array}$ & $5 / 15 / 96$ & KD Johnson \\
\hline 10. Develop a listing and description of databases. & $5 / 15 / 96$ & KD Johnson \\
\hline $\begin{array}{l}\text { 11. Develop a list of current work order services or other } \\
\text { form of agreement to provide work for other } \\
\text { organizations, on and offsite. }\end{array}$ & $6 / 01 / 96$ & KD Johnson \\
\hline $\begin{array}{l}\text { 12. Develop a list of current work order services or other } \\
\text { form of agreement to obtain work from other } \\
\text { organizations, on and offsite. }\end{array}$ & $6 / 01 / 96$ & KD Johnson \\
\hline $\begin{array}{l}\text { 13. Develop a list of permits governing facilities or } \\
\text { operation, and provide a description of each as to } \\
\text { where and how it applies. }\end{array}$ & $6 / 01 / 96$ & KD Johnson \\
\hline $\begin{array}{l}\text { 14. Verify the accuracy of property assignments and } \\
\text { correct any deficiencies. }\end{array}$ & $6 / 01 / 96$ & KD Johnson \\
\hline Post-Award Actions & & \\
\hline 1. Provide briefing(s) to the PHMC. & $7 / 01 / 96-7 / 15 / 96$ & KD Johnson \\
\hline 2. Provide tour(s) to the PHMC. & $7 / 01 / 96-7 / 15 / 96$ & KD Johnson \\
\hline $\begin{array}{l}\text { 3. Provide the PHMC access to key documentation, pre- } \\
\text { existing condition information, commitment data, } \\
\text { inventory data, etc. (as established in the pre-award } \\
\text { phase). Provide copies upon request. }\end{array}$ & by $6 / 15 / 96$ & KD Johnson \\
\hline
\end{tabular}




\begin{tabular}{|l|c|l|}
\hline \multicolumn{1}{|c|}{ Post-Award Actions } \\
\hline $\begin{array}{l}\text { 4. Provide any additional documentation or information } \\
\text { requested by the PHMC, if available, that was not } \\
\text { identified during the pre-award phase. }\end{array}$ & by $7 / 01 / 96$ & KD Johnson \\
\hline $\begin{array}{l}\text { 5. PHMC identify any recurring reports that they would } \\
\text { like to receive during transition. }\end{array}$ & by $7 / 15 / 96$ & KD Johnson \\
\hline 6. Provide necessary training to PHMC personnel. & $7 / 01 / 96-8 / 01 / 96$ & KD Johneon \\
\hline $\begin{array}{l}\text { 7. Provide an updated stanis of ongoing work prior to } \\
\text { contract turnover. }\end{array}$ & by $9 / 30 / 96$ & KD Johnson \\
\hline $\begin{array}{l}\text { 8. Perform a joint property and equipment inventory with } \\
\text { the PHMC. }\end{array}$ & by $8 / 30 / 96$ & KD Johnson \\
\hline $\begin{array}{l}\text { 9. Provide a categorized inventory of wastes or hazardous } \\
\text { materials. }\end{array}$ & by $9 / 30 / 96$ & KD Johnson \\
\hline $\begin{array}{l}\text { 10. Assist the PHMC in the conduct of pre-existing } \\
\text { condition assessments. }\end{array}$ & $7 / 15 / 96-8 / 30 / 96$ & KD Johnson \\
\hline
\end{tabular}

\subsubsection{Site Crane \& Rigging Services}

The Mission of ICF KH Site Crane and Rigging Services is to support various programs dedicated to the maintenance, cleanup, and restoration of the Hanford Site. This support is provided through the lifting, handling, movement, and placement of material and equipment; inspection and maintenance of lifting equipment; erection, inspection, and removal of scaffold; fabrication, testing, and control of below-the-hook rigging equipment; and the management of mobile lifting equipment. All of this support is provided within the boundaries and scope of WHC, ICF KH, or Hanford procedures, directives and orders.

\begin{tabular}{|l|c|l|}
\hline \multicolumn{1}{|c|}{ Pre-Award Actions } & $\begin{array}{c}\text { Scheduled } \\
\text { Date }\end{array}$ & Actionee(s) \\
\hline $\begin{array}{l}\text { 1. Identify number of briefings, and prepare briefing(s) } \\
\text { for the PHMC. (Provide for RL POC review.) }\end{array}$ & $5 / 15 / 96$ & WA Ferree \\
\hline $\begin{array}{l}\text { 2. Identify key documents for the facility (or operations). } \\
\text { (Provide for RL POC review.) }\end{array}$ & $5 / 01 / 96$ & CE Brewer \\
\hline $\begin{array}{l}\text { 3. Assemble key documentation and org. data, or identify } \\
\text { its location, for use by the PHMC. Includes } \\
\text { preparation of a "Smart Book," to include information } \\
\text { develop in the following actions. }\end{array}$ & $6 / 01 / 96$ & CE Brewer \\
\hline 4. Develop a listing and description of subcontracts. \\
$\begin{array}{l}\text { Include any subcontracts planned to be executed prior } \\
\text { to contract takeover. }\end{array}$ & $5 / 15 / 96$ & WA Ferree \\
\hline
\end{tabular}




\begin{tabular}{|c|c|c|}
\hline Pre-Award Actions & Schedoled. & teponere \\
\hline $\begin{array}{l}\text { 5. Using the pre-existing checklist, compile pre-existing } \\
\text { condition information on facilities and/or major } \\
\text { equipment. Conduct, or schedule as a separate post- } \\
\text { award action(s), any assessments required to adequately } \\
\text { address all relevant items on the checklist. Provide } \\
\text { completed checklist for RL POC review. }\end{array}$ & $6 / 01 / 96$ & WA Ferree \\
\hline $\begin{array}{l}\text { 6. Develop a list of open action items and commitments, } \\
\text { including status (Environmental, Tri-Party Agreement, } \\
\text { DNFSB as examples). }\end{array}$ & $6 / 01 / 96$ & WA Ferree \\
\hline $\begin{array}{l}\text { 7. Develop a list of facility (or operation)-specific } \\
\text { mandatory training and qualification requirements for } \\
\text { employees. }\end{array}$ & $6 / 01 / 96$ & SM Holloman \\
\hline $\begin{array}{l}\text { 8. Develop a listing of special agreement services } \\
\text { provided to } \mathbf{R L} \text {, other DOE sites, and other entities. }\end{array}$ & $6 / 01 / 96$ & WA Ferree \\
\hline $\begin{array}{l}\text { 9. Develop a listing of POCs to be available for the } \\
\text { transition phase. }\end{array}$ & $5 / 15 / 96$ & WA Ferree \\
\hline 10. Develop a listing and description of databases. & $5 / 15 / 96$ & WA Ferree \\
\hline $\begin{array}{l}\text { 11. Develop a list of current work order services or other } \\
\text { form of agreement to provide work for other } \\
\text { organizations, on and offsite. }\end{array}$ & $6 / 01 / 96$ & WA Ferree \\
\hline $\begin{array}{l}\text { 12. Develop a list of current work order services or other } \\
\text { form of agreement to obtain work from other } \\
\text { organizations, on and offsite. }\end{array}$ & $6 / 01 / 96$ & WA Ferree \\
\hline $\begin{array}{l}\text { 13. Develop a list of permits governing facilities or } \\
\text { operation, and provide a description of each as to } \\
\text { where and how it applies. }\end{array}$ & $6 / 01 / 96$ & WA Ferree \\
\hline $\begin{array}{l}\text { 14. Verify the accuracy of property assignments and } \\
\text { correct any deficiencies. }\end{array}$ & $6 / 01 / 96$ & WA Ferree \\
\hline Post-Award Actions & & \\
\hline 1. Provide briefing(s) to the PHMC. & $7 / 01 / 96-7 / 15 / 96$ & WA Ferree \\
\hline 2. Provide tour(s) to the PHMC. & 7/01/96-7/15/96 & WA Ferree \\
\hline $\begin{array}{l}\text { 3. Provide the PHMC access to key documentation, pre- } \\
\text { existing condition information, commitment data, } \\
\text { inventory data, etc. (as established in the pre-award } \\
\text { phase). Provide copies upon request. }\end{array}$ & by $6 / 15 / 96$ & WA Ferree \\
\hline $\begin{array}{l}\text { 4. Provide any additional documentation or information } \\
\text { requested by the PHMC, if available, that was not } \\
\text { identified during the pre-award phase. }\end{array}$ & by $7 / 01 / 96$ & WA Ferree \\
\hline $\begin{array}{l}\text { 5. PHMC identify any recurring reports that they would } \\
\text { like to receive during transition. }\end{array}$ & by $7 / 15 / 96$ & WA Ferree \\
\hline 6. Provide necessary training to PHMC personnel. & 7/01/96-8/01/96 & WA Ferree \\
\hline $\begin{array}{l}\text { 7. Provide an updated status of ongoing work prior to } \\
\text { contract turnover. }\end{array}$ & by $9 / 30 / 96$ & WA Ferree \\
\hline
\end{tabular}




\begin{tabular}{|l|c|c|}
\hline $\begin{array}{l}\text { 8. Perform a joint property and equipment inventory with } \\
\text { the PHMC. }\end{array}$ & by $8 / 30 / 96$ & WA Ferree \\
\hline $\begin{array}{l}\text { 9. Provide a categorized inventory of wastes or hazardous } \\
\text { materials. }\end{array}$ & by $9 / 30 / 96$ & WA Ferree \\
\hline 10. Provide overview of financial situation and work plans. & $8 / 30 / 96$ & WA Ferree \\
\hline 11. Status audit/surveillance items & $8 / 30 / 96$ & WA Ferree \\
\hline $\begin{array}{l}\text { 12. Assist the PHMC in the conduct of pre-existing } \\
\text { condition assessments. }\end{array}$ & $7 / 15 / 96-8 / 30 / 96$ & WA Ferree \\
\hline
\end{tabular}

\subsubsection{Landlord Program}

The Landlord Program (LP) replaces, repairs, or upgrades the existing Hanford Site (WHC, PNNL, HEHF, and ICF KH) general purpose infrastructure in support of the Hanford Cleanup and other missions. The general purpose infrastructure consists of buildings, systems, and equipment that, by design or use are not essentially dedicated to a single program mission. The LP provides capital funding for ongoing replacements or repairs to general purpose equipment and facilities to sustain support to the Hanford cleanup and other missions.

\begin{tabular}{|c|c|c|}
\hline Pre-Award Actions & $\begin{array}{l}\text { Scheduled } \\
\text { Date }\end{array}$ & Actionet $(9)$ \\
\hline $\begin{array}{l}\text { 1. Develop a list of key program documentation. Provide } \\
\text { for RL POC review. }\end{array}$ & $5 / 15 / 96$ & AD Gadeken \\
\hline $\begin{array}{l}\text { 2. Assemble key program documentation, or identify its } \\
\text { location, for use by the PHMC. Includes development } \\
\text { of a program/project "Smart Book," including listings } \\
\text { and descriptions developed below. }\end{array}$ & $6 / 01 / 96$ & AD Gadeken \\
\hline $\begin{array}{l}\text { 3. Identify number of briefings, and prepare program } \\
\text { briefing(s) for presentation to PHMC. (Provide for } \\
\text { coordination of number and content with RL POC.) }\end{array}$ & $6 / 01 / 96$ & AD Gadeken \\
\hline $\begin{array}{l}\text { 4. Develop a listing of Key program individuals as POCs, } \\
\text { including facility and operations personnel. }\end{array}$ & $6 / 01 / 96$ & AD Gadeken \\
\hline $\begin{array}{l}\text { 5. Develop listing and description of program subcontracts. } \\
\text { Include any subcontracts still planned to be executed } \\
\text { prior to contract takeover. }\end{array}$ & $6 / 01 / 96$ & AD Gadeken \\
\hline 6. Develop listing and description of program databases. & $6 / 01 / 96$ & AD Gadeken \\
\hline $\begin{array}{l}\text { 7. Verify the accuracy of property assignments and correct } \\
\text { any deficiencies. }\end{array}$ & $6 / 01 / 96$ & AD Gadeken \\
\hline $\begin{array}{l}\text { 8. Identify space and equipment within program area and } \\
\text { facilities for use by the PHMC. }\end{array}$ & $6 / 01 / 96$ & AD Gadeken \\
\hline $\begin{array}{l}\text { 9. Develop a listing and description of ongoing and } \\
\text { planned construction projects. }\end{array}$ & $6 / 01 / 96$ & AD Gadeken \\
\hline
\end{tabular}




\begin{tabular}{|c|c|c|}
\hline ( 1.4 Post-Award Actions & $\begin{array}{l}\text { Scheduled } \\
\text { Date. }\end{array}$ & retionecto \\
\hline $\begin{array}{l}\text { 1. Provide the PHMC access to the key program } \\
\text { documentation, listing of POCs, and listing of } \\
\text { subcontracts. Provide copies upon request. }\end{array}$ & prior to $6 / 15 / 96$ & AD Gadeken \\
\hline $\begin{array}{l}\text { 2. PHMC identify space and equipment needs within } \\
\text { program/project area and facilities. }\end{array}$ & by $6 / 8 / 96$ & PHMC \\
\hline $\begin{array}{l}\text { 3. Program facility space and services available for use by } \\
\text { the PHMC. }\end{array}$ & $6 / 15 / 96$ & AD Gadeken \\
\hline 4. Provide program briefing(s) to the PHMC. & 6/15/96-7/01/96 & AD Gadeken \\
\hline 5. Provide program level tour to the PHMC. & $6 / 15 / 96-7 / 01 / 96$ & AD Gadeken \\
\hline $\begin{array}{l}\text { 6. Perform a joint program level property and equipment } \\
\text { inventory with PHMC personnel. }\end{array}$ & by $8 / 30 / 96$ & AD Gadeken \\
\hline
\end{tabular}

\subsubsection{Resource and Energy Management}

Resource \& Energy Management (R\&EM) has the responsibility to reduce mortgage costs by sponsoring and validating life-cycle, cost effective, energy efficient projects. R\&EM promotes employee culture change for efficient resource use onsite. Documented resource and energy savings show progress towards reducing mortgage costs, allows more taxpayer dollars to be focused into the actual cleanup of the Hanford Site, and validates payback for the program.

Assumptions:

- Program will support Energy Savings Performance Contract in FY 1997 and outyears.

- Program will receive Landlord funding for life-cycle cost effective project development.

- Program will receive In-house Energy Management funding to support some energy related duties.

- Bonneville Power Administration will provide some level of project funding for the FY 1997 program.

- Related areas, such as electrical and other resource metering, will continue to be supported by RL and Site programs.

\begin{tabular}{|c|c|c|}
\hline Pre-Award Aetions & Scheduled & Actionee $(s)$ \\
\hline $\begin{array}{l}\text { 1. Develop a list of key program documentation. Provide } \\
\text { for RL POC review. }\end{array}$ & $5 / 15 / 96$ & WS Dunnivant \\
\hline
\end{tabular}




\begin{tabular}{|c|c|c|}
\hline Pre-Award Actions & $\begin{array}{l}\text { Scheduled } \\
\text { Date }\end{array}$ & Actionee(s) \\
\hline $\begin{array}{l}\text { 2. Assemble key program documentation, or identify its } \\
\text { location, for use by the PHMC. Includes development } \\
\text { of a program/project "Smart Book," including listings } \\
\text { and descriptions developed below. }\end{array}$ & 6/01/96 & WS Dunnivant \\
\hline $\begin{array}{l}\text { 3. Identify number of briefings, and prepare program } \\
\text { briefing(s) for presentation to PHMC. (Provide for } \\
\text { coordination of number and content with RL POC.) }\end{array}$ & 6/01/96 & WS Dunnivant \\
\hline $\begin{array}{l}\text { 4. Develop a listing of Key program individuals as POCs, } \\
\text { including facility and operations personnel. }\end{array}$ & $6 / 01 / 96$ & WS Dunnivant \\
\hline $\begin{array}{l}\text { 5. Develop listing and description of program subcontracts. } \\
\text { Include any subcontracts still planned to be executed } \\
\text { prior to contract takeover. }\end{array}$ & $6 / 01 / 96$ & WS Dunnivant \\
\hline 6. Develop listing and description of program databases. & $6 / 01 / 96$ & WS Dunnivant \\
\hline $\begin{array}{l}\text { 7. Verify the accuracy of property assignments and correct } \\
\text { any deficiencies. }\end{array}$ & $6 / 01 / 96$ & WS Dunnivant \\
\hline $\begin{array}{l}\text { 8. Identify space and equipment within program area and } \\
\text { facilities for use by the PHMC. }\end{array}$ & $6 / 01 / 96$ & WS Dunnivant \\
\hline 9. Conduct an energy assessment of site facilities. & $5 / 15 / 96$ & WS Dunnivant \\
\hline 44 Post-A ward Actions & & $\sqrt[3]{ }$ \\
\hline $\begin{array}{l}\text { 1. Provide the PHMC access to the key program } \\
\text { documentation, listing of POCs, and listing of } \\
\text { subcontracts. Provide copies upon request. }\end{array}$ & prior to $6 / 15 / 96$ & WS Dunnivant \\
\hline $\begin{array}{l}\text { 2. PHMC identify space and equipment needs within } \\
\text { program/project area and facilities. }\end{array}$ & by $6 / 08 / 96$ & PHMC \\
\hline $\begin{array}{l}\text { 3. Program facility space and services available for use by } \\
\text { the PHMC. }\end{array}$ & $6 / 15 / 96$ & WS Dunnivant \\
\hline 4. Provide program briefing(s) to the PHMC. & 6/15/96-7/01/96 & WS Dunnivant \\
\hline $\begin{array}{l}\text { 5. Perform a joint program level property and equipment } \\
\text { inventory with PHMC personnel. }\end{array}$ & by $8 / 30 / 96$ & WS Dunnivant \\
\hline
\end{tabular}

\subsection{REAL ESTATE AND MATERIAL MANAGEMENT}

\section{(Also see Section 3.6, Procurement and Materials Management.)}

\subsubsection{Land-Use Planning and Management}

This activity consists of the integration of land use activities at the 1,450 square kilometers (560 square miles) Hanford Site. This includes (1) site selection for individual construction projects; (2) site plans for major complexes and master plans within each operating area at Hanford; (3) comprehensive land use planning for the overall Site including integration with other sitewide plans such as environmental, strategic, systems engineering, 
ecologic/cultural, infrastructure, demolition, economic development/transition/asset conversion, site restoration, facility use/upgrades, safety, security, coordination of construction schedules, excavation permit approval, etc.; (4) development and operation of automated planning and mapping systems; and (5) information management, including building numbering system.

Regulatory Drivers:

- 48 CFR 970.5204-60, "Facilities Management"

- Secretary of Energy "Land and Facility Use Policy," dated December 21, 1994

- DOE 4320.2, "Capital Asset Management Process," dated March 13, 1992

- DOE 4300.1C, "Real Property Management," dated June 28, 1992

- DOE 4320.1B, "Site Development Planning," dated March 26, 1992

- DOE 6430.1A, "General Design Criteria," dated April 6, 1989

- Draft DOE 4310.00, "Land and Facility Use Management," dated May 1, 1995

- DOE 0 430.1, "Life Cycle Asset Management," dated August 24, 1995

- RL 4310.1B, "Policy for Use of Land on the Crest of Rattlesnake Mountain," dated November 17, 1988

- RL 4320.2C, "Site Selection," dated March 16, 1990

- RL Letter to President of Westinghouse Hanford Company, "Interim Process for RL Review/Approval of Site Selection Requests," dated October 16, 1995

- WHC-CM-8-7, Section 905, Rev. 2, "Site Selection"

Note: All of the regulatory drivers remain in effect until incorporated into the new operating contract, via DOE Order 430.1. Even though some of the orders may be canceled, the specific work related to these orders will continue. 


\begin{tabular}{|c|c|c|}
\hline Pre-Award Actions & $\begin{array}{l}\text { Scheduled } \\
\text { Date }\end{array}$ & Actionee(s) \\
\hline $\begin{array}{l}\text { 1. Develop a list of key program documentation. Provide } \\
\text { for RL POC review. }\end{array}$ & $5 / 15 / 96$ & EF Yancey \\
\hline $\begin{array}{l}\text { 2. Assemble key program documentation, or identify its } \\
\text { location, for use by the PHMC. Includes development } \\
\text { of a program/project "Smart Book," including listings } \\
\text { and descriptions developed below. }\end{array}$ & $6 / 01 / 96$ & EF Yancey \\
\hline $\begin{array}{l}\text { 3. Identify number of briefings, and prepare program } \\
\text { briefing(s) for presentation to PHMC. (Provide for } \\
\text { coordination of number and content with RL POC.) }\end{array}$ & $6 / 01 / 96$ & EF Yancey \\
\hline $\begin{array}{l}\text { 4. Develop a listing of Key program individuals as POCs, } \\
\text { including facility and operations personnel. }\end{array}$ & 6/01/96 & EF Yancey \\
\hline $\begin{array}{l}\text { 5. Develop listing and description of program subcontracts. } \\
\text { Include any subcontracts still planned to be executed } \\
\text { prior to contract takeover. }\end{array}$ & $6 / 01 / 96$ & EF Yancey \\
\hline 6. Develop listing and description of program databases. & 6/01/96 & EF Yancey \\
\hline $\begin{array}{l}\text { 7. Verify the accuracy of property assignments and correct } \\
\text { any deficiencies. }\end{array}$ & $6 / 01 / 96$ & EF Yancey \\
\hline $\begin{array}{l}\text { 8. Identify space and equipment within program area and } \\
\text { facilities for use by the PHMC. }\end{array}$ & 6/01/96 & EF Yancey \\
\hline 4 Post-Award Actions: & & \\
\hline $\begin{array}{l}\text { 1. Provide the PHMC access to the key program } \\
\text { documentation, listing of POCs, and listing of } \\
\text { subcontracts. Provide copies upon request. }\end{array}$ & prior to $6 / 15 / 96$ & EF Yancey \\
\hline $\begin{array}{l}\text { 2. PHMC identify space and equipment needs within } \\
\text { program/project area and facilities. }\end{array}$ & by $6 / 08 / 96$ & PHMC \\
\hline $\begin{array}{l}\text { 3. Program facility space and services available for use by } \\
\text { the PHMC. }\end{array}$ & $6 / 15 / 96$ & EF Yancey \\
\hline 4. Provide program briefing(s) to the PHMC. & $6 / 15 / 96-7 / 01 / 96$ & EF Yancey \\
\hline 5. Provide program level tour to the PHMC. & $6 / 15 / 96-7 / 01 / 96$ & EF Yancey \\
\hline $\begin{array}{l}\text { 6. Perform a joint program level property and equipment } \\
\text { inventory with PHMC personnel. }\end{array}$ & by $8 / 30 / 96$ & EF Yancey \\
\hline
\end{tabular}

\subsubsection{Calibration and Engineering Laboratories}

5.2.2.1 Engineering Laboratories. The objective of the Engineering Laboratories (EL) is to be the supplier of choice for EL services for development, assembly, testing, and evaluation to support the resolution of WHC, Hanford, and DOE complex-wide engineering issues for which we have a unique experience or capability and are competitively priced. Presently (March 1996), the ELs are staffed with 34 exempt technical staff and 30 nonexempt staff. The EL organizations operate under a laboratory oriented $\mathrm{COO}$, operate two major testing facilities and offer use of their services and facilities at competitive rates, 
approximately $\$ 90 / \mathrm{hr}$ exempt, and $\$ 55 / \mathrm{hr}$ non-exempt; no additional charge is made for use of the facilities and its resources if EL labor is used for a job.

The ELs provide "one-of-a-kind" in-house prototype process and system development or multiple unit fabrication outsourcing experience and skills, model/test unit and pilot plant design/fabrication capability to determine limitations/operability and ALARA impact of new designs. Acceptance test procedure (ATP) testing capability of first unit production, both inhouse or outsourced, to assure operability, before introduction into a contaminated environment where the item may be lost if it does not work as designed.

Operator/Maintenance start-up training on mock-up unit prior to permanent installation is also provided for customers. EL staff have the training and practical experience to prepare fabrication specifications and offsite work job surveillance.

The ELs have an efficient record of responding to emergency field problems, e.g., recent 101-SY support and providing rapid turn-around design, machining, assembly, testing, debugging, redesign/alteration, process modification and analyses, mock-up testing, and operator training capability for either the PHMC.

\begin{tabular}{|l|c|c|}
\hline & Scheduled & Date \\
\hline $\begin{array}{l}\text { 1. Provide an EL business review briefing to the PHMC major } \\
\text { subcontractors responsible for the major programmatic efforts } \\
\text { onsite. This would include TWRS, SNF and transition projects. }\end{array}$ & $7 / 26 / 96$ & LC Brown \\
\hline $\begin{array}{l}\text { 2. Tour PHMC through ELs for their understanding of capabilities } \\
\text { as part of their FY 1997 "make-buy" decision evaiuation. }\end{array}$ & $8 / 23 / 96$ & DG Panther \\
\hline
\end{tabular}

5.2.2.2 Calibration Laboratory (AKA Standards Laboratory). The objective of the Standards Laboratory (SL) is to manage the Site's centralized Calibration and Repair Program to ensure safe, cost efficient and responsive services in support of all Hanford mission areas that will assure the necessary data quality and tractability for the Site's highly regulated environment.

The Standards Laboratory operates using a Self-Liquidating Pool concept. Funding sources are provided through users, approximately 500 plus customers annually, of the calibration program. Calibration services also extends to all other RL contractors and a few DOE approved outside groups. The Standards Laboratory provides physical and electrical calibration services directly traceable to the National Institute of Standards and Technology (NIST). The laboratory also provides pick-up and delivery, recall and records services to assist customers ensure their equipment is ready and able to provide accurate measurements and the necessary data quality traceability. 


\begin{tabular}{|c|c|c|}
\hline Poet - werd/lotions & $\begin{array}{l}\text { Schediled } \\
\text { Date }\end{array}$ & Actiones $(6)$ \\
\hline $\begin{array}{l}\text { 1. Provide a SL business review briefing to the PHMC major } \\
\text { subcontractors responsible for the major programmatic efforts } \\
\text { onsite for their understanding of capabilities as part of their FY } \\
1997 \text { "make-buy" decision evaluation. }\end{array}$ & $7 / 26 / 96$ & JC Krogness \\
\hline
\end{tabular}

\subsection{INFORMATION AND COMMUNICATION}

Information and Communication includes the workscope currently performed by BCSR, as well as two areas performed in WHC.

BCSR provides RL and the Hanford Site contractors the ability to generate, store, access, and communicate information quickly, reliably, and cost effectively. BCSR plans, implements, and operates electronic communication, computing information management systems that enable effective operation of the Hanford Site.

The Transition Plan is compiled by major processes within BCSR (Sections 5.3.1 through 5.3.7), and the WHC areas (Sections 5.3.8 and 5.3.9). To simplify the plan, several of the smaller processes or organizations were consolidated into Section 5.3.3, "President's Office/Business Management/Technical Staff." A brief description of each area precedes the pre- and post-award actions. The primary pre-award deliverable is a baseline document for each area that fully documents the condition of the area as of May 1996.

\subsubsection{Operations and Support}

Boeing Operations and Support operates, maintains, and supports centralized and distributed computing and network infrastructure at Hanford.

\begin{tabular}{|c|c|c|}
\hline Pre-Award Actions & $\begin{array}{l}\text { Scheduled } \\
\text { Date }\end{array}$ & Actionee (s) \\
\hline $\begin{array}{l}\text { 1. Complete a baseline summary as defined by the outline in 5.3.7. } \\
\text { Prepare copies and briefing material. }\end{array}$ & $5 / 31 / 96$ & SA Estey \\
\hline 2. Conduct 5S. & $5 / 31 / 96$ & SA Estey \\
\hline $\begin{array}{l}\text { 3. Conduct and reconcile an inventory of all assigned property } \\
\text { including software. }\end{array}$ & $5 / 31 / 96$ & SA Estey \\
\hline 4. Consolidate enterprise server implementation. & $5 / 31 / 96$ & SA Estey \\
\hline $\begin{array}{l}\text { 5. Develop a plan for PHMC transition support including } \\
\text { computers and telephones. }\end{array}$ & $5 / 31 / 96$ & SA Estey \\
\hline Post-Award Actions & & \\
\hline 1. Applicable policies/procedures selected and in place. & 9/30/96 & SA Estey \\
\hline
\end{tabular}




\begin{tabular}{|c|c|c|}
\hline Post-Award /ctions & sonedipd & fetophes) \\
\hline 2. Identify performance measures for FY 1997. & $9 / 30 / 96$ & SA Estey \\
\hline 3. Finalize closeout agreements. & $9 / 30 / 96$ & SA Estey \\
\hline 4. Finalize scope assignments. & $8 / 01 / 96$ & SA Estey \\
\hline 5. Monitor contract performance during transition. & ongoing & SA Estey \\
\hline $\begin{array}{l}\text { 6. Finalize Memorandum of Understanding for services with other } \\
\text { contractors. }\end{array}$ & $9 / 30 / 96$ & SA Estey \\
\hline 7. Assign software licenses. & $9 / 30 / 96$ & SA Estey \\
\hline 8. Assign hardware/software maintenance agreements. & $9 / 30 / 96$ & SA Estey \\
\hline
\end{tabular}

\subsubsection{Information and Scientific Systems}

Information and Scientific Systems (ISS) provides planning, development, maintenance, and enhancement of automated information systems. It provides dedicated mainframe and microcomputer software applications support to programmatic, functional overhead, and general/administrative activities across the Hanford Site, as well as support to several identified national programs, using a wide range of hardware and software technologies.

\begin{tabular}{|c|c|c|}
\hline Pre-Awerd Actions & $\begin{array}{l}\text { Scheduled } \\
\text { Date }\end{array}$ & Actionee(s) \\
\hline $\begin{array}{l}\text { 1. Complete a baseline cummary a defined by the outline in 5.3.7. } \\
\text { Prepare copies and briefing material. }\end{array}$ & $5 / 31 / 96$ & RL Larson \\
\hline 2. Conduct $5 S$. & $5 / 31 / 96$ & RL Lareon \\
\hline $\begin{array}{l}\text { 3. Conduct and reconcile an iavenory of all assigned property } \\
\text { including software. }\end{array}$ & $5 / 31 / 96$ & RL Larson \\
\hline Pon-Awerd Actions & & \\
\hline 1. Applicable policies prinedure - lected and in place. & $9 / 30 / 96$ & RL Larson \\
\hline 2. Select systems for FY 1997 and identify support plan. & $7 / 01 / 96$ & RL Larson \\
\hline 3. Identify performance measures for FY 1997. & $9 / 30 / 96$ & RL Larson \\
\hline 4. Finalize closeout agreements. & $9 / 30 / 96$ & RL Larson \\
\hline 5. Finalize scope assignments. & $8 / 01 / 96$ & RL Larson \\
\hline 6. Monitor contract performance during transition. & ongoing & RL Larson \\
\hline 7. Finalize MOU for services with other contractors. & $9 / 30 / 96$ & RL Larson \\
\hline 8. Complete a plan for year-end closing and support. & $8 / 01 / 96$ & RL Larson \\
\hline
\end{tabular}




\subsubsection{President's Office/Technical Staff/Business Management}

The President's Office includes the BCSR President and Chief Information Officer (CIO), a small support staff, and Business Area Managers, acting as CIO's for the major WHC programs.

The Business Area Managers are members of the senior staff responsible for providing information management, planning, and integration services within assigned business areas. Business Area Managers focus on meeting the mission needs for effective electronic communication, computing, and information system capabilities.

Business Management's processes span the entire BCSR organization, providing centralized contract management, budget and pricing support, administrative liaison among contracting organizations, continuous quality improvement, and program planning and control functions.

\begin{tabular}{|c|c|c|}
\hline Pre-Award Actions & $\begin{array}{l}\text { Scheduled, } \\
\text { Date }\end{array}$ & Actiones $(s)$ \\
\hline $\begin{array}{l}\text { 1. Complete a baseline summary as defined by the outline in } 5.3 .7 \text {. } \\
\text { Prepare copies and briefing material. }\end{array}$ & $5 / 31 / 96$ & JO Perkins \\
\hline 2. Conduct 5 S. & $5 / 31 / 96$ & JO Perkins \\
\hline $\begin{array}{l}\text { 3. Conduct and reconcile an inventory of all assigned property } \\
\text { including software. }\end{array}$ & $5 / 31 / 96$ & JO Perkins \\
\hline (1) Pon-Awerd Actions & & \\
\hline 1. Submit SSPP. & $9 / 01 / 96$ & Jo Perkins \\
\hline 2. Issue preliminary rates for FY 1907 . & $7 / 10 / 96$ & JO Perkins \\
\hline 3. Issue final rates for FY 1097 . & $9 / 01 / 96$ & JO Perkins \\
\hline 4. Applicable policies/proceduren mincted and in place. & $9 / 30 / 96$ & JO Perkins \\
\hline 5. Identify performance menure & $9 / 30 / 96$ & JO Perkins \\
\hline 6. Finalize closeout agreemans & $9 / 30 / 96$ & JO Perkins \\
\hline 7. Identify a closeout manmerer & $8 / 01 / 96$ & CM Riddell \\
\hline 8. Finalize scope assignmenus. & $8 / 01 / 96$ & Jo Perkins \\
\hline 9. Monitor contract performance during transition. & ongoing & JO Perkins \\
\hline 10. Finalize MOU for services with otber contractors. & $9 / 30 / 96$ & JO Perkins \\
\hline
\end{tabular}

\subsubsection{Documentation and Records Management}

Establish a Records Management and Document Control Program that provides document receipt, release, clearance, and distribution; records identification, inventory, scheduling, transfer, collection, indexing, and disposition; vault storage; classified and 
unclassified document control; site forms administration and design; special project files management; database development and management; oversight of technology development and integration for electronic records and documents; and company policy, plans, and procedure oversight to ensure company and agency level compliance with all requirements (National Archives and Records Administration, DOE, Ecology, State of Washington, TriParty Agreement) governing the records management and document control program at Hanford.

\begin{tabular}{|c|c|c|}
\hline Pre-Avrard Actions & Sehediled & setionos $(0)$ \\
\hline $\begin{array}{l}\text { 1. Complete a baseline summary as defined by the outline in 5.3.7. } \\
\text { Prepare copies and briefing material. }\end{array}$ & $5 / 31 / 96$ & CN Potter \\
\hline 2. Conduct 5 S. & $5 / 31 / 96$ & CN Potter \\
\hline 3. Conduct and reconcile a classified document inventory. & TBD & CN Potter \\
\hline $\begin{array}{l}\text { 4. Conduct and reconcile an inventory of all assigned property } \\
\text { including software. }\end{array}$ & $5 / 31 / 96$ & CN Potter \\
\hline (ै) Post-Award Actions & & \\
\hline 1. Applicable policies/procedures selected and in place. & $9 / 30 / 96$ & CN Potter \\
\hline 2. Identify performance measures for FY 1997. & $9 / 30 / 96$ & CN Potter \\
\hline 3. Finalize closeout agreements. & $9 / 30 / 96$ & CN Potter \\
\hline 4. Finalize scope assignments. & $8 / 01 / 96$ & CN Potter \\
\hline 5. Monitor contract performance during transition. & ongoing & CN Potter \\
\hline 6. Finalize MOU for services with other contractors. & $9 / 30 / 96$ & CN Potter \\
\hline
\end{tabular}

\subsubsection{Media Management Systems}

Media Management Systems (MMS) offers multimedia solutions through integrated product development of computer-generated media products to meet the communication needs of the cleanup mission.

MMS develops, manages, and operates delivery systems using various media, including photographs, text, art, video, and sound. MMS also provides computer-generated, fully integrated information support and communication products and services, including technical illustration and graphic design, three-dimensional design, exhibit and display 
design, still photography, video production, technical writing and editing, custom photographic processing, digital imaging, macro writing, desktop publishing, internet publishing, and document analysis and data set tagging using Standard Generalized Markup Language (SGML).

MMS process analysis and automated work flow save time, money, and resources, and provide easy access to, and use of, information. MMS is helping today's users manage information for tomorrow.

\begin{tabular}{|c|c|c|}
\hline Pre-Award Actions & $\begin{array}{l}\text { Scheduled } \\
\text { Date }\end{array}$ & Actiono: \\
\hline $\begin{array}{l}\text { 1. Complete a baseline summary as defined by the outline in 5.3.7. } \\
\text { Prepare copies and briefing material. }\end{array}$ & $5 / 31 / 96$ & NE Kenny \\
\hline 2. Conduct 5 S. & $5 / 31 / 96$ & NE Kenny \\
\hline $\begin{array}{l}\text { 3. Conduct and reconcile an inventory of all assigned property } \\
\text { including software. }\end{array}$ & $5 / 31 / 96$ & NE Kenny \\
\hline $\begin{array}{l}\text { 4. Identify and inventory all physical and electronic information } \\
\text { repositories. }\end{array}$ & $5 / 31 / 96$ & NE Kenny \\
\hline Post-Award Actions & & \\
\hline 1. Applicable policies/procedures selected and in place. & $9 / 30 / 96$ & NE Kenny \\
\hline 2. Identify performance measures for FY 1997. & 9/30/96 & NE Kenny \\
\hline 3. Finalize closeout agreements. & 9/30/96 & NE Kenny \\
\hline 4. Finalize scope assignments. & $8 / 01 / 96$ & NE Kenny \\
\hline 5. Monitor contract performance during transition. & ongoing & NE Kenny \\
\hline 6. Finalize MOU for services with other contractors. & 9/30/96 & NE Kenny \\
\hline $\begin{array}{l}\text { 7. Identify redundant manuals, guides, and procedures that need to } \\
\text { be consolidated/integrated. }\end{array}$ & $9 / 30 / 96$ & NE Kenny \\
\hline $\begin{array}{l}\text { 8. Establish plan for printing/distribution of information with } \\
\text { company logo; i.e., letterhead, forms, document cover/title } \\
\text { pages, etc. }\end{array}$ & 9/30/96 & NE Kenny \\
\hline
\end{tabular}




\subsubsection{Telecommunications and Network Engineering}

The Telecommunications and Network Engineering Process plans, evaluates, designs, integrates, implements, and upgrades voice, video, data network, radio and computer systems. This process is responsible for technology infusion and upgrades to (1) Physical Infrastructure (i.e., inside and outside cable plant, facilities (building, electrical, UPS), site fiber infrastructure grid, and GIS formatted database), (2) Operation and Control Systems (i.e., logical and physical drawings, cable assignment systems, software libraries, billing/usage tracking systems, monitoring systems, and asset management systems), and (3) Logical Systems (i.e., electronics - bridges, routers, concentrators, microwave, PAX, paging radio base stations; operating software - MS NTAS, WFW, OS2/Lanman, protocols, name services, domain controllers; client/server software services - MS SQL SVR, Oracle, Sybase SQL SVR; applications [i.e., voice mail, electronic mail, alpha numeric paging, software distribution, office automation applications]). This process additionally provides technical oversight of third party service-provider contracts, ADP and Telecommunications Information and Work Flow Management.

\begin{tabular}{|c|c|c|}
\hline Pre-Award Actions & $\begin{array}{l}\text { Scheduled } \\
\text { Date }\end{array}$ & Actionee(s) \\
\hline $\begin{array}{l}\text { 1. Complete a baseline summary as defined by the outline in 5.3.7. } \\
\text { Prepare copies and briefing material. }\end{array}$ & $5 / 31 / 96$ & RE Harding \\
\hline 2. Conduct $5 \mathrm{~s}$. & $5 / 31 / 96$ & RE Harding \\
\hline $\begin{array}{l}\text { 3. Conduct and reconcile an inventory of all assigned property } \\
\text { including software. }\end{array}$ & $5 / 31 / 96$ & RE Harding \\
\hline Post-Award Actions & & s. \\
\hline 1. Applicable policies/procedures selected and in place. & $9 / 30 / 96$ & RE Harding \\
\hline 2. Identify performance measures for FY 1997. & 9/30/96 & RE Harding \\
\hline 3. Finalize closeout agreements. & 9/30/96 & RE Harding \\
\hline 4. Finalize scope assignments. & $8 / 01 / 96$ & RE Harding \\
\hline 5. Monitor contract performance during transition. & ongoing & RE Harding \\
\hline 6. Finalize MOU for services with other contractors. & 9/30/96 & RE Harding \\
\hline
\end{tabular}

\subsubsection{Baseline Summary Outline}

The following outline will be used for preparation of baseline summaries called for under each pre-award item $\# 1$ :

\subsection{Program Guidance and Direction}

1.1 Workscope Description

1.2 Performance Standards (Service/Commitment Levels)

1.3 Applicable DOE Orders, Directives, Letters, other

1.4 Identification of open Audit or other non-compliance items (HATS) 
2.0 Program/Operations Management Systems

2.1 Automated Systems (hardware/software/applications)

2.2 Current Projects

2.3 Status of Acquisitions

2.4 Liquidation Methods

2.5 Budgets/Cost

3.0 Organization and Personnel

3.1 Workforce Characterization

3.2 EEO Statistics

4.0 Resource Management

4.1 Equipment

4.2 Facilities

5.0 Interfaces

5.1 RL Contacts

5.2 WHC Contacts - Monitors, customers

5.3 Other Contractors, others

5.4 Memorandums of Understanding

6.0 Reporting Requirements

6.1 WHC

$6.2 \mathrm{RL}$

6.3 Other

7.0 Environmental, Safety and Health

7.1 Safety Program and Performance

7.2 Hazardous Waste Streams

7.3 Work Restrictions

8.0 Quality Assurance (if applicable)

8.1 Description and Implications

9.0 Safeguards and Security (if applicable)

9.1 Security Program and Performance

9.2 Classified Information

\subsubsection{Hanford Information System Inventory}

The Hanford Information System Inventory (HISI) is a valuable tool that provides a catalog of data about the information systems used at the Hanford Site. Registration of all Hanford systems in HISI is required by RL. HISI helps avoid redundancy of applications, and is a tool that can be used to help define the site information architecture. 


\begin{tabular}{|c|c|c|}
\hline Pre-Award Actinns & Sohediled & Actionee $(\mathrm{s})$ \\
\hline $\begin{array}{l}\text { 1. Update HISI data on key systems. Verify key systems with RL } \\
\text { POC. }\end{array}$ & $5 / 20 / 96$ & $\begin{array}{l}\text { MK Britton } \\
\text { JM Knoll, Jr. }\end{array}$ \\
\hline $\begin{array}{l}\text { 2. Publish HSI System Document, documenting system design and } \\
\text { processes. }\end{array}$ & $4 / 22 / 96$ & $\begin{array}{l}\text { MK Britton } \\
\text { JM Knoll, Jr. }\end{array}$ \\
\hline $\begin{array}{l}\text { 3. Develop status of action items from the Hanford Information } \\
\text { System Inventory Analysis of Potentially Duplicate Functionality. }\end{array}$ & $5 / 20 / 96$ & $\begin{array}{l}\text { MK Britton } \\
\text { JM Knoll, Jr. }\end{array}$ \\
\hline Post-Award Actions & & \\
\hline $\begin{array}{l}\text { 1. Conduct a presentation covering the HISI database and process, } \\
\text { completed and open actions from the duplicate system study. }\end{array}$ & $9 / 30 / 96$ & $\begin{array}{l}\text { MR Britton } \\
\text { JM Knoll, Jr. }\end{array}$ \\
\hline $\begin{array}{l}\text { 2. Provide listing of all registered systems in HISI, including list of } \\
\text { key systems. }\end{array}$ & $6 / 15 / 96$ & $? ?$ \\
\hline
\end{tabular}

\subsubsection{Data Management and Standards}

Data Management guidance and Data Standards facilitate integration and help provide consistency in data usage among systems, functions and contractors. Data Management guidance is provided through strategic and operational tools and resources. RL requires compliance by all contractors to the Data Standards listed in the Data Standards Library.

\begin{tabular}{|l|c|l|}
\hline \multicolumn{1}{|c|}{ Pre-Award Actions } & $\begin{array}{c}\text { Scheduled } \\
\text { Date }\end{array}$ & Actionee(s) \\
\hline 1. Update the Data Standards Library. & $5 / 20 / 96$ & $\begin{array}{l}\text { MK Britton } \\
\text { JM Knoll, Jr. }\end{array}$ \\
\hline $\begin{array}{l}\text { 2. Develop a presentation describing Data Management concepts } \\
\text { and resources. }\end{array}$ & $5 / 20 / 96$ & $\begin{array}{l}\text { MK Britton } \\
\text { JM Knoll, Jr. }\end{array}$ \\
\hline $\begin{array}{l}\text { 1. Conduct a presentation covering the Data Standards process and } \\
\text { Library. }\end{array}$ & $7 / 15 / 96$ & $\begin{array}{l}\text { MR Britton } \\
\text { JM Knoll, Jr. }\end{array}$ \\
\hline $\begin{array}{l}\text { 2. Conduct a presentation covering Data Management concepts and } \\
\text { resources. }\end{array}$ & $7 / 15 / 96$ & $\begin{array}{l}\text { MK Britton } \\
\text { JM Knoll, Jr. }\end{array}$ \\
\hline
\end{tabular}


WHC-SP-1181

This page intentionally left blank. 


\subsection{OTHER CROSSCUTTING SERVICES}

\subsection{SAFEGUARDS AND SECURITY}

Safeguards and Security (SAS) maintains a standardized Hanford Site security program consistent with current DOE Orders, RL's Directives and company security policies and procedures. SAS program elements are designed to:

- $\quad$ Physically protect special nuclear material (SNM), classified matter, U.S. Government property, and personnel located within the confines of the Hanford Site and leased facilities.

- Maintain an armed protective force capable of responding to security alarms and incidents throughout the Site.

- Develop, maintain, and implement policies and procedures governing the use, control, and accountability of nuclear materials.

- Provide guidance, training, and procedures to ensure proper protection is afforded to classified matter and sensitive unclassified information which is processed, stored, transmitted, or discussed throughout the Site.

- Manage the Classification Program to assure the identification and proper classification of information, documents, or material.

- Manage the Declassification Project to meet the requirements of the RL LargeScale Declassification Review Plan.

- Provide technical and analytical services which assist DOE in establishing and maintaining uniform and cost-effective safeguards and security programs.

- Coordinate and implement new security programs, systems, and upgrades for newly-constructed and existing facilities in order to protect Hanford interests.

- Provide protection program planning, evaluation, and validation testing to ensure that levels of protection and risk are appropriate to Site security interests that affect the protection of SNM and the health and safety of the public, onsite employees, and the environment.

- Develop, implement, and maintain policies and procedures governing the use and operation of government vehicles, roadways, and pedestrian walkways onsite in compliance with DOE Orders and Federal regulations. 


\begin{tabular}{|c|c|c|}
\hline Pre-Award Actions & sonedinled & Hetionee( $)$ \\
\hline $\begin{array}{l}\text { 1. Using the pre-existing checklist, compile pre-existing } \\
\text { condition information on the facility and/or major equipment. } \\
\text { Conduct, or schedule as a separate post-award action(s), any } \\
\text { assessments required to adequately address all relevant items } \\
\text { on the checklist. Provide completed checklist to RL POC for } \\
\text { review. }\end{array}$ & $6 / 01 / 96$ & VG Heiman \\
\hline $\begin{array}{l}\text { 2. Prepare SAS mission briefing to include manpower, budget } \\
\text { and organizational roles. }\end{array}$ & $5 / 15 / 96$ & VG Heiman \\
\hline $\begin{array}{l}\text { 3. Prepare copy of SAS Training Plan to include Training } \\
\text { Matrix. }\end{array}$ & $5 / 15 / 96$ & VG Heiman \\
\hline $\begin{array}{l}\text { 4. Prepare list of key SAS documents such as DOE Orders, } \\
\text { Manuals, and RIIDs. }\end{array}$ & $5 / 05 / 96$ & VG Heiman \\
\hline \multicolumn{3}{|l|}{ Post-Award Actions } \\
\hline 1. Conduct comprehensive SAS overview and mission briefing. & $6 / 13 / 96$ & VG Heiman \\
\hline 2. Provide copies of assembled documents. & $6 / 13 / 96$ & VG Heiman \\
\hline $\begin{array}{l}\text { 3. Identify space and make ready a functional office within } \\
\text { existing facilities for the PHMC. }\end{array}$ & $6 / 13 / 96$ & VG Heiman \\
\hline $\begin{array}{l}\text { 4. Verify accuracy of various facility, equipment, and vehicle } \\
\text { inventories. }\end{array}$ & $6 / 13 / 96$ & VG Heiman \\
\hline 5. Provide list of key SAS documents. & $6 / 13 / 96$ & VG Heiman \\
\hline
\end{tabular}

\subsubsection{Security Operations}

\begin{tabular}{|c|c|c|}
\hline Pre-Awari Actions & $\begin{array}{l}\text { Scheduled } \\
\text { Date }\end{array}$ & Sctionse(s) \\
\hline $\begin{array}{l}\text { 1. Collect listing and locations of all security areas (SCIF, PPA, } \\
\text { LA, PA) as well as access control requirements. }\end{array}$ & $5 / 15 / 96$ & $\begin{array}{l}\text { D Haskins } \\
\text { VG Heiman }\end{array}$ \\
\hline $\begin{array}{l}\text { 2. Collect a sample copy of the Asset Protection Agreement and } \\
\text { a list of all high, medium, and low asset protection facilities } \\
\text { with a schedule of asset protection surveys. }\end{array}$ & $5 / 15 / 96$ & $\begin{array}{l}\text { D Haskins } \\
\text { VG Heiman }\end{array}$ \\
\hline $\begin{array}{l}\text { 3. Collect list of ongoing or planned security projects or } \\
\text { upgrades. }\end{array}$ & $5 / 15 / 96$ & $\begin{array}{l}\text { D Haskins } \\
\text { VG Heiman }\end{array}$ \\
\hline $\begin{array}{l}\text { 4. Prepare a list of key SAS documents such as Controlled } \\
\text { Manuals, internal procedures, and MOUs. }\end{array}$ & $5 / 15 / 96$ & $\begin{array}{l}\text { D Haskins } \\
\text { VG Heiman }\end{array}$ \\
\hline (ै. + Post-Award Actions & & \\
\hline 1. Conduct comprehensive briefing of SAS operations. & $6 / 13 / 96$ & $\begin{array}{l}\text { D Haskins } \\
\text { VG Heiman }\end{array}$ \\
\hline 2. Conduct tours of SAS areas. & $6 / 13 / 96$ & $\begin{array}{l}\text { D Haskins } \\
\text { VG Heiman }\end{array}$ \\
\hline
\end{tabular}




\begin{tabular}{|c|c|c|c|}
\hline & rost-Amard hetions & senedired & hetionee ( ) \\
\hline & Provide copies of key SAS documents. & $6 / 13 / 96$ & $\begin{array}{l}\text { D Haskins } \\
\text { VG Heiman }\end{array}$ \\
\hline 4. & $\begin{array}{l}\text { Provide special briefing of security investigations to include } \\
\text { SEMS data base and FY } 1996 \text { quarterly trends. }\end{array}$ & $6 / 13 / 96$ & $\begin{array}{l}\text { D Haskins } \\
\text { VG Heiman }\end{array}$ \\
\hline
\end{tabular}

\subsubsection{Protective Forces}

\begin{tabular}{|c|c|c|}
\hline Pro-Award Actions & $\begin{array}{l}\text { Sohedinled: } \\
\text { Date }\end{array}$ & Letionee (6) \\
\hline $\begin{array}{l}\text { 1. Provide copies of Patrol Training Plans to include initial, } \\
\text { recurring, firearms, Special Response Team and Field } \\
\text { Training Officer operations. }\end{array}$ & $5 / 15 / 96$ & G McDowell \\
\hline $\begin{array}{l}\text { 2. Prepare briefing on contingency plans, weapons and } \\
\text { equipment, and organization. }\end{array}$ & $5 / 15 / 96$ & G McDowell \\
\hline $\begin{array}{l}\text { 3. Prepare a list of post orders and prepare tours of all posts } \\
\text { and patrols. }\end{array}$ & $5 / 15 / 96$ & G McDowell \\
\hline 4. Prepare briefing on explosive detector dog program. & $5 / 15 / 96$ & G McDowell \\
\hline $\begin{array}{l}\text { 5. Prepare inventory of unique protective force equipment such } \\
\text { as weapons, ammunition, weht vision devices, } \\
\text { training/exercise aids, etc. }\end{array}$ & $5 / 15 / 96$ & G McDowell \\
\hline $\begin{array}{l}\text { 6. Prepare listing of certified perwonnel such as instructors, } \\
\text { armorers, SPOII, etc. }\end{array}$ & $5 / 15 / 96$ & G McDowell \\
\hline Post-Awer Actions & & \\
\hline $\begin{array}{l}\text { 1. Prepare briefing and coun on operation and capability of the } \\
\text { Patrol Operations Center, Petrot Traning Academy, and } \\
\text { Central Alarm Station. }\end{array}$ & $6 / 13 / 96$ & G McDowell \\
\hline $\begin{array}{l}\text { 2. Conduct comprehenuve bre fiac os protective force } \\
\text { operations and capahilition }\end{array}$ & $6 / 20 / 96$ & G McDowell \\
\hline 3. Conduct tours of all Patrol operauns. & $6 / 20 / 96$ & G McDowell \\
\hline 4. Provide list of all key protecure force documents. & $6 / 20 / 96$ & G McDowell \\
\hline $\begin{array}{l}\text { 5. Facilitate introductions and tour of Benton County Sheriff's } \\
\text { Office substation. }\end{array}$ & $6 / 20 / 96$ & G McDowell \\
\hline
\end{tabular}

\subsubsection{Information Security}

\begin{tabular}{|c|c|c|}
\hline Pre-Award Actions & Scheduled & Actionet $(\mathrm{s})$ \\
\hline $\begin{array}{l}\text { 1. Prepare listing and location of all repositories for classified } \\
\text { information to include names of custodians. }\end{array}$ & $5 / 15 / 96$ & I Stowe \\
\hline
\end{tabular}




\begin{tabular}{|c|c|c|}
\hline +Pre-4ward Alations & Salediled & Nenplese $(1)$ \\
\hline $\begin{array}{l}\text { 2. Prepare listing of special position appointments such as } \\
\text { Classification Officer, Authorized Derivative Classifiers and } \\
\text { Declassifiers, Computer Security Site Manager, Computer } \\
\text { System Security Officer, Computer Protection Program } \\
\text { Manager, and Classified Matter Protection and Control } \\
\text { Coordinator, COMSEC, TSCM, TEMPEST, AND PDS. }\end{array}$ & $5 / 15 / 96$ & J Stowe \\
\hline $\begin{array}{l}\text { 3. Prepare for transfer of classified holdings to the new } \\
\text { contractor. }\end{array}$ & $5 / 15 / 96$ & J Stowe \\
\hline $\begin{array}{l}\text { 4. Prepare for certification of possession or non-possession of } \\
\text { classified material. }\end{array}$ & $5 / 15 / 96$ & J Stowe \\
\hline $\begin{array}{l}\text { 5. Establish an agreement with RL to transfer the Large Scale } \\
\text { Declassification Review project to the PHMC. }\end{array}$ & $5 / 31 / 96$ & J Stowe \\
\hline Post-Award Actions & & \\
\hline $\begin{array}{l}\text { 1. Conduct comprehensive briefing of Information Security } \\
\text { activities. }\end{array}$ & $6 / 13 / 96$ & J Stowe \\
\hline 2. Conduct tour of Information Security Areas. & $6 / 13 / 96$ & J Stowe \\
\hline 3. Prepare certificates of possession and/or non-possession. & $9 / 30 / 96$ & J Stowe \\
\hline $\begin{array}{l}\text { 4. Provide guidance for facility inventories of classified } \\
\text { repositories and help complete transfer of classified } \\
\text { documents. }\end{array}$ & $9 / 30 / 96$ & J Stowe \\
\hline $\begin{array}{l}\text { 5. Conduct status briefing for } \mathrm{RL} \text { and the PHMC on the Large } \\
\text { Scale Declassification Review project and arrange for project } \\
\text { transfer. }\end{array}$ & $8 / 31 / 96$ & Stowe \\
\hline
\end{tabular}

\subsubsection{Personnel Security and Central Badging}

\begin{tabular}{|l|l|l|}
\hline $\begin{array}{l}\text { 1. Prepare for issuance of security badges for PHMC } \\
\text { employees. }\end{array}$ & $5 / 15 / 96$ & W Hawkins \\
\hline 2. Prepare security awareness briefings for PHMC employees. & $6 / 03 / 96$ & W Hawkins \\
\hline $\begin{array}{l}\text { 1. Initiate actions to facilitate security clearance processing for } \\
\text { PHMC employees. }\end{array}$ & $6 / 13 / 96$ & W Hawkins \\
\hline 2. Start issuance of site security badges to PHMC employees. & $6 / 03 / 96$ & W Hawkins \\
\hline $\begin{array}{l}\text { 3. Start providing security awareness briefings for PHMC } \\
\text { employees. }\end{array}$ & $6 / 03 / 96$ & W Hawkins \\
\hline $\begin{array}{l}\text { 4. Conduct comprehensive briefing and tour for personnel } \\
\text { security and badging operations. }\end{array}$ & $6 / 13 / 96$ & W Hawkins \\
\hline
\end{tabular}




\subsubsection{Protection Program Management}

\begin{tabular}{|c|c|c|}
\hline Pre-Award Actions & $\begin{array}{l}\text { Scheduled } \\
\text { Date }\end{array}$ & Actioneds) \\
\hline $\begin{array}{l}\text { 1. Prepare briefing on DOE Threat Guidance, Site Safeguards } \\
\text { and Security Plan, Vulnerability Assessment (VA) process } \\
\text { and schedule to include risk results for each area. }\end{array}$ & $5 / 15 / 96$ & S Udell \\
\hline $\begin{array}{l}\text { 2. Collect copy of last HQ DOE and RL/SAS audit and survey } \\
\text { to include corrective action plans. }\end{array}$ & $5 / 15 / 96$ & S UdelI \\
\hline $\begin{array}{l}\text { 3. Collect copies of most recent internal SAS survey reports to } \\
\text { include corrective action plans. }\end{array}$ & $5 / 15 / 96$ & S Udell \\
\hline 4. Collect copies of most recent MBA audit reports. & $5 / 15 / 96$ & S Udell \\
\hline $\begin{array}{l}\text { 5. Collect listing and schedule of performance tests and copies } \\
\text { of performance test reports. }\end{array}$ & $5 / 15 / 96$ & S Udell \\
\hline 6. Collect listing of all current SAS deviations. & $5 / 15 / 96$ & S Udell \\
\hline $\begin{array}{l}\text { 7. Collect copies of Site Safeguards and Security Plan and VA } \\
\text { reports. }\end{array}$ & $5 / 15 / 96$ & S Udell \\
\hline Post-A wrard Actions & & \\
\hline 1. Conduct comprehensive briefing of PPM activities. & $6 / 13 / 96$ & S Udell \\
\hline 2. Conduct tour of PPM areas. & $6 / 13 / 96$ & S Udell \\
\hline $\begin{array}{l}\text { 3. Provide access to key documents, to include classified } \\
\text { documents as appropriate (classified documents will require } \\
\text { appropriate protection, clearances, and dissemination). }\end{array}$ & $6 / 13 / 96$ & S Udell \\
\hline $\begin{array}{l}\text { 4. Conduct a risk assessment briefing for each area on the } \\
\text { existing risk levels and planned corrective/upgrade actions. }\end{array}$ & $6 / 13 / 96$ & S Udell \\
\hline $\begin{array}{l}\text { 5. Allow PHMC employees to observe surveys and performance } \\
\text { tests conducted during transition period. }\end{array}$ & 9/30/96 & S Udell \\
\hline
\end{tabular}

\subsubsection{Material Control and Accountability}

\begin{tabular}{|l|c|l|}
\hline \multicolumn{1}{|c|}{ Pre-Award Actions } & $\begin{array}{c}\text { Scheduled } \\
\text { Date }\end{array}$ & Actionee(s) \\
\hline $\begin{array}{l}\text { 1. Prepare draft of Nuclear Material Transfer Plan for RL } \\
\text { review. }\end{array}$ & $5 / 15 / 96$ & B Fitzpatrick \\
\hline 2. Prepare copy of Material Control and Accountability Plan. & $5 / 15 / 96$ & B Fitzpatrick \\
\hline $\begin{array}{l}\text { 3. Prepare listing of all Material Balance Areas to include } \\
\text { location, program, category of material, name of custodian } \\
\text { and MBA representative. }\end{array}$ & $5 / 15 / 96$ & B Fitzpatrick \\
\hline 4. Prepare schedule of MBA inventories for FY 1997. & $5 / 15 / 96$ & B Fitzpatrick \\
\hline $\begin{array}{l}\text { 1. Conduct comprehensive briefing on MC\&A activities to } \\
\text { include HANMAS and IAEA procedures. }\end{array}$ & $6 / 13 / 96$ & B Fitzpatrick \\
\hline
\end{tabular}




\begin{tabular}{|l|l|l|}
\hline \multicolumn{1}{|c|}{ Post-Award Actions } & $\begin{array}{l}\text { Scheduled } \\
\text { Date }\end{array}$ & retionee( \\
\hline 2. Conduct tours of MC\&A areas to include all MBAs. & $6 / 13 / 96$ & B Fitzpatrick \\
\hline 3. Provide copies of assembled documents. & $6 / 13 / 96$ & B Fitzpatrick \\
\hline $\begin{array}{l}\text { 4. Allow PHMC personnel to observe IAEA visits during the } \\
\text { transition period. }\end{array}$ & $9 / 30 / 96$ & B Fitzpatrick \\
\hline $\begin{array}{l}\text { 5. Conduct joint inventory and complete transfer of special } \\
\text { nuclear material. }\end{array}$ & $9 / 30 / 96$ & B Fitzpatrick \\
\hline
\end{tabular}

\subsubsection{Technical Security}

\begin{tabular}{|l|c|l|}
\hline & $\begin{array}{c}\text { Scheduled } \\
\text { Date }\end{array}$ & Actionee(s). \\
\hline 1. Prepare overview briefing of ongoing projects. & $5 / 15 / 96$ & D Palmer \\
\hline 2. Organize tour and appropriate demonstrations of projects. & $5 / 15 / 96$ & D Palmer \\
\hline Post-Award Actions & & \\
\hline $\begin{array}{l}\text { 1. Conduct overview briefing of ongoing projects and how they } \\
\text { are integrated in the Site protection programs. }\end{array}$ & $6 / 13 / 96$ & D Palmer \\
\hline $\begin{array}{l}\text { 2. Conduct tour and demonstrations of Technical Security } \\
\text { areas/projects. }\end{array}$ & $6 / 13 / 96$ & D Palmer \\
\hline
\end{tabular}

\subsection{HANFORD ANALYTICAL SERVICES PROGRAM}

The Hanford Analytical Services Program (HASP) will provide analytical, field support and process development services for the Hanford mission. HASP will manage and integrate onsite capabilities as well as commercially contracted services. Operations of onsite facilities will be organizationally independent from the management and evaluation function. HASP will oversee analytical facility operations to assure safe and effective use of resources and to assure cost effective services are provided. HASP will establish and maintain a Quality Assurance Program for all analytical services and provide sitewide integration of the Data Quality Objectives (DQO) process.

HASP consists of the following elements:

- Site Sample Management Office

- Including integration of all site contractors

- High-level analytical services

- Low-level analytical services. 


\subsubsection{Sample Management Office}

A centralized Hanford Sample Management Office (SMO) is required by DOE. The SMO will be responsible for developing site strategies for the Analytical Services Program, including managing site sampling and analytical needs and capabilities, consolidating commercial contracts, overseeing data quality and providing data management services. All Hanford contractors will be integrated under the SMO.

\begin{tabular}{|c|c|c|}
\hline Pre-Award Actions & $\begin{array}{l}\text { Scheduled } \\
\text { Date }\end{array}$ & Actionee(s) \\
\hline $\begin{array}{l}\text { 1. Isaue letter to contractors formalizing the Sample } \\
\text { Management Working Group and directing SMO } \\
\text { Consolidation. }\end{array}$ & $4 / 15 / 96$ & RP Carter \\
\hline $\begin{array}{l}\text { 2. Sample Management Working Group to recommend } \\
\text { Consolidation Strategy. }\end{array}$ & $5 / 10 / 96$ & CR Stroup \\
\hline $\begin{array}{l}\text { 3. Consolidation Strategy and pre-award actions approved } \\
\text { by RL. }\end{array}$ & $5 / 15 / 96$ & RP Carter \\
\hline $\begin{array}{l}\text { 4. Submit the SMO Consolidation Strategy to DOE-HQ } \\
\text { complying with } 2 / 29 / 96 \text { DOE-HQ directive. }\end{array}$ & $5 / 21 / 96$ & RP Carter \\
\hline $\begin{array}{l}\text { 5. Memoranda of Understanding or equivalent approved } \\
\text { by ERC and PNNL for SMO consolidation. }\end{array}$ & $5 / 15 / 96$ & RP Carter \\
\hline $\begin{array}{l}\text { 6. Develop a list of key Analytical Services Program } \\
\text { documentation. Provide to RL POC for review. }\end{array}$ & $5 / 15 / 96$ & AG King \\
\hline $\begin{array}{l}\text { 7. Assemble key Analytical Services Program } \\
\text { documentation and identify its location, for use by the } \\
\text { PHMC. Includes development of a Analytical Services } \\
\text { Program "Smart Book." }\end{array}$ & $6 / 01 / 96$ & AG King \\
\hline $\begin{array}{l}\text { 8. Prepare Analytical Services Program briefing for } \\
\text { presentation to PHMC (coordinate with RL POC.) }\end{array}$ & $6 / 01 / 96$ & CR Stroup \\
\hline $\begin{array}{l}\text { 9. Develop a listing of key Analytical Services Program } \\
\text { individuals as POCs. Include facility and operations } \\
\text { POC list. }\end{array}$ & $6 / 01 / 96$ & AG King \\
\hline $\begin{array}{l}\text { 10. Develop listing and description of Analytical Services } \\
\text { Program subcontracts, including facility subcontracts. } \\
\text { Include any subcontracts still planned to be executed } \\
\text { prior to contract takeover. }\end{array}$ & $6 / 01 / 96$ & JL Deichman \\
\hline $\begin{array}{l}\text { 11. Develop listing and description of Analytical Services } \\
\text { Program databases, including facility databases. }\end{array}$ & $6 / 01 / 96$ & SL Brey \\
\hline $\begin{array}{l}\text { 12. Verify the accuracy of property assignments at the } \\
\text { Analytical Services Program organizational level and } \\
\text { correct any deficiencies. }\end{array}$ & $6 / 01 / 96$ & AG King \\
\hline $\begin{array}{l}\text { 13. Identify space and equipment within the Analytical } \\
\text { Services Program area and facilities for use by the } \\
\text { PHMC. }\end{array}$ & $6 / 01 / 96$ & AG King \\
\hline
\end{tabular}




\begin{tabular}{|c|c|c|}
\hline Pre-Award Actions & Scheduled & Actionee(s) \\
\hline $\begin{array}{l}\text { 14. Using the pre-existing condition checklist, compile pre- } \\
\text { existing condition information on } 1706 \mathrm{KE} \text {. Conduct, or } \\
\text { schedule as a separate post-closure action, any } \\
\text { assessments required to adequately address all relevant } \\
\text { items on the checklist. Provide completed checklist to } \\
\text { RL POC for review. }\end{array}$ & $6 / 01 / 96$ & AG King \\
\hline Post-Award Actions & & \\
\hline $\begin{array}{l}\text { 1. Finalized SMO Transition and post-award actions } \\
\text { approved by PHMC and RL. }\end{array}$ & TBD & RP Carter \\
\hline $\begin{array}{l}\text { 2. Provide the PHMC access to the key Analytical } \\
\text { Services Program documentation, any program level } \\
\text { generated pre-existing conditions information, listing of } \\
\text { POCs, and listing of subcontracts. Provide copies } \\
\text { upon request. }\end{array}$ & prior to $6 / 15 / 96$ & AG King \\
\hline $\begin{array}{l}\text { 3. PHMC identify space and equipment needs within } \\
\text { Analytical Services Program area and facilities. }\end{array}$ & by $6 / 08 / 96$ & PHMC \\
\hline $\begin{array}{l}\text { 4. Analytical Services Program and facility space and } \\
\text { services available for use by the PHMC. }\end{array}$ & $6 / 15 / 96$ & AG King \\
\hline $\begin{array}{l}\text { 5. Provide Analytical Services Program briefing to the } \\
\text { PHMC. }\end{array}$ & $6 / 15 / 96-7 / 01 / 96$ & AG King \\
\hline $\begin{array}{l}\text { 6. Brief PHMC on the Hanford Analytical Services } \\
\text { Quality Systems. }\end{array}$ & $6 / 15 / 96-7 / 01 / 96$ & JE Hyatt \\
\hline $\begin{array}{l}\text { 7. Assist the PHMC in the conduct of a Pre-Existing } \\
\text { Condition Assessment for the } 1706 K E \text { Facility. }\end{array}$ & $7 / 15 / 96-8 / 30 / 96$ & AG King \\
\hline $\begin{array}{l}\text { 8. Brief appropriate regulatory agencies and establish } \\
\text { method for transition of laboratory } \\
\text { accreditations/certifications to PHMC. }\end{array}$ & TBD & RP Carter \\
\hline
\end{tabular}

\subsubsection{High Level Analytical Services}

The 222-S Laboratory and associated facilities provide high-activity analytical and development services. The 222-S Laboratory includes hot cells, gloveboxes shielded hoods for analysis of highly radioactive materials.

\begin{tabular}{|l|c|c|}
\hline \multicolumn{1}{|c|}{ Pre-Award Actions } & $\begin{array}{c}\text { Scheduled } \\
\text { Date }\end{array}$ & Actionee(s) \\
\hline $\begin{array}{l}\text { 1. Prepare 222-S Laboratory complex briefing for the } \\
\text { PHMC (coordinate with RL POC). }\end{array}$ & $6 / 01 / 96$ & AG King \\
\hline $\begin{array}{l}\text { 2. Identify key documents for the 222-S Laboratory } \\
\text { complex and review with RL. Provide list for } \\
\text { consolidation with the Analytical Services Program list. }\end{array}$ & $5 / 01 / 96$ & AG King \\
\hline
\end{tabular}




\begin{tabular}{|c|c|c|}
\hline Pre-Award Actions & $\begin{array}{l}\text { Scheduled } \\
\text { Date }\end{array}$ & Actionee (s) \\
\hline $\begin{array}{l}\text { 3. Assemble key documentation, or identify its location, } \\
\text { for use by the PHMC. Includes preparation of a } \\
222-\$ \text { Laboratory complex "Smart Book." }\end{array}$ & $6 / 01 / 96$ & AG King \\
\hline $\begin{array}{l}\text { 4. Identify POCs for the 222-S Laboratory complex and } \\
\text { provide for consolidation with the Analytical Services } \\
\text { Program list. }\end{array}$ & $5 / 17 / 96$ & AG King \\
\hline $\begin{array}{l}\text { 5. Develop a listing and description of subcontracts } \\
\text { supporting the 222-S Laboratory complex and } \\
\text { consolidate with the Analytical Services Program list. } \\
\text { Include any subcontracts planned to be executed prior } \\
\text { to contract takeover. }\end{array}$ & $5 / 15 / 96$ & JL Deichman \\
\hline $\begin{array}{l}\text { 6. Using the pre-existing condition checklist, compile pre- } \\
\text { existing condition information on the } 222-S \text { Laboratory } \\
\text { complex. Conduct, or schedule as a separate post- } \\
\text { award action(s), any assessments required to adequately } \\
\text { address all relevant items on the checklist. Provide } \\
\text { completed checklist to RL POC for review. }\end{array}$ & $6 / 01 / 96$ & GB Griffin \\
\hline $\begin{array}{l}\text { 7. Develop a list of open action items and commitments, } \\
\text { including status (Environmental, Tri-Party Agreement, } \\
\text { DNFSB as examples). Provide to RL POC for review. }\end{array}$ & $6 / 01 / 96$ & IL Deichman \\
\hline $\begin{array}{l}\text { 8. Develop a list of 222-S specific mandatory training and } \\
\text { qualification requirements for employees. }\end{array}$ & $6 / 01 / 96$ & AG King \\
\hline $\begin{array}{l}\text { 9. Develop a listing of information which constitutes the } \\
\text { "authorization basis" for the } 222-5 \text { Laboratory. }\end{array}$ & $6 / 01 / 96$ & AG King \\
\hline $\begin{array}{l}\text { 10. Develop a description of ongoing and planned } \\
\text { construction projects and upgrades at the } \\
222-5 \text { Laboratory complex. }\end{array}$ & $6 / 01 / 96$ & SL Brey \\
\hline $\begin{array}{l}\text { 11. Develop a listing of special agreement services } \\
\text { provided to RL, other DOE sites, and other entities. }\end{array}$ & $6 / 01 / 96$ & AG King \\
\hline $\begin{array}{l}\text { 12. Develop a listing and description of } 222-5 \text { Laboratory } \\
\text { complex databases. Include information on } \\
\text { LABCORE. Provide list to the Analytical Services } \\
\text { Program level for consolidation. }\end{array}$ & $5 / 15 / 96$ & SL Brey \\
\hline $\begin{array}{l}\text { 13. Develop a list of current work order services or other } \\
\text { form of agreement to provide work for other } \\
\text { organizations, on and offsite. }\end{array}$ & $6 / 01 / 96$ & JL Deichman \\
\hline $\begin{array}{l}\text { 14. Develop a list of current work order services or other } \\
\text { form of agreement to obtain work from other } \\
\text { organizations, on and offsite. }\end{array}$ & $6 / 01 / 96$ & JL Deichman \\
\hline $\begin{array}{l}\text { 15. Develop a list of permits governing the } \\
222-S \text { Laboratory complex, and provide a description } \\
\text { of each as to where and how it applies. }\end{array}$ & $6 / 01 / 96$ & MJ Hall \\
\hline $\begin{array}{l}\text { 16. Verify the accuracy of property assignments for the } \\
222-5 \text { Laboratory complex and correct any } \\
\text { deficiencies. }\end{array}$ & $6 / 01 / 96$ & AG King \\
\hline
\end{tabular}




\begin{tabular}{|c|c|c|}
\hline Pre-Award Actions & Scheduled & $1+t_{0}+n_{p},(s)$ \\
\hline $\begin{array}{l}\text { 17. Develop a comprehensive list of open-ended } \\
\text { compliance issues at the } 222-5 \text { Laboratory complex } \\
\text { (HATS as an example). Provide to RL POC for } \\
\text { review. }\end{array}$ & $5 / 01 / 96$ & AG King \\
\hline \multicolumn{3}{|l|}{ Post-Award Actions } \\
\hline $\begin{array}{l}\text { 1. Provide 222-S Laboratory complex briefing to the } \\
\text { PHMC. }\end{array}$ & $7 / 01 / 96-7 / 15 / 96$ & AG King \\
\hline $\begin{array}{l}\text { 2. Provide a } 2225 \text { Laboratory complex tour to the } \\
\text { PHMC. }\end{array}$ & $7 / 01 / 96-7 / 15 / 96$ & GB Griffin \\
\hline $\begin{array}{l}\text { 3. Provide the PHMC access to key documentation, pre- } \\
\text { existing condition information, commitment data, } \\
\text { inventory data, etc. (as established in the pre-award } \\
\text { phase). Provide copies upon request. }\end{array}$ & by $6 / 15 / 96$ & AG King \\
\hline $\begin{array}{l}\text { 4. Provide any additional documentation or information } \\
\text { requested by the PHMC, if available, that was not } \\
\text { identified during the pre-award phase. }\end{array}$ & by $7 / 01 / 96$ & AG King \\
\hline $\begin{array}{l}\text { 5. PHMC identify any recurring reports that they would } \\
\text { like to receive during transition. }\end{array}$ & by $7 / 15 / 96$ & PHMC \\
\hline 6. Provide necessary training to PHMC personnel. & $7 / 01 / 96-8 / 01 / 96$ & AG King \\
\hline $\begin{array}{l}\text { 7. Provide an updated status of ongoing work at the } \\
222 \text { S Laboratory complex prior to contract turnover. }\end{array}$ & by $9 / 30 / 96$ & JL Deichman \\
\hline $\begin{array}{l}\text { 8. Perform a joint property and equipment inventory of } \\
\text { the 222-S Laboratory complex with the PHMC. }\end{array}$ & by $8 / 30 / 96$ & AG King \\
\hline $\begin{array}{l}\text { 9. Provide a categorized inventory of wastes at the } \\
222-5 \text { Laboratory complex. }\end{array}$ & by $9 / 30 / 96$ & MJ Hall \\
\hline $\begin{array}{l}\text { 10. Assist the PHMC in the conduct of a Pre-Existing } \\
\text { Condition Assessment for the } 222-5 \text { Laboratory. }\end{array}$ & $7 / 15 / 96-8 / 30 / 96$ & GB Griffin \\
\hline
\end{tabular}

\subsubsection{Low-Level Analytical Services}

The WSCF Complex provides low-level analytical, development and field services to Hanford. Emphasis is placed on faster turn around support to programs and includes process control and environmental analysis, field screening and analysis, stack counting and industrial hygiene services.

\begin{tabular}{|l|c|c|}
\hline \multicolumn{1}{|c|}{ Pre-Award Actions } & Scheduled & Date \\
\hline $\begin{array}{l}\text { 1. Prepare WSCF complex briefing for the PHMC } \\
\text { (coordinate with RL POC). }\end{array}$ & $6 / 01 / 96$ & AG King \\
\hline 2. $\begin{array}{l}\text { Identify key documents for the WSCF complex and } \\
\text { review with DOE-RL. Provide iist for consolidation } \\
\text { with the Analytical Services Program list. }\end{array}$ & AG King \\
\hline
\end{tabular}




\begin{tabular}{|c|c|c|}
\hline Pre-Award Actions & $\begin{array}{l}\text { Scheduled } \\
\text { Date }\end{array}$ & Actioner $(s)$ \\
\hline $\begin{array}{l}\text { 3. Assemble key documentation, or identify its location, } \\
\text { for use by the PHMC. Includes preparation of a } \\
\text { WSCF complex "Smart Book." }\end{array}$ & $6 / 01 / 96$ & AG King \\
\hline $\begin{array}{l}\text { 4. Identify POCs for the WSCF complex and provide } \\
\text { for consolidation with the Analytical Services } \\
\text { Program list. }\end{array}$ & $5 / 17 / 96$ & AG King \\
\hline $\begin{array}{l}\text { 5. Develop a listing and description of subcontracts } \\
\text { supporting the WSCF complex and consolidate with } \\
\text { the Analytical Services Program list. Include any } \\
\text { subcontracts planned to be executed prior to contract } \\
\text { takeover. }\end{array}$ & $5 / 15 / 96$ & JL Deichman \\
\hline $\begin{array}{l}\text { Using the pre-existing checklist, compile pre-existing } \\
\text { condition information on the WSCF complex. } \\
\text { Conduct, or schedule as a separate post-award } \\
\text { action(s), any assessments required to adequately } \\
\text { address all relevant items on the checklist. Provide } \\
\text { completed checklist to RL POC for review. }\end{array}$ & $6 / 01 / 96$ & CR Stroup \\
\hline $\begin{array}{l}\text { 7. Develop a list of open action items and commitments, } \\
\text { including status (Environmental, Tri-Party } \\
\text { Agreement, DNFSB as examples). Provide to RL } \\
\text { POC for review. }\end{array}$ & $6 / 01 / 96$ & JL Deichman \\
\hline $\begin{array}{l}\text { 8. Develop a list of WSCF specific mandatory training } \\
\text { and qualification requirements for employees. }\end{array}$ & $6 / 01 / 96$ & CR Stroup \\
\hline $\begin{array}{l}\text { 9. Develop a listing of information which constitutes the } \\
\text { "authorization basis" for the WSCF. }\end{array}$ & $6 / 01 / 96$ & CR Stroup \\
\hline $\begin{array}{l}\text { 10. Develop a description of ongoing and planned facility } \\
\text { upgrades at the WSCF complex. }\end{array}$ & $6 / 01 / 96$ & SL Brey \\
\hline $\begin{array}{l}\text { 11. Develop a listing of special agreement services } \\
\text { provided to RL, other DOE sites, and other entities. }\end{array}$ & $6 / 01 / 96$ & AG King \\
\hline $\begin{array}{l}\text { 12. Develop a listing and description of WSCF complex } \\
\text { databases. Include information on LABCORE. } \\
\text { Provide list for consolidation with the Analytical } \\
\text { Services Program list. }\end{array}$ & $5 / 15 / 96$ & SL Brey \\
\hline $\begin{array}{l}\text { 13. Develop a list of current work order services or other } \\
\text { form of agreement to provide work for other } \\
\text { organizations, on and offsite. }\end{array}$ & $6 / 01 / 96$ & IL Deichman \\
\hline $\begin{array}{l}\text { 14. Develop a list of current work order services or other } \\
\text { form of agreement to obtain work from other } \\
\text { organizations, on and offsite. }\end{array}$ & $6 / 01 / 96$ & JL Deichman \\
\hline $\begin{array}{l}\text { 15. Develop a list of permits governing the WSCF } \\
\text { complex and provide a description of each as to } \\
\text { where and how it applies. }\end{array}$ & $6 / 01 / 96$ & MJ Hall \\
\hline $\begin{array}{l}\text { 16. Verify the accuracy of property assignments for the } \\
\text { WSCF complex and correct any deficiencies. }\end{array}$ & $6 / 01 / 96$ & AG King \\
\hline
\end{tabular}




\begin{tabular}{|c|c|c|c|}
\hline & Pre-Award Actions & $\begin{array}{l}\text { Scheduled } \\
\text { pate }\end{array}$ & 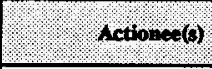 \\
\hline \multirow[t]{2}{*}{17.} & $\begin{array}{l}\text { Develop a comprehensive list of open-ended } \\
\text { compliance issues at the WSCF complex. (HATS as } \\
\text { an example). Provide to RL POC for review. }\end{array}$ & $5 / 01 / 96$ & AG King \\
\hline & Post-Award Actions & & \\
\hline 1. & Provide WSCF complex briefing to the PHMC. & $7 / 01 / 96-7 / 15 / 96$ & AG King \\
\hline 2. & Provide a WSCF complex tour to the PHMC. & $7 / 01 / 96-7 / 15 / 96$ & CR Stroup \\
\hline 3. & $\begin{array}{l}\text { Provide the PHMC access to key documentation, pre- } \\
\text { existing condition information, commitment data, } \\
\text { inventory data, etc. (as established in the pre-award } \\
\text { phase). Provide copies upon request. }\end{array}$ & by $6 / 15 / 96$ & AG King \\
\hline 4. & $\begin{array}{l}\text { Provide any additional documentation or information } \\
\text { requested by the PHMC, if available, that was not } \\
\text { identified during the pre-award phase. }\end{array}$ & by $7 / 01 / 96$ & AG King \\
\hline 5. & $\begin{array}{l}\text { PHMC identify any recurring reports that they would } \\
\text { like to receive during transition. }\end{array}$ & by $7 / 15 / 96$ & PHMC \\
\hline 6. & Provide necessary training to PHMC personnel. & $7 / 01 / 96-8 / 01 / 96$ & AG King \\
\hline 7. & $\begin{array}{l}\text { Provide an updated status of ongoing work at the } \\
\text { WSCF complex prior to contract turnover. }\end{array}$ & by $9 / 30 / 96$ & JL Deichman \\
\hline 8. & $\begin{array}{l}\text { Perform a joint property and equipment inventory of } \\
\text { the WSCF complex with the PHMC. }\end{array}$ & by $8 / 30 / 96$ & AG King \\
\hline 9. & $\begin{array}{l}\text { Provide a categorized inventivy of wastes at the } \\
\text { WSCF complex. }\end{array}$ & by $9 / 30 / 96$ & MJ Hall \\
\hline 10. & $\begin{array}{l}\text { Assist the PHMC in the conumet of a Pre-existing } \\
\text { Condition Assessment for W SCF. }\end{array}$ & $7 / 15 / 96-8 / 30 / 96$ & CR Stroup \\
\hline
\end{tabular}

\subsection{HANFORD SITE. TRAFTIC MANAGER}

The PHMC will need to establish and maintain sufficient organization, staffing and expertise to provide the following cross-cutting services that have significant safety and regulatory impact. This organization not only supports site missions but also provides unique technical support to DOE Headquarters and DOE contractors throughout the nationwide complex, and to other non-DOE entities through the Work For Others Program.

\subsubsection{Hanford Site Transportation and Packaging Support}

The mission of this organization (Transportation and Packaging) is to manage a comprehensive transportation and packaging program that ensures safe, efficient and cost effective movement of materials, especially hazardous materials and hazardous waste, to, across, or from the Hanford Site in support of all site mission areas. In this capacity the organization acts as the sole designated shipping agent for DOE at the Hanford Site. This effort includes site operational support, packaging and transportation system development, 
and a full range of transportation and traffic management services, it does not include actual fleet operations or maintenance. All Transportation and Packaging activities are conducted in accordance with DOE Orders, State Regulations, applicable company directives and procedures, and Federal laws and regulations codified in Title 10 and Title 49 of the CFR.

The WHC-CM-2-14, "Hazardous Material Packaging and Shipping" manual, implements the site transportation safety program through overall policies and procedures and through Safety Analysis Reports for Packaging (SARPs) and Safety Evaluations for Packagings (SEPs) for individual packaging systems. The technical content of these documents evaluates site hazardous materials packaging in accordance with applicable Federal regulations and DOE directives. Specifically, these evaluations include package and transport system design, assessment of authorized payload, structural, thermal, shielding, criticality, risk assessment and containment analyses, and performance test data.

Package design and engineering support is provided for packaging and transportation systems development, procurement and maintenance, and package testing.

Facilities are provided specific operational support on package selection and use, classification of hazards, preparation of containers and shipments, and coordination of support services required for the movement.

Transportation and traffic management services are available to all Hanford Site contractors. Activities include support for the movement of general freight, hazardous materials, import and export. freight rate negotiations, carrier selection, freight bill prepayment auditing and processing, and the movement of household goods of new and transferring employees. As the designated shipping agent for DOE, Transportation Packaging manages all inbound and outhound hazardous material shipments and cross site transfers.

The following pre-an ard and post-award actions will be completed to ensure a complete and orderly turnover of the Transportation and Packaging organization to the PHMC.

\begin{tabular}{|c|c|c|}
\hline $4: 4$ Pre-Awerd Artmen & $\begin{array}{l}\text { Scheduled } \\
\text { Date }\end{array}$ & Actionee(s) \\
\hline $\begin{array}{l}\text { 1. Develop a list of key protram documentation, } \\
\text { including regulatory drivers, workscope and charter, } \\
\text { customer and site contacts, staffing requirements and } \\
\text { funding sources/requirements. Provide to RL POC for } \\
\text { review. }\end{array}$ & $5 / 15 / 96$ & WF Irvine \\
\hline $\begin{array}{l}\text { 2. Assemble key program documentation, or identify its } \\
\text { location, for use by the PHMC. Includes development } \\
\text { of a program "Smart Book." }\end{array}$ & $6 / 01 / 96$ & WF Irvine \\
\hline $\begin{array}{l}\text { 3. Identify number of briefings, and prepare program } \\
\text { briefing(s) for presentation to PHMC. (Coordinate } \\
\text { number and content with RL POC.) }\end{array}$ & $6 / 01 / 96$ & WF Irvine \\
\hline 4. Develop a listing of Key program individuals as POCs. & $6 / 01 / 96$ & WF Irvine \\
\hline
\end{tabular}




\begin{tabular}{|c|c|c|}
\hline 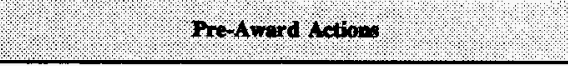 & $\begin{array}{l}\text { Scheduled } \\
\text { Dabe }\end{array}$ & Actiones (6) \\
\hline $\begin{array}{l}\text { 5. Develop listing and description of program } \\
\text { subcontracts. Include any subcontracts still planned to } \\
\text { be executed prior to contract takeover. }\end{array}$ & 6/01/96 & WF Irvine \\
\hline 6. Develop listing and description of program databases. & 6/01/96 & WF Irvine \\
\hline $\begin{array}{l}\text { 7. Verify the accuracy of property assignments at the } \\
\text { program organizational level and correct any } \\
\text { deficiencies. }\end{array}$ & $6 / 01 / 96$ & WF Irvine \\
\hline $\begin{array}{l}\text { 8. Identify space and equipment within facility for use by } \\
\text { the PHMC. }\end{array}$ & $6 / 01 / 96$ & WF Irvine \\
\hline $\begin{array}{l}\text { 9. Prepare a white paper describing how services are } \\
\text { provided across the site between multiple site facilities } \\
\text { and contractors, and identify strategies for } \\
\text { improvement. }\end{array}$ & $6 / 01 / 96$ & WF Irvine \\
\hline $\begin{array}{l}\text { 10. Prepare a white paper discussing strategies to leverage } \\
\text { transportation and packaging expertise prior to and } \\
\text { after contract award. }\end{array}$ & 6/01/96 & WF Irvine \\
\hline 4 (4ost-Award Actions & & (4) \\
\hline $\begin{array}{l}\text { 1. Provide the PHMC access to the key program } \\
\text { documentation to include listing of POCs, listing of } \\
\text { subcontracts, etc. Provide copies upon request. }\end{array}$ & prior to $6 / 15 / 96$ & WF Irvine \\
\hline 2. Provide space and services for use by the PHMC. & $6 / 15 / 96$ & WF Irvine \\
\hline 3. Provide program briefing(s) to the PHMC. & $6 / 15 / 96-7 / 01 / 96$ & WF Irvine \\
\hline $\begin{array}{l}\text { 4. Prepare and deliver briefing on how services are } \\
\text { provided across the site between multiple site facilities } \\
\text { and contractors, and identify strategies for } \\
\text { improvement. }\end{array}$ & $6 / 15 / 96-7 / 01 / 96$ & WF Irvine \\
\hline 5. Provide program level tour to the PHMC. & 6/15/96-7/01/96 & WF Irvine \\
\hline $\begin{array}{l}\text { 6. Prepare and deliver management briefing on strategies } \\
\text { to leverage Transportation and Packaging expertise } \\
\text { thorugh the WFO Program or outsourcing } \\
\text { (privatization). }\end{array}$ & $6 / 15 / 96-7 / 01 / 96$ & WF Irvine \\
\hline $\begin{array}{l}\text { 7. Perform a joint program level property and equipment } \\
\text { inventory with PHMC personnel. }\end{array}$ & by $8 / 30 / 96$ & WF Irvine \\
\hline
\end{tabular}

\subsubsection{DOE Headquarters EM Transportation Program Support}

The objective of this activity is to leverage the expertise and experience of the Transportation and Packaging staff to provide technical support to the DOE-wide transportation program managed by DOE-HQ EM-76. Related products and services provided are as follows. 
Transportation Logistics - motor carrier evaluations, transportation plans for unique shipment campaigns, logistics services to smaller DOE sites, and site operational assessments upon request.

Transportation Training - safety and regulatory compliance training to DOE and contractor personnel, other state and Federal agency personnel, tribal and local officials, commercial carrier employees, and others.

Transportation Automation - development and implementation of automated systems to manage overall DOE transportation operations.

Regulatory Compliance and Technical Assessment - review and analysis of regulatory changes and high-visibility issues that affect DOE transportation management. Assessment teams are also provided to evaluate field element and contractor transportation operations.

Explosives Classification and DOE Explosives Program Management - explosive classification reviews, registration with DOT, site safety assessments, information management support, and other related activities.

Packaging Engineering and Analysis - design, analysis, and testing of packaging systems needed across the DOE. This includes assessment of packaging system needs throughout DOE.

Packaging Safety Assessment - risk assessment and NEPA documentation. Includes package proof-testing, confirmatory analysis, and technical design reviews.

Package Testing - DOT-7A Type A package testing program for the DOE complex. Both Type A and Type B package testing capability exists.

\subsubsection{Transportation and Packaging Work For Others Services}

The objective of this activity is to leverage the expertise and experience of the Transportation and Packaging staff to provide a number of products and services to other DOE contractors across the country within the framework of DOE's Work For Others program, and to other government agencies and the commercial sector as authorized by DOE.

This activity benefits the site, the community and the entire DOE complex. The site benefits from the national experience of staff which translates to effective Hanford Site solutions. It preserves key personnel to meet site needs and benefits the community by retaining the core competency for potential business ventures. It benefits the DOE complex through effective utilization of resources and unique expertise. 
Products and services are provided in four specific areas.

- Packaging and transportation system development (package design, analysis and testing).

- Operations support, shipment coordination and planning.

- Traffic and transportation management and automated management systems.

- Transportation safety and compliance training.

\subsection{ARCHITECT ENGINEER/CONSTRUCTION MANAGEMENT}

Scope of Support and Administrative Services - Furnish the administrative services described below, subject to such further detailed requirements as may be issued from time to time. These services are provided by ICF KH to support the ICF KH scope of work.

- Financial management - Including general and cost accounting, payroll, internal audit, and budget functions.

- Property management - Maintain a property management program for personal property and equipment assigned.

- Shop and warehouse operations - Operate centralized shops and warehouses in support of construction. Warehouse activities will generally be limited to receiving and distribution in support of specific project activities.

- Facility custodianship - Perform maintenance and upgrading of facilities and equipment as required for assigned Government furnished buildings and grounds.

- Project management system - Provide a project management system for controlling the engineering and construction work including a project cost and schedule control system, an estimating system, and a scheduling system.

- Quality assurance - Perform those planned and systematic actions necessary to provide adequate confidence that a structure, facility, system, or component will perform satisfactorily in service. Quality assurance is to assure that components, systems, and processes are designed, developed, constructed, and tested according to sound engineering standards, quality practices, and technical specifications.

- Safety - Maintain effective environmental, safety, health, and emergency preparedness programs.

- Personnel management and labor relations - Provide work force mobilization and demobilization, labor-management relations, collective bargaining, etc. 
Maintenance of a qualified central and field staff commensurate with the workload.

- Procurement and Subcontracting - Solicit, award, and administer AE and construction subcontracts and agreements, including coordinating and expediting the work of suppliers/subcontractors. Develop and utilize a small/small disadvantaged business subcontracting program.

- Value Engineering (VE) - Maintain an effective VE program and perform VE analyses and studies.

\begin{tabular}{|c|c|c|}
\hline Pre-Award Actions & $\begin{array}{l}\text { Scheduled } \\
\text { Date }\end{array}$ & Actioned $(s)$ \\
\hline 1. Provide design authority and design agent staff listing. & $6 / 01 / 96$ & $\begin{array}{l}\text { RL Benedetti } \\
\text { SE Gregg }\end{array}$ \\
\hline
\end{tabular}

\subsubsection{Architect Engineer Services}

Maintain and manage an engineering staff for the purpose of providing engineering support and such technical services as may be specifically required to support the Hanford Site.

Perform various onsite engineering services and such other related technical services as may be required and assigned. These engineering services will include Title I Preliminary Design Services, Title II - Definitive Design Services, and Title III Engineering and Inspection during Construction. In addition, perform engineering studies and conceptual design in support of anticipated construction projects; maintain an up to date and accurate engineering catalog file; maintain accurate and up to date Hanford Standards and Specifications; and provide field survey services. Provide necessary administrative support services to accomplish this scope.

The preponderance of the work assigned may be generated via work orders from other onsite contractors. Accordingly, for each assigned task it will be necessary to work with, receive direction and guidance from, and maintain liaison with the other onsite contractors.

\begin{tabular}{|c|c|c|}
\hline Pre-Award Actions & $\begin{array}{l}\text { Seheduled } \\
\text { Date }\end{array}$ & Actionee(s) \\
\hline $\begin{array}{l}\text { 1. Identify number of briefings, and prepare briefing(s) } \\
\text { for the PHMC. Provide for RL POC review. }\end{array}$ & $6 / 01 / 96$ & $\begin{array}{l}\text { CD Maxson } \\
\text { CE Anderson }\end{array}$ \\
\hline 2. Identify key documents. Provide for RL POC review. & $5 / 01 / 96$ & $\begin{array}{l}\text { CD Maxson } \\
\text { CE Anderson } \\
\text { MD Olsen }\end{array}$ \\
\hline
\end{tabular}




\begin{tabular}{|c|c|c|}
\hline Pre-Award letions & Scheduled & Atriouee (s) \\
\hline $\begin{array}{l}\text { 3. Assemble key documentation, or identify its location, } \\
\text { for use by the PHMC. Includes preparation of a } \\
\text { "Smart Book," to include information develop in the } \\
\text { following actions. }\end{array}$ & $6 / 01 / 96$ & $\begin{array}{l}\text { CD Maxson } \\
\text { CE Anderson }\end{array}$ \\
\hline $\begin{array}{l}\text { 4. Identify POCs and input to consolidated } \\
\text { program/project listing. }\end{array}$ & $5 / 15 / 96$ & CD Maxson \\
\hline $\begin{array}{l}\text { 5. Develop a listing and description of supportive } \\
\text { subcontracts and input to the consolidated listing. } \\
\text { Include any subcontracts planned to be executed prior } \\
\text { to contract takeover. }\end{array}$ & $5 / 15 / 96$ & $\begin{array}{l}\text { CD Maxson } \\
\text { CE Anderson }\end{array}$ \\
\hline $\begin{array}{l}\text { Using the pre-existing checklist, compile pre-existing } \\
\text { condition information. Conduct, or schedule as a } \\
\text { separate post-award action(s), any assessments required } \\
\text { to adequately address all relevant items on the } \\
\text { checklist. Provide completed checklist for RL POC } \\
\text { review. }\end{array}$ & $6 / 01 / 96$ & $\begin{array}{l}\text { CD Maxson } \\
\text { CE Anderson } \\
\text { MD Olsen }\end{array}$ \\
\hline $\begin{array}{l}\text { 7. Develop a list of open action items and commitments, } \\
\text { including status. Provide to WHC for RL review. }\end{array}$ & $6 / 01 / 96$ & $\begin{array}{l}\text { CD Maxson } \\
\text { CE Anderson } \\
\text { JH Arslanian } \\
\text { MD Olsen } \\
\end{array}$ \\
\hline $\begin{array}{l}\text { 8. Develop a list of specific mandatory training and } \\
\text { qualification requirements for employees. }\end{array}$ & $6 / 01 / 96$ & $\begin{array}{l}\text { CD Maxson } \\
\text { CE Anderson }\end{array}$ \\
\hline 9. Develop a description of ongoing and planned projects. & $6 / 01 / 96$ & $\begin{array}{l}\text { CE Anderson } \\
\text { CD Maxson }\end{array}$ \\
\hline $\begin{array}{l}\text { 10. Develop a listing of special agreement services } \\
\text { provided to RL, other DOE sites, and other entities. }\end{array}$ & $6 / 01 / 96$ & $\begin{array}{l}\text { CD Maxson } \\
\text { CE Anderson } \\
\end{array}$ \\
\hline $\begin{array}{l}\text { 11. Develop a listing and description of databases. Provide } \\
\text { for consolidation include developed software. }\end{array}$ & $5 / 15 / 96$ & $\begin{array}{l}\text { CE Anderson } \\
\text { MD Olsen } \\
\end{array}$ \\
\hline $\begin{array}{l}\text { 12. Develop a list of current work order services or other } \\
\text { form of agreement to provide work for other } \\
\text { organizations, on and offsite. }\end{array}$ & $6 / 01 / 96$ & $\begin{array}{l}\text { CE Anderson } \\
\text { CL Stice }\end{array}$ \\
\hline $\begin{array}{l}\text { 13. Develop list of accountability and litigation issues and } \\
\text { provide to WHC. }\end{array}$ & $6 / 01 / 96$ & MD Olsen \\
\hline 14. Develop a list of patents and intellectual property. & $6 / 01 / 96$ & $\begin{array}{l}\text { CD Maxson } \\
\text { MD Olsen } \\
\text { CE Anderson }\end{array}$ \\
\hline $\begin{array}{l}\text { 15. Develop a list of current work order services or other } \\
\text { form of agreement to obtain work from other } \\
\text { organizations, on and offsite. }\end{array}$ & $6 / 01 / 96$ & $\begin{array}{l}\text { CE Anderson } \\
\text { CL Stice }\end{array}$ \\
\hline $\begin{array}{l}\text { 16. Develop a list of licenses, permits, and code } \\
\text { certifications and provide a description of each as to } \\
\text { where and how it applies. }\end{array}$ & $6 / 01 / 96$ & $\begin{array}{l}\text { CD Maxson } \\
\text { CE Anderson }\end{array}$ \\
\hline $\begin{array}{l}\text { 17. Verify the accuracy of property assignments and } \\
\text { correct any deficiencies. }\end{array}$ & $6 / 01 / 96$ & CD Maxson \\
\hline
\end{tabular}




\begin{tabular}{|c|c|c|}
\hline Post-Avard Actions & $\begin{array}{l}\text { Scheduled } \\
\text { Date }\end{array}$ & Actionee(s) \\
\hline 1. Provide briefing(s) to the PHMC. & $7 / 01 / 96-7 / 15 / 96$ & $\begin{array}{l}\text { CD Maxson } \\
\text { CE Anderson }\end{array}$ \\
\hline 2. Provide tour(s) to the PHMC. & $7 / 01 / 96-7 / 15 / 96$ & $\begin{array}{l}\text { CD Maxson } \\
\text { CE Anderson }\end{array}$ \\
\hline $\begin{array}{l}\text { 3. Provide the PHMC access to key documentation, pre- } \\
\text { existing condition information, commitment data, } \\
\text { inventory data, etc. (as established in the pre-award } \\
\text { phase). Provide copies upon request. }\end{array}$ & by $6 / 15 / 96$ & $\begin{array}{l}\text { CD Maxson } \\
\text { CE Anderson }\end{array}$ \\
\hline $\begin{array}{l}\text { 4. Provide any additional documentation or information } \\
\text { requested by the PHMC, if available, that was not } \\
\text { identified during the pre-award phase. }\end{array}$ & by $7 / 01 / 96$ & CD Maxson \\
\hline $\begin{array}{l}\text { 5. PHMC identify any recurring reports that they would } \\
\text { like to receive during transition. }\end{array}$ & by $7 / 15 / 96$ & CD Maxson \\
\hline 6. Provide necessary training to PHMC personnel. & $7 / 01 / 96-8 / 01 / 96$ & CD Maxson \\
\hline $\begin{array}{l}\text { 7. Provide an updated status of ongoing work prior to } \\
\text { contract turnover. }\end{array}$ & by $9 / 30 / 96$ & $\begin{array}{l}\text { CD Maxson } \\
\text { CE Anderson }\end{array}$ \\
\hline $\begin{array}{l}\text { 8. Perform a joint property and equipment inventory with } \\
\text { the PHMC. }\end{array}$ & by $8 / 30 / 96$ & CD Maxson \\
\hline $\begin{array}{l}\text { 9. Provide a categorized inventory of wastes or hazardous } \\
\text { materials. }\end{array}$ & by $9 / 30 / 96$ & $\begin{array}{l}\text { CD Maxson } \\
\text { CE Anderson }\end{array}$ \\
\hline $\begin{array}{l}\text { 10. Update and disposition accountability and litigation } \\
\text { issues. }\end{array}$ & by $9 / 30 / 96$ & $\begin{array}{l}\text { CD Maxson } \\
\text { MD Olsen }\end{array}$ \\
\hline
\end{tabular}

\subsubsection{Construction Management Services}

- Furnish all labor, material, management, and supervision necessary for the performance of construction, construction management, maintenance, repair, and other construction related services. Provide necessary administrative support services to accomplish this scope.

- The construction work shall consist of two distinct types: construction and construction management.

- Perform work involving force account construction under radiological and nonradiological conditions and/or during scheduled and unscheduled plant shutdowns. These conditions often make scheduling difficult and preparation of definitive design and competitive subcontracting sometimes impractical. However, cost-type work assigned shall, when appropriate and to the extent possible, be subcontracted as competitive fixed-price subcontracts. 
- In addition to industrial grade construction, the work will include fabrication of nuclear vessels, piping, and appurtenances which are subject to very stringent requirements. The work entails providing highly skilled craftsmen (electricians, pipefitters, boilermakers, millwright, sheetmetal workers, ironworkers, etc.) as necessary to support force account work.

- Perform construction management (CM) services on projects and provide dedicated CM staff and services on major projects which will include providing a wide range of professional services relating to management of a project during the predesign, design, and/or construction phases.

- The preponderance of the work assigned may be generated via work orders from other onsite contractors. Accordingly, for each assigned task it will be necessary to work with, receive direction and guidance from, and maintain liaison with the other onsite contractors.

\begin{tabular}{|c|c|c|}
\hline 4 Pre-Award Actions & $\begin{array}{l}\text { Scheduled } \\
\text { Date }\end{array}$ & Actionee(s) \\
\hline $\begin{array}{l}\text { 1. Identify number of briefings, and prepare briefing(s) } \\
\text { for the PHMC. Provide for RL POC review. }\end{array}$ & $6 / 01 / 96$ & $\begin{array}{l}\text { CD Maxson } \\
\text { ME Tenvooren }\end{array}$ \\
\hline 2. Identify key documents. Provide for RL POC review. & $5 / 01 / 96$ & $\begin{array}{l}\text { CD Maxson } \\
\text { ME Tenvooren }\end{array}$ \\
\hline $\begin{array}{l}\text { 3. Assemble key documentation, or identify its location, } \\
\text { for use by the PHMC. Includes preparation of a } \\
\text { "Smart Book," to include information develop in the } \\
\text { following actions. }\end{array}$ & 6/01/96 & $\begin{array}{l}\text { CD Maxson } \\
\text { ME Tenvooren }\end{array}$ \\
\hline 4. Identify POCs and input to consolidated listing. & $5 / 15 / 96$ & $\begin{array}{l}\text { CD Maxson } \\
\text { ME Tenvooren }\end{array}$ \\
\hline $\begin{array}{l}\text { 5. Develop a listing and description of supporting } \\
\text { subcontracts and input to consolidated listing. Include } \\
\text { any subcontracts planned to be executed prior to } \\
\text { contract takeover. }\end{array}$ & $5 / 15 / 96$ & $\begin{array}{l}\text { CD Maxson } \\
\text { ME Tenvooren }\end{array}$ \\
\hline $\begin{array}{l}\text { 6. Using the pre-existing checklist, compile pre-existing } \\
\text { condition information. Conduct, or schedule as a } \\
\text { separate post-award action(s), any assessments required } \\
\text { to adequately address all relevant items on the } \\
\text { checklist. Provide completed checklist to WHC for } \\
\text { review and consolidation. }\end{array}$ & $6 / 01 / 96$ & $\begin{array}{l}\text { CD Maxson } \\
\text { ME Tenvooren } \\
\text { MD Olsen } \\
\text { JH Arslanian }\end{array}$ \\
\hline $\begin{array}{l}\text { 7. Develop a list of open action items and commitments, } \\
\text { including status (Environmental, Tri-Party Agreement, } \\
\text { DNFSB as examples). }\end{array}$ & $6 / 01 / 96$ & $\begin{array}{l}\text { CD Maxson } \\
\text { ME Tenvooren }\end{array}$ \\
\hline $\begin{array}{l}\text { 8. Develop a list of specific mandatory training and } \\
\text { qualification requirements for employees. }\end{array}$ & $6 / 01 / 96$ & $\begin{array}{l}\text { CD Maxson } \\
\text { ME Tenvooren } \\
\end{array}$ \\
\hline $\begin{array}{l}\text { 9. Develop a description of ongoing and planned } \\
\text { construction projects. }\end{array}$ & $6 / 01 / 96$ & $\begin{array}{l}\text { CD Maxson } \\
\text { ME Tenvooren }\end{array}$ \\
\hline $\begin{array}{l}\text { 10. Develop a listing of special agreement services } \\
\text { provided to RL, other DOE sites, and other entities. }\end{array}$ & 6/01/96 & $\begin{array}{l}\text { CD Maxson } \\
\text { ME Tenvooren }\end{array}$ \\
\hline
\end{tabular}




\begin{tabular}{|c|c|c|}
\hline Sre-Aword Actions & Scheduled & Actiones $(s)$ \\
\hline $\begin{array}{l}\text { 11. Develop a listing and description of databases. Provide } \\
\text { for consolidation. }\end{array}$ & $5 / 15 / 96$ & $\begin{array}{l}\text { CD Maxson } \\
\text { ME Tenvooren }\end{array}$ \\
\hline $\begin{array}{l}\text { 12. Develop a list of current work order services or other } \\
\text { form of agreement to provide work for other } \\
\text { organizations, on and offsite. }\end{array}$ & $6 / 01 / 96$ & $\begin{array}{l}\text { CD Maxson } \\
\text { ME Tenvooren }\end{array}$ \\
\hline $\begin{array}{l}\text { 13. Develop a list of current work order services or other } \\
\text { form of agreement to obtain work from other } \\
\text { organizations, on and offsite. }\end{array}$ & $6 / 01 / 96$ & $\begin{array}{l}\text { CD Maxson } \\
\text { ME Tenvooren }\end{array}$ \\
\hline $\begin{array}{l}\text { 14. Develop a list of permits and provide a description of } \\
\text { each as to where and how it applies. }\end{array}$ & $6 / 01 / 96$ & $\begin{array}{l}\text { CD Maxson } \\
\text { ME Tenvooren }\end{array}$ \\
\hline $\begin{array}{l}\text { 15. Verify the accuracy of property assignments and } \\
\text { correct any deficiencies. }\end{array}$ & $6 / 01 / 96$ & $\begin{array}{l}\text { CD Maxson } \\
\text { ME Tenvooren }\end{array}$ \\
\hline \multicolumn{3}{|l|}{ Post-Award Actions } \\
\hline 1. Provide briefing(s) to the PHMC. & $7 / 01 / 96-7 / 15 / 96$ & $\begin{array}{l}\text { CD Maxson } \\
\text { ME Tenvooren }\end{array}$ \\
\hline 2. Provide tour(s) to the PHMC. & $7 / 01 / 96-7 / 15 / 96$ & $\begin{array}{l}\text { CD Maxson } \\
\text { ME Tenvooren }\end{array}$ \\
\hline $\begin{array}{l}\text { 3. Provide the PHMC access to key documentation, pre- } \\
\text { existing condition information, commitment data, } \\
\text { inventory data, etc. (as established in the pre-award } \\
\text { phase). Provide copies upon request. }\end{array}$ & by $6 / 15 / 96$ & $\begin{array}{l}\text { CD Maxson } \\
\text { ME Tenvooren }\end{array}$ \\
\hline $\begin{array}{l}\text { 4. Provide any additional documentation or information } \\
\text { requested by the PHMC, if available, that was not } \\
\text { identified during the pre-award phase. }\end{array}$ & by $7 / 01 / 96$ & $\begin{array}{l}\text { CD Maxson } \\
\text { ME Tenvooren }\end{array}$ \\
\hline $\begin{array}{l}\text { 5. PHMC identify any recurring reports that they would } \\
\text { like to receive during transition. }\end{array}$ & by $7 / 15 / 96$ & $\begin{array}{l}\text { CD Maxson } \\
\text { ME Tenvooren }\end{array}$ \\
\hline 6. Provide necessary training to PHMC personnel. & $7 / 01 / 96-8 / 01 / 96$ & $\begin{array}{l}\text { CD Maxson } \\
\text { ME Tenvooren }\end{array}$ \\
\hline $\begin{array}{l}\text { 7. Provide an updated status of ongoing work prior to } \\
\text { contract turnover. }\end{array}$ & by $9 / 30 / 96$ & $\begin{array}{l}\text { CD Maxson } \\
\text { ME Tenvooren }\end{array}$ \\
\hline $\begin{array}{l}\text { 8. Perform a joint property and equipment inventory with } \\
\text { the PHMC. }\end{array}$ & by $8 / 30 / 96$ & $\begin{array}{l}\text { CD Maxson } \\
\text { ME Tenvooren }\end{array}$ \\
\hline $\begin{array}{l}\text { 9. Provide a categorized inventory of wastes or hazardous } \\
\text { materials. }\end{array}$ & by $9 / 30 / 96$ & $\begin{array}{l}\text { CD Maxson } \\
\text { ME Tenvooren }\end{array}$ \\
\hline
\end{tabular}

\subsection{CONSTRUCTION PROJECT MANAGEMENT}

Construction Projects typically involved capital funded activities involving design, procurement, and construction efforts governed by DOE 4700.1A "Project Management System" and covered by WHC-CM-6-2, "Project Management." 
This scope includes the expense funded conceptual design phase where the project design criteria/requirements are established (scope baseline) and the associated cost and schedule baselines are developed. These three baselines are typically documented in the Conceptual Design Report (CDR) and are "validated" by RL (and HQ for Line Item projects, $>$ \$2.0M Total Estimated Cost [TEC]). Construction Projects are also responsible for the expense funded "Other Project Costs" (OPC) as reported on the Project Data Sheet approved by Congress. The TEC plus the OPC constitute the Total Project Cost (TPC).

Project Management involves either the onsite architect Engineer (ICF KH) or procurement of an offsite Architect-Engineer (A-E) through WHC Procurement. Typically, construction management services are provided by ICF KH for all projects except a very large project (MSA) which might involve a separate construction management contract strategy.

An exception is a project funded with expense dollars, (e.g., an in-tank demonstration scope). A second significant scope of work are "project-like" activities that are not formal authorized projects but involve design and construction activities. Support activities such as maintenance of the project management process/procedures, financial management, forecasts, etc., are also required.

\begin{tabular}{|c|c|c|}
\hline Pre-Award Actions & $\begin{array}{l}\text { Scheduled } \\
\text { Date }\end{array}$ & Actionee (8) \\
\hline $\begin{array}{l}\text { 1. Prepare Overview Package contanning all active formal } \\
\text { construction projects and supporning activities. }\end{array}$ & 6/01/96 & RR Gadd \\
\hline 2. Prepare a Significant Issues Lia & $6 / 01 / 96$ & RR Gadd \\
\hline $\begin{array}{l}\text { 3. Provide Project Mandgement Process Road Map and significant } \\
\text { issues, if any. }\end{array}$ & $6 / 01 / 96$ & RR Gadd \\
\hline $\begin{array}{l}\text { 4. Provide list of all existing entertnal contracts (e.g., A-E's, WHC } \\
\text { procurement or construstem cromerests, consultants). }\end{array}$ & $6 / 01 / 96$ & RR Gadd \\
\hline 5. Provide accurate properti ceenments for each organization. & $6 / 01 / 96$ & RR Gadd \\
\hline Poul-A-med Actions & 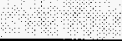 & W \\
\hline 1. Provide Project Review, far tre propects. & $8 / 02 / 96$ & RR Gadd \\
\hline 2. Provide Site walk-through for profects in construction. & 9/06/96 & RR Gadd \\
\hline
\end{tabular}




\subsection{ENVIRONMENTAL MONITORING AND SERVICES}

The following section address the scope of work as currently assigned to each contractor (M\&O, BHI, and PNNL), and do not reflect any potential transfer of scope between contractors.

\subsubsection{Hanford Technical Services}

Hanford Technical Services (HTS) conducts the RCRA Operational Monitoring Program (ROM) and provides technical expertise to support DOE Waste Management, Environmental, and Technology Development Programs. Services include: Well installation, monitoring, and maintenance, environmental sampling, geoscience expertise, environmental monitoring, geotechnical design and installation and geophysics monitoring/characterization. Pest Management (i.e., herbicide and pesticide program) is also provided and is addressed in Secton 5.1.6.

6.6.1.1 RCRA Operational Monitoring Office. The RCRA Operational Monitoring program office manages the ROM program activities, expense and capital equipment budgets, develops and status schedules and cost accounts, and administers subcontracts through task ordering agreements. The program office is the prime interface for the ROM program with the RL.

\begin{tabular}{|l|c|l|}
\hline \multicolumn{1}{|c|}{ Pre-Award Actions } & $\begin{array}{c}\text { Scheduled } \\
\text { Date }\end{array}$ & \\
\hline $\begin{array}{l}\text { 1. Identify key documents for the ROM program. (Provide a } \\
\text { listing of key program documents to RL for review). }\end{array}$ & $5 / 01 / 96$ & FT Green \\
\hline 2. Obtain copies of key program documents and assemble. & $5 / 15 / 96$ & FT Green \\
\hline $\begin{array}{l}\text { 3. Identify number of briefings, and prepare program briefing(s) } \\
\text { for presentation to PHMC. (Coordinate number and content } \\
\text { with RL POC.) }\end{array}$ & $6 / 01 / 96$ & FT Green \\
\hline $\begin{array}{l}\text { 4. Develop a listing of key program individuals as POCs, } \\
\text { including facility and operations personnel. }\end{array}$ & $6 / 01 / 96$ & FT Green \\
\hline $\begin{array}{l}\text { 5. Develop listing and description of program databases, } \\
\text { including facility (or operations) databases. }\end{array}$ & $6 / 01 / 96$ & FT Green \\
\hline $\begin{array}{l}\text { Post-Award Actions } \\
\text { 1. Make the key program documents available to the PHMC. } \\
\text { Provide copies upon request. }\end{array}$ & $6 / 15 / 96$ & FT Green \\
\hline \begin{tabular}{l} 
2. Provide program briefing(s) to the PHMC. \\
\hline
\end{tabular} & $\begin{array}{c}7 / 01 / 96- \\
7 / 15 / 96\end{array}$ & FT Green \\
\hline
\end{tabular}

6.6.1.2 Geosciences. Geosciences is responsible for geochemistry and hydrochemistry activities conducted under RCRA and other projects. The organization ensures quality control and environmental protection requirements are met for sampling and analysis associated with groundwater monitoring and other programs. Other environmental and earth 
sciences related projects are conducted on a work order or contract basis. Services include field operations personnel and equipment, preparing reports, mapping and graphics services, seismic monitoring, and natural hazards analysis.

Groundwater management (GWM) supports design, installation and testing of groundwater monitoring wells and networks and provides technical services to support geologic, hydrologic, and geotechnical investigations. The group maintains a large data base and provides expertise in investigating, mapping, and documenting Hanford geology and hydrogeology. The group also assembles and reports groundwater data to regulatory agencies as required.

\begin{tabular}{|c|c|c|}
\hline Pre-Award Actions & $\begin{array}{l}\text { Scheduled } \\
\text { Date }\end{array}$ & Actionee(d) \\
\hline $\begin{array}{l}\text { 1. Prepare for a comprehensive briefing for the PHMC } \\
\text { describing all operational aspects of the organization. }\end{array}$ & $5 / 01 / 96$ & D Horton \\
\hline $\begin{array}{l}\text { 2. Identify all key documents relevant to the organization's } \\
\text { operations, including a listing of key facility and field } \\
\text { documents. }\end{array}$ & $5 / 01 / 96$ & D Horton \\
\hline $\begin{array}{l}\text { 3. Obtain copies of key facility documents and assemble, or } \\
\text { identify their location for access. Develop operations } \\
\text { "Smartbook." }\end{array}$ & $6 / 01 / 96$ & D Horton \\
\hline 4. Identify points-of-contact for the organization. & $5 / 15 / 96$ & D Horton \\
\hline $\begin{array}{l}\text { 5. Using the pre-existing condition checklist, evaluate pre- } \\
\text { existing conditions. Conduct assessments as necessary to } \\
\text { adequately address the checklist. }\end{array}$ & $6 / 01 / 96$ & D Horton \\
\hline $\begin{array}{l}\text { 6. Identify applicable milestones and commitments (e.g., Tri- } \\
\text { Party Agreement and Consent Order milestones), and } \\
\text { determine their status. Describe the form of the deliverable } \\
\text { and what constitutes completion. }\end{array}$ & $5 / 15 / 96$ & D Horton \\
\hline $\begin{array}{l}\text { 7. Verify the accuracy of property assignments for the facility } \\
\text { and correct any deficiencies. }\end{array}$ & $5 / 15 / 96$ & D Horton \\
\hline $\begin{array}{l}\text { 8. Verify the completeness of all RIDS and correct any } \\
\text { deficiencies. }\end{array}$ & $5 / 15 / 96$ & D Horton \\
\hline Post-Award Actions & & \\
\hline $\begin{array}{l}\text { 1. Provide a comprehensive briefing for the PHMC complete } \\
\text { with all facility, personnel, and organizational documentation. } \\
\text { Provide copies upon request. }\end{array}$ & $6 / 15 / 96$ & D Horton \\
\hline $\begin{array}{l}\text { 2. Conduct a comprehensive tour of all facilities and field } \\
\text { operations relevant to the organization. }\end{array}$ & $6 / 15 / 96$ & D Horton \\
\hline $\begin{array}{l}\text { 3. Assist the PHMC in a survey of pre-existing conditions at the } \\
\text { facility. }\end{array}$ & $8 / 01 / 96$ & D Horton \\
\hline $\begin{array}{l}\text { 4. Provide an update on work completed and work in progress } \\
\text { during the transition to the PHMC. }\end{array}$ & 9/15/96 & D Horton \\
\hline $\begin{array}{l}\text { 5. Complete a joint property and equipment inventory with the } \\
\text { PHMC. }\end{array}$ & $8 / 30 / 96$ & D Horton \\
\hline
\end{tabular}




\begin{tabular}{|l|l|l|}
\hline Post Avard Actions & $\begin{array}{c}\text { Scheduled } \\
\text { Date }\end{array}$ & sctionee(s) \\
\hline $\begin{array}{l}\text { 6. Provide any additional documentation requested by the } \\
\text { PHMC. }\end{array}$ & ongoing & D Horton \\
\hline 7. Provide necessary training to PHMC personnel. & $8 / 01 / 96$ & D Horton \\
\hline 8. Provide a categorized inventory of wastes. & $9 / 30 / 96$ & D Horton \\
\hline
\end{tabular}

6.6.1.3 Well Services. Well services plans, designs, and provides field support to install, refurbish, and abandon groundwater and vadose zone monitoring wells. The organization maintains a database of wells installed at Hanford and coordinates use of these wells. The database includes information on well design and construction, lithographic and stratigraphic logs, and responsible parties.

\begin{tabular}{|c|c|c|}
\hline Pre-Award Actions. & $\begin{array}{c}\text { Scheduled } \\
\text { Date }\end{array}$ & Actionee(s) \\
\hline $\begin{array}{l}\text { 1. Prepare for a comprebensive briefing for the PHMC } \\
\text { contractor describing all operational aspects of the } \\
\text { organization. }\end{array}$ & $5 / 01 / 96$ & MG Gardner \\
\hline $\begin{array}{l}\text { 2. Identify all key documents relevant to the organization's } \\
\text { operations, including a listing of key facility and field } \\
\text { documents. }\end{array}$ & $5 / 01 / 96$ & MG Gardner \\
\hline $\begin{array}{l}\text { 3. Obtain copies of key facility documents and assemble, or } \\
\text { identify their location for access. Develop operations "Smart } \\
\text { Book". }\end{array}$ & $6 / 01 / 96$ & MG Gardner \\
\hline 4. Identify points-of-contact for the organization. & $5 / 15 / 96$ & MG Gardner \\
\hline $\begin{array}{l}\text { 5. Using the pre-existing conditions checklist, evaluate pre- } \\
\text { existing conditions. Conduct assessments as necessary to } \\
\text { adequately address the checklist. }\end{array}$ & $6 / 01 / 96$ & MG Gardner \\
\hline $\begin{array}{l}\text { 6. Identify applicable milestones and commitments (e.g., Tri- } \\
\text { Party Agreement and Consent Order milestones), and } \\
\text { determine their status. Describe the form of the deliverable } \\
\text { and what constitutes completion. }\end{array}$ & $5 / 15 / 96$ & MG Gardner \\
\hline $\begin{array}{l}\text { 7. Verify the accuracy of property assignments for the facility } \\
\text { and correct any deficiencies. }\end{array}$ & $5 / 15 / 96$ & MG Gardner \\
\hline $\begin{array}{l}\text { 8. Verify the completeness of all RIDS and correct any } \\
\text { deficiencies. }\end{array}$ & $5 / 15 / 96$ & MG Gardner \\
\hline Post-Award Actions & & \\
\hline $\begin{array}{l}\text { 1. Provide a comprehensive briefing for the PHMC complete } \\
\text { with all facility, personnel, and organizational documentation. } \\
\text { Provide copies upon request. }\end{array}$ & $6 / 15 / 96$ & MG Gardner \\
\hline $\begin{array}{l}\text { 2. Conduct a comprehensive tour of all facilities and field } \\
\text { operations relevant to the organization. }\end{array}$ & $6 / 15 / 96$ & MG Gardner \\
\hline $\begin{array}{l}\text { 3. Assist the PHMC in a survey of pre-existing conditions at the } \\
\text { facility. }\end{array}$ & $8 / 01 / 96$ & MG Gardner \\
\hline
\end{tabular}




\begin{tabular}{|c|c|c|}
\hline Post A word Actions & $\begin{array}{l}\text { Scheduled } \\
\text { Date }\end{array}$ & Actionee(f) \\
\hline $\begin{array}{l}\text { 4. Provide an update on work completed and work in progress } \\
\text { during the transition to the PHMC. }\end{array}$ & $9 / 15 / 96$ & MG Gardner \\
\hline $\begin{array}{l}\text { 5. Complete a joint property and equipment inventory with the } \\
\text { PHMC. }\end{array}$ & $8 / 30 / 96$ & MG Gardner \\
\hline $\begin{array}{l}\text { 6. Provide any additional documentation requested by the } \\
\text { PHMC. }\end{array}$ & ongoing & MG Gardner \\
\hline 7. Provide necessary training to PHMC personnel. & $8 / 01 / 96$ & MG Gardner \\
\hline 8. Provide a categorized inventory of wastes. & $9 / 30 / 96$ & MG Gardner \\
\hline
\end{tabular}

6.6.1.4 Near Field Monitoring. Near Field Monitoring directs facility specific monitoring of environmental media and performs engineering evaluations/studies pertaining to the monitoring and control of environmental pollutants.

\begin{tabular}{|l|l|l|}
\hline & Scheduled & Date \\
\hline $\begin{array}{l}\text { 1. Prepare for a comprehensive briefing for the PHMC } \\
\text { contractor describing all operational aspects of the } \\
\text { organization. }\end{array}$ & $5 / 01 / 96$ & $\mathrm{JJ}$ Dorian \\
\hline $\begin{array}{l}\text { 2. Identify all key documents relevant to the organization's } \\
\text { operations, including a listing of key facility and field } \\
\text { documents. }\end{array}$ & $5 / 01 / 96$ & $\mathrm{JJ}$ Dorian \\
\hline $\begin{array}{l}\text { 3. Obtain copies of key facility documents and assemble, or } \\
\text { identify their location for access. Develop operations "Smart } \\
\text { Book." }\end{array}$ & $6 / 01 / 96$ & $\mathrm{JJ}$ Dorian \\
\hline 4. Identify points-of-contact for the organization. & $5 / 15 / 96$ & $\mathrm{JJ}$ Dorian \\
\hline $\begin{array}{l}\text { 5. Using the pre-existing conditions checklist, evaluate pre- } \\
\text { existing conditions. Conduct assessments as necessary to } \\
\text { adequately address the checklist. }\end{array}$ & $6 / 01 / 96$ & $\mathrm{JJ}$ Dorian \\
\hline $\begin{array}{l}\text { 6. Identify applicable milestones and commitments (e.g., Tri- } \\
\text { Party Agreement and Consent Order milestones), and } \\
\text { determine their status. Describe the form of the deliverable } \\
\text { and what constitutes completion. }\end{array}$ & $5 / 15 / 96$ & $\mathrm{JJ}$ Dorian \\
\hline $\begin{array}{l}\text { 7. Verify the accuracy of property assignments for the facility } \\
\text { and correct any deficiencies. }\end{array}$ & $5 / 15 / 96$ & $\mathrm{JJ}$ Dorian \\
\hline $\begin{array}{l}\text { 8. Verify the completeness of all RIDS and correct any } \\
\text { deficiencies. }\end{array}$ & $5 / 15 / 96$ & $\mathrm{JJ}$ Dorian \\
\hline $\begin{array}{l}\text { 1. Provide a comprehensive briefing for the PHMC complete } \\
\text { with all facility, personnel, and organizational documentation. } \\
\text { Provide copies upon request. }\end{array}$ & $6 / 15 / 96$ & $\mathrm{JJ}$ Dorian \\
\hline $\begin{array}{l}\text { 2. Conduct a comprehensive tour of all facilities and field } \\
\text { operations relevant to the organization. }\end{array}$ & $6 / 15 / 96$ & $\mathrm{JJ}$ Dorian \\
\hline
\end{tabular}




\begin{tabular}{|c|c|c|}
\hline Poot Awrard Actions & $\begin{array}{l}\text { Scheduled } \\
\text { Date: }\end{array}$ & Yctionee(s) \\
\hline $\begin{array}{l}\text { 3. Assist the PHMC in a survey of pre-existing conditions at the } \\
\text { facility. }\end{array}$ & $8 / 01 / 96$ & JJ Dorian \\
\hline $\begin{array}{l}\text { 4. Provide an update on work completed and work in progress } \\
\text { during the transition to the PHMC. }\end{array}$ & $9 / 15 / 96$ & JJ Dorian \\
\hline $\begin{array}{l}\text { 5. Complete a joint property and equipment inventory with the } \\
\text { PHMC. }\end{array}$ & $8 / 30 / 96$ & JJ Dorian \\
\hline $\begin{array}{l}\text { 6. Provide any additional documentation requested by the } \\
\text { PHMC. }\end{array}$ & ongoing & JJ Dorian \\
\hline 7. Provide necessary training to PHMC personnel. & $8 / 01 / 96$ & IJ Dorian \\
\hline 8. Provide a categorized inventory of wastes. & 9/30/96 & JJ Dorian \\
\hline
\end{tabular}

6.6.1.5 Geophysics Investigations. The geophysics investigations team conducts surface and vadose zone geophysical testing. Primary support is to the RCRA Operational Monitoring program and Environmental Remediation Program.

\begin{tabular}{|c|c|c|}
\hline Pre-Award Actions & $\begin{array}{l}\text { Scheduled } \\
\text { Date }\end{array}$ & Actionee(s) \\
\hline $\begin{array}{l}\text { 1. Prepare for a comprehensive briefing for the PHMC } \\
\text { contractor describing all operational aspects of the } \\
\text { organization. }\end{array}$ & $5 / 01 / 96$ & JW Fassett \\
\hline $\begin{array}{l}\text { 2. Identify all key documents relevant to the organization's } \\
\text { operations, including a listing of key facility and field } \\
\text { documents. }\end{array}$ & $5 / 01 / 96$ & JW Fassett \\
\hline $\begin{array}{l}\text { 3. Obtain copies of key facility documents and assemble, or } \\
\text { identify their location for access. Develop operations "Smart } \\
\text { Book." }\end{array}$ & $6 / 01 / 96$ & JW Fassett \\
\hline 4. Identify POCs for the organization. & $5 / 15 / 96$ & JW Fassett \\
\hline $\begin{array}{l}\text { 5. Using the pre-existing conditions checklist, evaluate pre- } \\
\text { existing conditions. Conduct assessments as necessary to } \\
\text { adequately address the checklist. }\end{array}$ & $6 / 01 / 96$ & JW Fassett \\
\hline $\begin{array}{l}\text { 6. Identify applicable milestones and commitments (e.g., Tri- } \\
\text { Party Agreement and Consent Order milestones), and } \\
\text { determine their status. Describe the form of the deliverable } \\
\text { and what constitutes completion. }\end{array}$ & $5 / 15 / 96$ & JW Fassett \\
\hline $\begin{array}{l}\text { 7. Verify the accuracy of property assignments for the facility } \\
\text { and correct any deficiencies. }\end{array}$ & $5 / 15 / 96$ & JW Fassett \\
\hline $\begin{array}{l}\text { 8. Verify the completeness of all RIDS and correct any } \\
\text { deficiencies. }\end{array}$ & $5 / 15 / 96$ & JW Fassett \\
\hline Post-Award Actions & r & 1 \\
\hline $\begin{array}{l}\text { 1. Provide a comprehensive briefing for the PHMC complete } \\
\text { with all facility, personnel, and organizational documentation. } \\
\text { Provide copies upon request. }\end{array}$ & $6 / 15 / 96$ & JW Fassett \\
\hline
\end{tabular}




\begin{tabular}{|c|c|c|}
\hline Post-Anard Actions & Schedaled & petontoe $(1)$ \\
\hline $\begin{array}{l}\text { 2. Conduct a comprehensive tour of all facilities and field } \\
\text { operations relevant to the organization. }\end{array}$ & $6 / 15 / 96$ & JW Fassett \\
\hline $\begin{array}{l}\text { 3. Assist the PHMC in a survey of pre-existing conditions at the } \\
\text { facility. }\end{array}$ & $8 / 01 / 96$ & JW Fassett \\
\hline $\begin{array}{l}\text { 4. Provide an update on work completed and work in progress } \\
\text { during the transition to the PHMC. }\end{array}$ & $9 / 15 / 96$ & JW Fasectt \\
\hline $\begin{array}{l}\text { 5. Complete a joint property and equipment inventory with the } \\
\text { PHMC. }\end{array}$ & $8 / 30 / 96$ & JW Fassett \\
\hline $\begin{array}{l}\text { 6. Provide any additional documentation requested by the } \\
\text { PHMC. }\end{array}$ & ongoing & JW Fassett \\
\hline 7. Provide necessary training to PHMC personnel. & $8 / 01 / 96$ & JW Fassett \\
\hline 8. Provide a categorized inventory of wastes. & $9 / 30 / 96$ & JW Fassett \\
\hline
\end{tabular}

6.6.1.6 Environmental Operations Sampling. The environmental operations sampling team conducts regulatory driven protocol sampling. Sampling applications include characterization, waste designation, recycling, and safety screening. Media sampled include but is not limited to soil, water, gas, vapor, oil, sludge, tar, concrete, facility effluents, filters, and asbestos. The team collects samples from wells, boreholes, cribs, ponds, ditches, drums, tanks, inside water lines, sewer lines, ducts, ion exchange columns, burial boxes, orphan containers, etc.

\begin{tabular}{|c|c|c|}
\hline Pre-Award Actions & Scheduled & Actionee(s) \\
\hline $\begin{array}{l}\text { 1. Prepare for a comprehensive briefing for the PHMC } \\
\text { contractor describing all operational aspects of the } \\
\text { organization. }\end{array}$ & $5 / 01 / 96$ & DL Edwards \\
\hline $\begin{array}{l}\text { 2. Identify all key documents relevant to the organization's } \\
\text { operations, including a listing of key facility and field } \\
\text { documents. }\end{array}$ & $5 / 01 / 96$ & DL Edwards \\
\hline $\begin{array}{l}\text { 3. Obtain copies of key facility documents and assemble, or } \\
\text { identify their location for access. Develop operations "Smart } \\
\text { Book." }\end{array}$ & $6 / 01 / 96$ & DL Edwards \\
\hline 4. Identify POCs for the organization. & $5 / 15 / 96$ & DL Edwards \\
\hline $\begin{array}{l}\text { 5. Using the pre-existing conditions checklist, evaluate pre- } \\
\text { existing conditions. Conduct assessments as necessary to } \\
\text { adequately address the checklist. }\end{array}$ & $6 / 01 / 96$ & DL Edwards \\
\hline $\begin{array}{l}\text { 6. Identify applicable milestones and commitments (e.g., Tri- } \\
\text { Party Agreement and Consent Order milestones), and } \\
\text { determine their status. Describe the form of the deliverable } \\
\text { and what constitutes completion. }\end{array}$ & $5 / 15 / 96$ & DL Edwards \\
\hline $\begin{array}{l}\text { 7. Verify the accuracy of property assignments for the facility } \\
\text { and correct any deficiencies. }\end{array}$ & $5 / 15 / 96$ & DL Edwards \\
\hline
\end{tabular}




\begin{tabular}{|c|c|c|}
\hline Pre-Award Actions & $\begin{array}{l}\text { Scheduled } \\
\text { Date }\end{array}$ & Actionee(s) \\
\hline $\begin{array}{l}\text { 8. Verify the completeness of all RIDS and correct any } \\
\text { deficiencies. }\end{array}$ & $5 / 15 / 96$ & DL Edwards \\
\hline Post-Award Actions & & \\
\hline $\begin{array}{l}\text { 1. Provide a comprehensive briefing for the PHMC complete } \\
\text { with all facility, personnel, and organizational documentation. } \\
\text { Provide copies upon request. }\end{array}$ & 6/15/96 & DL Edwards \\
\hline $\begin{array}{l}\text { 2. Conduct a comprehensive tour of all facilities and field } \\
\text { operations relevant to the organization. }\end{array}$ & $6 / 15 / 96$ & DL Edwards \\
\hline $\begin{array}{l}\text { 3. Assist the PHMC in a survey of pre-existing conditions at the } \\
\text { facility. }\end{array}$ & $8 / 01 / 96$ & DL Edwards \\
\hline $\begin{array}{l}\text { 4. Provide an update on work completed and work in progress } \\
\text { during the transition to the PHMC. }\end{array}$ & $9 / 15 / 96$ & DL Edwards \\
\hline $\begin{array}{l}\text { 5. Complete a joint property and equipment inventory with the } \\
\text { PHMC. }\end{array}$ & $8 / 30 / 96$ & DL Edwards \\
\hline $\begin{array}{l}\text { 6. Provide any additional documentation requested by the } \\
\text { PHMC. }\end{array}$ & ongoing & DL Edwards \\
\hline 7. Provide necessary training to PHMC personnel. & $8 / 01 / 96$ & DL Edwards \\
\hline 8. Provide a categorized listing of wastes. & 9/30/96 & DL Edwards \\
\hline
\end{tabular}

6.6.1.7 Special Projects. The Indian Nation Program coordinates and facilitates projects at Hanford with participating tribes. Two projects currently being supported are (1) fish rearing projects at 100-K Area water purification pools in which salmonid, sturgeon, and warm water species are being reared for enhancing Columbia River Basin fisheries and commercial purposes and (2) a revegetation project to restore disturbed soil and the environment to the natural shrubbe step state.

\begin{tabular}{|l|l|l|}
\hline \multicolumn{1}{|c|}{ Pre-Award Actions } & $\begin{array}{c}\text { Scheduled } \\
\text { Date }\end{array}$ & $5 / 01 / 96$ \\
\hline $\begin{array}{l}\text { 1. Prepare for a comprehensive briefing for the PHMC } \\
\text { contractor describing all planning, implementation, and } \\
\text { operational aspects of the project. }\end{array}$ & DI Herborn \\
\hline $\begin{array}{l}\text { 2. Identify all key documents relevant to the project, including a } \\
\text { listing of key agreements and plans. }\end{array}$ & $5 / 01 / 96$ & DI Herborn \\
\hline $\begin{array}{l}\text { 3. Identify points-of-contact for all internal and external } \\
\text { organizations involved in the projects. }\end{array}$ & $5 / 15 / 96$ & DI Herborn \\
\hline $\begin{array}{l}\text { 4. Identify applicable commitments and projects and determine } \\
\text { their status. Describe the form of the deliverable and what } \\
\text { constitutes completion. }\end{array}$ & $5 / 15 / 96$ & DI Herborn \\
\hline $\begin{array}{l}\text { 5. Verify the accuracy of property assignments for the project } \\
\text { and correct deficiencies. }\end{array}$ & $5 / 15 / 96$ & DI Herborn \\
\hline
\end{tabular}




\begin{tabular}{|c|c|c|}
\hline Post-A ward Actions & $\begin{array}{l}\text { Scheduled } \\
\text { Date }\end{array}$ & Actionee $(\mathrm{O})$ \\
\hline $\begin{array}{l}\text { 1. Provide a comprehensive briefing for the PHMC complete } \\
\text { with all project documentation. Provide copies upon request. }\end{array}$ & $6 / 15 / 96$ & DI Herborn \\
\hline $\begin{array}{l}\text { 2. Conduct a comprehensive tour of all facilities and field } \\
\text { operations relevant to the projects. }\end{array}$ & $6 / 15 / 96$ & DI Herborn \\
\hline $\begin{array}{l}\text { 3. Provide an update on work completed and work in progress } \\
\text { during the transition to the PHMC. }\end{array}$ & $9 / 15 / 96$ & DI Herborn \\
\hline $\begin{array}{l}\text { 4. Provide any additional documentation requested by the } \\
\text { PHMC. }\end{array}$ & ongoing & DI Herborn \\
\hline
\end{tabular}

\subsubsection{CERCLA Monitoring}

Under its separate prime contract with RL BHI has responsibility for the management and coordination of Sitewide Comprehensive Environmental Response, Compensation, and Liability Act (CERCLA) monitoring activities. The scope includes groundwater characterization and monitoring services conducted by $\mathrm{BHI}$ to support investigations under the CERCLA program. CERCLA investigations are underway for a number of areas where known or suspected environmental contamination may have resulted from past Site practices.

PHMC Transition activities relevant to the CERCLA Monitoring program should focus on understanding the working relationships between PHMC, PNNL, and BHI for environmental monitoring; continuation of support services to BHI through select work orders; and PHMC assuming responsibility for applying the data collected by these activities for sitewide integration.

\begin{tabular}{|c|c|c|}
\hline Pre-Award Actions & $\begin{array}{c}\text { Scheduled } \\
\text { Date }\end{array}$ & Actionee(s) \\
\hline $\begin{array}{l}\text { 1. Prepare a briefing for the PHMC's environmental monitoring } \\
\text { programs representatives, describing interactions between } \\
\text { BHI, PNNL, and the PHMC, and relevant operational } \\
\text { aspects of BHI's organization. }\end{array}$ & $5 / 30 / 96$ & $\begin{array}{l}\text { SC Foelber } \\
\text { WL Pamplin }\end{array}$ \\
\hline $\begin{array}{l}\text { 2. Identify all key documents relevant to the workscopes and } \\
\text { interactions between the PHMC and BHI organizations for } \\
\text { environmental monitoring activities (including Sitewide } \\
\text { agreements and MOUs). }\end{array}$ & $5 / 01 / 96$ & WL Pamplin \\
\hline 3. Identify appropriate BHI points-of-contact for the PHMC. & $5 / 01 / 96$ & SC Foelber \\
\hline 4. List active work orders and their expiration dates. & $4 / 22 / 96$ & WL Pamplin \\
\hline $\begin{array}{l}\text { 5. Determine which support services work orders will be } \\
\text { continued and/or renewed, and which are candidates for } \\
\text { discontinuation. Document rationale. }\end{array}$ & $5 / 01 / 96$ & $\begin{array}{l}\text { SC Foelber } \\
\text { WL Pamplin }\end{array}$ \\
\hline
\end{tabular}




\begin{tabular}{|c|c|c|}
\hline Preaward fetions & ocheduled & 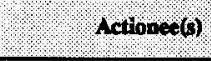 \\
\hline $\begin{array}{l}\text { 6. Identify applicable in-process milestones and commitments } \\
\text { for which PHMC must pick up supporting or contributing } \\
\text { responsibility (e.g., Tri-Party Agreement and Consent Order } \\
\text { milestones), and determine their status. Describe the form of } \\
\text { the support/involvement and what constitutes completion. }\end{array}$ & $5 / 10 / 96$ & WL Pamplin \\
\hline 7. Identify pre-existing conditions, as applicable. & $5 / 24 / 96$ & WL Pamplin \\
\hline Poot Award Actions & & \\
\hline $\begin{array}{l}\text { 1. Provide a briefing for the PHMC relevant to interactions } \\
\text { between BHI and PHMC, and PHMC support services for } \\
\text { ongoing and planned CERCLA monitoring activities. }\end{array}$ & $6 / 15 / 96$ & $\begin{array}{l}\text { SC Foelber } \\
\text { WL Pamplin }\end{array}$ \\
\hline $\begin{array}{l}\text { 2. Identify to PHMC those work orders for PHMC services that } \\
\text { BHI will continue and/or renew, and any work orders BHI is } \\
\text { considering discontinuing (offer PHMC opportunity to } \\
\text { improve by specified date). }\end{array}$ & $6 / 15 / 96$ & $\begin{array}{l}\text { SC Foelber } \\
\text { WL Pamplin }\end{array}$ \\
\hline $\begin{array}{l}\text { 3. Establish routine points of contact and agreed method and } \\
\text { frequency of interface between PHMC and BHI. }\end{array}$ & $6 / 15 / 96$ & WL Pamplin \\
\hline $\begin{array}{l}\text { 4. Reach concurrence and document agreement on continuation } \\
\text { of all work in-progress involving support from PHMC. }\end{array}$ & $7 / 26 / 96$ & (Numerous) \\
\hline $\begin{array}{l}\text { 5. If requested, provide the PHMC a tour of field sites to clarify } \\
\text { areas of BHI program responsibility (vs. PHMC and PNNL), } \\
\text { including discussion of relevant pre-existing site conditions. }\end{array}$ & TBD & WL Pamplin \\
\hline 6. Update/renew active work orders. as appropriate. & $9 / 13 / 96$ & WL Pamplin \\
\hline
\end{tabular}

\subsubsection{Groundwater Surveillance Monitoring Project}

\begin{tabular}{|c|c|c|}
\hline Fre-Awerd Actions & $\begin{array}{l}\text { Scheduled } \\
\text { Datie }\end{array}$ & Actonos (s) \\
\hline $\begin{array}{l}\text { 1. Prepare briefing for PHMC. decrnbiag relevant scope and } \\
\text { operational aspects of PNNL prosram and interactions } \\
\text { between PNNL, BHI. and PHMC. }\end{array}$ & $6 / 01 / 96$ & TL Page \\
\hline Podt-Awerd Actions & & \\
\hline $\begin{array}{l}\text { 1. Provide a briefing for PHMC, describing relevant scope and } \\
\text { operational aspects of PNNL program and interactions } \\
\text { between PNNL, BHI, and PHMC. }\end{array}$ & $6 / 14 / 96$ & TL Page \\
\hline $\begin{array}{l}\text { 2. Establish routine points of contact and agreed method and } \\
\text { frequency of interface between PNNL and PHMC. }\end{array}$ & $6 / 14 / 96$ & TL Page \\
\hline
\end{tabular}




\subsection{EXTERNAL/INTERNAL COMMUNICATIONS}

\subsubsection{Interface with Site Communications/Public Affairs}

The PHMC will be responsible for its own public relations and communications activities.

\begin{tabular}{|c|c|c|}
\hline Post- A ward Actions & $\begin{array}{l}\text { Sdiednled } \\
\text { Date }\end{array}$ & Actionee (s) \\
\hline $\begin{array}{l}\text { 1. Arrange meeting of PHMC public relations/communications } \\
\text { otaff with the Hanford Public Affairs Cabinet (all Hanford } \\
\text { Site public affairs/communications managers) and welcome } \\
\text { them to the cabinet. }\end{array}$ & $6 / 10 / 96$ & KK Randolph \\
\hline
\end{tabular}

\subsubsection{Integration of WHC/ICF KH/BCSR Program Components Into the PHMC Program}

Coordinate with the PHMC to integrate the existing employee communications, media relations, public involvement and community relations programs into the PHMC's program.

\begin{tabular}{|l|c|c|}
\hline & Scheduled & Date \\
\hline $\begin{array}{l}\text { 1. Arrange a meeting with RL and the PHMC to discuss the } \\
\text { WHC/ICF KH/BCSR communications program and potential } \\
\text { integration of the existing program. }\end{array}$ & $6 / 15 / 96$ & $\begin{array}{l}\text { PM Phelps } \\
\text { LE Love } \\
\text { KK Randolph }\end{array}$ \\
\hline $\begin{array}{l}\text { 2. Ensure that WHC/ICF KH/BCSR staff managers discuss } \\
\text { departmental responsibilities and activities with the PHMC. }\end{array}$ & 7/01/96 & $\begin{array}{l}\text { PM Phelps } \\
\text { LE Love }\end{array}$ \\
\hline
\end{tabular}

\subsubsection{Tri-Party Agreement Community Relations Plan}

WHC has responsibility for providing direct support to the RL Office of External Affairs, which has responsibility for implementing the Tri-Party Agreement Community Relations Plan (Ecology et al. 1990). The PHMC will be expected to take over these responsibilities.

\begin{tabular}{|c|c|c|}
\hline Post-Award Actions & $\begin{array}{l}\text { Scheduled } \\
\text { Dote }\end{array}$ & Aefionees $(\beta)$ \\
\hline $\begin{array}{l}\text { 1. Use regularly schedule monthly meetings of } \mathrm{RL} \text { and WHC } \\
\text { Project Managers and Public Information Officers to brief the } \\
\text { PHMC POCs on the Community Relations Plan schedule, } \\
\text { delineate responsibilities, and establish mechanisms for } \\
\text { ongoing support from the PHMC. }\end{array}$ & $6 / 03 / 96$ & $\begin{array}{l}\text { KK Randolph } \\
\text { JK Yerxa } \\
\text { PJ Bengtson }\end{array}$ \\
\hline
\end{tabular}




\begin{tabular}{|c|c|c|}
\hline Post-Award Actions & $\begin{array}{l}\text { Schediled } \\
\text { Depte }\end{array}$ & Hetionee (s) \\
\hline $\begin{array}{l}\text { 2. Recommend the PHMC submit material to the quarterly } \\
\text { Hanford Update Tri-Party Agreement newsletter and attend } \\
\text { public meetings to introduce key PHMC officials to interested } \\
\text { stakeholders. }\end{array}$ & $6 / 03 / 96$ & PHMC \\
\hline
\end{tabular}

\subsubsection{Introduce the PHMC Personnel to Hanford's External Publics}

Arrange for the PHMC personnel to meet key RL, Hanford Site contractors; neighbors, business and local government officials; the news media; and special-interest groups.

\begin{tabular}{|c|c|c|}
\hline Post-Award Actions & Scheduled & Actionee(s) \\
\hline $\begin{array}{l}\text { 1. Arrange a meeting to introduce the PHMC to key RL staff } \\
\text { and contractor principals. }\end{array}$ & $6 / 10 / 96$ & $\begin{array}{l}\text { JD Wagoner } \\
\text { KK Randolph }\end{array}$ \\
\hline $\begin{array}{l}\text { 2. Arrange a meeting to introduce the PHMC to local } \\
\text { government and business officials. }\end{array}$ & $6 / 10 / 96$ & $\begin{array}{l}\text { JD Wagoner } \\
\text { KK Randolph }\end{array}$ \\
\hline $\begin{array}{l}\text { 3. Coordinate with the PHMC to arrange an onsite state } \\
\text { legislator and congressional briefing as directed by RL. }\end{array}$ & $6 / 10 / 96$ & KK Randolph \\
\hline 4. Recommend the PHMC schedule a media briefing. & $6 / 10 / 96$ & $\begin{array}{l}\text { KK Randolph } \\
\text { PM Phelps } \\
\text { LE Love }\end{array}$ \\
\hline 5. Brief the PHMC on the Hanford Advisory Board. & $6 / 15 / 96$ & $\begin{array}{l}\text { JK Yerxa } \\
\text { WJ Sanderson }\end{array}$ \\
\hline
\end{tabular}

\subsubsection{Provide Employees With Necessary Information During Transition} activities.

Keep WHC/ICF KH/BCSR employees informed about the PHMC and transition

\begin{tabular}{|c|c|c|}
\hline Pre-Award Actions & $\begin{array}{c}\text { Scheduled } \\
\text { Date }\end{array}$ & Actionee(s) \\
\hline $\begin{array}{l}\text { 1. Continue to address WHC/ICF KH/BCSR employees' } \\
\text { concerns and information needs regarding the transition } \\
\text { through Management Network and Hanford Reach articles } \\
\text { addressing key questions and answers. }\end{array}$ & $6 / 01 / 96$ & $\begin{array}{l}\text { TL Cozzens } \\
\text { DL Cresswell } \\
\text { LE Love } \\
\text { ML Jackson } \\
\text { PS Hale }\end{array}$ \\
\hline
\end{tabular}




\begin{tabular}{|c|c|c|}
\hline Post-Avard Actions & $\begin{array}{l}\text { Scheduled } \\
\text { Date }\end{array}$ & Xctionee( \\
\hline $\begin{array}{l}\text { 1. Hold all-manager meeting for WHC/ICF } \mathrm{KH} / \mathrm{BCSR} \\
\text { managers to discuss transition actions, schedule and } \\
\text { responsibilities. }\end{array}$ & $6 / 15 / 96$ & $\begin{array}{l}\text { TL Cozzens } \\
\text { SA Woody } \\
\text { LE Love } \\
\text { ML Jackson }\end{array}$ \\
\hline $\begin{array}{l}\text { 2. Provide special Hanford Reach and Management Network } \\
\text { coverage for the transition, including coverage of the } \\
\text { turnover meetings/receptions (6.7.8) and articles about the } \\
\text { new contractors and their parent firms. }\end{array}$ & $6 / 10 / 96$ & $\begin{array}{l}\text { TL Cozzens } \\
\text { DL Cresswell } \\
\text { PS Hale } \\
\text { ML Jackson }\end{array}$ \\
\hline $\begin{array}{l}\text { 3. Evaluate employee suggestions and concerns regarding the } \\
\text { transition and adjust communications plan, if necessary. }\end{array}$ & $6 / 15 / 96$ & $\begin{array}{l}\text { TL Cozzens } \\
\text { WHC Communications } \\
\text { Council } \\
\text { LE Love } \\
\text { ML Jackson }\end{array}$ \\
\hline $\begin{array}{l}\text { 4. Provide assistance to the PHMC in scheduling and presenting } \\
\text { briefing sessions for employees on benefits package and to } \\
\text { answer questions. Include evening/off-shift sessions which } \\
\text { spouses of affected employees can attend. }\end{array}$ & $6 / 15 / 96$ & $\begin{array}{l}\text { HA Sieber } \\
\text { PHMC }\end{array}$ \\
\hline $\begin{array}{l}\text { 5. Publish a "key contacts" list for employees to use to raise } \\
\text { ongoing or unresolved issues, questions. }\end{array}$ & $\begin{array}{c}6 / 15 / 96 \\
10 / 01 / 96\end{array}$ & $\begin{array}{l}\text { TL Cozens } \\
\text { HA Sieber } \\
\text { PHMC } \\
\text { LE Love }\end{array}$ \\
\hline $\begin{array}{l}\text { 6. Provide a form for submitting written employee questions, } \\
\text { and identify response paths (such as general-interest questions } \\
\text { in Management Network or Hanford Reach, and individual } \\
\text { questions addressed directly to employees). }\end{array}$ & $6 / 03 / 96$ & $\begin{array}{l}\text { HA Sieber } \\
\text { TL Cozzens } \\
\text { PHMC } \\
\text { ML Jackson }\end{array}$ \\
\hline
\end{tabular}

\subsubsection{U.S. Department of Energy "Hanford Reach" and "DOE This Month" Communications}

WHC has responsibility for producing the Hanford Reach, an employee site newspaper, for RL. Since November 1994, WHC also has had responsibility for producing DOE This Month, a monthly publication for DOE Headquarters. The PHMC will be expected to take over these responsibilities.

\begin{tabular}{|l|c|l|}
\hline \multicolumn{1}{|c|}{ Post-Award Aetions } & $\begin{array}{c}\text { Scheduled } \\
\text { Date }\end{array}$ & Actionee(s) \\
\hline $\begin{array}{l}\text { 1. Develop and implement a plan for take-over of the Hanford } \\
\text { Reach weekly site newspaper, so that production continues } \\
\text { uninterrupted. }\end{array}$ & $\begin{array}{c}6 / 15 / 96 \\
10 / 01 / 96\end{array}$ & DL Cresswell \\
\hline $\begin{array}{l}\text { 2. Develop and implement a plan for take-over of DOE This } \\
\text { Month. }\end{array}$ & $6 / 15 / 96$ & TL Cozzens \\
\end{tabular}




\subsubsection{Hanford Site Emergency Preparedness Communications}

WHC has responsibility for implementing the Hanford Site Emergency Preparedness Plan. Accordingly, WHC Communications has the lead in implementing Emergency Preparedness Communications and staffing for the Joint Information Center (JIC). Communications staff from all Hanford Site prime contractors participate in the JIC and related emergency communications efforts. The PHMC will need to take over this responsibility.

\begin{tabular}{|c|c|c|}
\hline $\mathrm{Pos}+\mathrm{Aw}$ nrd fothens & Solecinited & 1 tetones \\
\hline $\begin{array}{l}\text { 1. Develop and implement a plan for take-over of Emergency } \\
\text { Preparedness Communications activities and plans. }\end{array}$ & $\begin{array}{l}6 / 15 / 96 \\
10 / 01 / 96\end{array}$ & SA Woody \\
\hline
\end{tabular}

\subsubsection{WHC/ICF Kaiser/BCSR Farewell Activities}

Arrange activities for WHC/ICF KH/BCSR employees, to bid farewell to coworkers moving to new contractors and be recognized by WHC management for their contributions, in order that the transition and continued teaming will be positive. Arrange farewell activities for external audiences.

\begin{tabular}{|c|c|c|}
\hline Post-Award Actions & $\begin{array}{l}\text { Scheduled } \\
\text { Date }\end{array}$ & Actionee(s) \\
\hline $\begin{array}{l}\text { 1. Schedule and conduct a series of meetings/receptions for all } \\
\text { employees to introduce them to their new management team, } \\
\text { and recognizing them for past contributions and } \\
\text { achievements. }\end{array}$ & $7 / 15 / 96$ & $\begin{array}{l}\text { TL Cozzens } \\
\text { SA Woody } \\
\text { MJ Auckland } \\
\text { LE Love } \\
\end{array}$ \\
\hline $\begin{array}{l}\text { 2. Place an advertisement in the Tri-City Herald and Yakima } \\
\text { Herald-Republic thanking WHC/ICF KH/BCSR employees } \\
\text { transferring to the new PHMC for their past contributions } \\
\text { and anticipated continued contributions as a member of the } \\
\text { Hanford Site team under their new employer. }\end{array}$ & $10 / 01 / 96$ & $\begin{array}{l}\text { TL Cozzens } \\
\text { PM Phelps } \\
\text { LE Love }\end{array}$ \\
\hline 3. Schedule a final news media interview for LaMar Trego. & $10 / 01 / 96$ & JC Britton \\
\hline $\begin{array}{l}\text { 4. Mail WHC/ICF KH/BCSR farewell letter to employees and } \\
\text { retirees. }\end{array}$ & 9/15/96 & $\begin{array}{l}\text { TL Cozzens } \\
\text { LE Love }\end{array}$ \\
\hline $\begin{array}{l}\text { 5. Mail WHC/CF KH/BCSR farewell letter to neighbors, } \\
\text { community leaders, activists, school contacts and } \\
\text { congressional representatives. }\end{array}$ & $9 / 15 / 96$ & $\begin{array}{l}\text { PM Phelps } \\
\text { LE Love }\end{array}$ \\
\hline $\begin{array}{l}\text { 6. Recommend the PHMC host a turnover reception for the } \\
\text { media and community. }\end{array}$ & $10 / 01 / 96$ & $\begin{array}{l}\text { KK Randolph } \\
\text { PM Phelps } \\
\text { LE Love }\end{array}$ \\
\hline
\end{tabular}




\subsection{TRAINING}

\begin{tabular}{|c|c|c|}
\hline Pre-Award Actions & $\begin{array}{l}\text { Scheduled } \\
\text { Dete }\end{array}$ & Actiones (s) \\
\hline $\begin{array}{l}\text { 1. Identify space and equipment within training areas and facilities } \\
\text { for use by the PHMC. }\end{array}$ & 6/01/96 & CL Nansen \\
\hline $\begin{array}{l}\text { 2. In outline form for all facilities/operations to follow, define the } \\
\text { appropriate contents and level of detail to be included in training } \\
\text { briefings to the PHMC (review with RL POC). }\end{array}$ & $5 / 01 / 96$ & CL Nansen \\
\hline Post-Award Actions & & \\
\hline $\begin{array}{l}\text { 1. PHMC identify spsce and equipment needs within } \\
\text { program/project area and facilities. }\end{array}$ & by $6 / 08 / 96$ & PHMC \\
\hline $\begin{array}{l}\text { 2. Provide training facility space and services for use by the } \\
\text { PHMC. }\end{array}$ & $6 / 15 / 96$ & CL Nansen \\
\hline 3. Provide training briefing(s) to the PHMC. & $6 / 15 / 96-7 / 01 / 96$ & CL Nansen \\
\hline 4. Provide training level tour to the PHMC. & $6 / 15 / 96-7 / 01 / 96$ & CL Nansen \\
\hline
\end{tabular}

\subsubsection{Training Services}

Training Services (TRS) provides training that facilitates excellence in radiological safety, industrial safety and health, and EM, and contributes to the improvement of technical and administrative performance. It also provides resources and services that assist line management in providing facility-specific training on processes and tools used to improve those processes. TRS provides administrative support to the training process with the Training Matrix (which identifies position-specific training needs for individual employees), training scheduling and registration, and training completion records. Functions of training audit and evaluation are also provided, as well as training partnerships with community educational institutions.

\begin{tabular}{|c|c|c|}
\hline Pre-Award Actions & $\begin{array}{l}\text { Scheduled } \\
\text { Date }\end{array}$ & Actionet(s) \\
\hline $\begin{array}{l}\text { 1. Prepare Training Services briefing for the PHMC } \\
\text { (coordinate content with RL POC). }\end{array}$ & $6 / 01 / 96$ & CP Wick \\
\hline $\begin{array}{l}\text { 2. Identify key documents for Training Services. Provide } \\
\text { listing to CL Nansen for consolidation and RL review. }\end{array}$ & $5 / 01 / 96$ & CP Wick \\
\hline $\begin{array}{l}\text { 3. Assemble Training Services documentation, or identify } \\
\text { its location, for use by the PHMC. Includes } \\
\text { preparation of a Training Services "Smart Book," to } \\
\text { include information developed in the following actions. }\end{array}$ & $6 / 01 / 96$ & CP Wick \\
\hline $\begin{array}{l}\text { 4. Identify POCs for Training Services and input to } \\
\text { CL Nansen. }\end{array}$ & $5 / 15 / 96$ & CP Wick \\
\hline $\begin{array}{l}\text { 5. Develop a listing and description of subcontracts } \\
\text { supporting Training Services and input to CL Nansen. } \\
\text { Include any subcontracts planned to be executed prior } \\
\text { to contract takeover. }\end{array}$ & $5 / 15 / 96$ & CP Wick \\
\hline
\end{tabular}




\begin{tabular}{|c|c|c|}
\hline Pret Award Actions & $\begin{array}{l}\text { Scheduled } \\
\text { Date }\end{array}$ & Actioner(s) \\
\hline $\begin{array}{l}\text { 6. Using the pre-existing checklist, compile pre-existing } \\
\text { condition information on Training Services facilities } \\
\text { and/or major equipment. Conduct, or schedule as a } \\
\text { separate post-award action(s), any assessments required } \\
\text { to adequately address all relevant items on the } \\
\text { checklist. Provide completed checklist to RL POC for } \\
\text { review. }\end{array}$ & 6/01/96 & RG Slocum \\
\hline $\begin{array}{l}\text { 7. Identify applicable milestones and commitments (e.g., } \\
\text { Tri-Party Agreement, DNFSB and consent order } \\
\text { milestones), and determine their status. Describe the } \\
\text { form of the deliverable and what constitutes } \\
\text { completion. Provide to RL POC for review. }\end{array}$ & $6 / 01 / 96$ & CP Wick \\
\hline $\begin{array}{l}\text { 8. Develop a listing of special agreement services } \\
\text { provided to RL, other DOE sites, and other entities. }\end{array}$ & $6 / 01 / 96$ & CP Wick \\
\hline $\begin{array}{l}\text { 9. Develop a listing and description of TRS databases. } \\
\text { Provide list to CL Nansen for consolidation. }\end{array}$ & $5 / 15 / 96$ & CP Wick \\
\hline $\begin{array}{l}\text { 10. Develop a list of current work order services or other } \\
\text { form of agreement to provide work for other } \\
\text { organizations, on and offsite. }\end{array}$ & $6 / 01 / 96$ & CP Wick \\
\hline $\begin{array}{l}\text { 11. Develop a list of current work order services or other } \\
\text { form of agreement to obtain work from other } \\
\text { organizations, on and offsite. }\end{array}$ & $6 / 01 / 96$ & CP Wick \\
\hline $\begin{array}{l}\text { 12. Verify the accuracy of property assignments for the } \\
\text { TRS organization and correct any deficiencies. }\end{array}$ & 6/01/96 & CP Wick \\
\hline Post-A ward Actions & 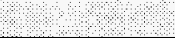 & 14 \\
\hline 1. Provide TRS briefing(s) to the PHMC. & $7 / 01 / 96-7 / 15 / 96$ & RG Slocum \\
\hline 2. Provide a TRS tour to the PHMC. & $7 / 01 / 96-7 / 15 / 96$ & RG Slocum \\
\hline $\begin{array}{l}\text { 3. Provide the PHMC access to key documentation, pre- } \\
\text { existing condition information, commitment data, } \\
\text { inventory data, etc. (as established in the pre-award } \\
\text { phase) Provide copies upon request. }\end{array}$ & by $6 / 15 / 96$ & CP Wick \\
\hline $\begin{array}{l}\text { 4. Provide any additional documentation or information } \\
\text { requested by the PHMC, if available, that was not } \\
\text { identified during the pre-award phase. }\end{array}$ & by $7 / 01 / 96$ & CP Wick \\
\hline $\begin{array}{l}\text { 5. PHMC identify any recurring reports that they would } \\
\text { like to receive during transition. }\end{array}$ & by $7 / 15 / 96$ & PHMC \\
\hline $\begin{array}{l}\text { 6. Provide an updated status of ongoing work prior to } \\
\text { contract turnover. }\end{array}$ & by $9 / 30 / 96$ & CP Wick \\
\hline $\begin{array}{l}\text { 7. Perform a joint property and equipment inventory with } \\
\text { the PHMC. }\end{array}$ & by $8 / 30 / 96$ & CP Wick \\
\hline $\begin{array}{l}\text { 8. Assist the PHMC in the conduct of a Pre-Existing } \\
\text { Condition Assessment. }\end{array}$ & $7 / 15 / 96-8 / 30 / 96$ & CP Wick \\
\hline $\begin{array}{l}\text { 9. Provide a list of PHMC personnel requiring HGET and } \\
\text { other general training. }\end{array}$ & $6 / 15 / 96$ & PHMC \\
\hline $\begin{array}{l}\text { 10. Provide HGET and other general site training to } \\
\text { PHMC personnel as needed for transition. }\end{array}$ & 6/15/96-7/15/96 & CP Wick \\
\hline
\end{tabular}




\subsubsection{Hazardous Materials Management and Emergency Response}

Hazardous Materials Management and Emergency Response (HAMMER) is a worker training and education program for hazardous material, waste management, and emergency response workers. The HAMMER facility is operated as a user center which hosts, brokers, and integrates the capabilities of its partnering organizations to ensure the delivery of state of the art training and education curricula. Its main emphasis is the delivery of its products and services for DOE and DOE contractor workers, managers, and emergency responders. HAMMER will host training focused on improving safety and health while reducing training costs and establishing a new training industry for the region.

\begin{tabular}{|c|c|c|}
\hline Pre-Award Actions & $\begin{array}{l}\text { Scheduled } \\
\text { Date }\end{array}$ & Actionde(s) \\
\hline $\begin{array}{l}\text { 1. Prepare HAMMER briefing for the PHMC (coordinate } \\
\text { content with RL POC). }\end{array}$ & $6 / 01 / 96$ & PJ Vandervert \\
\hline $\begin{array}{l}\text { 2. Identify key documents for the HAMMER } \\
\text { organization. Provide to RL POC for review. }\end{array}$ & $5 / 01 / 96$ & PJ Vandervert \\
\hline $\begin{array}{l}\text { 3. Assemble key HAMMER documentation, or identify } \\
\text { its location, for use by the PHMC. Includes } \\
\text { preparation of a HAMMER "Smart Book," to include } \\
\text { information developed in the following actions. }\end{array}$ & $6 / 01 / 96$ & PJ Vandervert \\
\hline 4. Identify POCs for HAMMER. & $6 / 01 / 96$ & PJ Vandervert \\
\hline $\begin{array}{l}\text { 5. Develop a listing and description of subcontracts } \\
\text { supporting HAMMER. Include any subcontracts } \\
\text { planned to be executed prior to contract takeover. }\end{array}$ & $6 / 01 / 96$ & PJ Vandervert \\
\hline $\begin{array}{l}\text { 6. Using the pre-existing checklist, compile pre-existing } \\
\text { condition information on HAMMER Facilities and/or } \\
\text { major equipment. Conduct, or schedule as a separate } \\
\text { post-award action(s), any assessments required to } \\
\text { adequately address all relevant items on the checklist. } \\
\text { Provide completed checklist to RL POC for review. }\end{array}$ & $6 / 01 / 96$ & PJ Vandervert \\
\hline $\begin{array}{l}\text { 7. Identify applicable milestones and commitments (e.g., } \\
\text { Tri-Party Agreement, DNFSB and consent order } \\
\text { milestones), and determine their status. Describe the } \\
\text { form of the deliverable and what constitutes } \\
\text { completion. Provide to RL POC for review. }\end{array}$ & 6/01/96 & PJ Vandervert \\
\hline $\begin{array}{l}\text { 8. Develop a description of ongoing and planned } \\
\text { construction projects. }\end{array}$ & $6 / 01 / 96$ & PJ Vandervert \\
\hline $\begin{array}{l}\text { 9. Develop a listing of special agreement services } \\
\text { provided to RL, other DOE sites, and other entities. }\end{array}$ & $6 / 01 / 96$ & PJ Vandervert \\
\hline $\begin{array}{l}\text { 10. Develop a listing and description of HAMMER } \\
\text { databases. }\end{array}$ & $6 / 01 / 96$ & PJ Vandervert \\
\hline $\begin{array}{l}\text { 11. Develop a list of current work order services or other } \\
\text { form of agreement to provide work for other } \\
\text { organizations, on and offsite. }\end{array}$ & $6 / 01 / 96$ & PJ Vandervert \\
\hline
\end{tabular}




\begin{tabular}{|c|c|c|}
\hline Pre Award Actions & $\begin{array}{l}\text { Soneduled } \\
\text { phos }\end{array}$ & $10010 \mathrm{sec}(\mathrm{s})$ \\
\hline $\begin{array}{l}\text { 12. Develop a list of current work order services or other } \\
\text { form of agreement to obtain work from other } \\
\text { organizations, on and offsite. }\end{array}$ & $6 / 01 / 96$ & PJ Vandervert \\
\hline $\begin{array}{l}\text { 13. Verify the accuracy of property assignments for the } \\
\text { HAMMER organization and correct any deficiencies. }\end{array}$ & $6 / 01 / 96$ & PJ Vandervert \\
\hline Post-Award Actions & & \\
\hline 1. Provide a HAMMER briefing to the PHMC. & $7 / 01 /-7 / 15 / 96$ & KA McGinnis \\
\hline 2. Provide a HAMMER tour to the PHMC. & $7 / 01-7 / 15 / 96$ & KA McGinnis \\
\hline $\begin{array}{l}\text { 3. Provide the PHMC access to key documentation, pre- } \\
\text { existing condition information, commitment data, } \\
\text { inventory data, etc. (as established in the pre-award } \\
\text { phase). Provide copies upon request. }\end{array}$ & prior to $6 / 15 / 96$ & PJ Vandervert \\
\hline $\begin{array}{l}\text { 4. Provide any additional documentation or information } \\
\text { requested by the PHMC, if available, that was not } \\
\text { identified during the pre-award phase. }\end{array}$ & by $7 / 01 / 96$ & PJ Vandervert \\
\hline $\begin{array}{l}\text { 5. PHMC identify any recurring reports that they would } \\
\text { like to receive during transition. }\end{array}$ & by $7 / 15 / 96$ & PHMC \\
\hline $\begin{array}{l}\text { 6. Provide an updated status of ongoing work prior to } \\
\text { contract turnover. }\end{array}$ & by $9 / 30 / 96$ & PJ Vandervert \\
\hline $\begin{array}{l}\text { 7. Perform a joint property and equipment inventory with } \\
\text { the PHMC. }\end{array}$ & by $8 / 30 / 96$ & PJ Vandervert \\
\hline $\begin{array}{l}\text { 8. Assist the PHMC in the conduct of a Pre-existing } \\
\text { Condition Assessment. }\end{array}$ & $7 / 15-8 / 30 / 96$ & PJ Vandervert \\
\hline
\end{tabular}

\subsection{EMERGENCY PREPAREDNESS}

\subsubsection{Emergency Management}

The Emergency Preparedness Program provides an emergency management system including development, coordination, and direction of planning, preparedness, and readiness assurance for response to emergency events on the Hanford Site; and emergency management support to RL.

The Emergency Preparedness Program meets the requirements for an integrated Hanford Site emergency program detailed in DOE/RL-94-02 Hanford Emergency Response Plan. This plan implements the DOE emergency management orders as well as applicable state and Federal regulations to ensure the health and safety of Site employees and the general public. 
Responsibilities of the organization include hazards assessments that form the basis of the emergency management program, planning to ensure integrated onsite emergency response, assisting $\mathrm{RL}$ with planning with state and local agencies to ensure that the public can be protected, developing and maintaining RL and contractor emergency plans and procedures, staffing and maintaining RL and contractor emergency response facilities, emergency notifications, maintaining the readiness of emergency response organizations through training and drills, and validating the Site Emergency Management program through exercises, evaluation and surveillances.

The Emergency Preparedness Program has the lead for a Hanford Site Emergency Response Organization Re-engineering project. This effort will provide a streamlined emergency response organization, while maintaining a strong response capability for the Site.

\begin{tabular}{|c|c|c|}
\hline Pre-Award Actions & $\begin{array}{l}\text { Scheduled } \\
\text { Date }\end{array}$ & Actionee(s) \\
\hline $\begin{array}{l}\text { 1. Develop list of documentation for the Emergency Preparedness } \\
\text { Program. Provide to RL POC for review. }\end{array}$ & $6 / 01 / 96$ & DA Marsh \\
\hline $\begin{array}{l}\text { 2. Assemble key progran (or project) documentation, or identify } \\
\text { its location, for use by the PHMC. Includes development of a } \\
\text { program/project "Smart Book." including listings and } \\
\text { descriptions developed above. }\end{array}$ & 6/01/96 & DA Marsh \\
\hline $\begin{array}{l}\text { 3. Develop an Emergency Preparedness Program Point of Contact } \\
\text { List. }\end{array}$ & $6 / 01 / 96$ & DA Marsh \\
\hline $\begin{array}{l}\text { 4. Develop Emergency Preparedoes Program Review management } \\
\text { presentation (coordinute conted with RL POC) } \\
\text { - Technical Program } \\
\text { - Budget } \\
\text { - Organization } \\
\text { - Status of Milestones and Other Commitments. }\end{array}$ & 6/01/96 & DA Marsh \\
\hline 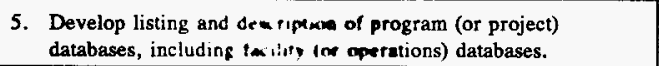 & $6 / 01 / 96$ & DA Marsh \\
\hline 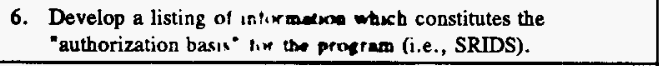 & $6 / 01 / 96$ & DA Marsh \\
\hline $\begin{array}{l}\text { 7. Develop a description of ongonas and planned construction } \\
\text { projects. }\end{array}$ & $6 / 01 / 96$ & DA Marsh \\
\hline $\begin{array}{l}\text { 8. Develop a listing of spectul agreement services provided to RL, } \\
\text { other DOE sites, and other entities. }\end{array}$ & $6 / 01 / 96$ & DA Marsh \\
\hline $\begin{array}{l}\text { 9. Develop a list of current work order services or other form of } \\
\text { agreement to provide work, or obtain work, for other } \\
\text { organizations, on and offsite. }\end{array}$ & 6/01/96 & DA Marsh \\
\hline $\begin{array}{l}\text { 10. Verify the accuracy of property assignments at the } \\
\text { program/project organizational level and correct any } \\
\text { deficiencies. }\end{array}$ & 6/01/96 & DA Marsh \\
\hline $\begin{array}{l}\text { 11. Identify space and equipment within program (or project) area } \\
\text { and facilities for use by the PHMC. }\end{array}$ & $6 / 01 / 96$ & DA Marsh \\
\hline 12. Define preexisting conditions. & 6/01/96 & DA Marsh \\
\hline
\end{tabular}




\begin{tabular}{|c|c|c|}
\hline 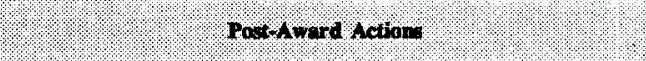 & sonedned & 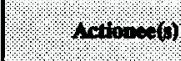 \\
\hline $\begin{array}{l}\text { 1. Provide the PHMC access to the key program (or project) } \\
\text { documentation, any program level generated pre-existing } \\
\text { conditions information, listing of POCs, and listing of } \\
\text { subcontracts. Provide copies upon request. }\end{array}$ & prior to $6 / 15 / 96$ & DA Marsh \\
\hline $\begin{array}{l}\text { 2. PHMC identify epace and equipment needs within } \\
\text { program/project area and facilities. }\end{array}$ & by $6 / 8 / 96$ & DA Marah \\
\hline $\begin{array}{l}\text { 3. Ascertain impact of PHMC contract to current emergency } \\
\text { response organization staffing. }\end{array}$ & $7 / 30 / 96$ & DA Marsh \\
\hline $\begin{array}{l}\text { 4. Provide Emergency Preparedness Program briefings and } \\
\text { emergency response facility tours to PHMC management. }\end{array}$ & $9 / 30 / 96$ & DA Markh \\
\hline $\begin{array}{l}\text { 5. Perform a joint program/project level property and equipment } \\
\text { inventory with PHMC personnel. }\end{array}$ & by $8 / 30 / 96$ & DA Marsh \\
\hline $\begin{array}{l}\text { 6. Identify and train new emergency response organization } \\
\text { members as required. }\end{array}$ & $9 / 30 / 96$ & DA Marsh \\
\hline $\begin{array}{l}\text { 7. In concert with the PHMC, develop a plan to revise emergency } \\
\text { management plans and procedures to reflect PHMC contract. }\end{array}$ & $9 / 30 / 96$ & DA Marsh \\
\hline
\end{tabular}

\subsubsection{Radiological Assistance Program}

The Radiological Assistance Program (RAP) is a direct funded Federal emergency response asset assisting other Federal, tribal, state and local authorities in radiological monitoring and assessment activities associated with radiological incidents or emergencies. Upon request, RAP resources are made available and assistance will be provided until it is no longer needed or there are sufficient resources to handle the situation. RL is the Regional Coordinating Office for DOE Region 8 (Alaska, Oregon, Washington).

\begin{tabular}{|c|c|c|}
\hline Pro-Aeard Actions. & $\begin{array}{l}\text { Scheduled } \\
\text { Date }\end{array}$ & Actionee(s) \\
\hline $\begin{array}{l}\text { 1. Develop list of documedeuce (olens, work authorization, } \\
\text { program review). }\end{array}$ & $6 / 01 / 96$ & DE Webb \\
\hline $\begin{array}{l}\text { 2. Assemble key program (or propect) documentation, or identify its } \\
\text { location, for use by the PHMC. Includes development of a } \\
\text { program/project "Smart Book," including listings and } \\
\text { descriptions developed above. }\end{array}$ & $6 / 01 / 96$ & DE Webb \\
\hline 3. Establish Point of Contact. & $6 / 01 / 96$ & DE Webb \\
\hline $\begin{array}{l}\text { 4. Develop management presentation on RAP } \\
\text { - Program Overview and Direction } \\
\text { - Budget Overview } \\
\text { - Program Status and Planned Activities. }\end{array}$ & $6 / 01 / 96$ & DE Webb \\
\hline $\begin{array}{l}\text { 5. Develop a listing of information which constitutes the } \\
\text { "authorization basis" for the program. }\end{array}$ & $6 / 01 / 96$ & DE Webb \\
\hline
\end{tabular}




\begin{tabular}{|c|c|c|}
\hline Pretirard sctions & $\begin{array}{l}\text { Scheduled } \\
\text { Dote }\end{array}$ & Hathonee (s) \\
\hline $\begin{array}{l}\text { 6. Develop a listing of special agreement services provided to } \mathrm{RL} \text {, } \\
\text { other DOE sites, and other regional entities. }\end{array}$ & 6/01/96 & DE Webb \\
\hline $\begin{array}{l}\text { 7. Develop a list of current work order services or other form of } \\
\text { agreement to provide work, or obtain work, for other } \\
\text { organizations, on and offsite. }\end{array}$ & $6 / 01 / 96$ & DE Webb \\
\hline $\begin{array}{l}\text { 8. Verify the accuracy of property assignments at the } \\
\text { program/project organizational level and correct any deficiencies. }\end{array}$ & 6/01/96 & DE Webb \\
\hline 9. Define preexisting conditions. & 6/01/96 & DE Webb \\
\hline Post-Award Actions & & \\
\hline $\begin{array}{l}\text { 1. Provide the PHMC access to the key program (or project) } \\
\text { documentation, any program level generated pre-existing } \\
\text { conditions information, listing of POCs, and listing of } \\
\text { subcontracts. Provide copies upon request. }\end{array}$ & prior to $6 / 15 / 96$ & DE Webb \\
\hline $\begin{array}{l}\text { 2. Ascertain impact of PHMC contract to the RAP program and } \\
\text { team. }\end{array}$ & $7 / 30 / 96$ & DE Webb \\
\hline 3. Provide RAP briefings to new management. & $9 / 30 / 96$ & DE Webb \\
\hline 4. Tour RAP facilities. & $8 / 30 / 96$ & DE Webb \\
\hline $\begin{array}{l}\text { 5. Perform a joint program/project property and equipment } \\
\text { inventory with PHMC personnel. }\end{array}$ & by $8 / 30 / 96$ & DE Webb \\
\hline $\begin{array}{l}\text { 6. Develop plan to revise affected documentation to include } \\
\text { DOE/RL-92-49, RAP Plan and Procedure Document and other } \\
\text { documents and work plans. }\end{array}$ & 9/30/96 & DE Webb \\
\hline 7. Identify and train new RAP team members. & 9/30/96 & DE Webb \\
\hline
\end{tabular}

\subsubsection{ESH Management Plan}

DOE Order 151.1 Comprehensive Emergency Management System, listed in Section J, Appendix C of the PHMC Request for Proposal has been defined as an ESH order. The PHMC ESH Management Plan must address how the projects will implement the requirements of DOE Order 151.1, as defined in DOE/RL-94-02 Hanford Emergency Response Plan, and support the Site emergency response organization with management and support personnel. WHC Emergency Preparedness performs oversight of the facility emergency management programs and since the transition of these activities is a part of the overall transition, the following pre- and post-award activities are necessary.

\begin{tabular}{|l|c|l|}
\hline \multicolumn{1}{|c|}{ Post-Award Actions } & $\begin{array}{c}\text { Scheduled } \\
\text { Date }\end{array}$ & Actionce(s) \\
\hline $\begin{array}{l}\text { 1. Review project transition plans for emergency preparedness } \\
\text { activities to ensure continuity of emergency response capabilities. }\end{array}$ & $6 / 30 / 96$ & DA Marsh \\
\hline $\begin{array}{l}\text { 2. Review ESH Management Plan for project implementation of } \\
\text { DOE/RL-94-02 Hanford Emergency Response Plan. }\end{array}$ & $9 / 30 / 96$ & DA Marsh \\
\hline
\end{tabular}




\subsection{DIRECT SUPPORT TO RL}

The M\&O Contractor currently provides a range of services (e.g., document control) to $R L$ which are budgeted and costed to RL financial accounts. It is anticipated that this support to RL will continue under the PHMC.

\begin{tabular}{|c|c|c|}
\hline Pre-Award Actions & $\begin{array}{l}\text { Schedoled } \\
\text { Date }\end{array}$ & Actioneds) \\
\hline $\begin{array}{l}\text { 1. Develop a consolidated list and description of services } \\
\text { and support activities provided to RL by the M\&O } \\
\text { Contractor. }\end{array}$ & $6 / 01 / 96$ & $\begin{array}{l}\text { JL Waite } \\
\text { RJ Light }\end{array}$ \\
\hline Post-Award Aetions & & \\
\hline 1. Provide listing of support to RI to the PHMC. & $6 / 15 / 96$ & RJ Light \\
\hline
\end{tabular}


WHC-SP-1181

This page intentionally left blank. 